\title{
Guide illustré \\ des produits renommés \\ des monts et mers du Japon
}

Nippon sankai meisan zue

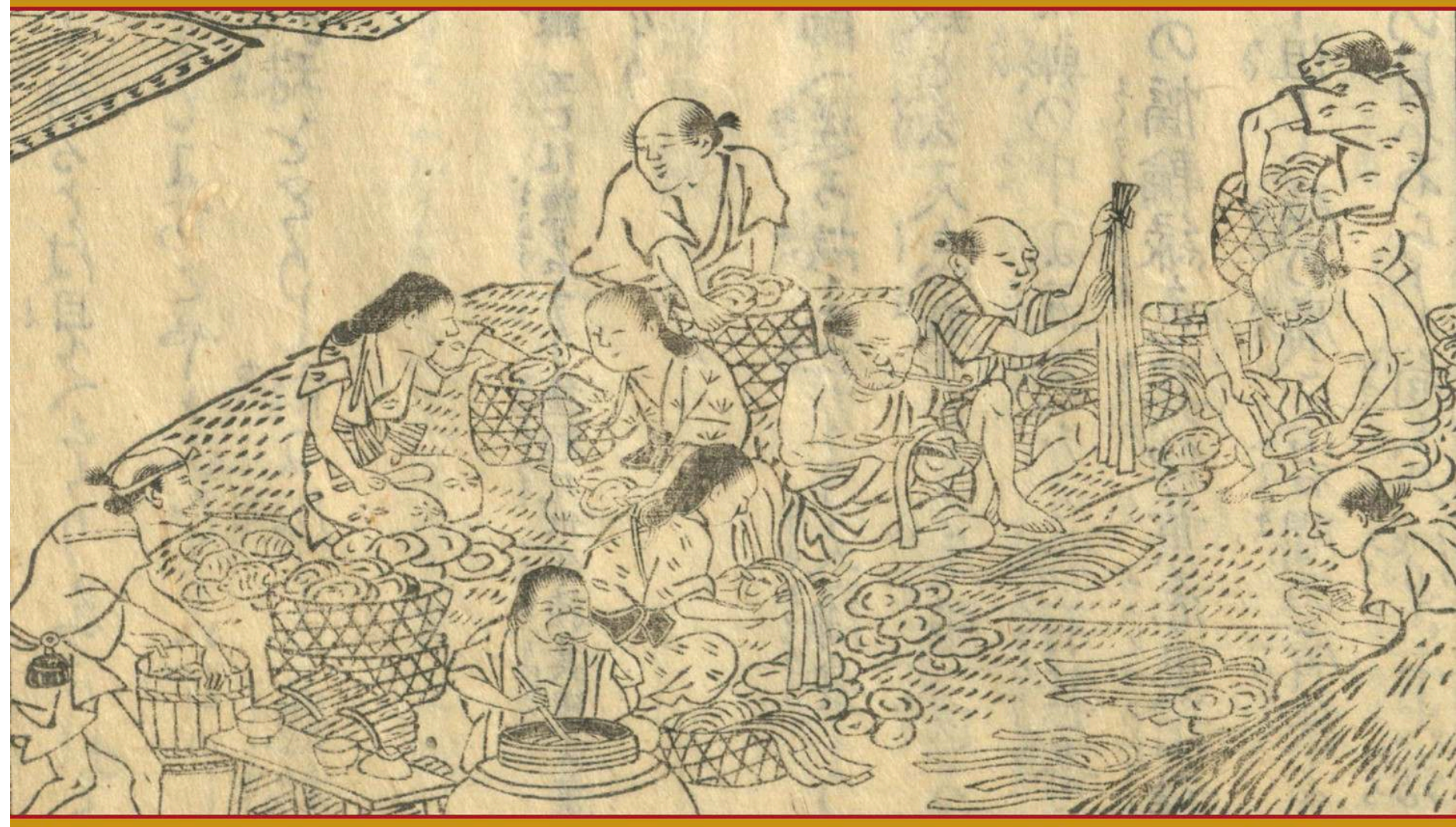

\section{Traduit et annoté par Annick HORIUCHI, Daniel STRUVE, Charlotte von VERSCHUER}

"If

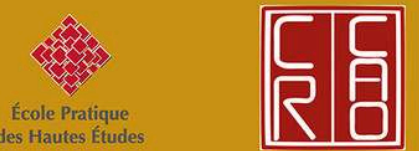

Centre de recherche sur les civilisations de l'Asie orientale 


\section{Guide illustré des produits renommés des monts et mers du Japon}

Famous Products of Japan from Mountains and Seas. An Illustrated Guide

Nippon sankai meisan zue

Annick Horiuchi, Daniel Struve et Charlotte von Verschuer (éd.)

Traducteur : Annick Horiuchi, Daniel Struve et Charlotte von Verschuer

DOI : 10.4000/books.cdf.8306

Éditeur: Collège de France

Lieu d'édition : Paris

Année d'édition : 2020

Date de mise en ligne : 2 juin 2020

Collection : Institut des civilisations

EAN électronique : 9782722605428

\section{OpenEdition}

\section{Books}

https://books.openedition.org

\section{Référence électronique}

HORIUCHI, Annick (dir.) ; STRUVE, Daniel (dir.) ; et VON VERSCHUER, Charlotte (dir.). Guide illustré des produits renommés des monts et mers du Japon. Nouvelle édition [en ligne]. Paris : Collège de France, 2020 (généré le 28 janvier 2022). Disponible sur Internet : <http://books.openedition.org/cdf/8306> ISBN : 9782722605428. DOI : https://doi.org/10.4000/books.cdf.8306. 


\section{RÉSUMÉS}

Le Guide illustré des produits renommés des monts et mers du Japon relève du genre des albums illustrés qui connaît une grande vogue au Japon à l'époque d'Edo (1600-1868). Composé de cinq fascicules, le livre est imprimé en 1799 et publié à Ōsaka. Les produits répertoriés sont aussi bien des produits naturels, issus de la chasse ou de la pêche, que des produits fabriqués ou transformés comme la porcelaine, le saké, la chaux, la bonite séchée, etc. L'une des raisons du succès de cet ouvrage réside dans les illustrations de grand format, réalisées par le peintre Shitomi Kangetsu, qui pourrait également avoir été l'auteur du texte. Elles offrent un tableau particulièrement vivant des scènes de fabrication, de récolte ou de capture de ces produits, qui vient en appui aux descriptions détaillées et techniques du texte.

\section{ANNICK HORIUCHI (TRADUCTION)}

Professeure à l'Université de Paris, membre du CRCAO, ses recherches portent sur l'histoire des savoirs du Japon prémoderne (XVII $-\mathrm{XIX}^{\mathrm{e}}$ siècles). Elle a co-dirigé avec Matthias Hayek, Listen, Copy, Read: Popular Learning in Early Modern Japan (Brill, 2014).

\section{DANIEL STRUVE (TRADUCTION)}

Professeur à l'Université de Paris, membre du CRCAO, ses recherches portent sur l'œuvre d'Ihara Saikaku, la littérature de l'époque d'Edo (XVII ${ }^{\mathrm{e}}-\mathrm{XIX}^{\mathrm{e}}$ siècles) et l'histoire de la prose classique japonaise. Il est l'auteur de Ihara Saikaku, un romancier japonais du XVII siècle (PUF, 2001) et co-auteur avec Jean-Jacques Tschudin du Littérature japonaise (coll. «Que-sais-je ?», 2016).

\section{CHARLOTTE VON VERSCHUER (TRADUCTION)}

Directrice d'études à l'École pratique des hautes études PSL, membre du CRCAO, ses recherches portent sur l'histoire du Japon ancien et médiéval et ses livres traitent des relations diplomatiques et commerciales du Japon avec la Chine et de l'histoire agricole du Japon. 


\section{NOTE DE L'ÉDITEUR}

Cet ouvrage a été traduit et annoté par Annick Horiuchi, Daniel Struve et Charlotte von Verschuer. Il a été édité par Annick Horiuchi.

Cet ouvrage a bénéficié du concours du Centre de recherche sur les civilisations de l'Asie orientale (CRCAO-UMR8155), CNRS, EPHE-PSL, Collège de France, Université de Paris. 


\section{Partie I - Présentation du volume}

Introduction générale

Annick Horiuchi

Le genre des guides illustrés

Le Nippon sankai meibutsu zue : le double du Meisan zue

La connaissance de la nature dans le Japon de l'époque d'Edo

Les auteurs

Le Tiangong kaiwu (Exploitation des œuvres de la nature)

Nota bene

\section{Avertissement}

Traduction

Abréviations

Sommaires de fascicules

Illustrations

Présentation des fascicules

Fascicule 1

Fascicule 2

Fascicule 3

Fascicule 4

Fascicule 5

Partie II - Traduction du Guide illustré des produits renommés des monts et mers du Japon

\section{Préface}

\section{Fascicule 1}

Fabrication du saké

\section{Fascicule 2}

Sommaire du fascicule 2

Variétés de pierres

Variétés de champignons

Miel

Salamandre (poisson-poivre)

Puéraire (kuzu)

Grenouille rouge (Grenouille de montagne)

Insecte de la Vitis ficifolia

Variétés de gibier

\section{Fascicule 3}

Sommaire du fascicule 3

Produits de la pêche 
Fascicule 4

Sommaire du fascicule 4

[Produits de la pêche : suite]

\section{Fascicule 5}

Sommaire du fascicule 5

[Produits de la pêche : suite]

Chaux de pierre

Céramique

Tissage des toiles

Otaries (ottotsujū

Laminaire (konbu)

Produits étrangers

Bateaux hollandais

Postface

Partie III - Annexes

Liste des anciennes provinces

Tableau des unités de mesure

Bibliographies

Liste des œuvres citées dans le Nippon sankai meisan zue

Tableau des noms propres

Glossaire des termes techniques

Tableau des toponymes 
Partie I - Présentation du volume 


\title{
Introduction générale
}

\author{
Annick Horiuchi
}

\section{Le genre des guides illustrés}

Le Nippon sankai meisan zue 日本山海名産図会 (Guide illustré des produits renommés des monts et mers du Japon) ${ }^{1}$ relève du genre des albums illustrés (zue 図会). C'est un genre qui connaît une grande vogue à l'époque d'Edo (1600-1868) et qui a largement contribué à la diffusion des savoirs à cette époque. Composé de cinq fascicules, le livre est imprimé en l'an 11 de l'ère Kansei (1799) et mis en vente par des libraires d'ōsaka ${ }^{2}$. Il connait un succès certain dont témoigne le nombre important de copies conservées ${ }^{3}$. Signe que le libraire se croyait en possession d'un filon profitable, il annonce dès les premiers tirages la publication prochaine d'une « suite» (zokuhen) $)^{4}$.

2 L'ouvrage se propose de faire connaître les "produits renommés » du Japon, une initiative qui porte la marque de la ville où elle voit le jour. Ōsaka joue en effet le rôle de plaque tournante dans l'économie japonaise de l'époque et c'est là que sont acheminés et entreposés nombre de produits régionaux avant d'être cotés puis redéployés dans le pays ou à l'étranger.

Le terme san 産 ou bussan 物産, traduit ici par «produit» mais que l'on aurait pu également traduire par "production ", englobe aussi bien des produits naturels, issus de la chasse ou de la pêche, que des produits fabriqués ou transformés comme la porcelaine, le saké, la chaux, la bonite séchée, etc. Si l'auteur ne précise pas sur quels critères il s'est fondé pour les sélectionner, les références aux diverses qualités du produit donnent à penser que son intérêt se porte sur les produits commercialisés à une grande échelle.

4 Les entrées sont assez librement composées et évoquent différents aspects des produits : les différentes dénominations - surtout s'il s'agit d'espèces naturelles -, les provinces renommées pour la production, le mode d'acheminement vers les régions centrales, les variétés existantes, leur qualité respective, la manière de distinguer les produits authentiques des contrefaçons, les techniques de production, etc. Un des traits remarquables de l'ouvrage réside dans le soin apporté aux illustrations (voir Fig. 1). 
Non seulement leur présence est systématique mais elles procurent de nombreux renseignements sur le site, les acteurs et le processus de production.

Fig.1 : Scène du lavage des grains de riz, Nippon sankai meisan zue, Fascicule 1

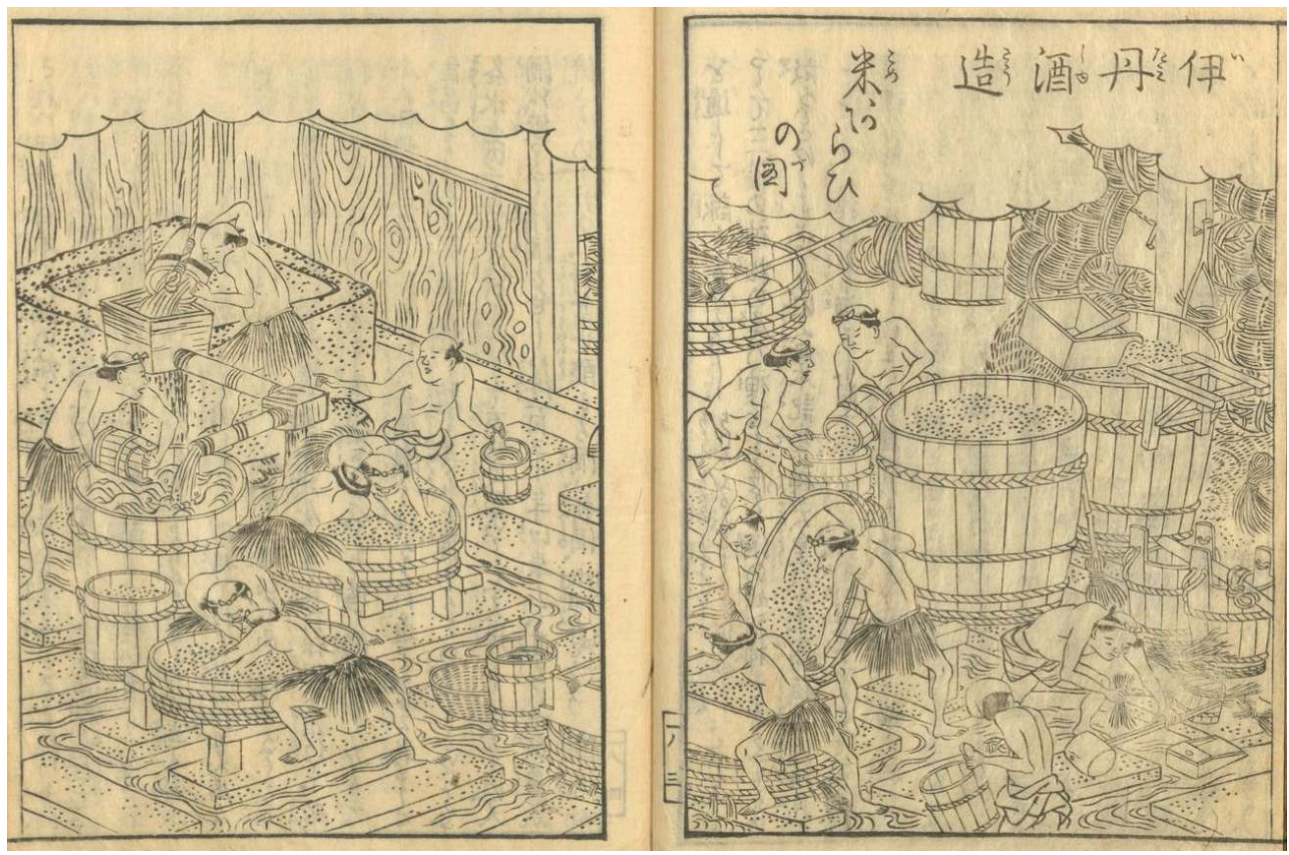

National Institute of Japanese Literature. Lien vers l'illustration

5 Le contenu des notices varie d'un produit à l'autre, mais il répond à un double souci, quelque peu contradictoire : celui de donner accès à la réalité du terrain, en rendant compte du processus de fabrication le plus contemporain, et celui de rattacher le produit à une tradition très ancienne attestée par les Classiques.

6 On note aussi que l'auteur prend toujours soin de sélectionner une région connue pour la production d'une spécialité. En ce sens, ce livre s'inscrit dans la même veine que les Guides illustrés des lieux célèbres (meisho zue), un genre qui rencontre un succès foudroyant à partir de la seconde moitié du XVIII ${ }^{\mathrm{e}}$ siècle ${ }^{5}$. À travers un ouvrage de cette sorte, il s'agit de promouvoir les régions et les provinces éloignées, de les faire découvrir à un public qui, dans sa grande majorité, a assez peu d'occasions de quitter sa terre natale.

\section{Guides illustrés des lieux célèbres}

7 L'auteur du Miyako meisho zue 都名所図会 (Guide illustré des lieux célèbres de la capitale ; 1780), Akisato Ritō 秋里篗島 (actif 1780-1814), joue un rôle non négligeable dans le succès de ces guides «touristiques ». Non seulement son livre est une réussite mais il exploitera avec talent le filon en s'associant avec les peintres les plus en vue. Ainsi, l'Ise sangū meisho zue 伊勢参宮名所図会 (Guide illustré des lieux célèbres du sanctuaire d'Ise), publié par Akisato en 1796 et mis en vente par des libraires de Kyōto et d'Ōsaka, est une collaboration avec Shitomi Kangetsu, peintre en vogue de l'époque, que l'on retrouve comme illustrateur (et auteur? voir infra) de l'album qui nous occupe ici. 
On note que la publication du Meisan zue est concomitante avec la publication de la série des guides illustrés des lieux célèbres de la région d'ōsaka édités par le même Akisato：Settsu meishozue 掑津名所图会 (1796-98), Kawachi meishozue 河内名所図会 (1801), et Izumi meishozue 和泉名所図会 (1796), respectivement dédiés à la province de Settsu, de Kawachi et d'Izumi. La publication de notre livre s'inscrit donc dans une période d'activité intense de la part des libraires d'ōsaka pour faire connaître les lieux renommés de leur région.

Mettre l'accent sur la célébrité est une technique employée par les libraires pour piquer la curiosité du nouveau public des livres. Ce dernier est issu de tous les milieux, d'origines géographiques variées, et a pour caractéristiques d'être avide de s'instruire et de partager la culture des couches aisées. Les dernières décades $d u \mathrm{xVIII}^{\mathrm{e}}$ siècle correspondent en effet au moment où la population japonaise accède massivement à la lecture. Les libraires, très actifs dans les trois grandes villes du Japon - Edo, Kyōto, et Ōsaka - répondent à cette demande en proposant des ouvrages écrits en langue vernaculaire dans lesquels les caractères chinois sont systématiquement assortis de lectures en syllabaire phonétique (furigana) (Fig. 2). De cette manière, des livres au contenu savant, réservés jusqu'alors à une élite, devenaient accessibles « aux femmes et aux enfants ", comme s'en félicite d'ailleurs le préfacier de notre ouvrage. C'est dans ce contexte que s'inscrit la vogue des encyclopédies domestiques et des guides illustrés de tout genre, permettant à chacun d'assouvir sa curiosité et de rêver à un meilleur statut $^{6}$.

Fig. 2 : Nippon sankai meisan zue, Fascicule 1

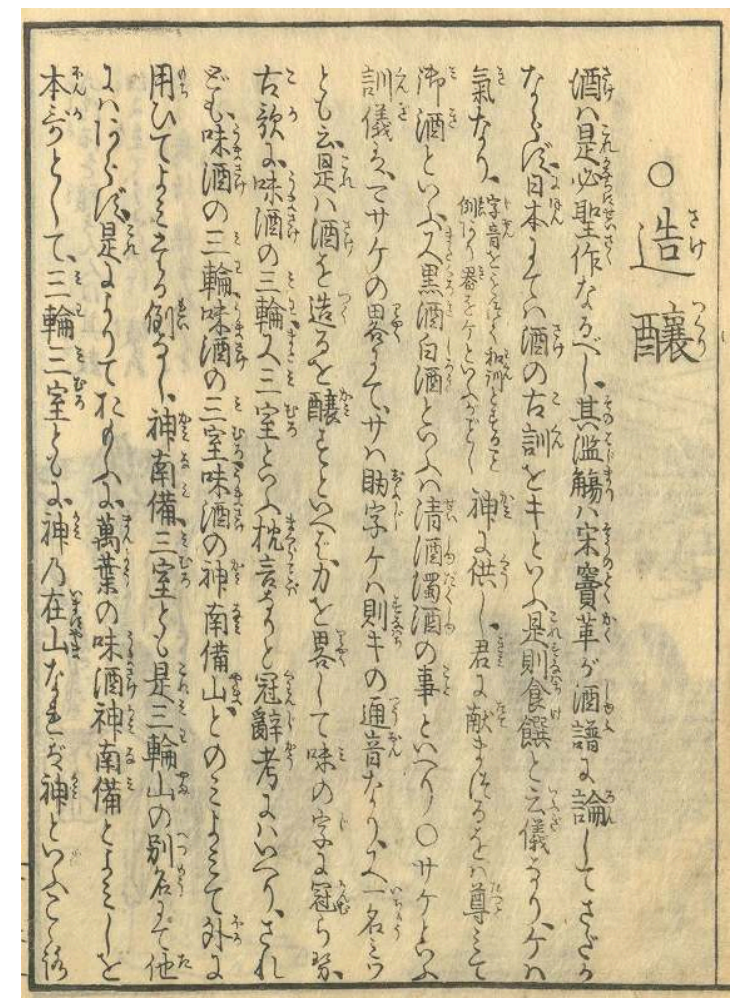

National Institute of Japanese Literature. Lien vers l'illustration 


\section{Le Nippon sankai meibutsu zue : le double du Meisan zue}

10 Mais notre Guide illustré des produits renommés des monts et mers du Japon a un modèle beaucoup plus proche. Il s'agit du Nippon sankai meibutsu zue $e^{7}$ 日本山海名物図会 (Guide illustré des spécialités des monts et mers du Japon), également composé de cinq fascicules, publié en 1754 à Ōsaka par Hirase Tessai 平瀬徹斎 (actif 1748-1780) avec les illustrations de Hasegawa Mitsunobu 長谷川光信 (actif 1724-1754). Il a été réédité en 1797 par le même groupe d'éditeurs-libraires d'Ōsaka que le Meisan zue, sous un format identique avec une couverture gaufrée évoquant les monts et les mers ${ }^{8}$. La postface est rédigée par le fils de l'auteur Hirase Kibō 平瀬鬼望 qui loue l'idée de son père de faire découvrir au public non averti les lieux et conditions de production des marchandises, et notamment des métaux (or, argent, cuivre et fer).

11 Les deux ouvrages, comme l'a noté l'anthropologue Chiba Tokuji qui en propose une édition moderne ${ }^{9}$, ont beaucoup en commun et peuvent être vus comme un diptyque. Tout d'abord, leurs titres ne diffèrent que par un seul caractère. On se réfère dans l'un au meibutsu, dans l'autre, au meisan, deux termes quasiment synonymes et renvoyant aux spécialités des régions, de fabrication humaine ou d'origine naturelle. Le premier terme associé aussi à la notion d'excellence sera traduit par "spécialité » et le second par " produits renommés », mais la différence est à peine perceptible en japonais.

Higuchi Hideo, auteur d'une édition fac-similé des deux ouvrages ${ }^{10}$, fait l'hypothèse que les deux ouvrages s'inscriraient dans le même projet éditorial et que le Meisan zue aurait été conçu comme une suite du Meibutsu zue ${ }^{11}$. Higuchi argumente de manière convaincante que le Meisan zue, tout comme le Meibutsu zue, émanent d'une même famille d'éditeurs d'ouvrages, les Hirase, ayant comme nom de marchand chigusaya 千 種屋. C'est cette maison qui détenait initialement les planches du Meibutsu zue et des éléments portent à croire que le Meisan zue n'est autre que la suite (kōhen), dont la publication était annoncée comme prochaine dans son catalogue daté de 1768 . Mais clairement cette publication a été retardée et les planches sont passées aux mains d'autres libraires. Lorsque le Meisan zue voit le jour en 1799, l'auteur Tessai comme l'illustrateur sont morts et l'on peut imaginer que ce retard est dû à la nécessité de retravailler un manuscrit laissé inachevé. Cela pourrait expliquer l'absence du nom d'auteur et l'accent mis sur l'illustrateur, Shitomi Kangetsu, dont la contribution pourrait être plus importante qu'on le ne croit $^{12}$. Nous reviendrons plus loin sur cet illustrateur.

Dans les deux ouvrages, on retrouve le même souci de mettre en valeur le terroir et de décrire avec la plus grande précision les étapes du processus de fabrication, de récolte ou de capture. Preuve que le lien entre les deux ouvrages n'avait pas échappé aux éditeurs tardifs de ces deux ouvrages, on trouve à la fin de l'édition tardive du Meibutsu zue un encart publicitaire vantant la complémentarité du Meisan zue et du Meibutsu zue (Fig. 3). Les deux ouvrages sont-ils pour autant identiques? 
Fig. 3 : Colophon du Nippon sankai meibutsu zue, édition de Kansei 9 (1797) avec la publicité du Meisan zue et les noms des libraires d'Ōsaka

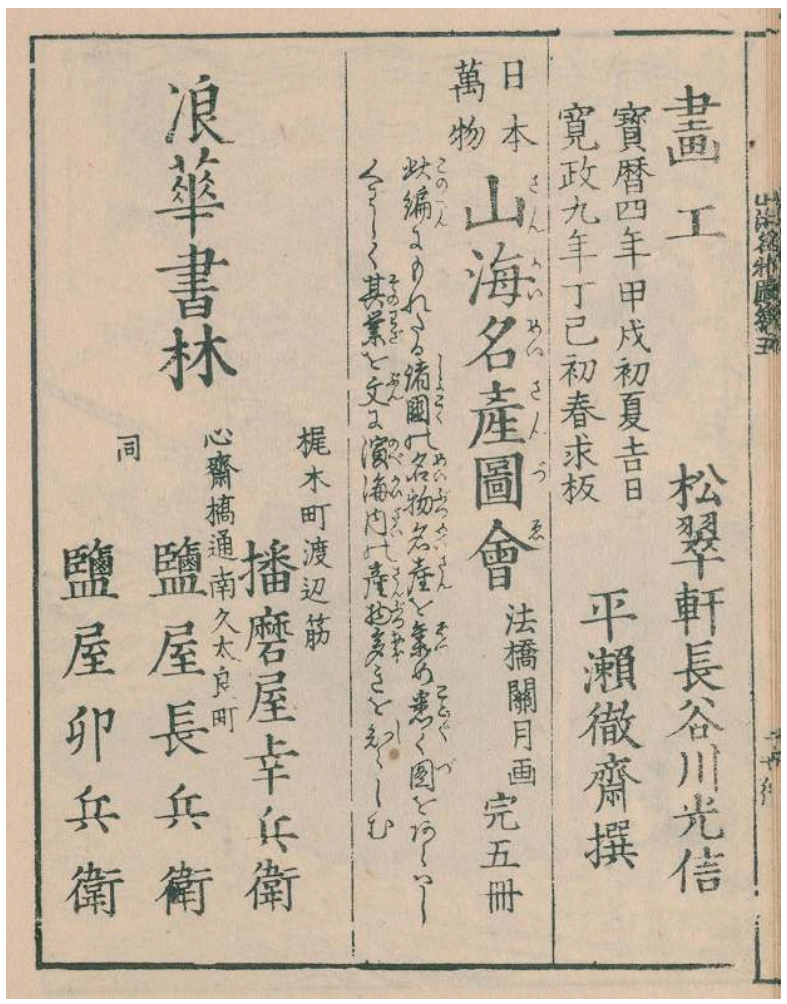

National Diet Library, Japan. Lien vers l'illustration

Regardons d'abord la place réservée aux illustrations dans chacun d'eux. Dans les deux cas, elles occupent une place essentielle. Dans la postface du Meibutsu zue, il est dit qu'elles ont été réalisées en situation, «en s'enquérant auprès des personnes qui, dans les provinces, tiraient leurs ressources de l'exploitation des rivières, des mers et des montagnes ", et en les rétribuant en conséquence (atai o hodokoshite). ${ }^{13}$ L'idée d'offrir au lecteur des vues fidèles de la scène de production est également adoptée par le Meisan $z u e$ où les illustrations occupent deux pleines pages et sont plus détaillées encore.

Le rapport texte/image a toutefois évolué entre les deux ouvrages. Dans le Meibutsu zue, les entrées se présentent selon un format unique de deux pages en regard: le texte n'occupe qu'une fraction de la page de droite, l'illustration remplissant le reste de la page de droite et la totalité de la page de gauche (Fig. 4). Le texte accompagne donc l'image qui détient le premier rôle, mais son contenu n'en est pas moins fourni. Il se réfère aussi bien aux grandes étapes de fabrication, à l'origine historique, aux variétés régionales, aux qualités du produit qu'aux classiques et poèmes évoquant le sujet, etc. 
Fig. 4 : Grandes bardanes (gobō) d'lyo, Nippon sankai meibutsu zue (1797) de Hirase Tessai, fascicule 4

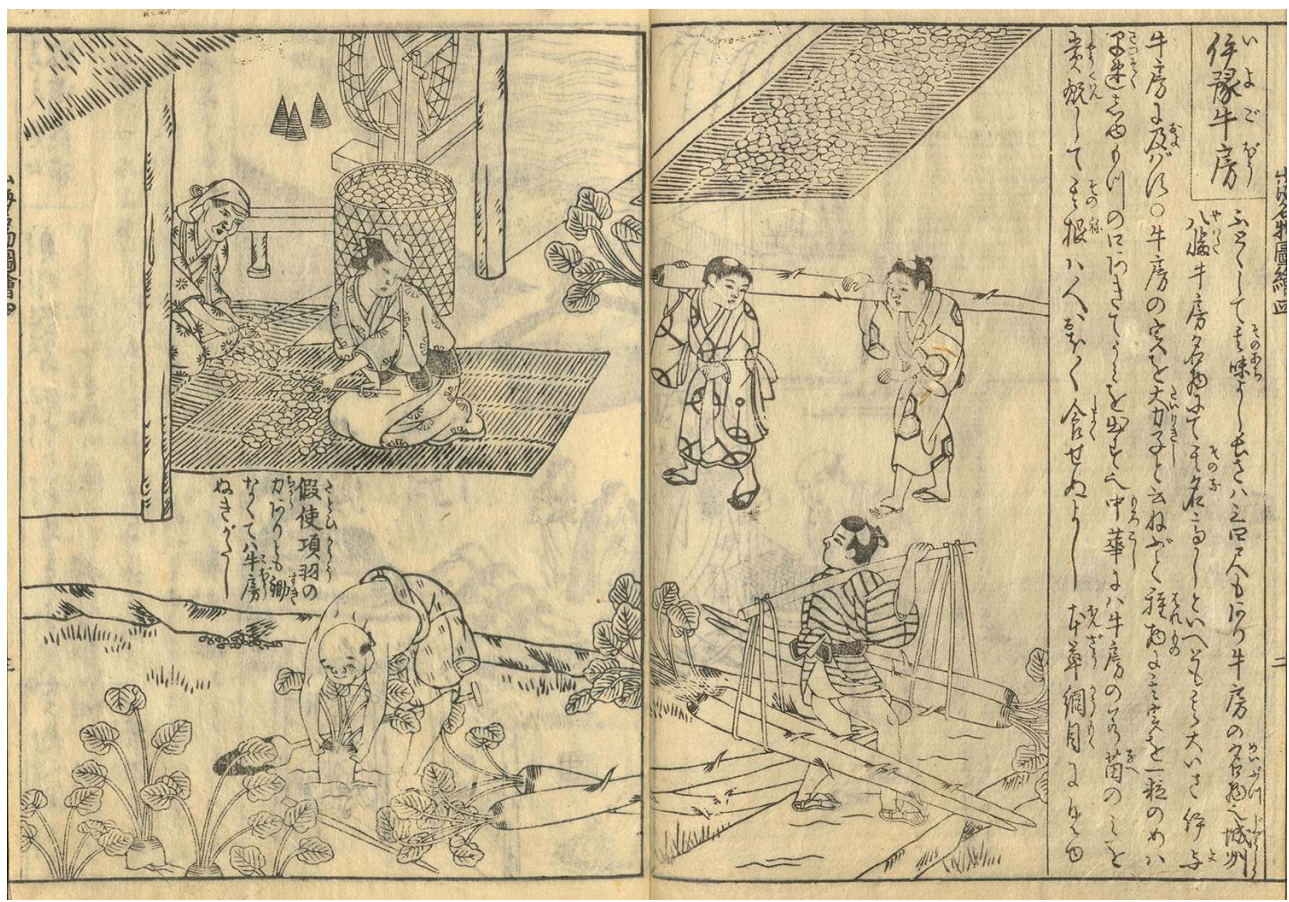

National Institute of Japanese Literature. Lien vers l'illustration

Le Meisan zue opte, pour sa part, pour un format moins rigide. Les planches sont moins nombreuses ${ }^{14}$, mais elles occupent à chaque fois deux pleines pages. Le texte est plus développé et donne à l'ouvrage un caractère plus érudit que le Meibutsu zue. Par son contenu et par les préoccupations qu'il révèle, le livre se rapproche du genre des ouvrages savants (mono no hon) s'adressant aux lettrés. Les références aux ouvrages anciens et chinois y sont plus nombreuses, comme les considérations sur les noms des espèces. L'auteur s'interroge davantage sur le crédit que l'on peut faire à telle ou telle légende et manifeste en ce faisant son souci d'étayer ses conclusions par des arguments solides, à la manière des lettrés chinois de son temps (kaozheng, j. kōshō) ${ }^{15}$.

17 Les deux livres traitent des mêmes genres de produits: spécialités culinaires, spécialités artisanales, produits d'origine animale, végétale ou minérale. Mais tout se passe comme si les sujets avaient été soigneusement répartis entre les deux pour éviter la redondance. Dans le Meibutsu zue, un fascicule entier (fascicule 1), abondamment illustré, est dédié au processus d'extraction et d'affinage des métaux (or, argent, cuivre, plomb). Le sujet n'est en revanche pas abordé dans le Meisan zue qui s'intéresse davantage à l'extraction et à l'utilisation des pierres et aux champignons (fascicule 2). De même, le Meibutsu zue réserve plusieurs entrées à la fabrication du thé, alors que le Meisan zue dédie un fascicule entier (fascicule 1) à celle du saké d'Itami. Enfin, parmi les curiosités, on note que le Meibutsu zue ouvre une large place à la chasse aux baleines de la région de Kii alors que le Meisan zue s'intéresse, pour sa part, à la capture des otaries par les Ainu d'Ezo.

Chiba note, dans son étude, que d'une manière générale, 70 \% des entrées du Meibutsu zue concernent des productions des régions centrales du Japon (Kinki), et que les produits de la pêche et les minéraux constituent des catégories bien représentées ( $20 \%$ des entrées pour chacune). Le Meisan zue, couvre un territoire plus large allant de 
Nagasaki à Ezo (actuel Hokkaidō) avec une préférence pour des produits naturels rares et une faible représentation des produits agricoles ou forestiers ${ }^{16}$.

Ainsi, le sentiment d'avoir affaire à une sélection lacunaire ou arbitraire que l'on ressent au premier contact disparait si l'on admet que notre ouvrage est un complément au Meibutsu zue. Le souci d'exhaustivité est donc bien présent derrière l'initiative du Meisan zue.

\section{La connaissance de la nature dans le Japon de l'époque d'Edo}

20 L'un des traits les plus frappants du Meisan zue est le vif intérêt porté aux produits « naturels », étant entendu que la distinction entre nature et artefact n'est aucunement perceptible dans le vocabulaire utilisé par nos auteurs qui l'englobent sous la même dénomination de sanbutsu (produit). Hormis le fascicule premier, entièrement consacré à la fabrication du saké, le contenu du Meisan zue présente beaucoup de similarités avec les traités d'histoire naturelle. Regroupées en grandes catégories (pierres, champignons, produits de la chasse pour le fascicule 2, produits de la pêche pour les fascicules 3 et 4), les entrées se succèdent, réunissant des informations les plus diverses sur l'espèce naturelle considérée. Elles reflètent les préoccupations et les centres d'intérêt des spécialistes du honzō 本草 (bencao en chinois), un domaine d'étude dont l'origine remonte au début de notre ère et dont le rôle a été de procurer des informations pratiques sur la materia medica, et par extension, sur toutes sortes de substances d'origine végétale, animale ou minérale.

21 Comme la plupart des savoirs japonais au début du XVII siècle, la science japonaise $d u$ honzō prend son envol grâce à des ouvrages chinois, et plus particulièrement grâce au Bencao gangmu 本草綱目 (Classement raisonné de la materia medica) de Li Shizhen 李時 珍 (1518-1593) ${ }^{17}$. La publication de cet ouvrage avait créé la surprise en Chine en raison du grand nombre de substances répertoriées (1892 en tout) et de l'immense érudition de son auteur. Le Bencao gangmu reposait en effet sur une parfaite maîtrise des ouvrages anciens et sur des données d'observation recueillies sur le terrain. Sous chaque entrée, on trouvait les différents noms de la substance (chacune d'entre elles se devait d'être identifiée par son nom " correct»), des indications morphologiques, les lieux où elle est recueillie, les usages connus, ses qualités, les erreurs des prédécesseurs, etc. Imprimé en 1596, il avait suffi de quelques années pour que le Bencao gangmu arrive au Japon où il est étudié avec ferveur par les lettrés confucéens comme par les médecins. Son influence est encore très nettement perceptible dans le Meisan zue qui le cite abondamment. Le livre de Li Shizhen n'en est pas moins l'objet de lectures critiques, soucieuses d'adapter cet immense savoir à l'environnement japonais.

À cet égard, le Yamato honzōo 大和本草 (La materia medica du Japon), publié en 1709 par Kaibara Ekiken 貝原益軒 (1630-1714), est révélateur de l'approche des savants japonais. Écrit en langue vernaculaire, le traité comporte 1362 entrées, limitées aux seules substances connues au Japon. Des espèces absentes du Bencao gangmu sont introduites et le livre est enrichi de témoignages et de descriptions que Kaibara a recueillis au cours de ses voyages. Le nombre de poissons a été sensiblement étoffé par rapport au modèle chinois et les notices sont agrémentées de conseils culinaires. De cette manière, 
Kaibara contribue grandement à populariser la connaissance des substances naturelles auprès de ses contemporains.

Au sein de la tradition du honzō, il existe des ouvrages spécialisés dans les substances comestibles. Là encore, des œuvres chinoises en sont à l'origine et certaines d'entre elles semblent avoir été connues de longue date par les médecins japonais. Dans ce domaine, la publication du Honchō shokkan 本朝食鑑 (Miroir des aliments de notre empire) de Hitomi Hitsudai 人見必大 (vers 1642-1701) constitue un événement majeur. Achevé en 1692 et imprimé en 1697, le livre réunit sur chacune des substances répertoriées dans le Bencao gangmu une somme de connaissances portant aussi bien sur les qualités culinaires ou pharmacologiques, que sur les techniques de préparation. Bien que contemporain de Kaibara Ekiken, Hitomi est plus proche du modèle du Bencao gangmu dont il suit scrupuleusement la structure. Mais le contenu est déjà fortement adapté au lectorat japonais, ce qui explique qu'il constitue l'une des sources principales du Meisan zue quand il s'agit de produits comestibles.

Tout au long du xvIII ${ }^{e}$ siècle, le honzō s'enrichit de nouvelles pratiques. Forts de l'intérêt manifesté par les autorités shogunales, certains savants se consacrent exclusivement à la discipline. Tokugawa Yoshimune 徳川吉宗 (1684-1751), qui prend les commandes du pays en 1716, orchestre avec l'aide de son personnel, un vaste inventaire des espèces disponibles dans le pays, achevant le travail de compilation entamé par Inō Jakusui 稲 生若水 $(1655-1715)^{18}$. Dans le même temps, avec la construction du jardin botanique de Koishikawa, les savants sont incités à acclimater les espèces végétales afin de réduire la dépendance du pays vis-à-vis du commerce extérieur ${ }^{19}$.

La seconde moitié du XvIII ${ }^{e}$ siècle voit également le succès des expositions de substances médicinales (yakuhinkai 薬品会). Il est intéressant de noter que les premières expositions sont organisées à Ōsaka par Tsushima Tsunenoshin 津島恒之進 (1701-1755), le maître de honzō de Kimura Kenkadō 木村䓦臀堂 (1736-1802), le préfacier de notre ouvrage. Tamura Ransui 田村藍水 (1718-1776), et son assistant, Hiraga Gennai 平賀源内 (1728-1780), en organisent cinq entre 1757 et $1762^{20}$ à Edo. Toutes ces expositions sont l'occasion de mondanités et d'échanges entre savants venus parfois de loin. Les objets exposés sont extrêmement variés. Il s'agit de piquer la curiosité du public par des objets exotiques, étranges ou rares.

La préface signée par Kimura Kenkadō inscrit clairement cet ouvrage dans la tradition du honzō. Les savants de Kyōto dont il mentionne le nom: Mukai Genshō 向井元升 (1609-1677), Inō Jakusui, Matsuoka Joan 松岡恕庵 (1668-1746), Tsushima Tsunenoshin, constituent une lignée intellectuelle à laquelle il se rattache par le biais de son dernier représentant, Tsushima Tsunenoshin, son maître en honzō. Alors que sa carrière est au plus haut, Kimura découvre un nouveau maître en la personne d'Ono Ranzan 小野蘭山 (1729-1810), un spécialiste de Kyōto, qui sera sollicité en 1799 par le shogun pour enseigner dans l'école de médecine de la capitale shogunale ${ }^{21}$. Le Honzō kōmoku keimō 本 草綱目啓蒙 (Introduction au Bencao gangmu) qu'Ono publie en 1803 est reconnu comme le plus important traité de honzō produit au Japon.

On voit ainsi que le honzō est un champ de recherche en pleine effervescence au seuil du $\mathrm{XIX}^{\mathrm{e}}$ siècle. L'étude ne se limite plus à la materia medica, mais à tous les produits (bussan) de consommation. Le shogun comme les seigneurs sont conscients que le salut du régime passe par une meilleure exploitation des ressources naturelles. Ainsi, l'ouvrage que nous nous proposons de traduire peut être considéré comme relevant du champ de honzō élargi. 


\section{La production en milieu rural au seuil du XIX siècle}

à nous interroger sur le contexte économique da ouvrages. Le Meibutsu zue et le Meisan zue sont en effet révélateurs d'une profonde mutation de l'économie japonaise que nous ne pouvons ici qu'esquisser. Tout d'abord, il faut noter que les productions évoquées ici prennent place en milieu rural, comme le sous-entend d'ailleurs la référence aux monts et aux mers. C'est peut-être dans cette limitation que réside la nouveauté de ces ouvrages. En effet, au début du XvII ${ }^{\mathrm{e}}$ siècle, le monde rural évoque avant tout le travail de la terre et la culture des céréales. Les premiers traités agricoles (nōsho 農書) publiés au Japon sous les Tokugawa consacrent l'essentiel de leurs pages à la manière de labourer, semer, récolter, décortiquer les grains, etc. Mais déjà, à l'époque de la publication du Nōgyō zensho 農業全書 (Traité complet d'agriculture ; 1697) ${ }^{22}$, à la fin du XVII siècle, on peut déceler l'intérêt porté aux cultures qui, comme le coton, sont destinées à être transformées puis commercialisées. Dans ce registre, les régions autour d'ōsaka concentraient les techniques les plus avancées, et c'était l'un des objectifs du Nōgyō zensho que de faire connaître largement dans le pays ces pratiques ayant fait leurs preuves ${ }^{23}$. Ainsi, il existait à la fin du XVII ${ }^{\mathrm{e}}$ siècle, un écart sensible entre le potentiel économique des régions centrales et celui des régions périphériques. Ce que le Meisan zue nous apprend, c'est que non seulement cet écart s'est réduit en un siècle, mais que grâce à la diffusion des techniques, de nouveaux centres de production régionaux ont émergé et qu'ils sont en mesure de concurrencer les anciens sites de production.

Ainsi, ce que ces deux ouvrages nous révèlent, c'est le spectaculaire développement régional de ces cultures industrielles au cours $\mathrm{du}_{\mathrm{XvIII}} \mathrm{e}^{\mathrm{e}}$ siècle, développement auquel les marchands des régions centrales (Kinai), et plus particulièrement d'Ōsaka ont largement contribué. On peut imaginer, même si le texte n'en dit rien, que ces derniers sont présents sur certains de ces sites de production, en tant qu'investisseurs, ou en tant que transporteurs des marchandises vers Ōsaka. En somme, en chantant les louanges des productions régionales, les auteurs de ces ouvrages ne feraient pas autre chose que de célébrer leur propre succès commercial.

On est également frappé, en lisant ces deux livres, de l'importance accordée à la qualité des produits. Ce fait est sans doute à rapprocher de l'évolution du marché et de l'augmentation de marchands indépendants, originaires des zones rurales, et non soumis aux règlements imposés par les corporations des métiers ${ }^{24}$. Ces marchands sont soucieux de fournir les clients privés, les restaurants, en mets raffinés. En ce sens, le Meisan zue est aussi et même avant tout un guide pour gourmets qui se propose d'informer le consommateur sur la manière de distinguer les produits authentiques des faux, sur les saisons où ces produits sont disponibles, etc. C'est à la fin du XVIII ${ }^{e}$ siècle, que les restaurants commencent à proliférer, et qu'un commerce parallèle se développe, au point que les Tokugawa furent obligés de publier des décrets pour mieux contrôler le marché alimentaire et sécuriser ses propres ressources ${ }^{25}$.

\section{Les auteurs}

31 On a vu que parmi les différences existant entre les deux ouvrages, il y avait le niveau d'érudition. Le Meisan zue propose non seulement de présenter le processus de 
production, mais il accorde aussi une large place à des considérations sur les différentes variétés, les origines de la production, les références aux classiques, qui donnent à penser que l'auteur cherche à aborder avec méthode et rigueur l'histoire du produit considéré. Ce penchant marqué pour l'érudition nous amène à nous interroger sur l'identité de son auteur, dont le nom ne figure nulle part dans l'ouvrage. Le colophon, en effet, n'indique que le nom de l'illustrateur: Shitomi Kangetsu 蔀関月 $(1747-1797)^{26}$.

Les publications anonymes ne sont pas rares à cette époque, lorsqu'il s'agit d'encyclopédies ou de guides qui se contentent de reproduire ou d'augmenter par petites touches des titres tombés dans le domaine public. Mais notre ouvrage ne correspond pas à ce cas de figure. L'anonymat suscite des interrogations qui ne sont que partiellement levées par l'explication, déjà évoquée, selon laquelle le Nippon sankai meisan zue serait la suite du Meibutsu zue, composée par le même auteur. Notre ouvrage comporte cependant une préface et une postface qui offrent des pistes de réflexion.

La préface rédigée entièrement en chinois classique, sans signe diacritique ${ }^{27}$, est signée par une sommité de l'époque : Kimura Kōkyō 木村孔恭, plus connu aujourd'hui sous le nom de Kimura Kenkadō 木村萧葭堂 (1736-1802). La postface quant à elle n'est pas signée et est écrite dans un japonais archaïsant inspiré de la littérature classique, ce qui traduit aussi une forme d'érudition peu commune. Dans une écriture riche en métaphores, la postface évoque le contexte de production du Meisan zue. Elle souligne le rôle du peintre Shitomi Kangetsu à la mort de Hirase Tessai ainsi que celui du peintre Nakai Rankō 中井藍江, disciple de Kangetsu, qui aurait repris le flambeau à la mort de son maître en 1797. L'auteur de la postface aurait apporté les dernières corrections à l'ouvrage. Si on attribue la postface à Kimura, on peut conclure que l'ouvrage est une œuvre collective, écrite à plusieurs mains, à laquelle auraient contribué Hirase Tessai, Shitomi Kangetsu, Nakai Rankō et Kimura Kenkadō. Ce qui expliquerait son caractère quelque peu disparate. Si la postface n'est pas de Kenkadō, il faut imaginer un auteuréditeur supplémentaire, qui serait intervenu sur le texte.

Examinons d'un peu plus près ces personnages. On a déjà évoqué la figure de Hirase Tessai sur laquelle on sait peu de choses, en dehors du fait qu'il était à la fois auteur et éditeur d'ouvrages à Ōsaka, connu sous le nom de Chigusaya Shin'emon. Shitomi Kangetsu commence sa carrière comme imprimeur à Ōsaka et comme peintre d'estampes à l'école de Tsukioka Settei 月岡雪鼎 (1726-87). Suivant les tendances de son temps, il se forme aussi à la peinture de lettrés dans le style chinois, ce qui l'amène à rencontrer le cercle des amateurs de lettres et de peintures chinoises d'Ōsaka, dont Kimura Kenkadō est un éminent représentant. Shitomi contribue par ses illustrations au succès des deux guides illustrés de lieux célèbres consacrés au sanctuaire d'Ise et à la région d'Ōmi ${ }^{28}$, tous deux publiés par des libraires d'Ōsaka. Pour ces deux ouvrages, Shitomi s'est associé avec Akisato Ritō, un éditeur prolifique de l'époque, déjà évoquéer Dans les années 1780, il se voit attribuer le titre de Hokkyō 法橋, une distinction qui rehausse encore son prestige déjà bien établi de peintre de personnages et de paysages.

Les illustrations de Shitomi dans notre ouvrage se distinguent par leur grand format (une double page). Elles offrent une vue à vol d'oiseau du site de production, illustrant les étapes du processus, les différents acteurs (hommes et femmes), leurs gestes, les moyens utilisés (dispositifs de capture des animaux, ustensiles, outils, etc.), ainsi que le cadre intérieur ou extérieur dans lequel la production prend place. 

travail n'est pas une nouveauté. On en trouve de nombreux exemples dans des répertoires illustrés publiés à l'époque Genroku, un siècle auparavant. Un ouvrage comme le Jinrin kinmōzui 人倫訓蒙龱彙 (Dictionnaire illustré de l'humanité ; 1690) se présentait comme un catalogue de statuts sociaux et de métiers, dans lequel les personnages étaient représentés dans leur cadre de vie avec, auprès d'eux, les outils et ustensiles dont ils se servaient au quotidien. Certains ouvrages étaient centrés sur les artisans. C'est le cas du Wakoku shoshoku e zukushi 和国諸職絵尽 (Catalogue illustré des métiers du Japon; 1685) dont les illustrations, réalisées par le peintre d'estampes Hishikawa Moronobu 菱川師宣 (?-1694), se distinguaient déjà par la qualité et la taille des images occupant les deux tiers de la page (Fig.5). Le même Moronobu est également l'illustrateur du Wakoku hyakujo 和国百女 (Cent femmes du Japon; 1695) représentant divers profils de femmes au travail. On notera que déjà, les auteurs de cette époque étaient soucieux de recentrer le propos sur les hommes et les femmes de l'archipel, par opposition à la Chine. La présence du mot Wakoku (le Japon) dans le titre témoignait du souci de transposer la culture, jusqu'alors très marquée par l'héritage chinois.

Fig. 5 : « Enduiseur » et « couvreur » dans Wakoku shoshoku e zukushi (1685)

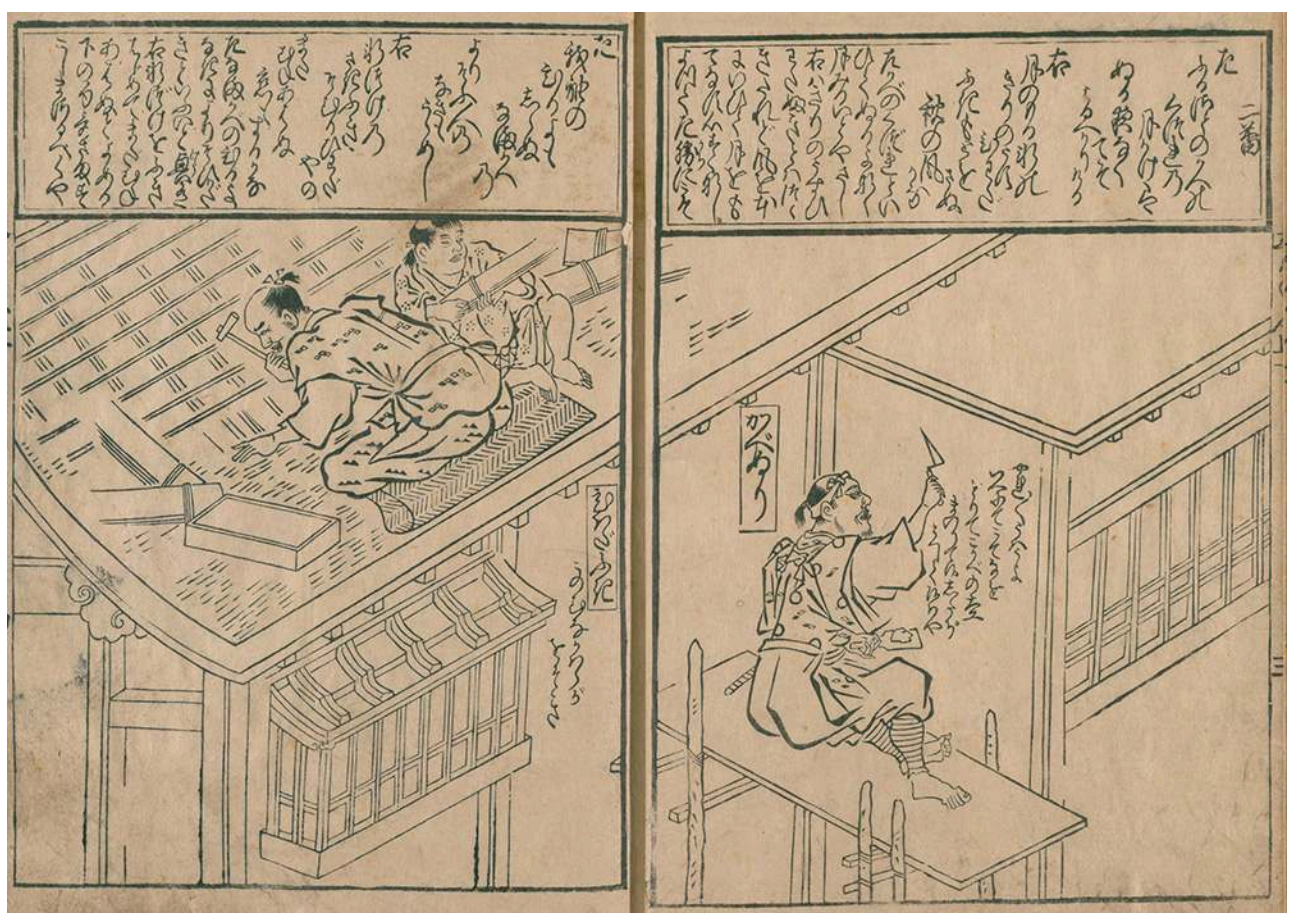

National Diet Library, Japan. Lien vers l'illustration

Les illustrations de Shitomi dans le Meisan zue s'inscrivent donc dans une tradition très riche. S'il ne peut être considéré comme novateur par ses thèmes, on peut dire que le secret de son succès réside dans sa capacité à réunir en une seule œuvre tous les ingrédients des ouvrages précédemment cités. Les vues à vol d'oiseau de grand format rappellent plutôt le style des illustrations des lieux renommés. Les représentations des hommes et des femmes en train d'accomplir leurs tâches s'inscrivent dans la droite ligne des catalogues illustrés des métiers. À la différence des ouvrages précédents qui mettaient en relief la figure de l'artisan (shokunin), il s'agit désormais de braquer le 
projecteur sur l'espace de production lui-même, avec ses installations, son matériel, ainsi que ses ouvriers, qui apparaissent désormais comme des pièces d'un rouage bien rodé. Les illustrations montrent clairement que certains ateliers de production au seuil $\mathrm{du} \mathrm{XIX}^{\mathrm{e}}$ siècle fonctionnent comme de véritables manufactures où le personnel travaille à plein temps.

\section{Kimura Kenkadō (1736-1802)}

Fig. 6 : Portrait de Kimura Kenkadō par Tani Bunchō, 1802

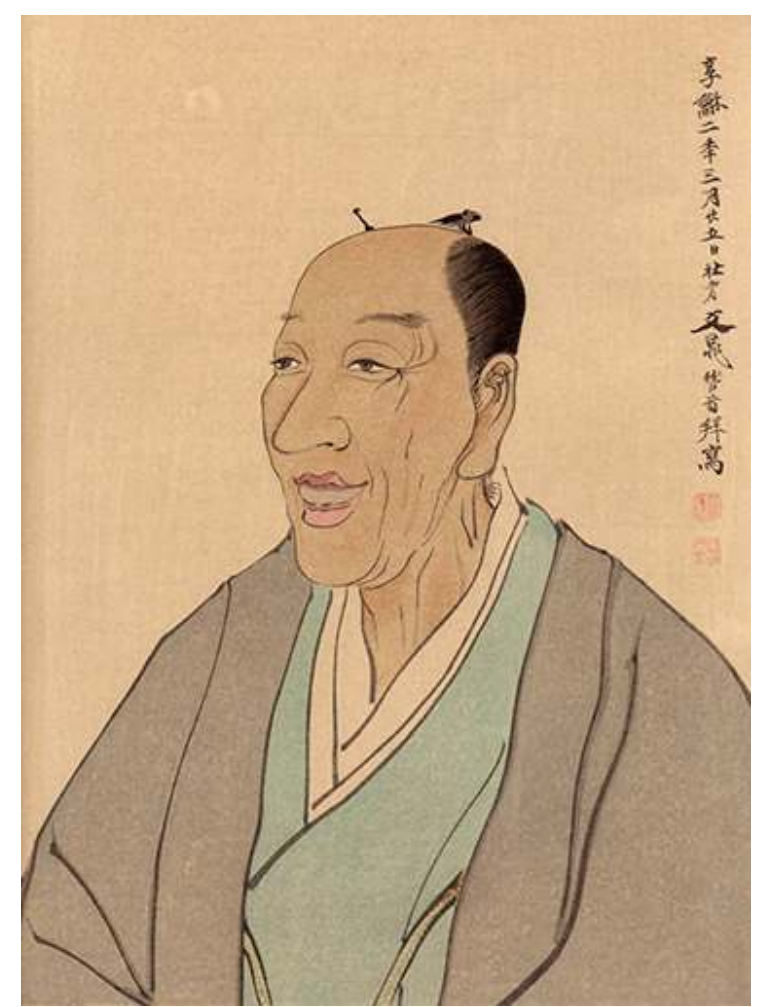

Tani Bunchō (1763-1841) 谷文昆筆 [Domaine public]. Lien vers l'illustration

Tournons-nous à présent vers Kimura Kenkadō. Ce marchand, producteur de saké d'Ōsaka, est aussi un grand lettré, expert dans les arts et lettres de la Chine (bunjin 文 人 $)^{30}$ : il pratique lui-même avec talent la poésie, la peinture et la gravure de sceaux. Cet esthète est aussi un passionné des produits de la nature (bussan 物産) et de la materia medica (honzō 本草), deux domaines auxquels il a été initié depuis son jeune âge par des savants réputés de Kyōto. Mais il n'y a pas que son érudition qui fait l'admiration de ses contemporains. Kenkadō est aussi un collectionneur. Son cabinet de curiosités, qui renferme de nombreux livres et cartes, est une véritable attraction pour les savants de cette époque. Plus que par le nombre des pièces, il semble que ce soit leur rareté qui suscitait l'étonnement de ses contemporains ${ }^{31}$. Il est difficile d'évaluer aujourd'hui l'ampleur de cette collection car elle fut éparpillée aussitôt après sa mort. Une part significative de sa collection de livres rares chinois a toutefois été acquise en 1803 par l'école shogunale du Shōheik $\overline{0}^{32}$. De son cabinet de curiosités, il ne nous reste guère que des spécimens de fossiles, de roches et de coquillages, soigneusement rangés dans des boîtes en laque. Ces dernières témoignent du soin dont Kimura entourait ses pièces et 
de l'attention qu'il portait à leur désignation ${ }^{33}$. On aurait tort cependant de le considérer comme un original. Cette personnalité d'esthète, qu'il a cultivée à l'extrême, et cette curiosité se retrouvent en effet chez nombre de lettrés de son époque. C'est une curiosité tournée aussi bien vers le monde présent que vers le passé le plus ancien, vers le Japon autant que vers les pays étrangers, comme en témoignent les mappemondes et les récits de voyage figurant dans sa collection.

Kimura a tissé au fil des années un réseau exceptionnel de relations et d'amis qui s'étendait bien au-delà des villes d'Ōsaka et de Kyōto. Les savants d'Edo en voyage vers l'Ouest ne manquaient pas de faire le crochet par sa résidence d'Ōsaka, où ils étaient certains d'être bien accueillis. Son journal, dont les volumes correspondant aux années 1779-1802 sont conservés, porte témoignage des échanges et mondanités qui constituaient l'essentiel de son quotidien ${ }^{34}$. Parmi ses amis les plus proches, on relève les noms des lettrés et poètes Ueda Akinari 上田秋成 (1734-1809) et Ōta Nanpo 大田南 㽞 (1749-1823), le lettré confucéen et futur maître du Shōheikō, Satō Issai 佐藤一斎 (1772-1859), le lithologue Kino'uchi Sekitei 木内石亭 (1724-1808), les peintres Tani Bunchō 谷文晁 (1763-1840), auteur du célèbre portrait de Kenkadō (voir Fig. 6), et Shiba Kōkan 司馬江漢 (1747-1818), le spécialiste de materia medica Ono Ranzan 小野蘭 山 (1729-1810), le géographe et explorateur Mogami Tokunai 最上徳内 (1755-1836), le spécialiste des études hollandaises Ōtsuki Gentaku 大槻立沢 (1757-1827) ou encore le daimyō de Hirado, Matsura Seizan 松浦静山 (1760-1841), un fin lettré, collectionneur comme lui de livres de toutes origines, autrement dit les meilleurs spécialistes dans leur domaine.

Kimura a laissé peu d'ouvrages imprimés. Le plus connu est sans doute son Ikkaku sankō 一角纂考 (Somme de recherches sur le monocéros; 1795), composé d'extraits d'ouvrages chinois et hollandais se rapportant à ce produit longtemps présenté comme la corne d'un quadrupède mais qui s'avéra être la défense du narval. L'ouvrage est issu d'une collaboration avec Ōtsuki Gentaku, spécialiste des études hollandaises. Kimura laisse aussi beaucoup de manuscrits inachevés qui reflètent son intérêt prononcé pour l'histoire naturelle et pour les espèces rares entourées de mystères. L'un de ses manuscrits intitulé Kenkadō kinfu 萧臀堂菌譜 (Traité des champignons de Kenkadō; date inconnue) porte sur les variétés de champignons, un sujet auquel le deuxième fascicule du Meisan zue fait une large place ${ }^{35}$.

41 La vie de Kenkadō connaît un revers de fortune brutal en 1789 lorsque le gérant de son atelier de production de saké est surpris en situation de dépassement du quota de production fixé par les autorités. Bien qu'aucune charge ne soit retenue contre lui, il ne jouit plus des mêmes faveurs. Il s'installe pour un temps loin de sa ville natale, sans pour autant rompre les liens avec ses amis. Il regagne Ōsaka en 1793 où il vivra jusqu'à sa mort dans des conditions plus spartiates, tout en restant très actif dans ses différents domaines de compétence ${ }^{36}$.

42 Revenons à la préface. Comme le veut la tradition, le préfacier explique qu'il a été sollicité par un libraire pour rédiger la préface d'un ouvrage et qu'à cette occasion il a été heureux de découvrir que non seulement l'ouvrage était illustré par son ami défunt Shitomi Kangetsu, mais qu'il rejoignait par son esprit un ancien ouvrage qu'il avait commencé à composer et qu'il avait été forcé d'abandonner en raison des malheurs qui se sont abattus sur sa maisonnée ${ }^{37}$. La préface est curieuse car elle ne fait pas véritablement l'éloge de l'auteur, ni celui du libraire dont le nom est également passé 
sous silence. Elle n'évoque pas non plus d'intervention directe de sa part sur la composition du livre.

On conclura donc que de nombreux signes donnent à penser que Kenkadō a été étroitement associé à l'étape éditoriale. Le livre s'appuie sur une vaste érudition en ouvrages chinois et en ouvrages japonais, mais aussi en histoire naturelle. Il traduit une forte fascination de son auteur pour les questions de philologie, d'archéologie et pour les antiquités, une curiosité pour les coutumes locales, le terrain de la production, la qualité des produits. Enfin, le livre révèle un goût prononcé pour le dessin, comme moyen de représentation, et la poésie ancienne. Autant de champs d'investigation et de penchants de Kimura dont témoignent les manuscrits conservés ${ }^{38}$.

\section{Le Tiangong kaiwu (Exploitation des œuvres de la nature)}

Le fait que Kimura Kenkadō soit associé au projet de publication de cet ouvrage nous invite également à accorder une attention particulière aux sources d'origine chinoise, notamment au Tiangong kaiwu 天工開物 (j. Tenkō kaibutsu, Exploitation des œuvres de la nature ; 1637) de Song Yingxing 株応星, qui semble avoir inspiré l'auteur du Meibutsu zue, comme celui du Meisan zue ${ }^{39}$.

Cet ouvrage publié très peu de temps avant la chute de l'empire Ming, constitue aujourd'hui une référence majeure pour les historiens des techniques. Composé de trois fascicules, se divisant en dix-huit sections, le Tiangong kaiwu couvre la quasi-totalité des grands domaines de production de l'époque ${ }^{40}$. L'ouvrage est d'autant plus précieux que ces domaines n'avaient que très peu retenu l'attention des lettrés fonctionnaires chinois, plus soucieux des techniques directement liées au fonctionnement de l'État, comme l'agriculture. On trouve parmi les domaines couverts par le Tiangong kaiwu : la culture des céréales, le tissage de la soie et du coton, la teinture, la production du sel, du sucre, de la céramique, la fonte des métaux, la construction navale, les techniques de la forge, la fabrication de l'huile ou du papier, les techniques d'affinage, de fermentation, la taille des pierres, la fabrication d'armes, etc.

Bien qu'écrit à une époque où des techniques occidentales d'origine jésuite étaient déjà connues et diffusées, Song Yingxing n'y fait que très peu allusion ${ }^{41}$. Le livre est surtout connu pour ses techniques traditionnelles dont la connaissance aurait été recueillie en se rendant sur le terrain. Si les propos de Song Yingxing ne peuvent être tous pris à la lettre, notamment lorsqu'il affirme qu'il a rejeté toutes les croyances infondées, le livre n'en reste pas moins une source très fiable, d'autant qu'il comporte de nombreuses illustrations et données chiffrées ${ }^{42}$. Les illustrations des premières éditions du livre sont d'une facture plutôt naïve (Fig. 7) mais elles témoignent de la volonté de l'auteur de rendre compte du cadre naturel, des installations, des outils, des ouvriers et ouvrières, ainsi que de l'organisation du travail. C'est une orientation que l'on retrouvera dans le Meisan zue. 
Fig. 7 : Tiangong kaiwu (Exploitation des œuvres de la nature) de Song Yingxing

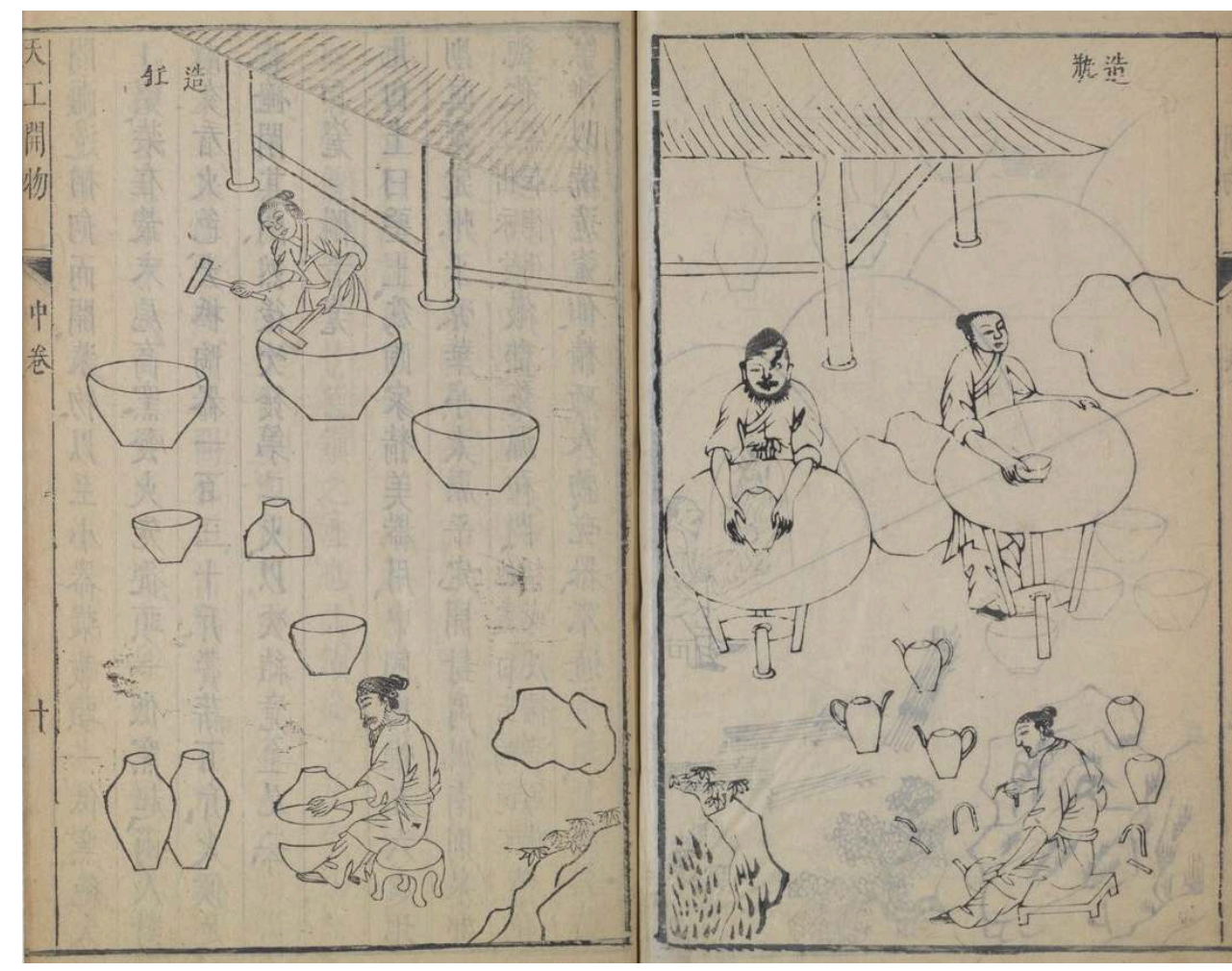

Bibliothèque nationale de France. Département des manuscrits chinois 5563. Lien vers l'illustration

47 On a déjà souligné l'importance des illustrations dans le Meisan zue. Ces dernières reflètent une maîtrise technique incontestable par rapport à celles des ouvrages de l'époque des Ming. Un auteur comme Kimura Kenkadō est en possession d'ouvrages hollandais, notamment d'encyclopédies techniques, et l'on doit sans doute aussi prendre en compte ces modèles-là pour analyser ces illustrations.

Cet ouvrage a reçu un bien meilleur accueil au Japon qu'en Chine où l'on ne trouve que sporadiquement des citations ici et là et où l'on finit par en perdre la trace ${ }^{43}$. Au Japon, à l'opposé, il a largement circulé et est mentionné par différents auteurs parmi lesquels Kaibara Ekiken ${ }^{44}$ et Hiraga Gennai ${ }^{45}$. Plus significatif pour notre propos, on sait que le nom de Kimura Kenkadō est étroitement lié aux deux éditions imprimées les plus anciennes de ce livre, conservées au Japon ${ }^{46}$. En effet, on sait que ces deux éditions faisaient partie de sa collection de livres et que la plus ancienne aurait servi de base à l'édition japonaise du livre chinois, publiée en 1771 à Ōsaka munie de signes diacritiques et de lectures en $\mathrm{kana}^{47}$. Comme on le verra plus loin dans notre traduction du Meisan zue, des passages entiers du Tiangong kaiwu sont cités, et l'auteur parfois se dispense de le signaler. L'ouvrage de Song Yingxing est donc présent comme toile de fond, même lorsqu'il n'est pas explicitement mentionné. On peut dire pour conclure que le Meisan zue se situe au confluent de nombreux courants éditoriaux, et que le personnage de Kimura Kenkadō associé aux libraires d'ōsaka, a pu jouer un rôle important dans l'achèvement de ce projet de publication. 


\section{Nota bene}

Le fait que le Nippon sankai meisan zue ne mentionne aucun nom d'auteur suscite de nombreuses interrogations. On a vu que la préface était signée par Kimura Kenkadō. Le livre comporte également une postface, écrite en japonais dans un style archaïsant, non signé. La Bibliothèque de Kyōu shooku 杏雨書屋 située à Ōsaka, conserve un manuscrit intitulé Hiraga Gennai bussankō 平賀源内物産考 (Réflexions de Hiraga Gennai sur les produits) qui entretient un lien très étroit avec notre guide illustré. En effet, il s'ouvre sur le texte de la postface du Meisan zue calligraphié avec soin, et portant la signature de Hiraga Kyūkei (nom de peintre de Gennai), et la date de la première année de Meiwa, soit 1764. Cette attribution est douteuse car la postface mentionne des noms de peintres que Gennai ne peut avoir connus. Le manuscrit présente des extraits du Meisan $z u e$, agencés selon un ordre différent. La présence de lectures en encre rouge à côté des kanjis donne à penser qu'il s'agit d'une version de travail préparée en vue de l'édition finale. Que Hiraga Gennai soit l'auteur de certains passages du texte n'est pas à exclure, compte tenu de l'importance des entrées portant sur les produits de la région d'Awa dont il est originaire. Il existe également une forte proximité entre les sources utilisées par Hiraga Gennai lors de la compilation de son ouvrage de honzō, le Butsurui hinshitsu 物類品潐, et celles citées dans le Meisan zue. Mais une étude plus approfondie est nécessaire pour déterminer quel a été le rôle de Hiraga Gennai dans la composition du Meisan zue et la signification à donner à ce manuscrit.

\section{NOTES}

1. Abrégé en Meisan zue par la suite.

2. Le livre a fait l'objet de plusieurs tirages. À partir des copies conservées, fort nombreuses, dont certaines sont accessibles en ligne, on peut constater que lors de sa première édition, datée de Kansei 11 (1799), les planches étaient en possession de Takagi Senkyōdō et vendues par le libraire d'Ōsaka Shioya Chōbee. Lors d'une édition postérieure datée de l'an 13 de l'ère Bunsei (1830), le livre était vendu conjointement par les libraires d'ōsaka, Shioya Chōbee et Harimaya Kōbee.

3. À partir des nombreux exemplaires conservés, on constate que le champ de diffusion n'a cessé de s'étendre. Vendu d'abord à Ōsaka, puis dans les trois grandes villes que sont Ōsaka, Kyōto et Edo, il a fini par être vendu très largement sur tout le territoire. Un des exemplaires conservés à la BULAC (Bibliothèque universitaire des langues et civilisations, Paris) indique en dernière page douze noms de libraires dont sept se trouvent à Edo, deux à Okayama, un à Kurashiki, un à Kyōto et un à Ōsaka. Le contenu du livre n'a pas été modifié d'une édition à l'autre, et les exemplaires diffèrent seulement par le colophon qui porte des noms de libraires et des publicités différents. Le colophon indique rarement la date de la réimpression. Les dernières réimpressions datent de l'époque Meiji. On remarque que le livre a retenu l'attention de nombreux acheteurs occidentaux. Quatre copies complètes font partie du fonds ancien de la BULAC et un fascicule 5 isolé figure également dans un autre don. Un exemplaire du livre, très bien conservé, se trouve à la Bibliothèque du Collège de France (fonds Castillon).

4. Voir par exemple, l'exemplaire conservé au Collège de France (fonds Castillon). 
5. Voir par exemple la liste établie par SHIRAHATA Yōzaburō dans "The Printing of Illustrated Travelogues in $18^{\text {th }}$ century Japan", in Written Texts - Visual Texts, Woodblock-printed Media in Early Modern Japan, FORMANEK Susanne and LINHART Sepp (ed.), Amsterdam, Hotei Publishing, 2005, p. 206-209. Shirahata inclut dans la liste le Meisan zue.

6. Pour les généralités concernant la popularisation de la culture, voir l'introduction, dans HAYEK Matthias and HORIUCHI Annick (ed.), Listen, Copy, Read: Popular Learning in Early Modern Japan, Leiden, Brill, “Japanese Studies Library", 2014, p. 1-22. Sur les encyclopédies domestiques, voir KINSKI Michael, "Treasure Boxes and Mirrors: On the Contents and the Classification of Popular Encyclopedias from Early Modern Japan", in Listen, Copy, Read: Popular Learning in Early Modern Japan, ibid., p. 71-90.

7. Abrégé en Meibutsu zue par la suite.

8. C'est ce que nous avons pu constater sur les exemplaires conservés à la Bibliothèque de la BULAC. Voir l'exemplaire du Nippon sankai meibutsu zue, répertorié sous la cote JAP331 bis, et l'exemplaire Nippon sankai meisan zue JAP331, rassemblés dans une même boîte. Ce n'est pas cependant le cas de tous les exemplaires.

9. Voir son commentaire dans CHIBA Tokuji (éd.), Nippon sankai meisan meibutsu zue, Tokyo, Shakai Shisōsha, 1970, p. 290-308. Les annotations de Chiba nous ont été utiles dans notre travail mais la traduction a été réalisée en consultant en permanence le livre original. Chiba s'appuyait luimême sur une transcription antérieure du Meisan zue datée de 1929, publiée dans la collection Nippon zue zenshū, en même temps que le Nippon sankai meibutsu zue déjà mentionné.

10. Voir Nippon sankai meibutsu zue, introduction de HIGUCHI Hideo, Tokyo, Meicho kankōkai, 1969 et Nippon sankai meisan zue, introduction de HIGUCHI Hideo, Tokyo, Meicho kankōkai, 1969.

11. Sa position est sensiblement différente de celle de Chiba qui considère que l'auteur du Meisan zue n'est autre que Kimura Kenkadō, auteur de la préface (voir plus loin). Les divergences proviennent en partie de ce que Chiba ignore que la première édition du Meibutsu zue date de 1754, et considère que les deux ouvrages ont été publiés dans la même foulée en 1797 et 1799 . Voir chiBA (éd.), Nippon sankai meisan meibutsu zue, op. cit., p. 295.

12. Nippon sankai meibutsu zue, op.cit., p. 1-11. Voir aussi la postface du Meisan zue, qui souligne son rôle.

13. Voir CHIBA (éd.), Nippon sankai meisan meibutsu zue, op. cit., p. 194.

14. Le Meibutsu zue comporte 161 entrées, donc 161 figures, là où le Meisan zue ne comporte que 71 planches.

15. Kaozheng ou kaozhengxue 考証学 (école d'études critiques) renvoie à une pratique des études confucéennes qui a pris son essor sous la dynastie des Qing en Chine, en réaction aux tendances spéculatives et philosophiques du confucianisme de l'époque précédente. Il s'agit pour les lettrés d'établir la connaissance du passé et des textes qui y donnent accès sur des fondements solides, en faisant appel notamment aux méthodes philologiques les plus rigoureuses. Les études qui s'inscrivent dans ce courant commencent à être appréciées au Japon vers la seconde moitié du $\mathrm{XVIII}^{\mathrm{e}}$ siècle, à l'époque précisément où le Meisan zue est composé. Voir ELMAN Benjamin A., From Philosophy to Philology: Intellectual and Social Aspects of Change in Late Imperial China, Cambridge Mass./London, Harvard University Press, 1984.

16. Voir CHIBA (éd.), Nippon sankai meisan meibutsu zue, op. cit., p. 300-301.

17. Sur l'histoire très longue de cette science en Chine, voir MÉTAILIÉ Georges, Science and civilisation in China Volume 6 Biology and biological technology Part IV Traditional botany and ethnobotanical approach, LLOYD Janet (trad.), Cambridge, Cambridge University Press, 2015, ainsi qu'Unschuld Paul Ulrich, Medicine in China. A History of Pharmaceutics, Berkeley/Los Angeles/ London, University of California Press, 1986, p. 145-163. Kaibara Ekiken, mentionné plus loin, est à l'origine d'une édition japonaise « révisée » du Bencao gangmu, publiée vers 1672. 
18. Inō Jakusui est un médecin qui entreprend, sous le patronage du seigneur Maeda du fief de Kaga, une vaste compilation érudite des connaissances relatives aux plantes. Il décède alors que son ouvrage, le Shobutsu ruisan (Répertoire des myriades de substances) est encore inachevé. À l'initiative de Yoshimune, Niwa Shōhaku 丹羽正伯 (1691-1756) reprend le flambeau et fait aboutir le projet. En 1738, le Shobutsu ruisan est achevé avec 1054 fascicules. Il aura été réalisé en trois étapes, la première étant le fait d'Inō Jakusui. Voir MARcon Federico, The Knowledge of Nature and the Nature of Knowledge in Early Modern Japan, Chicago/London, The University of Chicago Press, 2015, p. 115-139 et YABE Ichirō, Edo no honzō: yakubutsugaku to hakubutsugaku (Le honzō d'Edo: pharmacopée et histoire naturelle), «coll. Raiburari kagakushi 6 », Tokyo, Saiensusha, 1984, p. 66-71.

19. YABE, Edo no honzō : yakubutsugaku to hakubutsugaku, ibid, p. 82.

20. MARCON, The Knowledge of Nature and the Nature of Knowledge in Early Modern Japan, op. cit., p. 207-227.

21. Ono Ranzan est l'auteur du Honzō kōmoku keimō (Introduction au Bencao gangmu). Cet ouvrage de 43 fascicules publié en 1803 lui vaudra le surnom de « Linné du Japon » attribué par Siebold.

22. L'ouvrage a été composé par Miyazaki Yasusada 宮崎安貞, un guerrier au service du seigneur Kuroda du fief de Fukuoka, qui décide de se consacrer à l'agriculture. Il réunit les connaissances les plus en pointe au terme de nombreux voyages, notamment dans les régions centrales où il découvre les techniques les plus sophistiquées.

23. TSUKUBA Tsuneharu, Nihon no nōsho, nōgyō wa naze kinsei ni hatten shitaka (Les traités agricoles du Japon. Pourquoi l'agriculture s'est-elle développée à l'époque prémoderne ?), Tokyo, coll. «Chūkō shinsho », 1987, p. 123-124.

24. Voir par exemple SMITH Thomas C., Native Sources of Japanese Industrialization 1750-1920, Berkeley/Los Angeles/London, University of California Press, 1988, p. 15-47.

25. YoSHIDA Nobuyuki, "Dentō toshi no shūen » (À la périphérie de la ville traditionnelle), in Kinsei no kaitai (Déconstruction du prémoderne), vol.7, Nihonshi kōza, Tokyō daigaku shuppankai, 2005.

26. Le colophon indique en réalité Hokkyō Kangetsu, une désignation qui rappelle le titre honorifique qu'il s'est vu attribuer. Le peintre est également connu sous le nom de Yanagihara Genjirō 柳原源次郎.

27. Cet accompagnement est habituel et vise à faciliter la lecture. En ce faisant, notre auteur démontre une parfaite maîtrise de la langue chinoise et son excellence en tant que lettré.

28. Le Ise sangū meisho zue 伊勢参宮名所圖會 (1813) et le Ōmi meisho zue 近江名所図会 (1814), Ōmi désignant une ancienne province s'étendant autour du lac Biwa.

29. Voir SHIRAHATA, "The Printing of Illustrated Travelogues in $18^{\text {th }}$ century Japan", op. cit., p. 199-214.

30. Sur Kimura Kenkadō, on pourra consulter : ŌSAKA REKISHI HAKUBUTSUKAN (éd.), Kimura Kenkadō : naniwa chi no kyojin. Tokubetsuten botsugo 200 nen kinen (Kimura Kenkadō, le géant d'Ōsaka: Exposition spéciale en commémoration du deuxième centenaire de sa mort), Kyoto, Shibunkaku shuppan, 2003. Sur ses activités en tant que lettré et marchand, voir ARISAKA Michiko, "Toshi bunjin" (le lettré citadin) in Chishiki to gakumon o ninau hitobito (Les hommes chargés de la connaissance et des savoirs), чокотА Fuyuhiko (éd.), coll. « Mibunteki shūen to Kinsei shakai » 5, Tokyo, Yoshikawa kōbunkan, 2007, p. 143-174.

31. Un catalogue posthume de sa collection de livres, le Kenkadō shomoku comprend plus de 2000 titres. Voir INOUE, « Kenkadō no zōsho ni tsuite », in Kimura Kenkadō..., ōSAKA REKISHI HAKUBUTSUKAN (éd.), op. cit., p. 151-167.

32. Ibid.

33. Voir la page dédiée à sa collection sur le site du Musée municipal d'Histoire des sciences naturelles d'ōsaka, http://www.mus-nh.city.ōsaka.jp/collection/kenkado/stones/5/index.html 
ainsi que le chapitre 3, KAZU Tsuguto, chapitre 3 «Bussanka Kenkadō» (Kenkadō, le spécialiste des productions), in Kimura Kenkadō, ōSAKA REKISHI HAKUBUTSUKAN (éd.), op. cit., p. 89-90.

34. À ce sujet, on pourra consulter: MIZUTA Norihisa (éd.), Mizu no chūō ni ari; Kimura Kenkadō kenkyū (Une existence au cœur de l'eau: recherches sur Kimura Kenkadō), Tokyo, Iwanami shoten, 2002, p. 41-121.

35. L'original est conservé à la bibliothèque Kyōu sho'oku à Ōsaka. Voir ŌSAKA REKISHI HAKUBUTSUKAN (éd.), Kimura Kenkadō ..., op. cit., p. 80, pour deux reproductions. On peut consulter une copie établie en 1809 sur le site de la Bibliothèque nationale de la Diète : http://dl.ndl.go.jp/ info:ndljp/pid/2535633.

36. Sur la vie de Kenkadō, on pourra consulter son texte autobiographique, inclus dans le Kenkadō zatsuroku, reproduit dans MIZUTA, Mizu no chūō ni ari ; Kimura Kenkadō kenkyū, op. cit, p. 289-291. Sur ses sources de revenus, voir TSUKADA Takashi, « Kimura Kenkadō to Kita horie go chōme - Kinsei Ōsaka no toshi shakai kōzō to no kanren de » (Kimura Kenkadō et le quartier Kita horie 5 chōme : en lien avec la structure sociale de la ville d'Ōsaka), in Ōsaka ni okeru toshi no hatten to kōzō, TSUKADA Takashi (éd.), Tokyo, Yamagawa shuppan, 2004, p. 113-137.

37. L'ouvrage en question aurait porté le nom de Meibutsu dokudan (Mes vues sur les spécialités renommées).

38. Voir ōSAKA REKISHI HAKUBUTSUKAN (éd.), Kimura Kenkadō ...., op. cit., p. 212-219

39. L'ouvrage est plus particulièrement cité dans le fascicule $5 \mathrm{du}$ Meisan zue. Le titre est traduit "Works of Heaven and the Inception of Things" in SCHÄFER Dagmar, The Crafting of the 10,000 things: knowledge and Technology in Seventeenth-Century China, Chicago/London, The University of Chicago Press, 2011.

40. On peut consulter l'édition de 1637 sur le site de la Bibliothèque nationale de France qui en conserve un exemplaire. http://gallica.bnf.fr/ark:/12148/btv1b90029713

41. YABUUCHI Kiyoshi, "Kaisetsu ", (Présentation) in Tenkō kaibutsu, song Yingxing (auteur), YABUUCHI Kiyoshi (trad. et éd.), Tokyo, Heibonsha, coll. " Tōyō bunko », 1988 (première édition 1969), p. 372.

42. Voir SAEgUSA Hiroto "Tenkō kaibutsu no kenkyū» (Recherches sur le Tiangong kaiwu), in Saegusa Hiroto chosakushū (Collection d'œuvres de Saegusa Hiroto), vol. 11, Tokyo, Chūō kōronsha, 1973, p.120-180 ; YABUUCHI Kiyoshi, "kaisetsu» (Commentaire), in Tenkō kaibutsu, op. cit., p. 361-379 et YABUUCHI Kiyoshi (éd.), Tenkō kaibutsu no kenkyū (Recherches sur le Tiangong kaiwu), Tokyo, Kōseisha kōseikaku, coll. « Kyōto daigaku jinbun kagaku kenkyūjo kenkyū hōkoku », 1953. 43. Le fait que l'ouvrage n'ait pas été retenu lors de la compilation du Siku quanshu 四庫全書 y contribue sans doute. On remarque aussi que les premières traductions de passages de ce livre sont dues à Stanislas Julien. YABUUCHI, Tenkō kaibutsu, op. cit., p. 386-387.

44. INOUE Tadashi, Kaibara Ekiken, Tokyo, Yoshikawa kōbunkan, 1963, p. 186.

45. oKUMURA Seiji, Hiraga Gennai o aruku: Edo no kagaku o tazunete (Marcher sur les traces de Hiraga Gennai : à la recherche de la science de l'époque d'Edo), Tokyo, Iwanami shoten, 2003, p. 57.

46. L'une d'elles a brûlé lors du tremblement de terre de 1923 mais des copies en avaient été établies.

47. YABUUCHI (éd.), Tenkō kaibutsu, op. cit., p. 375. Une réimpression de cet ouvrage intervient en l'an 13 de Bunsei, 1830. On sait que Kenkadō s'est illustré à de nombreuses reprises pour rééditer au Japon des ouvrages rares jugés importants. Voir ōSAKA REKISHI HAKUBUTSUKAN (éd.), Kimura Kenkadō..., op. cit., p. 112. 


\section{Avertissement}

\section{Traduction}

1 La traduction du Meisan zue est le résultat d'un travail collectif qui a débuté en 2010 et s'est déroulé sous la forme d'un séminaire de travail mensuel. Les principaux artisans de la traduction française sont, dans l'ordre alphabétique: Annick Horiuchi, Josef Kyburz, Daniel Struve et Charlotte von Verschuer. Chaque membre du groupe a assuré les recherches préliminaires, la mise en forme et la rédaction des notes d'un ou deux fascicules (voir Tab. 1).

2 La traduction repose de manière significative sur les compétences en langue et en poésie classiques de Daniel Struve, ainsi que sur l'expertise en histoire des techniques de Charlotte von Verschuer, en ethnographie de Josef Kyburz et en histoire des savoirs d'Annick Horiuchi.

3 Une expertise sur les pratiques culturelles de la région de Kyōto a été apportée par le professeur Umeyama Hideyuki de l'université Momoyama gakuin durant son séjour au sein du laboratoire en 2016.

4 Enfin, François Lachaud a apporté son concours à la traduction de la préface.

\begin{tabular}{|l|l|}
\hline Fascicule 1 & C. von Verschuer \\
\hline Fascicule 2 & D. Struve \\
\hline Fascicule 3 & C. von Verschuer \\
\hline Fascicule 4 & J. Kyburz \\
\hline Fascicule 5 & A. Horiuchi \\
\hline
\end{tabular}

Tab. 1 : répartition des responsabilités entre les membres du groupe 


\section{Abréviations}

$5 \quad$ j. lecture japonaise d'un terme ou d'un titre d'ouvrage

6 c. lecture chinoise d'un terme d'origine chinoise

7 1. terme latin

\section{Sommaires de fascicules}

8 Le texte de chaque fascicule débute par un sommaire qui donne une vue d'ensemble du fascicule et reproduit, à quelques détails près, les titres des parties et des sous-parties. Il est à noter que ce sommaire n'a pas vocation à être utilisé pour circuler à l'intérieur du Meisan zue. Pour ce faire, le lecteur pourra utiliser toutes les fonctionnalités propres à l'édition en ligne de la plateforme OpenEdition Books.

\section{Illustrations}

- Le Meisan zue contient deux sortes d'illustrations. La première catégorie qui concerne la grande majorité d'entre elles se présentent sous la forme de deux pages pleines en vis-à-vis. Elles comportent toutes des légendes qui ont été traduites. Elles sont désignées par " planche » et numérotées sous la forme : Pl. 1, Pl. 2, etc.

- La deuxième catégorie d'illustrations, de moindre envergure et sans légende, ont été ordonnées par ordre alphabétique : Fig. A, Fig. B, etc.

- Le texte du Meisan zue ne mentionnant pas nécessairement les planches auxquelles il se réfère, les éditeurs ont inséré tout au long du texte des renvois sous la forme : [voir Pl.1], [voir Pl. 2], etc.

- La numérotation des planches respecte l'ordre d'apparition dans l'ouvrage original.

- Comme les planches étaient parfois insérées dans l'ouvrage original à des emplacements éloignés des textes correspondants, elles ont été déplacées dans la traduction pour qu'elles puissent jouer pleinement leur rôle.

- Les illustrations reproduites dans l'édition en ligne sont extraites de la copie du Meisan zue conservée à l'Institut national de littérature japonaise (Kokubungaku kenkyū shiryōkan, DOI 10.20730/200021673).

- Le lien vers la page numérisée de chaque illustration permettra au lecteur d'examiner chaque détail avec une très grande précision. 


\section{Présentation des fascicules}

\section{Fascicule 1}

Le saké est une boisson alcoolisée obtenue par la fermentation du riz. C'est un « vin de riz ». Sa fabrication englobe la culture de la moisissure moyashi (Aspergillus orizae qui est le ferment de saccharification), la préparation du kōji, celle du levain (moto), ainsi que le processus de fermentation alcoolique. C'est le levain, introduit au XvII siècle, qui constitue l'élément nouveau par rapport au saké médiéval. La qualité du saké dépend grandement de la qualité du riz (japonaica non gluant), de son traitement, c'est-à-dire du degré de pilage, du blanchiment, du lavage, de la durée de trempage et de détrempage, de la durée de cuisson (toujours à la vapeur), ou encore du dosage des ingrédients. Le saké varie aussi en fonction des conditions climatiques et de la qualité de l'eau'.

Depuis l'aube des temps, le saké est loué comme boisson divine. Dans le Nihon shoki (Chronique du Japon), achevé en 720, le saké participe du mythe fondateur de l'État japonais. Tout commence par la descente sur l'archipel du dieu Hononinigi, petit-fils de la déesse du soleil Amaterasu. Il y épouse une princesse qui lui donne trois fils. La princesse cultive du riz dans une rizière sacrée et brasse du "saké divin » qu'elle offre au dieu son époux. Jinmu, issu de leur union, est le premier empereur du Japon. Il monte sur le trône en 660 avant notre ère. Depuis lors, le saké divin (miwa) est chanté dans la poésie antique japonaise ${ }^{2}$.

Dans l'État régi par les codes, un bureau du saké est chargé de la fabrication et de l'approvisionnement en saké de la cour. Cette boisson est servie lors de toutes les cérémonies et des banquets officiels ${ }^{3}$ et, à partir de l'époque Nara (710-784), elle occupe un rôle prédominant lors des festivités. Bientôt, la consommation de saké, intégrée dans les rites shintō, se voit attribuer une fonction rituelle et un caractère sacré. Le Engishiki (Règlements de l'ère Engi ; 927) de la cour de Heian (794-1192) a codifié ce statut. Ce document encadre la fabrication et les quantités produites du saké, énumère ustensiles et récipients, et définit le protocole des services des banquets de cour. Il donne une liste de plus d'une centaine de rites shintō dans lesquels le saké est présenté en offrande aux divinités. Ces offrandes se déroulent annuellement dans la capitale comme dans les sanctuaires des soixante-cinq provinces du pays. C'est la naissance du 
caractère sacré du saké. Son rôle religieux s'est maintenu jusqu'à nos jours, avec la pratique des banquets accompagnés de cette boisson ${ }^{4}$.

4 À partir de l'époque Kamakura (1185-1333), la coutume des banquets arrosés de saké s'étend aux milieux guerriers. Ces derniers sont très rapidement rejoints par les milieux ecclésiastiques des grands monastères du Zen. Par la suite, quand le gouvernement militaire établit son siège à Kyōto dans le quartier Muromachi (1336-1573), les trois élites, guerrière, monastique et aristocratique, s'y trouvent réunies et se concurrencent pour offrir au shogun des «banquets solennels» (onari). À partir du XIV siècle le saké occupe une place centrale dans le protocole de ces banquets : les dîners commencent par un apéritif formel sous forme de toasts répétés et se terminent, après les services des mets, par une séance de saké qui finit souvent en beuverie se prolongeant jusqu'à l'aube. Le nombre de banquets arrosés auxquels participent les shoguns à l'invitation de leurs vassaux peut atteindre soixante à quatrevingt-dix par an. Cela signifie qu'ils passent l'équivalent de deux à trois mois de l'année à boire du saké. Les moines zen sont tout aussi friands de cette boisson: on trinque volontiers après un dîner végétarien. Par exemple, le supérieur du monastère Chōrakuji, situé dans l'actuel département de Gunma, a participé à cent cinq séances arrosées dans la seule année 1565. De manière générale, la consommation de saké lors des banquets monastiques atteint des volumes colossaux. On sait par exemple que la quantité de saké consommée au cours d'un banquet peut dépasser cent litres à Kyōto. C'est dire que le saké joue un rôle essentiel dans la société de la capitale ${ }^{5}$.

5 Il en découle un essor extraordinaire pour la production et le commerce du saké. Plus de 340 fabricants sont enregistrés dans la ville de Kyōto au milieu du $\mathrm{Xv}^{\mathrm{e}}$ siècle. Ils représentent un revenu fiscal important pour le shogun, d'autant plus que leur activité est menée de pair avec une activité bancaire. De nombreuses maisons de saké se spécialisent dans le crédit. Le saké fait ainsi son entrée dans le marché financier et connaît un essor économique à l'échelle du pays ${ }^{6}$.

6 À l'époque d'Edo (1600-1867), la production et la consommation de saké atteignent des niveaux sans précédent et représentent un enjeu économique considérable. Les producteurs de cette boisson sont soumis à une rude concurrence. Désormais, les plus gros marchands se concentrent dans la province de Settsu (qui couvre en partie les départements actuels d'ōsaka et de Hyōgo). Leur succès repose sur l'approvisionnement en saké de la capitale des shogun. Le Meisan zue indique que c'est la maison Yamanaka du village Kōnoike, à proximité d'Itami, qui se serait lancée la première dans ce commerce juteux. Elle aurait été suivie par Manganjiya d'Ikeda qui aurait innové en optant pour la livraison par bateau. À la fin du XvIII , ce sont les producteurs de Nishinomiya, Hyōgo, Nada et Imazu qui ont le vent en poupe alors qu'Itami et Ikeda amorcent leur déclin. Le succès de leur entreprise dépend en premier lieu de la facilité de communication avec la capitale Edo ${ }^{7}$.

7 À la fin du $\mathrm{XVII}^{\mathrm{e}}$ siècle, le volume de saké livré à Edo, appelé kudarizake, est évalué à 640000 taru par an, soit plus de 46 millions de litres. Dans les années 1820, ce montant a quasiment doublé et s'élève à 1200000 taru. Chaque année, l'arrivée du "nouveau saké » est un événement qui met en effervescence la population de la capitale de l'Est ${ }^{8}$. Mais derrière ces chiffres impressionnants, se cache une réalité en demi-teinte. Les productions de saké subissent de plein fouet les fluctuations des récoltes de riz. Le Japon des Tokugawa a été périodiquement frappé par des catastrophes climatiques qui se sont soldées par de terribles famines. Le gouvernement des shoguns a dû à chaque 
fois intervenir pour freiner la production de saké et veiller à ce que la population ait assez de riz pour se nourrir. À l'époque d'Edo, seuls les producteurs de saké pourvus d'une licence (sake kabu) sont autorisés à en produire. Pour chacun, le plafond du volume de riz qu'il peut transformer en saké est fixé. Ce plafond n'était pas toujours respecté, comme on le voit avec Kimura Kenkadō, le lettré d'Ōsaka auteur de la préface du Meisan zue, dont la fortune a brusquement chuté à la suite d'un contrôle du bakufu' Dans ce contexte, on mesure le statut exceptionnel du saké d'Itami dont la production, au plus fort de son succès, atteignait 100000 koku par an, soit 18 millions de litres, et qui assurait plus de $20 \%$ de l'approvisionnement de la capitale ${ }^{10}$. Le saké d'Itami était aussi servi à la table du shogun, ce qui ajoutait à son prestige. La principale raison de ce succès réside dans son savoir-faire. La description que donne le Meisan zue de la fabrication chez un brasseur d'Itami en donne un aperçu.

Le saké dont il est ici question est le morohaku, littéralement "tous blanchis », terme renvoyant au fait que les ingrédients utilisés pour la fabrication, qu'il s'agisse du riz étuvé ou du kōji, sont obtenus à partir de grains de riz blanchis au pilon. Le morohaku désigne aussi le saké parfaitement clair, opposé au nigorizake ou saké trouble, qui était le produit le plus courant jusqu'au $\mathrm{XvI}^{\mathrm{e}}$ siècle et qui continuera à être fabriqué dans les campagnes. Si le morohaku d'Itami est célèbre au point que le nom de la ville suffit à désigner ce type de saké, la technique de fabrication n'est pas née à Itami mais à Nara, au XVI ${ }^{e}$ siècle, dans les monastères bouddhiques du Kōfukuji. C'est en effet à Nara que l'on aurait adopté le système des ajouts successifs (soe) et que la préférence aurait été donnée au saké produit en hiver ${ }^{11}$. Mais la renommée du morohaku de Nara est éphémère et ne tarde pas à être supplantée par celle d'Itami.

Le saké était à l'origine produit en toute saison. Pour la fermentation, une température élevée était souhaitable, mais les risques de prolifération des bactéries étaient alors plus élevés si bien que la saison froide a fini par avoir la préférence, même si la durée de la fabrication s'en est trouvée allongée. Le Meisan zue évoque la distinction entre les quatre types de saké dont la fabrication s'échelonne entre l'automne et l'hiver: le «saké nouveau », le «saké d'entre-deux », le « saké d'avant les froids » et le "saké des froids », en notant que " plus la saison avance, plus la durée de fabrication est longue et le prix élevé ».

10 Il convient toutefois de souligner que la technique de fabrication exposée ici n'a rien d'un mystère pour les contemporains. Au tournant du XIX siècle, il n'est en effet pas de fief qui ne brasse son saké de qualité et certains brasseurs de "campagne ", méprisés auparavant, sont parvenus à gagner une certaine reconnaissance de la haute société d'Edo. Cela répondait d'ailleurs aux vœux du shogun qui souhaitait mettre fin au monopole des brasseurs du Kansai, à l'origine des prix élevés du saké dans la capitale. Il n'en reste pas moins que ce fascicule du Meisan zue, avec ses cinq somptueuses illustrations, retient l'attention par le caractère industriel que revêt la production de saké dans la région d'Ōsaka au seuil du XIX ${ }^{\mathrm{e}}$ siècle, avec des ouvriers spécialisés dont chaque geste est parfaitement maitrisé. C'est un niveau de perfection que les autres fiefs de l'Est étaient sans doute loin d'avoir atteint.

\section{Fascicule 2}

11 Le fascicule 2 est entièrement consacré aux produits de la montagne. À la différence du fascicule 1, qui s'attache à décrire la fabrication du saké en atelier, on s'intéresse 
essentiellement ici à des produits naturels, mis sur le marché sans grandes transformations. Des parties dédiées à des classes de produits, comme la pierre, le champignon, ou le gibier, côtoient des sujets plus ciblés, comme les abeilles, la cire, le puéraire ou la grenouille rouge.

Les pierres présentées ici sont des pierres extraites dans les régions centrales du Japon (autour de Kyōto et d'Ōsaka) et travaillées sur les lieux mêmes de leur extraction avant leur mise sur le marché. Les minéraux (seki), associés aux métaux (kin), constituent une catégorie (rui) présente dans les ouvrages de honzō. Dans le Bencao gangmu, cette catégorie englobe plus de 160 substances, se déclinant en "métaux», "jades", «minéraux » et "minéraux salés ». Comme le remarque G. Métailié, les critères sur lesquels cette classification se base sont assez flous ${ }^{12}$. Dans le Yamato honzō (1709), Kaibara Ekiken relève 67 substances dans une catégorie appelée "métaux, pierres précieuses, terres et pierres " dans laquelle les frontières entre les sous-catégories demeurent toujours aussi peu explicites. Toutes les concrétions qui se trouvent dans le sol s'y retrouvent. À la différence des plantes ou des animaux traités dans le livre, on note qu'il relève peu de variétés spécifiquement japonaises. À partir du XvIII ${ }^{\mathrm{e}}$ siècle, les pierres font l'objet d'un véritable engouement parmi les savants de l'archipel, alimenté par le développement d'une proto-archéologie qui pousse les savants à rechercher dans le sol les traces d'un passé lointain. Les pierres luminescentes, cristallines, ou aux formes animales, sont particulièrement appréciées des amateurs. Cette vogue est entretenue par les expositions successives de produits (bussankai) organisées à Ōsaka et à Edo, où les savants sont informés des dernières découvertes ou des dernières importations, car beaucoup de ces curiosités sont des produits d'origine étrangère. La publication dans les années 1773-1801 du monumental Unkonshi (Traité sur les roches) de Kino'uchi Sekitei (1724-1808) constitue un moment fort de la recherche dans le domaine des pierres. L'ouvrage répertorie environ deux mille espèces de roches, minéraux, fossiles et objets en pierre. L'auteur du Meisan zue renvoie le lecteur à cet ouvrage et annonce qu'il s'intéressera uniquement aux pierres servant de matériau à ustensiles, en laissant de côté les pièces de collection, appréciées pour leur beauté.

Parmi les pierres décrites en détail, on trouve la pierre de Teshima, nom d'une île située dans la mer du Japon, non loin de Takamatsu (préfecture de Kagawa). Il s'agit d'une pierre poreuse, connue pour sa résistance à la chaleur. On l'utilise notamment pour la fabrication des fourneaux de cuisine. Le site était exploité depuis l'époque Kamakura. L'auteur décrit en détail l'apparence de cette pierre, et la manière dont elle est extraite du sol, travaillée, puis transportée. La pierre de Mikage a des propriétés tout autres. Mikage est le nom d'un village localisé aujourd'hui dans la ville de Kōbe, où la pierre était taillée. Les carrières se situaient à l'époque d'Edo au pied des monts Rokkō, et les pierres étaient transportées sur des charrettes à bœufs. Roche granitique appréciée pour sa beauté et sa dureté, elle est destinée à de nombreux usages allant des statues bouddhiques, aux remparts, portiques et vasques de toutes tailles. La pierre de Tatsuyama, quant à elle, est une roche volcanique de la catégorie des rhyolites. Facile à tailler, mais résistante à la chaleur, son usage comme matériau de construction remonterait à l'époque des grands tertres. L'auteur mentionne ici des usages (remparts, murs, parapets, ...) qui s'expliquent par la taille massive des dalles. Le dernier paragraphe, dédié aux pierres à affûter, est particulièrement détaillé. L'auteur y déploie une grande expertise de la question. Il dresse une longue liste des pierres les plus adaptées pour chaque usage, selon le type de lame ou d'objet qu'il s'agit d'affûter 
ou de polir. Si les régions centrales autour de Kyōto jouissent d'un prestige inégalé pour la qualité de leurs pierres (uchigomori, asagi), certaines régions situées plus à l'Est ou au Nord, sont également productrices de pierres de qualité destinées à l'affûtage des sabres, et plus accessoirement des rasoirs. On observe que les progrès dans la fabrication des sabres ont été accompagnés par un approfondissement de la connaissance des pierres à affûter. Les sabres japonais pour atteindre leur coupant étaient polis au moyen de nombreuses variétés de pierres au grain de plus en plus fin.

Un autre sujet abondamment développé est celui des champignons, domaine où des écarts notables sont constatés avec les espèces connues en Chine. Comme le note Kaibara Ekiken : «Les champignons figurant dans le Bencao gangmu, ou dans le Xunpu (Traité des champignons) de Chen Renyu ${ }^{13}$, diffèrent grandement des espèces présentes dans notre pays ${ }^{14}$. Dans son Honchō shokkan (Miroir des aliments de notre empire ; 1697), Hitomi Hitsudai consacre une large place à la morphologie et aux lieux de production des espèces suivantes: matsutake, hatsutake ${ }^{15}$, shimejitake, shishitake (également appelé kawatake), shiitake, hiratake, enokitake, kikurage, iwatake, shōro ${ }^{16}$. À l'exception du dernier, ces champignons sont toujours parmi les plus appréciés de nos jours. Le matsutake, produit dans de nombreuses régions, est déjà considéré comme le roi des champignons en raison de sa chair parfumée. Des techniques existent pour les conserver au frais pendant le transport. Dans le Yamato honzō, la liste des champignons est un peu plus longue, mais les notices sont plus lapidaires et mêlent les espèces comestibles avec des espèces qui, telles le reishi (ganoderme luisant) ou le babotsu (vesse-de-loup perlée), ont un usage strictement médical. Le Meisan zue pour sa part ne recherche pas l'exhaustivité et se concentre sur quelques espèces : le saiwatake, de son autre nom reishi, un médicament considéré comme de bon augure, le shiitake, et l'iwatake. Un addenda final fait le point sur les nombreuses autres espèces consommées au Japon. L'auteur s'intéresse, dans le cas du shiitake, aux techniques de culture, différentes selon que l'on se situe dans la province de Hyūga (Kyūshū) où le champignon fut d'abord cultivé, ou dans les régions centrales. Pour l'iwatake, c'est le processus de cueillette, réalisée en escaladant des rochers escarpés, qui retient l'attention.

Dans la place qu'il accorde à l'abeille et au miel, le Meisan zue s'inscrit dans une longue tradition. Kaibara Ekiken s'attardait déjà en effet longuement sur les différents types de ruches, les imitations vendues dans le commerce, le comportement des abeilles ou encore la manière dont le miel est récolté de la ruche. Il mettait aussi son point d'honneur à rectifier l'erreur de Li Shizhen et de ses prédécesseurs qui expliquaient que le miel était le produit de la fermentation de fleurs au moyen d'excréments d'abeilles ou d'urine humaine ${ }^{17}$. L'évolution que fait apparaître le Meisan zue se situe dans le changement d'échelle intervenu dans la production. L'illustration représente un atelier où s'affairent un grand nombre de paysans, chargés chacun d'une tâche spécifique. Le comportement des abeilles comme leurs différents statuts au sein de la ruche, sont désormais mieux connus, de même que le phénomène d'essaimage. L'apiculteur dispose de techniques pour recueillir les essaims dans la nature ou pour éviter que des abeilles domestiquées aillent faire leur nid ailleurs. Si la production du miel ne constitue sans doute qu'un revenu d'appoint pour ces paysans, on perçoit néanmoins une recherche de rentabilisation dans la multiplication des récoltes sur l'année. La production de cire d'abeille constitue aussi une source de revenus pour ces producteurs. 

s'il n'a jamais été l'objet de domestication. Ce sont les oiseaux chassés dans la région d'Ōsaka qui sont considérés comme les plus savoureux. L'attention se concentre ici sur les différents modes de capture au filet. Si la notice fournit un luxe de détails sur les dispositifs utilisés, représentés au moyen de trois planches, c'est sans doute en raison de la proximité de ces terrains d'ōsaka. Les oies sauvages sont également appréciées, mais leur capture est jugée plus difficile, car elles ne se laissent pas facilement prendre au piège. L'auteur est discret sur le mode de traitement réservé aux volatiles une fois capturés. On ignore également si les chasseurs sont des villageois comme les autres ou si leur métier les place à la marge de la communauté.

Le fascicule s'achève par un long développement sur les méthodes de capture de trois sortes de gibiers destinés à des usages fort différents.

L'autour est en effet exclusivement destiné à la fauconnerie qui est une activité transmise depuis la Corée à l'époque ancienne et adoptée par l'aristocratie de cour, puis par les guerriers. À l'époque d'Edo, la possession de rapaces est un marqueur social et seuls le shogun, les daimyō et quelques vassaux, ont le droit d'organiser des chasses au vol sur des territoires réservés. À l'époque du shogun Yoshimune, dans la première moitié du xVIII ${ }^{e}$ siècle, la fauconnerie devient une véritable institution. Elle possède une administration dédiée et la pratique est ritualisée ${ }^{18}$. La description donnée dans le Meisan zue atteint un niveau de précision sans précédent ${ }^{19}$ et apporte un éclairage sur différents aspects de cette pratique. Parmi les modes de capture, c'est la chasse au filet pratiquée dans les montagnes du Shikoku et visant de jeunes oiseaux qui retient l'attention de l'auteur, probablement à cause de son exotisme. Les chasseurs qui s'y adonnent constituent en effet une population marginale ( $~$ ils ne se rasent pas le front et sont vêtus de lin même en hiver ») et la capture demande de la dextérité (" il ne s'agit pas d'une technique facile»). Le processus d'apprivoisement et de dressage de l'oiseau au moyen d'un conditionnement progressif (promenades nocturnes, alimentation carnée, utilisation de "proies de proximité ») est révélateur d'une sensibilité accrue au comportement animal. C'est un trait que l'on observe pour les deux autres espèces de gibier évoquées dans le fascicule.

La chasse à l'ours, pratiquée un peu partout au Japon, a pour but premier l'extraction de la vésicule biliaire (yūtan). La peau de l'ours, également commercialisée, ne donne lieu à aucun commentaire ${ }^{20}$ et la chair de l'animal, nous explique-t-on, est consommée uniquement à Tsugaru, dans l'extrême nord du Honshū, aux confins du Japon. La bile de l'ours est par contre un ingrédient majeur de la pharmacopée chinoise, vendu sans doute à un prix très élevé. L'auteur consacre une grande partie de la notice à l'explication d'une petite expérience qui permet de «tester » l'authenticité de la bile. Plus curieusement, il s'attarde sur la manière de fabriquer des contrefaçons. Quant aux techniques de capture, elles sont assez variées. L'ours peut être pris au piège, comme il peut être assailli dans sa tanière, mais dans un cas comme dans l'autre, l'entreprise n'est pas sans danger et fait appel à des hommes aguerris. Une certaine cruauté domine, sans qu'elle donne lieu à un sentiment de compassion de la part de l'auteur. À la différence de la chasse aux volatiles, précédemment évoquée, il s'agit d'un monde perçu comme plus lointain et sans doute moins civilisé, où les marchands d'ōsaka sont aussi moins présents. De ce fait, les produits mis sur le marché n'ont pas toujours la qualité attendue, d'où le long développement sur les contrefaçons. 


\section{Fascicule 3}

Le Japon est doté d'un milieu naturel favorable à la pêche. La richesse des eaux japonaises est due à la topographie de ce pays. L'archipel est caractérisé par l'extrême découpure $\mathrm{du}$ littoral et, de ce fait, présente une longueur côtière particulièrement importante par rapport à la superficie du pays. De plus, les rivages de l'archipel offrent d'innombrables abris pour les bateaux de pêche. C'est surtout la côte du Pacifique qui montre un enchevêtrement extraordinaire de caps, de baies et d'îles. Mais elle comporte aussi des régions hostiles à la pêche, composées de récifs abrupts et de profondeurs marines quasiment interdites. De plus, le littoral du Pacifique est fréquemment frappé par des tsunamis. En revanche, le littoral de la mer du Japon est beaucoup plus propice à la pêche. Les pêcheurs y trouvent une proximité relative de vastes fonds chalutables.

21 L'abondance et la variété des bancs de poissons qui fréquentent les eaux japonaises s'expliquent par leurs conditions hydrologiques favorables. Au Nord, un courant froid, l'Oyashiro, descend le long des côtes du continent asiatique. Ces eaux froides nourrissent de grands bancs de morue, de saumon et de hareng. Au Sud, le Japon est au contraire baigné par le courant tiède Kuroshio qui amène vers le nord bonites, thons, maquereaux, sardines et anchois. La juxtaposition et le mélange de masses hydrauliques à des températures contrastées et la composition très variée des fonds océaniques expliquent la grande abondance et l'étonnante diversité des prises. Aujourd'hui, la pêche japonaise enregistre quatre cents espèces de poissons, coquillages et crustacés. Par contre la pêche et la pisciculture en eau douce sont d'importance moindre.

Il est intéressant de remarquer que la petite pêche côtière conserve de nos jours des traits traditionnels. Elle est pratiquée par des équipes de pêcheurs montés à bord de petites embarcations. Plusieurs techniques modernes employées dans la pêche côtière ont leur origine à l'époque prémoderne :

23 1) La pêche à hameçons se pratique avec de simples lignes à partir des embarcations. Elle rapporte des poissons variés, des pieuvres et de la seiche.

2) La pêche au chalut racle les fonds de mer à proximité de la côte en utilisant des filets de 12 mètres de long.

3) La pêche de poissons migrateurs se pratique avec des filets fixes ou mobiles. Ces filets sont disposés perpendiculairement à la côte, en travers des passages des bancs de poissons. Cela concerne les maquereaux, thons, sardines et saumons. Quand ces poissons rencontrent le filet posé ou mobile, ils sont refoulés vers un filet-poche.

4) Le filet de senne joue un rôle particulièrement important. Les pêcheurs encerclent un banc de poissons avec un grand filet, puis ferment le fond avec un câble attaché à la base du filet. ${ }^{21}$ Les poissons pris au piège sont immédiatement reversés dans le bateau. Ces filets servent surtout pour capturer des poissons de surface comme la sardine et le hareng.

Notre ouvrage de 1799 nous renseigne sur deux autres techniques spécifiques : la pêche à la dorade d'Akashi à l'aide de planchettes secouées et la pêche nocturne du maquereau à l'aide des torches.

De manière générale, l'auteur traite dans ce fascicule de la capture ou de la collecte des espèces marines suivantes : les perles, la langouste, la sériole, le thon, la limande, le 
maquereau, les dorades et les huîtres. Il ne précise pas ce qui le guide dans ce choix, mais on note qu'il s'agit toujours de produits très populaires, pêchés en grandes quantités et dans diverses régions du Japon.

Dans ce fascicule, le lecteur est guidé à travers les provinces d'un point à l'autre du Japon, à la découverte de sites connus pour la production de différents poissons, crustacés et coquillages. Le texte renseigne sur les méthodes de pêche employées, notamment sur les techniques de capture et les savoir-faire anciens. Il décrit avec minutie les procédés de transformation des produits maritimes en vue de la conservation et de la consommation, notamment par salage et séchage. Chaque entrée de poisson comprend une note relative à ses différentes désignations et à leur étymologie, ainsi que des citations tirées d'ouvrages anciens de poésie. L'auteur évoque aussi des anecdotes relatives à certains produits, comme le rapport entre la crevette et les « hommes poilus du Nord", et prête une attention particulière aux usages rituels de certains produits, comme par exemple les rubans d'ormeau avec leur sens augural et leur histoire légendaire. Les ouvrages de materia medica (honzō) sont mis à contribution pour identifier les espèces.

30 Ce fascicule présente donc à la fois une dimension technique, littéraire et anthropologique. Mais c'est en premier lieu un traité technique d'une rare précision. C'est pourquoi le texte et surtout les images de ce fascicule sont cités et reproduits comme source de première main dans toute la littérature sur la pisciculture du Japon classique.

\section{Fascicule 4}

Le fascicule 4 dans son intégralité traite des poissons de mer et d'eau douce ainsi que d'autres animaux aquatiques, la plupart comestibles. On y trouve des informations non seulement sur les lieux de pêche, les modes de capture, de préparation et de consommation, mais aussi sur la qualité des différentes productions. À propos de chaque espèce, l'auteur se fait fort de discourir - sources chinoises et japonaises à l'appui -, sur les noms propres et leurs variantes locales, leur étymologie, graphies et sens différents, sans hésiter ici ou là à s'opposer à telle autorité classique, occasion de faire montre de cette érudition philologique dont se targuaient les cercles des lettrés de l'époque.

La partie la mieux renseignée, qui occupe près d'un quart de ce fascicule, est dévolue à la bonite. Est-ce le signe de la place notable occupée par ce poisson dans le régime alimentaire de la population, toutes classes sociales confondues? Si l'on en juge par la cohérence des informations que le compilateur est capable de nous livrer - en comparaison avec les renseignements quelque peu chancelants qu'il offre sur d'autres espèces -, l'hypothèse paraît probable. À condition toutefois de faire abstraction des nouveaux modes de consommation adoptés de nos jours, le plus notable étant de la manger crue. Car c'est bien la lecture attentive du tableau que nous y trouvons brossé de la vie quotidienne de cette fin du $\mathrm{XvIII}^{\mathrm{e}}$ siècle qui nous fait comprendre que les moyens de conservation et de transport de l'époque nécessitaient de tout autres préparations.

33 Ainsi l'auteur insiste-t-il, à grand renfort de références puisées dans les sources littéraires de toute époque, à commencer par les documents historiques du Japon 
ancien, sur le fait que la bonite a toujours été consommée séchée, sous forme de ces filets d'une dureté sonnante appelés katsuo-bushi qui, rabotés en flocons, bouillis et réduits, aboutissent à ce fond (dashi) qui continue même aujourd'hui de servir de base d'assaisonnement à la cuisine traditionnelle. Le processus de fabrication, de cuisson en séchage par les étapes intermédiaires de fumage et de fermentation, est décrit dans les moindres détails, les illustrations ajoutant un complément précieux à la compréhension fine du texte, allant parfois jusqu'à l'enrichir de précisions supplémentaires. Pour ce qui est des katsuo-bushi, tataki, et des produits dérivés comme les entrailles salées (shiokara) ou le bouillon concentré (nitori), force est de constater que les préparations telles que cette description les retrace sur plus d'un millénaire n'ont guère changé, à l'exception notable de la bonite séchée qui s'achète désormais déjà râpée, conditionnée en copeaux ensachés, suivant les derniers procédés de fabrication industrielle.

La tradition se perpétue aussi pour nombre d'autres produits de la mer dont il est question ici : l'holothurie (namako), l'oursin (uni), la palourde (hamaguri), les truites (masu et ayu), la lamproie (yatsume-unagi), la pieuvre (tako) et le poulpe (iidako), animaux que l'on apprête et consomme aujourd'hui encore sensiblement de la même façon que celles décrites ici. Et cela jusqu'aux entrailles - les abats de la bonite (shiokara), les viscères de l'holothurie (konowata), le corail de l'oursin, les œufs de pieuvre (heriko), tous macérés dans le sel pour la longue conservation -, gourmandises toujours très appréciées comme accompagnements du saké et qui, du fait de leur rareté, restent des produits réservés aux gourmets.

Autant les informations concernant la bonite sont claires et aisément compréhensibles - cela tiendrait-il aussi au fait que la pêche et la consommation de ce poisson sont répandues d'un bout à l'autre de l'archipel ? - autant les descriptions des différentes espèces de gobies (shirouo et gori) prêtent à confusion et présentent des difficultés à la traduction. On pourrait l'expliquer par l'aire très limitée dans laquelle ces menus habitants des lacs et rivières sont capturés, à savoir principalement la région du Kansai et les cours d'eau douce sortant du lac Biwa pour se jeter en fin de course dans la baie d'Ōsaka ; c'est du moins tout ce que notre guide semble connaître. Et sans doute ces petits poissons étaient-ils destinés à la consommation locale, immédiate, ne se prêtant ni à la conservation ni au transport. On peut ajouter que les noms locaux aussi bien que l'identification taxinomique de ces poissons devaient échapper aux savants de salon d'Ōsaka, dont les sources étaient principalement livresques.

Par contre, la description que l'on trouve des techniques et instruments de pêche est d'un intérêt ethnographique certain, d'autant que les pratiques et matériaux modernes les ont depuis longtemps relégués dans l'oubli. À en juger par le texte et l'image, ceci est particulièrement vrai des embarcations, dont la taille et l'équipement devaient restreindre la navigation à la seule pêche côtière. On admire cependant l'ingéniosité de certains dispositifs, tel ce leurre confectionné avec une dent de cachalot ou une corne de vache et garni de plumes de coq pour imiter le frétillement des sardines dont la bonite fait sa proie, ou telle planchette pourvue d'une carcasse de crabe pour piéger la pieuvre.

37 Si nombreuses sont dans ce genre d'ouvrage les citations et références, qu'une des tâches les plus ardues du traducteur est de suivre à la trace et de sonder les textes dont sont tirées les informations. Les sources dont nos bourgeois lettrés font aussi volontiers que profusément étalage sont souvent des ouvrages chinois et, exceptionnellement, 
coréens (tel le Tongŭi pogam de Hŏ Chun), qui s'imposent comme gage de crédibilité, d'autorité et de légitimité. Mais ils ont tout aussi fréquemment recours aux compositions de leurs compatriotes, encore que celles-ci se trouvent dans bien de cas être des traductions, des rééditions, des mises à jour ou des adaptations à la situation locale de classiques d'origine continentale. Mais les citations sont la preuve que dans le domaine des sciences naturelles - englobant en l'occurrence aussi bien la botanique et la zoologie que la géographie, la médecine et la pharmacopée, l'alimentation et l'hygiène, les us et des coutumes locaux, la façon de vivre et de s'habiller de la population -, les compendiums japonais, inventoriant l'état des provinces de l'archipel, ne le cèdent en rien aux modèles chinois. Tout le savoir de l'époque était contenu dans ces compilations encyclopédiques, les Shokumotsu honzō, Honchō shokkan, Honzō kōmoku, Yamato honzō, Kinmō zui et autres Wakan sanzai zue, illustrées pour la plupart, qui apparaissent comme les principaux outils de travail des auteurs de notre genre de guide. Sans oublier, non plus, les annales et chroniques anciennes (Nihongi, Kojiki), l'immense trésor de la littérature poétique (les anthologies Man'yōshū, Kokinshū, etc.), les dictionnaires de langue (Wamyōshō, Tōga) dissertant sur l'étymologie de tel mot ou nom, les histoires et topographies locales, les pharmacopées et autres materia medica, dont des lettrés comme Kimura Kenkadō disposaient dans leurs collections. Cela dit, le procédé consistant à puiser dans cette abondante littérature sans toujours vérifier à quoi la référence citée correspond dans la réalité peut aussi aboutir à une fausse science fondée sur les seuls nombre et prestige des autorités invoquées.

Si des doutes persistent sur l'auteur du Meisan zue, tout porte à croire qu'il devait appartenir à la bourgeoisie lettrée d'Ōsaka. La proportion des renseignements donnés par le Meisan zue penche lourdement en faveur des faits et pratiques localisés dans le Kansai et les régions adjacentes de la mer Intérieure. Toutes les régions situées à l'est de la ville de Kyōto (c'est-à-dire de la barrière d'Ōsaka, jadis installée sur la frontière entre les provinces de Yamashiro et d'Ōmi), sont succinctement désignées comme étant «à l'est d'Ōmi » (Kōtō 江東), les noms de la région du Kantō et de sa capitale d'Edo étant superbement ignorés. Comme l'est d'ailleurs la société des samouraïs avec ses us et ses coutumes, tout juste gratifiée, en passant, d'une seule et brève mention.

La région du Kansai, et Ōsaka en particulier, jouissant d'une solide réputation de culture culinaire, il n'est pas surprenant que les animaux aquatiques qui font l'objet de ce fascicule, soient abordés avant tout sous l'angle de la consommation. Notons que Kimura Kenkadō, quel que soit le rôle qu'il a pu jouer dans l'édition du Meisan zue, était fabricant de saké. Il est également connu pour son réseau de fins connaisseurs en toute matière qu'il recevait chez lui, et on imagine qu'à sa table les mets servis en accompagnement du saké (sakana 有), comme ceux dont il est question plus haut, ne manquaient pas d'être à l'honneur.

\section{Fascicule 5}

Le fascicule 5 du Meisan zue a un contenu à première vue assez hétéroclite : les sections sont respectivement consacrées à la méduse (comme préparation alimentaire), à la chaux (comme matériau de construction), à la porcelaine, au tissage du crêpe d'Echigo, à l'otarie (comme animal dont les organes génitaux sont prélevés pour un usage médicinal), à la laminaire (konbu), aux produits d'outre-mer, et enfin aux navires hollandais. 
41 Il est probable, même si cela n'est pas évident pour le lecteur d'aujourd'hui, que le thème unificateur $\mathrm{du}$ fascicule soit le caractère exotique des produits. L'origine étrangère est parfois explicitement soulignée : c'est le cas de la préparation de la méduse, dont il est dit qu'elle était primitivement importée de Chine et de Corée, mais qu'elle est désormais une spécialité de certaines provinces du sud du Japon. L'origine étrangère ne fait aucun doute quand il s'agit de l'otarie, un animal chassé par les Ainu d'Ezo et rarement aperçu ailleurs, des laminaires récoltées à Ezo, ou encore des cargaisons des navires hollandais. Parfois, l'origine étrangère est passée sous silence alors que l'auteur ne peut l'ignorer. C'est le cas par exemple de la porcelaine d'Imari qui a pris son envol au début du XvII ${ }^{\mathrm{e}}$ siècle, sous l'impulsion de potiers coréens déplacés lors des campagnes militaires de Hideyoshi.

On peut se demander alors les raisons qui ont conduit l'auteur à les inclure dans son livre, supposé vanter les produits renommés du Japon. Mais cette distinction entre produits d'origine japonaise et produits d'origine étrangère n'est peut-être pas le propos de notre auteur. Il s'agirait plutôt de célébrer l'excellence du travail des artisans, marchands, paysans et pêcheurs des quatre coins du Japon qui contribuent chaque jour à la prospérité de l'empire. C'est pourquoi, si Ezo ou la Hollande sont bien perçus comme des terres étrangères, il appartient aux commerçants japonais d'acheminer ces produits et de les rendre disponibles dans les régions centrales. Ce que l'auteur retient, c'est que ces productions ont un ancrage local et qu'elles font partie intégrante du paysage japonais.

L'auteur n'est pas pour autant indifférent au lien que certains produits entretiennent avec le lointain passé. Ce dernier est évoqué presque systématiquement au travers de poèmes ou de citations tirés de chroniques ou d'anthologies anciennes du Japon. Ces références aux temps des dieux ou des premiers empereurs confèrent à ces produits un prestige qui permet d'atténuer, voire de masquer la réalité que représente l'assimilation des techniques chinoises. Les ouvrages chinois, et plus particulièrement le Tiangong kaiwu (Exploitation des œuvres de la nature) de Song Yingxing, sont présents en filigrane, mais la Chine est rarement évoquée comme la source des techniques employées. Ce tiraillement est assez typique d'une époque où les lettrés sont tout à la fois pétris de culture chinoise et enclins à glorifier leur pays.

La notice consacrée à la méduse de Bizen est relativement brève. Elle se résume à la description de cet animal aquatique et des deux façons de la consommer. Dans un cas, on la fait blanchir dans de la chaux et de l'alun, c'est la méthode d'origine chinoise. Dans le second cas, elle est mise à tremper avec des feuilles de chêne pilées. L'illustration représente la pêche des méduses et leur transport dans des tonneaux jusqu'au village où elles sont mises à macérer.

La chaux fait l'objet d'une notice plus détaillée, avec une référence au caractère ancien de son usage, remontant à l'époque de l'empereur Kanmu, au viII siècle, dont le mobilier en était enduit. L'auteur cite abondamment ici, sans le nommer, le Tiangong kaiwu (Exploitation des œuvres de la nature) de Song Yingxing. La technique employée pour extraire les pierres et les cuire dans les fours emprunte clairement à la Chine, de même que la référence à la chaux obtenue en brûlant des coquilles d'huîtres. L'important est ici d'évoquer la qualité et la couleur de la pierre extraite sur le mont Ibuki, et de donner à voir le travail des ouvriers dans les carrières, un spectacle sans doute curieux pour les lecteurs du livre. 
(Exploitation des œuvres de la nature), ce qui laisse à penser que l'auteur savait parfaitement que les mêmes techniques étaient employées en Chine. Le crêpe d'Echigo qui concentre toute l'attention ici est une production récente qui a démarré au milieu du XVII ${ }^{e}$ siècle et qui connaît un grand essor à l'heure où le livre est publié, essor qui contraste avec le déclin de l'ancien centre de production de Nara. C'est sans doute cet essor récent qui justifie son statut de production renommée.

51 La récolte des laminaires pour sa part est spécifique à la région d'Ezo et ne doit rien à la Chine. Elle est attestée dès l'époque ancienne, mais elle connaît un essor remarquable à l'époque d'Edo, où les marchands des régions centrales du Japon sont installés à demeure à Ezo pour la superviser. L'auteur s'intéresse à celles qui sont ramassées 
autour du port de Hakodate, au sud de l'actuelle île de Hokkaidō, une catégorie connue aujourd'hui sous le nom de makonbu. C'est un exemple de produit qui s'inscrit à l'origine dans une sphère culturelle étrangère, mais qui est perçu comme une spécialité japonaise au seuil du xix siècle. Le sujet est traité également dans le Meibutsu zue, et s'accompagne d'une illustration (voir Fig. 1) qui pourrait parfaitement convenir pour la notice du Meisan zue qui en est dépourvue. Les deux ouvrages expliquent que la laminaire est récoltée et séchée sur place, mais qu'elle est transformée en métropole.

Fig. 8 : Les laminaires de Matsumae. Nippon sankai meibutsu zue, fascicule 4

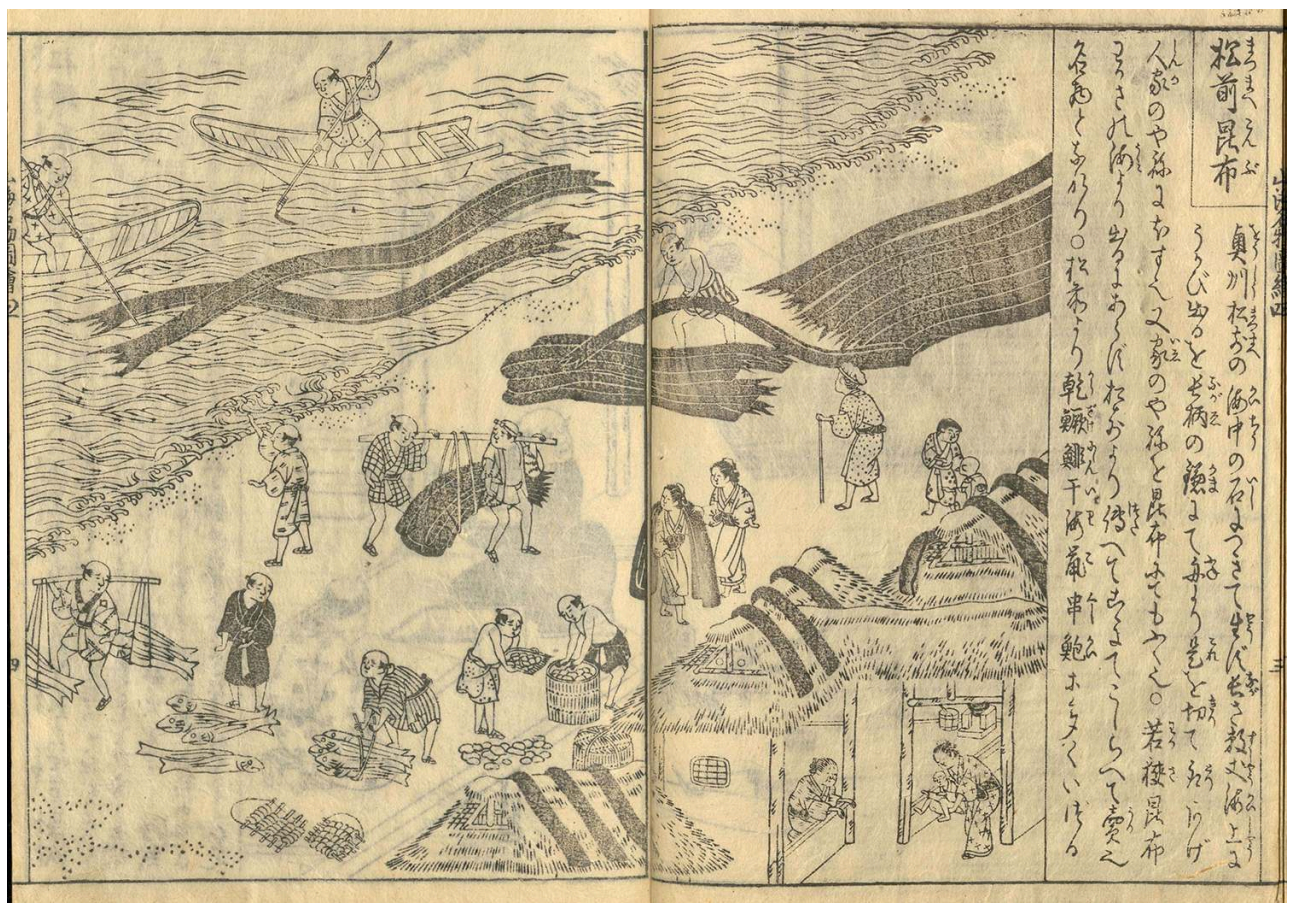

National Institute of Japanese Literature. Lien vers l'illustration

La dernière partie du fascicule n'est pas véritablement dédiée à des produits renommés. Il s'agit d'évoquer le commerce avec les Chinois et les Hollandais qui se déroule à Nagasaki et la curiosité que représente la présence de ces étrangers et de leurs bateaux.

\section{NOTES}

1. ABÉ Yoshio, «La fabrication du saké : processus biochimique et procédé technique ", in Des mérites comparés du saké et du riz, BÉRANGER Véronique, BRISSET Claire-Akiko et LEGGERI-BAUER Estelle (éd.), Paris, Diane de Selliers, 2015, p. 233-237.

2. Nihon shoki, fasc. 2, vol.67, Tokyo, Iwanami shoten, coll. "Nihon Koten Bungaku Taikei», p. 152-156 ; ASTON William George (trad.), Nihongi Chronicles of Japan, Tokyo, Charles Tuttle Comp., 1972, p. 83, 86 ; Man'yōshu, poème n 202, fasc. 2, in Man'yōshū, SATAKE Akihiro, YAMADA Hideo, KUDō Rikio, ōTANI Masao, YAMAZAKI Yoshiyuki (éd.), vol. 1, Tokyo, Iwanami shoten, coll. «Shin Nihon 
koten bungaku taikei », p. 149-150 ; ANTONI Klaus, Miwa - der heilige Trank, Wiesbaden, Franz Steiner Verlag, 1988.

3. Ritsuryō, 'Shikiin-ryō' 47. Voir Ritsuryō, Inoue Mitsusada et al. (éd.), Iwanami shoten, coll. « Nihon shisōtaikei » 3, 1982, p. 182.

4. Engishiki, fasc. 1-4, Fukyūhen, vol. jō, Tokyo, Yoshikawa kōbunkan, coll. « Shintei zōhō kokushi taikei », 1974, p. 9-97 et fasc. 40, Fukyūhen, vol. 3, coll. « Shintei zōhō kokushi taikei », p. 886-896.

5. ono Masatoshi, GOMI Fumihiko, HAGIHARA Mitsuo (éd.), Utage no chūsei : ba, kawarake, kenryoku (Le Moyen Âge des banquets : lieux, vaisselle, pouvoir), Tokyo, Kōshi Shoin, 2008 ; pour le Chōrakuji, voir NAKAI Atsushi, "Kyōen bunka to hajiki », in Utage no chūsei, ono Masatoshi (éd.), ibid., p. 134-140; pour les monastères Zen, voir l'exemple du Daitokuji en 1599, dans Dainihon komonjo, vol. Iewake 17, p. 3 : une commande de 135 litres de saké pour un seul dîner.

6. GAY Suzanne, The Moneylenders of Late Medieval Kyoto, Honolulu, University of Hawai'i Press, 2001.

7. YoSHIDA Hajime, Sake, Tokyo, Hōsei daigaku shuppankyoku, coll. « Mono to ningen no bunkashi »172, 2015, p. 134-138. Voir également BAUMERT Nicolas Le saké, une exception japonaise, Rennes, Presses universitaires de Rennes, 2011, p. 163-164.

8. YosHIDA, Sake, ibid., p. 154-156.

9. Voir l'introduction du présent ouvrage.

10. Voir YosHid, Sake, ibid., p. 156.

11. Le saké de Nara était apprécié notamment par les seigneurs de la guerre à l'époque des guerres civiles de la fin du XVI ${ }^{\mathrm{e}}$ siècle. YOSHIDA, Sake, ibid., p. 105. BAUMERT, Le saké, une exception japonaise, op. cit., 2011, p. 144-146.

12. "The criteria for distinguishing between these four lei are sometimes the same as those a layperson currently makes in daily life between metals, precious stones, minerals, and salts, on the grounds partly of their appearance, partly of their use, and partly of the value society attributes to them, but it seems difficult, if not impossible, to find generally valid criteria." MÉTAILIÉ Georges, "The Bencao ganmu of Li Shizhen: an innovation in natural history?", in Innovation in Chinese Medicine, HSU Elisabeth (ed.), Cambridge, Cambridge University Press, 2001, p. 230.

13. Lettré de l'époque des Song du sud, sur lequel on connaît peu de choses. Son Traité des champignons, daté de 1245 d'après la préface, décrit onze champignons de la région de Taizhou, dont l'auteur est originaire.

14. Yamato honzō, KAIBARA Ekiken, in Ekiken zenshū (Euvres complètes d'Ekiken), vol. 6, Tokyo, Kokusho kankōkai, 1973, (première édition 1911), p. 235.

15. Lactarius hatsudake, champignon consommé dans la région d'Edo, où les matsutake étaient plus rares.

16. Rhizopogon roseolus, champignon abondamment consommé, produit un peu partout au Japon, si l'on en croit l'auteur, mais devenu aujourd'hui une denrée rare. Voir Honchō shokkan, vol. 1, op. cit., p. 233-243.

17. Yamato honzō, KAIBARA, op. cit., p. 361.

18. NESAKI Mitsuo, Inu to taka no Edojidai: "Inu kubō" Tsunayoshi to "Taka shōgun" Yoshimune (L'époque d'Edo des chiens et autours : le shogun des chiens Tsunayoshi et le shogun des autours Yoshimune), Tokyo, Yoshikawa kōbunkan, 2016, p. 114-163.

19. Notons toutefois que le Honchō shokkan s'intéressait déjà à cet oiseau. HITOMI Hitsudai, SHIMADA Isao (trad. et éd.), Honchō shokkan (Miroir des aliments de notre empire), vol. 3, Tokyo, Heibonsha, coll. « Tōyō bunko », p. 142-143.

20. Il semblerait qu'à la différence des Chinois, les Japonais n'aient pas eu une attirance particulière pour les fourrures. 
21. HuetZ DE LemPS Alain, «L'expansion de la pêche japonaise ", Cahiers d'outre-mer, vol. 13, n 49, janvier-mars 1960, p. 5-27 ; TAJIMA Yoshiya, « Kinsei Kishū gyohō no tenkai », in Seisan no gijutsu, HAYAMA Teisaku (éd.), Tokyo, Chūō koronsha, « coll. Nihon no kinsei » vol. 4, 1992. 
Partie II - Traduction du Guide illustré des produits renommés des monts et mers du Japon 


\section{Préface}

Traduction : François Lachaud

Depuis longtemps, les honnêtes hommes se sont pour l'essentiel appuyés sur la materia medica (honz $\overline{0}^{1}$ ) dans leur étude des produits des régions. Ils ont identifié ceux des montagnes et ceux des mers, sans négligence ni oubli. Telle est l'opinion commune. Depuis [la génération] de Kō Kansui ${ }^{2}$ (1609-1677) et Tō Jakusui ${ }^{3}$ (1665-1715) jusqu'à Shō Igan $^{4}$ (1668-1746) et Tō Hōsui ${ }^{5}$ (1701-1755), il ne manque pas de savants talentueux en ces matières. Moi-même, j'ai très tôt profité de leurs lumières, et, durant plusieurs décennies, au terme de patients efforts, je suis parvenu à reconnaître ce qui était encore inconnu des hommes, et à distinguer ce qui jusqu'alors échappait à la classification. Au vrai, je ne ménageai point mes efforts en ma quête des choses cachées et ma recherche des choses singulières. J'avais, voici un certain temps déjà, ouï-dire que Tō Jakusui avait composé un ouvrage intitulé Saiyaku dokudan (Mes vues sur les collections de simples) ouvrage dans lequel il exprimait avec clarté ses conceptions, tel qu'à son ordinaire. Pourtant, en conçut-il quelque regret ou repentir, le voulut-il garder secret? Ou bien encore l'avait-il conservé en quelque recès obscur parmi les monts? Toujours est-il que ce texte ne nous est pas parvenu. C'est fort regrettable. D'ailleurs, je ne laissai en mon for intérieur de m'en lamenter. C'est pourquoi je pris en secret la décision de suivre son modèle, et composai ainsi plusieurs livres d'un ouvrage à venir. Je lui donnai pour titre Meibutsu dokudan (Mes vues sur les spécialités renommées). Je ne ménageai pas mes labeurs et ajoutai détail après détail. J'avais l'impression d'être parvenu à une source dont les eaux couleraient sans la moindre cesse. Telle fut la raison pour laquelle je décidai que, des infinies variétés des produits de la nature, je me contenterai, au fil des saisons, de ne retenir que les produits les plus propices à la conservation pour en exposer les modes de fabrication. Hélas! À peine avais-je mis la dernière main au manuscrit que ma demeure fit l'épreuve de nombreux troubles auxquels se devaient ajouter incendies et avanies diverses, tant et si bien que mon texte fut livré aux quatre vents et presque réduit en cendres ${ }^{6}$. Aujourd'hui encore, tout comme au moment de cette catastrophe, je ne puis songer à cet événement sans une grande amertume. Par hasard, un certain-libraire-imprimeur me fit mander par l'un de ses employés un livre illustré, et, avec la dernière des politesses, me demanda d'en bien vouloir composer la préface. L'ouvrage a pour titre Sankai meisan zue (Guide illustré des produits renommés des monts et mers). L'ayant pris en main, à mesure que j'avançais dans sa lecture, il me sembla que le livre traitait, pour chacun des produits 
régionaux de notre pays $^{7}$, des variétés rares et des saveurs étranges et que les spécialités les plus renommées faisaient toutes l'objet d'une illustration. Par ailleurs, les détails de la fabrication [de ces produits], l'attestation historique et philologique de leur existence faisaient également l'objet d'élucidations ajoutées à la fin [de chaque entrée]. Le texte se donnait pour ambition d'en faciliter l'accès aux femmes et aux enfants ${ }^{8}$. C'était comme une réponse à ce que j'avais désiré autrefois. Les illustrations étaient de la main de mon regretté ami Shitomi Kangetsu (1747-1797). Sans doute est-ce à ce moment que je pris conscience qu'il m'était impossible de ne pas rédiger cette préface. Voici donc les raisons pour lesquelles j'ai exposé ici à la fois les dispositions qui jadis avaient guidé mon désir d'exprimer mes opinions sur ces sujets, et les origines du présent livre. Que dire si l'on songe que les graines (de) moutarde brune ${ }^{9}$, tout comme l'ambre, la pierre d'aimant aussi bien que le fer, trouvent mêmement leur principe dans la nature dont elles procèdent. Le principe en est insaisissable mais il n'en est que plus vigoureux. Entre ciel et terre, une variété infinie de produits existe. Il est essentiel d'en rendre compte de la manière la plus étendue. Depuis les cent remèdes jusqu'aux plus infimes produits alimentaires, leur collecte ne tolère aucune erreur. Que dire alors de l'homme de bien qui dévoile les secrets du monde et les partage avec ceux [qui vivent] sous le ciel?

2 Première décade de la douzième lune, année de l'aîné terre cheval ${ }^{10}$ de l'ère Kansei (1798).

3 Kimura Kōkyō [Kenkadō] scripsit.

\section{NOTES}

1. Par le terme honzō (c. bencao), l'auteur renvoie ici au domaine des savoirs relatifs aux substances entrant dans la composition des remèdes. Les substances sont d'origine végétale, minérale ou animale. Les premiers traités chinois remontent à l'époque des Han. Le Classique fondateur a pour nom Shennong bencaojing, le Classique de la materia medica du divin laboureur.

2. Il s'agit du nom sinisé du lettré confucéen de Nagasaki, Mukai Genshō. Médecin et bon connaisseur de la pharmacopée, il est l'auteur du premier traité japonais de honzō. Il terminera sa carrière de médecin à Kyōto.

3. Il s'agit d'Inō Jakusui, lettré-médecin originaire d'Edo. Il est connu pour avoir conçu le plan de son monumental Répertoire des myriades de substances (Shobutsu ruisan), en réponse à la demande de son seigneur, Maeda Tsunanori de Kaga. L'ouvrage inachevé au moment de son décès sera achevé sous le gouvernement du shogun Tokugawa Yoshimune.

4. Il s'agit de Matsuoka Jo'an, également connu sous le nom de Gentatsu, lettré-médecin, ayant formé de nombreux spécialistes de honzō dans son école de Kyōto. Il est appelé à Edo, par Tokugawa Yoshimune, pour collaborer à l'entreprise d'inventaire des substances (bussan) du Japon.

5. Il s'agit de Tsushima Tsunenoshin, connu pour avoir organisé à un rythme annuel des expositions de produits régionaux à Ōsaka. C'est aussi le maître de Kimura Kenkadō en science du honzō.

6. Kimura fait ici allusion au revers que sa fortune connaît en 1789 . Voir notre introduction. 
7. Tōhō, littéralement régions de l'Est, signifiant ici le Japon.

8. C'est une manière conventionnelle de dire que l'imprimeur a adopté un style de graphie où les kanjis sont accompagnés de leur lecture en syllabaire.

9. Également appelée : moutarde indienne, moutarde chinoise (l. Brassica juncea).

10. Il s'agit de la dixième année de Kansei. 


\section{Fascicule 1}

PI. 1 : Poème du saké augural.

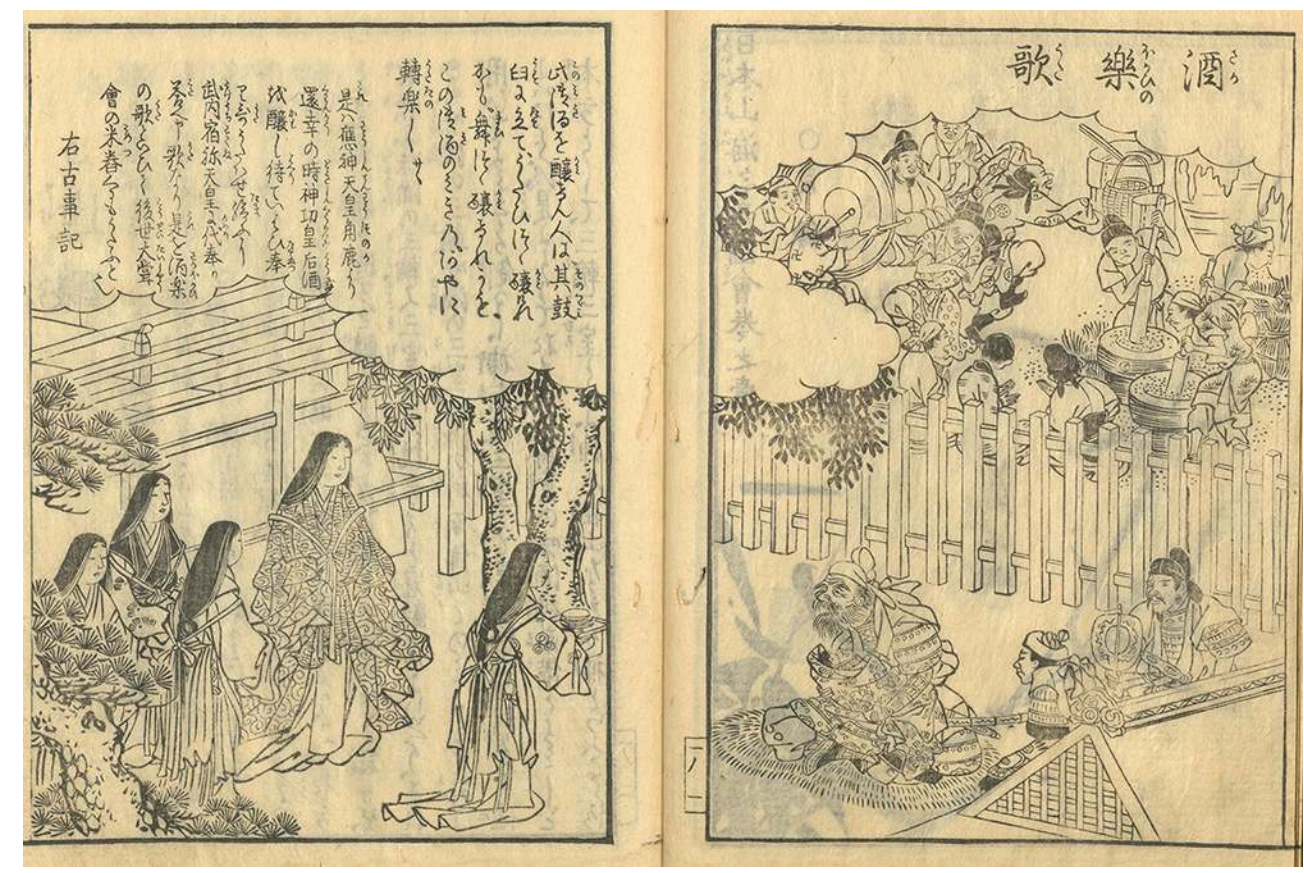

National Institute of Japanese Literature. Lien vers l'illustration

Ceux qui ont préparé le saké divin, ont dressé des tambours, ils ont dressé des mortiers ; ils chantent, voilà qu'ils l'ont préparé, ils dansent, voilà qu'ils l'ont préparé, ce saké divin, ce saké divin, quelle merveille, quel délice!

Lorsque l'empereur Ōjin ${ }^{1}$, arriva à Yamato par Tsuruga ${ }^{2}$, la reine Jingū fit brasser du saké pour l'honorer et lui présenta un poème. Alors Takeuchi no sukune lui offrit en réponse de la part du jeune souverain, ce poème sur le saké augural. Le poème en vint par la suite à être chanté lors du pilage du riz pour les Grandes prémices des souverains. Poème consigné dans le Kojiki (Chroniques des choses anciennes) ${ }^{3}$. 


\section{Fabrication du saké}

2 Le sakét doit certainement être une fabrication des Saints de l'Antiquité. Selon le Jiupu (Traité du vin) de Dou Ge 幊革 des Song ${ }^{5}$ son origine serait incertaine. Au Japon, sake 酒 se lisait jadis $k i$, c'est-à-dire $k e$ 饌 la collation. Le son $k e$ vient du chinois $k i$ 気, le souffle. (Il est fréquent que la lecture chinoise d'un caractère soit adoptée comme lecture japonaise. C'est le cas par exemple de l'ustensile $k i$ 器 qui est lu ke en japonais). Le saké, présenté aux dieux ou offert au souverain, est appelé miki 御酒 en signe de déférence. Par ailleurs, par les noms de "saké noir » et « saké blanc », on entend respectivement le saké clair et le saké trouble.

3 La lecture japonaise sake est une abréviation de ma-sa-ke, sa étant un terme auxiliaire et ke une lecture alternative de $k i$. Il existe une autre désignation, miwa: comme "fabriquer le saké » se dit kamosu, on a supprimé le son ka de kamosu de manière à ce que le son $m i$, qui signifie "bouquet", vienne en tête du nom miwa. Le Kanjikō (Réflexions sur les mots initiateurs) affirme que le terme "saké exquis " (umasake) que l'on trouve devant les noms de lieu Miwa ou Mimuro dans la poésie ancienne est un mot initiateur 6 . Toutefois, la poésie ancienne n'associe le terme «saké exquis » qu'à Miwa, Mimuro ainsi qu'au mont Kan'nami, et à aucun autre lieu'. Kan'nami et Mimuro ne sont que des noms différents du mont Miwa et rien d'autre. On peut en déduire que le poème du Man'yōshū (Recueil des dix mille feuilles) ${ }^{8}$, où figure l'expression « umasake no Kan'nami ", est le poème le plus ancien. Les termes Miwa et Mimuro renferment eux aussi l'idée sous-jacente de divinité (kami) puisqu'ils désignent une montagne où la divinité réside et c'est ainsi qu'on les chantait dans les poèmes. (Il en va de même lorsqu'on chante dans les poèmes, au lieu de la "puissante divinité » (chihayahuru kami), le "puissant kamo» (chihayaburu kamo) ou le "puissant » homme (uji)). Ce n'est donc pas sans raison que l'on considère le dieu Matsunoo de Miwa comme le dieu fondateur du saké. Selon le Nihongi (Chronique du Japon) ${ }^{9}$, à la huitième année du règne de l'empereur Sujin, Ikuhi du village de Takahashi fut désigné préposé au saké du dieu $\bar{O}$ miwa ${ }^{10}$, et à la douzième lune, Ōta Taneko reçut l'ordre de présenter l'offrande de saké au dieu Ōkunitama de Yamato. Ōkunitama est Ōmononushi, le dieu de Miwa. C'est, semble-t-il, à cette occasion que fut créée la fonction de préposé au saké, et ce fut le début de l'offrande de saké aux dieux ${ }^{11}$. (Cette tradition est à l'origine de l'utilisation du cryptomère comme enseigne par les fabricants de saké ${ }^{12}$ ). Plus tard, à l'époque de l'empereur Ōsazaki ${ }^{13}$, arrivèrent des royaumes de Corée les frères Sohori, spécialistes de la fabrication du saké. On leur conféra le titre de Maro et le surnom de Jeunes gens Sakami. Ils reçurent des princesses de Yamaka pour épouses et celles-ci furent nommées Demoiselles Sakami. C'est ainsi que sont apparus les noms des clans Sakami et Sakabe. Depuis lors les procédés de fabrication du saké n'ont cessé de se perfectionner et aujourd'hui aucun saké au monde n'égale celui du Japon. En effet, notre pays béni des Dieux est celui où pousse le riz de la plus haute qualité. Entre tous, le saké d'Itami de la province de Settsu ${ }^{14}$ est réputé comme le plus exquis. Ce saké est acheminé partout, par voie de mer ou par voie de terre, et fait l'objet de commandes des autorités. Aussi, les brasseurs d'Itami ont reçu l'autorisation d'apposer un sceau officiel sur leur production. Encore aujourd'hui, dans les provinces lointaines, les sakés de première qualité (morohaku) ${ }^{15}$ ne sont désignés que par le nom d'Itami. On peut donc considérer que le saké de qualité supérieure du Japon commence avec Itami et ne remonte pas à une époque si ancienne. On a commencé à produire ce saké dans les 
années Bunroku et Keichōo ${ }^{16}$, et ce sont les hommes de la famille Yamanaka du village de Kōnoike ${ }^{17}$ près d'Itami qui les premiers l'ont vendu à Edo. Au début, ils ne brassaient guère que $5 \mathrm{to}^{18}$ à $1 \mathrm{koku}^{19}$ et le vendaient par colportage. Lorsque la production atteignit 20 à $30 \mathrm{koku}$, la vente dans les provinces voisines ne permit plus d'écouler le surplus, si bien que, le chargeant sur des chevaux, ils l'emmenèrent pour en faire commerce dans la lointaine Edo. Ce qui leur rapporta des bénéfices inattendus et, chargeant les sommes obtenues sur le dos de leurs chevaux, ils s'en retournèrent chez eux. Edo devenant un marché de plus en plus florissant, les fabricants purent y vendre d'énormes quantités et devinrent immensément riches. Ensuite apparurent les maisons Inateraya et Masuya installées à Itami. Le transport par bateau fut initié par la maison Manganjiya d'Ikeda ${ }^{20}$ et les producteurs de saké se multiplièrent. Aujourd'hui on fabrique de l'excellent saké non seulement à Itami et à Ikeda, mais également à Nishinomiya, Hyōgo, Nada et Imazu ${ }^{21}$, de la même province de Settsu. À part ça, nombreux sont les fabricants qui se firent un nom dans d'autres provinces: tous ces sakés diffèrent selon les particularités du terroir et les savoir-faire si bien qu'il y a autant de saveurs que de visages humains et il est impossible d'en rendre compte en détail. Depuis cent trente ans, on obtient le saké clair exclusivement par pressurage, tandis qu'auparavant il était simplement filtré à l'aide d'un égouttoir en bambou.

Aujourd'hui, on fabrique :

- le «saké nouveau » (fabriqué à partir de la période de l'équinoxe d'automne ${ }^{22}$ ),

• le « saké intermédiaire » (entre le « saké nouveau » et celui « d'avant les froids »),

- le « saké d'avant les froids » et

- le « saké des froids ». (Plus la saison avance, plus la durée de fabrication est longue et le prix élevé).

Parmi toutes ces variétés, le «saké nouveau » d'Itami est un produit particulièrement réputé pour son bouquet subtil. On choisit un jour faste dans la période de l'équinoxe d'automne à la huitième lune, et, quatre jours auparavant, on commence à laver le riz destiné à la fabrication du kōji qui se fera le jour faste (depuis peu, on commence à la neuvième lune $e^{23}$, autour des premières gelées).

\section{Le kōji de saké (sake kōji)}

6 Jadis le blé était utilisé pour faire le kōji, ce qui explique que la graphie du caractère kōji 麦米 contient la clé du blé 麦. Par rapport à la méthode japonaise, très commode, le procédé chinois de fabrication est très compliqué.

7 Au matin du quatrième jour avant le jour choisi, on lave le riz et on le fait tremper dans l'eau pendant toute la journée. Le jour suivant, on le cuit à la vapeur, puis on le dépose sur des nattes de paille sur lesquelles on le remue et l'étale de façon égale à l'aide d'un râteau. Quand on juge que la masse est à la température corporelle, on transfère l'ensemble dans des cuves en bois (sorte de boîtes à riz cuit, toko) ${ }^{24}$. On recouvre alors les cuves de nattes de paille qu'on place ensuite dans une chambre de fermentation en argile pendant une demi-journée environ. À l'heure du cheval ${ }^{25}$, on écrase les grumeaux de la masse et on y ajoute de la moisissure de riz (moyashi) à raison de 2 gō de moisissure pour 1 koku de riz. À la huitième heure ${ }^{26}$ de la nuit, on retire toute la masse des cuves et on la dispose en monticules sur des plateaux à kōji que l'on superpose par dix. Le lendemain matin, on retourne une fois la masse dans chaque plateau, puis le soir, on l'étale en l'égalisant sur toute la surface des plateaux. On superpose les 
plateaux alternativement dans le sens de la longueur et dans le sens de la largeur. À la septième heure ${ }^{27}$ du jour suivant, on obtient un kōji jaune et blanc.

\section{Moisissure de riz (moyashi) $)^{28}$}

Il faut impérativement utiliser du riz d'une récolte ancienne. On le cuit à la vapeur, puis on incorpore de la cendre d'orme à raison de 2 gō environ pour un volume d' 1 shō de riz étuvé. On enveloppe le tout dans plusieurs épaisseurs de nattes de paille et on l'entrepose pendant dix jours sur des étagères dans une chambre de fermentation [voir Pl. 3]. Dès l'apparition de la moisissure, on forme avec l'ensemble de la masse des monticules sur des plateaux à kōji, que l'on mélange et égalise par la suite à deux reprises en remuant la masse au sein de chaque plateau, pour obtenir la moisissure de riz.

\section{PI. 3 : Fabrication du kōji de saké}

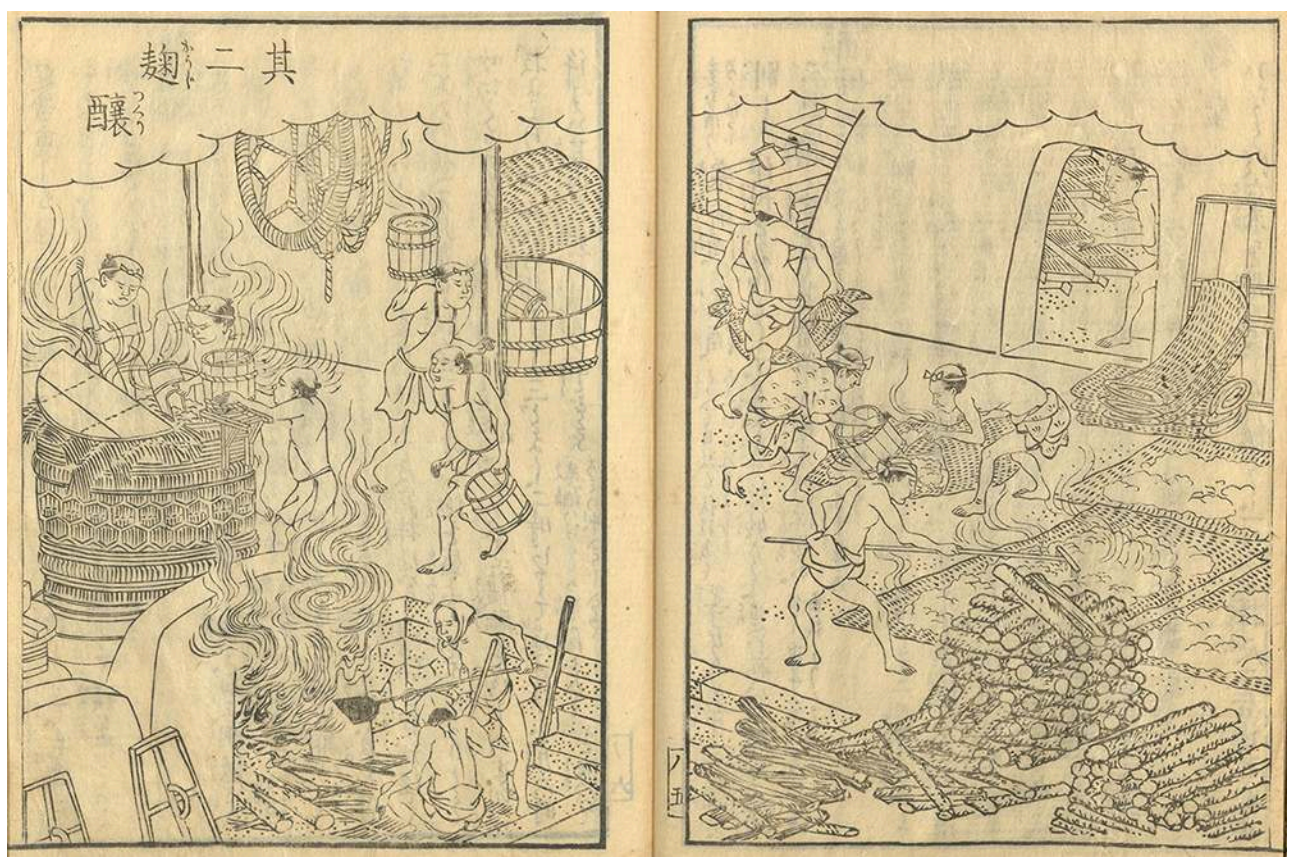

National Institute of Japanese Literature. Lien vers l'illustration

\section{Levain (moto) ${ }^{29}$}

5 to de riz correspondent à une portion de levain. On appelle un «cycle" (shimai) l'achèvement des opérations se déroulant sur une journée pour produire un moto. Pour ce qui est du nombre de moto produits, il dépend du statut du producteur.

Trois jours avant la date fixée ${ }^{30}$, on sort le riz des réserves. Le lendemain matin, on le lave puis on le met à tremper dans l'eau. Le matin suivant, on cuit le riz à la vapeur, on le dépose sur des nattes et on le laisse bien refroidir, puis on le répartit dans huit cuveaux (ou six cuveaux dans le cas du «saké des froids ») [voir Pl. 4]. On ajoute 1 to 7 shō de kōji et 4 to 8 shō d'eau respectivement pour un volume de 5 to de riz (mais les proportions varient selon les fabricants). Il faut compter environ une demi-journée pour que l'eau soit absorbée, puis on remue la masse à la main. Cette opération 
s'appelle «le travail à la main ». Le soir venu on écrase les grumeaux à l'aide d'une "pelle à mélanger ", cela s'appelle " défaire la montagne ». À partir de ce moment, on remue la masse toutes les deux heures, nuit et jour. (Cette opération s'appelle «le travail »). Trois jours plus tard, on rassemble le tout dans une cuve d'une capacité de $2 k o k u^{31}$. Au bout de trois jours, l'écume monte à la surface, cela s'appelle «la montée » agari ou « le souffle coupé » fukikiri. (Saisir ce moment est particulièrement important et exige un savoir-faire tout à fait remarquable $)^{32}$. On répartit alors cette masse dans deux cuveaux et une cuve de 2 koku, c'est-à-dire dans trois récipients au total, que l'on recouvre quatre heures plus tard avec des nattes de paille. Au bout de douze heures, lorsque l'on constate la chaleur naturelle qui s'en dégage (dans le cas du «saké des froids ", on procède en mettant de l'eau à chauffer dans une cuve que l'on verse dans l'épais liquide fermenté moromi), on remue le tout pendant deux ou trois jours avec une pelle à mélanger pour faire refroidir la masse. Là encore, on mélange à raison d'une fois toutes les deux heures. Le produit ainsi obtenu s'appelle le levain. ${ }^{33}$

\section{PI. 4 : Fabrication du levain de saké}

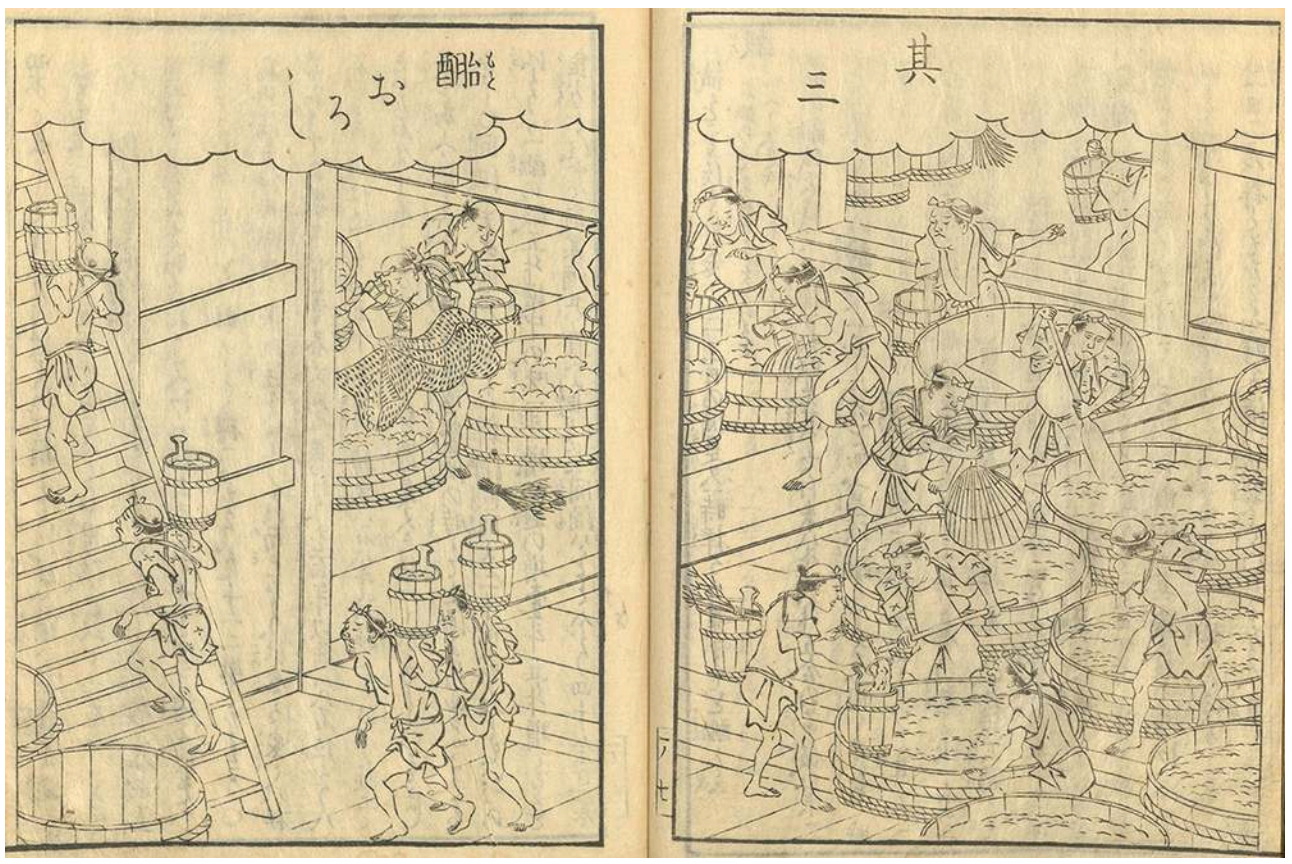

National Institute of Japanese Literature. Lien vers l'illustration

\section{Ajouts $^{34}$}

Les « ajouts » sont les opérations qui consistent à ajouter du riz, du kōji et de l'eau au levain ; c'est ce qu'on appelle « l'ajout du riz pour former le bouquet du saké ».

On rassemble la totalité du levain obtenu précédemment dans une cuve de 3 pieds de diamètre ${ }^{35}$. On y ajoute du riz étuvé obtenu à partir de 8 to 6 shō 5 gō de riz blanchi ${ }^{36}$ et du kōji obtenu à partir de 2 to 6 shō 5 gō de riz blanchi, après quoi on ajoute encore 7 to 2 shō d'eau. Cela s'appelle le "premier levain » hitomoto. On mélange ensuite la masse pendant un jour et une nuit, toutes les deux heures. Le troisième jour est appelé « milieu » (naka) [voir Pl. 5]. 


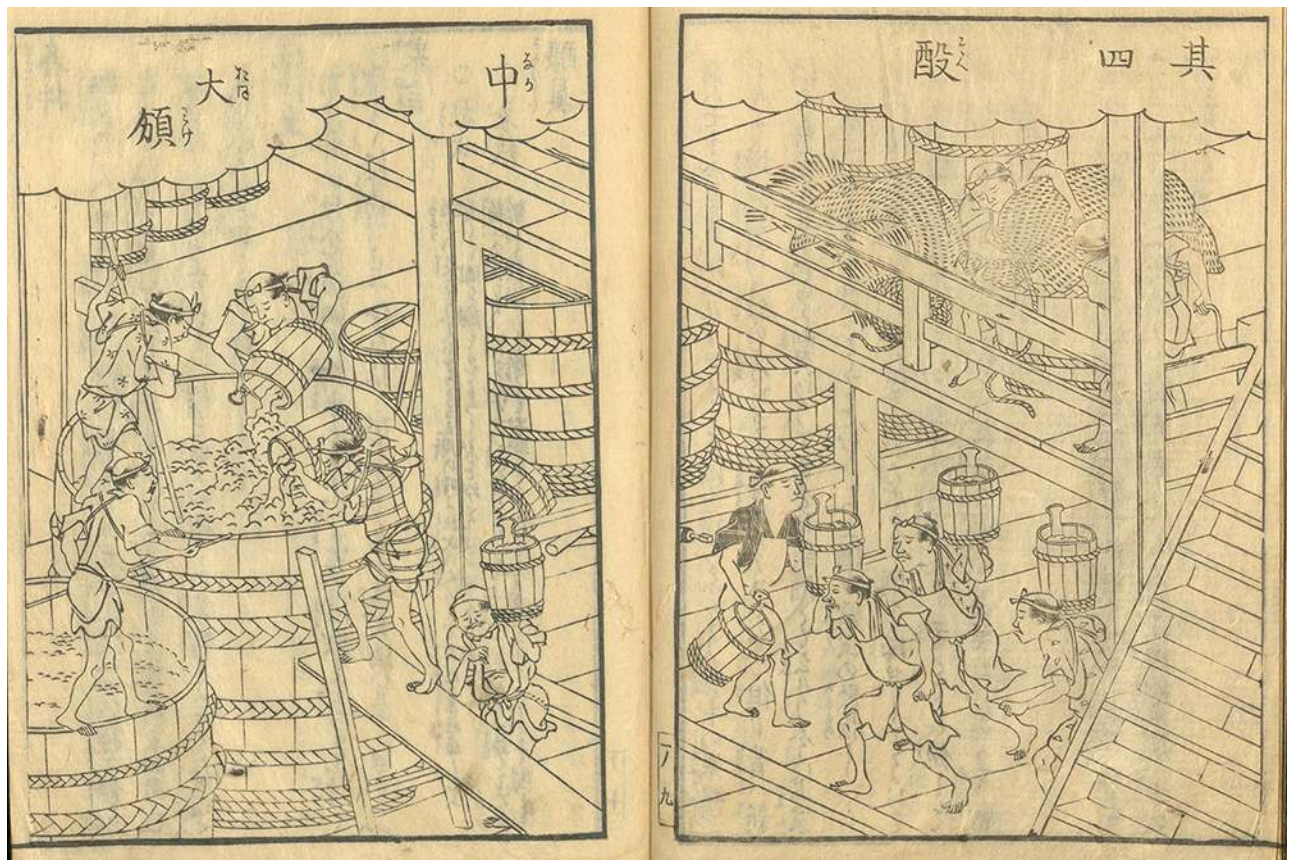

National Institute of Japanese Literature. Lien vers l'illustration

11 À ce moment, on répartit le tout dans deux cuves de 3 pieds de diamètre, et on y ajoute encore du riz étuvé obtenu à partir d'1 koku 7 to 2 shō de riz blanchi, du kōji obtenu à partir de 5 to 2 shō 5 gō de riz blanchi, et 1 koku, 2 to 8 shō d'eau. Puis on reprend l'opération consistant à mélanger toutes les deux heures. Le jour suivant, on sépare le contenu de chaque cuve en deux parts que l'on verse respectivement dans deux cuves. Cela s'appelle le " grand partage $"^{37}$. On continue à mélanger toutes les deux heures. Le jour suivant on ajoute : la quantité de riz étuvé obtenue à partir de 3 koku 4 to 4 shō de riz blanchi, la quantité de kōji obtenue à partir d'1 koku 6 to de riz blanchi, et un volume d'eau d'1 koku 9 to 2 shō. (Le volume obtenu correspond à vingt-cinq fois la contenance d'un cuveau-versoir de $8 s^{-38}$ ) [voir Pl. 3 et 4]. C'est ce qu'on appelle la fin du cycle des opérations (shimai) ${ }^{39}$. Le volume total de riz utilisé, riz et levain confondus, s'élève à $8 \mathrm{koku}, 5$ to, et le volume d'eau 4 koku et $4 \mathrm{to}^{40}$. On laisse reposer deux, trois voire quatre jours dans l'attente qu'il se produise un bouillonnement. Il est important de saisir ce moment pour commencer à remuer la masse. ${ }^{41}$ On la laisse refroidir doucement en la remuant toutes les deux heures et lorsque la masse achève de refroidir, on ne la remue plus que deux fois par jour. À ce moment-là, on considère que le saké est fait. On répartit alors le liquide dans quatre cuves de 3 pieds de diamètre. Après huit à neuf jours, on puise le liquide ${ }^{42}$ à l'aide de cuveaux puisoirs et on le verse dans des sacs, au nombre de trois cents à cinq cents à chaque fois, dont on remplit un pressoir (fune) [voir Pl.6]. On attache de nombreuses pierres à une poutre et on presse progressivement le liquide pour obtenir du saké clair. Du pressoir, le saké est versé dans une énorme cuve de clarification appelée «cuve à 7 pouces $»^{43}$. Après quatre ou cinq jours se forme un dépôt appelé « dépôt de lie brute » ${ }^{44}$. Enfin on verse le saké dans des fûts de 4 to, sachant que 7 to et 5 shō correspondent à une charge de cheval, ce qui nous donne deux fûts ${ }^{45}$. L'ensemble s'élève à onze ou douze charges ${ }^{46}$. La méthode décrite ci-dessus est celle d'une seule maison à Itami. Pour les autres fabricants, ils ont chacun leur secret, mais les proportions sont approximativement les mêmes. Il y a cent 
ans, on utilisait pour l'ajout entre 8 koku et 8 koku 4 o $5 t^{47}$; il y a quarante à cinquante ans, un volume de riz poli de 8 koku 8 to était considéré comme la quantité optimale. Aujourd'hui on considère que la perfection est atteinte avec 9 à 10 koku de riz. Mais il est impossible d'énumérer toutes les évolutions intervenues au cours du temps, tant elles sont nombreuses.

PI. 6 : Mélange de la masse moromi, mise en sacs, pressurage, obtention du saké, clarification

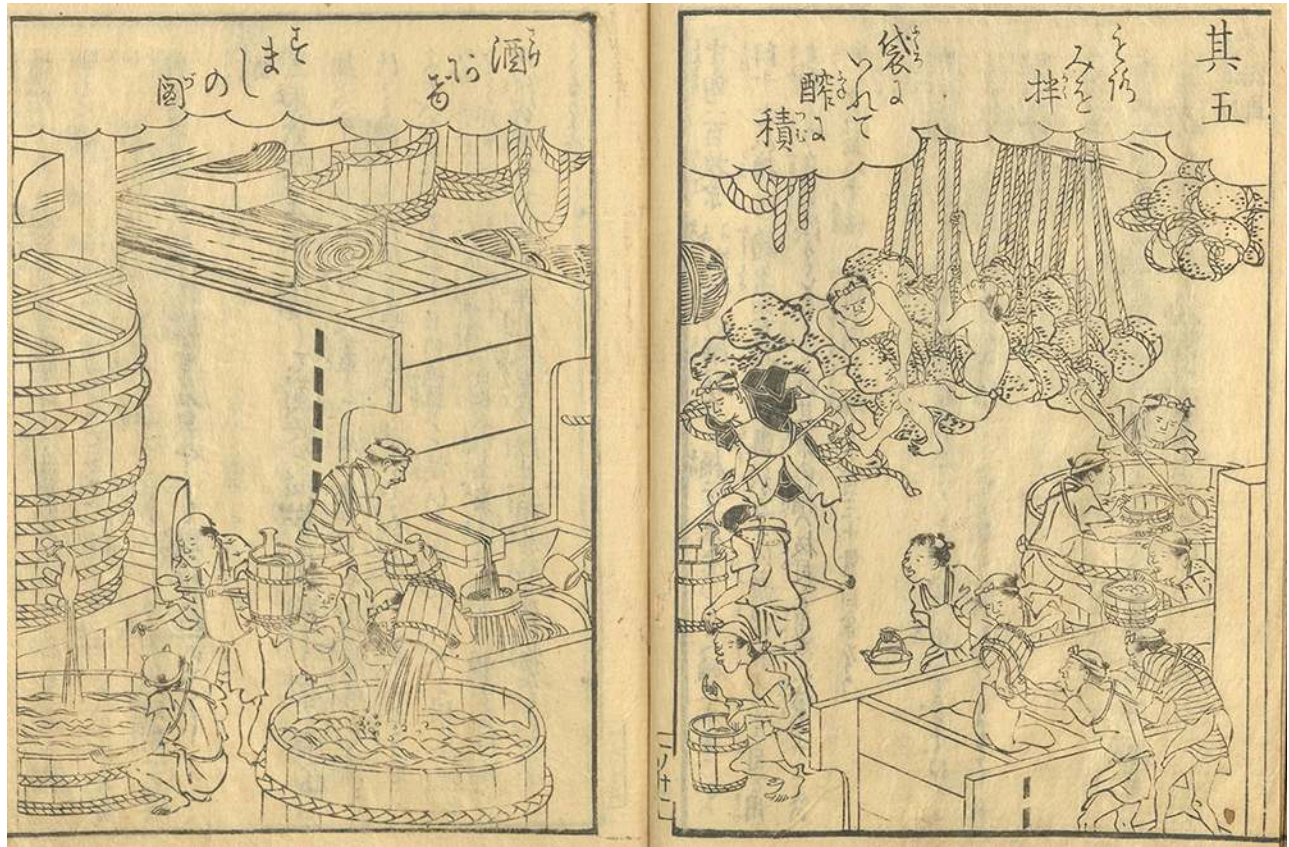

National Institute of Japanese Literature. Lien vers l'illustration

On ajoute des cendres pour clarifier le saké, quand il est de médiocre qualité, insipide ou encore altéré, mais jamais pour le saké supérieur. Pour le "saké d'entre-deux », on augmentait autrefois l'ajout de 3 to, tout comme pour le « saké nouveau $»^{48}$. Cependant, depuis une époque, on a jugé bon d'augmenter de 5 shō l'ajout du premier levain, d'1 to l'ajout du milieu, et d'1 to 5 shō l'ajout final du cycle des opérations ${ }^{49}$. Il en va de même pour le "saké d'avant les froids » et le "saké des froids ». Entre le moment où le levain est versé et celui où le saké est prêt, il faut compter environ quarante jours pour le "saké de l'entre deux », soixante-dix jours pour le « saké d'avant les froids », et quatrevingts à quatre-vingts dix jours pour le "saké des froids». Mais on dit qu'il est important de savoir adapter la durée [du processus] et le nombre de jours en fonction de la température de l'année.

Cependant, autrefois, avant le "saké nouveau », on produisait un saké dit botai, qu'on appelait parfois aussi "saké nouveau " ${ }^{50}$. C'est le saké qu'on produit aujourd'hui dans les villages de montagne. Autrefois, même à Ōsaka et dans les autres grandes villes, le saké de bonne qualité n'était pas une boisson destinée aux gens de condition humble. Ce que l'on appréciait à de rares occasions, c'est ce saké botai, produit par des fabricants des montagnes. Mais voilà deux cents ans que l'ordre règne dans le monde et même les gens qui vivotent chichement au jour le jour peuvent s'enivrer tout leur soûl, puis battre des mains et crier Vivat ! dans la rue. Nous devons être reconnaissants de vivre à une telle époque ! 
Riz

Pour le premier levain, on utilise le riz d'une récolte ancienne de régions limitrophes, par exemple celui de Kaga ${ }^{51}$, de Himeji ${ }^{52}$ ou d'Awaji ${ }^{53}$. Pour le riz ajouté, le riz d'une récolte ancienne des régions du nord est le meilleur, par exemple celui d'Akita ${ }^{54}$ ou de Kaga. Pour le levain du saké « d'avant les froids » et " des froids», on utilise le riz des entrepôts de Takatsuki, ou le riz nouveau des régions montagneuses du fief de Yodo ${ }^{55}$.

\section{Pilage du riz}

Pour le riz du levain, un homme pile la quantité de quatre mortiers par jour (à raison d'1 to 3 shō 5 gō de riz par mortier). Pour le riz ajouté, la quantité est de cinq mortiers, mais dans le cas du saké de qualité supérieure, elle est de quatre mortiers seulement, le riz étant pilé d'une manière extrêmement fine ${ }^{56}$. Pour cette opération, on évite les pilons usés et on utilise des pilons en bois de "pin à cinq aiguilles " de la province d'Owari ${ }^{57}$. Si le bout du pilon présente une concavité, les grains sont gravement abîmés. C'est pourquoi les hommes chargés du pilage l'inspectent périodiquement. Le bois d'Owari est apprécié parce qu'il est tendre ${ }^{58}$.

\section{Lavage du riz}

Il faut tout d'abord puiser toute l'eau dormante qui est restée dans le puits pour laisser venir l'eau nouvelle, puis enlever jusqu'aux moindres impuretés afin que l'eau soit parfaitement pure. Le riz est lavé par des équipes de trois hommes par cuveau et cela en renouvelant l'eau quarante fois [voir Pl. 2], voire cinquante fois dans le cas de "saké des froids ».

\section{PI. 2 : Fabrication du saké à Itami, lavage du riz}

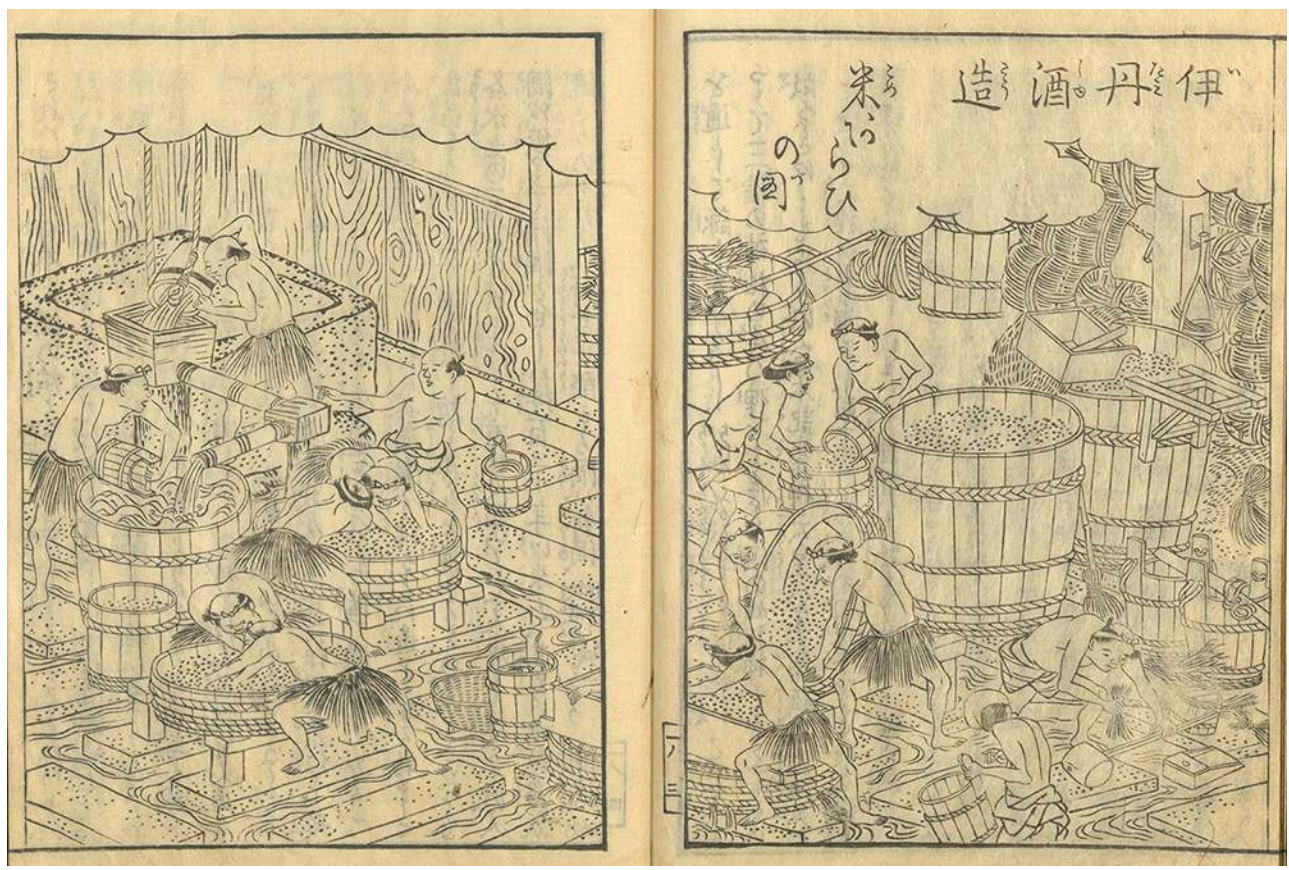

National Institute of Japanese Literature. Lien vers l'illustration 


\section{Traditions particulières}

17 « Maître Du » ${ }^{59}$ toji 杜氏 (Ce sont les chefs des opérations de fabrication de saké. On les appelle aussi " patrons » (oyaji). Il y avait déjà des maîtres brasseurs sous la dynastie des Zhou. Et plus tard, un certain She Du Kang est devenu fameux pour son habilité à brasser le saké. C'est de là que vient le nom de « Maître Du » tōji ${ }^{60}$.)

Emon (littéralement "motif de vêtement " ${ }^{61}$ (Ce sont les chefs des opérations de fabrication du kōji. Le terme emon ferait allusion à la fabrication de la «fleur » du kōji. Selon une autre explication, le terme viendrait de ce que les hommes qui fabriquaient le kōji en Chine ne quittaient pas leur poste près des plateaux à kōji pendant sept jours, sans jamais dormir, afin de surveiller l'entrée de la chambre de fermentation, tels les gardes des portes du palais (emon).)

\section{Ustensiles nécessaires à la fabrication du saké}

19 [Il faut] au moins deux cents cuveaux (pour l'ensemble des cycles d'opérations), au moins vingt cuves pour la fabrication du levain, au moins trois cuves de 3 pieds de diamètre, dix-sept à dix-huit mortiers avec pilon à levier, au moins quatre cents plateaux pour le kōji de saké. Pour les passoires à étuver, il faut utiliser du cèdre de Satsuma débité sur quartier (masame). Il est bon que la texture du bois soit perméable. Pour les autres cuves, on utilise le bois sur dosse (itame $)^{62}$. Il faut environ trois cent quatre-vingts sacs pour un pressoir à $12 k k^{6} u^{63}$, et au moins $130 k a n m e^{64}$ de combustible pour le premier levain.

\section{Fabrication des cendres}

On prend 1 to de cendres de bois produites dans la province Bungo et 4 shō 5 gō de chaux de pierre ${ }^{65}$ que l'on mélange et que l'on tamise bien, puis on verse l'ensemble dans un pot. Avec les premiers déchets du tamisage, on constitue une eau trouble que l'on utilise pour humidifier les cendres de clarification du saké. Sur ce point, il y a des transmissions secrètes.

\section{Cendres de rectification ${ }^{66}$}

21 On torréfie 1 to de chaux de pierre et 4 shō de cendres de Bungo dans un chaudron, puis on l'humidifie avec de l'eau trouble avant de l'utiliser.

22 On préfère chauffer (hi'ire $)^{67}$ le saké de garde (kakoizake) ${ }^{68}$ avant la saison des pluies.

\section{Alcool de riz (mirin)}

On met à fermenter dans un bac 10 koku d'alcool de riz (shōchū), 9 koku 2 to de riz gluant et 2 koku 8 to de $k \overline{o j j} i^{69}$. Le jour suivant, on remue la masse avec une pelle à mélanger et on mélange environ sept fois, tous les quatre ou cinq jours. Si l'opération est effectuée au printemps, elle s'étend sur une période de vingt-cinq jours. Jadis, on remuait tous les sept jours. Pour fabriquer l'alcool de riz hon'naoshi, on prend 10 koku d'alcool de riz 
(shōchū), 2 to 8 shō de riz gluant et 1 koku 2 to de kōji. Puis on poursuit selon le même procédé que pour le mirin.

\section{Vinaigre de riz}

24 On laisse tremper dans l'eau 2 to de riz complet pendant une nuit. Après l'avoir fait cuire à la vapeur, on le transvase, encore chaud, de la passoire à étuver dans une cuve et on ajoute 6 to de kōji et 1 koku d'eau. On met un couvercle et on enveloppe la cuve avec des nattes de paille de manière à ce que la moindre vapeur ne s'échappe. Après sept jours on soulève le couvercle, on mélange, et on referme. Tous les sept jours, on mélange sept à huit fois. On guette la fermentation qui intervient au bout de soixante à soixante-dix jours. Ensuite, on presse le vinaigre de la même manière que le saké. (Ce vinaigre sert rarement à la consommation, mais il est utilisé pour des pigments comme ceux extraits de carthame ou d'algues laminaria konbu).

Il existe de nombreuses variétés de vinaigre en fonction des terroirs et des recettes de chaque maison. Beaucoup de ces produits sont renommés tels que ceux d'Ogawa dans le Yamato, de Kokawa dans le Kii, de Kitakaze dans le Hyōgo, de Funai dans le Bungo, ou encore ceux de Sagami et de Suruga ${ }^{70}$.

\section{Lavage des sacs}

26 Quand le saké nouveau est prêt, on lave les sacs dans les eaux du fleuve Inagawa ${ }^{71}$. Les gueux des villages du voisinage guettent ce moment pour venir mendier les résidus. Le goût en est léger, comme celui du saké doux (amazake) ${ }^{72}$, mais différent des autres produits. Le poète de haikai Onitsura ${ }^{73}$ a composé le poème suivant :

Femmes gueuses

du suc s'écoule

des sacs lavés

\section{Fête d'Atago ${ }^{74}$}

27 Les feux d'Atago ont lieu le vingt-quatrième jour de la septième lune. On allume des lanternes depuis la rue principale d'Itami et on fabrique des effigies intéressantes dans un déploiement qui n'a rien à envier à la fête de purification dans la rivière, célébrée au sanctuaire de Tenjin à Ōsaka ${ }^{75}$. Ce jour-là, la foule vient en nombre admirer les immenses entrepôts des brasseurs de saké. Sur ce thème, Sōin ${ }^{76}$ a écrit ce poème :

Père lui aussi,

est enivré par les feux,

lanternes géantes d'Itami.

Ce jour-là, les futurs employés des maisons de saké, venus des provinces de Tanba et de Tango ${ }^{77}$, répondent, nombreux, à l'appel des fabricants, et concluent avec ces derniers, un contrat dont la période est fixée à cent jours. 
PI. 7a : Marques figurant sur les emballages en nattes de paille des maisons d'Itami. Liste abrégée.

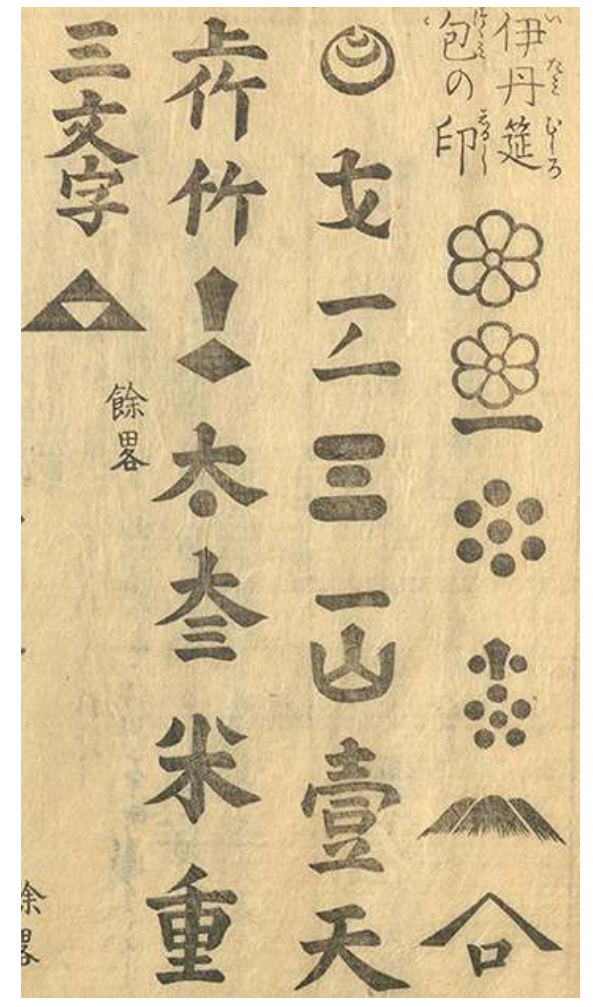

National Institute of Japanese Literature. Lien vers l'illustration

PI. 7b : Marques figurant sur les emballages de bâches de paille des maisons d'Itami. Liste abrégée.

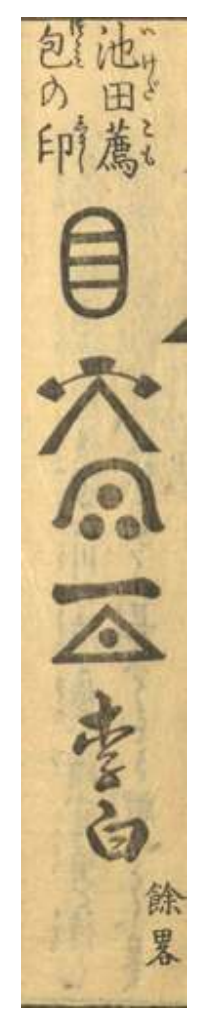

National Institute of Japanese Literature. Lien vers l'illustration 


\section{NOTES}

1. Ōjin (r. 270-320), empereur légendaire de la mythologie japonaise.

2. Tsuruga correspond à la ville actuelle de Tsuruga dans le département de Fukui.

3. Kojiki, première chronique du Japon, 712. Selon le Kojiki (fascicule Chū, épisode sur Ōjin), l'empereur mythique ōjin est né à Tsukushi (ancien nom de l'île de Kyūshū) de la reine Jingū alors qu'elle rentrait d'une expédition dans les contrées étrangères. Pour le protéger des conflits de pouvoir, la reine fit transporter le prince à Yamato en faisant un détour par Tsuruga (dans la province d'Echizen). Le poème de la reine Jingū chante l'entrée d'Ōjin à Yamato et Takeuchi no sukune a composé la réponse de la part du jeune souverain. Les Grandes prémices sont un rite qui marque l'intronisation de chaque empereur.

4. Il sera question, dans tout le fascicule, du saké fabriqué dans l'ancienne ville d'Itami, localisée dans l'actuel arrondissement d'Itami de la ville d'Ōsaka, département de Hyōgo.

5. Erreur pour Dou Ping 凟苹, auteur de l'époque des Song, 960-1127.

6. Kanjikō, dictionnaire des mots initiateurs de Kamo no Mabuchi (1697-1769) imprimé en 1757. Selon ce dictionnaire, la syllabe $m i$ serait un mot initiateur (makura-kotoba), c'est-à-dire un préfixe honorifique qui porte en même temps le sens d'« exquis " mi/umai.

7. Le mont Miwa, situé dans l'actuel département de Nara, était considéré, à l'époque ancienne, comme une montagne sacrée et comme siège de la divinité du saké.

8. Le Man'yōshū est une anthologie de 4500 poèmes datant du milieu du VII siècle à l'année 759. Le poème auquel l'auteur se réfère est le poème $n^{\circ} 3266$, fasc. 13, Man'yōshū, SATAKE Akihiro, YAMADA Hideo, KUDŌ Rikio, ōTANI Masao, YAMAZAKI Yoshiyuki (éd.), vol. 3, Tokyo, Iwanami shoten, coll. «Shin Nihon koten bungaku taikei », p. 249-250.

9. Le Nihongi est un autre nom du Nihon shoki, ouvrage achevé en 720 , première des six chroniques officielles du Japon.

10. Nihon shoki, Sujin huitième année, quatrième mois (90 avant $\mathrm{J}-\mathrm{C})$.

11. Il s'agit d'une interprétation personnelle de l'auteur de l'ouvrage concernant l'entrée du Nihon shoki, datée de Sujin huitième année, douzième mois. Celle-ci dit : «L'empereur ordonna à Ōta Taneko de présenter des offrandes à Ōmiwa. Ce jour, Ikuhi a présenté du saké divin miwa 神 酒 à l'empereur. »

12. Le mont Miwa est couvert d'une forêt de cryptomères.

13. Ōsazaki est l'empereur mythique Nintoku, r. 313-399, selon le Shinsen shōjiroku (Nouveau registre des familles ; 815).

14. Settsu, ancienne province située à cheval sur les départements d'ōsaka et de Hyōgo.

15. Le nom de morohaku, signifiant littéralement «tous blanchis", se réfère au fait que les ingrédients utilisés pour la fabrication, qu'il s'agisse du riz étuvé ou du kōji, sont obtenus à partir de grains blanchis au pilon. Le morohaku est le nom désignant le saké de qualité, totalement clair, produit notamment par les brasseurs d'Itami.

16. Bunroku (1592-1594) et Keichō (1596-1615).

17. Le village de Kōnoike, situé alors dans la province de Settsu, est localisé de nos jours dans la ville de Takarazuka, dans le département de Hyōgo. Il est fait référence ici à la maison Kōnoike qui deviendra plus tard l'une des familles les plus riches d'ōsaka. Il s'agit d'un véritable clan, comptant plus de dix branches, qui a bâti au départ sa fortune grâce à la production et à la vente de saké et qui s'est par la suite spécialisé dans les transports maritimes et dans le domaine financier (prêt et change). Les Kōnoike comptaient parmi leurs clients le bakufu et de très nombreux daimyō.

18. 18 litres.

19. 180 litres. 
20. Ikeda, ancienne ville, à l'emplacement de l'actuel arrondissement d'Ikeda d'Ōsaka.

21. Nishinomiya, Hyōgo, Nada et Imazu sont des noms d'anciennes villes situées dans l'ancienne province de Harima, l'actuel département de Hyōgo.

22. Aki no higan, période de sept jours autour de l'équinoxe d'automne en septembre, c'est-à-dire à la huitième lune du calendrier ancien.

23. Neuvième mois du calendrier ancien, octobre.

24. Voir planches 1 et 2 .

25. Entre $11 \mathrm{~h}$ et $13 \mathrm{~h}$. Dans la suite on traduira une heure ancienne (hito toki) par deux heures.

26. Entre $1 \mathrm{~h}$ et $3 \mathrm{~h}$ du matin.

27. Entre 3 et $5 \mathrm{~h}$ du matin du quatrième et dernier jour de cette étape de la fabrication.

28. Ce qui est appelé ici moisissure de riz n'est autre que l'aspergillus orizae, un agent qui provoque la fermentation. Ce procédé, qui dure une dizaine de jours, est la première étape dans la fabrication du saké, avant la fabrication du kōji. Voir ABÉ Yoshio, «La fabrication du saké : processus biochimique et procédé technique ", in Des mérites comparés du saké et du riz, op.cit., p. 233-238, au sujet de la bio-chimie du saké ; et ABÉ Yoshio, "Anthropologie comparée d'une céréale majeure asiatique : le riz ", Séminaire à l'EHESS, Centre d'études sur la Chine moderne et contemporaine, 2008-2009, 2009-2010, inédit, sur la fermentation du riz.

29. Nous traduisons moto par «levain» pour faciliter la compréhension. Le terme japonais signifie «base ». Pour l'interprétation du mode de fabrication, voir ABE Yoshio, «La fabrication du saké: processus biochimique et procédé technique », in LEGGERI-BAUER Estelle, BÉRANGER Véronique, BRISSET Claire-Akiko (éd.), Des mérites comparés du saké et du riz, Paris, Diane de Selliers, 2015, p. 233-237.

30. Le procédé de fabrication du levain dure trois jours.

31. Ni-koku iri no oke, grandes cuves d'une capacité de 360 litres, comme celles que l'on voit sur la planche 1.

32. Littéralement: un savoir-faire aussi difficile que la fabrication de l'oxyde de plomb, un précieux colorant.

33. La fabrication du levain moto, troisième étape dans la fabrication du saké, dure environ douze jours. Voir YUNOKI Manabu, Sakezukuri no rekishi (Histoire de la fabrication du sake), Tokyo, Yūzankaku Shuppan, 1987, p. 40.

34. Soe 酘, quatrième étape dans la fabrication du saké, il s'agit d'ajouts successifs de riz cuit, de kōji et d'eau, qui produisent un liquide épais fermenté appelé moromi.

35. Ce qui correspond à environ $90 \mathrm{~cm}$ de diamètre.

36. Le riz blanchi hakumai est décortiqué à un taux élevé de polissage, par exemple $50 \%$ ou plus.

37. Le contenu de deux cuves est versé dans quatre cuves au total; voir planche 4.

38. Les cuveaux versoirs bonburi sont des cuveaux d'une capacité de 8 shō (14,4 litres) et sont utilisés pour transvaser la masse dans de très grandes « cuves de 3 pieds » de diamètre (sanshakuoke).

39. Ce cycle d'opérations (quatrième étape dans la fabrication du saké) a pour objet l'ajout successif de trois ingrédients; il dure sept jours et produit un liquide épais fermenté moromi, voir YUNOKI, Sakezukuri no rekishi, op.cit., p. 39, 41.

40. 8,5 koku de riz étuvé et de kōji (1530 litres) et 4,4 koku (792 litres) d'eau donne un total de 12,9 koku (2 322 litres). Selon YUNOKI, Sakezukuri no rekishi, op.cit., p. 41, c'est le volume du liquide épais fermenté qui est ensuite pressuré pour donner du saké clair.

41. À partir d'ici il s'agit de la cinquième étape, celle du pressurage et de la clarification du saké.

42. C'est du saké cru dont la drèche (kasu) s'est déposée au fond de la cuve.

43. S'agit-il d'une cuve qui aura, au fond, un dépôt de 7 pouces $(21 \mathrm{~cm})$ ? 
44. Le dépôt de lie brute araori ou arabashiri marque la fin de la dernière étape de la fabrication du saké, après obtention du liquide épais fermenté. Voir YUNOKI, Sakezukuri no rekishi, op.cit., p. 41-42.

45. Ce sont des fûts de 4 to (72 litres). Avec 7 to et 5 shō (135 litres) par charge de cheval cela donne deux fûts par charge. Étant donné que l'ensemble s'élève à onze charges (1 485 litres) ou douze charges (1 620 litres), le volume de 12,9 koku (2 322 litres) pressurés donnerait 1620 litres de saké.

46. Le saké est mis dans des fûts de conservation pendant une période de cinquante ou soixante jours. C'est en automne que le saké est mis en fût de 72 litres pour le transport. Voir YUNOKI, Sakezukuri no rekishi, op.cit., p. 42.

47. Il est question du processus des ajouts. Le fabricant d'Itami, mentionné plus haut, utilisait au total traditionnellement 8,5 koku de riz étuvé et de kōji (1530 litres) et 4,4 koku (792 litres). Un siècle plus tôt (avant 1799, date du présent ouvrage), c'était donc entre 8 koku (1 440 litres) et 8 koku, 4 ou 5 to (1 530 litres) de riz étuvé et de kōji. Au moment de l'écriture du livre, c'est-à-dire en 1799, on comptait 9 à 10 koku de riz (1 620 à 1800 litres).

48. Pour le "saké d'entre-deux » et le «saké nouveau », on a majoré le volume des additions de riz cuit et de kōji de 3 to (54 litres) par rapport au standard évoqué ci-dessus.

49. Il s'agit des quantités de produits du riz (riz cuit et kōji) qui sont ajoutés au levain à trois reprises. Par rapport aux quantités données plus haut dans la section «Ajouts ", on a adopté des majorations respectivement de 5 shō (9 litres) pour les premiers ajouts au premier levain, de 1 to (18 litres) pour les ajouts intermédiaires et de 1,5 to (27 litres) pour les ajouts finaux.

50. Le botai a été développé par le temple Bodaisanji de Nara, mais il était considéré comme de mauvaise qualité à l'époque du Nihon sankai meisan zue (1799).

51. Il pourrait s'agir ici du fief de Kaga, et non de la province de Kaga, dans la mesure où la liste comporte deux autres noms de fiefs. Le fief de Kaga (également fief de Kanazawa), administré par la famille Maeda, couvrait à l'époque d'Edo les trois provinces de Kaga, Etchū et Noto, donnant toutes sur la mer du Japon. Sa production en riz était évaluée à 1200000 koku.

52. Himeji, ville castrale, chef-lieu du fief du même nom, située dans l'ancienne province de Harima (actuel département de Hyōgo), donnant sur la mer Intérieure.

53. Awaji est le nom d'un fief couvrant l'île d'Awaji, située dans la mer intérieure de Seto, et ayant une superficie de près de $600 \mathrm{~km}^{2}$.

54. Akita est le nom d'un fief situé dans la province de Dewa, au nord du Honshū, administré à l'époque d'Edo par la famille Satake. C'est aussi le nom de la ville castrale où siégeait son administration. Le fief tirait l'essentiel de ses revenus de sa production de riz.

55. C'est le riz produit par les fiefs Takatsuki (région d'Ōsaka) et Yodo (administré à partir de la ville du même nom située dans la province de Yamashiro, mais possédant également des terres dans des provinces éloignées) ; voir CHIBA, Nippon sankai meisan meibutsu zue, op.cit., note 36, p. 25.

56. Le taux de pilage s'élevait aux alentours de $50 \%$ pour le riz blanc destiné à la consommation, mais il était supérieur, de l'ordre de $70 \%$, pour le riz destiné à la fabrication du saké. La quantité moyenne était de 1 to, 3 shō, 5 gō (24,3 litres) de riz par mortier. Voir YUNOKI, Sakezukuri no rekishi, op.cit., p. 38.

57. Owari, ancienne province située dans l'actuel département d'Aichi. Il s'agit du bois du Pinus parviflora var. pentaphylla. Contrairement à ce que dit l'auteur, cette espèce ne se trouve pas dans la province d'Owari mais plutôt dans la province de Kiso, alors administrée par le fief d'Owari. CHIBA, Nippon sankai meisan meibutsu zue, op. cit., note 37, p. 25-26.

58. Voir YUNOKI, Sakezukuri no rekishi, op. cit., p. 38.

59. Terme désignant les maîtres brasseurs de saké qui dirigeaient les opérations de fabrication. Le terme est encore utilisé de nos jours.

60. She Du Kang 葉杜康 aurait vécu à la fin de l'époque du royaume de Zhou (1134-250 avant J-C). Il est désigné ici par son nom $\mathrm{Du}$ 杜, lu « to » en japonais. 
61. Le terme désigne les maîtres fabricants de kōji.

62. Voir YUNOKI, Sakezukuri no rekishi, op.cit., p. 40. Voir les veinures du bois dans MERTZ Mechtild, Wood and Traditional Woodworking in Japan, Otsu, Kaiseisha, 2011, p. 31-34, 60.

63. $12 \mathrm{koku}=2,160$ litres.

64. 130 kanme $=487,5 \mathrm{~kg}$.

65. Soit 18 litres de cendres de Bungo et 8,1 litres de chaux de pierre. Pour la chaux de pierre, on pourra consulter la notice correspondante dans le fascicule 5 du présent ouvrage.

66. Les cendres de rectification naoshibai sont utilisées pour rattraper le saké aigri. Les quantités indiquées correspondent à environ 18 litres ( 1 to ) de chaux et 7,2 litres $(4$ shō) de cendres.

67. Il s'agit d'une technique proche de la pasteurisation, consistant à chauffer le saké à une température peu élevée $\left(60^{\circ}\right)$ pour le conserver. YosHIDA, Sake, op.cit., p. 143.

68. Le terme renvoie au saké traité pour la conservation, différencié du namazaké ou saké frais.

69. Le mirinchū 味醂酎 ou mirin est utilisé de nos jours pour assaisonner les plats. Fabriqué à partir de l'alcool de riz shōchū, de riz gluant et de kōji, il était apprécié à l'origine comme alcool sucré à boire. Son utilisation en tant qu'assaisonnement s'est généralisée à partir du XIX siècle.

70. Yamato, ancienne province, située dans l'actuel département de Nara ; Kii, ancienne province située à cheval sur les départements actuels de Wakayama et de Mie.

71. Il s'agit d'un affluent du fleuve Yodo, situé à la frontière des départements de Hyōgo et de l'agglomération urbaine d'ōsaka.

72. Il s'agit d'une boisson à base de riz fermenté très peu alcoolisée.

73. Uejima Onitsura (1661-1738) est un poète de haikai de l'époque d'Edo, originaire d'Itami, né dans une famille de brasseurs de saké.

74. La fête d'Atago, avec le feu des torches Atago-bi, est célébré dans tous les sanctuaires d'Atago du pays sous l'égide du sanctuaire principal situé au mont Atago au nord-ouest de la ville de Kyōto. Cette fête est célébrée encore aujourd'hui tous les ans du 23 au 28 août.

75. Tenjin matsuri, grande fête célébrée à Ōsaka à l'époque d'Edo, le vingt-cinquième jour du septième mois, aujourd'hui le 25 juin.

76. Sōin désigne Nishiyama Sōin (1605-1683), célèbre poète de haikai (poésie en chaînes comique) de l'époque d'Edo, maître de renga (poésie en chaînes) attaché au sanctuaire Tenmangū d'Ōsaka. 77. Tanba, ancienne province située dans la partie sud de l'actuelle préfecture de Kyoto; Tango, ancienne province située dans la partie nord de l'actuelle préfecture de Kyōto. 


\section{Fascicule 2}

\section{NOTE DE L'ÉDITEUR}

Ce sommaire, placé en début de fascicule par l'auteur du Meisan zue, fait partie du texte d'origine. Ce sommaire n'a pas vocation à être utilisé pour circuler à l'intérieur du Meisan zue. Pour ce faire, le lecteur pourra utiliser toutes les fonctionnalités propres à l'édition en ligne de la plateforme OpenEdition Books, notamment sa table des matières générale.

\section{Sommaire du fascicule 2}

- Pierre de Teshima

- Pierre de Mikage

- Pierre de Tatsuyama

- Pierres à affûter

- Saiwatake

- Shiitake de Hyūga

- Champignon de roche de Kumano

- Miel de Kumano

- Cire d'abeille

- Cire d'Aizu

- Salamandre

- Puéraire de Yoshino

- Grenouille rouge

- Insecte de la Vitis ficifolia de Takagamine

- Filets à autours

- Filets à canards

- Canards volant au-dessus de sommets dans la province d'Iyo

- Filets « brume » de la province de Settsu

- Bascule sans pareille 


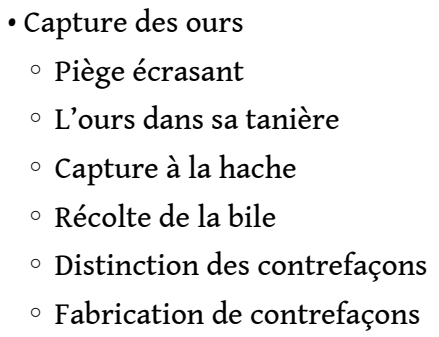

\section{Variétés de pierres}

1 Les pierres constituent l'ossature des montagnes. Selon le Wuli lun (Discours sur la raison des choses), « la pierre est une quintessence de terre, une concrétion de souffle vital ; les pierres que produit le souffle vital sont à la terre ce que sont les tendons, les vaisseaux, les ongles et les dents au corps humain ${ }^{1}$. La consistance des pierres est cependant loin d'être identique dans toutes les montagnes de toutes les provinces. Ceci tient à la variété des terrains et des climats, dont il est certain qu'ils influent sur la qualité du souffle vital ambiant. Il arrive aussi que des arbres et des herbes, des poissons et des coquillages se transforment et deviennent des pierres. Le Bencao gangmu (Classement raisonné de la materia medica) mentionne des pins, le Song shu (Livre des Song) ${ }^{2}$, des chênes transformés en pierre; dans les romans chinois (haishi) ${ }^{3}$, on trouve mention de bambous transformés en pierre. Selon le Daizuibian ${ }^{4}$ (Substitut à l'ivresse), au nord du Mont Fuyu dans le Yangquan se trouve un cours d'eau limpide : si on plonge $\mathrm{du}$ bois sur une distance de plusieurs dizaines de $b u^{5}$, il se transforme tout entier en pierre. Dans une province d'Italie aussi, se trouve une source étrange : tout ce qui y tombe au bout d'une quinzaine de jours commence à sécréter une peau en pierre qui l'entoure entièrement. Il est aussi un lac dans un pays à l'ouest de l'Europe. Lorsqu'on y immerge un arbre et qu'on l'enfonce dans le sol, il se transforme complètement et devient du fer. À ce qu'on dit, c'est sur la longueur immergée d'un tan, que le bois se transforme en fer. Au Japon aussi on trouve de nombreux lieux semblables à ceux-là, et il en va de même de tous les bords de mer ou bords de lac de pays froids. Il faut savoir que tous les ustensiles en matière fossilisée proviennent de ce genre d'endroits. Il y a aussi les pierres qui ont la forme d'organes sexuels mâles ou femelles qu'on frappe avec un fouet pour faire pleuvoir ou pour faire cesser la pluie. On en trouve des exemples dans l'Azuma kagami (Miroir de l'Est) ${ }^{6}$ pour l'an 7 de l'ère Hōki ou pour l'an 1 de l'ère Ninna et dans d'autres ouvrages. À Ishiyama dans la province d'ōmi ${ }^{7}$, on trouve une pierre qui correspond à ce que le Bencao gangmu désigne comme " pierre dressée yang ", un rocher qui est une merveille de l'Empire ${ }^{8}$. Dans le Nihon shoki (Chronique du Japon) on peut lire que la fille de l'empereur Yūryaku, alors qu'elle était vestale au sanctuaire d'Ise, fut soupçonnée d'adultère et que lorsque l'empereur fit ouvrir son ventre, on y trouva une matière semblable à de l'eau et dans cette eau il y avait une pierre. C'est ce que les ouvrages de médecine appellent sekka9. En somme, il s'agit dans tous ces cas d'agglomération de matière.

2 En ce qui concerne les espèces de pierres: stalactites, pierre d'aimant, arsénopyrite, stéatite, arsenic, salpêtre, calcite, marbre blanc, pierre ponce et autres pierres étranges, prodigieuses ou aux formes animales, l'Unkonshi (Traité sur les roches) ${ }^{10}$ compilé par le maître d'Ōmi ${ }^{11}$ en fait une liste exhaustive, si bien qu'il n'est pas besoin d'en traiter ici en détail. 
Quant à la lecture japonaise du caractère "pierre » ishi, c'est shi qui en est l'élément originel. Il peut être rapproché des mots shimari (fermeture, tension, fermeté), shizumu (aller au fond, couler) ou encore de l'expression vernaculaire shikkari (avec force) et signifie « accrétion ». Iha ${ }^{12}$ (rocher) signifie « dent de pierre » $i$-ha. Comme le montre le caractère ban 磐 (rocher) utilisé pour noter ce mot, il désigne nécessairement une pierre de grande taille, ayant la forme d'un croc, et veut dire «acéré ». Le mot ihaho (rocher) est noté avec le caractère gan 㦑 (falaise, abrupt) qu'on trouve dans un vers du Shijing (Livre des odes) : «les rocs se dressent en forme de pics》 (維石笼㦑, kore ishi gangan tari), et qui a aussi le sens de «se dresser en forme de pic ». Dans le Man'yōshū, le mot ihaho est noté avec les caractères 石穂 signifiant pierre (iha) et épi (ho), ce qui veut dire « qui se dresse en épis ». On dit aussi ihahoro. Que ce soit ishi, iha ou ihaho, tous ces différents termes s'emploient en un sens identique.

$\mathrm{Au}$ Japon il ne manque pas de pierres dont on fabrique des ustensiles. En particulier, parmi celles qu'on trouve dans les cinq provinces du Kinai ${ }^{13}$ ou dans les provinces de l'Ouest, les pierres de Mikage, Tatsuyama ou Teshima sont utilisées comme des matériaux pour servir aux besoins de l'homme et ne sont pas de simples pièces d'ornement. Je vais parler de trois ou quatre de ces variétés et laisserai de côté le reste. La pierre d'Izumi ${ }^{14}$ est toujours de couleur bleue, d'un grain fin, et on s'en sert pour les stèles inscrites. La pierre qu'on extrait depuis peu dans la province d'Awa ${ }^{15}$, lui est apparentée. Cette pierre ressemble à la pierre de Nabukawa, elle est de couleur verte et a la forme de fines planchettes. Sa consistance est tendre. Dans la province de Yamashiro ${ }^{16}$, on trouve aussi la pierre de Kurama, celle de la Kamogawa ou encore celle du Seikanji. On les utilise comme dalles de jardin ou rochers décoratifs, on en fait des vasques et on admire la couleur qu'elles prennent lorsqu'elles sont mouillées. D'une manière générale, on les utilise comme objets d'ornements à l'usage d'hommes de haute condition et d'amateurs de thé.

\section{Pierre de Teshima}

5 Il s'agit d'une île montagneuse d'une circonférence de 3 lieues, à 50 lieues d'ōsaka, à proximité de Shōdo shima ${ }^{17}$ dans la province de Sanuki ${ }^{18}$. Elle possède trois villages: Ieno'ura, Karutomura, Kōmura. Ieno'ura compte quelque 300 maisons, Karutomura et Kōmura, quelque 170 à 180 chacun. La pierre produite à Karōtomura est un peu plus dure que les autres et on l'utilise pour construire des portiques de sanctuaires shinto ou des quais en pierre. Cette montagne diffère des autres en ce que la pierre n'y est pas extraite à même la surface. On perce un trou dans la montagne semblable à l'entrée d'un puits de mine. Après avoir ouvert un accès, on creuse plus avant et on aménage une voie de 10 à 20 chō en enlevant la roche sur le côté et en hauteur. Comme les ouvriers s'éclairent à l'aide de torches, les galeries sont toutes noires au point qu'il est difficile de distinguer la roche de la terre et que les traits des ouvriers ne ressemblent pas à ceux d'hommes ordinaires [voir Pl. 8]. 


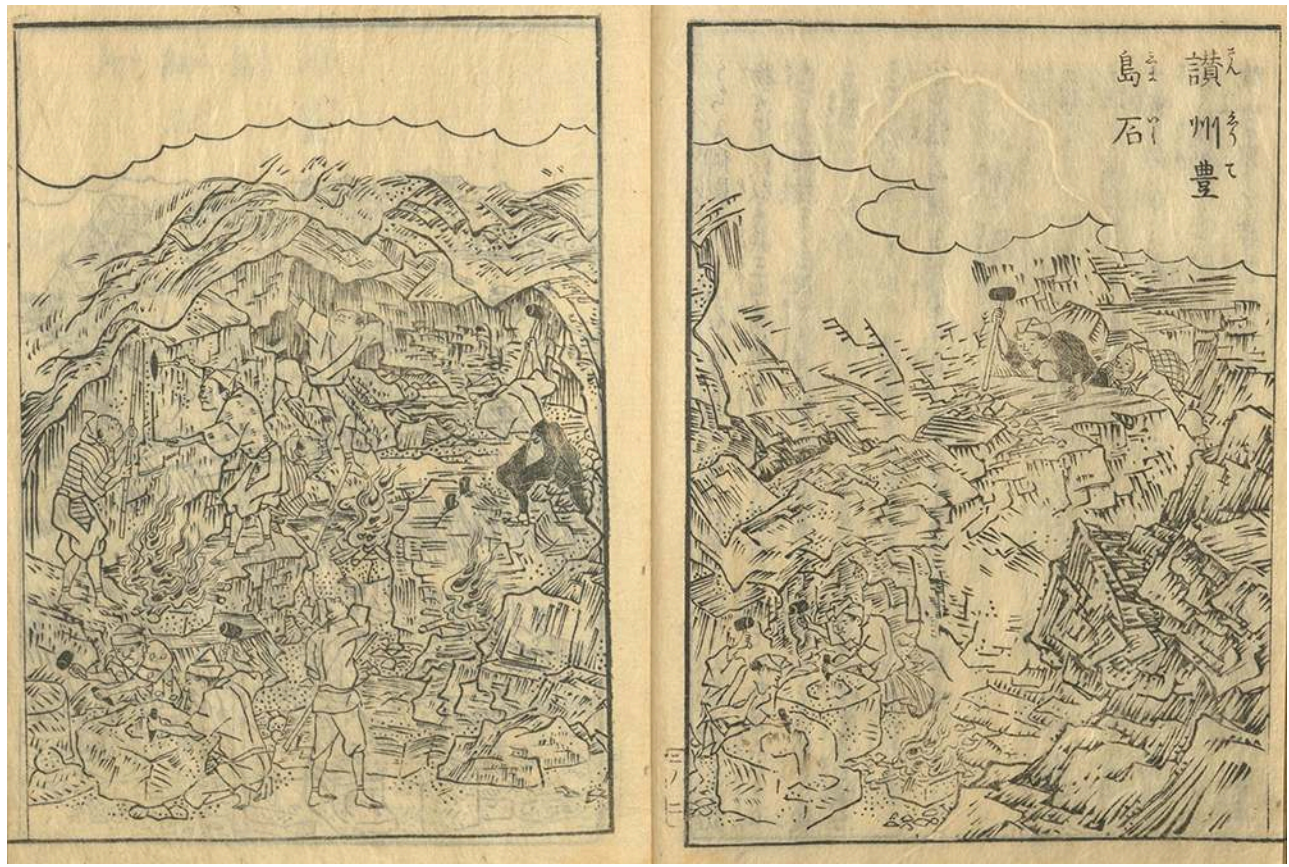

National Institute of Japanese Literature. Lien vers l'illustration

La raison pour laquelle on creuse ainsi est que cette pierre est enveloppée dans une gangue fort dure. C'est la pierre qu'on extrait sous le nom de nebukawa (la véritable pierre de Nebukawa ${ }^{19}$ se trouve dans la province d'Izu) et on évite d'utiliser des coins pour la fendre, mais on la découpe plutôt en plaques. La pierre de Teshima qui circule communément, est la partie qui se trouve à l'intérieur de la gangue. C'est pourquoi la bonne méthode est de creuser en évitant la gangue. À Ieno'ura il y a sept galeries. Mais comme il faut franchir une montagne pour revenir de cet endroit, la plupart des ustensiles sont fabriqués sur place et transportés ensuite. Il s'agit de margelles de puits, de rigoles, de foyers, de fourneaux, etc. mais pas des produits de très grande taille. Kōmura est un village de pêcheurs, mais on extrait aussi des pierres au sud de Karōto. Les ateliers des tailleurs sont groupés au pied de la montagne [voir Pl. 9]. 


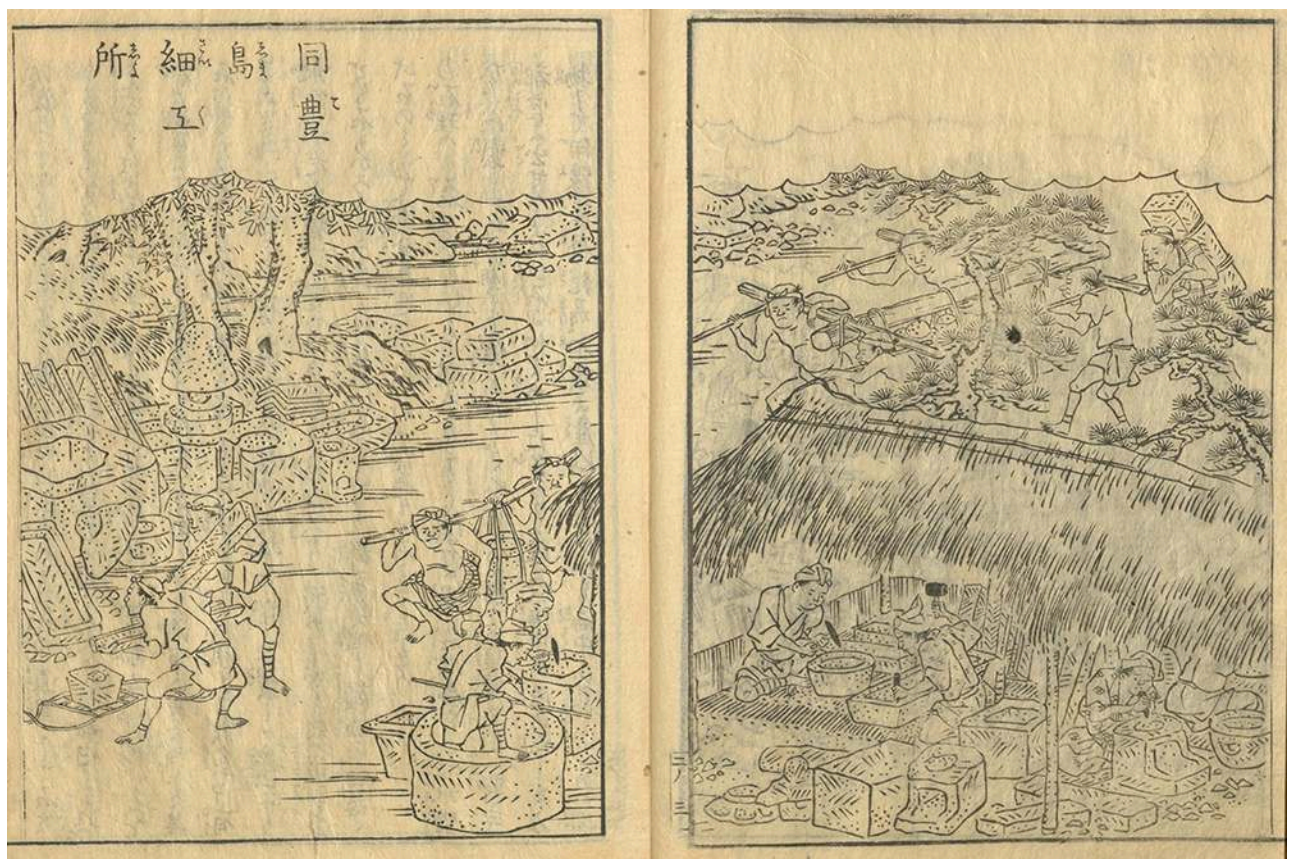

National Institute of Japanese Literature. Lien vers l'illustration

$7 \mathrm{Au}$ demeurant, cette pierre se trouve partout dans les montagnes de la province d'Izumi et c'est de cette pierre qu'est faite la grotte de Kōbō daishi du Zentsūji à Iyadani ${ }^{20}$.

8 Le grain de cette pierre est semblable à du gravier aggloméré. Elle ressemble à la pierre ponce et son grain est aussi grossier. Aussi lorsqu'on en fait des baquets à eau, elle laisse passer l'eau et ne la garde pas. En revanche, elle n'éclate pas au contact du feu. La pierre qu'on extrait à Utsunomiya dans la province de Shimotsuke ${ }^{21}$, lui ressemble, mais elle est un peu plus belle. La pierre ponce est une concrétion de l'écume de mer, et on la produit à Iyo, Satsuma, Kii, Sagami ${ }^{22}$. Cependant, comme ces montagnes s'élèvent aussi sur des îles au milieu de la mer, je me demande s'il ne s'agit pas d'eau de mer solidifiée depuis l'origine du monde. Est-ce que le nom "Shiaku " ne serait pas une déformation de Shioawa (littér. " écume de mer ») ? La pierre de Shiaku ${ }^{23}$ est produite dans ce qui est devenu une montagne réservée ${ }^{24}$, et beaucoup de celles qu'on appelle ainsi sont admirées parce qu'elles incorporent des coquillages. Elle y forme des rochers en bordure de mer, son grain est semblable à des grains de riz et sa consistance est dure. On l'utilise pour des dalles, des bassins, des pierres décoratives et elle a pour signe distinctif que la mousse y pousse vite. Les rochers en bord de mer sont creusés et déformés par les vagues et présentent des formes étranges, très recherchées.

\section{Pierre de Mikage (granit)}

On l'extrait dans les montagnes et les vallées des deux districts de $\mathrm{Muko}^{25}$ et d'Ubara ${ }^{26}$ dans la province de Settsu. Dans le village côtier de Mikage au pied de la montagne se trouvent des ateliers de tailleurs où l'on transforme cette pierre en ustensiles, et d'où on l'expédie : c'est pour cette raison qu'on l'appelle pierre de Mikage. La montagne dite Mikageyama se trouve à Kamo dans la province de Yamashiro, où l'on cueille des roses trémières, et il n'y a pas de montagne de ce nom dans la province de Settsu. Cependant 
dans le village, on montre un "pin de Mikage ", chanté par le seigneur Mototoshi ${ }^{27}$ dans un poème recueilli dans le Shoku kokinshū (Suite au Recueil des poèmes anciens et modernes) ${ }^{28}$. Dans les temps anciens, cette montagne était située au bord de la mer, si bien qu'autrefois il était inutile de charger les pierres sur des chariots à bœufs, mais aujourd'hui la baie s'est ensablée et le bord de mer s'est progressivement éloigné de la montagne. Par ailleurs, les carrières situées au pied de la montagne ont été épuisées, si bien qu'on va la chercher maintenant au fond de la montagne et qu'on la charge sur des charrettes à bœufs dans le village Sumiyoshi ${ }^{29}$ situé à 20 chō en amont pour la livrer au village de Mikage [voir Pl. 10].

\section{PI. 10 : Pierre de Mikage de la province de Settsu}

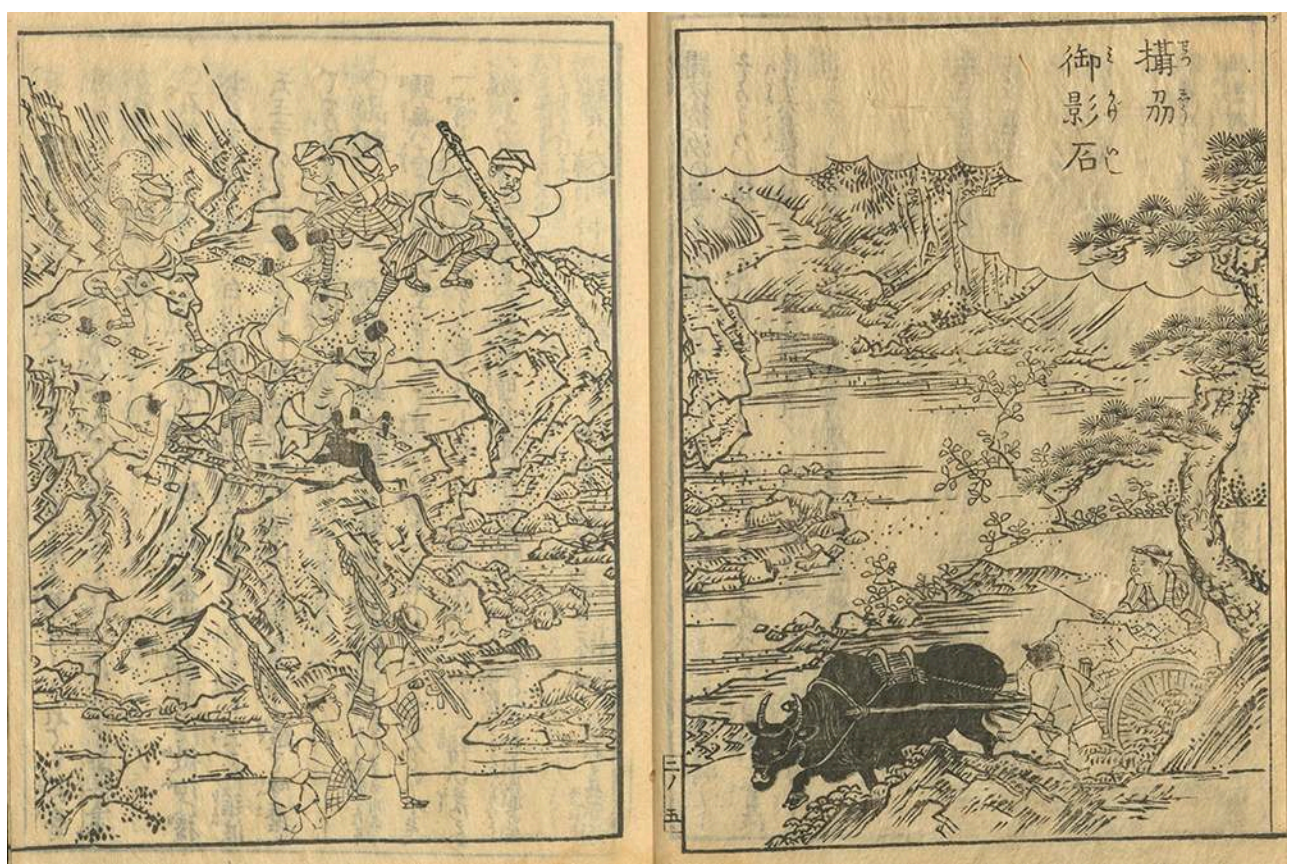

National Institute of Japanese Literature. Lien vers l'illustration

Les pierres de Namase et de Kawara sur la route d'Arima ${ }^{30}$ se trouvent au fond de ces mêmes montagnes. Celles de la meilleure qualité sont très blanches, sans taches noires. On en extrayait par le passé, mais aujourd'hui elles sont devenues rares. Toutefois si l'on ne regarde pas à la dépense, il n'est pas impossible d'en trouver en cherchant sur les hauts sommets ou dans les vallées profondes, mais il s'agit toujours d'endroits où l'on ne trouve guère d'hommes pour le transport.

\section{Caractéristiques de la pierre}

11 Il s'agit d'une pierre extrêmement dure dont le grain et la consistance ressemblent à la pierre de Shirakawa à Kyōto. C'est pourquoi, lorsqu'elle est utilisée pour fabriquer des ustensiles, elle permet au tailleur habile de sculpter les arêtes les plus fines. La pierre de Shirakawa, elle, s'effrite et ne se laisse pas tailler. Pour ce qui est des fabrications de grande taille, on en fait des objets comme le portique du temple Tennōji d'ōsaka, murailles, sarcophages, statues bouddhiques, stèles, murs d'enceinte. Polie, elle est lisse comme de la peau. C'est un matériau qui résiste au temps, un vrai trésor du pays. 


\section{Produits}

12 Les blocs non taillés peuvent être des "grandes vasques" (ōhachi), des "vasques moyennes» (chūhachi) ou des "petites vasques» (kohachi) (Cet emploi du mot hachi a pour origine l'expression « vasque à ablution » (chōzubachi), mais il est très large et peut désigner toutes sortes d'objets comme des fondations de piliers ou des conduits en pierre). On appelle zunashi ${ }^{31}$ les blocs ronds d'environ 1 pied 5 pouces de diamètre, ceux qui sont plus gros sont dits "pierre unique» (hitotsu ishi). On appelle encore "six hommes » (rokunin) les blocs qu'on charge par six dans un chariot. Les « châtaignes » (kuriishi) sont des pierres de petite taille: elles dévalent les montagnes jusqu'aux vallées par temps de fortes pluies, si bien qu'elles n'ont pas d'arêtes. On les utilise pour les pierres qu'on dispose autour de la vasque à ablution ou comme pierres d'ornement. (On trouve dans un chant de la "Chronique de l'empereur ōjin » du Nihon shoki le terme kuri au sens de récifs ${ }^{32}$. Dans le Man'yōshū, on peut lire l'expression okitsu ikuri3 ${ }^{33}$ qui serait une forme vernaculaire de la région San'in ${ }^{34}$. Cette expression ne serait pas en rapport avec la taille.)

13 Pour ce qui est des blocs fendus, on parle de " grande découpe ", " découpe moyenne ", "petite découpe ». On parle aussi de " découpe en longueur» (nobe, pour les blocs coupés longs) ou de "couvercle» (futaishi), il s'agit de blocs d'environ 2 pieds de longueur, d'1 pied 1 à 2 pouces de largeur et de 3 à 4 pouces d'épaisseur). Toutes ces pierres sont utilisées pour la construction de murs d'enceintes, de culées de ponts, de ponts en pierre, d'enceintes de jardin, de fortifications et de nombreux autres usages. Pour les ponts en pierre, on utilise spécialement une pierre extraite dans la province de Kawachi ${ }^{35}$. Pour la détacher, on creuse des trous où l'on introduit des coins qu'on enfonce en précipitant sur eux des blocs de pierre afin de provoquer des fissures, puis on sépare la pierre à l'aide de leviers. C'est la méthode dite " des chocs " (uchitsukewari). On appelle méthode par "levée » (sukuiwari), celle qui consiste à fissurer la pierre en suivant une ligne horizontale.

\section{Pierre de Tatsuyama ${ }^{36}$}

On en produit dans la province de Harima ${ }^{37}$. Comme ces montagnes sont faites d'un seul bloc de pierre, il y pousse très peu d'arbres. Il y en a beaucoup à différents endroits, mais on a commencé à extraire la pierre des endroits d'accès facile pour le transport, et ce sont ceux-là que l'on exploite aujourd'hui, alors que les montagnes d'accès plus difficile restent inexploitées. Le Sanctuaire de pierre ${ }^{38}$ est de la pierre de Tatsuyama, et on exploite intensivement les carrières des environs, l'endroit étant facile d'accès. La méthode d'extraction est partout identique, aussi me dispensé-je d'illustration. La couleur est un mélange des cinq teintes ${ }^{39}$. Les pierres extraites sont toujours taillées en longueur. On utilise cette pierre pour construire des rigoles, des quais de rivières ou de canaux ou bien encore pour des dalles servant à délimiter les jardins, des murs de résidences, des enceintes de jardins, mais pas pour d'autres usages. Leur taille va de 3-4 à 7-8 pieds. On appelle « 5-6» les pierres d'environ 5 pieds sur 6 , et « 5-7 ", celles de 5 pieds sur 7. Il existe également de nombreuses pierres encore plus grandes. (Des ateliers de tailleurs sont disposés dans le village de Shioichi ${ }^{40}$, au pied de la montagne. $\mathrm{Au}$ sud, à Shikazaki, se trouve une pierre en forme de tête de dragon appelée Tatsugahashi, « extrémité du dragon ». C'est pour cela que le lieu s'appelle Tatsuyama) 


\section{Pierres à affûter 砥礪}

On appelle shi 砥 les pierres à grain fin et rei 礪 les pierres à gros grain.

Le dicton dit : « Les pierres à affûter ne se trouvent pas plus loin qu'à 5 lieues autour du palais du souverain, c'est autour de la capitale qu'on les extrait ", ce qui est effectivement le cas. La pierre blanche que l'on extrayait autrefois derrière le Mont Kasuga, dans la province du Yamato, était utilisée comme pierre pour l'affinage final (awasedo) des lames de sabres. Aujourd'hui l'extraction a cessé et seul le site demeure.

Les pierres à affûter qu'on produit aujourd'hui dans la province du Yamashiro ou dans les environs de Saga, à Narutaki et à Takao, sont de la meilleure qualité dans tout l'Empire et en effet on en trouve rarement de la même qualité ailleurs. Celles qu'on extrait à Harayama, aux confins des provinces de Yamashiro et de Tanba ${ }^{41}$, sont appelées uchigumori ${ }^{42}$ ou asagi $^{43}$. On en extrait également à Shire dans la province de Tanba. Toutes ces pierres sont utilisées comme pierres pour polir des sabres, mais aussi pour affûter des rasoirs. S'en servent en outre tous les charpentiers ou menuisiers.

Il y a encore la pierre à affûter de Tosawa dans la province de Kōzuke ${ }^{44}$, une pierre de qualité supérieure, qu'on peut utiliser sans la mouiller. La pierre de Nakura en Mikawa $^{45}$ est blanchâtre avec des mouchetures. La pierre à affûter d'Echizen ${ }^{46}$ est communément appelée pierre de Jōkeiji ${ }^{47}$. Elle est de qualité inférieure à la pierre uchigumori. Toutes les pierres énumérées ci-dessus servent à l'affûtage final des lames de sabre. Dans le Bencao gangmu, elles sont désignées par le terme esshi 越砥 ${ }^{48}$. (Toutes ces pierres sont entourées de gangues. Lorsqu'on les extrait des carrières, on les taille en blocs rectangulaires et il est de règle de charger quatre blocs par cheval).

18 La pierre à affûter bleue (aoto, ardoise) se trouve à Hirao, Somata, Minamura, Monzen, Nakamura, Idenokuro, Yufune, etc. Minamura et Monzen, en particulier, sont situés à quelque 7 lieues au nord-est de Kyōto et forment une montagne de 7 lieues de tour. Dans la province de Tanba, on trouve les pierres d'Inokura, Saiki, Ashinoyama, Ōgitani, Nagatani, Ōbuchi, Iwatani, Miyakawa et beaucoup d'autres produits. Il y a aussi la pierre d'Amakusa dans le Hizen ${ }^{49}$, les pierres rouges et blanches d'Iyo, etc. Toutes ces pierres sont appelées aussi pierres à affûter de grain moyen (nakato). Cependant, il est impossible de parler en détail des qualités et des défauts de chaque produit.

Les pierres à polir à grain fin (awaseto) dont il a été question plus haut et les pierres à affûter de grain moyen (nakato), sont toujours mêlées à la terre et aux roches de la montagne. Aussi perce-t-on une galerie au pied de la montagne et creuse-t-on profondément, ouvrant ici et là des fenêtres, le long de la veine, en s'éclairant avec des lampes à huile en conque de turbot cornu qu'on tient à la main, tout comme on creuse une galerie dans les mines métalliques. Quand la veine est épuisée, on enlève les boisages et on laisse la montagne s'effondrer. Aussi, même en temps ordinaire, l'intérieur des galeries donne-t-il l'impression d'être sur le point de s'effondrer, spectacle effrayant qui fait se dresser les cheveux sur la tête de ceux qui ne sont pas du métier. J'énumère plus bas les pierres adaptées aux différents usages. Par exemple, la pierre mangée de «vers» (mushikuito) de Tsushima sert au polissage des miroirs et des nodosités des ustensiles en laque. Comme elle s'effrite quand on la plonge dans l'eau, on ne peut l'utiliser pour l'affûtage des sabres, mais elle est idéale pour polir les moules utilisés dans l'artisanat de l'argent. En revanche pour les moules servant à couler des poids de filets, on utilise plutôt la pierre d'affûtage blanche d'Iyo. Cette pierre d'affûtage blanche est également une matière étonnante, qui se forme par la concrétion 
au fil du temps de débris de roches qui s'accumulent dans une vallée et finissent par former de nouveau des blocs de pierre. C'est pourquoi il arrive parfois que des feuilles d'arbres soient prises entre les débris et qu'elles s'agrègent à la roche constituant des "pierres en feuilles d'arbres", pierres curieuses, qu'on trouve en quantité dans ces montagnes.

Les pierres à affûter à gros grain sont produites à Karatsu et Monkuchi dans le Hizen, à Kayaganaka et Mikogahama dans le Kii. Celles qu'on produit en Iyo sont d'un grain un peu plus fin. Toutes ces pierres ne sont pas extraites en creusant la terre, mais on en fait tomber des blocs en bas de la montagne, qu'on débite ensuite en milliers de pièces [voir Pl. 11].

\section{PI. 11 : Carrière de pierres à affûter}

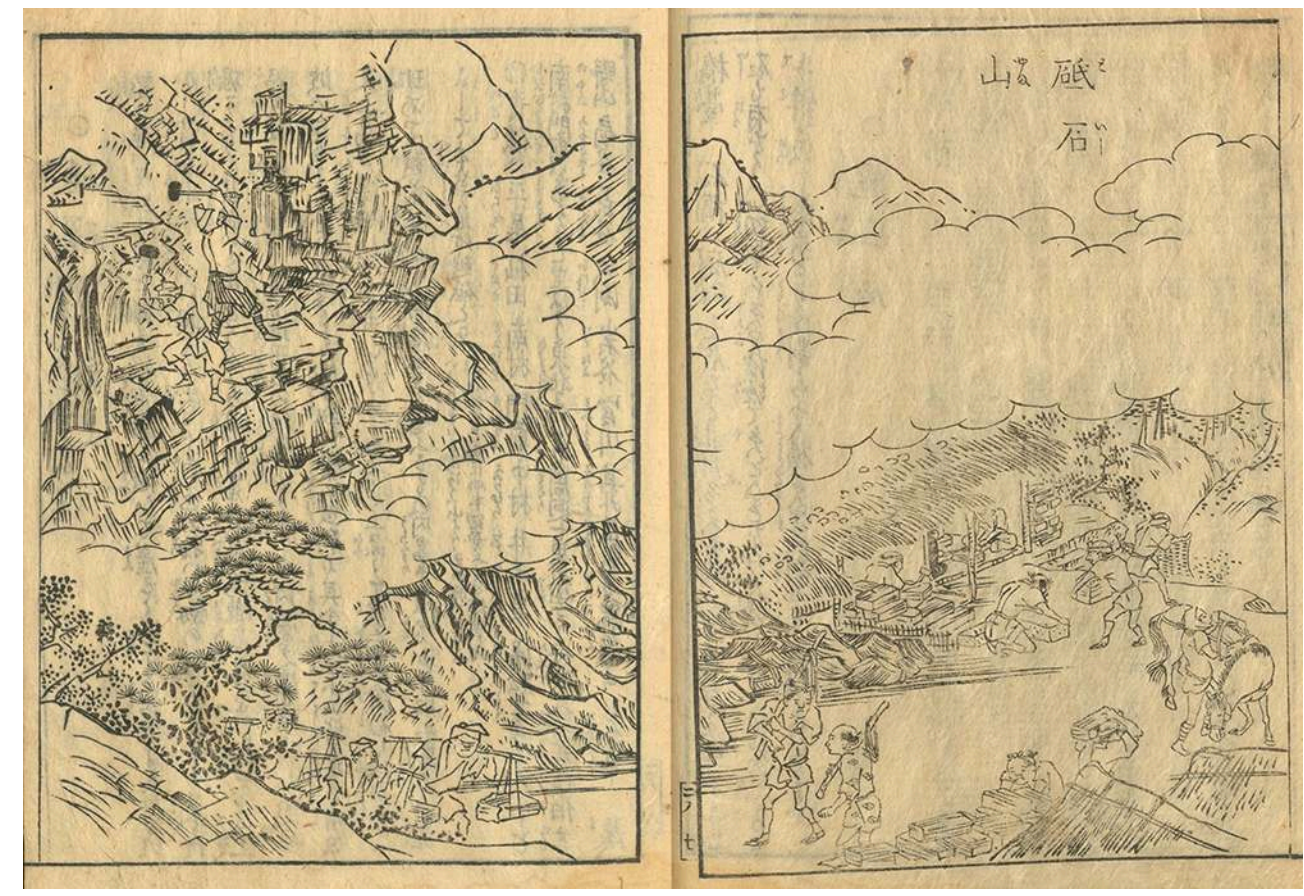

National Institute of Japanese Literature. Lien vers l'illustration

21 Pour ce qui est des pierres utilisées dans les différentes fabrications, les forgerons de sabre utilisent la pierre de Daikuchi, et les polisseurs, la pierre d'Aokaya, Shiro'uma, Chamiko, Amakusa, Iyo, ou encore de Jōkenji, en appliquant successivement des pierres de plus en plus fines. Après avoir affiné les lames avec la pierre d'Inokura ou la pierre uchigumori, on utilise de la pierre d'affûtage concassée (uwahiki) pour conférer à la surface un éclat ciel nuageux. (Le uwahiki est de la pierre uchigumori réduite en poudre. On l'appelle aussi Narutaki no jitsuya [terre à lustrer de Narutaki] et on l'utilise avant la pierre d'Inokura. Une autre appellation est kaki.)

Pour les rasoirs, on les dégrossit d'abord à la pierre de Karatsu, Shiro'uma, Aomiko, Chamiko ou Amakusa, puis on les polit à la pierre de Narutaki ou Takao. Quant aux couteaux, s'il agit de couteaux à tabac, on commence avec de la pierre Daikuchi, puis on les affûte avec de la pierre Hirao ou Somata, sans aller jusqu'à un polissage fin. Quant aux couteaux à légumes comme l'usuba (à deux tranchants) ou le nagatana (à un tranchant), on dégrossit au Daikuchi, Shirouma, Aomiko, Chamiko, Shiroiyo, puis on fait briller avec le uwahiki. Les pièces de cuivre sont dégrossies avec de la pierre de 
Karatsu ou de Mikogahama, puis affinées à la pierre rouge d'Iyo. Les charpentiers et les menuisiers tels que les fabricants de boîtes et de meubles, passent sur les ustensiles en bois de la pierre bleue de Monzen, Hirao ou Somata, puis les polissent avec de la pierre de Narutaki ou de Takao. Pour les couteaux de cuisine, on utilise la pierre bleue (aoto) de Yamashiro; pour les petits couteaux, la pierre de Minamura; pour les objets en bambou, la pierre d'Amakusa. S'agissant d'aiguilles ou de pinces à épiler, on les dégrossit à la pierre de $\operatorname{Tos}^{50}$, puis on les polit avec de la pierre rouge ou blanche de la province d'Iyo. Pour les poinçons de teinturiers (katahori), on utilise la pierre blanche d'Iyo. Pour les couteaux à papier (kamitachi), la pierre Somata. Voilà ce qu'il en est des principaux produits. En matière de façonnage, on dit qu'il convient d'appliquer une matière dure à une matière tendre et inversement, mais en fait certaines qualités de pierre conviennent naturellement à certaines qualités de métal et il est difficile de donner un principe général.

\section{Variétés de champignons}

On appelle kin 菌 ou jin 罩 les champignons qui poussent en terre et sen ceux qui poussent sur les arbres. Le dictionnaire Wamyōshō (Répertoire ordonné des noms du Yamato) $^{51}$ donne la lecture take pour le caractère kin, et ki no mimi (oreilles d'arbre) pour le caractère sen. Il existe de nombreuses espèces de champignons (kin). On distingue les champignons des arbres (comme les oreilles de Judas), les champignons de terre (qui sortent de terre), les champignons de roche (comme l'iwatake ${ }^{52}$ ) et il en existe d'innombrables variétés. Cela est décrit en détail dans l'ouvrage Junpu (j. Kinfu, Traité des champignons) ${ }^{53}$, rédigé par un maître de l'époque des Song, Chen Renyu (j. Chinjingyoku). Dans le Bencao gangmu on peut lire: «En général, les champignons qui croissent dans les montagnes durant les septième et huitième mois, quand la chaleur et l'humidité sont très importantes, ont un goût sucré, une consistance agréable et sont propres à être consommés ». Cependant les champignons qui figurent dans le Junpu et dans le Bencao gangmu sont souvent différents de ceux qu'on trouve dans notre pays, et il est difficile d'en traiter exhaustivement.

Bien que les champignons soient appelés couramment kusabira en japonais, dans le Wamyōshō c'est ainsi qu'on lit le mot saiso (légumes). Dans les dialectes du Chūgoku ${ }^{54}$ et du Kyūshū, on trouve le mot naba. Du côté de la province d'Owari ${ }^{55}$, on dit mimi.

\section{Saiwatake ${ }^{56}$}

L'appellation courante est reishi. On l'appelle aussi kameisō (herbe des mandarins), fushisō (herbe d'immortalité), fukusō (herbe de bonheur). Les lectures japonaises sont katode take, sakikusa.

Dans le Bencao gangmu, on trouve l'appellation goshikidake (champignons de cinq couleurs). Il s'agit d'un remède merveilleux. Depuis que les quatre vieillards chenus du Mont Shang ${ }^{57}$ ont ramassé et consommé ces champignons saiwatake, ils sont devenus la nourriture de tous les ascètes des montagnes. Outre les cinq couleurs, il y a des saiwatake de couleur mauve. Ainsi l'on distingue six variétés. Les mauves sont particulièrement nombreux. Ils poussent en terre, dans le sable ou sur les roches ou sous les pins, et une fois qu'ils apparaissent en un lieu, ils s'y maintiennent pendant des années. Au tout début, ils sont de couleur jaune, puis, les jours passant, ils prennent 
une teinte rouge, et à maturité, brun-mauve. Le pied est noir et luisant. L'envers du chapeau n'est pas découpé, mais lisse. Quant au goût, aux cinq couleurs s'ajoutent les cinq saveurs. Ce champignon est une plante faste, qui s'épanouit trois fois l'an. Le Engishiki (Règlements de l'ère Engi) le mentionne aussi dans le chapitre consacré aux présages heureux. Dans le Ruimingli ${ }^{58}$ il est dit que cette plante apparaît quand le souverain est humain et miséricordieux, ce qui va dans le même sens. Il pousse parfois isolément, parfois en groupe. Des champignons peuvent pousser en touffe à partir d'un seul pied comme pour la poule des bois (Grifola frondosa, polypore en touffes). Ou bien un pied développe des ramifications avec des chapeaux. Dans certains cas, au contraire il n'y a pas de chapeau et la tige seule croît et se ramifie jusqu'à une taille d'environ 3 pieds, comme la ramure d'un daim. On appelle cela saiwatake à ramure de daim. C'est une curiosité : l'an dernier on en a découvert dans les montagnes d'Ise. Il y a en tout quelque 600 variétés de saiwatake, dont les plus rares sont décrites en détail dans le Bencao gangmu. Dans la province de Tanba, on offre ces champignons pour célébrer un départ en voyage. À Ise, on les appelle mannen take («champignons de dix mille ans ») et ils font partie des décorations du Nouvel An. À Edo on les appelle neko jakushi, à Sendai mago jakushi et on les utilise pour faire tomber les pustules de la variole.

Saru no koshikake ${ }^{59}$

Il s'agit de la même espèce que les saiwatake. Il pousse sur les arbres et n'a pas de pied. Les plus grands peuvent atteindre 4 à 5 pieds.

\section{Shiitake}

Autres noms : kōshi kōkin shojin ${ }^{60}$

Ceux que l'on produit dans la province de Hyūga ${ }^{61}$ sont considérés de qualité supérieure. On en fait pousser en grand nombre dans la région de Kumano ${ }^{62}$. On considère que les véritables spécimens sont ceux qui croissent sur les troncs des arbres shii ${ }^{63}$. Cependant il en vient peu spontanément ${ }^{64}$. Pour en produire, on abat des arbres shii, on les laisse se décomposer sous la pluie, on les arrose d' " eau blanche ${ }^{65}$, puis on les recouvre de nattes tressées. Au bout d'un certain nombre de jours, les champignons viennent. Il arrive aussi qu'on en produise en abattant des chênes. Après les avoir cueillis, on les sèche non pas au soleil mais en les passant à la flamme pour qu'ils conservent leur parfum. On appelle kiboshi (séchage sur pied) la technique consistant à sécher les champignons alors même qu'ils poussent encore sur l'arbre : ils sont alors d'une saveur exquise. Leur nom chinois est kajin ("champignon domestique) ». La forme est semblable à celle du matsutake et les spécimens authentiques sont ceux dont le pied pousse au centre. En Chine, il existe également une variété appelée « champignon du tonnerre ». Je me demande s'il ne s'agit pas de champignons cultivés. Dans le Tongya ${ }^{66}$, on lit : " On incise des cedrela de Chine, des ormes, des chênes avec une cognée, puis on laisse leur écorce se dégrader lentement sous la pluie et on arrose d'eau de riz. Lorsqu'on entend le tonnerre gronder, les champignons se mettent à pousser. Si le tonnerre ne gronde pas, on prend une grande cognée et on en frappe les arbres. Aussitôt les champignons se mettent à pousser ». Cela ressemble à la méthode utilisée pour cultiver les shiitake (« champignons parfumés »). Aujourd'hui, les shiitake produits à Yoshino ${ }^{67}$ ou au Mont Ise dans la province du Yamato sont supérieurs à ceux du Hyūga. On y utilise la méthode suivante. On abat de nombreux charmes (shidenoki), on les entasse dans un endroit, on les enterre à moitié et on les entoure d'un enclos pour 
les protéger du vent. On les laisse pendant environ un an, exposés aux intempéries et au soleil. On guette le moment où ils seront décomposés à souhait, puis on se sert d'une grande cognée pour en frapper le bois et opérer des entailles. Cela suffit, et il n'est pas besoin d'arroser le bois avec de l'eau de riz. Cependant les champignons poussent d'abord en petit nombre, et ce n'est généralement qu'à la troisième année que la récolte est vraiment abondante. Mais par la suite, comme la production diminue d'année en année, on doit de nouveau pratiquer des entailles avec la cognée pour que la production se maintienne. Les champignons viennent du printemps jusqu'à l'automne, mais pas en hiver. La production obtenue au printemps est considérée comme étant de qualité supérieure entre toutes et on l'appelle "parfum du printemps». Les champignons venus en été ont des chapeaux plus fins et ne sont pas aussi bons. Par ailleurs, on met à part comme étant de toute première qualité et on appelle « parfum de neige » des champignons aux bords épais et à la forme parfaite. Ces champignons sont sélectionnés parmi ceux de la catégorie "parfum de printemps » et appelés ainsi à cause de leurs lamelles de blanc pur.

\section{Champignon de roche (iwatake) $)^{68}$}

Autre nom : sekishi

À Kumano, au sommet du Pic du Tengu ${ }^{69}$, se trouve un énorme rocher. Là poussent en grand nombre des champignons de roche. On les trouve toujours sur des pentes abruptes et rocheuses. Pendant les mois d'été, lorsqu'il fait très chaud, ils sont tout petits, tel le lichen des pins ${ }^{70}$. Le dessus est noir, le dessous, bleu, la forme, semblable à celles des oreilles de Judas (kikurage). Ils n'ont pas de pied. Ils poussent sur des roches, dans des endroits sombres. Pour les cueillir on utilise des échelles fixées par des cordes, ou bien on monte dans des nacelles de cordes suspendues aux branches d'un arbre, comme on peut le voir sur l'illustration. [voir Pl. 12] Quoique cela soit effrayant à voir de loin, c'est bien plus aisé que de grimper aux arbres à la manière des singes. C'est également ainsi, dit-on, qu'on recueille les œufs de fauvette. C'est la production du Yamato qu'on considère comme étant de qualité supérieure. 


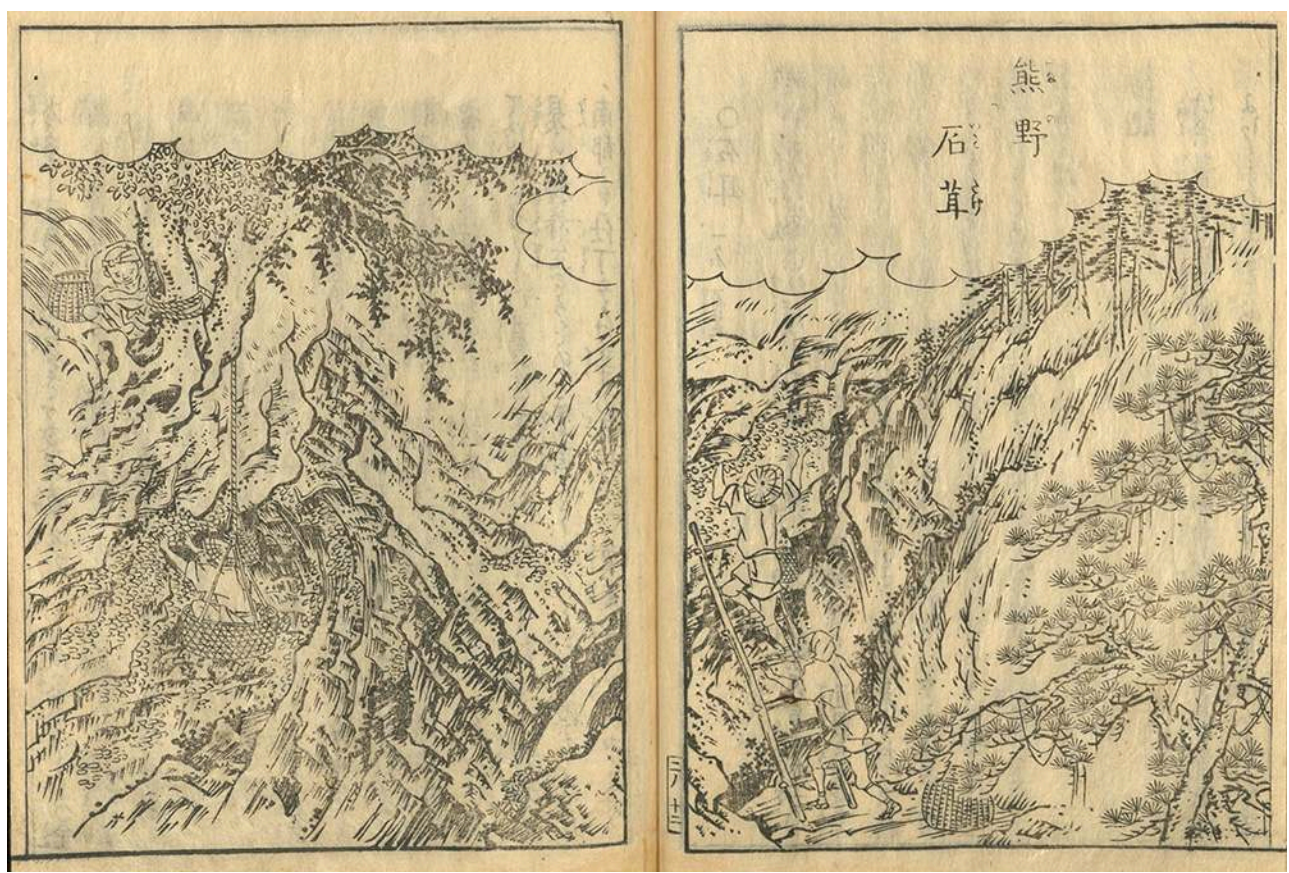

National Institute of Japanese Literature. Lien vers l'illustration

\section{Addenda}

Il existe beaucoup d'autres espèces de champignons.

- La province de Yamashiro produit de bons matsutake (champignon des pins). Ces champignons ne poussent que dans les endroits où se trouvent des pins femelles. Ainsi, comme il y a beaucoup de pins mâles dans les provinces de l'Ouest, on y trouve très peu de matsutake, mais de nombreux bukury $\overline{\bar{o}}^{71}$. Dans la région de la capitale, les pins femelles sont nombreux et les matsutake sont donc nombreux, tandis que les bukuryō sont rares.

- Le kintake (champignon d'or) ${ }^{72}$ pousse de l'hiver au printemps et ressemble au matsutake mais il est petit.

- Le shimeji $i^{73}$.

- Le nunobikidake.

- Le hatsutake ${ }^{74}$ a le dessous du chapeau verdâtre. Du côté d'Owari on l'appelle ōhachi.

- Le nametake, qu'on appelle mizutake dans les provinces de l'ouest. Il pousse en hiver.

- Le hiratake (pleurote en forme d'huître), produit en abondance au mont Kōya, pousse sous tous les arbres.

- Le maitake $e^{75}$ ressemble au hiratake. Plusieurs champignons poussent à partir d'un seul pied, telles des aiguilles. De petite taille, son sommet est de couleur mauve.

- Le kikurage (oreilles de Judas) se fixe sur l'écorce des arbres. Il est de couleur jaune pâle au début, puis prend une teinte rougeâtre. Cueilli et séché, sa couleur tourne au noir. Au Japon, on considère que ceux qui poussent sur les sureaux sont de qualité supérieure.

- Le kuwatake ("champignon de mûrier»). Il en existe deux espèces. Les durs, sont des polypores du mûrier. Les mous sont des oreilles de Judas destinées à la consommation. On trouve aussi des champignons sous les sophoras du Japon, les ormes, les saules, les deutzias. Le champignon du cyprès pousse sur des souches de cyprès du Japon coupés et ressemble aux pleurotes en forme d'huître. Ils poussent en abondance dans les montagnes reculées. 
- Le kuzutake («champignon de puéraire») est de l'extrait de fleur de puéraire. Le benikin en est une variété. Une autre variété produite au printemps est appelée «champignon de la fauvette" ou encore sakutake. À Tanba, on l'appelle akatake, à Nara, shichōtake. Le ogitake ( (champignon des roseaux») est un shimeji qui pousse dans les roseaux. Il pousse vers le neuvième mois.

- Le inokorotake est appelé aussi haritake (« champignon-aiguilles »), mais diffère de ce qu'on appelle habituellement par ce nom. Le véritable haritake développe en poussant un chapeau, au-dessous duquel se trouvent des aiguilles. Il est de couleur blanche et de goût amer.

- Le ushinokawatake («champignon cuir de bœuf», oreille de terre) pousse en abondance sur les collines ombragées, dans les racines des arbres. Le pied est court et ils poussent en grappes. Le dessus est noir, avec des filaments bruns. Le dessous est blanc, sans lamelles.

- Le kawatake (« champignon cuir ») est de couleur noire et de même espèce que le précédent. Il en est de même du kurokawatake (« champignon cuir noir »). place. Ensuite vient la province $\mathrm{d}^{\prime} \mathrm{Aki}^{\mathrm{7}}$. En outre, on en produit depuis les temps anciens dans les provinces d'Ise, d'Owari, de Tosa, d'Iwami ${ }^{77}$, de Chikuzen ${ }^{78}$, d'Iyo, de Tanba, de Tango, d'Izumo ${ }^{79}$, etc. Il y a aussi le miel importé. Il est de qualité inférieure. On le fabrique avec du sucre ou avec du sucre raffiné. Voici comment on fait pour les reconnaitre : si on chauffe du miel japonais, les abeilles sont attirées spontanément, tandis qu'elles ne sont pas attirées par le miel importé. C'est ainsi qu'on les distingue. Le miel s'accumule dans les nids des abeilles pendant l'été : elles en font leur provision de nourriture pour l'hiver. Certaines espèces construisent spontanément des nids dans les habitations humaines, et on appelle « miel de montagne» le miel qui s'y accumule. On appelle « miel d'arbres » celui qui s'accumule dans des nids construits dans les creux des grands arbres. À Kumano, ces miels sont appelés "miel de montagne» et sont considérés de qualité supérieure. On appelle "miel de roche », le miel qui se dépose dans les rochers. Quant au miel qu'on récolte d'abeilles élevées à la maison, comme on doit chaque année vider le contenu du nid, il a moins de goût. On l'appelle «miel domestique ». Celui qu'on obtient en séchant le nid au soleil et qu'on recueille en le laissant s'écouler dans un récipient, est appelé «miel d'écoulage » et il est de qualité supérieure. Le nom chinois est shōmitsu (« miel cru»). (Une autre méthode consiste à mettre le nid dans une cuve et en extraire le miel en le faisant chauffer sur du feu. Cependant il est essentiel de trouver très exactement la bonne chaleur.) On peut aussi prendre le nid, l'écraser et le découper avec les larves, ajouter de l'eau et porter à ébullition, puis extraire le miel par pressage. C'est ce qu'on appelle le «miel de pressage ». (En chinois on l'appelle jukumitsu « miel cuit »). D'une manière générale, le 
miel n'a pas une couleur déterminée. Il peut prendre une multitude de couleurs selon la nature des fleurs du lieu.

\section{Abeilles domestiques}

Noms chinois: kazoku «pirates des fleurs», mikkan «eunuques du miel », ōyōdo «porteurs royaux », kabai « marieuses des fleurs »

Lorsqu'on veut élever des abeilles chez soi, il faut d'abord fabriquer un nid avec un baquet ou une boîte. On y place du saké ou de l'eau sucrée et on perce de nombreux trous dans le couvercle. Quand on place le baquet près d'un nid installé dans le creux d'un grand arbre, les abeilles s'y transportent d'elles-mêmes. On les ramène alors chez soi, et après avoir bien refermé le couvercle, on accroche le baquet à l'avancée du toit ou à une fenêtre [voir Pl. 13].

\section{PI. 13 : Abeilles de Kumano}

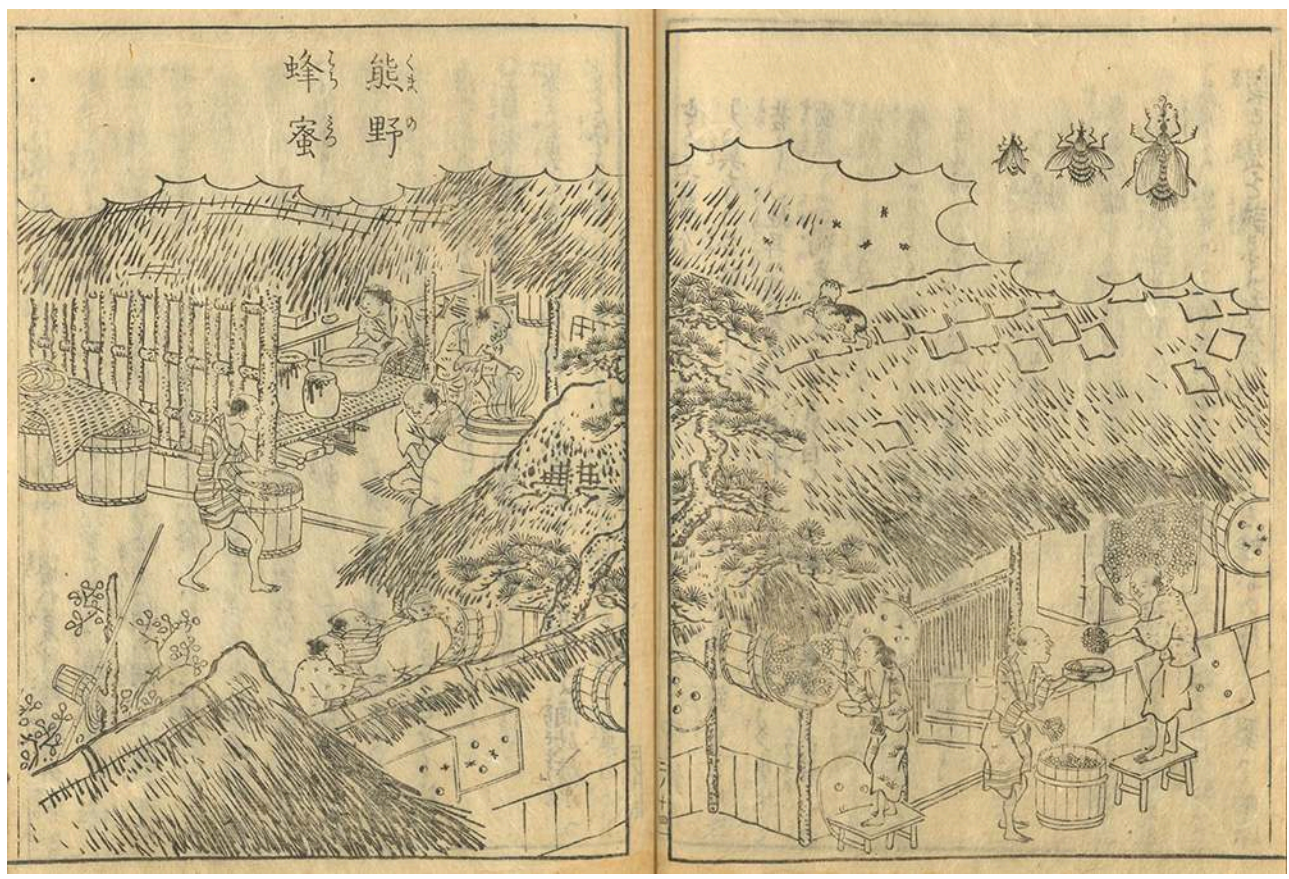

National Institute of Japanese Literature. Lien vers l'illustration

Il y a des règles concernant la dimension de ce baquet ou de cette boîte. Cependant elles sont différentes selon les provinces. Voilà ce que j'ai entendu au sujet d'une maison située dans le Kyūshū: la boîte doit avoir une face carrée de 9 pouces de côté, une hauteur de 2 pieds 9 pouces et on l'accroche verticalement. Il y a aussi des maisons où l'on préfère installer les boîtes obliquement. On s'abstient d'utiliser pour ces boîtes des essences parfumées et on utilise toujours du bois de vieux pin. On ne le travaille qu'à la scie, sans raboter. L'épaisseur des planches est d'environ 4 lignes. On fixe des deux côtés de la boîte des anses assez solides qu'on attache fermement avec de la corde, faute de quoi, la boîte devenant par la suite très lourde, il arrive qu'elle tombe et se fracasse. La porte comporte un panneau supérieur et un panneau inférieur et au-dessus de la partie inférieure on ménage une fente de 1 ligne 8 ri de hauteur et d'environ 4 pouces de largeur pour permettre aux abeilles d'entrer et de sortir. Si la fente est trop large d'1 ou $2 r$ i, des guêpes s'introduisent par l'ouverture et dérangent beaucoup les abeilles. 
C'est aussi par là que sort le roi qui est en général plus petit que la hauteur de la fente. Le nombre de boîtes est limité à trois ou quatre par maison. Pour élever d'autres abeilles, on emprunte des emplacements dans les maisons voisines.

\section{Fabrication des nids}

miel ou les fleurs qui servent de nourriture à leurs petits. Quand les petits sont devenus grands et se mettent à aller et venir en volant, elles stockent aussi du miel dans les alvéoles qu'ils ont libérées. Le miel est au début une liqueur très peu sucrée. Mais à mesure que les abeilles déposent le miel, avec le passage du temps, la saveur sucrée s'accroît de jour en jour, comme cela se passe quand on brasse du saké. Quand l'alvéole est remplie de cette liqueur, l'ouverture en est fermée de manière à ce que pas une goutte ne se perde et que le miel garde toute sa saveur. Plus le nombre d'abeilles est important, plus le miel est parfumé.

Les abeilles ne sont pas grandes ; leur taille est d'environ 5 bu et elles ressemblent aux maruhach ${ }^{80}$. Elles sont jaunes et noires. Elles se rassemblent en grand nombre, et celles qui butinent les fleurs ne construisent pas le nid, tandis que celles qui construisent le nid ne butinent pas les fleurs. Il leur arrive de temps en temps d'échanger leurs fonctions. Il y a parmi elles une grosse abeille qu'on nomme le roi. Ce roi se tient sur un trône aménagé en dessous du nid des abeilles noires, appelé utena. Les descendants de ce roi lui succèdent et ils deviennent rois à leur tour. Le roi ne butine pas les fleurs : les abeilles ordinaires vont chaque jour le faire à tour de rôle, et lui offrent leur récolte. Bien il n'y ait qu'un seul roi par baquet, il fait des petits, tout comme les espèces qui ont mâle et femelle. C'est quelque chose de merveilleux, qui sort tout à fait de l'ordinaire. Les abeilles ordinaires servent le roi avec la vénération d'un sujet pour l'Empereur. En outre, il y a une dizaine d'abeilles noires, qu'on appelle les ouvrières. Elles gardent l'entrée du nid et surveillent les mouvements des autres abeilles. Quand des sujets tentent de pénétrer dans la ruche alors qu'ils n'apportent pas de fleurs, elles leur reprochent leur manque de zèle et leur interdisent l'entrée dans la ruche. En cas de tentative répétée, elles finissent par tuer le coupable, tout à fait comme si elles exécutaient un ordre militaire. Sur ce point, les abeilles domestiques et les abeilles sauvages se comportent d'une manière identique. 


\section{Séparation de nids}

Quand un des enfants du roi parvient à l'âge adulte et sort en volant hors de la ruche, une partie des abeilles ordinaires se mettent à son service et assurent sa garde avec la plus grande vigilance, tout comme lors des déplacements de l'empereur. D'ordinaire ils s'éloignent de quelque 5 à 10 travées et vont se fixer sur la branche d'un arbre, les abeilles s'accrochant aux dos et aux ventres les unes des autres comme si elles pendaient à la branche. Elles se rassemblent en un groupe compact autour du roi, qui est comme le noyau au centre du groupe. Les apiculteurs les suivent et disposent un sac au-dessous de l'essaim, puis le font tomber en agitant un plumeau sous la branche de l'arbre. Le groupe tout entier tombe alors dans le sac avec un bruit comme lors de la chute d'un objet lourd. (Aujourd'hui ce sac est fabriqué en osier, pour permettre aux abeilles de respirer. Dans le cas contraire, beaucoup meurent.) L'essaim recueilli est transféré dans une boite préparée à l'avance. C'est ce qu'on appelle "séparation de nids ", un peu comme la formation de branches cadettes dans une famille. Si des abeilles s'envolent précipitamment et se séparent du groupe au moment où il tombe dans le sac, elles cessent d'appartenir à l'escorte du roi et ne savent plus où le rejoindre. Lorsqu'elles reviennent à leur nid précédent, les autres abeilles leur en interdisent strictement l'accès, les combattent et les tuent, comme si elles voulaient les punir pour leur manque de loyauté. C'est un spectacle horrible qui émeut jusqu'aux larmes les témoins.

Par ailleurs, il arrive que toutes les abeilles se montrent au dehors du baquet au milieu de la journée vers l'heure des huit coups ${ }^{81}$ et fassent résonner leurs ailes. On appelle cela "tapage de l'heure des huit coups». Au troisième mois, quand les abeilles essaiment, il y a toujours un roi au milieu de chaque essaim. Lorsqu'il y a trois rois dans un nid, les abeilles s'envolent en se divisant en trois groupes. Les éleveurs les arrosent avec de l'eau, si bien que les abeilles, dont les ailes sont mouillées, renoncent à essaimer et reviennent dans leur niche originale. Ainsi, dit-on, on peut les élever d'année en année.

\section{Découpage du nid et récolte du miel}

Le miel se récolte au moment où achève de fleurir le sarrasin, quand il est suffisamment sucré et parfumé. Quand on souhaite le récolter, on commence par frapper légèrement le couvercle : les abeilles se réfugient alors à l'arrière de la ruche. On coupe alors les deux tiers du nid, en en laissant un tiers, que les abeilles reconstruisent dans son état initial. En procédant ainsi, on peut effectuer un nombre indéterminé de récoltes. L'hiver venu on place le nid dans de l'eau bouillante pour obtenir du miel de pression.

Il existe aussi une variété appelée " abeilles de terre ${ }^{82}$, d'une taille d'environ $5 \mathrm{bu}$, qui creusent profondément la terre et y font leur nid. Elles aussi font du miel. À Nanbu, on les appelle detchi-sugari ${ }^{83}$. Or sugari est un vieux nom japonais pour "abeille». Dans le Kokinshū (Recueil des poèmes de jadis et de maintenant) ${ }^{84}$, dans la section "Séparation", on peut lire le poème suivant :

Voyageur qui au matin

t'en vas dans la lande

aux lespédèzes d'automne

Où bourdonnent les abeilles (sugaru)

Quand donc attendrai-je ton retour? 
On trouve encore sur les falaises au profond des montagnes du miel naturel vieilli au cours de nombreuses années. Les gens du pays, pour le récolter, le font s'écouler à l'aide de longues perches. On récolte aussi le miel jeune en escaladant les falaises. Quant aux abeilles d'élevage, on en obtient par boîte 20 kin de miel en incluant celui obtenu par pression ( $1 \mathrm{kin}$ faisant $160 \mathrm{me}$ ) et $2 \mathrm{kin}$ de cire. On considère que ces $2 \mathrm{kin}$ suffisent pour couvrir les frais de restauration de la boîte ou du baquet.

\section{Cire d'abeilles}

appelée aussi « cire jaune »

Il s'agit de ce que l'on appelle "cire jaune ", et qui n'est autre que le nid de l'abeille. C'est-à-dire, ce qui reste du nid lorsqu'on a pressé le miel. Voici comment on fait pour séparer le miel de la cire. Après avoir récolté le miel d'écoulage, on met le nid dans un chaudron rempli d'eau qu'on fait porter à ébullition. Alors on remplit un autre récipient d'eau froide, qu'on recouvre d'une natte de bambou. Lorsqu'on y transvase la cire, les résidus restent sur la natte tandis que la cire surnage à la surface de l'eau dans le récipient. Quand on l'introduit dans un récipient de céramique et qu'on chauffe au bain-marie, la cire se réunit spontanément en une masse compacte. Lorsqu'on recueille du miel de pression, si on le porte à ébullition dans un chaudron, le miel surnage en haut du chaudron tandis que la cire reste au milieu et les résidus se déposent au fond. Il suffit de recueillir la cire et de la faire refroidir pour qu'elle s'agrège d'elle-même pour former de la cire jaune.

\section{Cire d'Aizu ${ }^{85}$}

Il s'agit de la cire qu'on récolte à Aizu dans la province de Mutsu ${ }^{86}$, désignée dans le Bencao gangmu comme "cire blanche d'insecte ». On la produit en élevant un insecte nommé ibokurai qu'on lâche dans les branches de l'arbre ibota ${ }^{87}$ et qui sécrète spontanément sur les branches une cire de couleur très blanche. Cet insecte ne se trouve que dans la province de Mutsu et dans aucune autre. Aussi ignoré-je quelle est précisément sa forme. Aujourd'hui, dans les autres provinces, ce qu'on appelle «cire blanche » est de la cire d'arbre à laque blanchie par exposition au soleil. Quant à la pommade vendue par les apothicaires sous le nom de "cire blanche", c'est du miel d'abeille blanchi. Il ne s'agit pas là non plus de produit authentique. On trouve l'arbre ibota en abondance dans de nombreux endroits. Ses feuilles sont petites et ressemblent à celle du chèvrefeuille japonais ${ }^{88}$. En été, de petites fleurs blanches se forment aux extrémités des branches, puis des fruits. Quand ils mûrissent, ils deviennent noirs et ressemblent à des crottes de rats. En hiver, les feuilles tombent. Lorsqu'on enduit de cette cire la lame d'un sabre, elle ne rouille pas et se conserve longtemps. Si on en enduit des verrues, elles tombent d'elles-mêmes. Aussi l'appelle-t-on « tombe-verrue ». Il est difficile de savoir si la cire d'Aizu qu'on vend aujourd'hui chez les marchands de cire, est authentique ou contrefaite.

\section{Salamandre (poisson-poivre) ${ }^{89}$}

La salamandre habite dans les torrents de montagnes. Elle ressemble au poissoncrocodile $^{90}$, mais sa bouche est plus grande. Elle est de couleur brune et a des taches sur 
la peau. Elle sort volontiers de l'eau et se déplace sur la terre ferme. Les sujets les plus grands atteignent environ 3 pieds. Elle dégage une forte odeur poivrée. Elle monte sur les arbres à poivre et en consomme l'écorce. Quand on les élève, on les entend crier la nuit et leur voix ressemble à celle de jeunes enfants. C'est un animal à la nature extrêmement résistante. On les élève ordinairement dans des bassins et lorsqu'on les attrape, on coupe leur corps en deux. Si on remet la seconde moitié dans le bassin, la chair repousse d'elle-même et l'animal retrouve son aspect premier. C'est pourquoi on l'appelle hanzaki (littér. "coupé en deux») dans le dialecte de la province de Mimasaka ${ }^{91}$. On dit aussi qu'une peau de salamandre continue à bouger pendant une longue période. Il existe une autre espèce qu'on appelle salamandre de Hakone ${ }^{92}$. C'est une salamandre de petite taille. On l'appelle sengan-uo dans la province d'Echigo ${ }^{93}$. Elle ressemble au triton: son abdomen est rouge comme chez ce dernier, ce qui fait qu'on l'appelle aussi akahara (« ventre rouge ») $)^{94}$. On la vend séchée comme remède contre les terreurs nocturnes des enfants. C'est le même animal que le poisson noir qui habite dans les hautes sources du pays de Min, mentionné dans le Wuli xiaozhi (Aperçu sur le principe des choses ${ }^{95}$. Les variétés que l'on trouve dans la province de Sagami ou encore à Karuizawa et à Wada dans la province de Shinano, ressemblent également à ce triton. On peut les voir la nuit escalader les berges des torrents de montagnes. Pour attraper ces salamandres on utilise des sacs en coton, montés comme des épuisettes et fermés à l'une des extrémités à la manière d'une bourse. On les trouve en s'éclairant avec des torches et quand on tend le sac devant elles, elles y entrent d'elles-mêmes. On défait alors le cordon de la bourse et on les récupère dans des jarres [voir Pl. 14]. On en produit aussi dans les provinces de Tanba, Tajima et Tosa.

\section{PI. 14 : Salamandres}

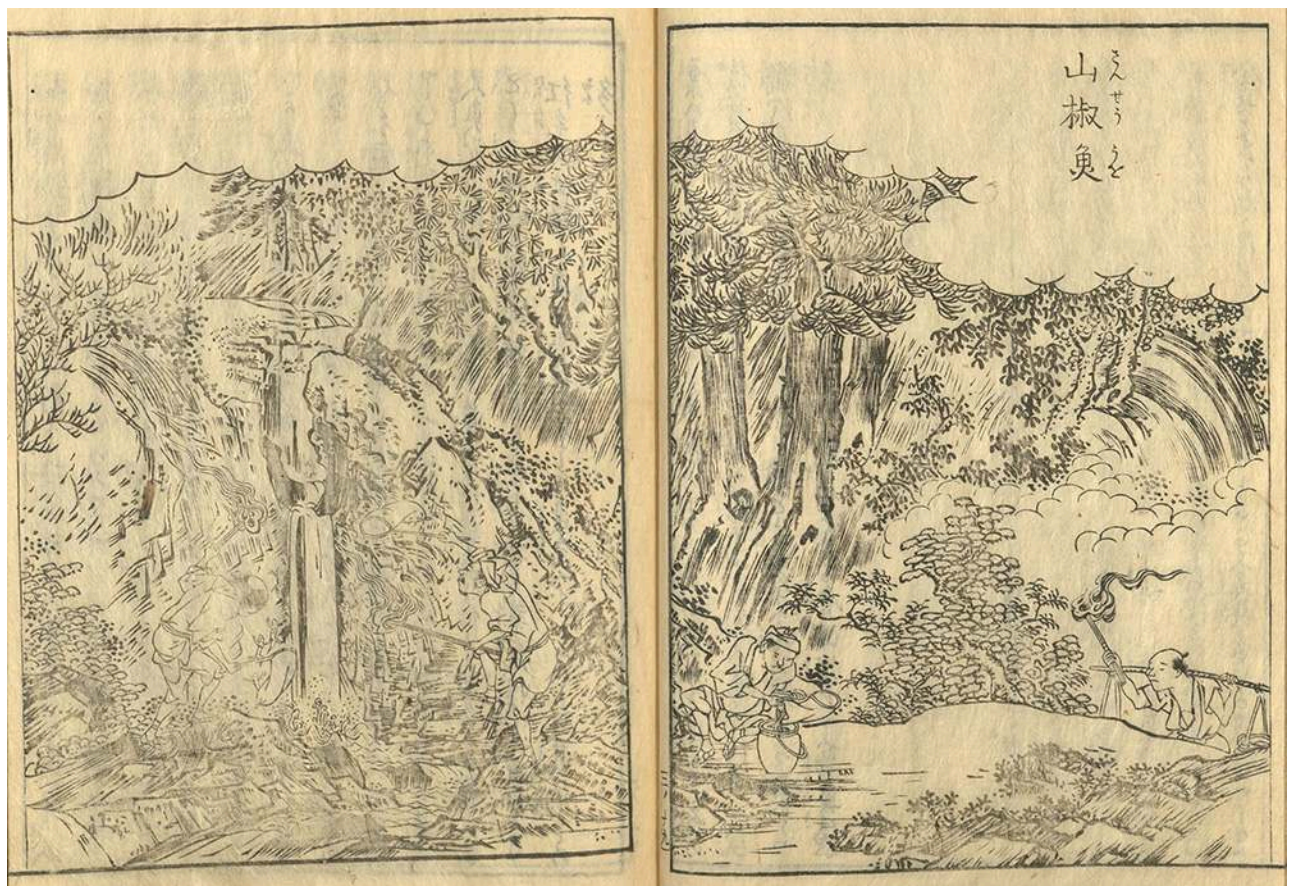

National Institute of Japanese Literature. Lien vers l'illustration

Dans le Bencao gangmu, il est question d'un poisson tiyu (j. teigyo) qui est aussi appelé " poisson poivré ", mais il s'agit de la sirène (j. ningyo) ${ }^{96}$. On le trouve dans les fleuves et dans les lacs. Son aspect ressemble à celui du poisson chat, il a de longs ailerons, tels 
des bras et des jambes. L'animal dont il est question dans le Qishenlu (Histoires pour réfléchir au merveilleux $)^{97}$ de Shizhen est la sirène des mers, qui est mentionné dans le Ka'itsūshōkō (Considérations sur les échanges commerciaux avec la Chine et les barbares $)^{98}$. Les Hollandais qui viennent au Japon possèdent des os de ces sirènes de mer, qu'ils appellent heishimuru ${ }^{99}$. Mais beaucoup sont des contrefaçons.

\section{Puéraire (kuzu)}

Kakkoku, autre nom : « haricot des daims » (shikamame)

Il s'agit d'une liane, dont la racine, appelée "racine de puéraire", est comestible. Réduite en poudre, on l'appelle "poudre de puéraire ». Celle qu'on récolte à Yoshino est réputée de qualité supérieure. On apprécie aussi la variété dite Rokurō dayü $\bar{u}^{100}$, récoltée dans la province de Kii, dont le goût est excellent, sans doute parce qu'on ne lui ajoute aucun autre produit. La plupart du temps, la puéraire pousse naturellement en montagne comme en plaine. Contrairement au Japon, en Chine on la cultive et on l'appelle alors puéraire domestique par opposition à la puéraire sauvage.

Les feuilles de la puéraire ressemblent à celle du haricot, à trois folioles. Elles ont parfois une forme triangulaire, comme celles du haricot azuki ${ }^{101}$. Les tiges comme les feuilles sont couvertes de poils et au septième mois donnent des fleurs d'un rouge mauve, semblables aux fleurs de la glycine. Elles sont en forme d'épis pendants, longs d'environ 3 pouces, et produisent des gousses, également couvertes de poils.

On déterre les racines en hiver et on les hache sur une plaque de pierre, puis, après avoir éliminé le jus, on les pile soigneusement avec un mortier en métal jusqu'à les réduire en une fine poudre, qu'on filtre par lévigation. Ensuite la poudre est exposée au soleil sur des plateaux, puis mise dans des seaux pour être vendue. [voir Pl. 15] (Le Wahōsho ${ }^{102}$ nomme cela "poudre lavée ", mizukona). La racine de puéraire est vendue chez les apothicaires sous deux formes : séchée ou blanchie au soleil. 


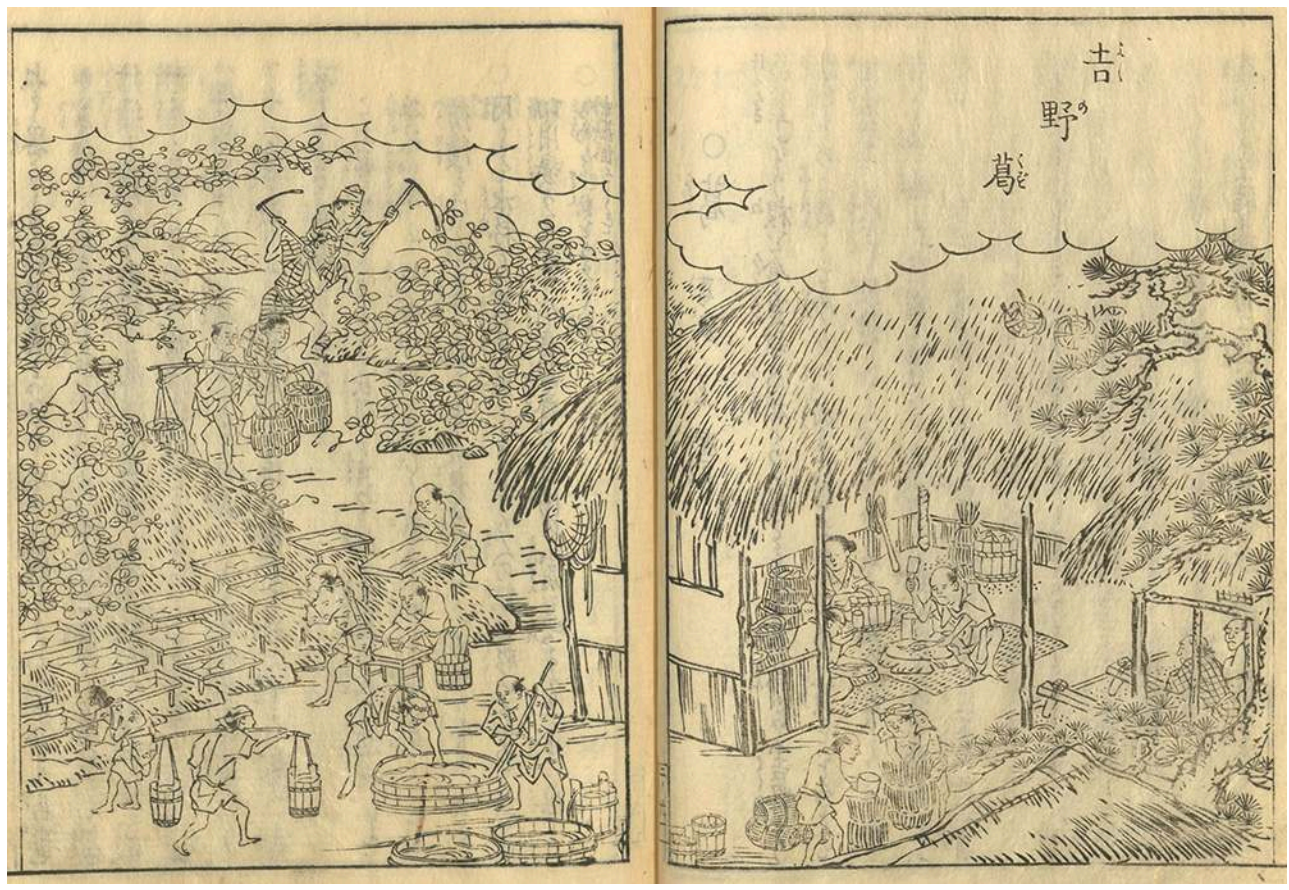

National Institute of Japanese Literature. Lien vers l'illustration

51 La liane est trempée dans l'eau pour en enlever l'écorce, puis tressée pour en faire des ustensiles. C'est ce qu'on produit à Minakuchi et ce qu'on appelle fuchikori (« coffres en vannerie »). On appelle tsuzura la liane tressée. Pour obtenir la toile de kuzu, on cuit la liane, puis on la déchire, la file et la tisse comme la ramie. Dans le Shijing (Livre des odes), on trouve le terme 絺綌 $c h i x i^{103}$. Chi désigne un fil fin, $x i$, un fil épais. Il semble qu'anciennement on tissait en Chine des toiles semblables au crêpe d'Echigo ${ }^{104}$ actuel.

Quant à l'appellation kuzu de la puéraire, le mot signifie « débris » et fait référence à la " poudre lavée ». Le nom originel de la plante était fuji, qui est le même mot que muchi, le fouet. Autrefois on fabriquait les fouets avec cette plante, d'où le nom. Si les vêtements de deuil sont appelés fujigoromo, c'est qu'ils étaient faits en toile de puéraire.

Liane, feuilles, racine, fleurs, écorce, tout dans cette plante a son utilité pour la population. C'est pourquoi les habitants des villages reculés, par familles entières pour s'entraider, vont vivre dans la montagne pour se nourrir des racines de puéraires qu'ils déterrent. Quand la plante a poussé haut et qu'il n'y a plus de poudre, ils descendent de la montagne et s'adonnent au filage et au tissage des fibres qu'ils ont recueillies. Le secours et le profit qu'apporte à tous cette plante la met juste après les cinq céréales. Après vient la « poudre lavée » qu'on fabrique à partir des racines de fougères. Même si c'est une denrée vile, elle est toujours d'une grande utilité quand il s'agit de remédier à la famine. C'est grâce à elle que Boyi et Shuqi eurent la vie sauve sur le Mont Shouyang ${ }^{105}$. (Un produit contrefait est obtenu par l'addition de gluten (namafu). Le goût en est très mauvais.)

54 La poudre de puéraire a de multiples autres usages. Elle sert à préparer des mochi (boulettes) ou des suiton (boulettes en potage). On peut l'ajouter à du fard blanc ou s'en servir comme glu, ou encore pour l'assaisonnement en cuisine : nombreux sont les profits qu'elle apporte aux gens. 
On peut lire dans un ouvrage : la puéraire est très efficace contre les poisons, mais il s'agit de la partie de sa racine qui s'enfonce à plus de 5-6 pouces sous terre et qu'on nomme "bile de puéraire ", qui en constitue la tête. Si on en fait prendre à quelqu'un, on le fait vomir.

\section{Grenouille rouge (Grenouille de montagne) ${ }^{106}$}

Beaucoup proviennent des provinces de Yamashiro et de Saga ou encore de celle de Tanba ou de la montagne de Sayo à Harima. On en trouve également à Kanzaki dans la province de Settsu, mais celles-ci sont d'une nature médiocre. D'une manière générale, elles habitent au fond de marécages plantés de bambou nains ou de roseaux. On les attrape en rabattant sur eux une épuisette, ou à l'aide d'une sorte d'épervier : on tient dans les deux mains la boucle de fermeture et on imprime au filet une torsion comme celle qu'on donne à une toupie ; le filet s'envole brusquement et s'étend sur un carré d'environ 3 pieds de côté [voir Pl. 16].

PI. 16 : Grenouille rouge

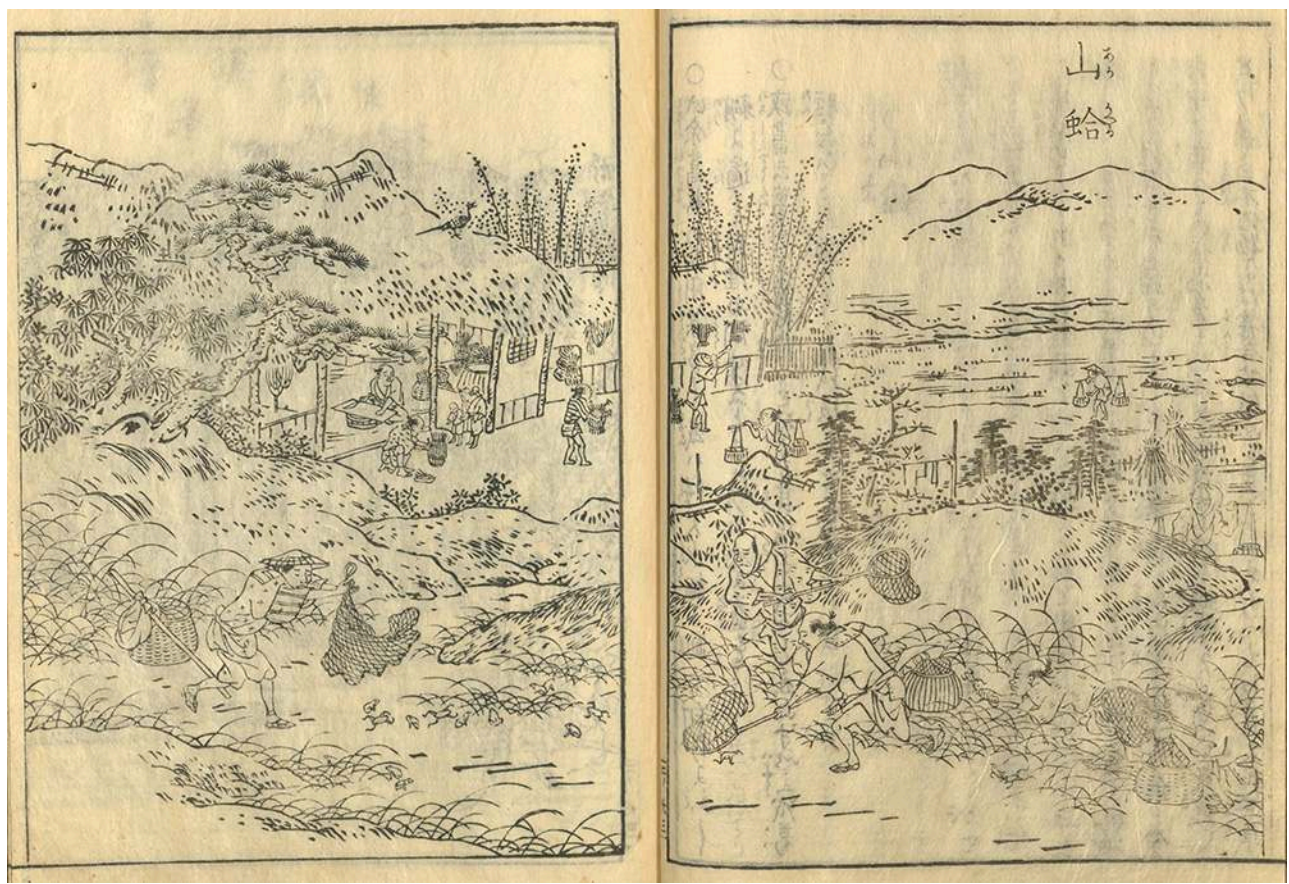

National Institute of Japanese Literature. Lien vers l'illustration

Une fois attrapées, on les éviscère et on les vend séchées. Elles sont de couleur pêche, satinées, avec des pattes supérieures et inférieures très longues, et des yeux semblables à des nœuds d'éventail. Souvent celles que l'on vend aujourd'hui sur les marchés sont fausses. Dans le Bencao gangmu, il est question d'une grenouille de montagne plus grande que le crapaud et de couleur jaune, ce qui ne correspond pas à ce que l'on appelle « grenouille de montagne » au Japon, sans doute parce qu'il s'agit d'animaux de deux pays différents. Le Yamato honzō cite le Mumyōshō (Notes sans titre) de Kamo no Chōmei ${ }^{107}$, où il est question de la grenouille d'Ide. Il s'agit bien de la grenouille rouge. Son cri nocturne diffère de celui des autres espèces. Selon le Yamato honzō, il semble 
qu'elle soit de couleur noire et de petite taille et son identification avec la grenouille de montagne est douteuse ${ }^{108}$.

\section{Insecte de la Vitis ficifolia109}

Autre nom de cette plante : vigne sauvage

Celui qui provient de Takagamine dans la province de Yamashiro est de qualité supérieure. La liane, les feuilles, les fleurs, les fruits de la Vitis ficifolia sont en tous points semblables à ceux de la vigne. C'est de cette plante qu'il est dit dans le Shijing (Livre des odes) que "au sixième mois, on mange le raisin $»^{110}$. Au printemps, des bourgeons se forment qui donnent de petites fleurs jaunes et blanches en forme d'épis. Les fruits se forment au cours du septième et du huitième mois. Ils sont petits, ronds, de couleur mauve pâle. Quand on souffle dans la tige, l'air la traverse. Le jus est semblable à celui de l'akébie. Sur la liane, on trouve ici ou là des renflements dont la forme rappelle celle des racines de la zizanie. À l'intérieur de ces renflements se trouvent des insectes blancs. On récolte ces lianes et on les vend au marché comme remède contre les convulsions des enfants [voir Pl. 17].

\section{PI. 17 : Insecte de la Vitis ficifolia de Takagamine}

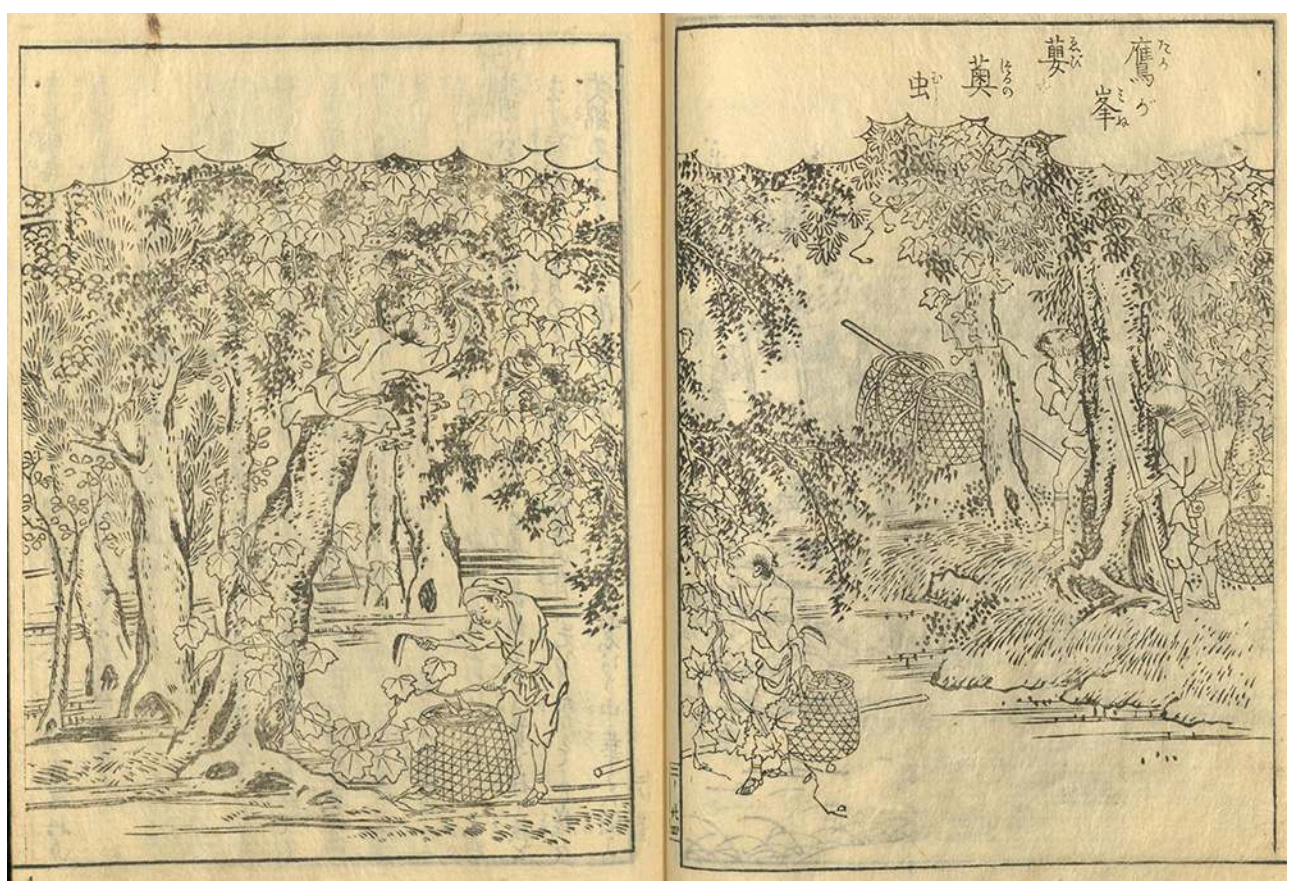

National Institute of Japanese Literature. Lien vers l'illustration

Cependant aucun ouvrage chinois ou japonais ne mentionne la présence de ces insectes dans les tiges de la Vitis ficifolia. On dit que ce remède est même supérieur à l'insecte du saule ou à celui du kusagi ${ }^{111}$, réputés pour la même propriété. À Nara, où il n'y pas de vraie vigne, on récolte le fruit de la Vitis ficifolia, on en retire les pépins et on les cuit jusqu'à l'évaporation du liquide pour en faire une pâte comestible. Quant aux feuilles, le revers en est velu. Séchées et bien malaxées, elles deviennent comme du moxa et sont utilisées pour guérir les verrues. Aussi les appelle-t-on « tue-verrues». En Chine, on en 
fait du vin : c'est le «vin exquis parfumé au curcuma » dont fait l'éloge le poème Tang ${ }^{112}$.

Le terme japonais ebizuru donne lieu depuis longtemps à un malentendu. Ce terme désigne la vigne (budō), tandis que les caractères chinois 顽䓴 désignent aussi bien la fausse vigne (inuebi) que la vraie (budō). Cependant la confusion s'est installée depuis les temps anciens.

\section{Variétés de gibier}

\section{Autour ${ }^{113}$}

61 Ceux qu'on capture dans les montagnes de la province de Ka'i, ou dans les provinces de Hyūga, Tango, Iyo, sont tous de petite taille. Le grand autour ${ }^{114}$ se capture à Kurokawa, Kami-Kurokawa, Ōsawa, Tomisawa, Aburata, Tokkai, Ōtsume, Yamata et d'autres endroits encore de la province de Mutsu ${ }^{115}$. Les autours qu'on capture dans le district de Shinobu de la province de Mutsu sont tous appelés "autours de Shinobu ». L'autour vient de Corée et on l'utilise pour chasser les grues et les oies sauvages. L'élevage de l'autour a pris son origine en Corée et il y a un ouvrage intitulé Yōkotsuhō (Méthode pour élever les autours et les faucons) ${ }^{116}$. Ainsi, lorsque sous le règne de l'empereur Nintoku, Ahiko du Domaine royal de Yosami présenta à la cour des autours, on n'y connaissait même pas le nom de cet oiseau. Alors le prince Shukun de Paekche déclara que cet oiseau était appelé kuchi en Corée. Il arma les autours de jets et de sonnettes et, après les avoir dressés, organisa des parties de chasse et prit de nombreux faisans dans la lande de Mozu. Aussi les gens de l'époque appelèrent-ils le village de Taka'i le lieu d'élevage des autours (taka-kai) : c'est encore aujourd'hui le village de Takaa'i dans le district de Sumiyoshi. Il semble donc qu'au début on ait utilisé au Japon les méthodes d'élevage coréennes.

Ceux qui élèvent et dressent les autours les capturent généralement quand ils sont encore dans leur nid. Cependant à Oyamada dans la province d'Iyo, on attrape les autours à l'aide de filets. C'est une grande montagne qui s'étend sur trois provinces : Iyo, Tosa et $\mathrm{Awa}^{117}$ et comme les autours lors de leurs migrations se dirigent vers les hautes montagnes, on les trouve toujours à cet endroit. On estime que les autours viennent généralement dans la région au tournant des septième et huitième mois, quand commencent à jaunir les fruits du citronnier yuzu.

On utilise des filets dits " tendus » (harikiri ami), aux mailles d' 1 ou 2 pouces, tressés en fil de carex ou de ramie, de 3 à 4 pieds de longueur et d'environ deux travées [12 pieds] de largeur. En dessous on installe un filet en forme de lanterne en fil de coton d'environ 3 pieds, où l'on introduit un passereau ${ }^{118}$, qu'on attache à un pieu. Non loin de là, on dispose à l'avance pendant la nuit un serpent fabriqué en bois, aussi ressemblant que possible, qu'on introduit dans un récipient cylindrique en bambou et auquel on attache un long fil. À l'aube quand l'autour quitte les cimes des arbres pour chasser sa nourriture, un chasseur dissimulé derrière une palissade tire sur le fil du serpent et le fait mouvoir dans la direction du passereau, qui prend peur et commence à s'ébattre. Il est alors aperçu par l'autour qui fond sur lui et se prend dans le filet. Les pieux en bambou disposés des deux côtés du filet sont enduits de laque de manière à ce que le filet glisse bien. Dès que l'autour touche le filet, ce dernier se resserre de lui-même et retient l'oiseau empêtré [voir Pl. 18]. 
PI. 18 : Capture d'un autour à l'aide d'un filet tendu

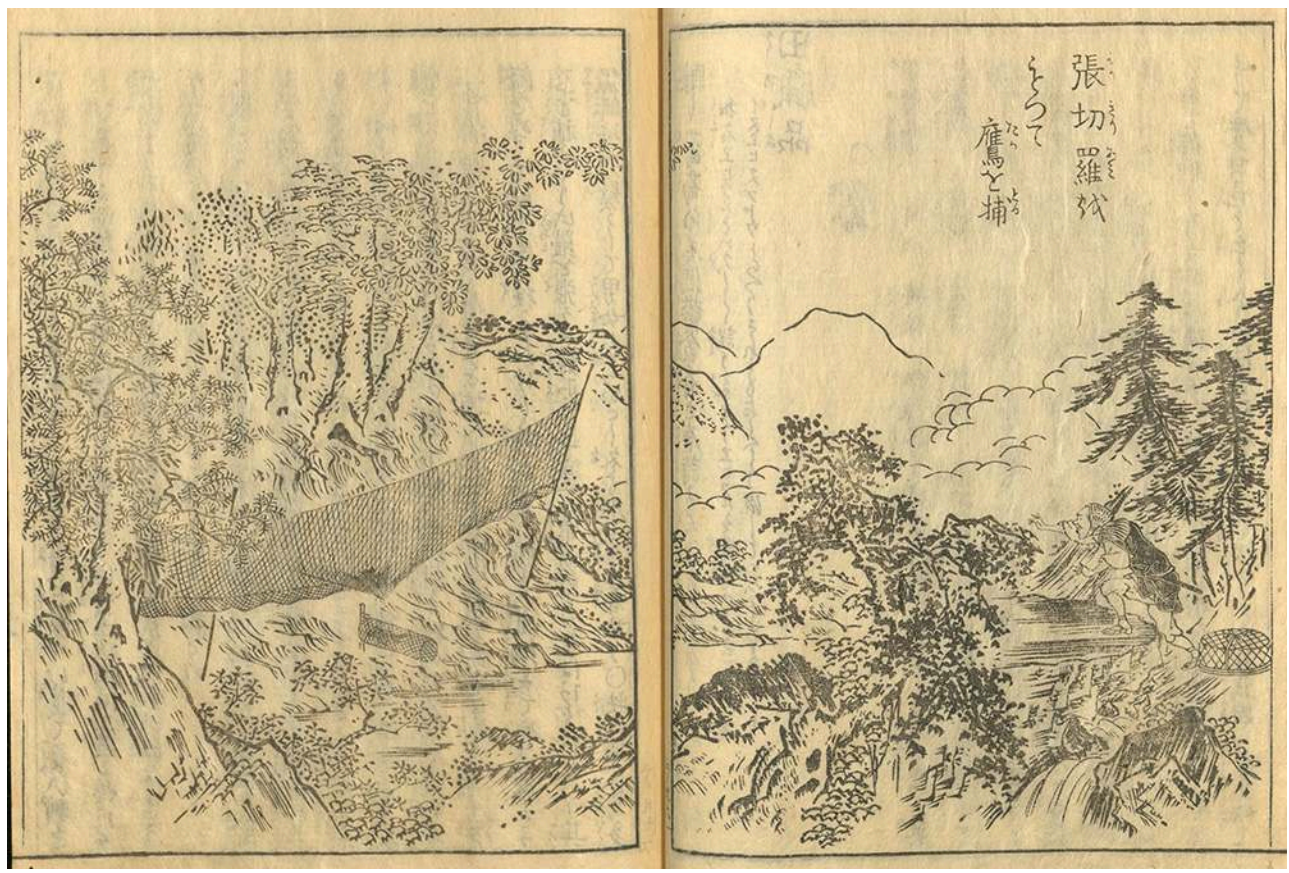

National Institute of Japanese Literature. Lien vers l'illustration

Il paraît qu'il y a toutes sortes de points délicats concernant la manière de tendre ce filet et qu'il ne s'agit pas d'une technique facile. Les gens qui s'adonnent à ce métier ne se rasent pas le front, et il est difficile de distinguer les hommes des femmes. Même en hiver, ils s'habillent de vêtements en lin qu'ils portent les uns sur les autres. Les oiseaux qu'on attrape en ce lieu sont souvent des haitaka ${ }^{119}$, dits aussi hashitaka, et ce sont souvent des femelles. Ils excellent à la chasse aux canards, aux hérons. Leur aspect est semblable à celui du gros autour, mais ils sont de taille plus petite. Leurs plumages comportent toutes sortes de motifs. Après les avoir ainsi capturés, on les arme de jets (yama ashio ou ōo), dont le cordon est toujours tressé en ramie. La partie qui est en contact avec la patte de l'oiseau est faite en cuir chamoisé. L'attache est en bambou ou en corne de daim.

On attache la queue et les ailes des petits autours avec du papier et on les met en cage pour les vendre au village.

Dans d'autres provinces et notamment à Mutsu, le grand autour est appelé sudaka (épervier de nid). C'est parce qu'on le capture dans son nid. Je ne connais pas les détails de cette technique.

67 La nourriture des autours leur est tendue sur une planche.

68 Pour les grands autours, on utilise des poches de protection pour les ailes et la queue faites dans une étoffe souple, qu'on fixe en les cousant en un point. La taille de ces poches varie selon les dimensions de la queue et des ailes.

\section{Différentes appellations selon l'âge de capture :}

- Akage (alezan) (Autres noms : akake (pris au filet), hatsukusa (première herbe), wakataka (jeune épervier). Il s'agit d'autours nés en été et capturés en automne).

- Sutaka (épervier de nid) (Autour capturé dans son nid). 
- Sumawari (alentours du nid) (Autour capturé au cinquième ou au sixième mois, au moment où il sort du nid).

- Nosaretaka (exposé à la lande) (On dit aussi yamasare (exposé à la montagne) ou kisare (exposé aux arbres). Autour capturé au dixième ou au onzième mois).

- Satoochitaka (autour ayant quitté sa demeure) (Capturé au douzième mois).

- Aratamataka (autour du Nouvel An) (Capturé au premier mois).

- Saohime kaeri (autour de printemps) (On dit aussi : otometaka (autour-jeune fille), koyamagaeri (autour de Koyama) autour capturé au deuxième ou troisième mois).

- Kaeri (jeune autour) (Dans les montagnes on dit : les plumes repoussent (kaeru). On appelle katakaeri, la première repousse, et morokaeri, la deuxième).

\section{Comment affaiter un autour}

L'autour qu'on vient de capturer est appelé uchi'oroshi (« frais descendu »). On lui lave soigneusement la queue, les ailes et le pourtour du bec avec de l'eau tiède pour nettoyer les parties tachées notamment par la nourriture. Ensuite on lui taille le bec et les ongles, on fixe les jets à ses pattes et on procède à des sorties nocturnes. De quoi s'agit-il ? Dès qu'on observe qu'un autour « frais descendu » commence à s'habituer aux gens, on ouvre sa cage la nuit et on l'installe sur le poing sans utiliser de lumière. Puis on parcourt plaines et montagnes et à mesure que la nuit s'écoule on lui montre de faibles lueurs de torches, qu'on rapproche progressivement les nuits suivantes. On procède ainsi pour éviter que l'autour ne soit effrayé par le feu des torches et n'en garde un vice, qui ne permettra pas de le sécher facilement au feu de bois lorsqu'il aura les plumes mouillées. Cela permet aussi d'éviter de nombreux autres inconvénients. Après des sorties nocturnes répétées, quand l'autour s'est rassuré et qu'on constate qu'il ne s'effraie pas quand on agite les bras ou qu'on fait des mouvements avec son corps, alors on passe aux sorties matinales. On commence d'abord avant l'aube, puis à mesure que les sorties se répètent, de plus en plus tard le matin, jusqu'à ce qu'on le sorte en plein jour. Quand on constate que l'autour s'est déjà développé et qu'il suit du regard les oiseaux sauvages, on lui montre des oiseaux qu'on a préparés à l'avance à cet effet et on les fait tourner devant lui en les tenant dans la main pour qu'il les attrape. On prend soin seulement d'en tailler ou attacher les becs, pour qu'ils ne blessent pas l'autour et qu'ils ne crient pas. En effet, en criant, les oiseaux pourraient effrayer l'autour, ce qui pourrait lui faire acquérir un vice. On appelle ces oiseaux «proies de proximité à becs arrondis » (koshi marubashi) et on dit qu'on leur "arrondit les becs » (kuchibashi o marobasu). Quand l'autour attrape bien ces oiseaux, on lui donne une petite quantité de "sang tiède" (nukuchi), c'est-à-dire de viande, mais pas trop. Si on lui donne trop de viande, l'autour risque de prendre de l'embonpoint, ce qui est mauvais. Il convient de prendre garde au développement de l'autour : s'il est gras ou maigre, si son plumage ou son allure sont corrects ou non, etc. Dans le cas du grand autour, on considère comme une qualité que ses yeux soient petits, et c'est le contraire pour le petit autour. On examine aussi les déjections et on en corrige la couleur (cela s'appelle régler la diète de l'autour). On élève également des oiseaux qu'on fait voler comme proies, dits tobinagashi : oiseaux dont on coud les yeux pour qu'ils s'élèvent tout droit vers le ciel. Lorsque l'autour devient habile à les prendre, on l'emmène en plaine ou en montagne pour qu'il prenne des oiseaux.

Un autour pris au nid est placé dans une cage tapissée de feuilles d'armoise ou de peau de lièvre. On lui donne de petits oiseaux coupés en fins morceaux, sans aucune 
adjonction d'eau. À peine né, il est appelé norige ou watage (duvet de coton), plus tard, à mesure qu'il grandit, murage (duvet en touffes), puis tsubanage (duvet sur les ailes). On établit l'âge de l'autour à partir de la croissance du balai : on dit " première poussée " (hitofu), puis « seconde poussée » (futafu). À la « troisième poussée » (mifu), on introduit un perchoir dans la cage. Dès le début, la cage est recouverte d'une moustiquaire, pour éviter les piqûres. On appelle autours aînés les mâles, et autours cadets les femelles. On les distingue par leur poids : les plus légers sont des aînés et les plus lourds sont des cadets. Quand la queue et les ailes ont poussé régulièrement et qu'elles sont devenues rigides, on fixe les jets aux pattes de l'autour et on le maintient attaché à son perchoir pendant environ cinq jours. On le laisse tranquille et pendant trois jours on lui fait prendre des bains chauds. S'il ne prend pas de bain on l'asperge à plusieurs reprises. On déploie ensuite les ailes froissées, puis on procède selon la méthode exposée plus haut en lui faisant attraper des oiseaux aux becs taillés et ainsi de suite.

\section{Liste succincte des différentes espèces d'autours}

- Ōtaka (grand autour) (On dit aussi autour bleu (aotaka) ou jaune (kitaka)).

- Hashitae (Les aînés et les cadets sont difficiles à distinguer).

- Faucon pèlerin (hayabusa 鶻) (Mâle. Il est de petite taille).

- Faucon pèlerin (hayabusa 隼) (Femelle. Il est de grande taille).

- Haitaka (Femelle).

- Konori (C'est le mâle du haitaka).

- Tsumi $^{120}$ (On le désigne aussi par le caractère 鵰 et il en existe de nombreuses variétés : kurotsumi, konohatsumi, kayoitsumi, kumatsumi, Kitayama-tsumi. Ils sont tous de la même espèce. On les distingue à leur plumage).

- Essai (Il est plus petit que le tsumi).

- Sashiba ${ }^{121}$ (On distingue : akasashiba, aosashiba, sokosashiba, shitasashiba, susogosashiba).

- Washi (aigle) (Son plumage est entièrement noir, des taches blanches de différentes formes apparaissent avec l'âge. Dans les poèmes on dit que son plumage est noir et ses yeux, bleus. Son bec est bleu. On le reconnaît au duvet qu'il a sur les pattes).

- Kumataka (aigle montagnard ${ }^{122}$ ) (Il est entièrement noir. Diverses taches noires apparaissent sur la queue avec l'âge. Dans les poèmes, le kumataka est connu pour son bec noir, ses serres bleues et le duvet qui couvre ses pattes).

On trouve dans le Yamato honzō le passage suivant:

Quand on examine les autours et les faucons, on peut distinguer trois espèces : les faucons, les autours, les aigles. En outre, on peut considérer que les shirataka (autours blancs), les haitaka, les ōtaka (grands autours) sont des autours. Les hayabusa (faucons pèlerins) et les sashiba (buses à joues grises) sont des faucons. Les aigles et les milans sont des aigles. On dresse les autours et les faucons pour chasser les oiseaux. On ne le fait pas avec les aigles. Par ailleurs, chez tous les oiseaux, le mâle est plus grand. C'est seulement chez les autours que les femelles sont plus grandes. On trouve cela dans les ouvrages chinois. ${ }^{125}$

Pour plus de détails, il faut se reporter aux sources. Ici je n'en parlerai pas. 


\section{Canard}

Parmi les canards, ceux qu'on prend dans les environs d'Ōsaka dans la province de Settsu sont les plus savoureux. Ceux de Kitanakajima sont considérés comme de qualité supérieure. Ensuite viennent ceux de Kawachi. Le filet utilisé pour les prendre est appelé partout ailleurs kamoami (filet à canard), mais dans la province de Settsu on dit shikiden. Ce sont des filets de fil fin, de quelque cinq à six travées de long et d'une travée de haut qu'on tend aux deux bouts sur des perches de bambou. On en tend trois ou quatre, en laissant entre eux une distance de quelque trois travées [voir Pl. 21].

\section{PI. 21 : Filets « brume » dans la province de Settsu}

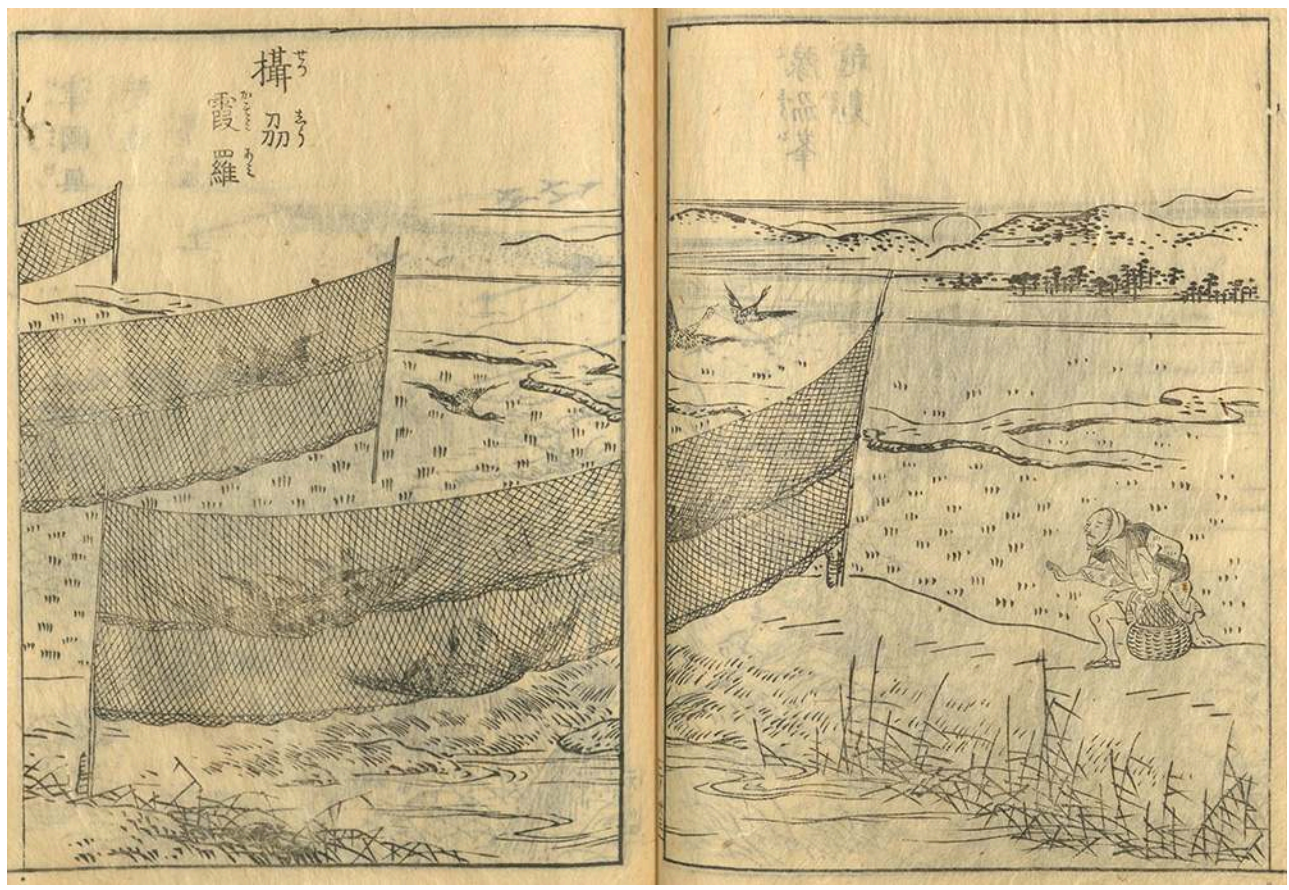

National Institute of Japanese Literature. Lien vers l'illustration

On appelle cela parfois "brume» (kasumi). Une autre méthode consiste à disposer horizontalement un grand nombre de tiges de bambou enduites de glu au bord d'un étang. Quand les canards viennent manger l'œnanthe aquatique ${ }^{126}$ qui pousse au bord de l'eau, ils passent sous les perches et se prennent dans la glu. Cette méthode s'appelle hago. Il existe une autre méthode pour prendre les oiseaux sur l'eau, dite "glu flottante ». On imprègne de glu de la paille qu'on met à l'eau en amont, puis on prend les oiseaux dont les ailes s'y engluent.

Il y a encore la méthode des " cordes hautes " (takanawa) qui permet d'attraper les oiseaux dans les étangs, les marais ou les rizières inondées [voir Pl. 19]. 


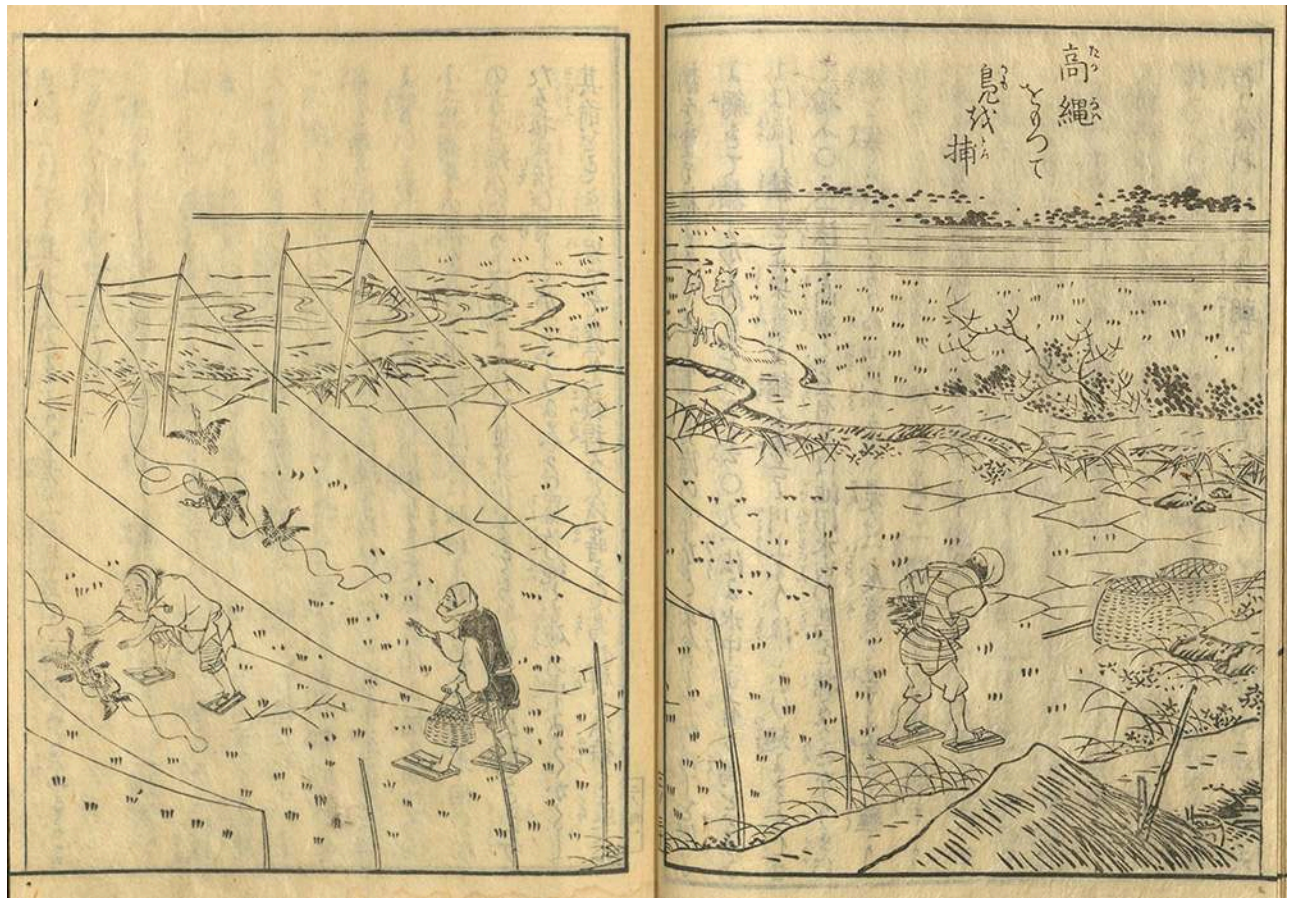

National Institute of Japanese Literature. Lien vers l'illustration

On commence par ajouter de l'huile à la glu pour l'empêcher de se figer sous l'effet du froid, puis on porte à ébullition et on enduit du mélange une corde de ramie, qu'on enroule sur une bobine. Puis on plante sur les deux bords des canes de bambou d'un ken disposées à une distance d'un ken et demi l'une de l'autre, et on y fixe la corde en la tendant comme sur l'image, tandis que l'autre extrémité en est passée dans l'encoche creusée à la pointe du poteau situé en face, qu'on enduit d'huile. Quand un oiseau touche la corde, l'extrémité de celle-ci sort de l'encoche et l'on capture l'oiseau qui s'y est pris. On dit que la corde " chute ». Quand le vent souffle d'ouest en est, on dispose les cordes du nord au sud et inversement : il s'agit d'attendre les canards, qui viennent toujours contre le vent. On dit sōmakuri (prise totale) quand vient une nuée d'oiseaux et que toutes les cordes chutent. Les chasseurs portent des chaussettes de cuir dites mizutabi (chaussettes d'eau), et attachent par-dessus des nanba (raquettes en forme de planche de bois), commodes pour marcher dans la boue des marécages ou des rizières marécageuses. On peut savoir, en observant l'apparence trouble ou limpide de l'eau, si les oiseaux se sont posés le soir ou le matin. Grâce à l'examen des traces de pas, on peut déterminer sans erreur si les oiseaux viendront ou ne viendront pas le soir.

On utilise aussi cette méthode des cordes hautes pour chasser les oies sauvages, mais les oies sont plus rusées que les canards et voient même dans l'obscurité. Aussi ne descendent-elles pas quand elles voient qu'on a mis beaucoup de nourriture ou que le sol a été remué. Lorsqu'un couple d'oies, s'étant approchées du lieu, poussent un cri et s'éloignent, tout le vol s'éloigne à sa suite. Quand par chance des oies se posent près de ces cordes hautes et que les chasseurs se jettent sur elles brusquement avec des bâtons de bambou, ce n'est qu'une fois sur dix que les oies effrayées se prennent dans les cordes. 

utilisée pour prendre les canards à Torigai, dans le district de Shimashimo de la province de Settsu [voir Pl. 22].

PI. 22 : Filets à canards « bascule sans pareille » dans la province de Settsu

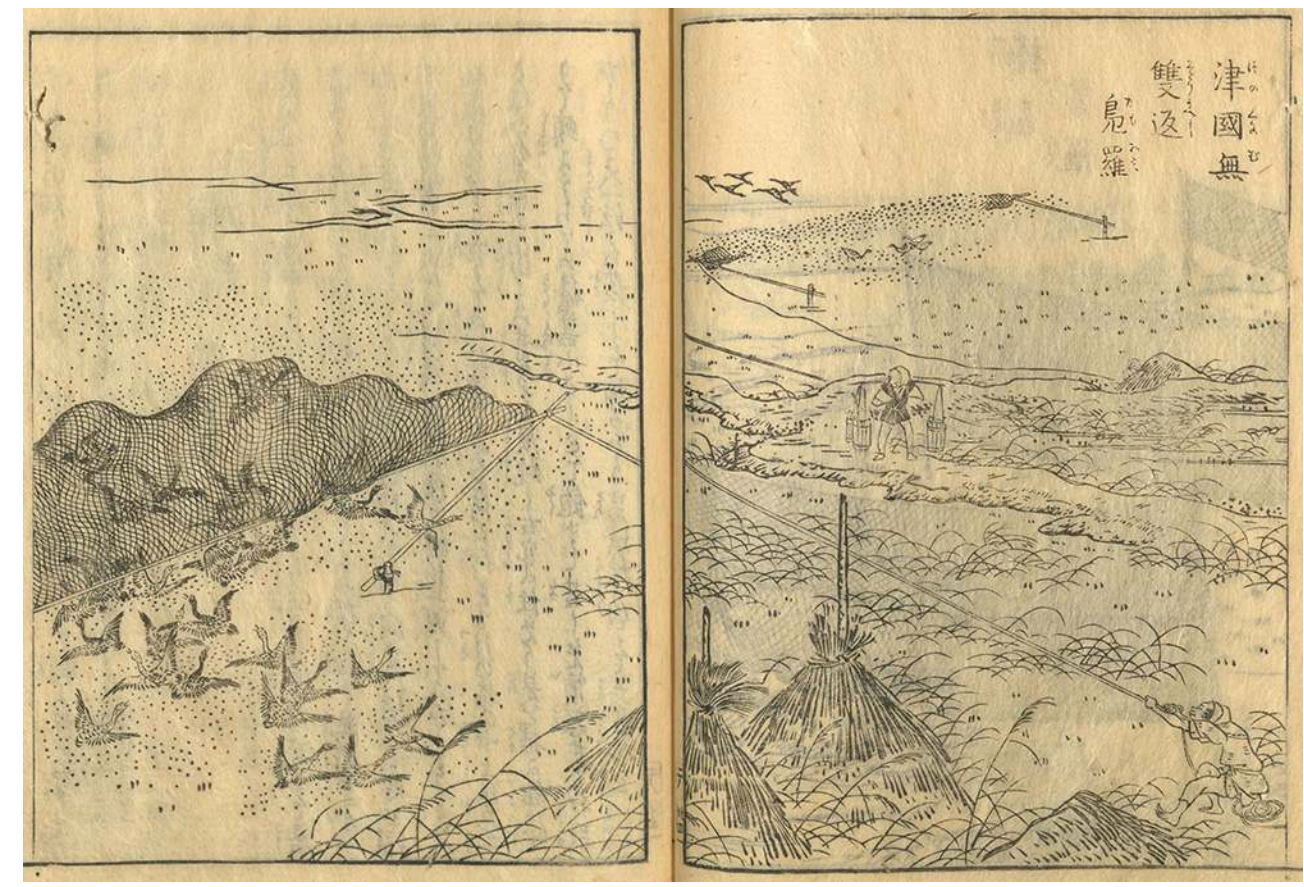

National Institute of Japanese Literature. Lien vers l'illustration

81 Autrefois on utilisait le filet recouvrant (öten) ou les cordes hautes, mais ces derniers temps on y a appris des chasseurs de la province d'Owari la méthode, très efficace, du «filet basculant» (kaeshiami). On utilise des filets de quelque six travées de long et deux travées de haut, auxquels on attache une corde d'environ vingt travées. On plante aussi deux pieux courts dans le sable ou bien dans la partie laissée à découvert par la marée. On fixe en l'attachant le bas du filet. En haut du filet sont fixés des bâtons de bambou croisés qu'on attache aux pieux qu'on a au préalable plantés de manière à ce que le filet bascule bien. Puis on dissimule soigneusement filet, bâtons et corde dans le sable. Le terrain devant le filet est légèrement creusé de manière à former un renfoncement. On y répand du riz ou du millet et on attend que les oiseaux s'assemblent. Quand deux chasseurs tirent de loin sur l'extrémité de la corde, le filet se rabat sur les oiseaux et n'en laisse échapper aucun. On peut en prendre plusieurs dizaines d'un coup. Si on retourne et croise les ailes des oiseaux, et qu'on les lâche sur la digue, ils ne peuvent s'envoler. C'est ce qu'on appelle le haga-ijime (attacher ailes croisées). Cette méthode est aussi utilisée pour chasser les oies sauvages. Mais il y a une manière particulière de dissimuler le dispositif dans le sable et de répandre la nourriture et cette méthode est difficile à pratiquer pour qui n'est pas bien entrainé.

Les canards sauvages habitent les vallées de montagne, les bords de mer ou les lacs. On ne les élève pas. En Chine, le canard à tête verte est considéré comme étant de qualité supérieure. Au Japon on l'appelle magamo (canard véritable). C'est pourquoi dans les poèmes du Man'yōshū le canard est associé à la couleur verte. Le canard osaki (queue 
pointue) vient immédiatement après et est dit ogamo (petit canard). Anciennement on l'appelait takae.

Kurokamo (canard noir); akagashira (tête rouge); hitori; yoshifuku; shimafuku ; kaitsuburi; shibaoshi; aisa; tōnaga; mikoai; hashihiro; ajikamo (ou ashikamo); onaga (longue queue). Il y a encore beaucoup d'autres variétés. Les canards aokubi, ogamo et aji sont savoureux. Les autres sont de goût médiocre.

\section{Canards volant par-dessus un sommet}

Le caractère 鴨 désigne le canard domestique : ahiru. C'est pourquoi, le canard sauvage kamo est parfois écrit «canard d'eau » 水鴨. Le caractère correct pour kamo est 鳥. Ici j'ai suivi l'usage courant au Japon.

Il s'agit d'une technique utilisée dans les montagnes de la province d'Iyo ${ }^{127}$. Aux huitième et neuvième mois, matin et soir, des nuées de canards franchissent les sommets, éraflant de leurs ailes les imperata, qui de ce fait ne poussent pas très haut. Les gens du pays creusent au milieu de ces herbes des trous d'environ 3 pieds de profondeur comme de circonférence, et s'y dissimulent. On fabrique des filets en forme d'éventails, à l'extrémité desquels on fixe un long manche en bambou. Quand les canards survolent le trou, on rabat sur eux le filet [voir Pl. 20].

PI. 20 : Capture de canards volant au-dessus d'un sommet dans la province d'lyo

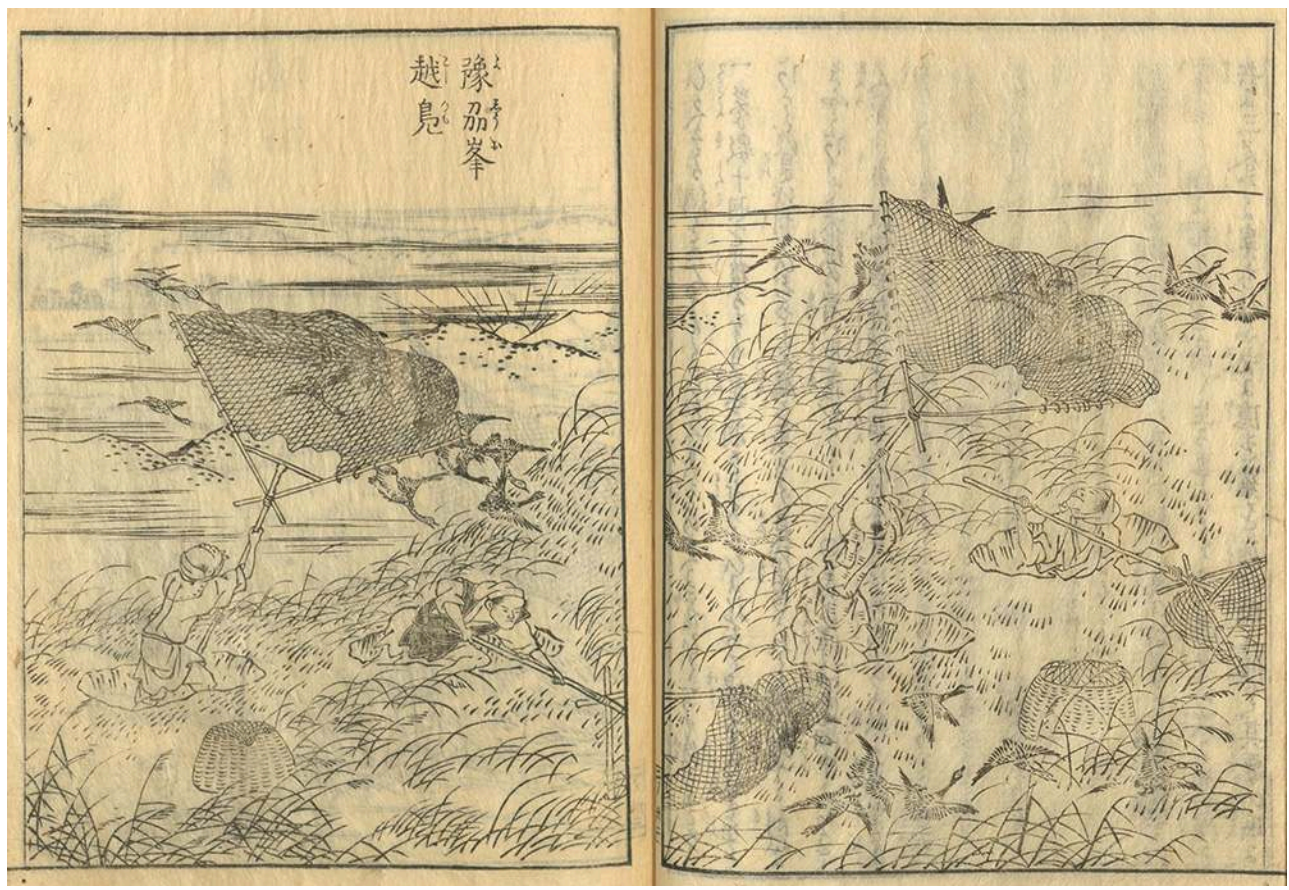

National Institute of Japanese Literature. Lien vers l'illustration

Celui-ci se referme sur les canards et il n'y a plus qu'à capturer les oiseaux qui y sont pris. Il est vrai que cette méthode exige un bon entraînement. (Il vaut mieux que la montagne soit entourée des deux côtés par des rizières. Qu'il s'agisse du soir ou du matin, cette chasse se pratique toujours dans l'obscurité. Ce type de filet est appelé sakaami « filet de côte »). 


\section{Chasse à l'ours}

L'ours est aussi appelé shiro (chin. zilu)

Les ours vivent toujours dans les creux de grands arbres et dorment beaucoup. On ferme l'entrée de la cavité avec des rondins assemblés à l'aide de lianes de glycine en forme de claie, on introduit dans le creux d'arbre un grand nombre de branches, préalablement coupées, puis on attend que l'ours apparaisse de lui-même à l'entrée de la cavité et on le tue, en tranchant ou en piquant [voir Pl. 24].

\section{PI. 24 : Capture d'un ours dans sa tanière}

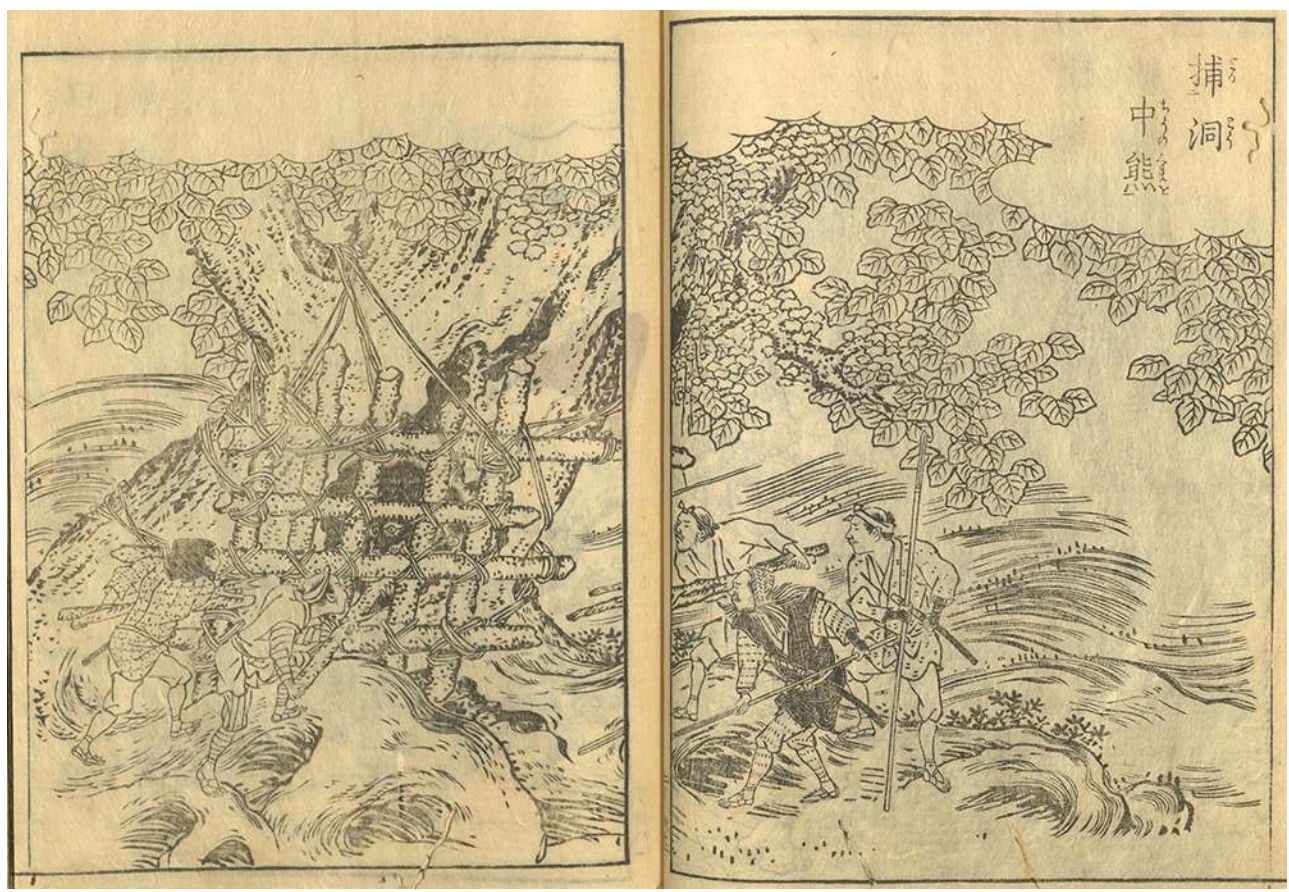

National Institute of Japanese Literature. Lien vers l'illustration

À Mino ${ }^{128}$ on utilise des lances de bambou, à Inaba ${ }^{129}$, des lances en métal, à Higo ${ }^{130}$, le mousquet, dans les provinces du Nord, une sorte de fauchard nommé hanataki. Dans tous les cas, on vise un peu au-dessus du croissant de lune que l'ours porte au front. Dans les montagnes de la province d'Iwami, les ours s'installent souvent dans les cavités qui ont servi auparavant à brûler du charbon de bois. Si on les tue incontinent à la lance ou au mousquet, leur foie sera tout petit. Pour cette raison on ne les tue qu'après les avoir tourmentés et rendus furieux. Une autre méthode consiste à utiliser des pièges. À Iyo, on appelle cela « piège-plafond » ou oso. À Awa, osu (qui est une forme ancienne pour oshi, « piège écrasant »). Il se présente comme sur la figure [voir Pl. 23]. 


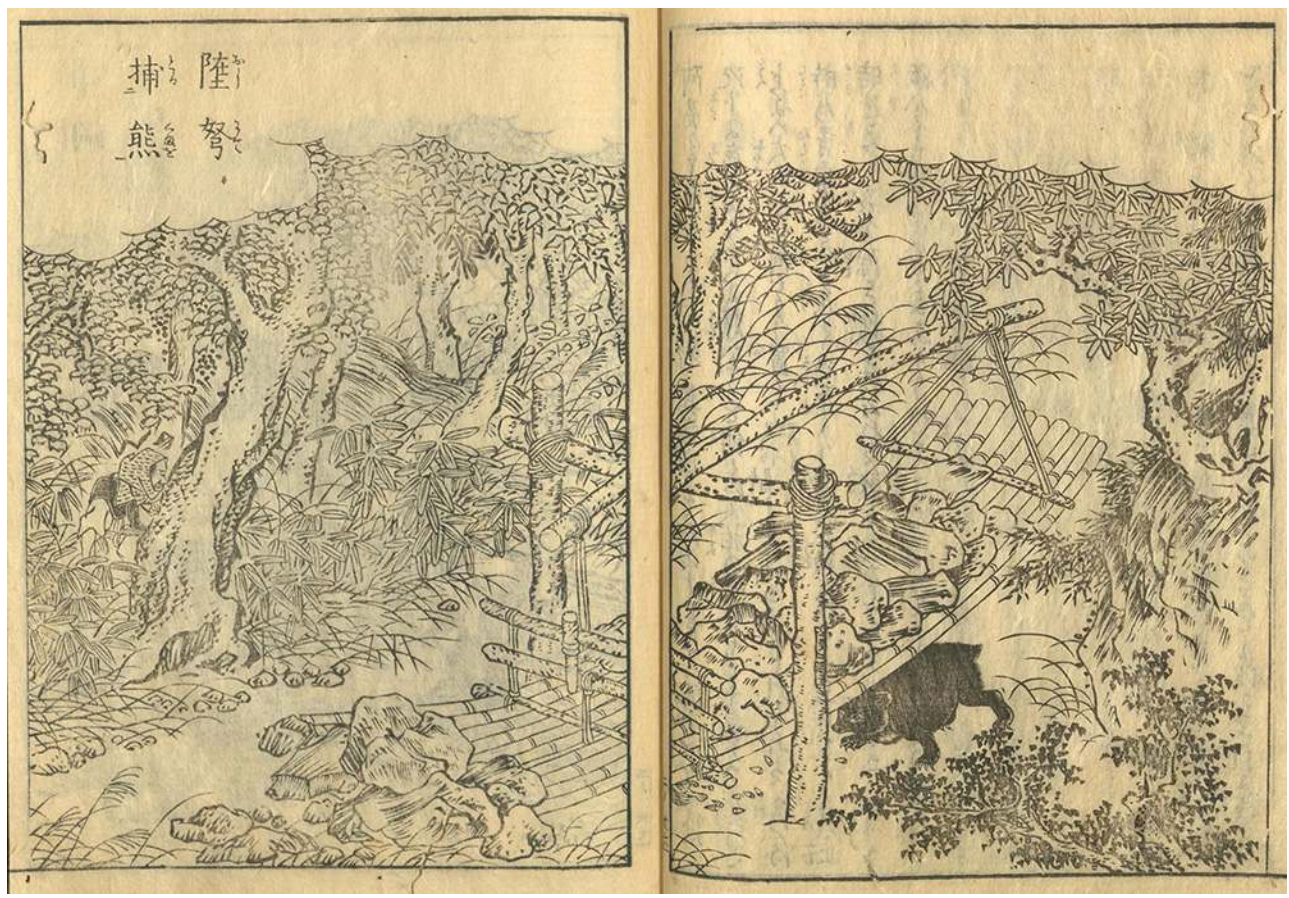

National Institute of Japanese Literature. Lien vers l'illustration on dispose en dessous de la viande de daim fumée en guise d'appât. On peut aussi semer des glands de chêne, des baies de shashanbo ${ }^{131}$, etc. Par-dessus on dispose quelque vingt grosses pierres (à Awa, soixante-quinze), si bien que lorsque le piège s'effondre, cela fait un bruit de tonnerre. Même après que le piège est tombé sur lui, l'ours continue à le remuer pendant environ trois jours. On attend que le mouvement cesse, puis on retire les pierres pour soulever le dispositif en bambou : souvent l'ours mort se tient debout, ses quatre pattes enfoncées d'1 pied dans le sol. Il y a aussi le piège à trou, mais il ressemble au piège écrasant. On attrape aussi les ours en les poursuivant avec de longues lances. Lorsque l'ours s'enfuit à toutes jambes, si on crie "Qu'il se retourne !", l'ours se retourne et affronte ses poursuivants. Si à ce moment on crie «Croissant de lune $! »^{132}$, il montre des signes de peur et on l'attaque aussitôt pour lui asséner des coups mortels. Mais ces procédés sont dangereux à moins que le chasseur ne soit aguerri et d'une grande dextérité.

Il existe encore une autre méthode, pratiquée à Fuchü ${ }^{133}$, dans la province de Suruga ${ }^{134}$. Deux hommes brandissant de grandes haches se tiennent des deux côtés du repaire de l'ours, tandis qu'une ou deux autres personnes coupent des branches d'arbres entières et s'en servent pour explorer l'intérieur du repaire. Comme l'ours se saisit de ces branches avec ses pattes et cherche à les attirer dans son repaire, ils ne le laissent pas faire, tirant les branches vers le côté. Pendant ce temps, les autres surveillent les mouvements désordonnés de l'ours qui cherche à s'emparer des branches et l'attaquant des deux côtés lui tranchent les pattes avec les haches [voir Pl. 25]. Comme une grande partie de la force de l'ours est dans ses membres, il s'en trouve affaibli et finit par se faire prendre. 


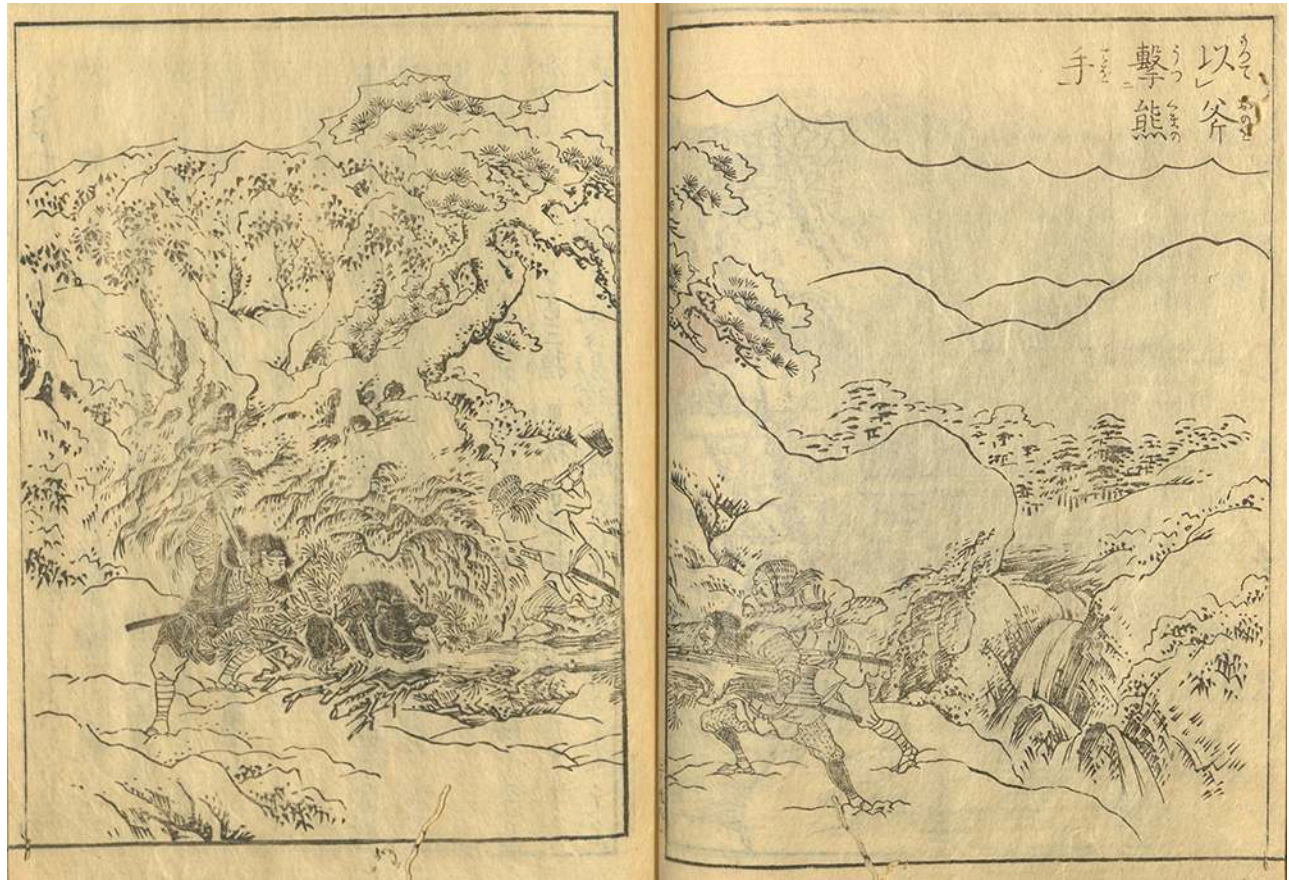

National Institute of Japanese Literature. Lien vers l'illustration

90 C'est ainsi qu'on récolte la vésicule biliaire et qu'on produit des peaux d'ours, principalement dans la province du Mutsu. À Tsugaru on mange la viande des pattes qu'on sert même à la table de nobles personnages. Les ours ont coutume de se nourrir de fourmis charpentières ${ }^{135}$, de pousses de bambous, de crabes de rivières (zugani, aujourd'hui mokuzugani ${ }^{136}$ ) et parmi les fruits des arbres, de ceux qui sont sucrés. Il lui arrive aussi de manger de la viande d'autres animaux. À Ezo, dit-on, on élève des ours en les nourrissant de lait humain.

\section{Récolte de la bile}

La bile d'ours de la province de Kaga est considérée de qualité supérieure. Vient ensuite la bile produite dans les provinces d'Echigo, d'Etchū, de Dewa. On en produit aussi dans le Shikoku, dans les provinces d'Inaba, de Higo, de Shinano, de Mino, de Kii et en beaucoup d'autres lieux. La bile produite à Matsumae et à Ezo, est souvent de qualité inférieure. Cependant la bile d'ours de Kaga n'est pas toujours non plus de qualité supérieure, ni non plus celle de Matsumae de qualité inférieure. La nature de l'ours, la saison, l'habileté de celui qui l'abat comptent aussi et il est difficile de parler en général. Parmi les biles de qualité supérieure de Kaga, on distingue trois variétés: " noire ", « jaune ", « ambre » (kohakute). De toutes ces variétés, la bile « ambre » est la meilleure. On dit «bile d'été » ou «bile d'hiver " pour distinguer le produit selon la saison de sa récolte. La membrane de la poche en été est épaisse et la bile est peu abondante: on la considère de qualité inférieure. Celle qu'on récolte à partir du huitième mois est dite "bile d'hiver». La membrane de la poche est fine et la bile, abondante. On la considère de qualité supérieure. Cependant, pour la variété " ambre ", la bile d'été est supérieure à la bile d'hiver. Elle est de couleur orangée et translucide. La variété « noire » n'est pas ainsi, mais d'un noir brillant, c'est la plus répandue. 


\section{Procédés pour reconnaître les contrefaçons}

92 Il semble qu'il y ait beaucoup de contrefaçons tant au Japon qu'en Chine. Le Bencao gangmu expose la méthode pour tester le produit. On y lit : «Lorsqu'on laisse tomber sur de l'eau une goutte de bile d'ours grosse comme un grain de riz, si elle tourne sur elle-même, évitant les impuretés, puis s'enfonce au fond de l'eau s'étirant comme un fil, c'est qu'il s'agit d'un produit authentique ». J'estime qu'il s'agit d'une méthode ancienne, encore insuffisante. La bile de tous les animaux, quels qu'ils soient, tourne à la surface de l'eau, ce n'est pas la propriété exclusive de la bile d'ours. L'expérience montre aussi que la suie d'une maison où l'on a abattu des animaux ou cuit de la viande, tourne elle aussi à la surface de l'eau. Cependant, c'est la seule méthode à la portée des amateurs. Si on ne peut faire autrement que de tester le produit en faisant tomber dans l'eau une goutte de bile et en examiner le fil qui se forme jusqu'au fond de l'eau, on considèrera comme bon un produit à réaction rapide, qui tourne vite, comme en bondissant, sur la surface de l'eau et produit un fil extrêmement fin. Celui qui tourne lentement, sans force, puis s'arrête, n'est pas bon. N'est pas bon non plus celui qui tourne vite, mais ne disparaît pas entièrement. Quand le produit n'est pas bon, sa force est affaiblie et le fil met du temps à se former. Si le produit descend au fond de l'eau sous forme de poudre, il doit aussi être considéré comme de qualité inférieure. Quand ce qui descend au fond de l'eau est de couleur orange, le produit est de qualité supérieure, quand la couleur est brune, c'est qu'il s'agit d'une grossière contrefaçon.

Les spécialistes reconnaissent la qualité à son goût. Lorsqu'on a affaire à un produit authentique de qualité supérieure, dès qu'on le place sur la langue il dégage aussitôt une puissante amertume. Les saveurs douces-amères pénètrent dans la bouche sans coller au palais, l'amertume se renforce progressivement, mais les différentes saveurs restent bien distinctes à l'intérieur de la bouche et celle-ci reste pure. Au contraire, lorsqu'il n'y a que de l'amertume, on a affaire à une contrefaçon. Un bon produit possède une saveur à la fois douce et amère. Par ailleurs, la présence d'un arrière-goût de chaire crue est un défaut. Mais cela peut tenir à la nature de l'ours, qui avait un régime carné, et ne signifie pas nécessairement qu'il s'agit d'une contrefaçon. Quand la saveur est d'abord douce, et amère par la suite, il s'agit d'un produit de moindre qualité. En revanche quand le produit a un goût de brûlé il est de bonne qualité. Mais c'est une méthode qui ne saurait s'enseigner. Même un homme d'expérience, s'il peut distinguer le vrai du faux, a du mal à apprécier la qualité d'un produit.

\section{Procédés de fabrication de produits contrefaits}

On réduit en poudre fine trois ingrédients : écorce de Phelllodendron amurense (ōbaku), baies de gardénia (sanshishi), racine de renoncule (keōren, umanoashigata, Ranunculus japonicus). On grille légèrement les baies de gardénia pour en ôter le parfum. Puis on mélange les trois, on ajoute de l'eau et on fait réduire au feu. On obtient une matière noire luisante qui, séchée, ressemble au produit authentique. Pour l'envelopper, on superpose deux feuilles de papier de Mino sur lesquelles on presse du jus de racines de narcisse, qu'on fait sécher : ainsi, au moment d'envelopper, le papier retient le liquide. Quand, après l'avoir enveloppé, on met le produit sous presse entre deux planches et qu'on le laisse sécher à l'abri de la lumière, le papier se froisse, s'imprègne de matière liquide, et prend un aspect semblable au produit authentique. S'il est préparé en hiver, le produit tend à se désagréger l'été venu, aussi le prépare-t-on toujours durant l'été. 
Il s'agit de la méthode utilisée dans la province de Bingo et ses environs. Dans les autres provinces, c'est ce même procédé qui prévaut et il est difficile de connaître les autres méthodes. On raconte qu'on utilise aussi des kakis amers appelés konerigaki qu'on a laissé mûrir sur l'arbre et qu'on enveloppe parfois dans du papier à parapluie usagé. Il arrive assez souvent que du produit de contrefaçon soit introduit dans de vraies membranes de poche à bile d'ours. C'est une ruse grossière pour abuser les gens.

\section{Addendum}

On dit que l'ours est appelé kuma, parce qu'il est noir (kuro), mais on ne peut l'affirmer avec certitude. Il semble que ce soit un mot vernaculaire coréen. Le toponyme Komogai, écrit avec les caractères kuma "ours " et gawa "rivière " est une déformation de kumagawa («Rivière aux ours »). Aujourd'hui encore « ours » se dit kom en coréen.

\section{NOTES}

1. Wuli lun (Discours sur la raison des choses) est un essai métaphysique chinois de Yang Quan (dates inconnues), de l'époque des trois dynasties. Composé originellement de 16 fascicules, seuls des fragments sont conservés.

2. Song shu (Livre des Song) est l'histoire officielle en 100 fascicules de la dynastie Song (420-479), fondée par Liu Yu et souvent désignée de Liu Song pour la distinguer de la grande dynastie Song.

3. Genre littéraire des anecdotes et des histoires officielles. Le terme désignait à l'origine, sous les Han, les notes et propos consignés par les fonctionnaires subalternes pour tenir l'Empereur au courant de la mentalité du peuple.

4. Abréviation de Langye daizuibian, 40 fascicules. Ouvrage de l'époque des Ming, de Zhang Dingsi (1543-1603).

5. Sachant qu'un bu, comme unité de distance vaut environ $1,8 \mathrm{~m}$, la distance dont il est question ici correspondrait à plusieurs vingtaines de mètres.

6. Azuma kagami est une chronique du bakufu de Kamakura en 52 fascicules d'auteur inconnu, relatant des événements survenus entre 1180 à 1266.

7. Ancienne province, située autour du lac Biwa, et correspondant à l'actuel département de Shiga.

8. Voir Bencao gangmu, Fascicule 10, section des minéraux et des pierres 4, catégorie des pierres, notice " yangqishi ».

9. Terme présent dans le Canon interne de l'Empereur jaune (Huangdi neijing) désignant les caillots durs formés dans l'utérus, sous l'effet du souffle froid qui empêche la bonne circulation du sang.

10. Unkonshi (Traité sur les roches), ouvrage en 16 fascicules imprimé entre 1773 et 1801 par le naturaliste japonais Kino'uchi Sekitei (1724-1808), répertoriant environ deux mille espèces de roches, de minéraux, de fossiles et objets en pierre.

11. Il s'agit de Kino'uchi Sekitei, l'auteur de l'Unkonshi, qui était effectivement originaire de cette province.

12. Prononcé et aussi parfois écrit iwa, qui est aussi l'orthographe moderne. 
13. Kinai, région centrale autour de la capitale Kyōto, comprenant les cinq anciennes provinces de Yamato (département de Nara), Yamashiro (partie sud de la préfecture de Kyōto), Settsu (préfecture d'Ōsaka) et Kawachi (partie est de la préfecture d'Ōsaka).

14. Ancienne province, correspondant à la partie sud de la préfecture d'ōsaka.

15. Ancienne province, correspondant à l'actuel département de Tokushima.

16. Ancienne province couvrant la partie sud de l'actuelle préfecture de Kyōto.

17. île d'environ $150 \mathrm{~km}^{2}$, située dans l'actuel département de Kagawa, dans la mer Intérieure.

18. Ancienne province correspondant à l'actuel département de Kagawa.

19. Pierre volcanique (andésite) extraite à Nebukawa dans l'actuelle ville d'Odawara.

20. À Iyadaniji, une dépendance du temple Zentsūji, on montre la grotte où le moine Kūkai (774-835), de son nom posthume Kōbō daishi, aurait fait ses études.

21. Ancienne province correspondant à l'actuel département de Tochigi.

22. Iyo, Satsuma, Kii, Sagami sont les noms d'anciennes provinces correspondant respectivement : 1) au département d'Ehime 2) à la partie ouest du département de Kagoshima 3) au département de Wakayama et à la partie sud du département de Mie, et enfin 4) au département de Kanagawa.

23. L'archipel de Shiaku, également lu Shiwaku, est situé dans la mer intérieure de Seto, entre l'actuel département de Kagawa (Shikoku) et celui d'Okayama (Honshū). Il compterait jusqu'à vingt-huit îles, mais les plus importantes sont au nombre de sept. Son nom proviendrait de ce que, à marée haute, la mer située entre les îles écumerait.

24. Le terme otomeyama (montagne réservée) désigne à l'époque d'Edo des zones de forêt dont l'exploitation du bois est soumise à un contrôle seigneurial.

25. L'ancien district de Muko couvrait un secteur englobant les villes d'Amagasaki, Nishinomiya et Takarazuka.

26. Le texte original indique la lecture mubara, mais il s'agit sans doute d'une erreur. L'ancien district d'Ubara couvrait un secteur englobant la ville d'Ashiya et une partie de la ville de Kōbe.

27. Le courtisan et poète Fujiwara no Mototoshi (1055-1142).

28. Shoku kokinshū, abréviation du Shoku kokinwakashū (Suite au Recueil des poèmes anciens et modernes), anthologie en vingt fascicules, compilée sur ordre impérial par le poète Fujiwara no Teika en 1265.

29. Le village de Sumiyoshi se trouvait dans un secteur aujourd'hui situé dans l'arrondissement Higashi nada de la ville de Kōbe.

30. Célèbre station thermale, connue depuis l'époque ancienne, bénéficiant d'une haute réputation à l'époque d'Edo.

31. Terme dialectal dont le sens n'est pas clair.

32. L'expression ikuri qu'on y rencontre désigne des pierres dans la mer.

33. Man'yōshū, poème $n^{\circ} 933$, fasc. 6, Man'yōshū, SATAKE Akihiro, YAMADA Hideo, KUDō Rikio, ōTANI Masao, YAMAZAKI Yoshiyuki (éd.), vol. 2, Tokyo, Iwanami shoten, coll. « Shin Nihon koten bungaku taikei », p. 30.

34. Un des sept «parcours" (shichidō) du Japon ancien : désigne un ensemble de provinces du nord-ouest, situées sur la Mer du Japon.

35. Kawachi est le nom d'une ancienne province, correspondant à la partie est de la préfecture d'Ōsaka.

36. Tuf volcanique provenant notamment de Tatsuyama à Takasago, dans le département de Hyōgo.

37. Ancienne province correspondant à la partie sud-ouest de l'actuel département de Hyōgo.

38. Ishi no hōden: célèbre monolithe de $5,7 \mathrm{~m}$ de haut en tuf volcanique, taillé en forme rectangulaire, qu'on peut voir à Takasago, dans le département de Hyōgo.

39. Bleu, jaune, rouge, blanc, noir. 
40. Village que l'on peut localiser dans l'actuelle ville de Takasago, dans le département de Hyōgo.

41. Ancienne province comprise dans les limites actuelles de la préfecture de Kyōto.

42. Ce terme désigne la couleur jaunâtre avec des membrures mauves utilisée pour faire apparaitre la ligne de trempe du sabre.

43. Le terme désigne une couleur jaune pâle.

44. Ancienne province correspondant à l'actuel département de Gunma.

45. Ancienne province recouvrant la partie orientale du département d'Aichi.

46. Ancienne province recouvrant la partie orientale du département de Fukui.

47. Déformation de Jōkyōji (浄教寺), dans l'actuel département de Fukui. Les pierres à affûter, produites aux abords de ce temple, étaient réputées et sont mentionnées, par exemple, dans le Wakan sanzai zue (Encyclopédie sino-japonaise des trois règnes ; 1712).

48. Bencao gangmu, section des minéraux et des pierres 4, notice « Yueshi ».

49. Nom d'une ancienne province couvrant partiellement le département de Saga et celui de Nagasaki.

50. Nom d'une ancienne province, correspondant à l'actuel département de Kōchi.

51. Wamyōshō, nom abrégé de Wamyō ruijushō (Répertoire ordonné des noms de Yamato), est un dictionnaire de l'époque de Heian de Minamoto no Shitagô (911-983), répertoriant environ 2600 termes chinois selon différentes catégories, allant des phénomènes célestes aux plantes et animaux. Deux versions existent, comptant respectivement 10 et 20 fascicules.

52. Voir plus loin.

53. Junpu (Traité des champignons), composé par Chen Renyu des Song. Selon sa préface, cet ouvrage décrit onze espèces de champignons poussant dans la région de son auteur, le Zhejiang (au sud de Shanghai), et décrivant notamment les lieux de production, l'aspect, la saveur et la manière d'en neutraliser le poison.

54. Renvoie à la partie occidentale du Honshū, englobant les cinq préfectures d'Okayama, Hiroshima, Yamaguchi, Shimane et Tottori.

55. Nom d'une ancienne province, qui recouvrait la partie occidentale de l'actuel département d'Aichi.

56. Ganoderme luisant, Ganoderma lucidum.

57. L'Histoire des Han (Hanshu, $\mathrm{I}^{\mathrm{er}}$ siècle) rapporte que, durant la dynastie des Qin, Dongyuan Gong, Qili Ji, Xia Huanggong and Luli Xiansheng se réfugièrent sur le Mont Shang où ils se nourrirent de champignons.

58. Il n'existe pas d'ouvrage de ce titre. Il s'agit d'une citation mal coupée du dictionnaire Erya zhushu (Song du Nord), elle-même citant un passage non identifié qui proviendrait du Classique des rites (Liji). (Ces indications nous ont été données par Frédéric Devienne).

59. Polypore, littér. siège à singes.

60. Littéralement « champignon parfumé ». Il s'agit du shiitake (Lentinula edodes).

61. Ancienne province correspondant à l'actuel département de Miyazaki dans le Kyūshū. C'est à partir de cette région que cette espèce aurait été diffusée sur tout le territoire. CHIBA, Nippon sankai meisan meibutsu zue, op. cit., p. 44-45.

62. Kumano est une région montagneuse située dans le département de Wakayama et connue pour ses épaisses forêts.

63. Castanopsis.

64. Le terme employé est shizensei, littéralement " génération spontanée ».

65. Shiromizu, eau blanchâtre, qui a servi à laver le riz.

66. J. Tsūga, ouvrage de lexicologie de Fang Yizhi (1579-1671) de la dynastie Ming en 52 volumes, réunissant sa réflexion sur un large spectre de termes, classés selon 25 domaines.

67. Désigne la partie sud de la province de Yamato, c'est-à-dire la partie de l'actuel département de Nara. 
68. Umbilicaria esculentia.

69. Montagne non identifiée.

70. Matsu non koke, aujourd'hui appelé saruogase (usnée).

71. Poria cocos. Champignon de couleur noir, qui pousse sous les pins noirs du Japon et est utilisé en pharmacopée chinoise.

72. Sans doute le samatsutake, variété hâtive (juin-juillet) et moins savoureuse du matsutake.

73. Plusieurs espèces sont désignées ainsi dont le honshimeji (« vrai shimeji », Lyophyllum shimeji).

74. Lactarius lividatus.

75. Polypore en touffes ou poule des bois (Grifola frondosa).

76. Ancienne province couvrant la moitié occidentale de l'actuel département de Hiroshima.

77. Ancienne province correspondant à la partie occidentale de l'actuel département de Shimane.

78. Ancienne province correspondant à la partie nord-ouest de l'actuel département de Fukuoka.

79. Ancienne province correspondant à la partie orientale de l'actuel département de Shimane.

80. Espèce non identifiée.

81. Yatsudoki. Vers 2 heures de l'après-midi.

82. Peut-être la guêpe kurosuzumebachi (Vespula flaviceps), dont on consomme les larves.

83. Le terme detchi désigne un jeune apprenti dans les maisons de commerce.

84. Première anthologie impériale du Japon, compilée vers 913-914 (ou vers 905) par Ki no Ki no Tsurayuki (?-?) et d'autres poètes. Il compte, dans sa version la plus répandue, 1111 poèmes, répartis en 20 livres et classés selon diverses rubriques.

85. Nom d'une localité, intégrée dans le fief du même nom, couvrant la partie occidentale de l'actuel département de Fukushima.

86. Ancienne province recouvrant l'actuel département d'Aomori, et une partie d'Iwate, dans le nord du Honshū.

87. Il s'agit du Toxicodendron succedaneum (Rhus succedanea L.).

88. Lonicera japonica.

89. Le terme sanshōuo (littér. " poisson poivre ») désigne notamment les salamandres géantes du Japon de la famille des Cryptobanchidae, évoquées au début du fascicule, ainsi que de petites salamandres appartenant à la famille des Hynobydés.

90. Kochi. Il s'agit d'une famille de poissons à tête plate, Platycephalus.

91. Ancienne province correspondant à la moitié nord du département d'okayama.

92. Onychodactyllus japonicus.

93. Ancienne province correspondant au département de Niigata.

94. C'est aussi le nom d'un triton, le triton à ventre de feu (akaraharaimori, Cynops pyrrhogaster)

95. Ouvrage encyclopédique en vingt fascicules de Fang Yizhi (1611-1671), organisé autour d'une classification en quinze domaines (Ciel, Histoire, Divination, Corps humain, Minéraux, Outils, Vêtements, Aliments, Animaux, Végétaux, etc.). Il est imprimé à titre posthume en 1664.

96. Le Shanhaijing (Classique des monts et des mers, $\mathrm{III}^{\mathrm{e}}-\mathrm{IV}^{\mathrm{e}}$ siècle) mentionne la sirène (renxing, $\mathrm{j}$. ningyō), créature vivant dans les rivières et ressemblant au poisson $t i$, qui serait la salamandre géante.

97. Qishenlu (Histoires pour réfléchir au merveilleux), recueil de contes chinois du XI ${ }^{\mathrm{e}}$ siècle de Xu Xuan. L'ouvrage n'est pas de Li Shizhen, mais le passage mentionné est bien cité dans le Bencao gangmu, section des animaux à écailles 4 , catégorie des poissons sans écailles, notice «tiyu ». Le passage sur la sirène est traduit par Alain Briot dans une note consacrée à ce sujet : " Détours des mondes » : detoursdesmondes.typepad.com/files/sirenes-1.docx (consulté le 29 avril 2017).

98. NISHIKAWA Joken, IJIMA Tadao et al. (éd.), Nihon suido kō, Suido kaiben, zōho kai tsūshōkō, Tokyo, Iwanami shoten, coll. « Iwanami bunko », 1944, p. 193.

99. Cette phrase concernant les Hollandais provient de Nishikawa Joken cité plus haut, qui précise qu'il s'agit d'un mot portugais. Par ailleurs, une créature mi-femme mi-poisson, dont le nom hollandais serait heishimureru (de l'espagnol pez mujer « femme poisson ») est mentionnée 
dans le Yamato honzō de Kaibara Ekiken. La synthèse complète des occurrences de la notion dans les ouvrages savants de Chine, du Japon et d'Europe, se trouve dans le Rokumotsu shinshi (Nouvelle description des six espèces) du spécialiste des études hollandaises Ōtsuki Gentaku (1757-1827), un ouvrage publié en 1787, à la publication duquel Kimura Kenkadō a joué une part non négligeable. Voir Rokumotsu shinshi, Ikkaku sankō, annoté par sōDA Hajime, coll. « Edo kagaku koten sōsho32 ", Tokyo, Kōwa shuppan, 1980, p. 121.

100. Ce nom signifie quelque chose comme «le sieur Rokurō», Rokurō étant un prénom signifiant « Sixième fils ».

101. Vigna angularis.

102. Sans doute l'ouvrage de médecine Wahō ichimanpō de Murai Kinzan (1733-1815).

103. Prononcé chigeki, en japonais, le terme désigne la toile de fibres de puéraire.

104. Technique de fabrication du tissu de ramie pratiquée dans l'actuel département de Niigata. Le crêpe d'Echigo est traité dans le fascicule 5 du présent ouvrage.

105. Pour protester contre la politique du souverain des Zhou les vertueux frères Boyi et Shuqi refusèrent de consommer les produits du royaume et se retirèrent sur le Mont Shouyang, où ils se nourrirent de fougères.

106. C'est le sens de la prononciation akagaeru proposée ici pour des caractères habituellement lus yamagaeru et signifiant "grenouille de montagne ». Plutôt que la " grenouille de montagnes » de couleur jaune et de grande taille décrite dans le Bencao gangmu, il s'agit d'une grenouille de plus petite taille communément appelée akagaeru (littéralement, "grenouille rouge ", genre Rana).

107. Recueil d'anecdotes et de réflexions sur la poésie par l'auteur du célèbre Hōjōki (Notes de ma cabane de moine). Concernant la grenouille d'Ide, voir KAMO NO CHÔMEI, Notes sans titre Mumyôshô: Propos sur les poètes et la poésie, traduit du japonais et annoté par le groupe Koten, Paris, Le Bruit du temps, 2010, p. 71-73.

108. Yamato honzō, KAIBARA Ekiken, op. cit., p. 368.

109. En japonais ebizuru.

110. Pièce 154, dans la section « Chansons de Bin » du Shijing (Livre des Odes).

111. Ou ordre du clergé (Clerodendrum trichotomum).

112. Allusion au poème "Fait en voyage ", de Li Bai, voir Florilège de Li Bai, traduit, présenté et annoté par Paul JACOB, Paris, Gallimard, coll. « Connaissance de l'Orient » no 58, 1985, p. 117.

113. Nous traduisons par " autour », le terme taka, qui peut désigner différents oiseaux de proie utilisés en fauconnerie.

114. Ōtaka, Accipiter gentilis, connu en France sous divers noms : épervier autour, autour des palombes ou épervier bleu.

115. Ancienne province recouvrant l'actuel département d'Aomori, et une partie d'Iwate, dans le nord du Honshū.

116. Ǔnggolbang, traité coréen sur l'élevage et le dressage des autours et des faucons, composé par Yi Yong, dont l'édition la plus ancienne remonte à 1444. Voir MIHO Tadao, « Riyō-sen Kohon Yōkotsuhō, Rien sen Shinzō Yōkotsuhōni tsuite - Kunaichō shoryōbu shozō takasho takakotoba no kenkyū » (À propos du Yōkotsuhō de Riyō ainsi que la nouvelle édition augmentée du Yōkotsuhō de Ri'en : recherches sur les manuels de fauconnerie conservés à la bibliothèque du Kunaichō et la terminologie de fauconnerie), Shinjodai kokubun, ${ }^{\circ} 25$ (2014), p. 23-40.

117. Nom d'une ancienne province correspondant à l'actuel département de Tokushima dans l'île de Shikoku.

118. Hiyodori, bulbul à oreillons bruns.

119. Accipiter nisus, épervier d'Europe.

120. Épervier du Japon, Accipiter gularis.

121. Buse à joues grises, Butastur indicus.

122. Nisaetus nipalensis. 
123. Faucon crécerelle, Falco tinnunculus. En japonais moderne, on l'appelle chōgenbō ou makusotaka.

124. Caprimulgus jotaka.

125. Yamato honzō (La materia medica du Japon), KAIBARA Ekiken, in Ekiken zenshū (๔uvres complètes d'Ekiken), vol. 6, Kokusho kankōkai, 1973, p. 394.

126. Oenanthe javanica, j. seri.

127. Nom d'une ancienne province correspondant à l'actuel département d'Ehime.

128. Nom d'une ancienne province recouvrant la partie sud de l'actuel département de Gifu.

129. Nom d'une ancienne province recouvrant la partie est de l'actuel département de Tottori.

130. Nom d'une ancienne province, correspondant à l'actuel département de Kumamoto.

131. Vaccinium bracteatum.

132. L'ours noir d'Asie ou ours à collier porte sur le poitrail une tache blanche en forme de croissant de lune.

133. Grande cité de l'époque d'Edo, administrée directement par les Tokugawa, choisie par le premier shogun pour se retirer après avoir cédé le titre à son fils.

134. Ancienne province située au milieu de l'actuel département de Shizuoka.

135. Camponotus japonicus.

136. Eriocheir japonica. 


\title{
Fascicule 3
}

\author{
NOTE DE L'ÉDITEUR
}

Ce sommaire, placé en début de fascicule par l'auteur du Meisan zue, fait partie du texte d'origine. Ce sommaire n'a pas vocation à être utilisé pour circuler à l'intérieur du Meisan zue. Pour ce faire, le lecteur pourra utiliser toutes les fonctionnalités propres à l'édition en ligne de la plateforme OpenEdition Books, notamment sa table des matières générale.

\section{Sommaire du fascicule 3}

- Ormeau d'Ise

- Rubans d'ormeau

- Addendum : les perles

- Langouste d'Ise

- Sériole de Tango

- Filet droit

- Filet vertical

- Addendum : la sériole des autres provinces

- Thon de Hirado

- Filet d'hiver

- Maquereau tacheté de Sanuki

- Filet flottant

- Dorade de Wakasa

- Limande de Wakasa

- Trempage en saumure et le séchage à l'air

- Addendum : la dorade des autres provinces

- Filets à planchettes secouées d'Emata en Sanuki

- Filets « des cinq sagesses »

- Maquereau de Noto

- Bateaux de pêche des autres provinces 
- Huîtres de Hiroshima

- Élevage de ces dernières

- Variétés

\section{Produits de la pêche}

\section{Ormeau (awabi 鰒)}

Fabrication des rubans d'ormeau. Complément : perles.

Selon certains, la graphie correcte du mot awabi serait 石決明, le caractère 鰒 se lisant tokobushi (petite haliotide).

Dans la province d'Ise ${ }^{1}$, on pêche l'ormeau dans trois baies, celles de Wagu, d'Omashi et d'Ōno, et on fabrique les rubans d'ormeau dans la baie de Futami et dans le nord de l'île de Tōse ${ }^{2}$. Ce sont des plongeuses qui pêchent les ormeaux (parce qu'elles peuvent retenir leur souffle plus longtemps que les hommes). Lorsqu'elles prennent le large, elles sont toujours accompagnées d'un membre de leur famille à qui elles confient la tâche de conduire le bateau et de tenir la corde qui les retient [voir Pl. 26].

PI. 26 : Ormeaux d'Ise

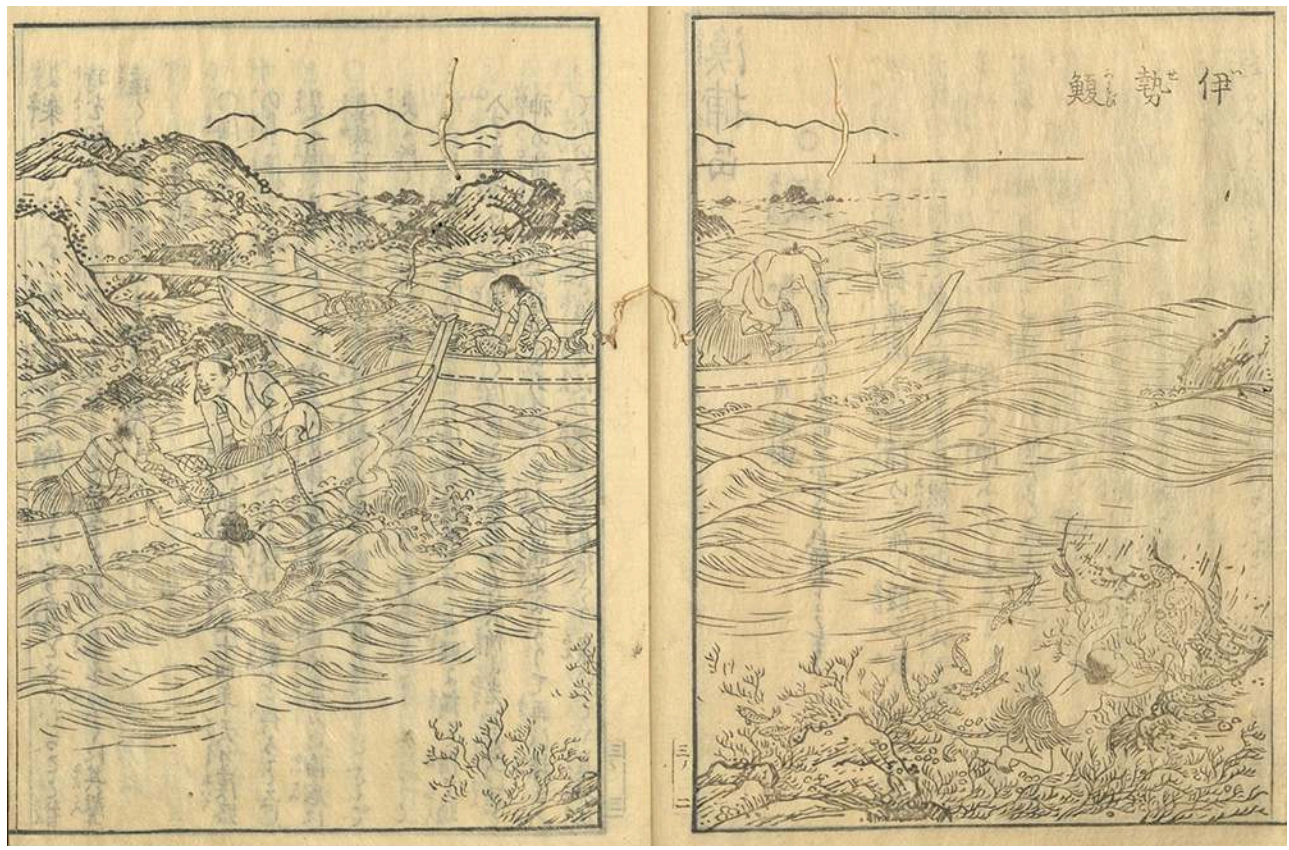

National Institute of Japanese Literature. Lien vers l'illustration

Les plongeuses sont équipées d'un sac tressé attaché à la hanche. Elles remontent à la surface quand elles l'ont rempli de trois ou quatre ormeaux, et même de deux s'ils sont grands. Dans les endroits peu profonds, elles remontent à l'aide d'une perche qu'on leur tend, appelée « perche amie ». Dans les endroits profonds, elles font signe en tirant sur la corde attachée à leur hanche et se font remonter par les personnes qui sont dans le bateau. Les plus jeunes peuvent plonger à une profondeur de cinq brasses et celles qui ont trente ans ou plus peuvent aller jusqu'à dix ou quinze brasses.

Elles plongent tête la première et nagent verticalement jusqu'au fond de la mer où elles prélèvent ce qui est attaché aux rochers. Elles prennent les ormeaux par surprise et les 
détachent à l'aide d'une spatule, puis elles les mettent dans leur sac tressé. Elles retiennent leur souffle pendant un long moment; et bien qu'elles aient l'habitude de ce métier qu'elles exercent matin et soir, leurs voix résonnent pitoyablement au loin quand elles reprennent leur respiration après être remontées à la surface.

\section{Addenda}

4 À propos de la pêche à l'ormeau au fond de la mer, le Nihon shoki (Chronique du Japon) ${ }^{3}$ relate, à la quatorzième année du règne de l'empereur Ingy $\bar{o}^{4}$, que le souverain alla à la chasse dans l'île d'Awaji ${ }^{5}$. Il y avait de nombreuses bêtes sauvages, mais il ne parvint à en capturer aucune de toute la journée. Il fit procéder à des divinations et reçut sur le champ un message divin disant ceci : « Il y a une perle dans la mer d'Akashi ${ }^{6}$. Si vous me présentez cette perle en offrande, vous pourrez vous emparer de la totalité du gibier. À ces paroles, le souverain convoqua les plongeurs des environs et leur fit examiner le fond de la mer. Mais celui-ci restait hors de portée. Alors un plongeur du nom d'Osashi du village de Nagamura de la province d' $\mathrm{Awa}^{7}$, attacha une corde à sa hanche et plongea dans la mer. Après quelque temps, il remonta en disant : « Il y a un ormeau gigantesque au fond de la mer qui émet une lumière autour de lui. " Comme les gens du lieu pensaient que ce que demandait la divinité se trouvait précisément dans le ventre de cet ormeau, le plongeur retourna une seconde fois au fond de la mer ; il saisit l'ormeau géant et le remonta au-dessus des vagues, puis rendit son dernier souffle. Comme prévu, il y avait une perle dans le ventre de l'ormeau, grande comme le noyau d'une pêche. Les gens, attristés par la mort d'Osashi, l'ensevelirent dans un tombeau qui existe encore aujourd'hui. La mer avait soixante brasses de profondeur à cet endroit, et les gens furent impressionnés par le travail du plongeur. Sur ce thème fut composée, plus tard, la ballade de la «Perle de Hakozaki », elle remonte à ces faits. (L'ormeau est le premier des coquillages. On l'apprécie hautement depuis les temps anciens. Les plus grands mesurent plus d'1 pied de diamètre, les plus petits 2 à 3 pouces. Sous l'eau, l'ormeau se meut avec la moitié du corps sorti de la coquille).

5 Dans les cinq provinces de la région centrale, Kinai ${ }^{8}$, on appelle communément l'ormeau «coquillage des plongeuses" parce qu'il est pêché par les plongeuses (ama). L'ormeau étant un coquillage à une valve qui se fixe sur une face, son nom signifie « fruit qui n'a pas son âme sœur ».

6 Le onzième fascicule du Man'yōshü (Recueil des dix mille feuilles) donne ce poème : «De l'awabi / que matin et soir plongeant / cueillent les pêcheurs / d'Ise dit-on coquille / dépareillée est mon amour » et le septième fascicule dit ceci : «De la mer d'Isé / quand le pêcheur des îles / a trouvé la perle / dans le coquillage / désir encore le tient $\$ .{ }^{9} \mathrm{Et} \mathrm{le}$ dix-septième fascicule écrit $a$-wa-bi avec les caractères 着石玉 («perle attachée au rocher »). L'ormeau mâle est mince et long, la femelle ronde, courte et plus charnue. Les coquilles d'ormeaux percées de neuf ou de sept perforations sont très rares.

\section{Fabrication des rubans d'ormeau}

La graphie courante noshi 尉斗 est erronée. Noshi est un outil utilisé par les ouvrières pour repasser les vêtements, hinoshi, c'est-à-dire le «fer chauffé aux braises ».

7 Tout d'abord on trie et on compte les ormeaux à débiter selon la taille; puis on entaille un grand nombre par le bord de la coquille et on les émince en fins rubans avec un couteau fin à partir de l'entaille, en faisant tourner l'ormeau dans la main, comme on 
peut le voir sur l'image. On met les rubans à sécher en rangées sur des nattes de paille de type Teshima fabriquées dans la province de $B u n g 0^{10}$; à la suite de quoi, ils sont imprimés du motif tressé de la paille [voir Pl. 27].

\section{PI. 27 : Fabrication des rubans d'ormeau}

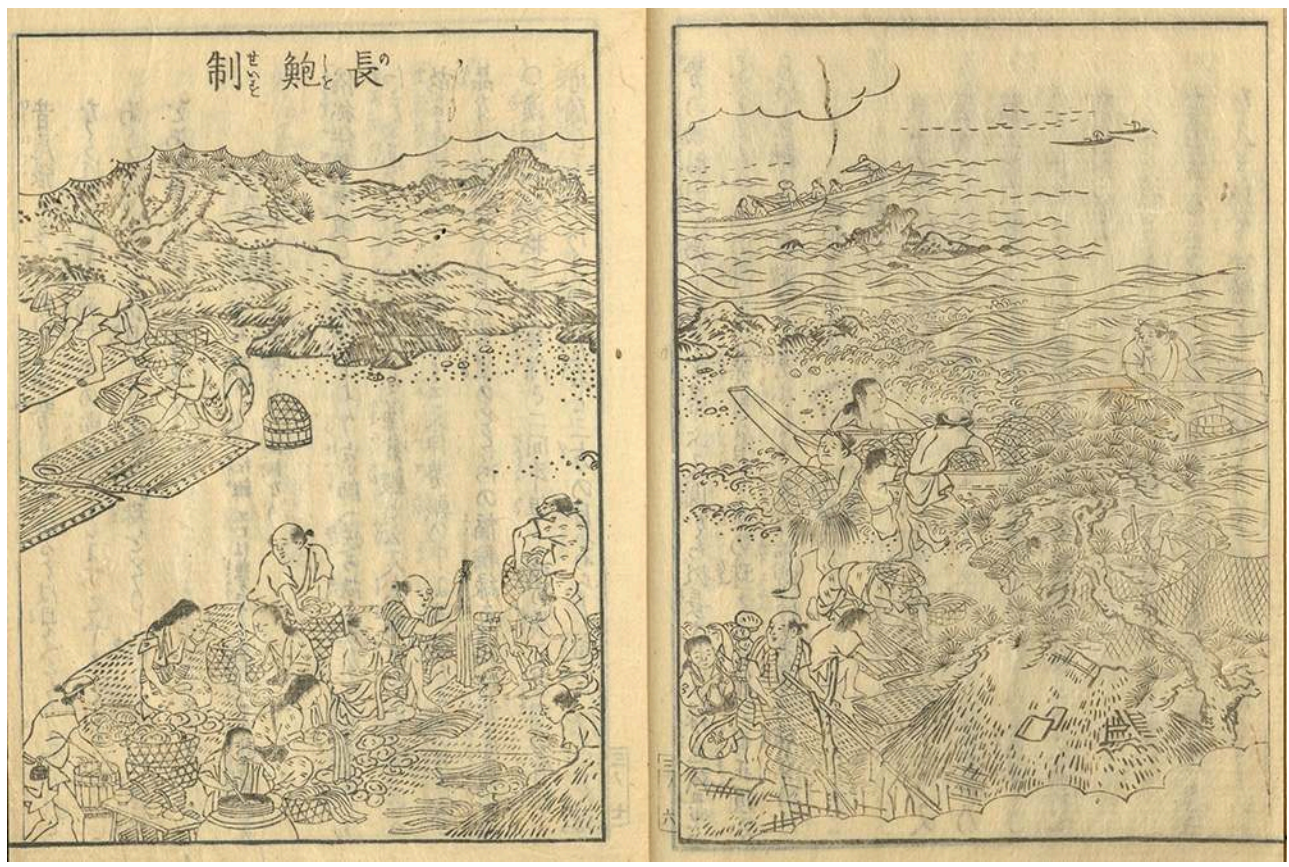

National Institute of Japanese Literature. Lien vers l'illustration

Cela permet ensuite de les lier en faisceaux par les deux bouts. Le mot «ruban d'ormeau » noshi provient du fait que, jadis, on battait (uchi) l'ormeau et on l'appelait alors « ormeau battu » (uchi awabi), de même qu'on parle de «châtaignes battues (ou pilées)» (uchi kuri). L'ormeau débité en rubans est appelé noshi ou encore «ormeau séché » (hoshi awabi) ${ }^{11}$.

9 L'ormeau séché et l'ormeau battu ont été consommés depuis les temps anciens. On les appelle aussi «ormeaux émincés ». Le Gōshidai (Protocole des célébrations selon la maison Ōe $)^{12}$ fait mention de quatre mets qui accompagnent le riz cuit au feu sacré, à savoir l'ormeau émincé, la dorade séchée, la sardine et le chinchard ${ }^{13}$. On lit dans le Sangi ittō (Trois principes - une autorité) ${ }^{14}$ que l'usage des rubans d'ormeau comme ornements ou petits mets d'accompagnement lors des banquets festifs remonterait au moment où, se réclamant d'Ashikaga Yoshimitsu ${ }^{15}$, Imagawa Ujiyori, préfet de la capitale section de gauche, Ogasawara Nagahide ${ }^{16}$, adjoint de l'office des Magasins d'armes, et Ise Mitsutada, gouverneur de Musashi, auraient ordonné les maisons guerrières selon onze rangs et exigé qu'elles intègrent l'ormeau dans les différents cérémonials de cour selon leur statut respectif de maison shogunale, maison seigneuriale, de gouverneur, de seigneur extérieur ou de membre du conseil des dignitaires.

Dans l'Antiquité, l'ormeau séché faisait partie des mets servis lors des Grandes prémices qui ont suivi l'intronisation de l'empereur Tenji ${ }^{17}$. Ce mets figure également parmi les offrandes de nombreux rites du Engishiki (Règlements de l'ère Engi). En premier lieu, la province d'Ise est la capitale des Dieux de notre empire et abrite le plus 
grand nombre de sièges de divinités. Il faut donc prendre en compte l'origine de la fabrication des rubans d'ormeaux dans cette province et comprendre qu'ils sont non pas une décoration, mais une denrée alimentaire. Jusqu'aux alentours de l'époque de Kamakura (1185-1336), on a toujours utilisé l'ormeau comme un mets. Une preuve en est donnée par l'épisode de l'exil de Yoritomo ${ }^{18}$ dans le Heiji monogatari (Récit épique de Heiji) :

Un soir, Sukedono fit halte dans la province d'ōmi ${ }^{19}$ et, toute la nuit, pria le dieu Takebe myōjin ${ }^{20}$ pour que le trajet se déroule sans encombre. Pendant que les gens se reposaient, le suivant Moriyasu s'adressa à Yoritomo : «À la capitale je vous ai dit de ne pas entrer en religion, parce que j'avais eu un rêve insolite. Vous êtes allé prier le dieu Hachiman ${ }^{21}$, vêtu de l'habit purifié, et vous vous teniez assis sur la coursive externe ${ }^{22} \mathrm{du}$ sanctuaire. Moi Moriyasu, je me tenais sur mes gardes sur des dalles en pierre, lorsque douze ou treize jeunes garçons, munis d'arcs et de flèches, vinrent les poser sur la coursive. Ils déclarèrent être venus avec le carquois et les flèches de Yoshitomo ${ }^{23}$. Alors une voix majestueuse se fit entendre de l'intérieur du sanctuaire disant: «Présentez-moi ses armes afin qu'elles soient déposées dans le sanctuaire! Afin qu'elles puissent enfin être attribuées à Yoritomo. Donnez ceci à manger à Yoritomo! ». Sur ce, les jeunes garçons célestes sortirent une chose qu'ils posèrent devant vous. Quand vous vous êtes penché pour voir ce que c'était, c'était des ormeaux [...] La voix vous ordonna de les manger. Vous en avez compté soixante-si $\mathrm{x}^{24}$. Vous avez serré les rubans d'ormeau entre vos deux mains et mangé trois bouchées du côté épais puis vous m'avez donné, à moi Moriyasu, le côté mince et je les ai mis dans le repli de mon vêtement...

11 Il faut apprécier le sens de ce récit. Par ailleurs, les provinces de l'Ouest se sont récemment mises à fabriquer des rubans de seiche, de crevette, ou encore d'holothurie. Il s'agit de fruits de mer débités en rubans très fins qui frappent par leur raffinement et leur mystère.

Chaque année, le premier jour du sixième mois, le village de Kunisaki ${ }^{25}$ de la province de Shima ${ }^{26}$ fournit aux deux grands sanctuaires d'Ise les « ormeaux longs » en rubans. C'est pourquoi cet endroit s'appelle aussi Cap des ormeaux Noshisaki ou Cap des turbos cornus Sazaesaki. Aujourd'hui on ne fait plus de turbo cornu en rubans. L'Engishiki mentionne « l'ormeau des cuisines des sanctuaires ». ${ }^{27}$ Par ailleurs, l'offrande annuelle du Nouvel An adressée au shogun d'Edo comprend des rubans d'ormeau de 3 pieds de long et d'1 pouce de large ainsi que d'autres variétés.

\section{Perles}

\section{Nom chinois lizangtu 李蔵㻌}

13 Ce sont les perles du coquillage akoyagai ${ }^{28}$. On les pêche à Ise d'où leur nom de perles d'Ise. Ces dernières sont estimées de qualité supérieure, celles d'Owari ${ }^{29}$, de qualité inférieure. Les perles des coquillages akoyagai pêchés à Ōmura ${ }^{30}$ de Hizen ${ }^{31}$ seraient de qualité supérieure mais elles ne sont pas vendues en pharmacie. L'akoyagai est aussi appelé "coquillage-manche » parce qu'il a la forme d'une manche. Dans la baie de Waka $^{32}$ on l'appelle "coquillage-papillon ». Il mesure de 1,5 à 2 pouces. Il est gris et certains sont couverts de petites taches noires. L'intérieur est blanc avec une épaisse couche [de nacre] d'un reflet bleu. Non pas que toutes les coquilles renferment une perle. Les perles d'Ise sont rondes et légèrement bleutées. Celles qui ne sont pas rondes mais allongées avec un reflet vert sont des perles d'ormeaux. Les apothicaires les appellent "perles de coquillage». Celles d'Owari ne sont pas tout à fait rondes, elles 
sont de couleur trouble, sans éclat et très petites. Ce sont des perles de palourdes, de corbiculas ou encore de moules. Voici la forme qu'elles ont :

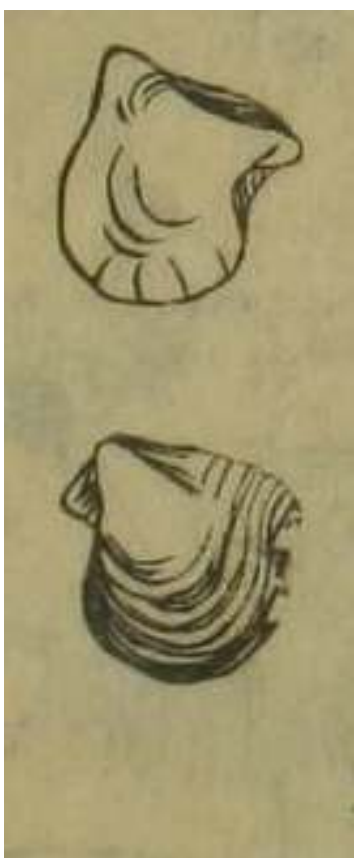

National Institute of Japanese Literature. Lien vers l'illustration

\section{Addenda}

14 Selon certains, Akoya serait un nom de lieu dans le district de Chita ${ }^{33}$ à Owari. Ce toponyme existe également à Mutsu ${ }^{34}$. Dans le Shinsarugakuki (Notes sur de nouveaux divertissements comiques) ${ }^{35}$, on parle de "perles d'Akuya » (akuyadama) ${ }^{36}$. Les perles d'ormeau (awabidama) du Man'yōshū sont glosées «perles d'Akoya» dans le Rokujō (Poèmes anciens et modernes en six livres) ${ }^{37}$. Par ailleurs, on raconte ces temps-ci qu'on peut prélever beaucoup de coquillages du nom de namima kashiwa ${ }^{38}$. Les perles de moules correspondent à celles d'Owari mentionnées plus haut.

Un poème de Saigyō dans le Sankashū (Recueil de l'ermitage de montagne) dit ceci :

On a entassé

les moules d'Akoya

dont on prend les perles

sans doute a-t-on voulu

montrer traces du trésor. ${ }^{39}$

On voit de ce qui précède qu'Akoya était jadis compris comme le nom d'un lieu à Owari et que les véritables perles n'étaient autres que celles d'Owari. Aujourd'hui les coquillages pêchés à Ise sont appelés akoya, parce que les nombreux coquillages collectés jadis à Owari n'existent aujourd'hui plus qu'à Ise. De plus, le fait que les "perles d'ormeau » du Rokujō et celles des moules du poème de Saigyō sont appelés akoya, montre que jadis on récoltait à Akoya beaucoup de perles de toutes sortes de coquillages qui étaient tous appelés akoya, et que c'est un terme général désignant différentes sortes de coquillages. 


\section{Langouste (ebi 海鰕) ${ }^{40}$}

Nom chinois: kakai 蝦魁 ("tête de crevette »); l'«Explication des mots » ${ }^{41}$ donne le nom kōka 紅鰕 (《crevette rouge »). Le mot [japonais] ebi est un terme générique. On en compte plus de trente variétés. Celle qui a pour nom chinois ryūka 龍鰕 ( crevette dragon ») n'est autre que le « serpent de mer » umi-hebi 海鰕42.

On les appelle communément "langoustes d'Ise » parce que c'est d'Ise qu'elles sont acheminées vers Kyōto. À Edo, on les appelle « crevettes de Kamakura » parce qu'elles sont expédiées de Kamakura. À Owari, on les appelle "crevettes de Shima » parce qu'elles sont expédiées de Shima. Il y a des langoustes de cinq couleurs parmi celles d'Ise, mais elles sont très rares. Elles ont des antennes blanches, un dos bleu et des somites verts là où ils se superposent. Ailleurs, le jaune, le rouge et le noir s'entremêlent.

Le filet de pêche a environ soixante-dix brasses de long et deux travées de large. [voir Pl. 28]

\section{PI. 28 : Filet à langoustes}

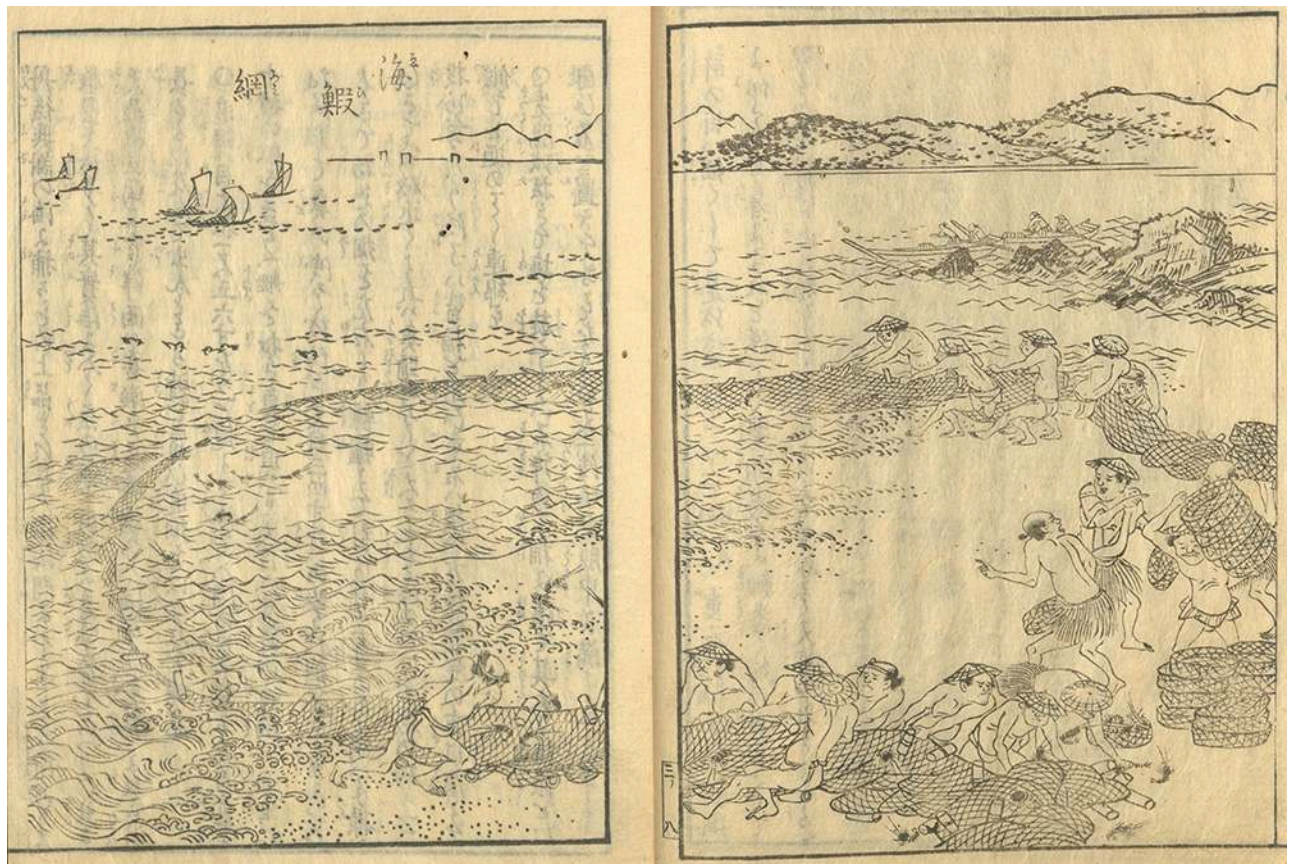

National Institute of Japanese Literature. Lien vers l'illustration

19 Mais il y a des variantes selon l'étendue du rivage et la distance entre les rochers. Les mailles de l'ouverture et des deux côtés du filet sont grandes, celles situées vers le fond, à quinze brasses de l'ouverture du filet, sont petites. C'est la partie appelée " poche $»^{43}$. Les flotteurs ( $a b a$ ) (c'est-à-dire des flotteurs uke, faits de baquets en bois) et les plombs (pour lesquels on utilise des bouteilles en terre cuite) sont semblables à ceux des filets de la dorade. On jette le filet au coucher du soleil et on le lève le matin suivant. Les crustacés sont alors tous pris dans les mailles du filet. Cela est dû au fait que, comme ils cherchent à s'échapper à reculons, ils restent accrochés par la queue. Il y en a même qui restent accrochés à l'extérieur du filet. 
Les entrailles des langoustes sont attachées au cerveau et les œufs se trouvent à l'extérieur sous l'abdomen. Les yeux sont violets-noirs avec une partie jaune sur le devant. Ils ressortent comme des verrues. Les langoustes ont quatre antennes à côté de la bouche, dont deux mesurent 1 à 2 pieds de long. Les pattes ont des nœuds comme des tiges de roseau. Sa carapace est dure comme une armure. La langouste saute et danse. C'est la puce de la mer. D'ailleurs, le corps de la puce est semblable à celui de la langouste.

21 La lecture japonaise ebi se décompose en $e$-bi 桶䰅 «manche-barbe ». Le manche $e$ signifie la branche eda. Qu'il s'agisse du $e$ 胞 ${ }^{44}$ (placenta) ou du $e$ 江 (confluent), $e$ renvoie aux branches (i.e. les membres) du corps humain ou à celles (i.e. le bras) de la mer. Le nom ebishi pour Ezo 蝦夷 («crevettes barbares ») vient de l'assimilation avec l'île des hommes poilus ${ }^{45}$. Par ailleurs, le choix des langoustes sur le plateau de Nouvel An est une manière de célébrer la graphie de ebi 海老 «mer-vieillard » ${ }^{46}$.

\section{Sériole (buri)}

La sériole pêchée dans la mer de Yosa dans la province de Tango ${ }^{47}$ est estimée de qualité supérieure. Cela s'explique par le fait qu'il y a, à l'une des extrémités de la baie, un endroit appelé Ine ${ }^{48}$, où poussent de nombreux arbres Shiia ${ }^{49}$ dont les faînes tombent dans la mer et servent de nourriture aux poissons. Ce serait la raison de l'excellent goût de leur chair. Cernée au nord par l'Amano-hashidate ${ }^{50}$, au sud par Miyazu, à l'ouest par Kiseto ${ }^{51}$ : c'est ainsi que se présente la baie de Yosa. Les poissons y circulent en permanence. On guette le moment où, devenus adultes, ils s'apprêtent à la quitter pour les pêcher avec un filet droit (oiami) ${ }^{52}$. Le filet droit est fait en corde, avec des mailles d'1 pied 5 à 6 pouces, et tendu à l'entrée de la baie. On aligne plusieurs dizaines de bateaux dans la mer et on chasse les poissons vers l'intérieur de la baie en tapant contre le flanc des bateaux. Puis on tend un autre filet en corde avec des mailles de 8 pouces, qui double le premier filet et empêche les poissons de s'échapper. Enfin on mouille une troisième rangée de filets en chanvre avec des mailles de 3 ou 4 pouces. À la suite de quoi, on ramène le premier filet vers la côte à l'aide de treuils installés de part et d'autre de la baie [voir Pl. 29], puis on tire les filets de chanvre en ramenant leurs câbles à la main ${ }^{53}$. Quand les poches formées par les filets s'approchent de la côte, les poissons en grand nombre commencent à se débattre ; on les attrape alors avec des grands crocs afin de les jeter sur la plage. Les flotteurs sont tous faits de tonnelets en bois. Les plombs du filet en corde sont faits en terre cuite et ceux des filets en chanvre sont en fer. Ils sont en forme de tuyaux que l'on a enfilés sur une corde [voir Pl. 30]. 
PI. 29 : Filet ramasseur à sérioles 1

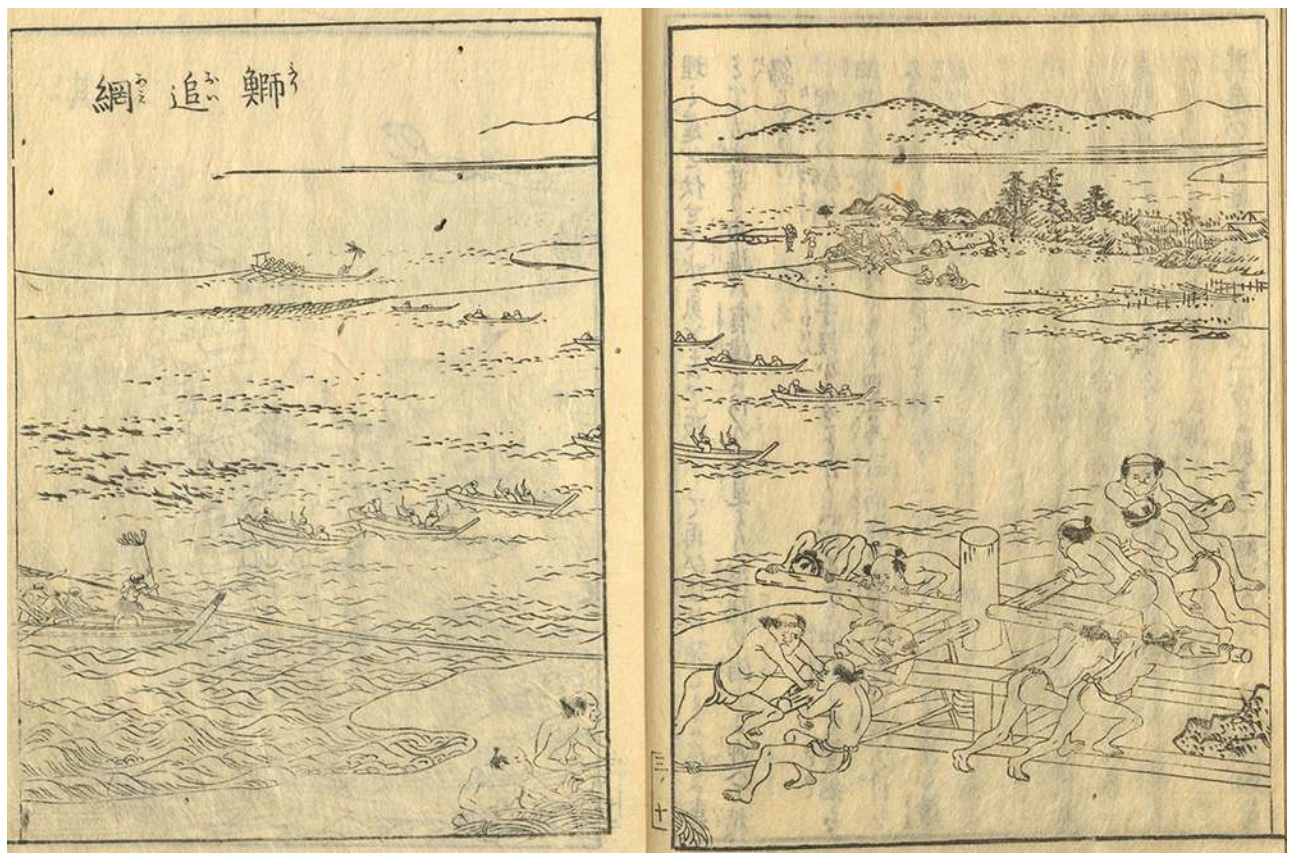

National Institute of Japanese Literature. Lien vers l'illustration

PI. 30 : Filet ramasseur à sérioles 2
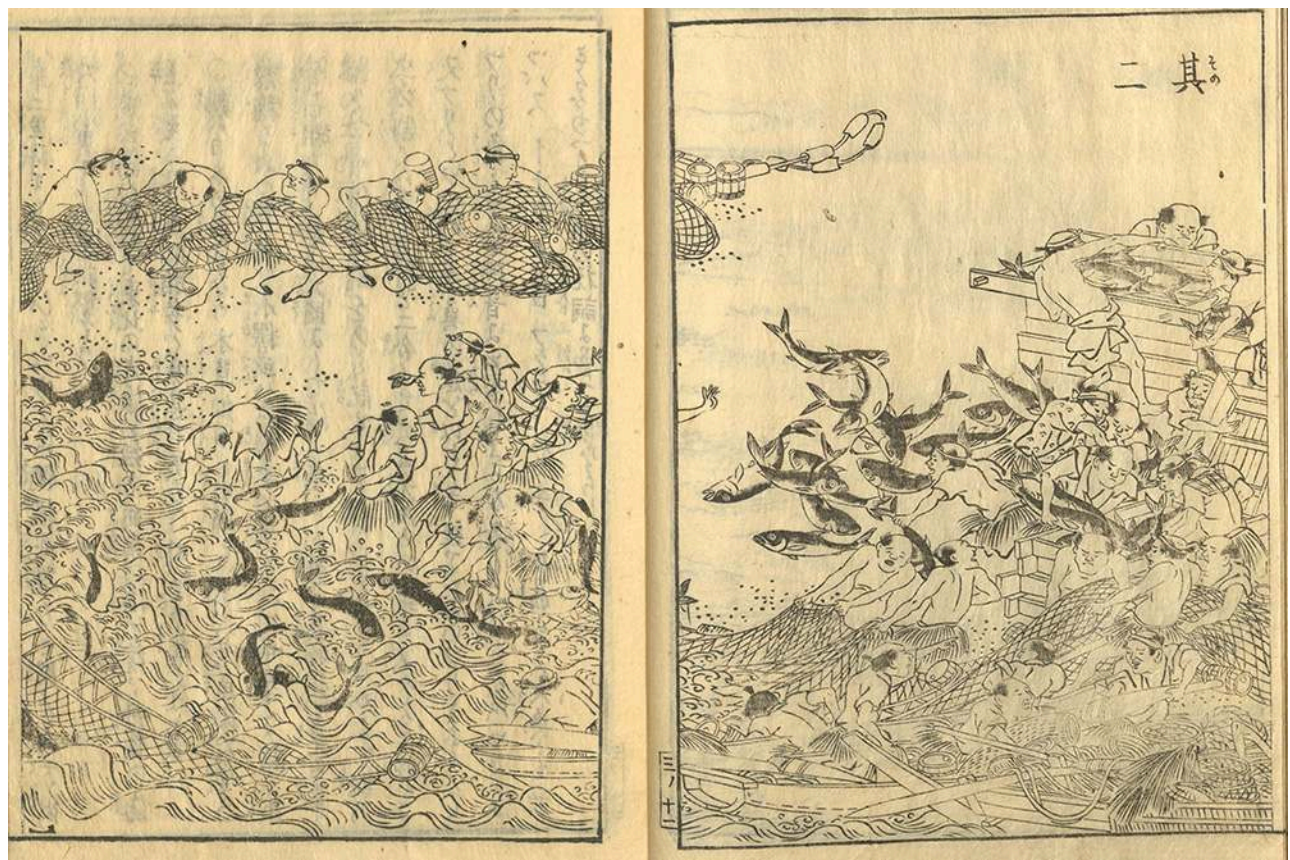

National Institute of Japanese Literature. Lien vers l'illustration

D'abord les poissons sont vidés et salés. On les place dans de très grandes cuves de $6 \mathrm{koku}$; on les recouvre avec des sacs de sel et on les presse avec des pierres. Une autre méthode consiste à farcir les poissons de sel, à les enfouir dans le sable et à les couvrir de nattes de paille pour les faire dégorger. Puis on les retire du sable, on les sale encore et les emballe dans des bâches de paille pour les transporter. Le marché se trouve à 
Miyazu et les commerçants viennent par la mer, à l'endroit de la prise, pour charger le poisson, puis s'en retourner.

Pour les filets de la pêche à la sériole utilisés dans les autres provinces, les procédés sont grosso modo les mêmes. C'est-à-dire qu'on utilise des filets de haute mer. Mais pour les filets verticaux [voir Pl. 31] on utilise des filets à mailles serrées d'une hauteur de 7 à 14 ou 15 brasses, en fonction de la profondeur de la mer.

PI. 31 : Le filet vertical à sérioles

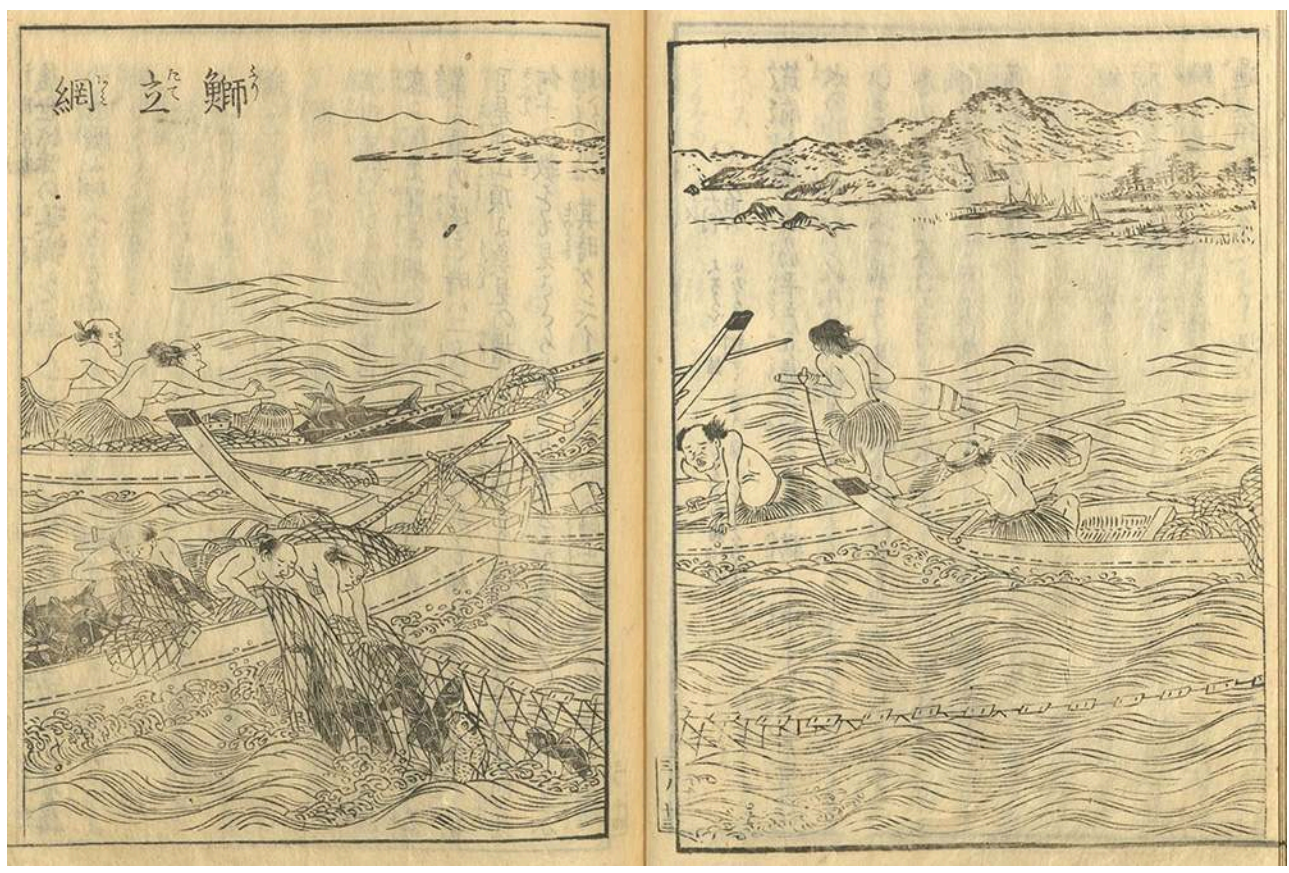

National Institute of Japanese Literature. Lien vers l'illustration

En hiver, jusqu'à la fin du premier mois, les mailles ont environ 7 pouces de large, et à partir du deuxième ou du troisième mois, 5 à 6 pouces. Il y a cinq hommes dans chaque bateau de pêche, quatre tirent le filet et le cinquième tient le gouvernail. Les flotteurs sont faits de cuveaux et les poids ressemblent à des pierres à aiguiser. Quand on pose des filets, on le fait à la manière des nasses utilisées dans les lacs ${ }^{54}$, ce qui empêche le poisson de ressortir. Puis une personne s'installe avec une longue-vue sur une hauteur près de la mer et guette l'arrivée des poissons. Leur présence se signale par une lueur dans la mer et par un niveau de l'eau qui paraît plus élevé. Quand on voit sauter un poisson, on sait qu'il y en a mille autres. La personne donne alors (du sommet de la montagne) le signal aux bateaux en agitant des fanions. Cela s'appelle «faire la sentinelle " ou "monter la garde » ou encore "quêter le poisson». Deux bateaux attendent en mer, et se dirigent dans un sens ou dans un autre selon les indications données à l'aide de fanions. Ils se séparent et s'éloignent l'un de l'autre et, tout en jetant les filets, ils parcourent à la rame une distance qui peut atteindre 2 lieues. Les filets sont relevés soit avec des treuils soit à la main. Les techniques varient légèrement d'une province à l'autre, mais sont globalement similaires. ${ }^{55}$

On dit aussi que les sérioles se déplacent en bancs et, laissant derrière elles la mer agitée du nord-est, parcourent la mer en direction du sud-ouest. Arrivées au large de la 
région de Tango, elles sont bien grasses et d'un goût exquis. C'est pourquoi on considère la sériole comme une spécialité de Tango.

La graphie buri 鯽 (sériole), composée des deux éléments "poisson» gyo 魚 et «maître "shi 師 a été inventée au Japon. Dans le Bencao gangmu (Classement raisonné de la materia medica), le mot gyoshi 魚師 《 maître-poisson » est un terme générique pour les poissons âgés ou grands et ne concerne pas la morphologie. On dit aussi que les explications des ouvrages chinois sur les poissons de mer sont toutes très sommaires. Comme leur pays est très grand et la mer éloignée, ils ont rarement la possibilité de voir les poissons, si bien qu'ils écrivent souvent d'après ouï-dire. Mais au Japon on a apparemment forgé le caractère buri composé par "poisson» et "maître » en lui donnant le sens de «très vieux ». La prononciation buri, signifie « vieux poisson » qui a vécu beaucoup d'années (furi), la prononciation furi étant voisée en buri, cette forme est entrée dans l'usage courant. La sériole, encore petite, est appelée wakanako, tsubasu, inada, mejiro, fukuraki ou hamachi. À Kyūshū on l'appelle aussi le "grand poisson », c'est pourquoi il figure parmi les vœux de bon augure du début de l'an.

\section{Thon (shibi) ${ }^{56}$}

Les grands sont désignés par oyu «thon-roi », les moyens par shukuy $\bar{u}$ «thon-cadet » (mekuro dans notre pays), et les petits par «thon-enfant» mejika 鮥子. Ils sont appelés maguro dans les provinces de l'est. ${ }^{57}$

Le thon est massivement pêché à Munakata ${ }^{58}$ dans la province de Chikuzen ${ }^{59}$, dans la province de Sanuki ${ }^{60}$, dans l'île de Hirado ${ }^{61}$ et dans l'archipel de Gotō ${ }^{62}$. Surtout les thons d'Iwashimizu à Hirado sont considérés comme de qualité supérieure. On commence à le pêcher à partir de l'équinoxe d'été, au huitième mois, et, jusqu'au dixième mois, il est appelé « longue nageoire " (hirenaga). Celui pêché entre le dixième mois et le doyo ${ }^{-63} \mathrm{~d}$ 'hiver est appelé « thon noir » et il est de grande taille. Celui pêché du doyō d'hiver au doyō du printemps est le hadara ${ }^{64}$, petit poisson d'à peine 1 pied 2-3 pouces; c'est le petit du «thon noir » (kuroshibi) de l'année précédente. Pour tous, la chair ressemble à celle de la bonite et la couleur est très rouge. Mais le goût ne vaut pas celui de la bonite. Une bonne prise de thon peut atteindre cinquante à soixante-dix mille poissons dans les meilleurs moments. On parle de hatsu no mi (première chair) parce que personne en ville n'achète un thon entier et que la chair est découpée, pesée et vendue en morceaux grands ou petits pour répondre à la demande de chacun. C'est ainsi que même dans les autres provinces, on appelle cela "découpe de la chair " (mikiri) du grand poisson. Le nom de hatsu (premier) provient du fait que jadis, la chair de ce poisson était tellement prisée, qu'à peine avait-on commencé à pêcher qu'elle était aussitôt mise en vente, et les gens se précipitaient et se battaient pour être les premiers à en acheter. Cela est comparable à ce qui se passe aujourd'hui quand on fait grand bruit de l'arrivée de la première bonite (hatsu gatsuo) à Edo. C'est ainsi qu'on appelle "premier", les produits de la première pêche. Plus tard, on est revenu de cet engouement pour la première bonite et la mode en est passée ; les premiers morceaux sont devenus une chose vulgaire et ne sont plus servis dans les banquets. Mais pour les gens de peu, ils représentent toujours l'une des huit merveilles et sont hautement appréciés. (On met aussi des poissons de petite taille à sécher pour en faire de la fausse bonite séchée.)

Poème du Man'yōshū : 
Telle la flamme

des pêcheurs

qui attirent les thons

mes sentiments secrets

finiront-ils par apparaître ${ }^{65}$.

Selon les procédures au fil du calendrier contenues dans le Liji (Mémoire sur les Rites), le souverain ferait à la fin du printemps (troisième lune) une offrande de thon shibi dans le sanctuaire des ancêtres. Mais il y a une incertitude sur le mot shibi [dans le texte chinois] et il est impossible de savoir s'il s'agit de ce que l'on appelle aujourd'hui " premier thon » hatsu. Nous y reviendrons plus loin.

Le filet [pour le thon] a des mailles de 8 pouces environ et une longueur de 20 chō environ. Il est fabriqué avec de la corde fine. Le filet est pourvu d'un fond en forme de van. À son extrémité il y a une poche ${ }^{6}$. Les câbles de halage des deux côtés du filet sont plus épais qu'1 pouce et restent immergés au fond de la mer [voir Pl. 32].

\section{PI. 32 : Filet à thon pour la pêche d'hiver}

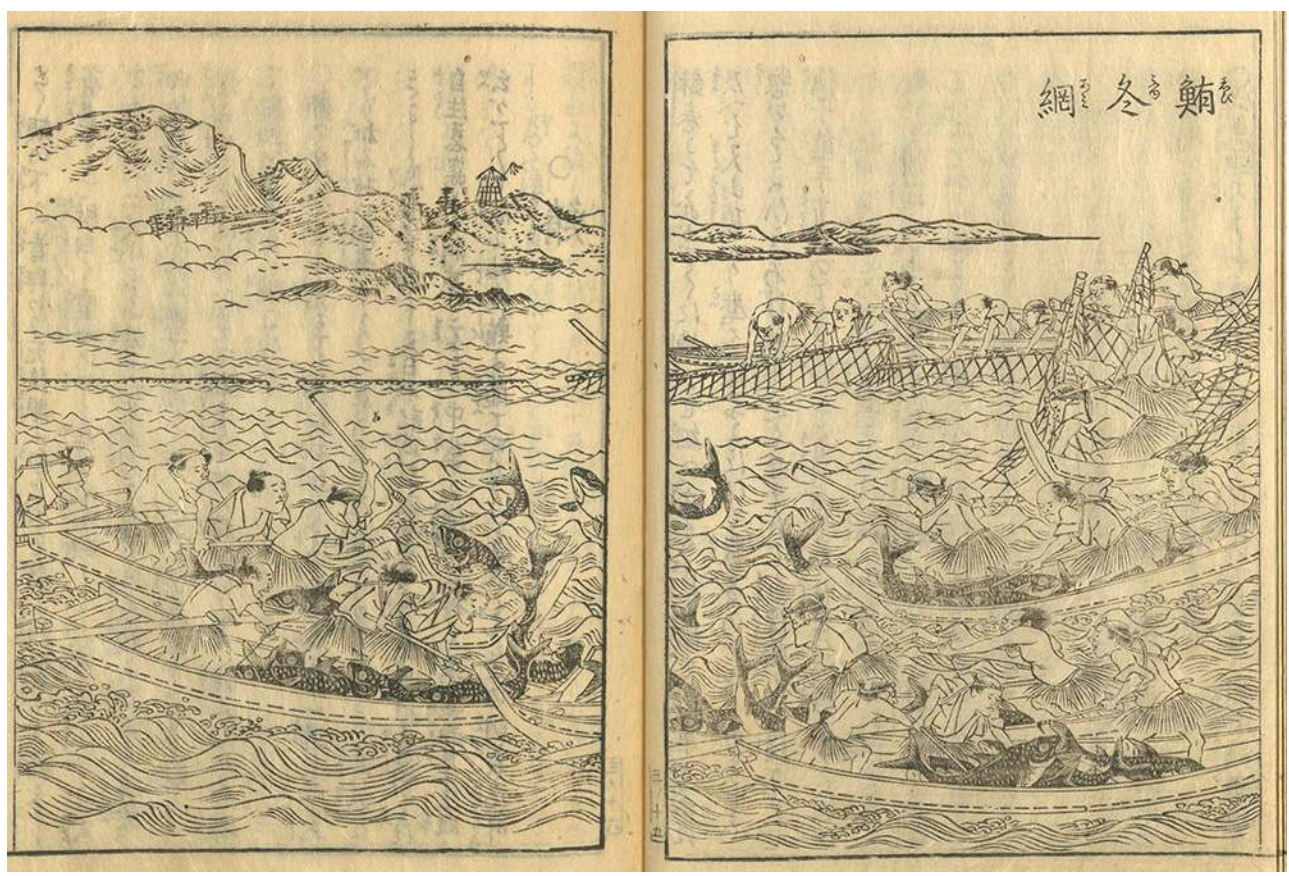

National Institute of Japanese Literature. Lien vers l'illustration

Chacune des extrémités est fixée à deux bateaux qui attendent que les poissons viennent nombreux. Quand les poissons tardent à s'attrouper, les bateaux passent jusqu'à deux ou trois mois à surveiller inutilement le filet. Au sommet de la montagne se trouve un poste d'observation à partir duquel on estime le nombre de poissons amassés en milliers ou en dizaines de milliers. On agite alors un fanion et on crie kamairo kama-iro (ce qui signifie kamaeyo «à vos gardes »). À ce moment on fait partir trois petites embarcations appelées danbei ${ }^{67}$. Dans chaque embarcation sont montés trois hommes, vêtus d'un pagne de paille, les manches relevées avec un cordon et la tête ceinte d'un bandeau. Ils rament aussi vite que le vent vers le filet. Ils se saisissent du [câble attaché au] fond du filet. Quand ils l'ont relevé à moitié68, on agite à nouveau le fanion du sommet de la montagne. De nombreuses autres embarcations danbei arrivent alors en ramant et tous ensemble ils relèvent le filet. Au fur et à mesure que les bateaux 
de halage se rapprochent, les poissons se mettent à grouiller comme si une source jaillissait du fond de la mer. Quand les pêcheurs frappent les poissons à la tête avec des râteaux ou des instruments semblables à des crocs de bûcheron, ils s'agitent de plus belle et sautent d'eux-mêmes dans les embarcations. Une fois tous les poissons dans les embarcations, les pêcheurs relâchent le filet dans la mer, et ramènent les embarcations vers la côte à la rame. La poche attachée au fond du filet est pleine de sardines, de quoi remplir deux bateaux, mais les pêcheurs ne s'intéressent qu'au thon. Comme le filet est resté plongé longtemps en mer, il est couvert de mousse, si bien que les poissons en ont fait comme leur nid. Voir l'image [Pl. 32]. Une autre méthode est la pêche à la ligne.

C'est une technique de la province de Wakasa ${ }^{69}$, pour laquelle on utilise des hameçons de 3 pouces et une corde en ramie de cent travées. Des liens en ramie d'une travée relient l'œillet des hameçons à la corde principale. Cela s'appelle des «queues de rat». Comme appâts, on utilise des entrailles de bonite. Les fils de ligne sont enroulés autour de baquets et ne sont jamais fixés à des cannes ${ }^{70}$.

Le thon a une grande tête, un bec pointu, un nez long et une bouche située sous la mâchoire inférieure ${ }^{71}$. Les branchies sont comme un casque de fer. Il a des taches bleues sous les joues. Quand il est mort, du sang coule de ses yeux. Il a le dos hérissé d'une crinière d'aiguilles. Il n'a pas d'écailles. Il est de couleur bleu noir avec un ventre blanc [brillant] comme du mica. La queue est fourchue et très rigide, la partie supérieure est grande et la partie inférieure petite. Les plus grands thons ont une taille d'une ou deux toises, les plus petits une taille de 7 à 8 pieds. La chair est épaisse et grasse et la tête très puissante. Quand il se trouve la tête tournée vers la côte, la queue vers le large, il est plus facile à prendre, parce que sa queue est faible. Lorsque, attiré par la chaleur, il remonte à la surface, il est ébloui par le soleil et forme des bancs. C'est alors que les pêcheurs le capturent, soit pour en recueillir l'huile, soit pour le sécher ${ }^{72}$.

Le fait d'employer le caractère 鮹 ${ }^{73}$, pour écrire le mot shibi (thon) n'est en accord ni avec le Bencao gangmu ni avec les dictionnaires. Mais cette lecture se fonde sans doute sur le fait que le Wamyōshō (Répertoire ordonné des noms du Yamato) ${ }^{74}$, citant le Minshu (Livre de Min) ${ }^{75}$, distingue les noms des thons selon qu'ils sont grands ou petits. De plus, une glose du chapitre sur l'empereur Buretsu du Nihon shoki, donne la lecture shibi pour le nom du fils du ministre Matori, en notant que cela se lit Shibi ${ }^{76}$. Comme mentionné plus haut, les traités chinois sont depuis toujours assez lacunaires quand il s'agit d'animaux de mer. On ne peut donc rien dire de plus au sujet du shibi pour le moment. La lecture et le sens de shibi demeurent incertains.

\section{Maquereau tacheté du Japon ${ }^{77}$ (sawara)}

On le pêche dans la province de Sanuki au filet flottant ${ }^{78}$. Les maquereaux tachetés $y$ sont particulièrement nombreux du cinquième au dixième mois. Les plus grands peuvent atteindre 6-7 pieds de longueur. Lorsqu'ils repèrent un attroupement, les pêcheurs les poursuivent à la rame, en rangée de plusieurs dizaines de bateaux, tant et si bien que les poissons finissent par s'épuiser, et tomber dans un état d'hébétude, comme s'ils étaient enivrés [voir Pl. 33]. 
PI. 33 : Filet flottant à maquereaux tachetés

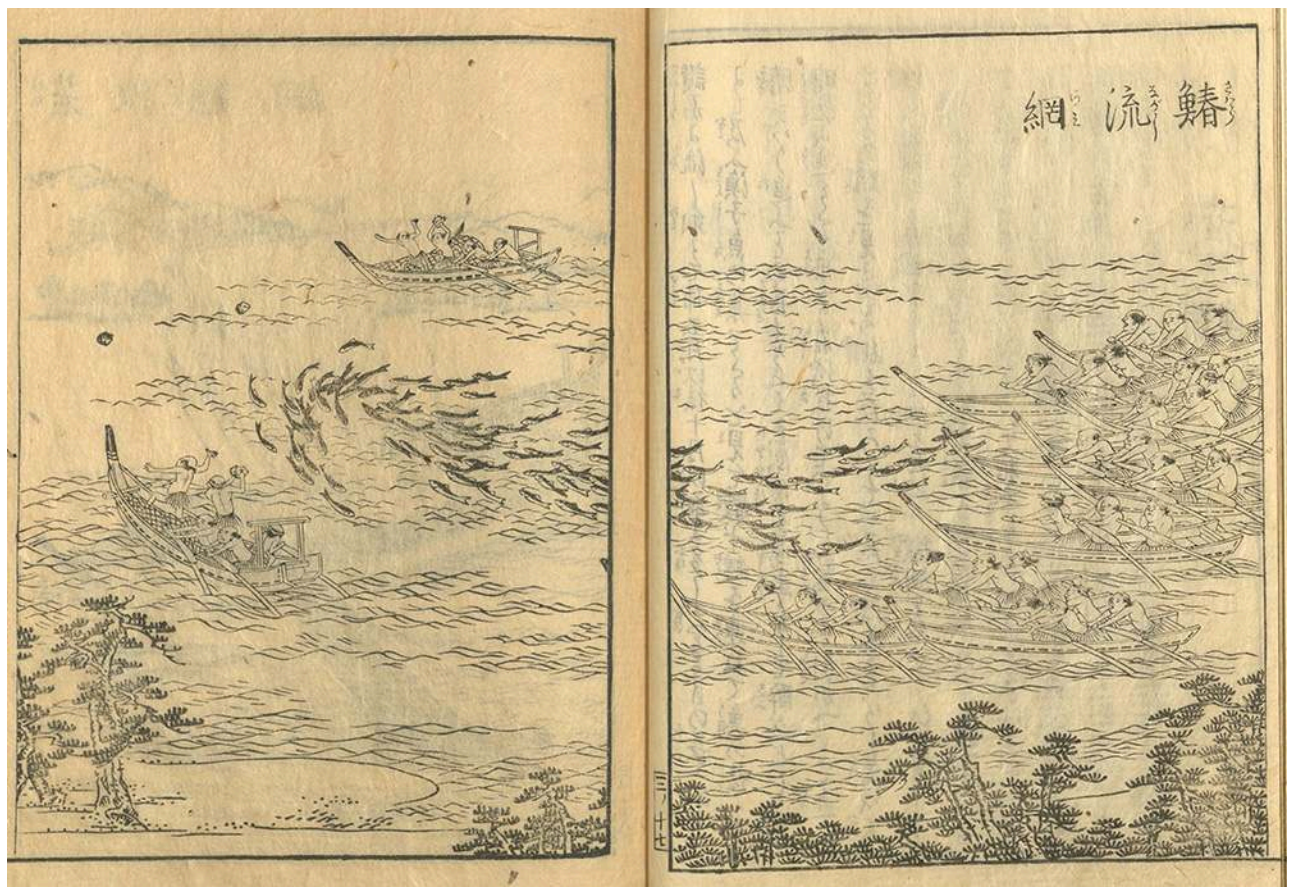

National Institute of Japanese Literature. Lien vers l'illustration

37 À ce moment, depuis les bateaux partis à l'avant vers le large, on jette des pierres pour les effrayer, et lorsque les poissons font demi-tour pour s'échapper, les pêcheurs d'autres bateaux, qui attendaient ce moment pour jeter le filet, les capturent sans en laisser échapper un seul. C'est ce qu'ils appellent "pratiquer le grand filet », ou bien « enserrer les poissons ». Ils ramènent alors les filets et puisent les poissons à l'aide de trubles.

Le caractère sawara 鰆 est une fabrication du peuple japonais. Les dictionnaires mentionnent bien un «poisson du printemps » 春魚, mais ce nom désigne un poisson minuscule de 2 ou 3 lignes si bien qu'il ne peut s'appliquer au sawara. Le Yamato honzō (Materia medica du Japon) ${ }^{79}$ écrit sawara 馬鮫魚 avec les caractères : cheval, requin et poisson, et note que ce poisson est grand, mais avec un ventre petit et mince, d'où l'appellation « mince $(s a)$ ventre (wara) » 狭腹, sa signifiant mince et petit. Le Minshu note : taches bleues, sans écailles, a des dents. Les petits sont appelés "flèches bleues » (sagoshi), etc ${ }^{80}$. Les œufs sont très gros. Séchés, on les appelle karasumi ${ }^{81}$, c'est-à-dire « œufs séchés » karashi-mi. Mais celui que l'on fabrique à partir du mulet est meilleur.

\section{Limande (karei) $)^{82}$ de Wakasa}

Elle est pêchée en haute mer à 30 lieues de la côte. Ces endroits sont appelés « habitats des limandes ». Les pêcheurs des trois provinces de Wakasa, d'Echizen ${ }^{83}$ et de Tsuruga ${ }^{84}$, utilisent des filets de chalut ${ }^{85}$ [voir Pl. 34]. 


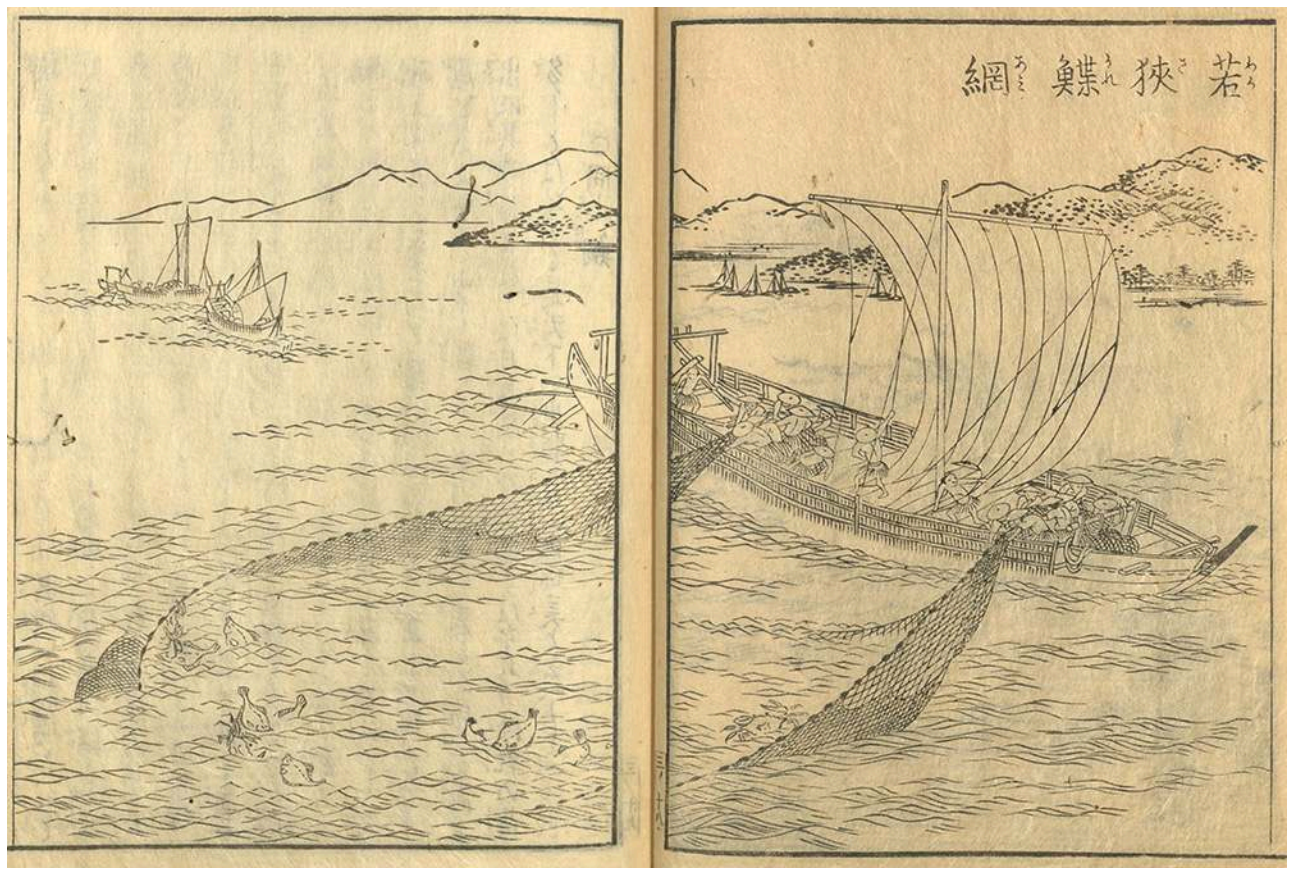

National Institute of Japanese Literature. Lien vers l'illustration

La mer y atteint cinquante brasses de profondeur ; la limande vit au fond de l'eau et vient rarement en surface. Douze à treize pêcheurs, montés à bord d'un bateau de 30 koku se répartissent de part et d'autre, à l'avant et à l'arrière, mettent la voile de côté et traînent le filet avec la force du vent sur le plein côtés ${ }^{86}$. Les flotteurs en bois demeurent à la surface, les plombs tombent au fond de l'eau et étendent ainsi le filet. On n'a pas plus de détails. Dans la prise sont mêlés quantités de crabes de grande taille.

Conservation par salage et séchage : par «limande étuvée " (mushi kare) on entend le poisson salé et cuit à la vapeur. Il ne s'agit pas d'un produit qui a été exposé au feu. D'abord on met le poisson fraîchement pêché à tremper une nuit dans la saumure, de manière à ce qu'il soit à moitié macéré, puis on le pose sur le sable, on le couvre de nattes de paille, et il est cuit sous l'effet de l'air chaud et humide. Ensuite on attache les poissons deux par deux par la queue avec une ficelle et on les met à sécher légèrement [voir Pl. 35]. 


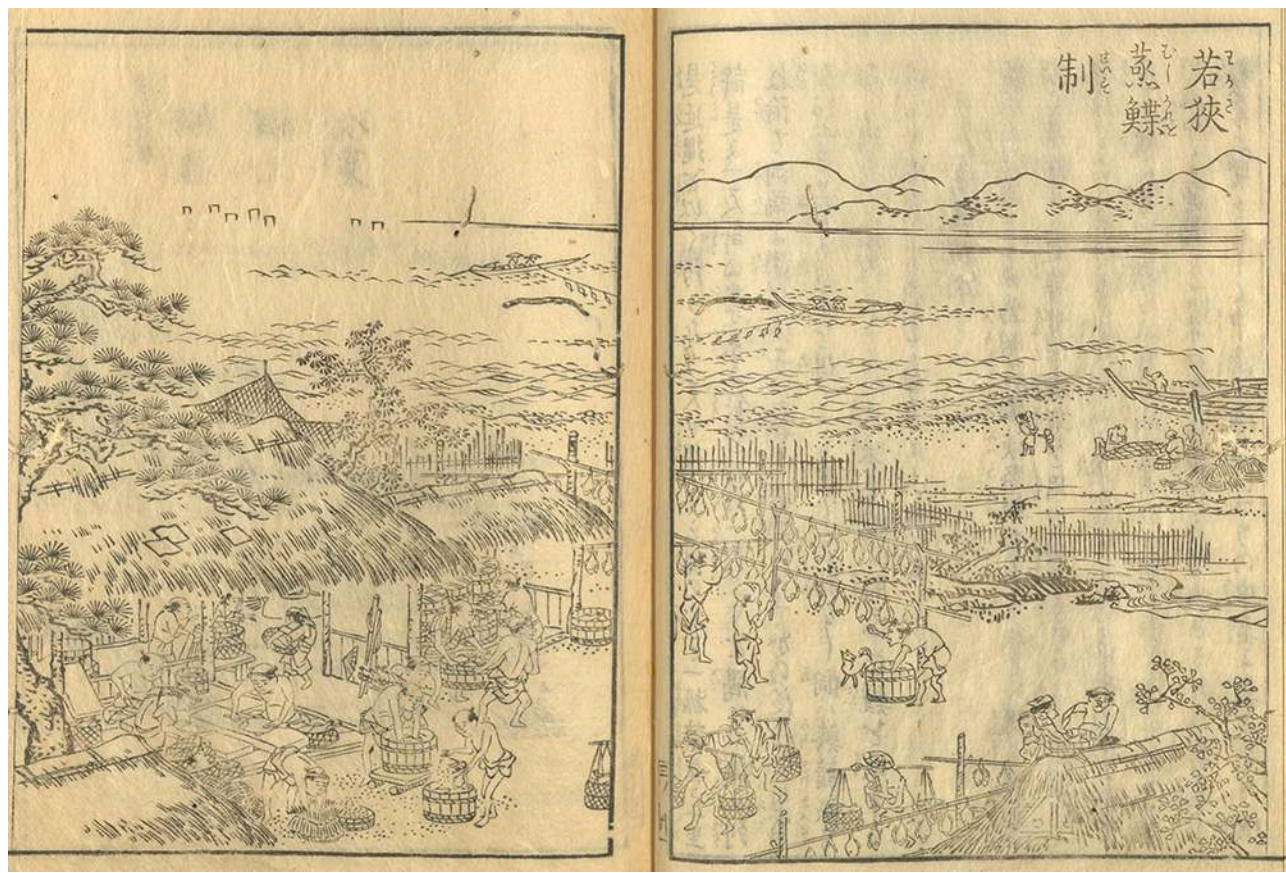

National Institute of Japanese Literature. Lien vers l'illustration

On les envoie à Kyōto avant qu'une journée ne s'écoule. En pleine saison, les trajets pour Kyōto se font tous les jours ou tous les deux jours. Il existe beaucoup de produits salés séchés dans le pays, mais parmi toutes ces productions, les limandes sont de saveur particulièrement sublime.

\section{Petite dorade (kodai) de Wakasa}

On la pêche à la ligne de traîne ${ }^{87}$, appelée aussi senawa. La corde nawa est grosse comme un poing et longue d'1 lieue. On y attache tout le long, espacés d'une brasse, des fils de ramie d'1 pied de long munis d'hameçons. Aux deux extrémités de la corde on attache des tonnelets comme flotteurs et, après un moment, en surveillant les tonnelets, on retire la corde de l'eau. En ce faisant on attrape cent poissons avec cent hameçons sans aucune perte. Comme appât on utilise le chinchard, le maquereau, la crevette, etc. Si on prépare les dorades selon la recette précédente, par salage et séchage, elles seront même meilleures que la limande. On utilise la même méthode pour pêcher la morue tara, dite « la vraie petite dorade » en certains endroits.

\section{Pêche au filet de la dorade dans les autres provinces}

La dorade d'Akashi et celle d'Awaji sont appréciées comme des articles de qualité dans les régions centrales [autour de Kyōto]. Mais on les pêche surtout à Emata ${ }^{88}$ dans la province de Sanuki. Dans ces différents lieux, on pêche les dorades avec un filet de chalut ${ }^{89}$. Dans les eaux, aux endroits où l'on compte de nombreux récifs, on les pourchasse avec des planchettes (buri $)^{90}$ pour les rassembler dans un endroit approprié. Les buri sont de fines planches reliées en grand nombre par des ficelles à une longue corde que l'on place en cercle tel un filet [voir Pl. 36]. 


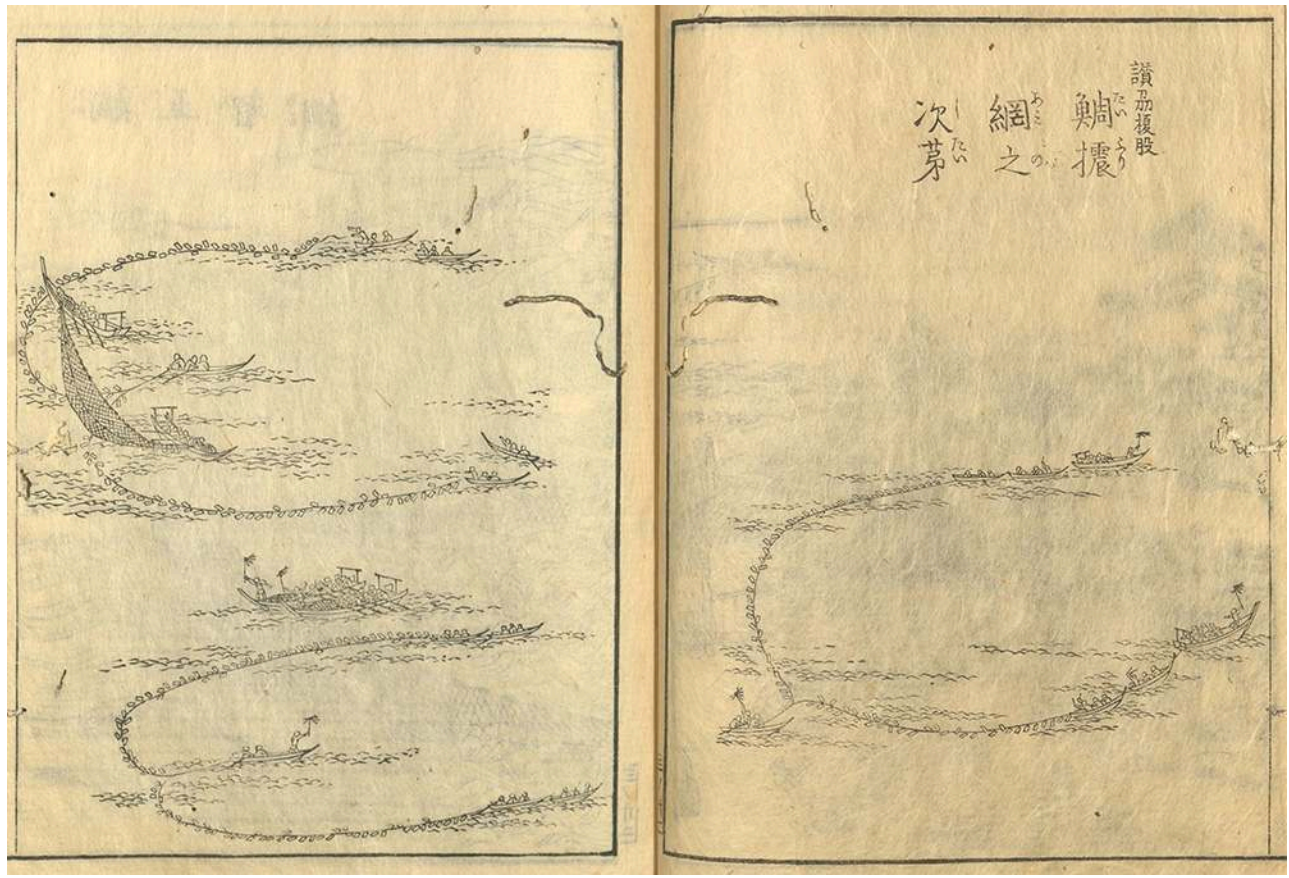

National Institute of Japanese Literature. Lien vers l'illustration

Les buri tournoient alors dans l'eau comme les feuilles dans le vent. Les poissons pourchassés sont surpris et effrayés, remontent plus haut dans l'eau et se rassemblent au sein du cercle des planchettes. À chaque extrémité de la longue corde, sont attachés respectivement trois bateaux. Trois pêcheurs sont respectivement à bord des deux bateaux de tête ; sur les trois, deux tiennent la corde pendant que le troisième frappe à l'aide d'un bâton de chêne ou d'un maillet sur le flanc du bateau pour empêcher les poissons de se disperser. Le dernier des trois bateaux de chaque côté s'appelle bateau de Katsura, le deuxième s'appelle "bateau du milieu », et celui en tête de chaque côté, qui fait avancer les deux files, s'appelle «bateau de halage» (amifune). Dans chaque bateau de halage se trouvent huit personnes dont une agite un fanion, les sept autres manient les rames. Dans le même temps, un autre bateau stabilisateur se place à l'extérieur du cercle à mi-longueur de la corde à planchettes, et pour l'empêcher de s'enfoncer, maintient la corde en la fixant au bateau. Parmi les trois hommes de ce bateau, l'un tient la corde, le deuxième tient la rame et le troisième agite un fanion avec lequel il signale le moment propice. Alors, les deux bateaux de halage s'avancent en premier, agitent leur fanion depuis le côté gauche de la corde aux planchettes, et rament d'une seule traite vers le bateau stabilisateur. Celui-ci s'avance à la rame et se dirige à l'intérieur [du cercle] de la corde à planchettes. Les deux bateaux de halage le suivent de part et d'autre de la corde et se font face. Puis, à la hauteur de la corde, au niveau du bateau stabilisateur, ils déploient le filet en prenant appui sur la corde aux planchettes, et laissent glisser le filet à l'extérieur. Enfin des deux côtés, ils tirent. Sur ce, les deux bateaux de halage, qui se trouvaient aux deux extrémités de la corde, la lâchent et le bateau stabilisateur la relève à la main. Ensuite, ils tirent sur le filet en rapprochant les deux bouts jusqu'à ce que les proues des deux bateaux de halage se croisent. En se rapprochant, le filet se resserre. Les poissons se mettent à grouiller, comme si une source jaillissait du fond de la mer, plongent dans le filet, sortent la tête par ci et agitent la queue par là, brillant de mille éclats. Les pêcheurs les prennent avec 
des trubles et les jettent dans des embarcations. Trois hommes sont à bord de ces dernières qui, à l'aide de perches, poussent le bateau vers le rivage. Cette technique s'appelle le « filet des cinq sagesses » [voir Pl. 37].

\section{PI. 37 : Filet « des cinq sagesses » pour pêcher la dorade}

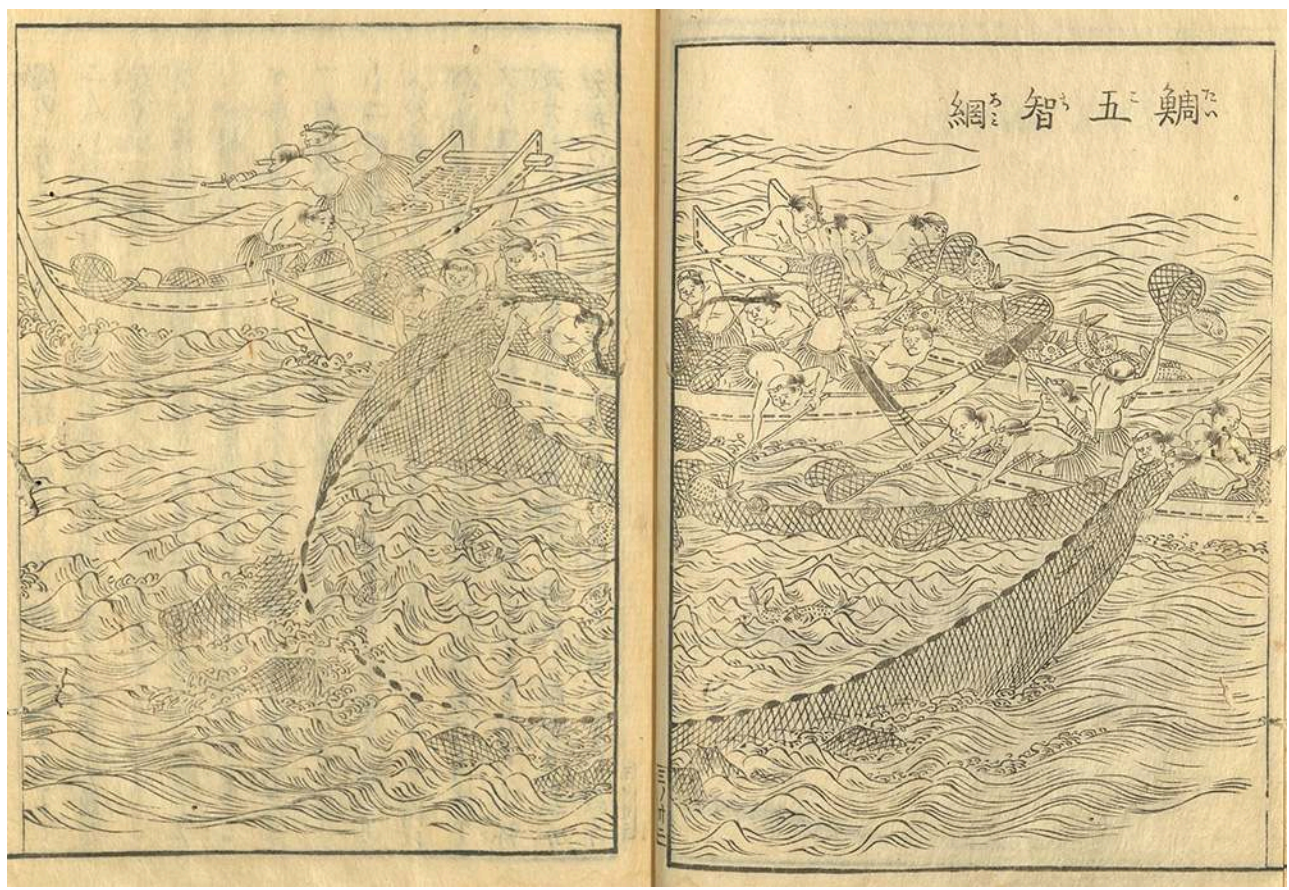

National Institute of Japanese Literature. Lien vers l'illustration

La corde à planchettes mentionnée plus haut mesure trois cent vingt brasses de long ; le grand filet fait quinze brasses de long et huit brasses de profondeur au milieu, et progressivement quatre, puis trois brasses de profondeur. On utilise du chanvre de qualité supérieure très fin avec des mailles d'une taille qui laisse passer sept doigts. Il y a des flotteurs en bois et pas d'autres flotteurs. Les plombs sont fabriqués à l'aide de tronçons de bambou que l'on a remplis de pierres, le tout étant maintenu avec une ficelle, comme un tambourin. Le filet est agrandi en raccordant une, deux ou plusieurs pièces appelées jō. Les pièces sont nouées ensemble avec dextérité en l'espace d'un instant. Chaque jō a quatre travées de largeur et dix travées de profondeur.

Sur la plage de sable blanc de cette province il y a beaucoup de ces choses appelées « os de serpent $»^{91}$. Ces os sont donc pris souvent dans les « filets des cinq sagesses ».

Himokugyo est le nom de la famille des limandes karei ${ }^{92}$. Le poisson appelé " poissonsemelle » (keiteigyo) dans l'« explication des noms » du Bencao gangmu ${ }^{93}$ est la variété dite ushinoshita («langue de bœuf ») ou encore kutsuzoko (« semelle de chaussure »). La graphie de la limande 鰈 (poisson plat comme la tête d'une rame) lui correspond bien. Le Yamato honzō explique en détail la limande étuvée de Wakasa. Dans l'est du pays on l'appelle hirame.

49 La dorade ne figure pas dans le Bencao gangmu ${ }^{94}$, mais elle est également décrite en détail dans le Yamato honz $\overline{0}^{95}$. C'est pourquoi je ne développe pas le sujet ici. C'est une pratique ancienne d'écrire "dorade " avec le caractère 鯛 mais les caractères corrects

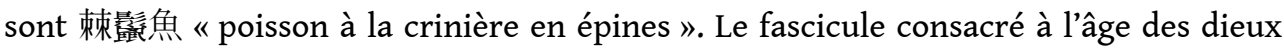
jindai du Nihon shoki, l'appelle "yeux rouges" (akame). Et le Engishiki écrit «poisson 
plat » (heigyo), ce qui évoque sa forme. Plus particulièrement, les dorades de Wakasa sont appelées hanaore ou renko, ce qui fait référence à sa petite taille et sa minceur. C'est une variété de couleur jaune pâle. Le sens de hanaore est obscur. On rencontre dans les dialectes des autres provinces des noms comme heike ou hyōdai, ils sont sans doute dérivés de heigyo. On trouve dans le fascicule $9 \mathrm{du}$ Man'yōshū le poème long suivant ${ }^{96}$ :

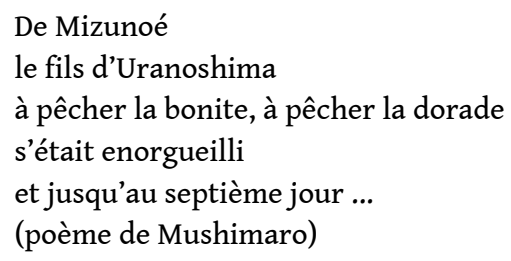

\section{Maquereau (saba)}

On en produit à Tanba ${ }^{97}$, à Tajima ${ }^{98}$ et à Kumano ${ }^{99}$ en Kishū (Kii) ${ }^{100}$. Le maquereau de Noto $^{101}$ est également réputé. La méthode de pêche est à peu près la même dans toutes les provinces. Lorsqu'un soir de printemps, d'été, ou d'automne, le ciel est couvert, que la marée est haute, et qu'une brume s'élève au-dessus de la mer, on dit que c'est un temps à maquereaux. Alors plusieurs centaines de bateaux vont s'aligner au large sur plus d'1 lieue, puis plus loin, à 1 lieue de distance, d'autres bateaux s'alignent comme les premiers. Avec deux braseros sur chaque bateau, des myriades de feux illuminent le ciel. Les pêcheurs ont tous des rouleaux de fils de chanvre d'une longueur de dix brasses et, comme pour les cordes de cithare, ils y attachent des poids en plomb de cinq monme $e^{102}$, puis y fixent des appâts de sardines et de crevettes, sans utiliser de cannes à pêche. À Tajima, ils n'utilisent même pas d'hameçons. Il suffit d'agiter les torches pour que, les poissons, sous l'effet des ombres creusant les vagues, se mettent à danser et à se jeter spontanément dans le bateau [voir Pl.38]. C'est là encore une technique singulière. Ces bateaux sont un peu plus grands et plus plats que les bateaux de pêche habituels. Ils sont encore plus grands à Echizen. 


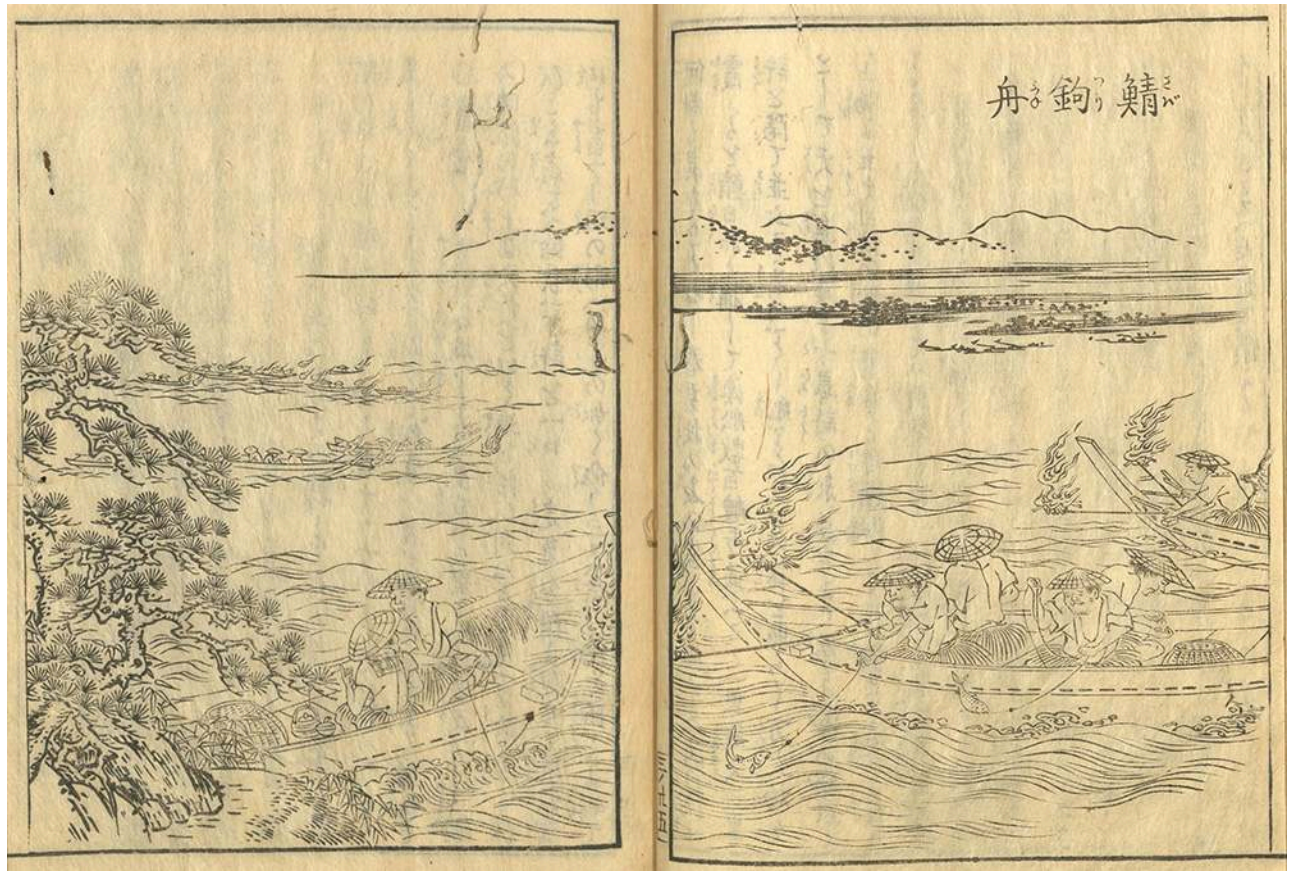

National Institute of Japanese Literature. Lien vers l'illustration

Le caractère 鯖103 du maquereau est lu ao-saba (« maquereau bleu ») dans le Wamyōshō. Le poisson noté 青魚 («poisson bleu») ou 鯖 dans le Bencao gangmu est celui que l'on appelle kado. Ce n'est autre que le hareng. Les œufs sont appelés kazunoko ou kadonoko. La graphie correcte du maquereau est inconnue ${ }^{104}$. Selon le Yamato honz $\overline{0}^{105}$, ce poisson a des petites dents. D'où son nom de $s a$ - $b a$ 狭歯 « dents-étroites ", où $s a$ (étroit) signifie petit. Le Tōga (Er'ya de l'Est) ${ }^{106}$ dit: dans la mesure où saha désigne les choses rassemblées en grand nombre dans la langue ancienne, cela pourrait en être la signification. Mais j'ignore laquelle de ces explications est la plus correcte.

\section{Huîtres (kaki)}

Autre nom : « fleurs des récifs »

Les huîtres consommées dans la région centrale de Kinai sont toutes produites à Hiroshima dans la province d'Aki ${ }^{107}$. Elles sont particulièrement renommées. Celles qui sont pêchées dans les provinces de Harima ${ }^{108}$, Kii et Izumi ${ }^{109}$ sont grandes et naturelles ${ }^{110}$. Elles n'ont pas bon goût. On trouve également des huîtres dans les provinces de Musashi ${ }^{111}$, Mikawa ${ }^{112}$ et Owari. Celles cultivées à Hiroshima et vendues à Ōsaka sont des huîtres de trois ans. Leur goût est donc irréprochable. Dans la région de Hiroshima, on en élève à cinq ou six endroits, comme à Kusatsu, dans la baie de Niho, à Tanna, à Ewa, à Hiuna et à Ōko ${ }^{113}$. Sur dix bateaux transportant des charges vers la baie d'Ōsaka, sept ou huit viennent de Kusatsu ou de Niho parce que les élevages y sont les plus nombreux. Les bateaux sont amarrés tous les ans au port d'Ōsaka à partir du dixième mois [novembre] et reprennent la mer à la fin du premier mois [février].

L'ostréiculture : les élevages s'étendent sur la côte, respectivement à distance d'1 à 3 lieues de la ville de Hiroshima. À marée basse, on installe sur le sable du rivage des palissades faites de troncs de bambous qui se dressent sur 1 lieue environ. Elles sont 
appelées hibi. On fixe les dimensions d'un lot à 1 toise de hauteur sur 1 chō de longueur et chacun cultive un nombre de lots correspondant à sa condition. Les palissades sont disposées en forme de $\uparrow$, en pratiquant ici et là des ouvertures de 3 pieds [voir Pl. 39] permettant d'attraper les poissons qui s'y rassemblent. De petites huîtres ou des algues se fixent sur les palissades à chaque marée ; entre le deuxième et le dixième mois, on en détache de temps en temps en les grattant avec une houe dentée de Bichū. Puis, on les transfère dans une sorte de vivier de poisson, également entouré d'une palissade de bambou, dont la surface carrée [au sol] fait 5 ou 10 travées de côté et la hauteur une toise. À l'intérieur, on enterre les huîtres dans le sable à 1 pied de profondeur, et c'est au bout de trois ans de culture qu'elles sont considérées comme adultes. Les algues cultivées à cet endroit jouissent du nom d'«algues de Hiroshima». On pêche aussi divers coquillages dont de nombreuses praires.

\section{PI. 39 : Ostréiculture à Hiroshima}

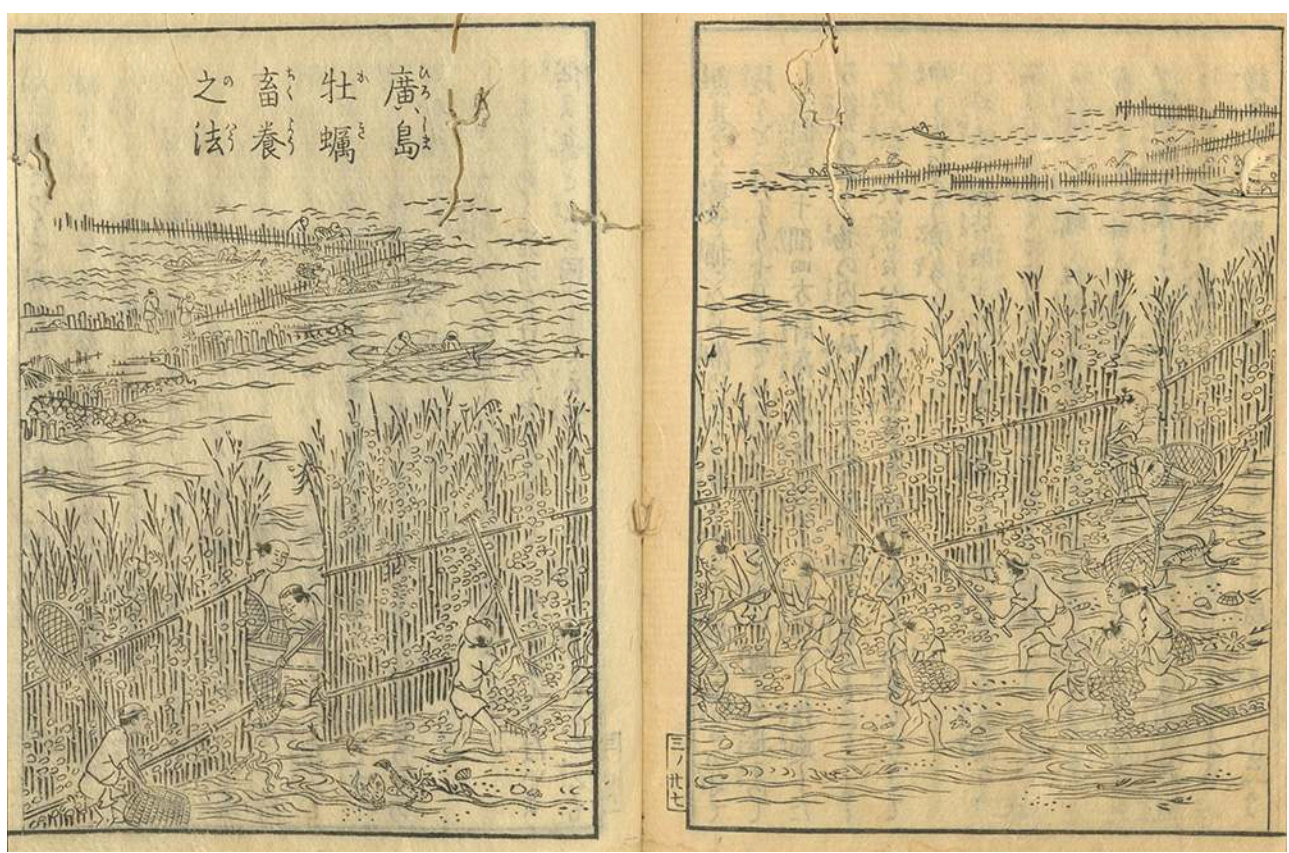

National Institute of Japanese Literature. Lien vers l'illustration

Les coquillages sont généralement vivipares ou ovipares. Seule, cette espèce [l'huître] se reproduit spontanément. Étant donné qu'elles sont accrochées aux rochers et ne bougent pas, il ne peut y avoir d'accouplement. Elles sont toutes mâles et sont donc appelées " huîtres mâles » (bo rei). La [graphie] de l'huître 蠇 (rei) exprime le caractère rugueux et grossier de la coquille. En se fixant à un rocher, elles s'agrègent et forment une sorte de chambre appelée " chambre d'huître » (rei bō). Au début, ces chambres ne dépassent pas plus qu'un poing en taille, mais leur surface peut s'étendre, jusqu'à atteindre une voire deux toises de côté. Chaque chambre [ou coquille] renferme un morceau de chair. La chair des grandes chambres a la taille d'un fer à cheval ; celle des petites chambres la taille d'un doigt. Quand la marée monte, toutes les chambres s'ouvrent et on raconte qu'elles remplissent leur estomac de tous les petits insectes qui entrent. On appelle aussi "monticules d'huîtres ", les chambres fixées aux rochers en bordure de mer qui, en se superposant en grand nombre, forment des monticules. Les huîtres petites et isolées sont appelées "huîtres fleurs de prunier ». Celles de 
Hiroshima sont de cette sorte. Le nom de « coquillages d'intérieur » qu'on leur attribue dans la province de Chikuzen est dû au fait qu'elles se trouvent sur les rochers à l'intérieur d'une baie. Les coquillages dits: "coquillages du large" (okigai) et "coquillages culbutants» (korobigai) ne s'accrochent pas au rocher; elles sont indépendantes et de grande taille. Il y a encore [la variété] que l'on appelle namima kashiwa. Elles sont nombreuses sur les plages et de forme ronde, plate et petite. À l'extérieur elles sont rouges et pourvues de petites pointes. Elles sont particulièrement belles. Les amateurs les conservent beaucoup comme objet de collection. Ce sont les huîtres furei mentionnées par Han Baosheng ${ }^{114}$. Ce que les livres de poésie appellent suma kashiwa désigne les coquilles d'huîtres. Il y a aussi [la variété] surnommée les «immortels». Ce sont des coquilles avec des épines larges. On appelle par ailleurs «chrysanthème de mer » l'espèce portant un grand nombre de longues épines d'1 pouce. Enfin il y a les huîtres qui, telles des nuages, n'ont pas d'épines. Elles peuvent être de différentes couleurs. (Pour tout ce qui vient d'être dit, j'ai suivi les explications du Bencao gangmu et celles sur les « chambres d'huitres ».)

La lecture kaki de l'huître doit être dérivée de kake. Le mot kake, qui est synonyme du mot «précipice » (gake) de la langue vernaculaire moderne, pourrait avoir son origine dans cette phrase d'un ancien poème : "Le sentier rocheux kake michi, dans les falaises de Miyoshino, piétiné et égalisé sous nos pieds... »"115. Il y a aussi le sens de "chose qui manque » (kake-taru), c'est-à-dire qui est imparfaite, ni ronde, ni carrée.

On grille la coquille d'huitre pour produire de la chaux dont on enduit les murs, comme on peut le lire dans le Bencao gangmu. Le Yamato honzō traite des coquilles d'huîtres fixées à des grands rochers de hautes falaises. Ce sujet est également évoqué dans le Bencao gangmu, dans le débat concernant les vieux hommes du mont Wushan ${ }^{116}$. Comme je ne saurais le trancher, je ne développe pas ce point. Mais il me semble que c'est une explication par trop détournée que de dire que les coquilles se seraient fixées sur les rochers au cours des nombreuses transformations que l'univers a connues depuis son apparition.

\section{NOTES}

1. Ise, ancienne province située dans l'actuel département de Mie.

2. Wagu, Omashi et Ōno sont des localités de l'actuelle ville de Shima; Tōse, près de la baie de Futami, correspondrait, selon Chiba, à l'île de Tōshi, dans la ville de Toba, les villes de Shima et Toba se trouvant dans le département de Mie.

3. Nihon shoki, première histoire du Japon, éditée par le prince Toneri (676-735), achevée en 720, première des six chroniques officielles (rikkokushi).

4. Empereur Ingyō, souverain mythique dont la légende date le règne de 412 à 453 de notre ère ; la quatorzième année correspondrait donc à l'an 425.

5. Awaji, grande île de la mer intérieure de Seto.

6. Akashi est une localité, située dans la partie sud-est du département de Hyōgo, donnant sur la mer intérieure, en face de l'île d'Awaji. 
7. Awa, ancienne province située dans l'actuel département de Tokushima dans l'île de Shikoku. Le village Nagamura est un toponyme inconnu.

8. Kinai, région centrale autour des capitales de Nara et Kyōto, comprenant les cinq anciennes provinces de Yamato (département de Nara), Yamashiro (préfecture de Kyōto), Settsu (préfecture d'Ōsaka) et Kawachi (partie est de la préfecture d'Ōsaka).

9. Poème $n^{\circ}$ 2798, fasc. 11, in Man'yōshū, SATAKE Akihiro, YAMADA Hideo, KUDō Rikio, ōTANi Masao, YAMAZAKI Yoshiyuki (éd.), vol.3, Tokyo, Iwanami shoten, coll. «Shin Nihon koten bungaku taikei », p. 108 ; Man.yôshû, SIEFFERT René (trad), Paris, Publications Orientalistes de France/ UNESCO, Livres X-XII, 2003, p. 202. Poème n 1322, fasc. 7 in Man'yōshū, SATAKE et al., op. cit., vol. 2, p. 173 ; Man.yôshû, SIEFFERT, op. cit., Livres VII-IX, 2001, p. 113.

10. Bungo, ancienne province située dans l'actuel département d'ōita dans l'île du Kyūshū. Les nattes de paille appelées «Teshima goza » étaient une spécialité répandue de Teshima à Settsu (Osaka).

11. Voir YANO Ken'ichi, Awabi (Ormeaux), Tokyo, Hōsei daigaku shuppankyoku, coll. « Mono to ningen no bunkashi » 62, 1989.

12. Le Gōshidai ou le Gōke shidai est un manuel des cérémonies de la cour, de date inconnue, compilé par Ōe no Masafusa (1041-1111).

13. Voir Gōke shidai, vol. 2, fasc. 7, Tokyo, Meiji tosho shuppan, coll. « Shintei zōhō kojitsu sōsho », 1951, entrée "Sixième lune » Rokugatsu. Le feu sacré imubi est, dans le cas d'Ise, le feu du fourneau dans le pavillon de l'Interdit où un cuisinier, qui a observé l'interdit, prépare les offrandes quotidiennes pour Amaterasu.

14. Manuel d'étiquette qu'Ogasawara Nagahide (?-1424) aurait écrit à l'intention de la classe guerrière de l'époque Muromachi. Il comprendrait notamment les règles relatives à l'équitation et le tir à l'arc.

15. Ashikaga Yoshimitsu (1358-1408) est le troisième shogun du gouvernement militaire (bakufu) de Muromachi, à Kyōto. Il a le titre de shogun de 1368 à 1394, mais dirige le bakufu jusqu'en 1408.

16. Ogasawara Nagahide, instructeur en tir à l'arc monté du shogun Yoshimitsu, aurait organisé le rituel guerrier. Les deux autres personnages ne sont pas identifiés.

17. Tenji (626-671, r. 668-671) a institué les structures étatiques de la cour impériale qui resteront en vigueur jusqu'en 1867.

18. Minamoto no Yoritomo (1147-1199), premier shogun, fondateur du gouvernement militaire (bakufu) de Kamakura en 1185. L'exil dont il est question ici intervient à l'âge de huit ans, à la suite de la défaite de son père Yoshitomo (1123-1160) lors des troubles de l'ère Heiji en 1159. Voir cet épisode dans Heiji monogatari, vol. 31, section "Yoritomo enryū no koto », Tokyo, Iwanami shoten, coll. « Nihon koten bungaku taikei », 1961, p. 292-293.

19. Ōmi, ancienne province située dans l'actuel département de Shiga.

20. Takebe, divinité tutélaire du sanctuaire Takebe jinja (actuellement à Ōtsu, département de Shiga) de la province d'Ōmi, Kurita-gun, connu du Engishiki (927), fasc. 10, op.cit., p. 244.

21. Hachiman, dieu de l'art militaire, divinité tutélaire de la maison Minamoto, qui a fondé le sanctuaire Hachiman à Kamakura en 1080, connu aujourd'hui sous le nom de Tsurugaoka Hachimangū.

22. Le terme employé est ōyuka (littér. " grand plancher »), qui désigne, dans un édifice shintō, la partie externe de plancher qui fait le tour du hisashi (la galerie). Le plus souvent, le hisashi peut être protégé des intempéries, lorsque l'on ferme des volets, ce qui n'est pas le cas du ōyuka (ou sunoko) qui est à l'extérieur et qui se trouve à un niveau plus bas que le plancher du hisashi. Il est fait le plus souvent de planches étroites et courtes, alors que celles du hisashi sont larges et longues. Nous remercions Nicolas Fiévé pour ces précisions.

23. Minamoto no Yoshitomo (1123-1160), père de Yoritomo, fut battu par les Taira lors des troubles de l'ère Heiji (1159) et tué, pendant sa fuite à Owari (Aichi-ken). 
24. Soixante-six est le nombre des provinces du Japon. Le rêve est le présage de la conquête du pays par Yoritomo.

25. Kunimura est Kunisaki, village actuellement situé sur la côte nord-est de la péninsule de Shima, département de Mie, lieu jadis réputé pour la pêche à l'ormeau et la récolte d'algues wakame, et qui fournit, jusqu'à ce jour, les ormeaux aux deux sanctuaires d'Ise.

26. Shima, ancienne province située dans la partie est de l'actuel département de Mie.

27. Voir Engishiki, op.cit., fasc. 4, règlement sur « Les sanctuaires d'Ise », p. 57-97.

28. Les huîtres akoya, Pinctada martensii fucata, étaient prisées pour le nacre et les perles. Elles se concentrent au sud de la péninsule de Bōsō et dans le Kyūshū.

29. Owari, ancienne province située dans l'actuel département d'Aichi.

30. Ōmura, ancien village, aujourd'hui commune d'Ōmura, dans le département de Nagasaki.

31. Hizen, ancienne province située dans l'actuel département de Saga dans l'île du Kyūshū.

32. Waka-ura, lieu non identifié.

33. Chita est encore aujourd'hui le nom d'un district situé dans le département d'Aichi. En revanche, aucune localité du nom d'Akoya (écrit en hiragana dans le texte) n'est attestée dans l'ancienne province d'Owari.

34. Mutsu, ancienne province qui comprenait tout le nord-est de l'île de Honshū.

35. Le Shinsarugakuki est une œuvre de fiction attribuée à Fujiwara no Akihira (989-1066), comportant des descriptions d'arts de divertissement et de différents métiers avec des listes d'objets et de termes techniques qui leur sont associés.

36. Les perles d'akoya sont mentionnées dans l'épisode « Hachirō no Mahito » du Shinsarugakuki. Voir Shinsarugakuki (Notes sur de nouveaux divertissements comiques), FUJIWARA no Akihira, Francine HÉRAIL (traduit et annoté par), Paris, Les Belles Lettres, 2014, p. 277.

37. Il s'agit du Kokin waka rokujō, une anthologie de compilateur inconnu, qui a vu le jour dans les années 976-987.

38. Namima kashiwa, Anamia chinensis namimakashiwa.

39. Saigyō (1118-1190), moine poète, proche des empereurs retirés de Heian, est connu pour ses pérégrinations à travers les provinces du Japon. Voir WATABE Tamotsu (éd.), Saigyō Sankashū zenchūkai (Le Sankashū de Saigyō intégralement annoté), Tokyo, Kazama shobō, 1979, poème 1478, Section Zō, p. 762. La traduction est de Daniel Struve.

40. L'auteur donne la liste des noms sous lesquels cette « espèce " apparaît dans les ouvrages chinois de bencao. L'ouvrage de référence qu'est le Bencao gangmu comporte les deux entrées 鰕 et 海鰕 dans la catégorie des animaux à écailles. La première entrée renvoie aux crevettes en général, la seconde aux langoustes. Le terme japonais ebi (aujourd'hui noté avec les caractères 海 老, soit «mer »+ « vieillard») désignerait l'espèce considérée dans la deuxième entrée, à savoir celles des langoustes. La note qui suit utilise plus souvent le caractère 蝦, dont le Bencao gangmu dit qu'il est une variante de 鰕.

41. Le terme renvoie à la rubrique de ce nom dans le Bencao gangmu. On trouve en effet ce nom dans la rubrique correspondante de l'entrée 海鰕.

42. Le terme umihebi désigne aujourd'hui les serpents de l'espèce des hydrophiinae. Il est probable que ce soit à cette espèce que l'auteur renvoie.

43. D'après cette description et la note sur la dorade, ci-après, il s'agit d'un filet traîné du genre du chalut, pourvu d'une poche au fond; voir plus bas, la section « La pêche au filet de la dorade dans les autres provinces ». Aujourd'hui, les crevettes sont couramment pêchées avec des filets, alors que les langoustes le sont avec des casiers; voir MUUS B. J. et DAHLSTRÖM Preben, Guide des poissons de mer et pêche, Paris, Delachaux \& Niestlé, 1981, p. 196-201.

44. Le terme peut être lu ena et signifie alors placenta.

45. Ezo, appelé ici Ebishi, c'est-à-dire Emishi, désigne une région englobant l'extrême nord-est de Honshū et l'île Hokkaidō. Elle était habitée par les Ainu qui étaient appelés « hommes poilus » à l'époque d'Edo. 
46. La forme courbée de la crevette est comparée, dans l'usage commun, au dos courbé d'un vieillard et on lui attribue le sens de longévité.

47. Tango est le nom d'une ancienne province, donnant sur la mer du Japon, correspondant à la partie nord de l'actuelle préfecture de Kyōto. Yosa est le nom d'un ancien village, situé dans l'actuel district de Yosa, préfecture de Kyōto.

48. Ine est un ancien village, actuelle commune Ine-chō à Yosa, située sur la mer du Japon.

49. Shii no ki : Shiia cuspidata Makino, castanopsis ou shiia, est une fagaceae très répandue dans tout le Japon.

50. Ama no hashidate (le pont du ciel) est un site célèbre situé dans la baie de Miyazu, ville de la préfecture de Kyōto, sur la mer du Japon. Il s'agit d'un mince banc de sable couvert de pins, de $3 \mathrm{~km}$ de long et de 40 à 110 mètres de large, reliant les deux extrémités de la baie.

51. Kiseto, lieu non identifié situé sur la baie de Yosa, dans l'actuelle préfecture de Kyōto.

52. D'après l'explication développée plus loin, le «filet ramasseur » oiami est un filet droit qui "pend verticalement et librement dans l'eau comme un rideau. On le tend en pleine mer... Généralement, on relie plusieurs filets bout à bout en un «fleet» de plusieurs kilomètres... Des flotteurs et des plombs de divers types maintiennent le filet à la surface ou à une profondeur déterminée. »; voir MUUS et DAHLSTRÖM, Guide des poissons..., op.cit., p. 217 ; NIHON GAKUSHIIN NIHON KAGAKUSHI KANKŌKAI (éd.), Meiji zen Nihon gyogyō gijutsushi (Histoire des techniques de pêche du Japon d'avant Meiji), Tokyo, Nihon gakujutsu shinkōkai, 1959, rééd. 1982, p. 223-225.

53. Les «filets en chanvre » sont manipulés par plusieurs dizaines de bateaux. Comme ils ont la forme d'un sac (CHIBA, Nippon sankai meisan meibutsu zue, op. cit., p. 96) et qu'ils sont posés dans la mer, on peut supposer qu'il s'agit de sennes. À la différence des filets traînés (comme les chaluts, voir plus bas, la limande), les sennes côtières sont posées en demi-cercle et sont donc immobiles. Quand elles sont pleines, elles sont hissées ou halées vers le rivage. La senne est constituée d'une longue nappe de mailles avec une poche au centre; elle est bordée de deux ralingues (cordes courant le long du filet) pourvues de flotteurs en haut et de plombs en bas, ainsi que de quatre très longs câbles de halage fixés sur les quatre coins du filet. Les câbles sont tirés par deux embarcations de chaque côté (voir MUUS et DAHLSTRÖM, Guide des poissons..., op. cit., p. 183, 220). La pêche à la senne était également pratiquée dans l'Occident médiéval (voir MANE Perrine, «Images médiévales de la pêche en eau douce ", Journal des savants, juillet-décembre 1991, p. 242, 254). Dans le Japon de l'époque d'Edo, ce genre de filet était fixé à deux bateaux ancrés en mer. Le filet se gonfle par le courant tout en étant maintenu sur place. Les poissons sont attirés, selon notre texte, par le tapage sur les flancs du bateau. Mais normalement, de jour, on jette des appâts, de nuit, on attire les poissons avec des flambeaux. Les poissons sont alors « ramassés " kakemawashi. Dès que les filets sont pleins, les câbles (tenawa) sont ramenés (taguru) par les deux bateaux. Les câbles du bas du filet sont tirés vers le haut (kuriageru) si bien que, lorsque les deux bateaux se rapprochent en avançant vers la côte, les filets sont graduellement resserrés à l'ouverture. Les filets sont ensuite jetés devant la plage et traînés jusqu'à la terre par les pêcheurs. (NIHON GAKUSHIN NIHON KAGAKUSHI KANKŌKAI (éd.), Meiji zen Nihon gyogyō gijutsushi, op. cit., p. 217, 344, 345).

54. Selon Chiba Tokuji, la pêche se faisait dans les lacs avec des palissades faites de bambou ou d'autres végétaux. Cette palissade était disposée en cercle avec, sur le côté, une ouverture en forme de nasse. L'ouverture, resserrée vers l'intérieur, permettait l'entrée, mais empêchait la sortie des poissons. Voir CHIBA, Nippon sankai meisan meibutsu zue, op. cit., p. 97, note 3. Pour la pêche à la sériole dans les provinces, évoquée dans le texte, on peut penser qu'il s'agissait d'un filet du genre de la bordigue, un filet en forme de nasse avec une ouverture en forme d'entonnoir. Les poissons sont guidés vers l'entrée par un long filet de barrage. (voir muUs et DAHLSTRÖM, Guide des poissons..., op.cit., p. 218).

55. Voir les filets et les treuils dans NIHON GAKUSHIIN NIHON KAGAKUSHI KANKŌKAI (éd.), Meiji zen Nihon gyogyō gijutsushi, op. cit., p. 217-225, 344, 345, et AKIYAMA Takashi et al. (éd.), Zuroku. Sangyoson 
seikatsu jiten (Encyclopédie de la vie dans les villages de montagne et de pêcheurs. Atlas), Tokyo, Kashiwa shobō, 1991, p. 134-157. Voir la pêche au buri dans AKIYAMA, Zuroku..., op. cit., p. 139-141.

56. Le caractère correspondant, prononcé également $y \bar{u}$, est le nom générique du thon. À l'époque d'Edo, ce nom est couramment employé dans les régions de l'Ouest, alors qu'à l'Est, le même poisson est désigné par maguro. Aujourd'hui, c'est sous ce dernier nom que le thon est généralement désigné au Japon. Les quatre variétés pêchées au large des côtes japonaises sont le kuromaguro (T. orientalis), le mebachi (T. obesus), le kihada (T. albacares) et le bin.naga (T. alalunga). Voir NIHON GYORUI GAKKAI (éd.), Nihonsan gyomei daijiten (Grand dictionnaire des noms de poissons produits au Japon), Tokyo, Sanseidō, 1981, p. 111, 135, 293, 407 ; ABE Tokiharu (éd.), Genshoku gyorui daizukan (Grand atlas des genres de poissons dans leurs couleurs d'origine), Tokyo, Hokurikukan, 1987, p. 886.

57. Cette petite note s'inspire d'un passage de la notice consacrée au poisson xun 俩髙 du Bencao gangmu. La désignation wei (j. y $\bar{u})$ 鮁 (lu shibi dans le Meisan zue) y est en effet donné comme un autre nom de ce poisson. Citant le lettré Guo pu (276-324), Li Shizhen écrit : « les grands sont appelés wangwei (j. o’i) «wei-roi», les petits, shuwei (j. shuku'i) «wei-cadet», et les tout petits, luozi (j. rakushi). Le Yamato honzō (Materia medica du Japon) note que la description du poisson wei dans le Bencao gangmu ne coïncide que partiellement avec le thon shibi pêché au Japon. Il préconise pour sa part de ne pas employer ce caractère pour éviter les confusions. Il conserve cependant cette idée de catégoriser le poisson selon la taille et établit une correspondance entre ces noms chinois et les désignations couramment utilisées au Japon. Il dit : « le ōyū est le shibi, le shukuyu est le maguro, et le rakushi est le mejika». S'ajoute ici une petite complication venant de ce que, pour l'auteur du Yamato honzō, le petit shibi est appelé maguro alors que pour l'auteur du Meisan zue, ce serait mekuro, maguro étant le nom employé dans l'est du Japon pour shibi. Voir Yamato honzō, op. cit., fascicule 13, entrée shibi.

58. Munakata, actuelle ville de Munakata au nord de Fukuoka dans le Kyūshū.

59. Chikuzen, ancienne province située dans l'actuel département de Fukuoka dans le Kyūshū.

60. Sanuki, ancienne province située dans l'actuel département de Kagawa dans l'île de Shikoku.

61. Hirado, île située à l'extrémité nord-ouest du Kyūshū.

62. Archipel de Gotō, situé à l'extrémité nord-ouest du Kyūshū.

63. Doyō d'hiver : période de dix-huit jours précédant le « début du printemps » selon l'ancien calendrier, soit approximativement la deuxième moitié de janvier ; doyō de printemps, période de dix-huit jours précédant le « début de l'été ", selon l'ancien calendrier, soit approximativement la deuxième moitié d'avril.

64. Il s'agit aujourd'hui d'une dénomination régionale pour la sardine japonaise (sardinella zunasi), sappa étant le nom japonais couramment employé. D'une longueur de 10 à $15 \mathrm{~cm}$, il s'agit d'une autre espèce que le thon.

65. Poème 4218, fasc. 19, attribué à Ōtomo no Yakamochi (718 ?-785), écrit en 750, au cinquième mois, in Man'yōshū, op. cit., vol. 4, p. 317.

66. D'après cette description et la planche 32 «Filet à thon pour la pêche d'hiver ", ce filet est de forme conique ouvert sur un côté (comme un van) et se termine au fond par une poche, voir muUs et DAHLSTRÖM, Guide des poissons..., op.cit., p. 183, 221. À la différence de la senne de la sériole, qui est visible en surface grâce aux flotteurs, ce filet est immergé et maintenu étendu par de longues tiges. Pour les filets à poches, voir AKIYAMA, Zuroku..., op. cit., p. 139-143, p. 151-152.

67. Danbei: bateaux de navigation fluviale utilisés pour le transport de pierres, ou grandes embarcations à fond plat en usage sur la rivière Yodogawa.

68. Hiki-guruma dans l'édition de CHIBA, Nippon sankai meisan meibutsu zue, op. cit., p. 101, mais hiku koto, dans l'original xylographique de 1799.

69. Wakasa, ancienne province située dans la partie occidentale de l'actuel département de Fukui qui donne sur la mer du Japon. 
70. Cette méthode ressemble à la pêche à la « ligne dormante » actuelle, avec une ligne composée d'un tronçon de fil très fort de cent à 300 mètres de long. Sur ce fil sont noués, à des intervalles de 2 à 3 mètres, un grand nombre de liens appelés "avançons" (bas de lignes) à hameçons. Plusieurs «lignes dormantes» sont attachées les unes aux autres pour former un long réseau. Cette méthode est pratiquée aujourd'hui en Europe pour la pêche au thon. Voir muUs et DAHLSTRÖM, Guide des poissons..., op.cit., p. 142, p. 215, p. 216. Comme il ne s'agit pas de cannes à pêche individuelles, on n'utilise pas de fil de ligne, mais seulement de la corde plus épaisse.

71. L'auteur veut signifier sans doute ici que le thon a une mâchoire supérieure longue, descendant en bas derrière la mâchoire inférieure.

72. Tout le passage est presque intégralement tiré du Honchō shokkan, HITOMI Hitsudai, SHIMADA Isao (trad. et éd.), Tokyo, coll. « Tōyō bunko », Heibonsha, 1980, vol. 4, p. 223-224.

73. Le caractère est composé des éléments « poisson » gyo 魚 et « avoir » yū 有.

74. Wamyōshō, titre abrégé de Wamyō ruijushō. Mentionné également plus loin, sous l'entrée «maquereau».

75. Traité géographique chinois en 150 fascicules portant sur la province du Fujian, compilé par He Qiaoyuan, achevé en 1520 et imprimé en 1631.

76. Nihon shoki, fasc. 16, règne de Buretsu, mentionne le fils Shibi de Matori no omi et note que «Shibi 鮁 se lit shibi », coll. « Nihon koten bungaku taikei », vol. 68, p. 8.

77. Le poisson étudié ici est l'espèce Scomberomorus niphonius, souvent désignée par «Japanese Spanish mackarel » ou "Spotted mackarel », de la famille du maquereau. NIHON GYORUI GAKKAI (éd.), Nihonsan gyomei daijiten, op. cit., p. 162 ; ABE, Genshoku gyorui daizukan, op. cit., p. 887.

78. Le maquereau est également pêché au filet flottant aujourd'hui en France, voir muUs et DAHLSTRÖM, Guide des poissons..., op.cit., p. 141.

79. Traité de honzō de Kaibara Ekiken (1630-1714), imprimé en 1709, réalisé sous l'influence du Bencao gangmu mais dont le propos est recentré sur les espèces naturelles observées au Japon. Composé de 16 fascicules, 2 fascicules annexes, et 2 fascicules d'illustrations.

80. Yamato honzō, in Ekiken zenshū (Æuvres complètes d'Ekiken), vol. 6, Tokyo, Kokusho kankōkai, 1973, p. 335, avec la citation du Minshu.

81. Équivalent japonais de la poutargue, il s'agit d'un mets apprécié, produit en mettant à tremper des poches d'œufs du mulet cabot ou encore de sawara dans le sel, puis en les mettant à sécher au soleil.

82. Le caractère est glosé kare dans le texte original, mais se lit karei aujourd'hui. Le terme japonais, traduit ici par « limande », englobe la famille des pleuronectidae.

83. Echizen, ancienne province située dans la partie orientale de l'actuel département de Fukui.

84. Tsuruga, ville portuaire, située sur la mer du Japon, à l'emplacement de l'actuelle ville de Tsuruga.

85. La limande est également pêchée au chalut aujourd'hui en France, voir MUUS et DAHLSTRÖM, Guide des poissons..., op. cit., p. 181-182. Teguru ou taguru signifie tirer, amener, ramener d'une main après l'autre, ou enrouler, embobiner. Le filet teguri ami correspond au chalut européen. Le chalut est un filet traîné et se distingue des filets posés (comme la senne).

86. Il s'agit d'un filet de haute mer, ou filet traîné hiki ami (ami o hiku dans le texte), et donc mobile, comme les chaluts. Le filet traîné se distingue du filet posé shiki ami immobile, qui est installé en mer et halé vers le rivage une fois rempli, comme les sennes ; NIHON GAKUSHIN NIHON KAGAKUSHI KANKŌKAI (éd.), Meiji zen Nihon gyogyō gijutsushi, op. cit., p. 217-222, p. 344-345.

87. La ligne de traîne est composée, selon le texte, d'une corde de presque $4 \mathrm{~km}$ de long portant une centaine d'hameçons. Elle est aujourd'hui pratiquée en France pour la morue et le thon, voir MUUS et DAHLSTRÖM, Guide des poissons..., op. cit., p. 115-116.

88. Emata, lieu non identifié, situé dans l'actuel département de Kagawa dans l'île Shikoku.

89. Pour le chalut (teguri ami) voir plus haut, l'entrée « limande». 
90. Buri en katakana dans le texte, en kanji sur la planche 36, est un dispositif de pêche, composé de planchettes qui tournent sur elles-mêmes, appelées « sinker » en anglais.

91. Au large de Wakasa (Fukui-ken), sur la mer du Japon, les os de mammouth, appelés traditionnellement " grand serpent ", se seraient pris fréquemment dans les filets des pêcheurs, à l'époque d'Edo ; voir CHIBA, Nippon sankai meisan meibutsu zue, op. cit., p. 111.

92. Les caractères 比目魚 correspondant à himokugyo sont lus karei dans le Yamato Honzō.

93. L'auteur fait référence ici à l'une des rubriques appelée "Explication des noms » (shakumei) du Bencao gangmu, où les diverses désignations sont discutées.

94. L'auteur se contente de dire bencao, comme quelques phrases plus haut, mais il veut dire Bencao gangmu.

95. KAIBARA Ekiken, Yamato honzō, op. cit., fasc 13, p. 327-329.

96. Poème 1740, fasc. 9, de Takahashi no Mushimaro (dates inconnues), in Man'yōshū, SATAKE et al., op. cit., vol. 2, p. 359 ; Man.yôshû, SIEFFERT, op.cit., vol. 3, p.327. De ce long poème, notre texte ne reproduit qu'une phrase.

97. Tanba, ancienne province située dans la partie orientale de l'actuel département de Hyōgo, qui donne sur la mer du Japon.

98. Tajima, ancienne province jouxtant Tanba, partie orientale de l'actuel département de Hyōgo, qui donne sur la mer du Japon.

99. Kumano, région couvrant la partie sud de l'actuel département de Wakayama et de l'actuel département de Mie et correspondant à la partie sud de l'ancienne province de Kii.

100. Kii, ancienne province située dans l'actuel département de Wakayama et dans la partie sud du département de Mie, dans la péninsule de Kii.

101. Nom d'une péninsule située dans le département d'Ishikawa, donnant sur la mer du Japon.

102. Monme, unité de poids valant $3,75 \mathrm{~g} ; 5$ monme pèsent environ $19 \mathrm{~g}$.

103. Ce caractère encore employé de nos jours se compose de la clé du poisson et du caractère signifiant « bleu ».

104. On se trouve ici dans le cas où un même caractère renvoie à deux poissons différents en Chine et au Japon. L'auteur en conclut que la graphie utilisée au Japon n'est pas correcte.

105. KAIBARA Ekiken, Yamato honzō, op. cit., fasc. 13, p. 335.

106. Tōga: dictionnaire en 20 fascicules donnant l'étymologie des caractères, composé par Arai Hakuseki (1657-1725) en 1717.

107. Aki, ancienne province située dans l'actuel département de Hiroshima et donnant sur la mer Intérieure.

108. Harima, ancienne province située dans la partie sud-ouest de l'actuel département de Hyōgo et donnant sur la mer Intérieure.

109. Izumi, ancienne province située dans la partie sud-ouest de l'actuel département d'Osaka et donnant sur la mer Intérieure

110. Le terme employé est shizensei, littéralement " génération spontanée ».

111. Musashi, ancienne province très vaste comprenant la ville de Tokyo et les départements de Saitama et de Kanagawa.

112. Mikawa, ancienne province située dans la partie orientale de l'actuel département d'Aichi.

113. Kusatsu, ancien port sur la mer Intérieure dans l'actuelle ville de Hiroshima; les autres toponymes ne sont pas identifiés.

114. Han Baosheng ( $\mathrm{X}^{\mathrm{e}}$ siècle), savant de l'académie Hanlin, a rédigé, sur ordre impérial, le Shu bencao 蜀本草 (Materia medica de Shu) qui est une réédition du Tang bencao 唐本草.

115. Poème de l'empereur retiré Gotoba (1180-1239) dans le Shoku Kokin wakashū, fasc. 4, section " Automne »; la citation est toutefois incorrecte et incomplète.

116. Le sens de ce passage est obscur. Cela fait référence à un phénomène naturel plus fréquemment observable au Japon qu'en Chine, où des coquilles d'huîtres se trouvent fossilisées sur les rochers. Voir Chiba, Nippon sankai meisan meibutsu zue, op. cit., p. 116. 


\title{
Fascicule 4
}

\author{
NOTE DE L'ÉDITEUR
}

Ce sommaire, placé en début de fascicule par l'auteur du Meisan zue, fait partie du texte d'origine. Ce sommaire n'a pas vocation à être utilisé pour circuler à l'intérieur du Meisan zue. Pour ce faire, le lecteur pourra utiliser toutes les fonctionnalités propres à l'édition en ligne de la plateforme OpenEdition Books, notamment sa table des matières générale.

\section{Sommaire du fascicule 4}

- Bonite de Tosa

- Kumano

- Holothurie de Sanuki

- Rôtie et séchée

- Entrailles marinées

- Oursin d'Echizen

- Gobie (shiro-uo) de Nishinomiya

- De Kuwana

$\circ$ Hio

- Addendum : Dorome'uo

- Palourde grillée de Kuwana

- Palourde « averse d'automne »

- Gobie (gori) de la rivière Kamo

- De la rivière Asano en Kaga

- Ishifushi d'Ōzu en Iyo

- Truite de la Jinzugawa

- Lamproie du lac de Suwa

- Vandoise

- Pieuvre d'Akashi

- De Nagahama 
- Pieuvre géante de Namerikawa

- Poulpe de Takasago

- Kajika

- De Yase

- Des chutes de Tsuzumi

- D'Ide

- Addendum : les différentes espèces de kajika

\section{[Produits de la pêche : suite]}

\section{Bonite (katsuo)}

1 On la pêche dans les provinces de Tosa, Awa ${ }^{1}$, Kii, Iyo, Suruga, Izu, Sagami, Awa ${ }^{2}$, Kazusa, Mutsu, Satsuma et dans quelques autres ${ }^{3}$. Vers les quatrième, cinquième mois, elle remonte des mers du sud-est et se déplace en banc le long des côtes est. C'est ainsi qu'on la rencontre en Sagami, Tosa, Kii. Elle est particulièrement abondante à Kamakura et à Kumano, mais c'est le produit de Tosa et de Satsuma qui est le plus réputé, pour sa chair grasse et savoureuse; séchée, elle est de qualité supérieure. Mangée fraîche, elle est délicieuse. Celle d'Awa et d'Ise s'en approche. Celle de Suruga, Izu, Sagami et Musashi est moins goûteuse et sa chair plus ferme : bonne à consommer fraîche, séchée, elle est un peu fade. Celles d'Awa, de Kazusa est de Mutsu sont de moindre qualité.

Quant aux différentes variétés de ce poisson, on parle, entre autres, de «bonite [à ventre] rayé » (suji-gatsuo), de "bonite à rayures transversales » (yokowa-gatsuo), de «bonite gâteau de riz» (mochi-gatsuo), de «bonite à cercles» (uzuwa-gatsuo), de «bonite plate » (hira-gatsuo). Parmi ces variétés, celle « à ventre rayé » est considérée comme la vraie bonite, celle « à rayures transversales » venant en second ${ }^{4}$. C'est de ces deux sortes que l'on fait le produit séché. Dans les provinces de l'Est on appelle mejika ${ }^{5}$ la bonite de petite taille.

Pour la pêcher, on la prend le plus souvent à l'hameçon, rarement au filet. On sort au large en toute saison. Cependant, entre le début et le milieu du troisième mois, on pêche la "première bonite " (hatsu-gatsuo) que l'on mange toujours fraîche. Le poisson pris jusqu'à la fin du cinquième mois donne ce qu'on appelle la «bonite du printemps » (haru-bushi) ${ }^{6}$, de première qualité. Celle pêchée jusqu'à la fin du huitième mois donne la «bonite d'automne»(aki-bushi). Étant donné que l'on appâte la bonite de sardines vivantes, on commence d'habitude par prendre celles-ci au filet [voir Pl.40]. On les plonge dans un panier large de plus de 6 mètres carrés ${ }^{7}$ plein d'eau de mer, puis on les transvase dans des cuves d'une contenance d'environ $3 k k^{8} u^{8}$ d'eau, que l'on charge à bord d'une barque de pêche jaugeant quelque 14 à 15 koku. Pendant qu'un homme puise dans le contenu de la cuve avec une louche à long manche, un autre à côté de lui ne cesse de rajouter de l'eau afin de garder les sardines frétillantes. Sur chaque barque douze hommes s'occupent de pêcher à l'aide d'une canne d'une travée et demie, et d'une ligne d'une travée environ de long, l'une et l'autre étant plus épaisses que la normale. La pointe de l'hameçon est sans ardillon. La barque est équipée de panneaux faits de lamelles de bambou ou de nattes pour couper les vagues [voir Pl. 40]. Alors, lorsqu'on se met à pêcher, on commence par lâcher quantité de sardines dans l'eau, appâtant ainsi la bonite qui approche en grouillant. Lorsqu'on lance sa ligne, une 
sardine attachée par la queue à l'hameçon, au milieu de ce tourbillon, les poissons mordent aussitôt et on n'arrête plus d'en attraper tant et tant, sans un moment de répit, si bien que malgré soi on en a capturé plusieurs dizaines: c'est comme le concours de tir à l'arc du Sanjūsangendōo .

PI. 40 : Pêche à la ligne de la bonite dans la province de Tosa

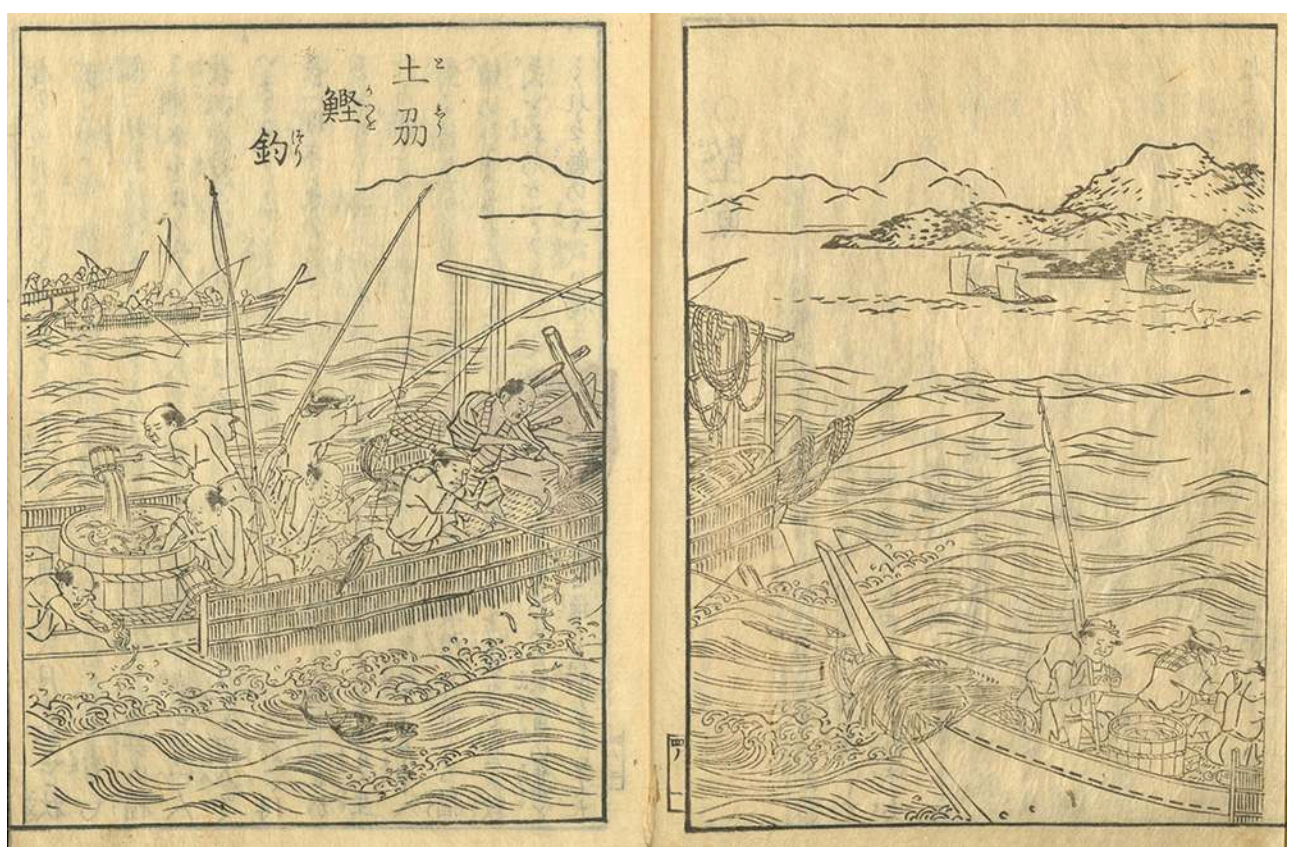

National Institute of Japanese Literature. Lien vers l'illustration

Il existe une autre méthode : quand on voit le poisson s'attrouper spontanément en eau peu profonde, on se sert d'une dent de cachalot ou d'une corne de vache, dans le creux de laquelle on fixe l'hameçon, ce qui permet d'attraper le poisson même sans appât. On parle alors de «leurrer» le poisson (dans l'eau, en effet, la corne scintille d'une lueur qui peut faire croire à un attroupement de sardines). De même, si l'on veut attirer la bonite, on fait danser à la surface de l'eau cette corne garnie de plumes de coq, dont l'éclat évoque plus encore le banc de sardines [voir fig. dans le cartouche de la Pl. 41]. 
PI. 41 : Les habitants d'un village côtier accueillent les bateaux revenant de la pêche et découpent la bonite sur le rivage.

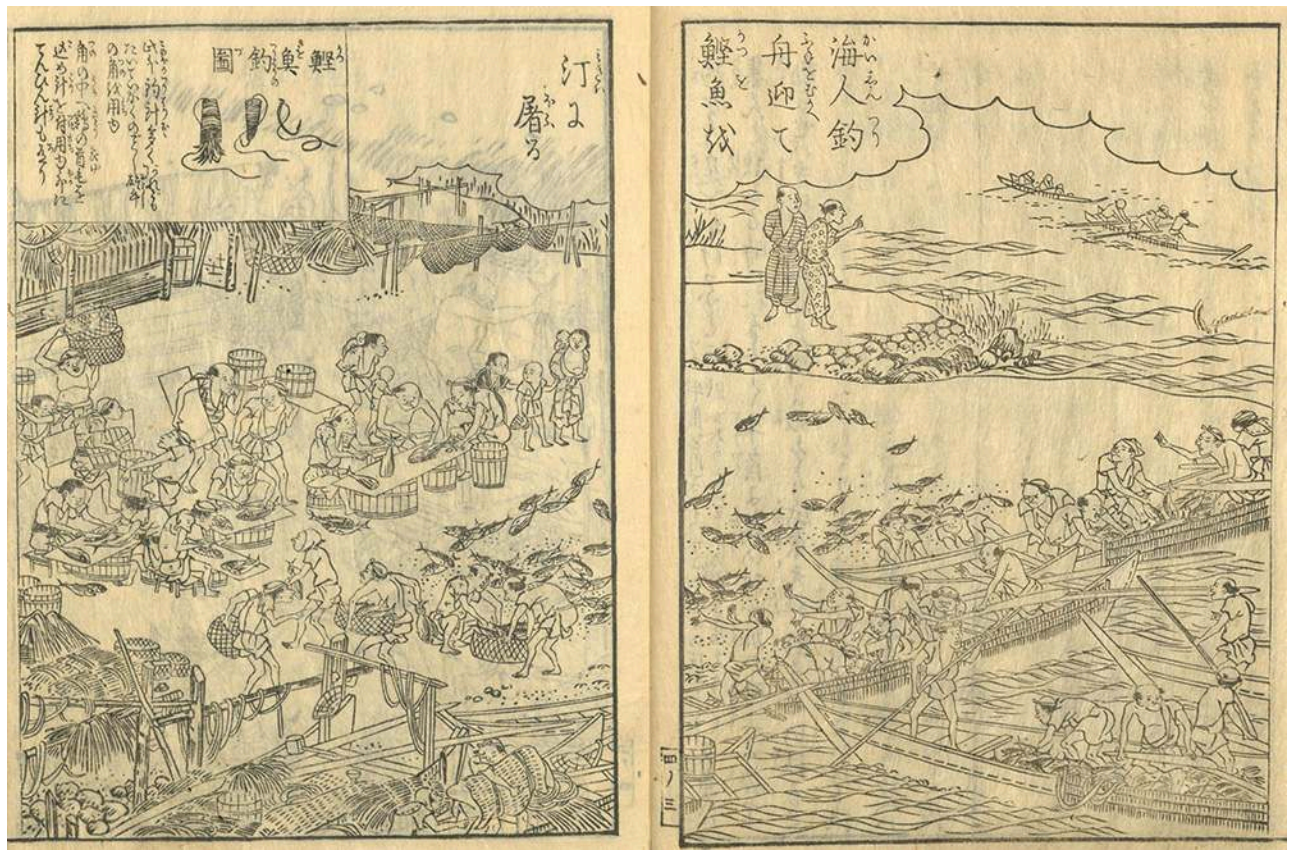

Dans le cartouche : « Illustration de l'hameçon pour la pêche à la bonite. Il en existe quantité d'autres sortes, mais en général on utilise une corne de vache comme on le voit dans l'image. On bourre la corne de plumes de coq parmi lesquelles on fixe l'hameçon. Il y a par ailleurs les hameçons fixés au fléau. »

National Institute of Japanese Literature. Lien vers l'illustration

emploie bien d'autres méthodes encore, telle la pêche « au fléau » ${ }^{10}$, mais, quoi qu'il en soit, ce sont toutes des passe-temps de gens de la terre qui ne sont pas en usage chez les vrais pêcheurs.

Quand on sort pêcher en bateau et que l'on rencontre au loin un banc de bonites poursuivant leur proie, il arrive que les poissons sautent tout seuls dans l'embarcation et avec un tel élan que l'équipage n'arrive pas à les en empêcher. Ils peuvent être si nombreux que le bateau risque de sombrer, raison pour laquelle, les apercevant au loin, on s'en éloigne à toutes rames et l'on attend que le banc passe son chemin.

\section{Préparation de la bonite par cuisson et séchage}

7 Quand le bateau de pêche aborde le rivage et que l'on a lancé le poisson sur la plage, les hommes et les femmes du village, jeunes et vieux sans distinction, se rassemblent à l'appel, munis qui de cuveaux, qui de planches ou de couteaux. Sur une planche posée sur des cuveaux en guise de tranchoir [voir Pl. 41], on commence par étêter et éviscérer le poisson, le couper en deux le long de l'arête et en diviser encore une fois chacune des moitiés, de sorte qu'on en obtient quatre morceaux. On jette arêtes et entrailles dans un baquet, et cela constitue la part des employés qui ne sont pas autrement rémunérés. Ces derniers font macérer ces abats dans le sel et en tirent profit en vendant le produit ${ }^{11}$ comme mets pour accompagner le saké ${ }^{12}$.

8 Ailleurs, on installe le site de traitement dans un endroit à l'écart, à une distance d'une lieue à peu près ${ }^{13}$. Deux personnes se font face de part et d'autre d'une grande planche à découper, l'un étêtant le poisson tandis que son vis-à-vis le soulève par la queue et le 
tranche à la verticale [voir Pl. 42]. Cette manœuvre s'accomplit avec dextérité et de manière très rapide. Dans la région de Kumano, cela se passe partout de cette façon.

PI. 42 : Découpe de la bonite dans l'atelier

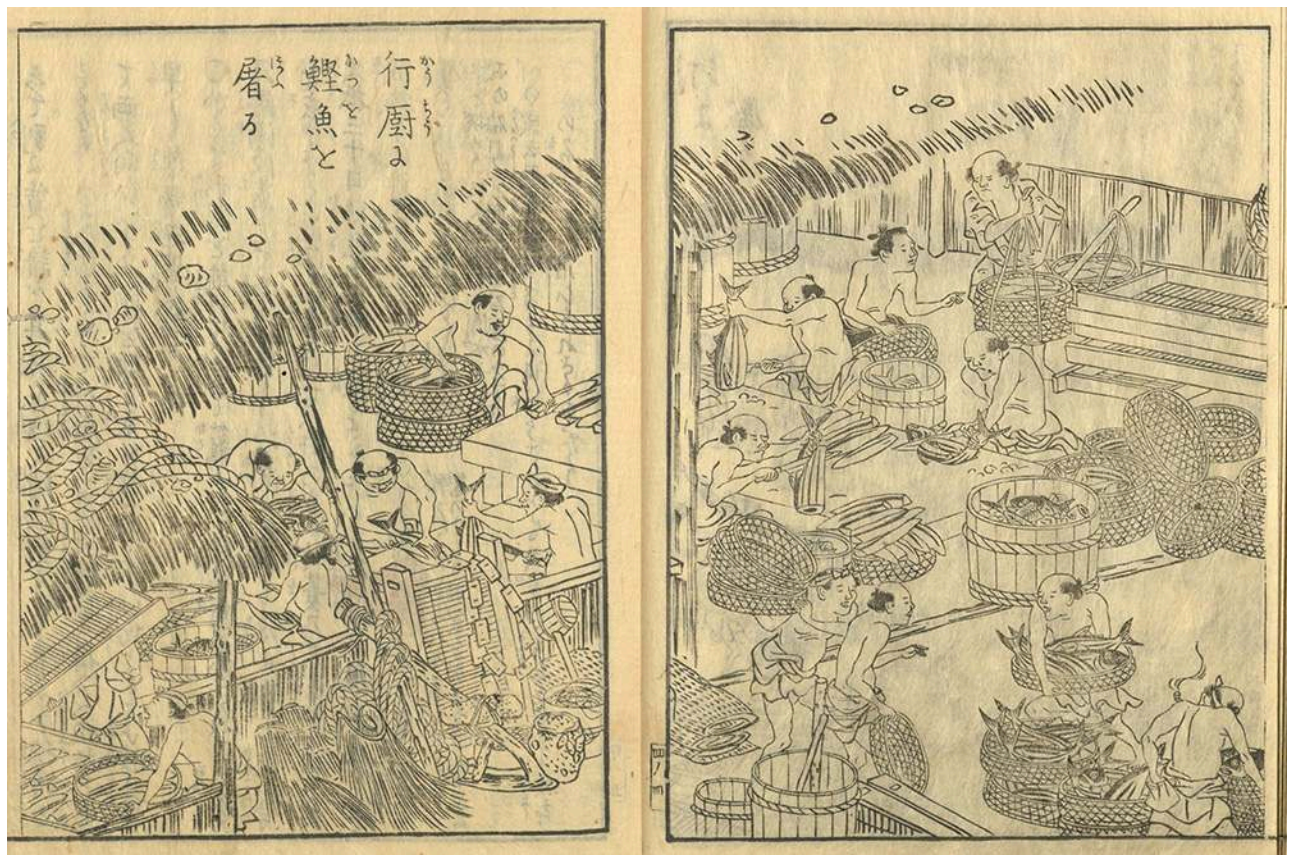

National Institute of Japanese Literature. Lien vers l'illustration

9 On en obtient ainsi des morceaux de bonne taille que, rangés dans des paniers, l'on superpose au-dessus d'un grand chaudron rempli d'eau bouillante pour les cuire à la vapeur $^{14}$ [voir Pl. 43]. On retire les paniers l'un après l'autre en commençant par ceux $\mathrm{du}$ bas, puis on refroidit les morceaux en les plongeant dans de l'eau froide, les débarrasse des menues arêtes, puis les rince avec soin. Après quoi on les aligne sur des claies de bambou de quelque 5 pieds de long et les met à sécher au soleil pendant une trentaine de jours. Ensuite on les râpe avec des peaux de requin, les racle et, pour finir, on les polit avec une corde de paille [voir Pl. 44]. En termes de qualité, les morceaux du «dos » (se-bushi) sont supérieurs à ceux du "ventre» (hara-bushi) ${ }^{15}$. Les morceaux du dos sont incurvés, ceux du ventre sont droits. Il existe des contrefaçons, faites, elles, avec de la chair de thon ${ }^{16}$, qui dégage une odeur extrêmement forte. (S'il pleut au cours du séchage, on élimine l'humidité en brûlant de la paille sous les claies. On choisit soigneusement l'eau pour le refroidissement : c'est surtout la qualité de l'eau qui fait la réputation du produit. Ainsi, la raison pour laquelle la bonite de Tosa est la plus renommée, c'est qu'on y utilise une eau fameuse provenant du lieu-dit Shimizu ${ }^{17}$. 
PI. 43 : La bonite est cuite et mise à sécher

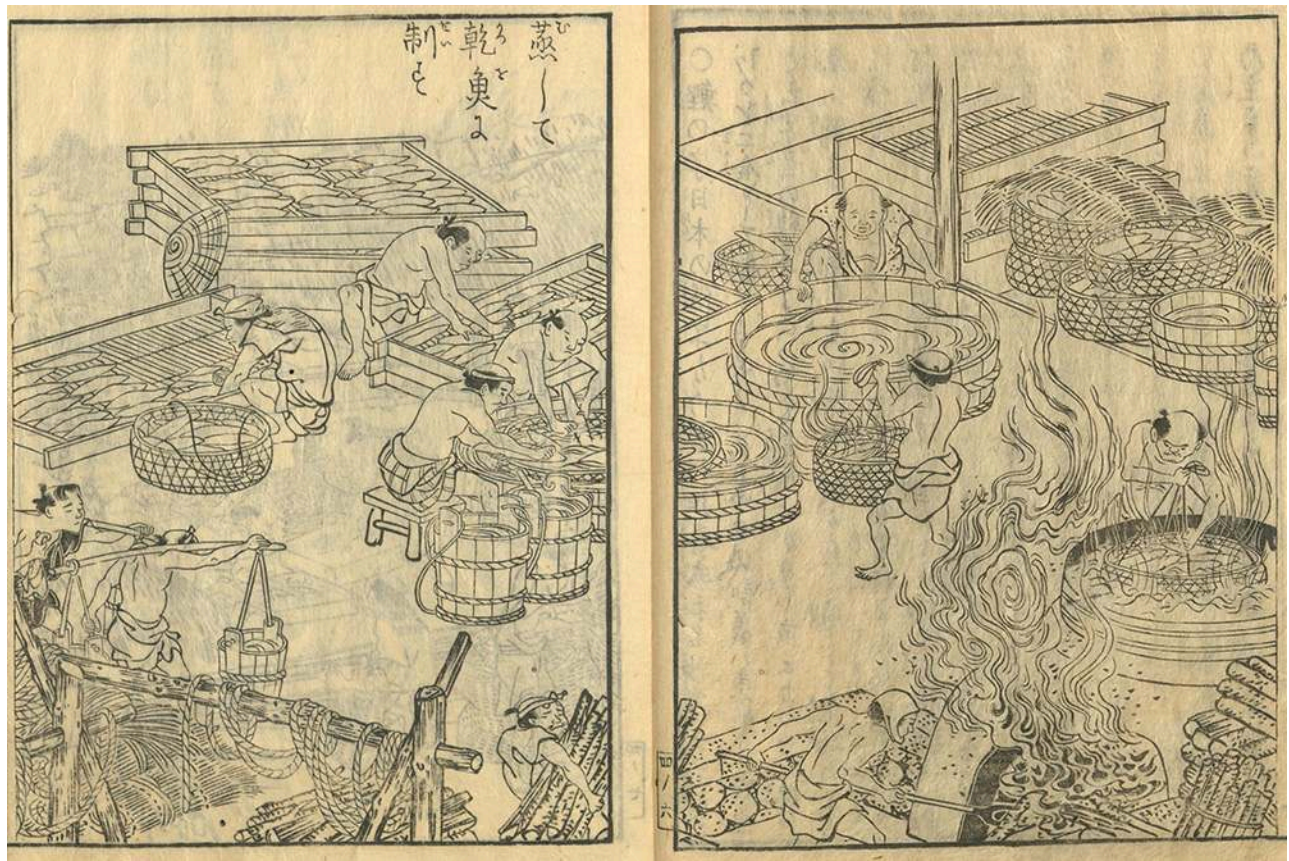

National Institute of Japanese Literature. Lien vers l'illustration

\section{PI. 44 : La bonite séchée est raclée et emballée}

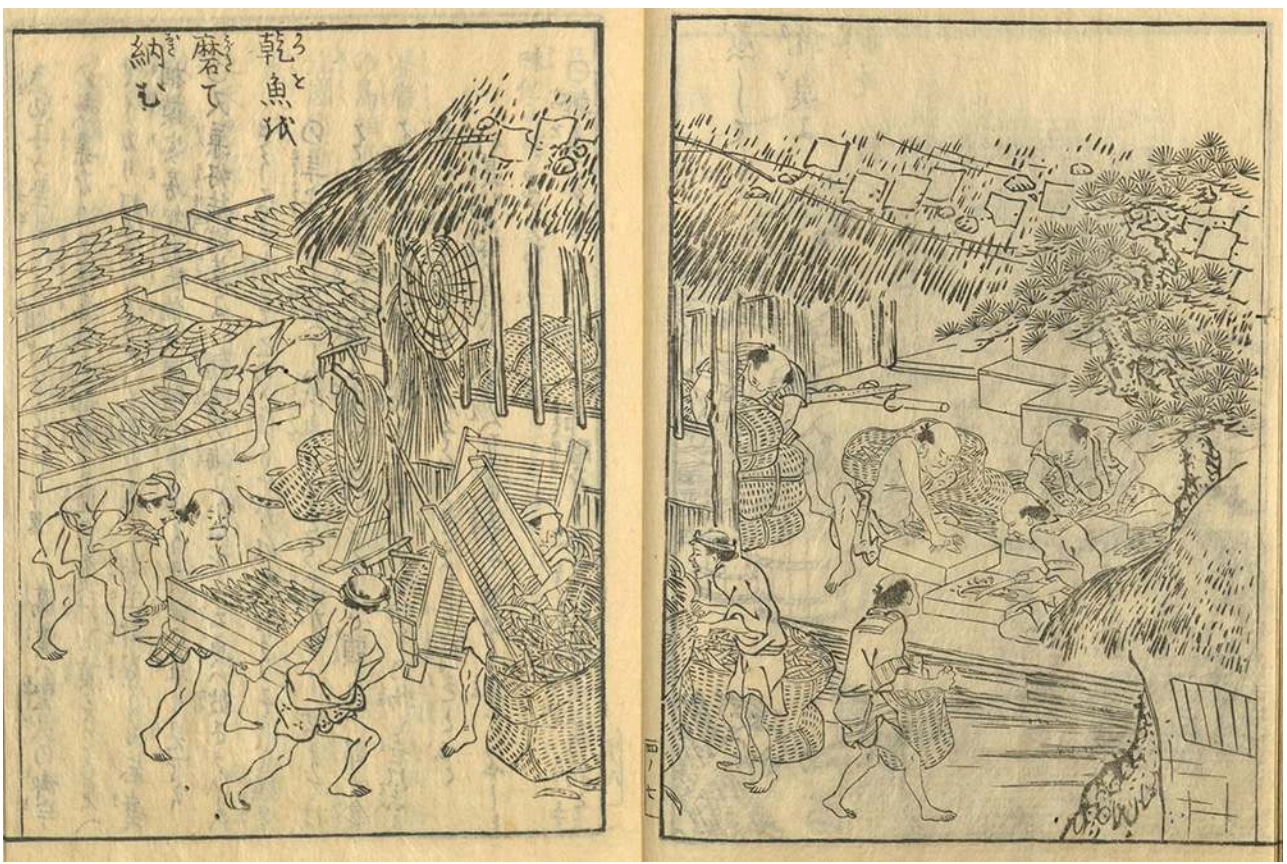

National Institute of Japanese Literature. Lien vers l'illustration

On dit aussi que, si les morceaux du «ventre » sont considérés comme étant de qualité inférieure, ce n'est pas une question de goût, mais parce que, chez les guerriers, on évite de faire cadeau ${ }^{18}$ de toute chose dont le nom rappelle le ventre ${ }^{19}$.

Le caractère 鰹 katsuo est une fabrication japonaise. Il a été obtenu en fondant en un seul caractère les deux idéogrammes 堅 kata («dur ») et 魚 uo (《poisson») composant le mot katsuo dans le Engishiki (Règlements de l'ère Engi) ${ }^{20}$ et dans le Wamyōshō 
(Répertoire ordonné des noms du Yamato) ${ }^{21}$. Selon le Tōga (Er'ya de l'Est) ${ }^{22}$, le mot katsuo serait une déformation phonétique du mot kotsugyo (魵魚 ${ }^{23}$ ), mais cela est peu crédible. D'autres y voient une déformation de kataki-uo (堅き魚, litt. «poisson dur ») désignant le «poisson séché » (乾魚, aussi lu katsuo). Ce nom aurait ensuite été étendu à l'animal vivant ${ }^{24}$.

11 Dans le Tongŭi pogam (Trésor de la médecine orientale) ${ }^{25}$, la bonite est désignée par le mot shōgyo 松魚 (《poisson de pin ») ${ }^{26}$. Cet ouvrage a été écrit par le médecin de la cour coréen Hŏ Chun. Cependant, d'après ce qu'a répondu un envoyé coréen venu dernièrement qu'on a interrogé à ce propos, il semblerait que shōgyo corresponde à notre « saumon" (sake). Sa chair est rouge tel un nœud de pin ${ }^{27}$. Mais, d'après un autre envoyé arrivé plus récemment ${ }^{28}$, le nom ancien kogyo 固魚 ("poisson dur») correspondrait à notre katsuo 堅魚 ${ }^{29}$. Mais là encore, cela a l'air d'être une appellation vernaculaire bien récente. Le kotsugyo 魵魚 dont il a été question plus haut correspondrait à ce que l'on nomme ici managatsuo, un autre nom serait shō 鯧 ou gyoyū 魚遊 ${ }^{30}$. Cela correspond à ce que dit Shunshui ${ }^{31}$ et c'est une erreur de vouloir écrire le nom managatsuo avec le caractère 魴 $(h \bar{o})^{32}$.

La bonite séchée est un produit d'usage courant dans notre pays, elle sert d'assaisonnement de base et permet d'harmoniser les cinq saveurs ${ }^{33}$. Le nom de katsuo est assurément ancien. Un poème long du Man'yōshū (Recueil des dix mille feuilles) ${ }^{34}$ chante : «... De Mizuno'e / le fils d'Uranoshima / à pêcher la bonite / à pêcher la dorade / s'était enorgueilli / ...35» Or ce Man'yōshū est une anthologie de poèmes qui date de l'auguste règne de l'Empereur Shōmu (724-749). En outre, dans les Règlements de l'ère Engi relatifs au département de la Population ${ }^{36}$, il est question de tribut fourni sous forme d'« extrait de bonite » (ikari). Cet ikari correspond sans doute à ce qu'on appelle maintenant nitori ${ }^{37}$. Et il y est également dit que l'office des Comptes recevait ce produit en tribut des provinces de Shima, Sagami, Awa, Kii, Tosa, Hyūga, Suruga et Bungo.

Kenkō dit dans le Tsurezuregusa (Les heures oisives) ${ }^{38}$ :

Ce qu'on pêche dans la mer de Kamakura sous le nom de bonite est un poisson quelconque, mais que l'on en est venu de nos jours à lui faire les honneurs de la table. Et selon les dires d'un vieillard de Kamakura : «ce poisson, au temps de ma jeunesse, on ne l'aurait jamais servi à un personnage de rang. Quant à la tête, même les domestiques n'en mangeaient pas. C'était quelque chose à couper et jeter. ${ }^{39} \mathrm{Il}$ faut vraiment que nous soyons arrivés à la fin du monde pour que pareil mets apparaisse jusque sur la table de nos maîtres ! ${ }^{40}$ celui d'Odawara en Sagami. Mais le tataki en provenance de Tanagura en Mutsu, de couleur blanche, dépasse en saveur tous les autres. Au point qu'on le présente en tribut au seigneur local ${ }^{43}$.

\section{Holothurie (namako)}

rôtie et séchée (iriko), et ses entrailles marinées (konowata) 
Miura en Sagami, à Kanazawa en Musashi, à l'ouest dans l'île de Shōdo en Sanuki ${ }^{46}$, mais on en recueille aussi par-ci par-là au nord [voir Pl. 45]. Comme elle est très rare en Chine, on y fabrique des contrefaçons avec des peaux ou des pénis d'âne ${ }^{47}$; aussi les envoyés et les marchands de ce pays en achètent-ils en grand nombre ici pour les ramener chez eux. Du fait qu'on l'utilise dans le traitement de l'émaciation infantile à la place du ginseng, le Shiwu bencao (Pharmacopée des aliments) ${ }^{48}$ de Shizhen la surnomme "ginseng marin ${ }^{49}$. En outre, celles que l'on recueille dans l'île de Kinkasan ${ }^{50}$ dans le Mutsu sont de forme cylindrique et de couleur jaune pâle. Du fait que leurs entrailles renferment de la poudre d'or on les nomme "holothuries d'or" $(\text { kinko })^{51}$.

\section{PI. 45 : Pêche à l'holothurie dans la province de Sanuki}

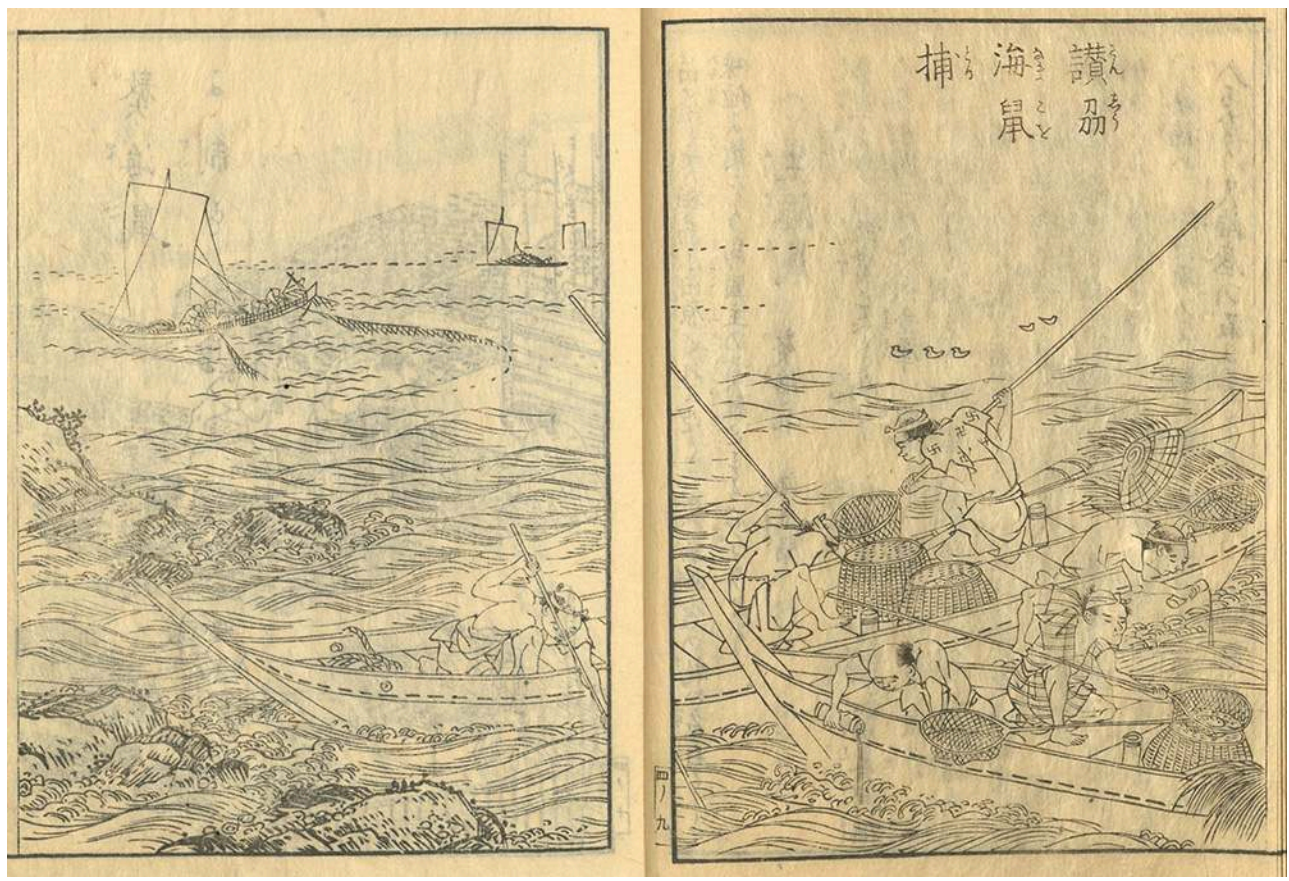

National Institute of Japanese Literature. Lien vers l'illustration

Il y a plusieurs façons de les pêcher. Pour les prendre au large, on attache un filet à la poupe du bateau et le traîne: elles s'y prennent d'elles-mêmes. Pour ramasser celles attachées aux roches du fond, on répand un peu de bouillon d'holothurie séchée ou de l'huile de baleine sur la surface de l'eau pour la rendre limpide [voir Pl. 45], tant et si bien qu'on voit le fond aussi clairement que dans un miroir. On n'a plus alors qu'à les remonter avec une épuisette.

Voici comment on fait pour les griller et les sécher [voir Pl. 46]. On les ouvre pour retirer les trois viscères, puis on en met plusieurs centaines dans un chaudron et on les fait bouillir à feu vif pendant une journée. Les holothuries dégorgent l'eau salée qu'elles contiennent, noircissent à la cuisson, sèchent, durcissent et rétrécissent. Alors on les fait bouillir à nouveau pendant une nuit, et lorsqu'elles ont regonflé un peu, on les sort, les met à refroidir puis à sécher sur un fil. On peut aussi les sécher en les embrochant sur des tiges de bambou, et on parle alors d'" holothuries en brochette» (kushiko). Les grandes sont enfilées sur des sarments de glycine. C'est le cas de celles produites à l'Est et en Echigo. Celles produites sur l'île de Shōdo sont de grande taille et d'un goût 
délicieux, alors que celles provenant des provinces de Satsuma, Chikuzen, Buzen et Bungo sont minuscules.

PI. 46 : L'holothurie est grillée et séchée

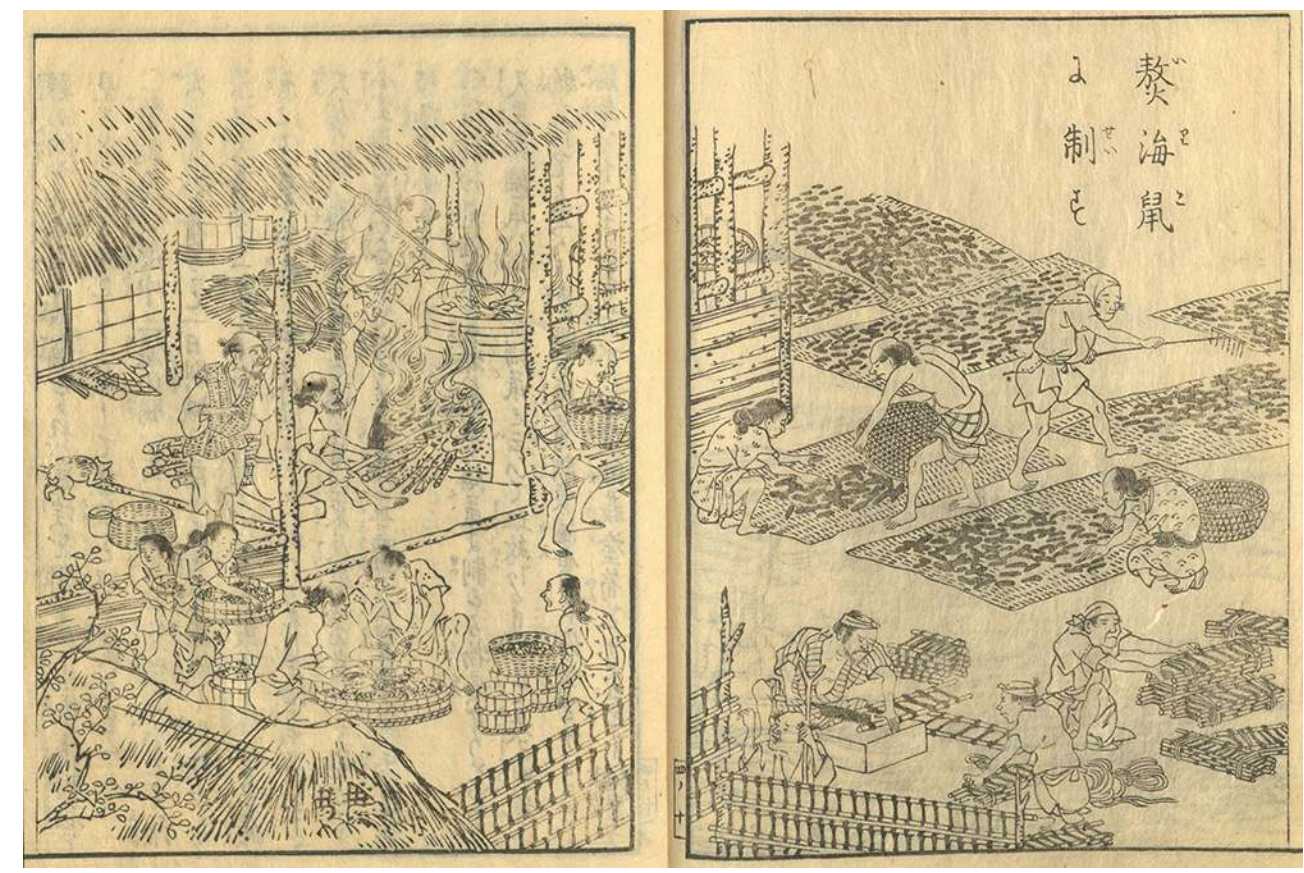

National Institute of Japanese Literature. Lien vers l'illustration

Selon le Wamyōshō, ce que l'on appelle hoya ${ }^{52}$ n'est autre que ce que l'on transforme en iriko. Par ailleurs, l'holothurie (namako) est communément appelée " holothurie tigrée » (torako) ; elle est tachetée, mais il paraît qu'il en existe d'autres espèces. Il est dit dans le Tōga : «dans le Kinmō zue (Encyclopédie illustrée) de Tekisai ${ }^{53}$, 沙僎 (shasun) ${ }^{54}$ est lu namako (holothurie), tandis que kaijin 海参 (" ginseng marin ») est lu iriko (holothurie rôtie et séchée). Pour Jakusui ${ }^{55}$, shasun (trépang), shasan 沙蒜 (anémone de mer) et tojun 塗荀 (siponcle) $)^{56}$ sont des namako, alors que kaidanshi ${ }^{57}$ et kaiso ${ }^{58}$ sont des iriko etc. » Mais dans tous ces cas, on ne sait pas ce qu'il en est vraiment. Cependant le kaidanshi, dont il est question dans le Wuzazu (Les cinq miscellanées) ${ }^{59}$, est nommé ainsi parce qu'il ressemble au membre viril.

\section{Les entrailles marinées (konowata)}

Le Honchō shokkan (Miroir des aliments du Japon) ${ }^{60}$ donne tawarago comme nom alternatif, mais à tort, semble-t-il. Tawarago est le nom de l'« holothurie tigrée ", séchée, et désigne l'holothurie en général ${ }^{61}$.

On retire les viscères, les rince des dizaines de fois à l'eau de mer claire, les fait macérer dans le sel et les laisse reposer. Celles qui sont jaunes, luisantes comme de l'ambre sont considérées comme de qualité supérieure, tandis que celles qui ont des tâches noires sont de qualité inférieure. Lorsqu'on expose au soleil un mélange aux trois couleurs et qu'on le remue fréquemment, elles finissent par devenir uniformément jaunes. On en rend la saveur plus délicate encore si l'on incorpore un jaune d'œuf par shō (près de 2 litres) d'entrailles. Jadis on pouvait même s'acquitter du tribut au moyen de ce produit, mais cela seulement dans les provinces de Noto, Owari et Mikawa, et nulle part 
ailleurs. Ceci en vertu de l'insigne rareté de leur produit, de couleur jaune pure. (Il existe une autre sorte dont les entrailles contiennent des parties orange de la couleur des algues nori et que l'on appelle «œufs d'holothurie » (konoko), mais le goût en est désagréable.)

\section{Oursin ${ }^{62}$}

\section{Autre nom : reiruishi}

L'oursin ${ }^{63}$ est le plus prisé des produits de mer macérés dans le sel. On en trouve dans certaines îles, mais ceux d'Echizen et de Satsuma sont les plus renommés [voir Pl. 47]. Sa carapace $^{64}$ est de forme arrondie comme une mandarine et il est hérissé de nombreux piquants qui le font ressembler à une bogue de châtaigne. Sur les grèves de Sumiyoshi, de Futami ou ailleurs, on en gratte les piquants afin d'en faire des jouets pour les enfants. Sa forme générale ressemble à un casque, et la bouche se trouve au beau milieu de la carapace. Il arrive qu'on en laque l'intérieur pour en faire un récipient. La chair ne remplit pas toute la carapace, elle est très peu abondante et grasse. Les pêcheurs la font macérer dans du sel et on la considère comme un excellent accompagnement du saké. On apprécie le plus ceux dont la chair est d'un jaune tirant sur le rouge. La production d'Ōmura, des îles Gotō et de Hirado est réputée. Ceux de couleur jaune violacée proviennent de Satsuma, domaine des Shimazu ${ }^{65}$. Leur chair est fondante et leur arôme incomparable. Les oursins d'Echizen sont glutineux et l'emportent sur tous les autres par leur éclat. Utilisés comme condiment à la place du miso, ils donnent aux mets un goût d'un grand raffinement. Il en est ainsi des oursins grillés ou des brochettes assaisonnées selon les préférences de chacun.

\section{PI. 47 : Oursins d'Echizen}

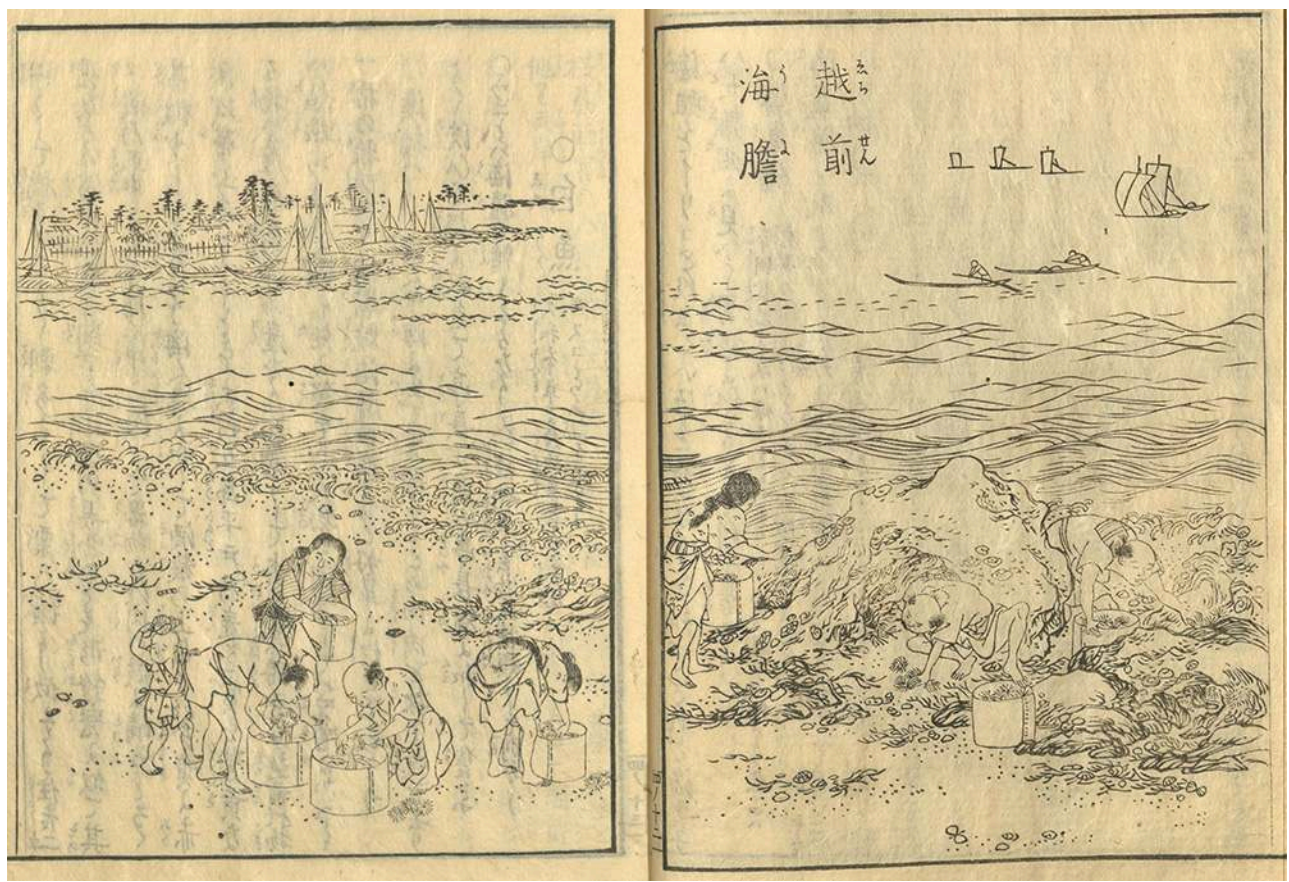

National Institute of Japanese Literature. Lien vers l'illustration 
gens de la mer se rendent sur la laisse et les ramassent entre les rochers. Ils les vident aussitôt de leur chair, jettent les coques, et les rincent soigneusement avant de les recueillir dans des baquets. Ils sont ensuite envoyés aux négociants qui les vendent après les avoir salés. Le nom uni dérive de un-i («bile de mer ») ${ }^{66}$. On trouve également la variété appelée « coquillage-casque »(kabutogai $)^{67}$, qui est de la même sorte sans être identique.

\section{Gobie (shiro'uo) ${ }^{68}$}

Le Yamato honzō (Materia medica du Japon) ${ }^{69}$ considère comme fausse la thèse selon laquelle kaisangyo 鱠残魚 ${ }^{70}$ serait le kisuko ${ }^{71} .^{72}$

Dans l'estuaire (de la Shukugawa) de Nishinomiya, dans la province de Settsu, vers la deuxième ou troisième lune, on dispose des deux côtés de la rive des huttes de paille en cinq points sur une centaine de mètres, à distance égale mais décalées les unes par rapport aux autres [voir Pl. 48] $]^{73}$.

\section{PI. 48 : Gobies de Nishinomiya}

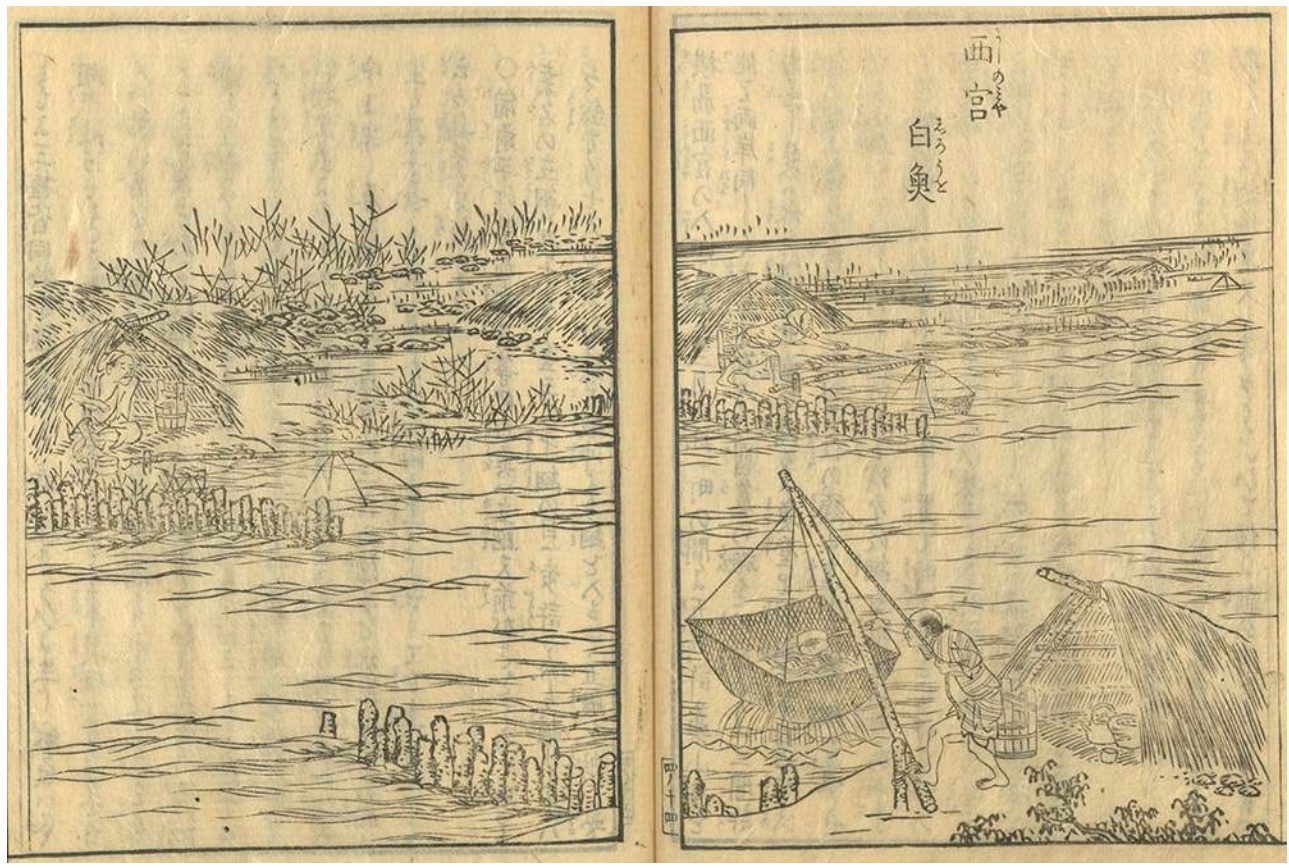

National Institute of Japanese Literature. Lien vers l'illustration

Puis on plante des pieux en forme de gord ${ }^{74}$ à partir des deux rives vers le milieu de la rivière sur une longueur d'une ou deux travées. On laisse l'eau inonder les gords et à marée haute les poissons remontent la rivière. Lorsqu'ils redescendent à marée basse, on attend qu'ils s'amassent derrière les gords et on (les prend) au carrelet. On lève et baisse celui-ci en maniant la corde qui passe par une petite roue fixée dans le trou aménagé à l'extrémité de la perche. Le carrelet, fait d'un tissu de moustiquaire, est laissé abaissé du côté des pieux où se rassemblent les poissons. On le remonte de temps en temps pour recueillir la prise avec une épuisette dont le fond est tendu de toile, comme on le voit sur l'image [Pl. 48]. Cela me rappelle cette façon de pêcher que l'on pratiquait avec les antiques «palissades » (ajiroki) plantées dans la rivière Uji. Celles-ci consistent en deux rangées de pieux enfoncés en angle fermé à la manière d'une nasse, 
de sorte que le pêcheur de garde n'a plus qu'à prendre au filet les " poissons de glace " $(\text { hio })^{75}$ retenus avec l'eau entre les palissades ${ }^{76}$. Le Man'yōshū chante le courant tumultueux de la rivière :

(De fiers guerriers

octante tribus) de l'Ujigawa

les vagues qui heurtent

les palissades du pêcheur

où vont-elles je ne sais ${ }^{77}$

Il est vrai que les gobies et les poissons de glace remontent en grand nombre de la mer vers la troisième lune. Doromesako ${ }^{78}$, avec ce que l'on appelle aussi shiro'uo et ce que l'on tient communément pour l'alevin de la truite ayu, sont trois variétés du même poisson qui naît au printemps dans les eaux saumâtres des estuaires, alors que le " poisson de glace " éclot en hiver dans les eaux troublées du lac Biwa, dans la rivière Uji ou à Tanakami ${ }^{79}$. Quant à dorome, c'est [un autre nom de] l'alevin de la truite $a y u^{80}$. Si [le nom de] cette dernière est " poisson annuel ${ }^{81}$, c'est parce que sa durée de vie est limitée à un an : en effet, les femelles qui commencent à se remplir d'œufs alors qu'elles vivent encore en amont, descendent l'automne venu vers la mer pour y frayer dans les eaux saumâtres ${ }^{82}$. C'est en quittant l'estuaire et en remontant la rivière, que les petits grandissent peu à peu. Dans l'estuaire de $\mathrm{Naniwa}^{83}$ on peut les pêcher pendant une dizaine de jours seulement. Par chirimen-zako et chirimen-koayu on entend le dorome salé et séché ${ }^{84}$, produit qu'on ne trouve pas dans l'Est ${ }^{85}$. Selon le Honchō shokkan, shiro'uo désigne le "poisson de glace» de grande taille ${ }^{86}$. Qu'il naisse dans l'estuaire d'une rivière, descende au printemps vers la mer et $\mathrm{y}$ fraye entre la deuxième et la troisième lune parmi les algues et les cailloux, que l'alevin en grandissant devienne " poisson de glace ", qu'il grandisse de plus belle en remontant l'estuaire et qu'il finisse ainsi par devenir shiro'uo : tout cela me semble relever d'une théorie bien douteuse.

Dans des endroits comme Hirae en Bizen et Kuwana en Ise $^{87}$, on prend les gobies au filet droit ou à l'haveneau. Le filet droit utilisé à Kuwana mesure sept toises de long et trois de haut, avec des mailles d'environ une ligne de côté, des baquets pour flotteurs et du plomb pour lest. Ailleurs, cinq embarcations avec chacune sept hommes à bord posent le filet au large puis le ramènent vers le rivage à la rame, reliées entre elles par des amarres. Avec ce filet qui mesure quelque cent brasses on prend environ 2 koku (env. 360 litres) de poissons, une quantité telle qu'on les vend au poids. Un autre produit typique de ce lieu est l'« enfilade ", une brochette de bambou sur laquelle on a enfilé un grand nombre de poissons par les yeux. Cette pêche est pratiquée dans des endroits comme Akasuga-Hamajizō ${ }^{88}$, Kametsu ${ }^{89}$, Fukusaki ${ }^{90}$, Toyota ${ }^{91}$ ou Isshiki ${ }^{92}$. Au milieu de cette portion de côte de 3 lieues, il y a un endroit dit Yokomakura, situé à la frontière entre les provinces d'Owari et d'Ise ${ }^{93}$. Tandis que du côté d'Owari il n'y a pas de gobies, du côté de Kuwana il n'y a pas d'huîtres. Et même si l'on en ramasse de temps en temps, le goût n'en est pas fameux. Les gens trouvent cela étonnant. À la réflexion il s'agit d'une de ces zones d'eau saumâtre dont on parlait plus haut. La mer d'Ise est à l'origine un estuaire et Kuwana et Fukushima sont situés à l'embouchure du fleuve. La rivière dont le cours inférieur se jette ici dans la mer, est appelée en amont la Kisogawa. Les poissons y fraient suivant le même principe qu'à Nishinomiya ${ }^{94}$.

Il y a ce qu'on appelle isaza, que l'on dit être l'alevin d'une espèce de sardine ${ }^{95}$. On connaît un poisson identique qui fraye dans l'eau de mer. Son autre nom est sanobori $i^{96}$ et on le pêche en hiver. On trouve encore un poisson similaire dans le Wakasa appelé amasagi $^{97}$ qu'on pêche entre le milieu de l'hiver et le début du printemps. Par ailleurs ce 
que l'on appelle shirouo dans le Chikuzen est un petit poisson long d'environ 1 pouce dont le ventre est marqué de sept points noirâtres. Ce nombre ne dépend pas de l'âge du poisson.

\section{Palourde grillée (yaki-hamaguri), palourde « averse d'automne » (shigure-hamaguri)} la province d'Ise ${ }^{99}$. On fait un feu de pommes de pin, sur lequel on grille les palourdes, le crochet vers le bas pour que le ligament se détache de la coquille. C'est un mets délicieux.

Ce qu'on appelle palourde "averse d'automne " (shigure-hamaguri) se cuisine comme suit : on mélange le bouillon des coquillages à la saumure qui flotte à la surface dans la cuve où l'on a mis les boules de miso à fermenter, on assaisonne de poivre du Japon (sanshō $^{100}$, d'oreilles de Judas ${ }^{101}$, de gingembre, etc., et on y cuit la chair jusqu'à réduction complète. Ce produit supporte de longs voyages sans s'abimer. Quant au miso fermenté, il se fabrique selon la méthode suivante : on fait cuire longtemps des graines de soja, les enveloppe dans de la paille et les suspend au-dessus du foyer pendant un mois. Puis on les pile dans un mortier, les sale et ajoute de l'eau. Le liquide qui se forme à la surface, on l'utilise comme du shōyu, tandis que ce qui se dépose au fond est le miso. (Si l'on cuit le poisson avec du shōyu, même s'il est un peu avarié, il retrouve toute sa saveur et le goût en est excellent. Aujourd'hui encore on en sert couramment dans les relais officiels.)

\section{Gobies (gori 鮴) ${ }^{102}$}

Ce caractère ne figure pas dans les dictionnaires. J'adopte ici le caractère en usage dans notre pays. Autre nom : 鮻 ishifushi

C'est un produit renommé de la rivière Kamo en Yamashiro. Le Yamato honzō en distingue deux espèces. L'une possède des nageoires rondes sur le ventre ${ }^{103}$. Ces nageoires ont une partie plate qui permet au poisson de s'accrocher aux rochers. Il s'agit du vrai gobie. C'est un poisson gras, délicieux en consommé. Il ressemble au chabot $^{104}$ mais en plus petit et porte sur le dos des marques noires et blanches. La thèse du maître Kaibara pour qui ishifushi ${ }^{105}$ est un autre nom de ce poisson, n'est pas justifiée. Il s'agit effectivement d'un poisson de la même espèce, mais il en diffère tout de même quelque peu par sa forme, comme cela ressort de l'illustration ci-dessous ${ }^{106}$.

31 Voici comment on les pêche [voir Pl. 49] : on coud deux nattes de paille, les immerge dans un gué et les leste d'un grand nombre de petits cailloux. Deux hommes se mettent d'un côté et soulèvent les nattes par les deux coins, tandis qu'un troisième, de l'autre côté, remonte vers ces nattes en ratissant le fond de la rivière avec un racloir en bois d'environ 3 pieds de large, poussant ainsi les poissons devant lui. Chassés de la sorte, les poissons vont se réfugier dans les galets posés sur les nattes, et on les attrape ainsi en soulevant le tout hors de l'eau. C'est ce qu'on appelle « pousser le gobie » (gori-oshi). 


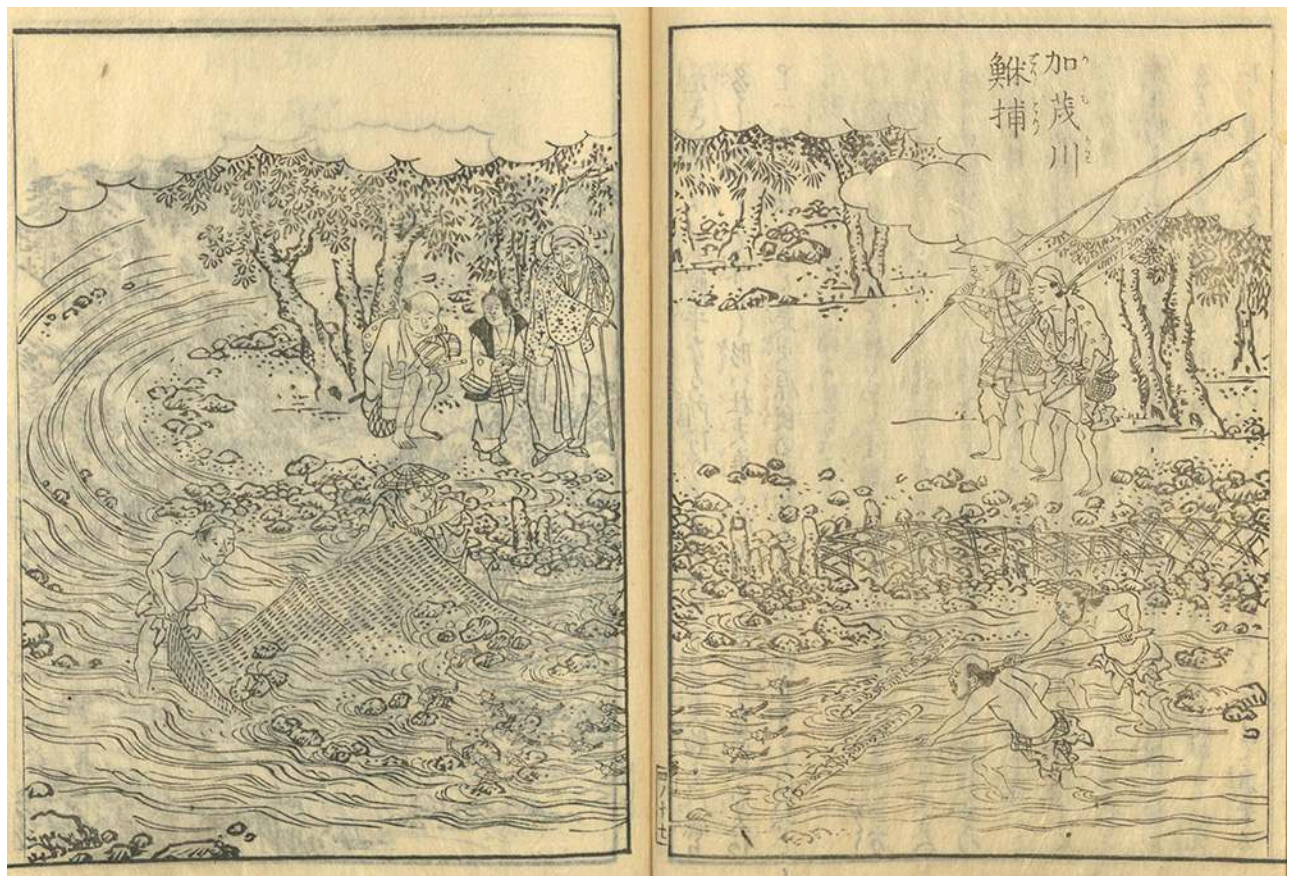

National Institute of Japanese Literature. Lien vers l'illustration

Les gobies de la rivière Asano en Kaga sont eux aussi renommés [voir Pl. 50] [voir aussi Pl.51]. On les pêche selon le même procédé que dans la rivière Kamo, la seule différence résidant dans ce que l'on nomme ici futtai et ita-oshiki ("plateau de planche »107. Le futtai est quelque chose comme un grand van fait de lames de bambou qu'on utilise à la place des nattes de la Kamogawa, alors que le plateau est constitué d'une épaisse planche d'environ 5 pieds de haut sur 3 pieds de large, prise entre des perches de bambou et pourvue à sa base d'un trou servant d'appui-pied. On y passe le pied, on saisit les perches de bambou par la partie qui dépasse, et on racle le fond parsemé de pierres, poussant ainsi les poissons selon le procédé vu précédemment. 


\section{PI. 50 : Pêche aux gobies dans la rivière Asano en Kaga}

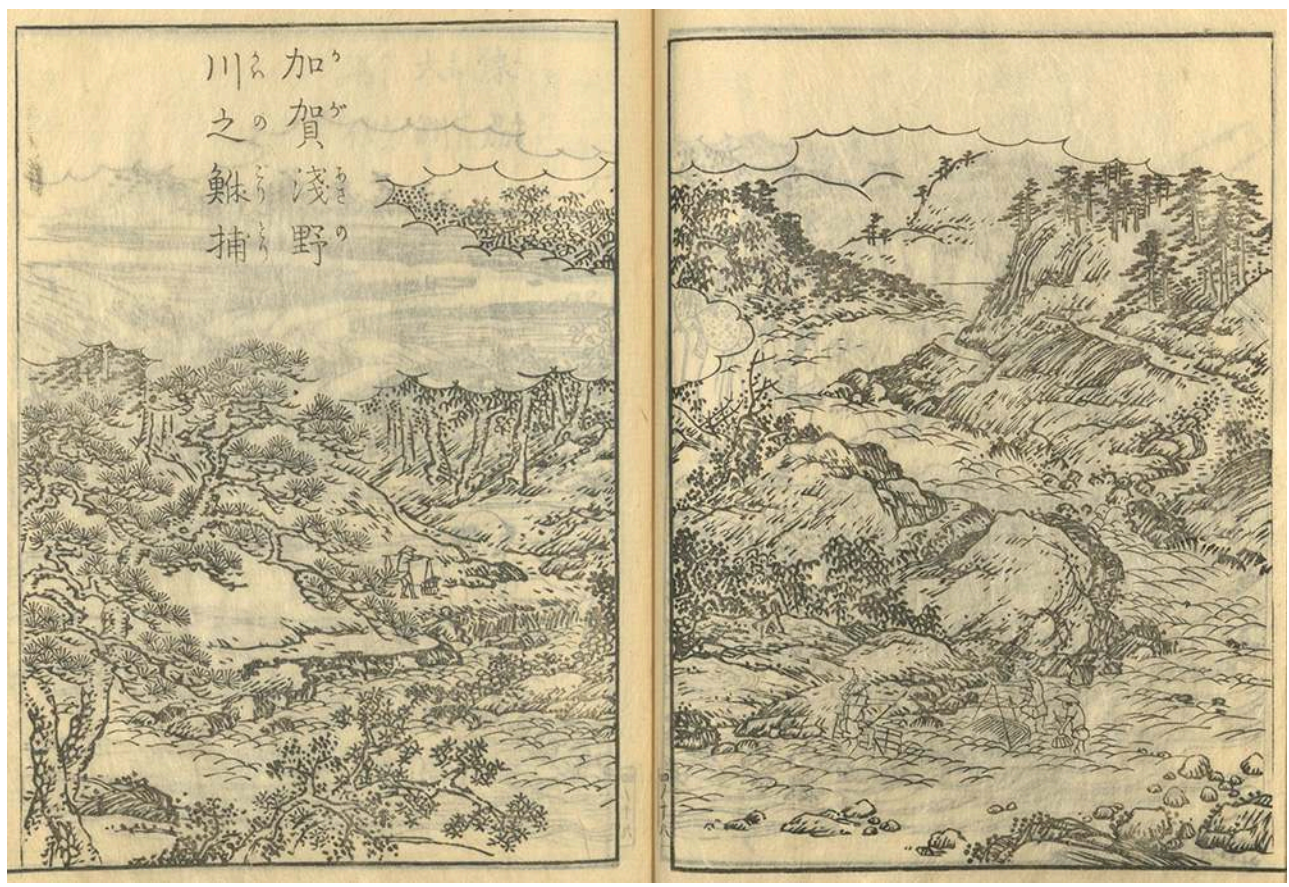

National Institute of Japanese Literature. Lien vers l'illustration

\section{PI. 51 : « Accroche-pierre » d'Ōzu en lyo}

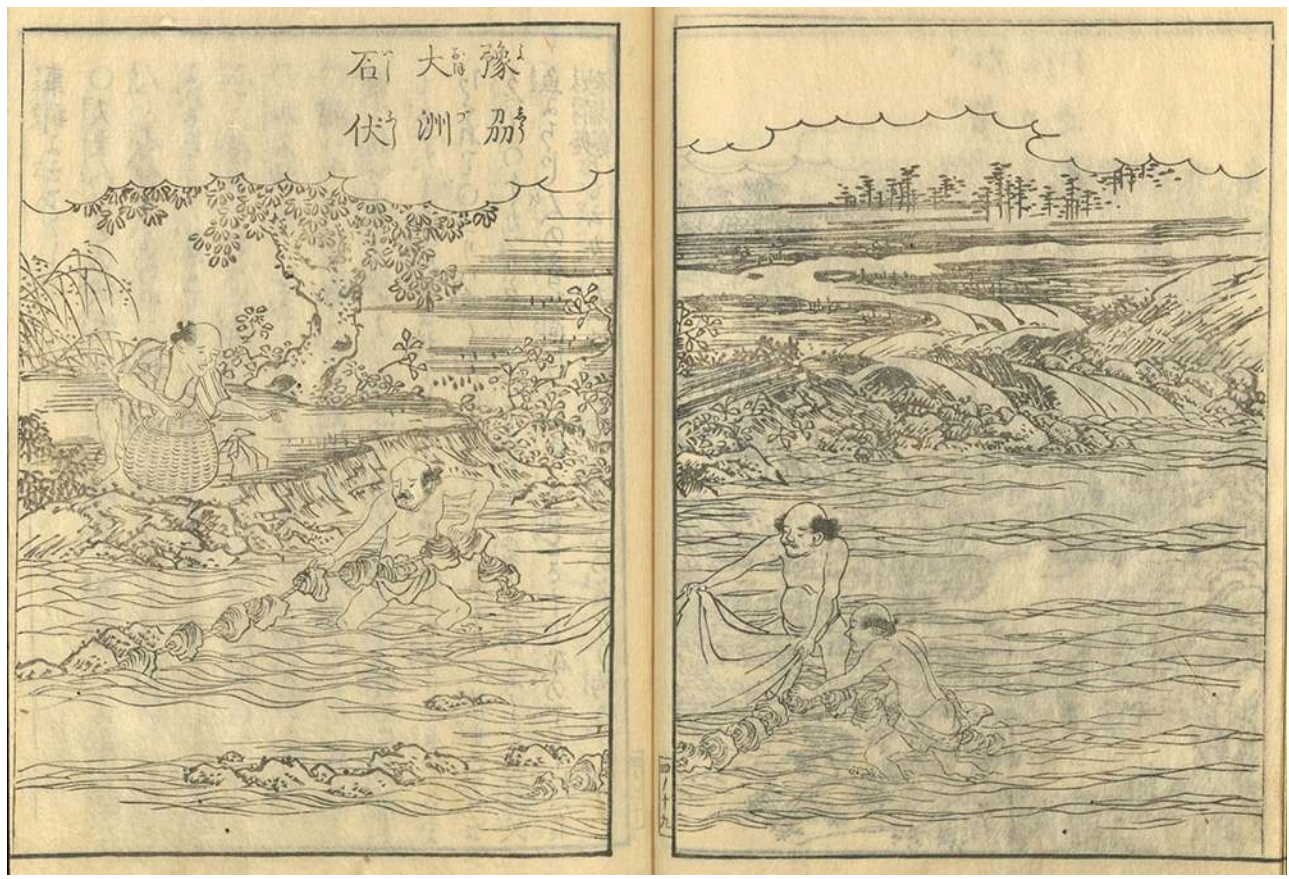

National Institute of Japanese Literature. Lien vers l'illustration

33 Par ailleurs, lorsque les villageois flânent le long de la rivière pour prendre le frais, ils pêchent ces poissons en plongeant dans l'eau leurs deux mains jointes dans lesquelles ils tiennent un appât odorant et en les appelant par leur nom "gori », les poissons s'attroupent et viennent dans le creux des mains. On peut aussi les attraper avec un 
panier. Toutefois ce n'est pas chose facile pour quelqu'un d'inexpérimenté. Même dans l'eau limpide et peu profonde, c'est un poisson difficile à distinguer. ressemble à l'ishifushi et qu'il a des piquants sur les joues. Mais à mon avis, on ne peut suivre à la lettre ce qu'expriment ces caractères chinois. Si l'on suit le sens de la lecture japonaise, ishifushi («se cache dans la pierre ») correspond au poisson qu'on appelle maintenant gori-ishifushi" ${ }^{113}$. Quant à gori, cela doit être une appellation récente venant du cri «gorigori » qu'émet ce poisson ${ }^{114}$. Chichi dans chichikaburi veut dire «terre » (tsuchi), et le sens du mot est « couvert de terre ». En cela il se rapproche du chabot (tofugyo). Lorsqu'il entend des voix humaines, il enfonce sa tête dans le sable telle une ancre. [C'est pour cela qu'] on l'appelle aussi "gobie creuseur de sable »115. Le nom de karakako vient du fait que ce poisson a des piquants ( $k \overline{0}$ 鉋) sur les joues, car ce mot kako est un ancien mot pour «piquant ». Le sens de kara est obscur. On dit encore que les poissons qui émettent un cri clignent immanquablement des yeux. C'est considéré comme une chose étonnante. On ne sait toutefois ce qu'il en est vraiment. Tous ces poissons sont parfois appelés kajika ${ }^{116}$ dans les parlers locaux. Il y a à ce sujet une explication. Nous verrons ce qu'il en est dans l'article consacré à ce poisson plus loin.

\section{Truite (masu) $)^{117}$}

On distingue la truite de rivière et la truite de mer. La truite de rivière a meilleur goût. On la trouve dans des régions comme Etchū, Echigo, Hida, Mutsu, Hitachi, mais la plus renommée serait celle de la rivière Shintō ${ }^{118}$ en Etchū. Elle nous arrive conservée dans le sel. Elle ressemble au saumon et vit dans les mêmes endroits. Ses écailles sont menues, ses yeux striés de veines rouges et sa chair est pleine de fines arêtes. Pour la pêcher, on se sert de ce que l'on appelle un "filet de rivière manœuvré par bateau » (nori-kawaami) ${ }^{119}$, une senne à poche de 7 ou 8 pieds de large et de 5 brasses de long, munie de flotteurs en haut et de lest en bas, espacés de 4 pouces à peine [voir Pl. 52]. Entre les flotteurs restant à la surface et le lest coulant au fond, le filet est maintenu ouvert. On fixe de longues perches de bambou aux deux extrémités du filet en laissant dépasser les bouts des perches. Deux petites barques montées par deux hommes chacune enserrent le filet des deux côtés. On attend que le poisson y entre puis on le soulève prestement tout en l'enroulant par les deux côtés. On peut capturer ainsi un, voire deux ou trois poissons. Comme les truites remontent la rivière à contre-courant, les tireurs assistent les pêcheurs en ramant à contre-courant. 


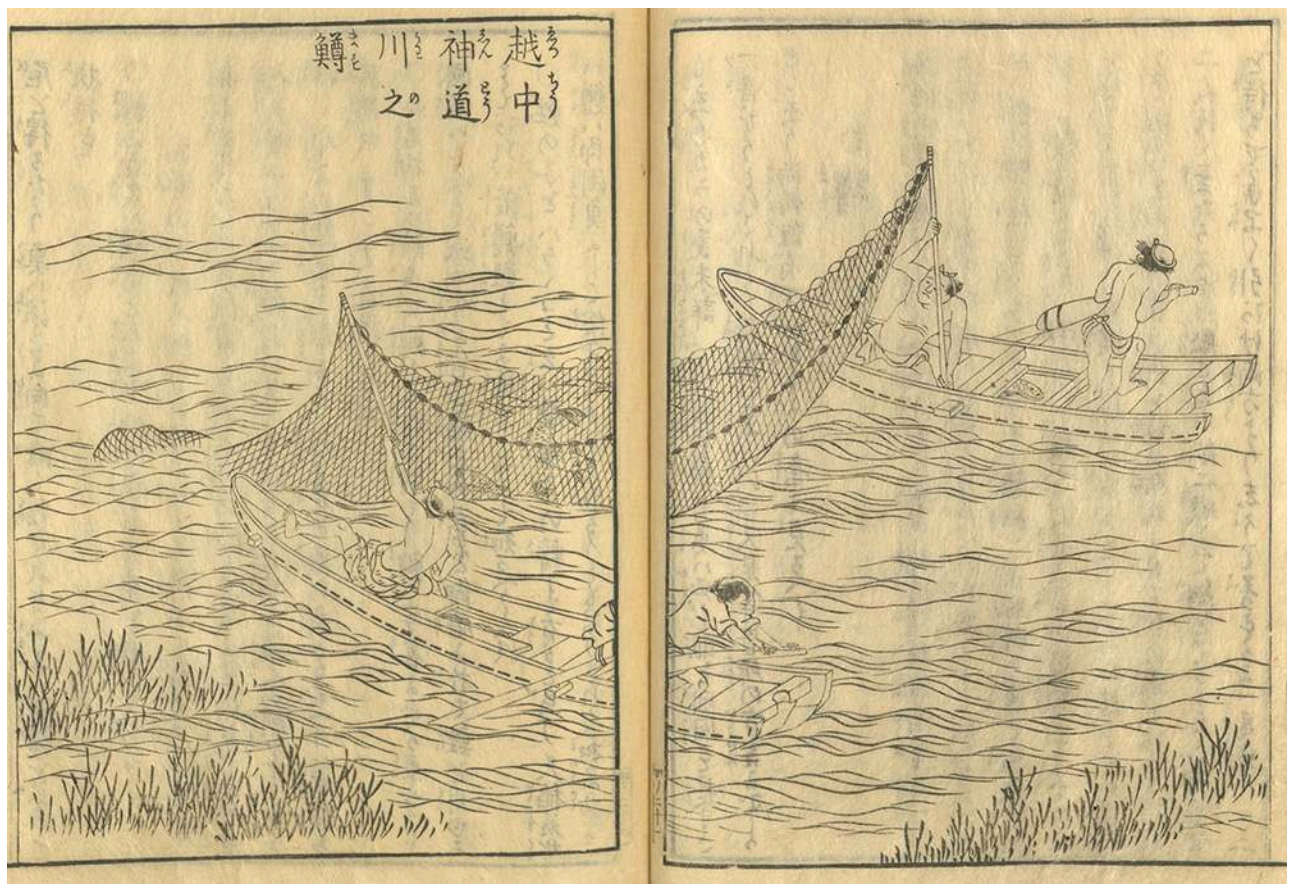

National Institute of Japanese Literature. Lien vers l'illustration

L'ancien nom de la truite est «ventre rouge $»^{120}$. On la présente en offrande lors des rites saisonniers. Comme le dit le poème :

Au début du printemps

les truites au ventre rouge pêchées sur la longue grève

de Nagahama,

présage d'un règne de mille générations,

sont pour mon souverain

Chaque année, le jour de l'an, on l'offre en tribut à l'empereur. Lorsque la livraison n'arrive pas à temps, on la présente le septième jour. Le Nihongi (Chronique du Japon) ${ }^{121}$ rapporte qu'en l'an 18 du règne de l'empereur Keiko ${ }^{122}$, au moment où il passait par le village de Tamakina, des pêcheurs lui avaient offert des truites. Depuis ce précédent, on n'a jamais cessé de faire cette offrande à l'empereur. De ce fait, on parle de la truite comme d'un poisson d'offrande. Quant à Nagahama, ce lieu se situe dans le district du même nom et s'appelle aussi Nagasu ${ }^{123}$.

Le Wamyōshō, cependant, distingue haraka 鮀魚 ${ }^{124}$ et la truite. L'idéogramme 鮀 ne figure pas dans les dictionnaires de caractères chinois et doit être propre à notre pays. Mais on peut l'expliquer autrement: dans la mesure où, aujourd'hui, au Nouvel An, il arrive que les cuisines impériales préparent cette offrande avec du saumon, il n'est pas impossible que le nom de haraka désigne aussi bien le saumon que la truite.

Quant au nom de hararago que l'on donne aux œufs de saumon, il se pourrait bien que ce soit là une déformation de haraakago ${ }^{125}$. Par ailleurs, selon Tō Jakusui ${ }^{126}$, la truite est un " poisson qui vit dans les creux des rivières ${ }^{127}$ ", et elle est appelée communément oikawa $^{128}$. De fait, n'est-ce pas pour cette raison que le Wamyōshō fait une distinction entre les deux poissons haraka et masu ${ }^{129}$ ? Les avis sont partagés et il est difficile de savoir ce qu'il en est. Il y a encore matière à réflexion. Le oikawa est un poisson au ventre rouge ${ }^{130}$. 


\section{Lamproie (yatsume-unagi) ${ }^{131}$}

La lamproie vit aussi bien dans la mer que dans les rivières. Celle pêchée dans la « mer de Suwa $»^{132}$, dans la province de Shinano, est un produit particulièrement renommé ${ }^{133}$. Sur 1 lieue environ, entre Kami-Suwa et Shimo-Suwa, le lac gèle complètement en hiver, la glace atteignant généralement une épaisseur de 2 à 3 pieds. Au plus fort des grands froids, sur toute cette distance d'1 lieue, apparaissent dans la glace d'étranges traces qui dessinent un chemin. On appelle cela le "passage des dieux ». Il marque le début de leurs allers et venues ${ }^{134}$. C'est à ce moment-là que s'ouvre la pêche de la lamproie. On commence par ériger des huttes sur la glace [voir Pl. 53]. Voici comment on procède : on perce des trous dans la glace en allumant des feux, pour y planter les piliers des abris destinés au repos des pêcheurs. On estime l'emplacement des ouvertures à pratiquer dans la glace pour y introduire un filet ou une corde. On fait aussi des feux de bois pour percer ici et là des brèches, puis on y plonge la palangre garnie d'appâts. On pêche ainsi d'énormes quantités de poissons. Lorsque le lac ne gèle pas, on se sert de gaffes à anguille. En outre, on trouve dans ce lac beaucoup de " poissons de roche tachetés ${ }^{135}$ que l'on appelle aussi « poissons rouges » (aka'uo) ou encore "ventres rouges» (akahara). On les pêche avec un filet que l'on attache à une perche de bambou et qu'on introduit par un trou dans la glace pour le faire ressortir plus loin par un autre.

PI. 53 : Pêche à la lamproie et à la vandoise dans le lac Suwa

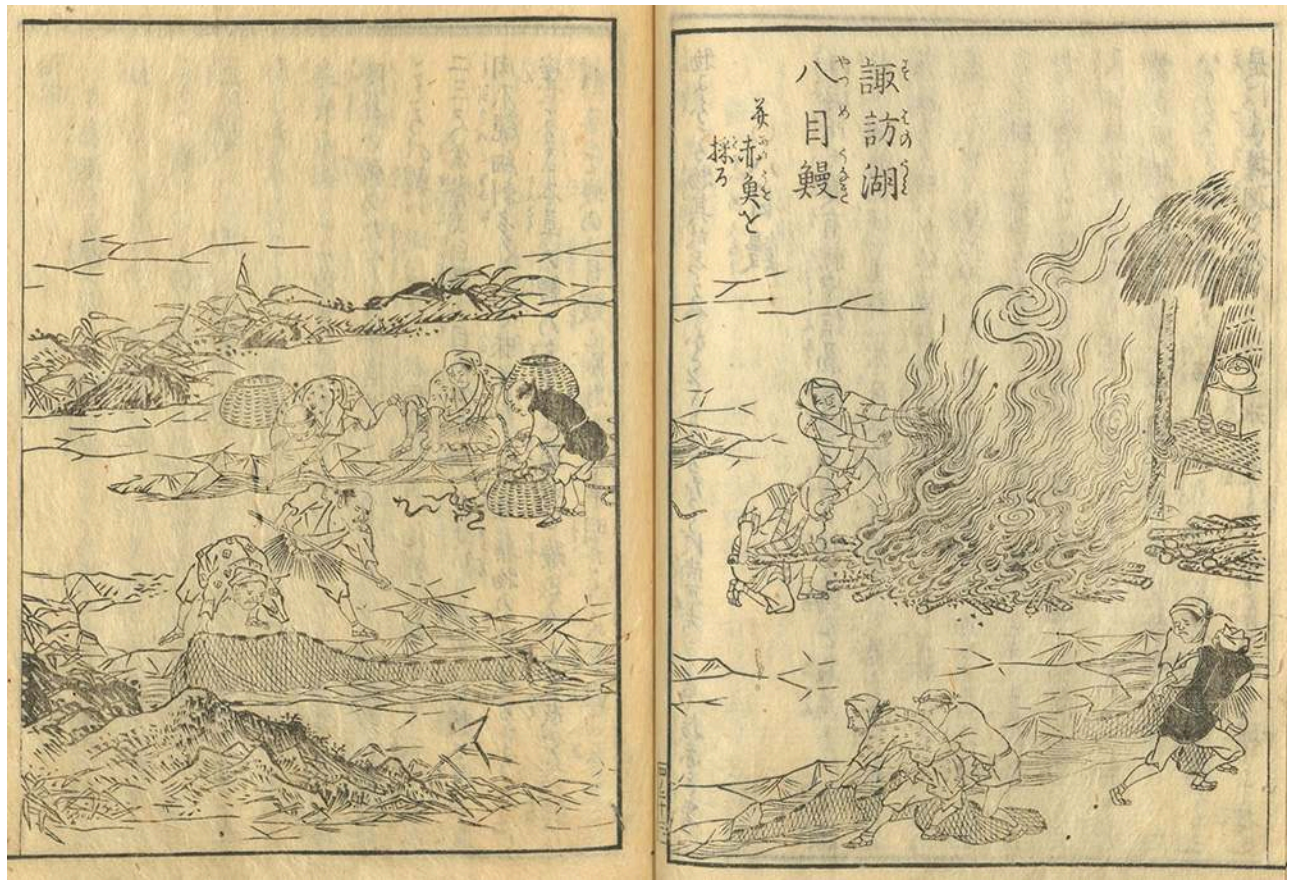

National Institute of Japanese Literature. Lien vers l'illustration

\section{Addenda}

[Certains,] en suivant le Bencao gangmu (Classement raisonné de la materia medica) ${ }^{136}$ qui dit du poisson rei 鱧 ${ }^{137} q u$ 'il a sept points à côté de ses yeux, donnent ce nom au poisson 
dont il est question ici ${ }^{138}$. D'autres disent que le rei, en provenance de Chine, est un autre nom pour « carpe noire » (kokurigyo), qui, elle, ressemblerait au mulet (bora) ${ }^{139} \mathrm{en}$ plus petit, et aurait de grandes écailles et sept points à côté de l'œil. Elle serait de couleur noire violacée avec des taches d'un noir profond. Les Chinois fréquentant Nagasaki l'appellent « poisson à neuf points ", même s'il n'en a que sept. On ne connaît pas ce rei dans les eaux japonaises. Maître Joan ${ }^{140}$ se trompe en l'identifiant à notre lamproie.

Dans la région du Nanbu ${ }^{141}$ on a pêché ces derniers temps une espèce de poisson avec sept "points» sur la tête que les gens du pays appellent "poisson à sept points » (shichiseigyo). Toutefois, on ignore si c'est le même genre de poisson que le rei dont on parle ici. La question ne serait pas encore élucidée. Le Honchō shokkan dit au sujet du rei que c'est un poisson très visqueux, qu'il ressemble plus ou moins à l'anguille ou à l'« anguille de mer " ${ }^{142}$, qu'il peut mesurer jusqu'à 2 ou 3 pieds de long et qu'il possède sur le dos neuf points blancs semblables à des yeux. C'est la raison pour laquelle on le nommerait "anguille à huit yeux" ${ }^{143}$. Sa chair, pleine de fines arêtes, manque de fondant et n'a pas bon goût. [Toujours d'après cette encyclopédie,] c'est un poisson que l'on ne pêche que pour son usage dans la pharmacopée ${ }^{144}$. À mon avis, si, dans l'article à propos de rei, le Bencao gangmu n'indique pas qu'il soigne les convulsions, c'est bien la preuve qu'il s'agit d'un autre poisson que la lamproie qui est notre sujet ici. Par ailleurs, le Honchō shokkan tient clairement la lamproie pour un remède aux convulsions ${ }^{145}$, c'est donc que l'identifier au rei est une erreur. Quoi qu'il en soit, il suffirait qu'il soit établi que la lamproie d'aujourd'hui ait des vertus curatives contre les convulsions pour que la discussion sur les dénominations chinoises devienne sans objet.

\section{Pieuvre (tako)}

Autres noms "poisson à huit branches " (hasshōgyo) ou encore "bonze de la mer" (kai'oshō) ; le caractère communément utilisé est 鮹 qui semble résulter de l'assemblage de deux éléments : [le caractère du " poisson » 魚, pour indiquer le genre, et] celui de la «branche» 梢, pour sa prononciation $[s h \bar{o}]^{146}$

On en trouve dans beaucoup de provinces. Elle est particulièrement abondante dans la baie d'Akashi en Harima où l'on a coutume de la pêcher avec deux ou trois pots en terre cuite ceints avec des cordes et jetés à l'eau, dans lesquels la pieuvre vient se loger d'elle-même ${ }^{147}$. On appelle ce récipient en terre cuite un "pot à pieuvre ", mais dans la ville les gens s'en servent aussi comme pot de fleurs. La pieuvre s'accroche à l'intérieur et ne s'en laisse pas facilement déloger. Mais si on racle le dos du fond avec un objet quelconque, elle sort toute seule et on peut rapidement l'en détacher. À Nagahama en Iyo, ce poisson est si foisonnant qu'on le vend au marché sous forme séchée ${ }^{148}$. On le pêche ici avec ce qu'on appelle le suichō, et un seul homme peut en prendre cinq ou six cents, un bateau jusqu'à mille ou deux mille [voir Pl. 54]. Quant à ce suichō, il s'agit d'une planchette de 4 sur 6 pouces environ, sur la surface de laquelle on a fixé deux hameçons à l'extrémité et un crabe à mitaines ${ }^{149}$ auquel on a retiré la carapace en lui laissant les pinces; on la leste d'un galet ficelé en deux points avec du chanvre et la lance dans l'eau attachée à trois filins de chanvre de quarante à cinquante brasses environ de long. Lorsqu'on sent que la pieuvre avide de chair de crabe grimpe sur la planchette, on la ramène. Arrivé près du rivage ou à la surface, la pieuvre est déconcertée et veut se sauver, mais finit par se prendre dans les hameçons. En Izumi, pour pêcher les petites pieuvres avec ce même dispositif, on se sert d'os de seiche ou de 
fleurs de sarrasin comme appât. Près d'Akamagaseki dans le Chōshü ${ }^{150}$, on allume à l'arrière du bateau des torchères au-dessous desquelles beaucoup de pieuvres viennent s'attrouper, leur tête ondulant à la surface. Les pêcheurs les prennent à la main, et là où elles sont hors de portée ils se servent d'une gaffe. L'adresse avec laquelle ils les attrapent est proprement étonnante.

\section{PI. 54 : Pieuvres de Nagahama en lyo}

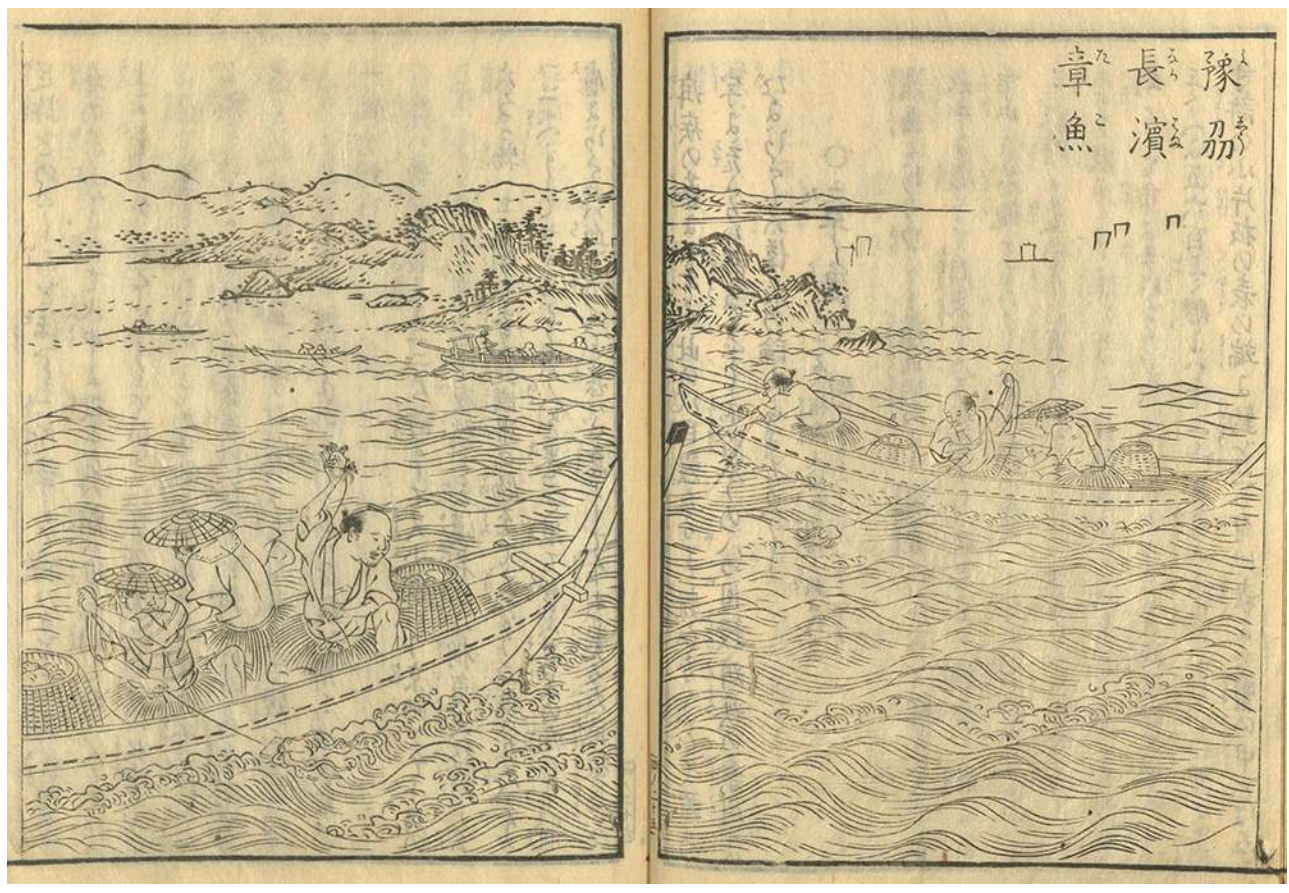

National Institute of Japanese Literature. Lien vers l'illustration

La pieuvre commune mesure entre 1 et 2 pieds environ, mais il existe une sorte plus petite. Celles que l'on vend en quantité sur le marché de la capitale aux alentours de la dixième lune portent le nom de "pieuvres des dix nuits $»^{151}$. Son nom chinois est shōhasshōgyo ("poisson à huit petites branches») 152 ou rakutei ("pieds qui se tortillent $»)^{153}$. La pieuvre de grande taille se nomme sekidako ${ }^{154}$. Quant à celles que l'on pêche dans les régions du Nord, elles sont énormes. Elles mesurent en général de 8 ou 9 pieds, jusqu'à 10 voire 20 pieds de long; il n'est pas rare qu'elles attrapent un homme en s'y enroulant et le dévorent. Si elles arrivent à fixer une ventouse de leurs tentacules sur la peau de quelqu'un, elles le vident de son sang avec une grande rapidité et il en succombe en quelques instants. De la même manière elles s'en prennent aussi aux chiens, rats, singes ou chevaux. La nuit, elles gagnent le rivage, relèvent leur ventre, dressent leur tête avec des yeux furibonds, se déplacent sur leurs huit tentacules en courant comme si elles volaient, envahissent les champs, y arrachent des patates et les mangent. Même le jour, elles se comportent de cette façon lorsqu'il n'y a personne. Il arrive, dit-on, qu'un paysan qui en est témoin, en abatte avec une gaule et s'en empare ${ }^{155}$. Le Yamato honzō rapporte qu'une grande pieuvre de Tajima lutta avec un énorme serpent enroulé autour d'une branche de pin et que pour finir elle le tira dans la mer avec la branche ${ }^{156}$.

Dans le fief de Toyama en Etchū, les pieuvres de Namerikawa s'emparent elles aussi de bétail et de chevaux pour les manger, font chavirer les bateaux de pêche et capturent 
l'équipage. Les pêcheurs n'ont aucun moyen pour les attraper. Pour cette raison ils feignent de dormir et attendent que la pieuvre, guettant l'occasion, s'approche, projette ses tentacules et grimpe sur l'embarcation; ils les lui coupent alors sur-lechamp avec une hallebarde, puis s'en retournent à toute vitesse à la rame ${ }^{157}$. Le danger est tel que de ce bref instant dépend la vie ou la mort. C'est véritablement comme le jeune valeureux qui, alors qu'il se rend sur le champ de bataille, fait aussi peu de cas de sa vie que de la poussière et pour qui la morale ne peut être qu'une affaire de loyauté et d'honneur, ou qui y va pour débarrasser ce monde de la violence. En comparaison avec ceux qui meurent à cause d'un seul tentacule de pieuvre, Ji Xin et Yoshiteru ${ }^{158}$ n'ont guère subi un sort pitoyable car ils ont péri sur le champ d'honneur.

Pendus à l'auvent des boutiques du marché, les tentacules de ces pieuvres géantes sont si longs qu'ils traînent par terre [voir Pl.55]. Une seule de ces ventouses suffit pour constituer le repas d'une journée. Les autres espèces de pieuvre étant bien connues, on n'en dira rien ici.

\section{PI. 55 : Pieuvres géantes de Namerikawa en Etchū}

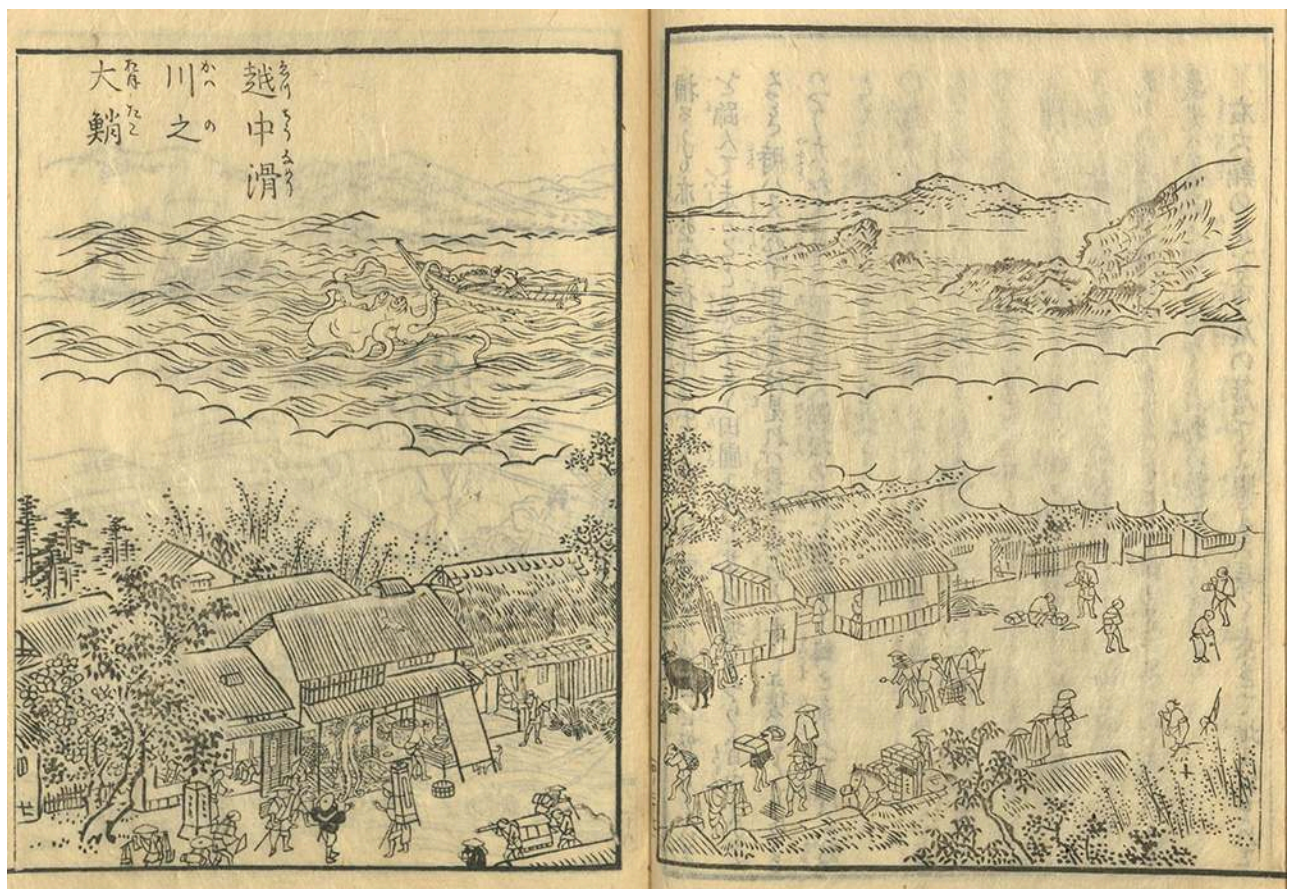

National Institute of Japanese Literature. Lien vers l'illustration

On appelle heriko les œufs accrochés en grappes aux roches, reliés par dizaines de milliers. Confits dans le sel, on les appelle « glycines de mer » (kaitōka) ${ }^{159}$.

La pieuvre doit son nom (tako) au grand nombre de ses tentacules. Ta- signifie «bras » 手 $(t e)$ tandis que -ko signifie «enfant» 子 $(k o)$ parce qu'elle a la tête lisse comme un nourrisson.

\section{Poulpe ocellé (iidako 飯蛸)}

Le nom chinois en est bōchōgyo 望潮魚 ${ }^{160}$

51 Il abonde dans les provinces de Settsu, Izumi, Kii et Harima. Celui de Takasago en Harima est un produit particulièrement renommé. C'est une espèce de pieuvre qui ne 
dépasse guère 3 ou 4 pouces. Ses entrailles sont remplies de ce qui ressemble à des grains de riz blanc cuit. D'après le Honchō shokkan, on n'en a jamais vu à l'est d'ōmi ${ }^{161}$, mais on en trouverait de temps en temps en Awa et Kazusa ${ }^{162}$, sans savoir toutefois s'il en est vraiment ainsi ${ }^{163}$.

Quant à la façon de le pêcher, on attache tout au long d'un câble de sept à huit travées de long un grand nombre de cordes plus fines, longues d'une brasse environ, au bout desquelles on attache une coquille d'escargot de mer Noire ${ }^{164}$ et on l'immerge dans l'eau [voir Pl. 56]. Quand la marée descendante provoque des remous, les poulpes cherchent des trous au fond de l'eau pour s'y réfugier et se cachent alors dans ces coquilles. Lorsqu'on remonte le câble et que les coquilles bougent, ils s'y retranchent davantage encore. Aussi ne faut-il pas tarder à ramener prestement le câble.

\section{PI. 56 : Poulpe ocellé de Takasago}

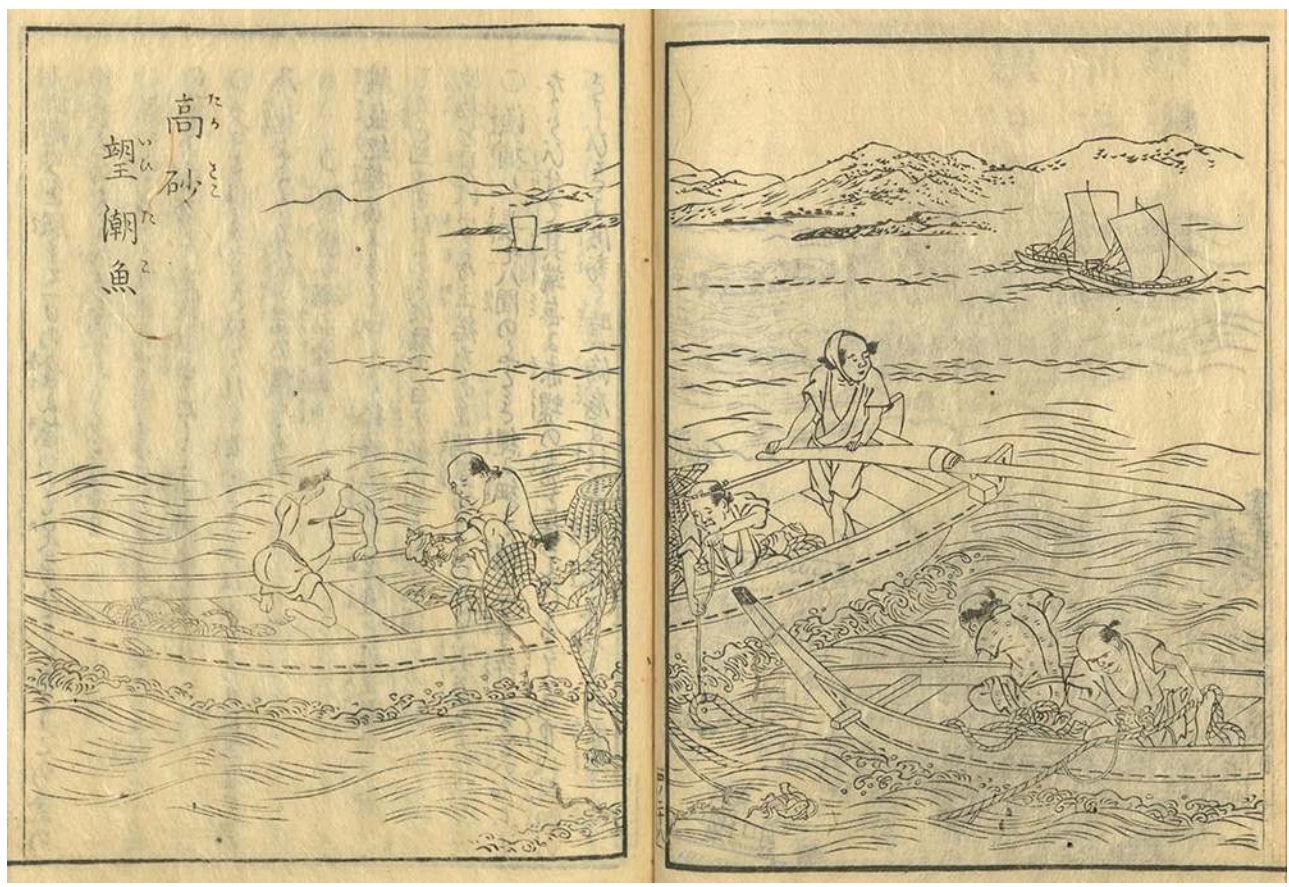

National Institute of Japanese Literature. Lien vers l'illustration

\section{Kajika}

Il y a quantité d'animaux dénommés kajika dans les provinces. Ici il désigne un poisson ${ }^{165}$, là une grenouille ${ }^{166}$, et si le terme est maintenant d'emploi courant, il n'est attesté ni dans les poèmes classiques ni dans les romans anciens. Seul le Onko jitsuroku (Notes journalières de choses anciennes actualisées) ${ }^{167}$, répertoire des termes de saison utilisés dans la poésie en chaîne, donne la lecture kashika pour le poisson appelé tofugyo ${ }^{168}$, sans plus de détail. On voit seulement qu'il figure dans la section de la " Huitième lune $~^{169}$. À part cela, d'autres répertoires de mots de saison utilisés dans la "poésie en chaîne plaisante» (haikai) ont paru ces derniers temps avec des commentaires qui identifient kajika avec les gori ou les ishifushi ${ }^{170}$ en se fondant sur des ouvrages populaires comme le Sansai zue (Encyclopédie illustrée des trois règnes) ${ }^{171}$, mais sans se référer aux écrits ou aux romans anciens. En outre, maître Kaibara écrit dans l'entrée « chabot » (tofugyo) de son Yamato honzō qu'il s'agit du kajika que chantent 
les poèmes anciens, mais c'est une grossière erreur. À mon avis, le nom de kajika a commencé à être employé de manière fantaisiste par les maîtres du haikai et s'est répandu dans les années Kan'ei (1624-1644) ; on l'a introduit dans des poèmes de Saigyō (1118-1190), ce qui a sans doute fini par l'imposer. Ainsi raconte-t-on que celui-ci aurait composé le poème suivant lorsqu'il habitait à Sarashina :

Près du torrent de montagne

voilà que j'ai senti

flux et reflux de la marée

dans la bise de l'automne

se lamente le kajika

Je ne sais pas de quel ouvrage provient ce poème, mais lorsque j'ai interrogé une certaine personne sur le sens de ces vers, elle m'a répondu : «Quand la marée est haute, les kajika s'orientent vers l'amont en criant gi-gi, quand elle est basse ils se tournent vers l'aval avec des cris de gori-gori ». Selon cette explication, le terme kajika désignerait un poisson nommé gori ou gigi ${ }^{172}$, mais il ne s'agit en rien d'une preuve fondée sur des écrits anciens. En fait d'animaux vivant dans l'eau et poussant des cris, je ne connais pas d'exemples chantés par les poètes depuis les temps anciens autres que les grenouilles et les oiseaux aquatiques. Comme Saigyō était quelqu'un qui composait des poèmes en toute liberté, il ne sert à rien de discuter des réalités qui ont pu l'inspirer. Cependant, il est probable que ce soit une fausse attribution. Par ailleurs, il est un poème que l'on prétend tiré du Man'yōshū (selon d'autres ce serait un poème composé à ōhara par l'impératrice entrée en religion Kenreimon'in au sujet de la cascade d'Ochiai) :

Gravier du torrent

roulant korokoro

comme le cri du kajika

à Ochiai dans la vallée

Mais là encore, il n'y a rien de tel dans le Man'yōshū. Je n'ai pas non plus entendu dire qu'il figure dans un autre ouvrage. On trouve encore, dans le Fubokushū (Sélection de poésie japonaise) $)^{173}$, fascicule 24 , "Poèmes divers", Section $6^{174}$, cette composition attribuée à l'empereur d'Okamoto ${ }^{175}$ :

Sur la route d'Ōmi / «En chemin à ta rencontre »

au mont de Toko / « au mont du lit »

rivière Isaya / " allons, venez ! "

korokoro / « des jours et des jours encore »

me faudra-t-il languir?

La tradition veut que ce korokoro / "des jours et des jours ", évoque le cri du kajika. C'est là encore une erreur grossière. Ceci correspond en fait au poème du fascicule $4 \mathrm{du}$ Man'yōshū :

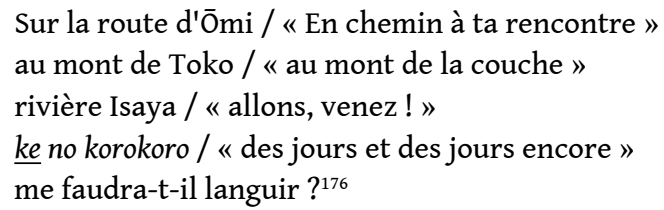

Selon le Daishōki ${ }^{177}$, commentaire du Man'yōshū, le terme ke dans ce poème signifie suiki (" humidité »), et il s'agirait donc du brouillard sur la rivière. Korokoro voudrait simplement dire koro ("jours»). Ce serait un emploi semblable à nemo korogoro (" de tout cœur ») que l'on trouve en poésie ${ }^{178}$ et il ne s'agit pas nécessairement d'un poème traitant du kajika. Il en va souvent ainsi en poésie, où le sens n'est pas toujours très 
précis, et comme dans ce cas particulier on ne dispose pas de preuves tangibles qu'il s'agisse du kajika, rien n'empêche que l'on donne le nom de "daim de rivière " (kawa no shika $=k a j i k a)^{179}$ à toute créature qui crie dans l'eau en automne, que ce soit poisson ou grenouille, en suivant la tendance la plus répandue. Néanmoins, il est désolant que l'on compose des poèmes en prenant pour sujet des créatures mal identifiées. Or si l'on veut vraiment trouver une référence dans les temps anciens, c'est la " grenouille d'Ide » que cite le Mumyōshō (Notes sans titre) de Chōmei ${ }^{180}$ qui correspond le mieux à ce que l'on appelle kajika aujourd'hui. On y lit :

Les grenouilles dites kawazu ne se rencontrent nulle part ailleurs. Il ne s'en trouve que dans la rivière Ide. De couleur noirâtre, elles ne sont pas très grandes. On ne les voit jamais sauter comme le font les grenouilles ordinaires. Vivant tapies dans l'eau, leur chant au cœur de la nuit est d'une remarquable pureté et il s'en dégage un charme pénétrant. ${ }^{181}$

Les grenouilles que l'on attrape de nos jours à Yase à la capitale, et celles que les gens de Naniwa vont chercher aux chutes de Tsuzumi en Arima, sont identiques aux grenouilles d'Ide. Il n'y a pas de doute que ce soit ce qu'on appelle aujourd'hui kajika. Ainsi donc, dans la poésie classique (waka), on chante la grenouille (kawazu) et non pas le poisson kajika. Quant à kajika, on ne se trompe pas en le considérant comme un terme vulgaire autorisé dans le haikai. On trouve dans de nombreux écrits l'amour immodéré porté jadis à la grenouille d'Ide. Celles que l'on attrape maintenant à Yase, Arima et Ide, ne chantent pas toutes de cette même façon. Celles dont le chant émeut n'ont pas la même apparence : c'est une sorte de crapaud (gama) noirâtre aux reflets verts. Les pattes avant ne sont pas palmées, tandis que les bouts des doigts sont tous arrondis. Elles habitent les eaux pures et leur chant nocturne ressemble au korogoro du rougegorge ${ }^{182}$. Les sixième et septième mois, elles chantent de concert une fois par nuit. Elles chantent aussi de jour, mais leur voix ressemble alors à celle de la pie-grièche ${ }^{183}$. Comme elles sont très agiles, elles sont difficiles à attraper. Aussi, pour les prendre attend-on la période des grandes chaleurs lorsqu'elles restent au fond de l'eau. Le fait qu'on les ait confondues avec des poissons, vient de ce que l'usage vulgaire de kajika dans la poésie haikai fait oublier le sens ancien de "grenouille». Les temps ayant changé maintes fois depuis l'époque de Chōmei, on ne se souvenait plus ces derniers temps que du nom de kajika. Tant et si bien que, se rendant au bord des rivières ou près des torrents, et en entendant les sons de gori et de gigi ${ }^{184}$, on s'est persuadé que cela ne pouvait être que le kajika, dans la mesure où le chant était le même. C'est ainsi que l'on a fini par perdre le fil.

\section{Révision}

Dans son Kokin uchigiki (Notes recueillies sur le Kokinshū), Kamo no Mabuchi ${ }^{185}$ dit :

La grenouille (kawazu), dont on trouve un autre nom tanikugu ${ }^{186}$ dans le Man'yōshū comme dans les norito (prières liturgiques du shintō), vit dans les rivières et se distingue par une voix fort plaisante. Étant donné qu'elle chante toujours de l'été à l'automne, elle apparaît dans le Man'yōshū dans les poèmes d'automne. Elle diffère des bêtes au coassement assourdissant qui peuplent de nos jours les rizières, les champs, les digues et les étangs. Si les poètes des temps postérieurs n'ont parlé que de ces dernières, c'est parce qu'ils ignoraient les poèmes anciens. Le poème suivant du Man'yōshū est un témoignage de plus de l'attrait de ce chant: Inespérée, certes, / fut votre visite, / mais pour écouter le chant / des grenouilles de la Sahogawa ${ }^{187}$ / je n'ai su vous retenir ! ${ }^{188}$ 
Aussi, ce que l'on appelle aujourd'hui couramment kajika, doit être le kawazu des temps anciens. Cette grenouille-là ne se fait pas entendre au printemps, mais elle chante du milieu de l'été et durant tout l'automne. ce rapport avec les rivières Yoshinogawa ${ }^{192}$, Kaminamigawa ${ }^{193}$, Kiyokigawara, ou le bassin de Mutsuta ${ }^{194}$, il s'agit de cours d'eau limpides, et il n'y a pas, dans tout le Man'yōshū, un seul exemple de poème évoquant les grenouilles chantant dans les rizières ou dans les champs. À l'origine, le nom d'Ide apparaît dans le Kokinshū (Recueil de poésies de jadis et de naguère), dans un poème qui a été repris par la suite dans le Rokujō ([Recueil en] en six cahiers) ${ }^{195}$. Dans tous les cas, il faut savoir que kajika désigne la grenouille (kawazu) et que c'est un terme de la langue vernaculaire utilisé dans le haikai.

Voilà ce que dit Mabuchi.

attestant que le nom de tanikugu désigne le kawazu, il faut considérer cela comme une hypothèse parmi d'autres. C'est bien dans la manière du Man'yōshū d'évoquer la grenouille en la situant dans les cours d'eau et en admirant son chant plaisant, mais on ne saurait affirmer qu'elle soit associée à l'automne d'un bout à l'autre de ce recueil. Aussi, c'est la manière dépouillée des Anciens de ne pas distinguer à tout prix les espèces voisines et de les chanter sous un même nom. Les exemples en sont nombreux, comme on peut le voir dans ce poème du fascicule 6 (du Man'yōshū) : «Inespérée, certes, / fut votre visite / mais pour écouter le chant / des grenouilles de la Sahogawa / je n'ai su vous retenir ". Ainsi que dans cet autre poème d'amour évoquant un animal qui chante en automne, et dont le thème d'ailleurs lui aussi est l'automne : Dans la brume matinale / j'entends la grenouille chanter / sous l'abri où se consume l'encens contre les moustiques / et je languis / de ne point entendre votre voix ${ }^{189}$. entendant une grenouille : La grenouille qui / partage mon gîte / est-ce la tombée de la nuit / qui rend son chant si triste?»

tant d'autres, la preuve que le chant de la grenouille était fort apprécié : «Dans le lit du ruisseau / de Saho aux ondes pures / le cri des pluviers / ni des grenouilles non plus / jamais ne pourrai oublier ${ }^{191}$.

Toutes les fois que, dans un poème, il est question de « la grenouille qui chante », que J'ai déjà expliqué plus haut comment l'aspect et la voix de la grenouille étaient chantés dans les poèmes. Kajika est un terme vulgaire que l'on n'emploie pas en poésie. Si on chante la grenouille dans un poème de style classique, il faut employer le terme kawazu.

J'en veux pour preuve le Man'yōshū (où il est dit) :

L'officier principal de l'office des Fabrications, Kuratsukuri no suguri Masuhito, avait préparé un banquet en l'honneur du directeur, le prince Sai ${ }^{196}$. Mais avant la tombée de la nuit, le prince s'était déjà retiré. C'est sur cela que Masuhito, affligé et déçu de ce départ hâtif, composa ce poème :

Inespérée, certes,

fut votre visite,

mais pour écouter le chant

des grenouilles de la Sahogawa

je n'ai su vous retenir ${ }^{197}$ 
Fig. non chiffrée : [La grenouille : kawazu]

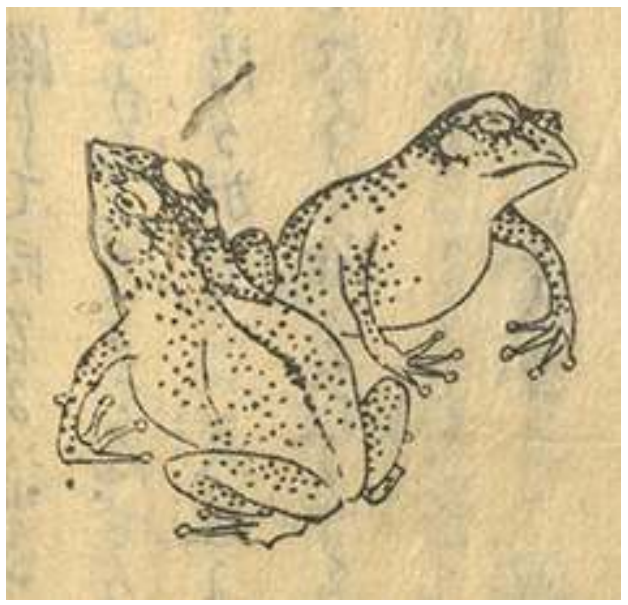

National Institute of Japanese Literature. Lien vers l'illustration

\section{Les poissons appelés kajika dans les différentes provinces}

67 Celui d'Ōzu en Iyo [Fig. A] ressemble, en un peu plus grand, à la loche des sables ${ }^{198}$. Sa voix ressemble au son produit lorsqu'on racle le fond d'un bol de thé. Elle se détache clairement dans la nuit. Quand il crie, ses deux joues vibrent. Dans l'entrée tofugyo ${ }^{199} \mathrm{du}$ Yamato honzō, il n'est pas question de sa voix.

Fig. A : [Kajika d'Ōzu en lyo]

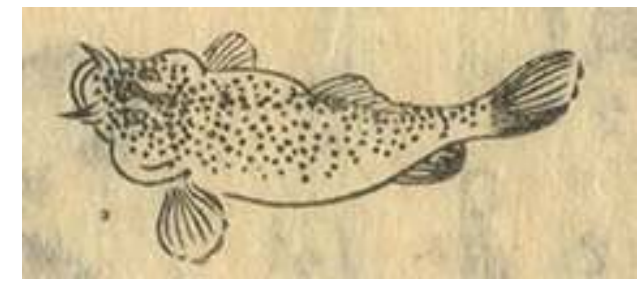

National Institute of Japanese Literature. Lien vers l'illustration

Celui de la province d'Echigo [Fig. B] a une grande tête et il est couvert de taches noires. Son ventre est blanc. Les plus petits mesurent 3 pouces, les plus grands 5 à 6 . Sa voix est celle $\mathrm{du}$ ver de terre, mais plus claire. Il chante la nuit. Toutefois, on le trouve également en grand nombre dans les rivières d'autres provinces. Dans l'île de Shikoku, on l'appelle yamadonko ${ }^{200}$, à Ōsaka, donguro-haze ${ }^{201}$. 
Fig. B : [Kajika de la province d'Echigo]

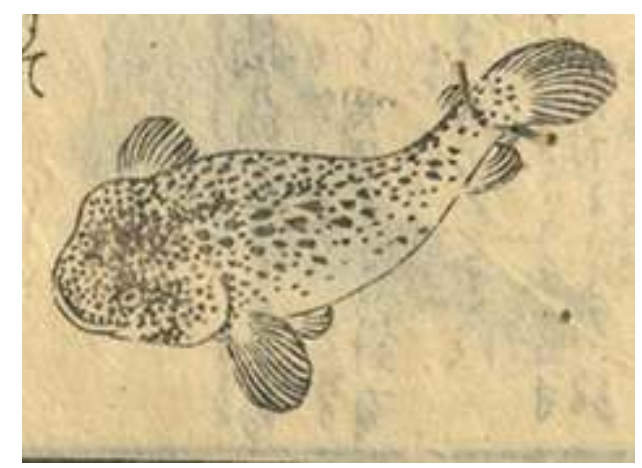

National Institute of Japanese Literature. Lien vers l'illustration

Celui de la province de Kaga [Fig. C] a une grande tête et une queue fourchue. Son dos est noir, son ventre blanc. Sa voix ressemble à celle de la souris. Il chante la nuit. Les plus petits mesurent 1 pouce à peine, les plus grands environ 2 pieds ${ }^{202}$. Les plus petits sont muets.

Fig. C : [Kajika de la province de Kaga]

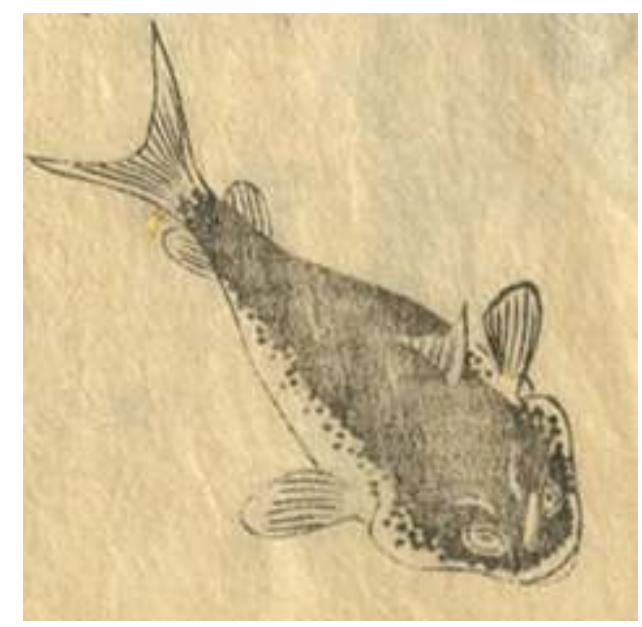

National Institute of Japanese Literature. Lien vers l'illustration

\section{Ishifushi («accroche-pierre ») gori de son autre nom [Fig. D et E]}

Il en existe deux espèces : l'une vivant dans l'eau de mer, l'autre dans l'eau douce. Les vrais " accroche-pierre " possèdent sous le ventre des nageoires au moyen desquelles ils s'accrochent aux pierres. L'accroche-pierre ressemble au chabot (tofugyo), mais il est plus petit. Il possède une voix et chante la nuit. Ses nageoires sont épineuses. Les épines sont souples pour l'espèce qui vit en mer, et acérées pour celle qui vit en eau douce. 
Fig. D : [Le vrai accroche-pierre, marin]

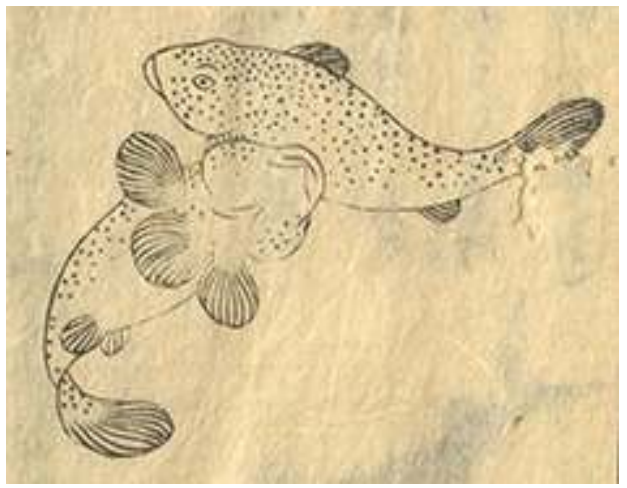

National Institute of Japanese Literature. Lien vers l'illustration

Fig. $\mathrm{E}$ : [Accroche-pierre de rivière]

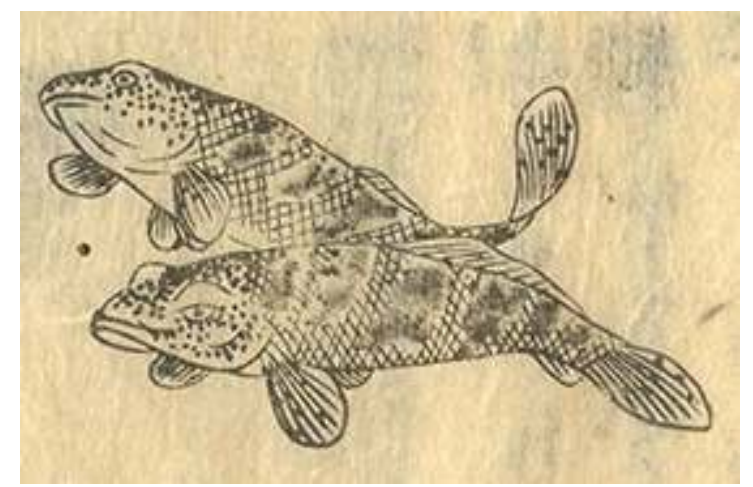

National Institute of Japanese Literature. Lien vers l'illustration

\section{Gigi [Fig. F]}

71 Il ressemble au poisson que l'on appelle "poisson-miko» (miko-uo ${ }^{203)}$ à Saga ${ }^{204}$ et « courtisane-miko» (miko-jorō) en Harima. De couleur rouge, il a des épines sous la gorge ainsi qu'aux nageoires. Le pêcheur s'y pique souvent la main. Le nom chinois est kōsōgyo (" poisson au front jaune »), et celui du poisson-miko, ōshigyo ${ }^{205}$. Il vit aussi bien en mer qu'en rivière (Petits, ils mesurent 3 pouces environ, grands 4 à 5 . Il a des nageoires sous les branchies. De couleur brun-jaune, il est tacheté de noir). 
Fig. F : [Gigi : « poisson-miko »]

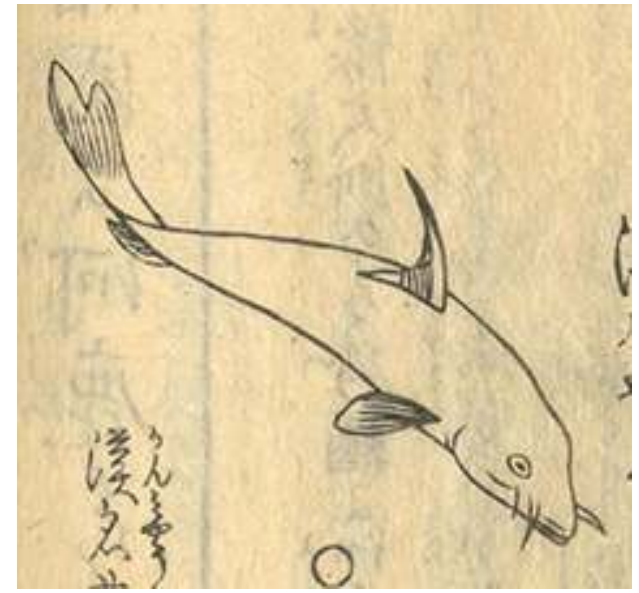

National Institute of Japanese Literature. Lien vers l'illustration

\section{Arare-uo («poisson-grêle ») d'Echizen [Fig. G]}

Selon certains, ce serait une erreur de l'identifier au tofugyo en arguant de ce qu'on le trouve dans aucune autre province. On dit que, lorsqu'il grêle, il se laisse emporter par le courant, le ventre tourné vers le haut. Un autre nom est kakubutsu' ${ }^{206}$. Il possède une voix. À bien y réfléchir, il s'agit d'une espèce de chabot tofu. Ce n'est donc pas une erreur de l'appeler par ce nom ${ }^{207}$.

Fig. G : [« Poisson-grêle » d'Echizen]

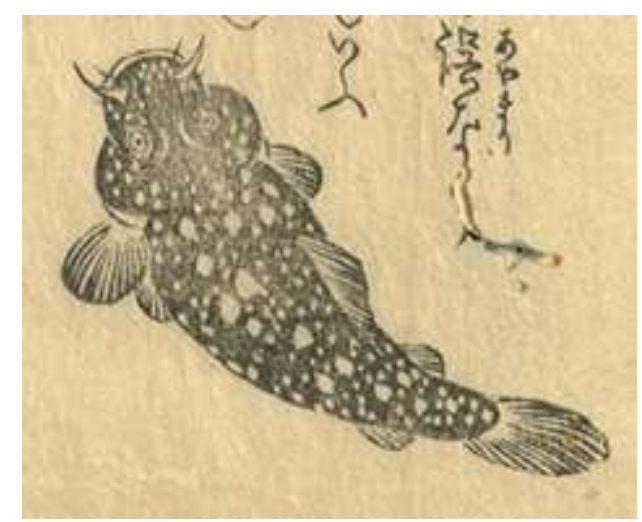

National Institute of Japanese Literature. Lien vers l'illustration

Ishigurai (« mord-pierre ») [Fig. H]

Appelé aussi donguro, il n'a pas d'épines aux nageoires. Nom chinois inconnu. 
Fig. $\mathrm{H}:$ [ "Mord-pierre »]

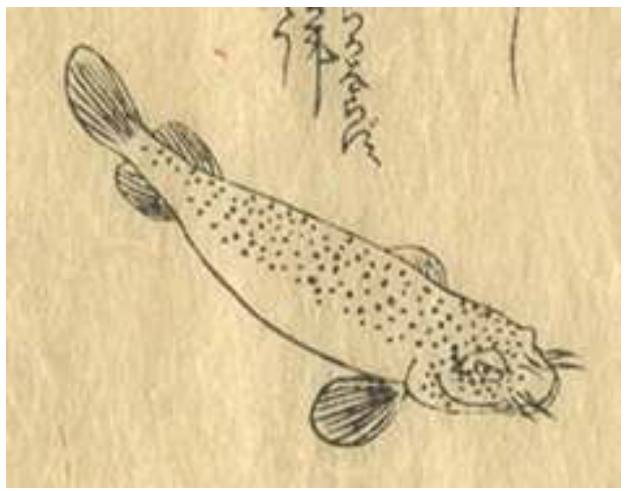

National Institute of Japanese Literature. Lien vers l'illustration

\section{Tofugyo [Fig. I]}

74 Appelé aussi ishimochi ("agrippe-pierre »), ou kawa-okoze (" okoze de rivière ») 208 à Fushimi, kuchinawadonko (« donko-serpent ») en Iyo, maru («poisson-hôte ») à Saga, muko (" poisson-gendre ») en Ōmi. Il vit au fond de l'eau et se fixe aux rochers comme les « accroche-pierre ». Tacheté de noir comme le kochi209, il abonde dans la Kamogawa. Il a des épines acérées à la tête et aux nageoires.

Fig. I : [Tofugyo]

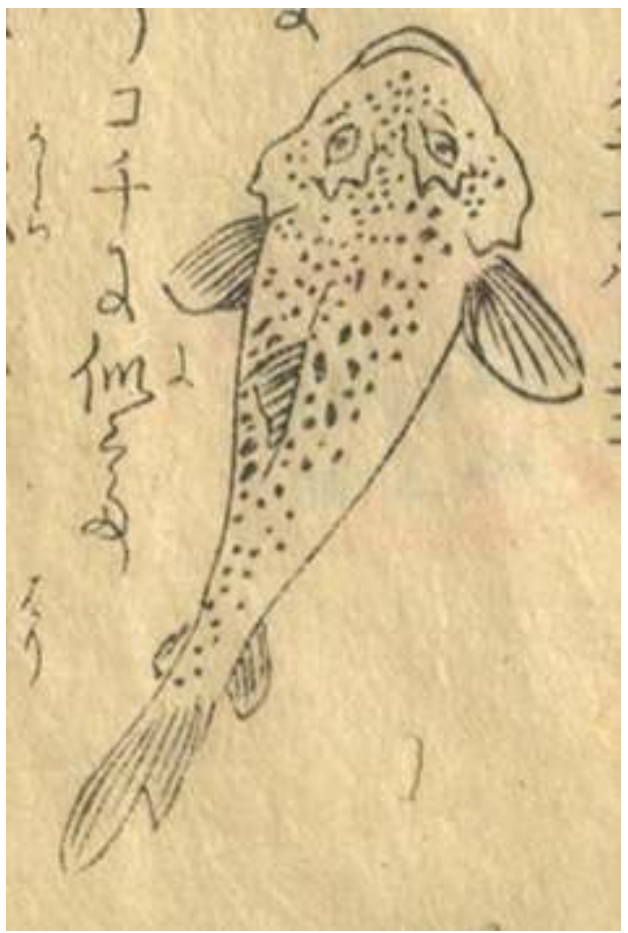

National Institute of Japanese Literature. Lien vers l'illustration 


\section{NOTES}

1. Awa 阿波, province de l'île de Shikoku, correspondant maintenant au département de Tokushima.

2. Awa 安房, province homophone occupant, avec celle de Kazusa, la péninsule de Bōsō et formant avec elle l'actuel département de Chiba.

3. Poisson migrateur, la bonite tourne dans les eaux chaudes du Pacifique, mais, avec le réchauffement estival, les bancs se laissent porter par le courant du Kuroshio et longent les côtes du Kyūshū (Satsuma), du Shikoku (Tosa, Awa), du Honshū (Kii, Iyo, Suruga, Izu, Sagami), et de la péninsule de Bōsō (Awa, Kazusa), remontant jusqu'au large du Hokkaidō.

4. D'un strict point de vue taxinomique, seulement la sujigatsuo est à classer dans le genre «bonite» (Euthynnus pelamis Linn.) et dans la famille des Katsuwonidae; il s'agit ici, bien évidemment, de la «bonite japonaise " (Katsuwonus pelamis), genre habitant le Pacifique (voir Ph. Fr. von, TEMMinCK C. J. and SCHLEGEl H., Fauna Japonica. 2. Pisces, apud Arnz et Socios, 1844, p. 96 et pl.XLIX ; KISHINOUYE Kamakichi, "Contributions to the Comparative Study of the So-called Scombroid Fishes", Journal of the College of Agriculture, vol. VIII, $\mathrm{n}^{\circ} 3$, 1923, p. 450-456). Les yokowa-, uzuwa- et hira-gatsuo font partie du genre des hirasōda-gatsuo, ou sōda-gatsuo (Auxis thazard Lacepède), les auxides. Ensemble, ces trois genres appartiennent à la famille des Scombridés dont font aussi partie les maquereaux et les thons. Mochi-gatsuo est l'appellation de la bonite de la première pêche de printemps, particulièrement appréciée pour sa consistance rappelant le mochi (gâteau de riz). Voir NIHON GYORUI GAKKAI ed., Nihonsan gyomei daijiten (Dictionary of Japanese Fish Names and Their Foreign Equivalents), Tokyo, Sanseidō, 1981, p. 88, 288 ; TSUDA Takeyoshi, Genshoku. Nihonkai gyorui zukan (Encyclopédie illustrée des poissons de mer), Toyama, Katsura shobō, 1990, p. 133, et, plus généralement, томіуама Tetsuo, Fisheries in Japan - Skipjack and Mackerel, Tokyo, Japan Marine Products Photo Materials Association, 1976.

5. Selon les régions, ce nom s'applique à des poissons fort différents, mais majoritairement aux auxides.

6. Le terme désignant la bonite, ici et partout ailleurs lorsqu'il est employé dans la même acception, est fushi/bushi, indiquant le filet qui, découpé et séché, fournira la fameuse «bonite séchée " (katsuo-bushi). Il faut comprendre que, hormis le poisson attrapé pendant la première moitié de la troisième lune, mangé frais, la bonite prise au cours des autres saisons est destinée à être séchée. Aussi, par «frais » faut-il entendre non pas crue, comme on consomme souvent la chair de ce poisson aujourd'hui, mais cuite ou grillée aussitôt après la pêche.

7. Pris à la lettre, 2 tsubo équivaudraient à $6,6 \mathrm{~m}^{2}$, indication à prendre avec précaution si l'on considère l'utilisation qui en est faite ici. Il n'est pas impossible que le caractère pour tsubo 坪 soit une erreur du graveur pour to 斗 en écriture cursive, mesure de capacité d'environ 18 litres. Des paniers d'un volume de 36-40 litres seraient certainement plus adaptés à l'usage que les pêcheurs sont censés en faire.

8. Mesure de contenance, soit, ici 3180 litres environ.

9. Il est fait allusion à un rite annuel qui a toujours lieu au «Temple aux trente-trois travées » (Sanjūsangendō) de Kyōto et qui consiste en une compétition de tir à l'arc (tōshiya) où l'archer doit réussir l'exploit d'atteindre la cible placée à 60 mètres de distance à l'autre bout de la véranda qui longe la façade occidentale de ce célèbre temple. Le vainqueur est celui qui compte le plus de tirs réussis pendant un laps de temps déterminé.

10. Cet instrument de pêche en forme de "fléau» de balance (tenbin), porteur aux deux extrémités d'une ligne garnie d'hameçon(s) qu'il empêche de s'emmêler, est depuis longtemps passé d'usage, remplacé au XIX ${ }^{e}$ siècle par la palangre. $\mathrm{Ph}$. F. von Siebold en préserve cependant la mémoire dans son livre Nippon, où il en donne une illustration dans la TAB. II du vol. VI (voir 
SIEBOLD, Philipp Franz von, Nippon - Archiv zur Beschreibung Japans, Leyden : bei dem Verfasser, 1832-1852, Tokyo, Yūshōdō, 1979, Fig. 335), suivant les informations recueillies pendant son séjour à Nagasaki de 1823 à 1829. C'est à bon escient que cette méthode de pêche ne soit mentionnée ici qu'en passant, car en raison de son poids le « fléau » est adapté à la pêche de fond et ne devait donc pas servir pour la bonite qui nage à la surface.

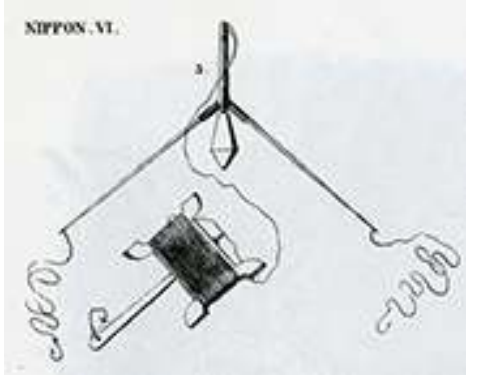

Illustration provenant de l'ouvrage de SIEBOLD, Philipp Franz von, Nippon.

11. Qui porte le nom de shutō (littéralement vol de saké), ou, plus généralement, de shiokara, abats (viscères) de poisson macérés dans le sel que l'on continue de consommer de nos jours (pour l'actuel procédé de fabrication, voir TomiYAmA, Fisheries in Japan..., op. cit., p. 122-123).

12. Ce produit est l'équivalent du garum, assaisonnement «tiré des entrailles marinées du scombre ou maquerau », fort prisé des anciens Romains, si l'on en croit Brillat-Savarin (BRILLATSAVARIN Jean Anthelme, Physiologie du goût, 1825).

13. Il y a plusieurs raisons qui pourraient expliquer que les villageois préféraient exécuter ces opérations à l'écart des habitations. La principale est sans doute l'odeur, la puanteur même qui s'y répand pendant tout le processus de conditionnement (cuisson, moisissure, marinage, séchage, etc.) dont la durée totale peut dépasser les trois mois. Il se peut que ce soit aussi pour retarder le temps du transport, l'opération de découpage, car la chair d'un poisson trop frais se contracte après débitage, de sorte qu'il est alors difficile d'obtenir des morceaux de forme appropriée au katsuo-bushi. L'état idéal est atteint après quelques heures, lorsque la chair s'est raffermie tout seule. Par contre, quand trop de temps s'est écoulé depuis la prise, c'est-à-dire quand le poisson n'est pas suffisamment frais, la chair se sera trop ramollie et aura alors tendance à se défaire à la cuisson (voir TomIYAMA, Fisheries in Japan..., op. cit., p. 157-158).

14. Le traducteur se trouve ici face à un problème. Si le terme employé par l'auteur pour cette méthode de cuisson est bien musu, c'est-à-dire " cuisson à la vapeur ", cela va à l'encontre non seulement du mode de cuisson que l'on voit illustré sur la planche 43 , mais également de ce que l'on connaît par ailleurs, et jusqu'à l'époque actuelle, de cette étape de préparation qui se fait bien par cuisson dans l'eau bouillante (voir TомгуAмA, Fisheries in Japan..., op. cit., p. 102-117, 157-158).

15. La chair du dos étant moins grasse que celle du ventre, le morceau du dos fournit un bouillon plus clair et au goût plus fin que le morceau du ventre. En cadeau, les deux sont souvent présentés par paire, le premier sous le nom de « mâle » (o-bushi), l'autre de « femelle » (me-bushi). 16. Shibi : voir l'entrée «thon » dans le fascicule 3.

17. Le toponyme Shimizu signifie " eau pure ». Il est à noter ici que cette phrase manque en totalité dans l'édition du texte établie par Chiba Tokuji.

18. La bonite séchée reste en effet à ce jour un cadeau de valeur et très apprécié, fait à certaines occasions rituelles.

19. Outre l'homophonie de bushi (節 copeaux) et bushi (武士 guerrier), cette réticence s'expliquerait par le fait que la lecture sino-japonaise inversée des caractères harabushi est seppuku 切腹 ( s'ouvrir le ventre»), terme désignant le suicide rituel du guerrier. Il semble cependant que ce soit là un genre de superstition populaire, car l'Almanach des maisons militaires de l'ère Kansei (Kansei bukan) des années 1790, inventoriant les articles offerts selon la 
saison en tribut officiel à la cour shogunale, dénombre plusieurs seigneurs de haut rang - dont les chūnagon de Kii, les Matsudaira de Bungo, les Matsudaira de Tosa, les Gotō d'Ōmi - devant présenter de la bonite séchée, en l'occurrence le produit renommé de leur domaine (voir Koji ruien (Encyclopédie des choses anciennes), Kojiruien kankōkai éd., Tokyo, Koji ruien kankōkai, 1936, vol. 51 : "Mets et boissons ", rubrique "Mets séchés ", p. 930). Qui plus est, une graphie homophone de katsuobushi, à savoir 勝男武士 signifiant " guerrier victorieux ", peut être prise pour un autre indice que la bonite séchée fut aussi un produit très apprécié de la classe des samouraïs.

20. Engishiki (901-930), édité par Fujiwara no Tokihira (871-909) et al., en 50 fascicules, expose le détail du fonctionnement de l'administration centrale.

21. Wamyōshō, également connu sous le nom de Wamyō ruijushō, est un dictionnaire de l'époque de Heian de Minamoto no Shitagō (911-983), achevé autour de 934, répertoriant environ 2600 termes chinois classés selon différents domaines, allant des phénomènes célestes aux plantes et animaux.

22. Tōga, dictionnaire en 20 fascicules composé par Arai Hakuseki (1657-1725) en 1717 et donnant l'étymologie des noms japonais.

23. Pour forger cette étymologie, Arai Hakuseki, l'auteur du Tōga, s'inspire sans doute du Wamyōshō, qui attribue au caractère 鮎 pris isolément, la lecture japonaise kotsuo, nom d'un poisson de la famille des requins.

24. En d'autres termes, l'étymologie vulgaire associe le nom de "poisson dur » à l'idée de la dureté presque métallique du morceau de bonite séchée. Les spécialistes la réfutent cependant, avançant l'argument que la lecture katsuo du nom écrit kata-uo ("poisson dur») est incontestablement attestée bien avant l'introduction de la technique de séchage (voir MIYASHITA Akira, Katsuo bushi (La bonite séchée), Tokyo, Hōsei daigaku shuppankyoku, coll. « Mono to ningen no bunkashi » 97, 2000, p. 93-105).

25. Tongŭi pogam, encyclopédie de médecine coréenne, réalisée sous la direction de Heo Jun au $\mathrm{XVI}^{\mathrm{e}}$ siècle. L'ouvrage composé de 25 fascicules fut abondamment consulté en Chine et au Japon à l'époque d'Edo.

26. Il s'agit d'un autre nom de la bonite, les mêmes caractères se lisant aussi bien katsuo que shōgyo, ce dernier nom étant toujours en usage à l'époque actuelle.

27. Matsu no fushi : difficile de dire à quoi ce « nœud de pin » fait référence. Il est possible que ce soit à cette structure en forme de nœud concentrique qui marque des deux côtés de la partie dorsale la chair des Scombridae dans sa coupe transversale. Une explication moderne du terme " poisson de pin », ignorant cette comparaison avec le « nœud » (fushi), affirme que ce nom est dû à ce que la chair de la bonite séchée (katsuo-bushi) partage avec le bois du pin la même nuance de couleur de cachou.

28. Si cette référence à un prétendu " envoyé » coréen relève d'un fait historique, il ne peut alors se rapporter qu'à un membre d'une des deux dernières ambassades dépêchées par ce pays avant la date de publication du présent ouvrage (1799), à savoir celles de 1748 pour le premier, et de 1764 pour le dernier.

29. L'argument repose sur le fait que les deux premiers caractères, 固 et 堅, peuvent se lire tous les deux kata-, signifiant « dur ».

30. Managatsuo est un poisson pélagique de la famille des Stromateidés, proche de la fiatole (Stromateus fiatola). Shō semble être un nom local de la fiatole argentée (Pampus argenteus), mais on peine à identifier le poisson qui se cache sous le nom de gyoyū.

31. Zhu Shunshui (1600-1682), lettré confucianiste chinois, originaire de Yuyao dans la province de Zhejiang. Réfugié au Japon après la chute des Ming, il fut le conseiller du seigneur de Mito, Tokugawa Mitsukuni (1628-1700). Son enseignement confucéen a durablement marqué l'école d'historiographie de ce fief. 
32. Un poisson de ce nom figure dans le Bencao gangmu, combiné avec le caractère poisson (hōgyo). Il s'agirait de la brême chinoise (Megalobrama terminalis), un poisson d'eau douce utilisé comme matière médicale. La raison de cette référence à Shunsui est obscure.

33. Rappelons à ce propos un fait en ce moment sur toutes les lèvres, à savoir qu'en plus de nos quatre saveurs fondamentales (le doux, l'acide, le salé, l'amer), auxquelles la médecine traditionnelle chinoise ajoute une cinquième : l'âcre, les Japonais en sont venus depuis un peu plus d'un siècle à en distinguer une nouvelle, appelée umami (le "savoureux »), goût dont justement le bouillon à base de laminaires et de flocons de bonite séchée exprime la quintessence.

34. Man'yōshū, anonyme, fin du VIII ${ }^{\mathrm{e}}$ siècle. Il s'agit de la plus ancienne anthologie de poésie conservée au Japon.

35. Il s'agit du «très célèbre et long poème qui raconte l'histoire du fils d'Urashima, lequel crut avoir passé trois ans auprès d'une déesse des mers et qui, revenu quelques siècles plus tard, ne retrouva plus son village. » (Man'yōshū, fasc. 9, nº 1740 ; Man.yôshû, SIEFFERT, op. cit., (fasc. 7, 8 et 9), 2001, p. 327). Voir aussi Man'yōshū, SATAKE Akihiro et al. (ed.), op.cit., vol. 2, p. 359. Il n'y saurait être question de «bonite séchée », mais katsuo y est écrit avec les caractères kata-uo 堅魚.

36. Voir Engishiki, Fasc. 22 et 23.

37. L'Engishiki fait effectivement mention d'« extrait de bonite », mais le Wamyōshō (934) précise que ce terme se prononçait katsuo-irori (堅魚煎汁). Les deux appellations d'ikari et de nitori sont inconnues des sources de l'époque de Heian. Cette phrase est tirée mot à mot du Tōga où il est dit que « irori est ce que l'on appelle maintenant vulgairement katsuo-nitori (堅魚煮取) ». Il s'agit en fait d'une décoction très concentrée du bouillon obtenu lors de la cuisson de la bonite préalable au séchage, comme on l'a vu ci-dessus. Pour sa préparation, toujours d'actualité, voir TомIYAMA, Fisheries in Japan..., op. cit., p. 126-127. Si le terme de nitori est aussi attesté dans d'autres sources de l'époque d'Edo, on ne trouve nulle part trace du nom ikari dans cette acception.

38. Le Tsurezuregusa est un essai au fil du pinceau en 2 fascicules du XIV ${ }^{\mathrm{e}}$ siècle, composé par Yoshida Kenkō (dates inconnues). Des spécialistes situent la date de sa composition autour de 1331.

39. Voir, par comparaison, la traduction de cette remarque dans Les heures oisives (Tsurezuregusa), URABE Kenkō, GROSBOIS Charles et YOSHIDA Tomiko (Traduction et commentaires de), suivi de Notes de ma cabane de moine (Hōjō-ki), KAMо no Chōmei, R. P. Sauveur Candau (trad.), Paris, Gallimard, 1980, CIX, p. 105-106.

40. Section 119 du Tsurezuregusa, in Hōjōki, Tsurezuregusa, SATAKE Akihiro et KUBOTA Jun (éd.), Tokyo, Iwanami shoten, coll. «Shin Nihon koten bungaku taikei » 39, 1989.

41. Sur la foi de ce que cet auteur semble rapporter ici, certains soutiennent - à tort selon MIYASHITA, Katsuo bushi, op. cit., p. 196-198 - qu'en son temps encore il n'y avait que les basses classes qui consommaient la bonite "fraîche », c'est-à-dire cuite, grillée ou salée, mais non pas séchée.

42. Contrairement au sens revêtu aujourd'hui par ce terme, la préparation à laquelle il est fait référence ici, plus connue sous le nom de shiokara, est fabriquée à partir d'entrailles de poisson macérées dans le sel. Voir la note 11 plus haut.

43. Nom d'une ancienne ville castrale, située dans l'actuel département de Fukushima (district de Shirakawa-Est), confinée dans une plaine cernée de montagnes et distante d'une cinquantaine de kilomètres de la mer. Il est peu probable qu'on ait pu y trouver de la bonite en tataki, et les commentateurs modernes s'accordent à dire que l'auteur s'est trompé.

44. namako : nom commun de cet animal marin appartenant aux échinodermes et à la classe des Holothuroideae, qui porte par ailleurs les noms de "concombre de mer", "bêche de mer", "vier marin", voire encore de "tripang» ou «trépang" (dérivés du malai). Comestible, l'holothurie peuple la mer Rouge et les océans Indien et Pacifique où les grosses espèces sont très 
appréciées - parfois pour leurs supposées vertus aphrodisiaques. On en dénombre environ mille cinq cents espèces à travers le monde, et des quelque deux cents présentes dans les eaux du Japon, trente à peu près (appelées manamako) sont consommées.

45. L'île de Saku se situe entre les bras des péninsules d'Atsumi et de Chita, entourée des baies de Mikawa et de Chita, dans l'actuel département d'Aichi.

46. Importante île dans la mer (dite) de Harima, elle-même partie de la mer Intérieure de Seto, département de Kagawa.

47. L'auteur se réfère sans doute ici au Shiwu bencao édité par Li Shizhen (voir note ci-dessous). Le Yamato honzō cite intégralement le passage correspondant sous l'entrée "namako ", Yamato honzō, KAIBARA Ekiken, fasc. 14.

48. Le Shiwu bencao (j. Shokumotsu honzō) est un ouvrage chinois en vingt-deux fascicules de Li Gao (1180-1251), édité par Li Shizhen et imprimé en 1638, dans lequel l'auteur ne retient des pharmacopées traditionnelles que les produits comestibles.

49. Le ginseng (Panax ginseng), ninjin en japonais, où le premier caractère signifie " homme ", dont le nom renvoie à l'allure anthropomorphe des racines de cette plante, fait figure en Extrême-Orient de panacée par excellence. Le nom kaijin où le premier caractère signifie «mer » attribue donc à l'holothurie des vertus toniques comparables.

50. Kinkasan, ou Kinkazan, où kin désigne le métal ou l'or, est une petite île au large d'Ishinomaki, dans l'actuel département de Miyagi, au nord-est du Japon. Elle est entièrement occupée par le mont Kogane, avec, au sommet, l'éponyme Koganeyama-jinja (sanctuaire Koganeyama), car, selon l'antique tradition, c'est ici que fut trouvé pour la première fois de l'or (kogane) au Japon, la découverte venant à point pour réaliser la dorure du Grand Bouddha du Tōdai-ji qui put ainsi être consacré en 752.

51. Bouilli et séché, conditionné en sacs de paille, ce produit était acheminé en grande quantité à Nagasaki, où il se vendait fort cher à des négociants chinois qui l'exportaient vers les marchés continentaux.

52. Hoya (ou maboya) désigne l'ascidie (classe des Ascidiaceae), ou le tunicier, animal marin différent de l'holothurie, qui vit fixé sur un substrat et colonise tous les milieux, des rochers de bord de mer aux fonds littoraux. En l'occurrence, il s'agit de Halocynthia roretzi, comestible et de nos jours élevé surtout en Corée, et dans une moindre mesure également au Japon où il est consommé aussi bien cru que mariné, fumé, grillé frit ou séché.

53. Il s'agit du Kinmō zui de Nakamura Tekisai (1629-1702) en 20 fascicules, imprimé en 1666, selon le modèle Ming du Sancai tuhui (1607). Il procure pour un large spectre d'objets, animaux ou phénomènes, les noms japonais et chinois, complétés d'une illustration et d'une courte description.

54. Apostichopus japonicus est l'espèce d'holothurie la plus courante au Japon, la plus consommée aussi, et de ce fait représentative du genre dans son ensemble (en français communément traduit par «trépang »), au point de porter le nom de «vraie holothurie » (ma-namako). Il en existe de couleur rouge, verte et noire.

55. Inō Jakusui (1655-1715), spécialiste japonais de materia medica, auteur du Shobutsu ruisan (Répertoire des myriades de substances). Voir l'Introduction du présent ouvrage.

56. Les siponcles (Phascolosoma esculenta) sont des animaux marins vermiformes. Vivant dans toutes les mers, sédentaires, cachés dans les sédiments et les anfractuosités rocheuses, Lamarck, en 1809 déjà, pensait pouvoir leur reconnaître "des rapports avec les holothuries ", mais en dépit de certaines ressemblances avec celles-ci, ils appartiennent à l'embranchement très différent des Annélidés. Bien plus qu'au Japon, ils servent de nourriture en Chine et en Corée.

57. Kaidanshi (c. hainanzi) est un autre nom de kaijin, vu plus haut.

58. C. haiqu : selon le Grand Ricci, le terme désigne des ligies, petits crustacés terrestres vivant au bord de la mer. Au Japon, il désigne aujourd'hui un genre de blatte appelée funamushi (船虫). 
59. Le Wuzazu (j. Gozasso) est un ouvrage de Xie Zhaozhe (1567-1624) des Ming, en 16 fascicules, imprimé en 1619, abordant des sujets relevant de la politique, de l'économie, des savoirs, et des arts, classés selon les cinq catégories : Ciel, Terre, Homme, Faits, et Choses.

60. Honchō shokkan, ouvrage de Hitomi Hitsudai (vers 1642-1701), en 12 fascicules, achevé en 1692 et imprimé en 1697. Inventorie 442 sortes d'aliments produits au Japon.

61. Honchō shokkan, catégorie «Espèces à écailles » 3, fasc. 9 ; SHIMADA Isao (trad. et éd.), Tokyo, Heibonsha, coll. « Tōyō bunko », 1980, vol. 4, p. 323-329.

62. Uni, littéralement «bile de mer». L'analogie avec la bile vient probablement de la couleur jaunâtre ou orangée des gonades (le « corail ») qui tapissent l'intérieur du test.

63. Animal marin de l'embranchement des échinodermes, de la famille des échinidés (Echinoidea), proches des concombres de mer, caractérisé par les piquants (radioles) qui en protègent la carapace. La ressemblance avec la bogue de la châtaigne est si évidente qu'elle se traduit aussi bien en japonais, dans la graphie moderne de uni 海栗, littéralement « châtaigne de mer ", qu'en français où le même sobriquet avait cours.

64. Le « test ", selon la terminologie zoologique.

65. Emploi inhabituel du composé «Satsuma-Shimazu», comme toponyme. Satsuma est le nom d'une province du sud du Kyūshū, domaine sur lequel régnait tout au long de l'époque prémoderne, la famille Shimazu.

66. Uni serait ainsi la contraction de umi-i, tels que sont lus les caractères 海膽 signifiant « bile de mer ».

67. Animal non identifié, à moins que l'on suive certains dictionnaires qui lisent ces deux caractères "uni» et en font une sorte $d^{d}$ ' oursin». Toutefois, le «Dictionnaire portugaisjaponais » (Vocabvlario da Lingoa de Iapam), œuvre composé par les missionnaires jésuites et publié à Nagasaki en 1603, explique que Cabutogai est une sorte de coquillage appelé « uni ».

68. 白魚: si l'on se fie à la seule transcription indiquée par l'auteur, ce nom se prononce shiro'uo, et désignerait donc une espèce de gobie (Leucopsarion petersii), de la famille des Gobiidae (voir JORDAN D. S., TANAKA S., SNYDER J. O., "A Catalogue of the Fishes of Japan", Journal of the College of Science, Imperial University of Tokyo, Tokyo, publ. by the University, 1913, p. 363, fig. 319). Les mêmes deux caractères peuvent toutefois se prononcer aussi shira'uo, auquel cas il s'agirait d'un poisson qui n'est pas sans rappeler une sorte d'« éperlan », dit « éperlan japonais » (Salangichthys microdon), de la famille des Salangidae (JORDAN, TANAKA, SNYDER, "A Catalogue of the Fishes of Japan”, ibid., 1913, p. 47). Mais comme ni l'un ni l'autre n'a vraiment d'équivalent en Europe, on voit souvent utilisé, à défaut d'un nom français, les termes anglais de «ice goby » pour le premier, et de "(Japanese) icefish" ou, en traduction, " poisson des glaces ", pour l'autre. Les deux espèces se ressemblent assez, de par leur petite taille (entre 7 et $15 \mathrm{~cm}$ de longueur), par leur habitat dans les eaux côtières et l'estuaire des rivières, ainsi que par leur corps, presque transparent lorsqu'ils sont vivants, tournant aussitôt au blanc après la mort (d'où leur nom japonais qui signifie dans les deux cas « poisson blanc »). On note qu'un poisson ayant pour nom 白魚 (poisson blanc) donné sans lecture fait l'objet d'une notice dans le Yamato Honzō de Kaibara Ekiken (fasc. 13, f. 39) ; il s'agirait d'un poisson semblable au bar japonais (suzuki) d'une longueur atteignant les 60 centimètres avec des écailles blanches.

69. Le Yamato honzō est un traité de Kaibara Ekiken 貝原益軒 (1630-1714), imprimé en 1709, réalisé sous l'influence du Bencao gangmu mais dont le propos est recentré sur les espèces naturelles observées au Japon. Composé de 16 fascicules, 2 fascicules annexes, et 2 fascicules d'illustrations.

70. Dans son Yamato honzō, fasc. 13, ff. 10-11, au sous-chapitre "Poissons de rivière ", Kaibara Ekiken consacre une notice à un poisson nommé avec les trois caractères 鱠残魚, qu'il lit shiro'uo (et non kaisangyo comme mentionné ici dans la note). Il est à noter que dans l'usage actuel, les mêmes caractères sont employés comme l'un des noms vernaculaires du shirauo. L'étymologie de ce nom se rapporte à la légende selon laquelle le roi du royaume chinois de Wu (585-473 av. J.-C.), 
monté en bateau, aurait mangé un silure (魚鱠) et jeté les restes dans la rivière. Ceux-ci se seraient alors transformés en « restes de silure » 鱠残 (ces deux caractères se lisant aussi kaisan). 71. Voir Yamato honzō, fasc. 13, sous-chapitre «Poissons de mer», f. 39, entrée kisugo. Kaibara y condamne une nouvelle fois l'usage courant consistant à lire kisugo le poisson noté 鱠残魚 alors qu'il s'agirait en réalité du shiro'uo. Le Kisuko (encore kisugo, ou kisu dans le Kantō), est un poisson à la chair très délicate, évoluant près du littoral. En français, le nom vernaculaire est donné comme "pêche-madame argenté" (Sillago sihama ou Sillago japonica), de la famille des Sillaginidae. (voir JORDAN, TANAKA, SNYDER, "A Catalogue of the Fishes of Japan", op. cit., 1913, p. 187).

72. Cette note est le premier indice de la confusion que commet l'auteur, confusion qui n'est toutefois que partiellement de son fait, puisqu'elle règne déjà dans l'ouvrage auquel il se réfère ici et si souvent ailleurs.

73. La pêche dans la baie de Nishinomiya, visant un genre de dorade de couleur rouge vif (Sacura margaritacea), se trouve illustrée dans le vol. VIII, p. 20, de Settsu meisho zue (Lieux renommés du Settsu), ouvrage paru un an à peine avant le Nippon sankai meisan zue, vers Kansei 10 (1798).

74. Pêcherie formée d'une double rangée de pieux enfoncés dans la rivière en angle obtus, avec une ouverture au milieu pour le libre passage des poissons en amont, mais qui peut être fermée par un filet pour les attraper en descendant. La caractéristique du dispositif japonais est que les pieux, en simple ou double rang, servent à maintenir à la verticale un clayonnage de bambou ou de branchages (ajiro), tressé suffisamment fin pour retenir le fretin.

75. Pour ce qui est de l'Ujigawa, rivière qui prend sa source dans le lac Biwa, on y attrape l'alevin de la truite ayu (Plecoglossus altivelis), de la famille des Plecoglossidae, présente au Japon, en Corée, en Chine et au Taiwan. Autour du lac et le long des rivières qui en découlent, on en apprécie tout particulièrement les alevins de 3 à $6 \mathrm{~cm}$, pêchés en hiver, de décembre à mars, d'où le nom de hio, ou hi'uo 水魚, littéralement « poisson de glace». Mais une autre raison pour cette appellation est que l'ayu à l'état d'alevin est, tout comme le gobie et l'éperlan, semi-transparent et devient blanc suite à la mort.

76. Une parfaite illustration de cette technique de pêche est donnée dans l'Ishiyama-dera engi emaki (Rouleaux illustrés de l'Histoire du monastère d'Ishiyama), œuvre datée autour de 1325.

77. Poème 264, fasc. 3 du Man'yōshū, « que composa Kakinomoto no Asomi Hitomaro quand, de la province d'Ōmi montant à la capitale, il arriva au bord de la rivière d'Uji ». Voir Man'yōshū, op. cit., vol. 1, p. 205. On en a adopté la traduction de René Sieffert (Man.yôshû, SIEFFERT, op.cit., 1997, I, p. 256-257), mais en substituant à «claies", mot par lequel il a traduit ajiroki, le terme de "palissades » qui paraît mieux correspondre au dispositif mis en œuvre dans l'Ujigawa (comme il ressort de façon patente des Rouleaux illustrés de l'histoire du monastère d'Ishiyama mentionnés dans la note précédente).

78. Ce poisson n'a pu être identifié. Un poisson de même nom figure dans le Yamato honzō (fasc. 13, f. 11), avec la prononciation shiro'uo. Il est présenté comme différent, plus petit et moins délicat que le shiro'uo précédent, écrit lui kaisangyo (voir supra, n. 62).

79. Tanakami (Tanokami) est le nom d'une localité située sur la rive gauche de la Setagawa, à $2 \mathrm{~km}$ à peine de l'endroit où celle-ci émerge du lac Biwa, à la hauteur mais de l'autre côté de la rivière, du monastère d'Ishiyama.

80. Dorome est le nom courant d'un poisson (Chasmichthys ou Chaenogobius gulosus) formant une autre espèce de la famille des Gobiidae, vivant près des côtes de Hakodate au nord, à Nagasaki au sud (voir TANAKA Shigeho, (Zōho kaitei) Nihon-san gyorui zusetsu - Figures and Descriptions of the Fishes of Japan - including Riukiu Islands, Bonin Islands, Formosa, Kurile Islands, Korea, and Southern Sakhalin, Tokyo, Kazama shobō, vol. I-XXX, 1951, p. 99-102, pl. XXIX, Fig. 114 ; HIYAMA Yoshio et YASUDA Fujirō, Nihon-san gyorui daizukan (Grand atlas des poissons produits au Japon), Tokyo, Kōdansha, 1971, p. 207, n² 283 ; JORDAN, TANAKA, SNYDER, “A Catalogue of the Fishes of Japan”, op. 
cit., 1913, p. 353, Fig. 306). Suivant la taxinomie ichthyologique moderne, dorome n'a donc rien à voir avec la truite ayu.

81. Le « poisson annuel » nengyo est en effet une autre désignation pour la truite ayu, toujours utilisée aujourd'hui.

82. Lorsqu'est venu le moment de frayer, le dos de l'ayu se couvre de taches de « rouille » et elle prend alors le nom de sabi-ayu que l'on pêchait au huitième mois dans un genre d'ajiro plus adapté à la pêche aux poissons adultes (illustré dans la pl. 3, fasc. 5, du Nippon sankai meibutsu zue).

83. Naniwa est l'ancien nom d'Ōsaka.

84. Le chirimen-zako, également prononcé chirimen-jako, ainsi que le chirimen-koayu, désignent aujourd'hui la blanchaille consistant en divers petits poissons tels le gobie, la truite ayu et toutes sortes de sardines. Après l'avoir trempé dans de l'eau bouillante salée puis étalé en plein air, le poisson prend sous le soleil un aspect ondulé qui rappelle le «crêpe » (chirimen). Les deux noms sont toujours en usage dans le Kansai.

85. Le terme employé ici est Tōbu, à savoir « l'est de Musashi ».

86. Voir Honchō shokkan, catégorie « Espèces à écailles » 3, fasc.9; HITOMI Hitsudai, SHIMADA Isao (trad. et éd.), op. cit., vol. 4, p. 287-290.

87. Hirae en Bizen n'a pas été identifié. Kuwana dans la province d'Ise est une ville du département de Mie, située au bord de l'estuaire de la Kisogawa.

88. Peut être localisé dans l'actuelle ville de Kuwana, dans le département d'Aichi.

89. Nom de lieu non identifié.

90. Lieu situé aujourd'hui dans la commune de Kisosaki, dans le district de Kuwana.

91. Nom de lieu non identifié. Il pourrait s'agir d'une confusion avec Toyodane, situé également dans la commune de Kisosaki.

92. Aujourd'hui nom d'un quartier donnant sur la baie de Mikawa, dans la ville de Nishio, département d'Aichi.

93. Cette frontière suivait à l'époque la Kisogawa qui se jette à mi-chemin entre les actuelles villes de Tōkai (département d'Aichi) et de Yokkaichi (département de Mie) dans la baie d'Ise.

94. On se rappelle ce qui avait été dit plus haut à propos de la pêche au carrelet dans l'embouchure de la Shukugawa, à Nishinomiya, ici comparé à Kuwana.

95. En l'occurrence il est probable qu'il s'agisse de Chaenogobius isaza, un genre de gobie d'eau douce endémique du lac Biwa, dont les alevins, sans avoir le moindre lien de parenté, présentent une certaine ressemblance avec ceux de la sardine. Connu par son nom japonais d'isaza, ce petit poisson de 5-8 cm de long se pêche pour la consommation locale pendant les mois d'hiver.

96. Aucun poisson de ce nom n'est actuellement répertorié.

97. Le petit (max. $12 \mathrm{~cm}$ ) wakasagi (Hypomesus olidus), parfois nommé «éperlan à petite bouche » en français, est un poisson comestible consommé au Japon. Natif des lacs et estuaires du Hokkaidō, il est répandu plus largement dans les eaux saumâtres et lacs salés du nord du Honshū, du Hokkaidō et jusqu'à la Corée du Nord.

98. Hamaguri: mollusque bivalve marin dont trois espèces (Meretrix lusoria, - lamarckii, petechialis) se trouvent couramment au Japon (voir SHIRAI Shōhei, Kai (Les coquillages), 3 vols., Hōsei daigaku shuppankyoku, coll. «Mono to ningen no bunkashi » 83, 1997, p. 757-813). La "palourde japonaise », originaire du Pacifique, a été introduite en France dans les années 1970, où, à cause de sa croissance rapide, on l'élève depuis pour la consommation.

99. C'est à la halte de Tomita, où la route du Tōkaidō traverse le bourg de Kuwana, que le voyageur pénétrait dans la province d'Ise. Ce quarante-troisième des « 53 relais du Tōkaidō" était alors célèbre pour ses palourdes grillées que le passant se faisait plaisir d'y consommer en casse-croûte (voir https://data.ukiyo-e.org/mfa/images/sc163549.jpg).

100. Clavalier, ou poivrier du Japon (Zanthoxylum piperitum), dont on utilise, au Japon, non pas les graines comme en Chine, mais les jeunes feuilles en assaisonnement. 
101. Kikurage (Auricularia auricula-judae), espèce de champignon comestible dont l'aspect rappelle vaguement celle d'une oreille.

102. Gori 鮴 (également écrit 䱃) est un des noms usuels d'un petit poisson d'eau douce de la grande famille des "gobies» (j. haze $k a$ ), semblable au shira'uo précédemment traité, et qui répond selon la région à des appellations fort différentes.

103. Voir Fig. D, à la fin du présent fascicule.

104. Tofugyo (杜夫魚, également écrit 杜父魚) est le nom chinois du chabot kajika (Cottus gobio) dont il sera question plus bas.

105. Ici écrit avec les caractères 石伏: «[qui se] cache dans les pierres ».

106. Voir Fig. E, à la fin du présent fascicule.

107. En d'autres termes, futtai correspondrait aux deux nattes cousues ensemble, et l'itaoshiki au racloir en bois.

108. Wamyōshō, fasc. 19 (Poissons), f. 8 recto, entrée 䱌: la lecture de ce caractère y est effectivement donnée comme « ishifushi ».

109. Littéralement : « qui se tapit dans les pierres [roches] ».

110. Wamyōshō, fasc. 19 (Poissons), f. 8 recto, entrée 鰎.

111. Selon NIHON GYORUI GAKKAI (éd.), Nihonsan gyomei daijiten, op. cit., p. 47, chichikaburi est le nom usuel, dans la région autour du lac Biwa où il est endémique, du "gobie flottant " (ukigori : Gymnogobius urotaenia ou isaza), mais il désigne aussi l'ishifushi ou le chabot kajika à Hirosaki, dans le département d'Aomori.

112. Wamyōshō, fasc. 19 (Poissons), f. 8 recto, entrée 䱩魚.

113. Serait-ce, comme l'affirme Chiba (сHIBA, Nippon sankai meisan meibutsu zue, op. cit., p. 139), le " gobie de lac » ou « gobie d'eau douce », yoshinobori en japonais (Rhinogobius brunneus)?

114. Gorigori serait l'onomatopée imitant le son qu'émet ce poisson exposé à l'air.

115. Sunabori-haze 砂堀魦.

116. Ce nom désigne habituellement le chabot.

117. La truite masu (Oncorhynchus masou Brevoort) est une espèce de la famille des Salmonidés.

118. Il est difficile de juger s'il faut attribuer la faute à l'auteur ou au copiste, mais la rivière qui s'écrit ici 神道川 et dont la prononciation est donnée comme Shintōgawa, ne saurait être autre que la Jinzugawa qui traverse la ville de Toyama et qui s'appelait déjà ainsi au Xvi siècle.

119. Nori-kawaami 乗川網.

120. Traduction littérale de haraaka.

121. Nihongi, également Nihon shoki : première histoire du Japon, en 30 fascicules, achevée en 720 , et attribuée au prince Toneri.

122. Douzième empereur de la dynastie de Yamato, dont le règne, légendaire, daterait de 71-130 de notre ère.

123. Il devrait s'agir de l'actuelle ville de Nagasu du district de Tamana, dans le département de Kumamoto. Pour son passage dans ce village, en l'an 88, voir Nihongi, fasc. 7 et 15.

124. Notre texte suit ici effectivement la transcription du Wamyōshō, fasc. 19, f. 3 recto, mais c'est normalement le premier caractère seul qui se prononce ainsi. Dans le Dictionary of Japanese Fish Names (NIHON GYORUI GAKKAI, op. cit., p. 47-48), les noms de haraka aussi bien que haraaka n'apparaissent qu'à propos de la vandoise japonaise (Tribolodon hakonensis Günther), ugui en japonais, petit poisson de la famille des carpes (Cyprinidae), dans certaines régions aussi connu sous le nom de akahara.

125. À savoir « œufs de ventre rouge ».

126. Il s'agit d'Inō Jakusui, déjà rencontré plus haut ( $\left.n^{\circ} 55\right)$.

127. Traduction littérale de engyo 淵魚, où le premier idéogramme signifie " eau profonde », le second « poisson ». 
128. Zacco platypus (Temminck et Schlegel) : chevesne ornithorynque, poisson d'eau douce de la famille des carpes, ressemblant à la vandoise ugui (supra $\left.\mathrm{n}^{\circ} 124\right)$, et, comme celle-ci, aussi connu dans certaines régions sous le nom de akahara ou akabara.

129. Dans ce dictionnaire, ces deux noms figurent effectivement sous une entrée séparée : Wamyō ruijyushō, fasc. 19, f. 3 recto, pour harako, et idem, f. 7 verso, pour masu.

130. Pendant la période de reproduction, de mai à août, le ventre du mâle se teint de couleur rose.

131. Dans la famille des lamproies, on distingue les deux genres de "lamproie fluviatile» (Lampetra japonica Martens) et la « lamproie de sable » (Lethenteron reissneri) qui, à la différence de la première, ne descend pas dans la mer pour frayer. C'est de ce genre dont il est question ici. Il est à noter qu'en dépit du nom japonais de yatsume-unagi, "anguille à huit yeux ", et de la ressemblance morphologique certaine, la lamproie et l'anguille appartiennent à deux classes complètement différentes. Les « huit yeux» - en réalité sept de chaque côté du vrai œil -, sont des fentes branchiales.

132. Il s'agit, bien sûr, du lac de Suwa.

133. La lamproie de sable (ou lamproie de ruisseau d'Extrême-Orient) est une espèce de petite taille (environ $20 \mathrm{~cm}$ ) vivant exclusivement en eau douce, dans certains lacs ou en amont des rivières du Japon et de la Corée méridionale. Les jeunes vivent enfouis dans le sable ou la boue. Quant à celles du lac Biwa, jadis les plus célèbres, on n'y en a plus observé depuis les années mille neuf cent soixante.

134. Ce que l'on appelle " passage des dieux » (kamiwatari) est un phénomène naturel distinctif du lac Suwa. La plupart des années ce lac gèle en effet sur toute sa surface et quelquefois sur plusieurs dizaines de centimètres d'épaisseur. La glace se dilatant et se contractant en fonction de la température, elle finit par se briser à grand fracas et à s'accumuler le long d'une ligne de fissure qui traverse le lac d'une rive à l'autre, en général suivant trois trajectoires distinctes. Le folklore local y voit le chemin que se frayent les dieux et déesses pour se rendre visite entre les deux sanctuaires supérieurs et les deux inférieurs qui, de chaque côté du lac, forment ensemble le Grand Sanctuaire de Suwa.

135. Le nom donné ici est sekihangyo, littéralement "poisson à taches [en forme de] cailloux », « rockfish » en anglais.

136. Bencao gangmu, traité de bencao (j. honzō) composé par Li Shizhen (1518-1593) vers les années 1590-1596, et considéré comme le sommet jamais atteint par les spécialistes du domaine au cours des XVII-XIX ${ }^{\mathrm{e}}$ siècles.

137. Bencao gangmu, fasc. 44, [Poissons à] écailles, Partie $4:$ «Shizhen dit que [le poisson appelé] li [鱧] a sept points sur la tête ». Le poisson dont il est ici question, écrit avec l'idéogramme 鱧 est la murène japonaise (Muraenesox cinereus), hamo en japonais.

138. Si la murène japonaise est bien un poisson serpentiforme comme la lamproie, elle appartient cependant à la famille des anguilles et ne possède nullement à côté de l'œil les sept taches noires caractéristiques de cette dernière. Ce qui n'empêche pas que l'une et l'autre soient consommées de façon fort similaire.

139. bora : mulet, aussi mule ou muge (Mugil cephalus), est un nom qui s'applique aussi bien aux familles des Mugilidae qu'aux Cyprinidae (telle la « vandoise »).

140. Matsuoka Jo'an (1668-1746), également connu sous le nom de Gentatsu, lettré-médecin, disciple du maître de pharmacopée de Kyōto Inō Jakusui, dont le nom a déjà été mentionné.

141. Nanbu est le nom du vaste territoire dévolu à l'époque féodale à la maison de même nom et qui s'étendait sur des parties des actuels départements d'Aomori, d'Akita et d'Iwate.

142. umi-unagi : nom commun du congre (Conger conger), espèce de poisson anguilliforme de la famille des congridés. 
143. L'auteur ne semble voir aucun inconvénient à accepter, dans ce cas, le nom "à huit yeux » pour un poisson qui en a neuf, alors qu'il vient de relever le fait que les Chinois de Nagasaki nomment « à neuf points » un autre qui n'en a que sept...

144. Voir Notice "Hamo (Rei)», Honchō shokkan, catégorie «Espèces à écailles » 3, fasc. 9 ; SHIMADA Isao (trad. et éd.), op. cit., vol. 4, p. 253-257.

145. Voir Notice « Yatsume unagi », SHIMADA Isao (trad. et éd.), op. cit., p. 257-258.

146. Étant entendu que la lecture sino-japonaise du composé 鮹 lui-même est shō.

147. Une belle illustration de cette façon de pêcher la pieuvre figure dans le Nippon sankai meibutsu zue, fasc. 5, Pl. 9.

148. Haritako: le manteau vidé, les tentacules embrochées et déployées, la pieuvre est tendue (haru) sur un cadre en bambou et exposé au soleil et au vent.

149. Ici zugani, ce qui semble être l'abréviation de mokuzu-gani ou de mozuku-gani: vraisemblablement Eriocheir japonicus de Haan 1835, plus connu sous le nom de « crabe chinois à mitaines ", du fait de sa ressemblance avec le "crabe de Shanghai », tous caractérisés par les longs poils qui couvrent les deux pinces, les faisant ressembler à des mitaines. Crabe de petite taille (carapace d'environ 5-12 cm), apprécié pour la saveur de sa chair et de son corail.

150. Akagamaseki est l'ancien nom de la ville portuaire de Shimonoseki tandis que Chōshū désigne la province de Nagato qui comprenait la partie occidentale et septentrionale de l'actuel département de Yamaguchi.

151. Jūya-dako, ainsi appelé à cause du rite saisonnier des «dix nuits» - du soir du cinquième jusqu'au matin du quinzième jour de la dixième lune -, lors desquelles, jadis, les fidèles de la secte Jōdo en particulier, en veillant, s'évertuaient à invoquer le nom du Buddha Amida.

152. Par branches, il faut comprendre tentacules.

153. Dans le composé rakutei 絡蹄, le deuxième caractère chinois signifie « sabot ».

154. sekidako : ce nom, dont on ignore le sens, n'est attesté dans aucune autre source, ni ancienne ni moderne.

155. Ces informations sont tirées presque verbalement du Honchō shokkan (1697), catégorie «Espèces à écailles » 3, fasc. 9. Voir Honchō shokkan, HITOMI Hitsudai, SHIMADA Isao (trad. et éd.), Tokyo, Heibonsha, coll. « Tōyō bunko », vol. 4, 1980, p. 294. Il s'agit peut-être de la pieuvre géante $\mathrm{du}$ Pacifique (Enteroctopus dofleini) vivant dans les eaux du Pacifique Nord, espèce de céphalopode dont on paraît avoir effectivement observé des spécimens avec des bras dépassant les 9 mètres de long.

156. Voir Yamato honzō, fasc. 13, f. 34 recto. À propos des histoires de pieuvres sortant de l'eau, arrachant des patates, luttant avec un serpent ou tuant chevaux et bétail, voir TONE, Tako, op. cit., p. 62-82.

157. Les rumeurs ici rapportées à propos des pieuvres géantes ont connu un retentissement digne de la frayeur que pouvait inspirer l'image de la planche 55. Au début de l'ère Meiji, par exemple, Utagawa Hiroshige III en a extrait le détail en haut à gauche, de la pieuvre attaquant les marins dans leur bateau, et fait, sous le même titre, une estampe qu'il fait figurer dans son Dainippon bussan zue (voir ici).

158. Ji Xin (mort en 204 av. notre ère), seigneur de guerre au service de Liu Bang (plus tard empereur Gaozu des Han antérieurs), et Murakami Yoshiteru (?-1333), célèbre guerrier de la fin de l'époque de Kamakura et un des neuf hommes-lige du prince Yoriyoshi, tous les deux, ayant sacrifié leur vie à la place de leur suzerain, sont cités ici en tant que parangons de loyauté.

159. La similitude avec la grappe de fleurs de glycine est frappante. Salés, les œufs de pieuvre sont encore aujourd'hui une spécialité d'Akashi (département de Hyōgo), sur la mer intérieure du Japon.

160. Ces caractères aussi se lisent actuellement "iidako». Le poulpe ocellé (aussi "pieuvre » ocellée), Octopus ocellatus (ou Amphioctopus fangsiao) est un petit mollusque abondant dans les eaux côtières peu profondes du Pacifique du nord-ouest, du Japon à la Corée et la Chine. Sa chair 
tendre est très appréciée dans toutes les cuisines de l'Asie orientale. Le caractère 飯 lu «ii » traduirait la ressemblance des granules blanches, dont tout le corps de la femelle se couvre peu de temps avant le frais, avec du « riz cuit » (ii).

161. C'est-à-dire dans le Kantō.

162. À savoir à l'est d'Edo, dans la péninsule de Bōsō, que recouvrent ces deux anciennes provinces.

163. Voir Honchō shokkan, catégorie « espèces à écailles » 3, fasc. 9 ; SHIMADA Isao (trad. et éd.), op. cit., vol. 4, p. 295-302.

164. akanishi: Rapana venosa est une espèce de mollusque gastéropode de la famille des Muricidae, originaire des mers de Chine et du Japon, qui s'est installée dans la mer Noire dans la deuxième moitié du xxe siècle. La taille de la coquille peut atteindre $15 \mathrm{~cm}$, sa couleur varie du brun-rouge au beige-orangé.

165. Aujourd'hui, le poisson appelé kajika (Cottus pollux Günther, ou Cottus japonicus) est un genre de chabot (voire de cotte ou de chaboisseau) de la famille des Cottidae, de petite taille, excédant rarement les 15 centimètres. Si la plupart des espèces de cette famille sont des poissons de mer, celui-ci est endémique d'eau douce (voir watanabe Masao, Fauna Japonica - Cottidae (Pisces), Tokyo, Biogeographical Society of Japan (Tokyo News Service), 1960, p. 119-123 ; HIYAMA \& YASUDA, Nihon-san gyorui daizukan, op. cit., 1971, p. 287, n 386).

166. Il s'agit d'une espèce d'amphibien (Buergeria buergeri) décrite pour la première fois en 1838 par Temminck et Schlegel, dans la Fauna japonica de Siebold où elle est illustrée sous le nom de Hyla buergeri. Ce nom provient de ce que l'espèce a été décrite par Heinrich Bürger (1804-1858), naturaliste allemand, assistant de Siebold à Nagasaki de 1825 à 1828. Endémique du Japon, et faute de nom plus répandu, on est réduit à l'appeler " grenouille kajika ».

167. Onko jitsuroku, également connu sous le nom de Onko nichiroku, est un traité de " poésie en chaîne » (renga) composé en 1739 par Sugimura Tomoharu.

168. 杜父魚: ces caractères se lisent alternativement tofugyo, kakubutsu ou kajika. Ce serait le nom chinois pour ce dernier.

169. Le texte original note八目 (yatsume), mais il est plus que probable que ce soit une erreur du graveur pour八月 (hachigatsu) comme nous l'avons traduit ici.

170. Voir ci-dessus le paragraphe sur les gobies (gori).

171. Le Sancai tuhui ou Encyclopédie illustrée des trois règnes (Ciel, Terre, Homme) est un ouvrage chinois en 106 fascicules, paru en 1609 sous la dynastie des Ming. C'est en prenant ce livre comme modèle que Terajima Ryōan a composé en 1712 son Wakan sansai zue ou Encyclopédie sino-japonaise des trois règnes, en 105 fascicules. C'est de cette dernière dont il est question ici. Les noms gori et ishifushi apparaissent dans le fascicule 48 (Wakan sansai zue, op. cit., volume jō, p. 534-540).

172. Pour comprendre cette allusion, il convient de se référer à la notice ci-dessus consacrée au poisson appelé gori.

173. Fuboku wakashō, également désigné dans le livre par Fubokushū. Recueil de plus de 17350 poèmes japonais (waka) édité en 1310 par Fujiwara no Nagakiyo.

174. Fascicule 24, poème $n^{\circ} 10864$. Fuboku wakashō (Sélection de poésie japonaise), Fujiwara Nagakiyo, Kunaichō shoryōbu (éd.), coll. «Zushoryō sōkan », vol. 4, Tokyo, Meiji shoin, 1987, p. 11.

175. L'empereur Jomei (r. 629-641) résida de 630 à 636 dans le palais d'Okamoto, à Asuka, dans le Yamato.

176. Poème 487, fasc. 4, Man'yōshū, op. cit., vol. 1, p. 327. Notre traduction est suivie, sur chaque ligne, de celle, entre guillemets, de Sieffert. Voir Man.yôshû, SIEFFERT, op. cit., 1998, p. 13.

177. Man'yō daishōki (Étude du Man'yōshū continuée à la place de son auteur), commentaire du Man'yōshū composé par Keichū (1640-1701) et achevé en 1690.

178. Tel que dans le poème $n^{\circ} 3284$ du Man'yōshū, par exemple.

179. 河鹿 : c'est avec ces caractères signifiant « daim de rivière » que s'écrit le nom kajika. 
180. Traité de poétique daté des années 1210 de Kamo no Chōmei (1155-1216).

181. La traduction est tirée de : Kamo no Chômei Notes sans titre: Mumyôshô : Propos sur les poètes et la poésie. Traduit du japonais et annoté par le Groupe Koten, Paris, Le Bruit du temps, 2010, p. 73.

182. komadori : parent japonais (Erithacus akahige) du rouge-gorge familier d'Europe.

183. Mozu : pie-grièche bucéphale (Lanius Bucephalus Temminck \& Schlegel 1847) est une espèce de passereau endémique de l'Asie du nord-est (Japon, Chine, Russie, Corée) connue pour les sons de « kiou » courts et rauques qu'émet le mâle à trois ou quatre reprises.

184. Un des noms locaux de kajika. Voir plus loin.

185. Kamo no Mabuchi (1697-1769), savant du mouvement dit des "études nationales", s'adonna, à l'instar de son disciple Motoori Norinaga, à l'étude de la poésie et à la philologie de la langue ancienne, rédigeant des commentaires notamment sur les norito, le Man'yōshū ainsi que sur le Kokin wakashū. Le Kokin wakashū uchigiki mentionné ici est un commentaire du Kokin wakashū.

186. Tanikugu : littéralement qui «se cache dans les creux». Si le nom de ce batracien est bien transcrit tanikugu ici, Péronny (P ÉRONNY Claude, Les animaux du Man.yô-shû, Paris, Collège de France - Institut des Hautes Études Japonaises, 2007, p. 151-153) ne semble pas avoir tort en transcrivant taniguku, puisque c'est ainsi qu'il est prononcé aussi bien dans le Man'yōshū que dans l'usage actuel. Le texte ne fait manifestement pas la différence, mais ce nom désigne bien le « crapaud» (Bufo bufo japonicus) et non pas la grenouille.

187. Affluent de la Yamatogawa, la rivière Saho traverse le territoire de la ville et ancienne capitale de Nara et son nom apparaît de ce fait souvent dans la poésie du viII ${ }^{\mathrm{e}}$ siècle.

188. Poème 1004, fasc. 6, de Kuratsukuri no suguri Masuhito. Voir Man'yōshū, op. cit., vol. 2, p. 64 ainsi que la traduction de Sieffert, Man.yôshû, SIEFFERT, op.cit., 1998, p. 335.

189. Poème 2265, anonyme, fasc. 10, du Man'yōshū. Voir Man'yōshū, op. cit., vol. 2, p. 529 et Man.yôshû, SIEFFERT, op.cit., 2003, p. 100, dont la traduction diffère sur plusieurs points.

190. Gosenshū : titre abrégé de Gosen wakashū (Recueil de poèmes sélectionnés postérieurement), anthologie de poésie japonaise, compilée sur ordre impérial entre 951 et 958 , en 20 fascicules, par Ōnakatomi no Yoshinobu, Kiyohara no Motosuke, Minamoto no Shitagō, Ki no Tokibumi, et Sakanoue no Mochiki.

191. Poème 1123, anonyme, fasc. 7, du Man'yōshū dont on suit la traduction de Sieffert, Man.yôshû, SIEFFERT, op.cit., 2001, p. 31. Voir aussi Man'yōshū, op. cit., vol. 2, p. 124.

192. Poème 1868, fasc. 10, Man'yōshū, op. cit., vol.2, p. 430, et Man.yôshû, SIEFFERT, op.cit., 2003, p. 19.

193. Poème 1435, fasc. 8, Man'yōshū, op. cit., vol. 2, p. 231 et Man.yôshû, SIEFFERT, op.cit., 2001, p. 163. 194. Poème 1105, fasc. 7, Man'yōshū, op. cit., vol. 2, p. 119, et Man.yôshû, SIEFFERT, op.cit., 2001, p. 23. Le bassin de Mutsuta, aujourd'hui appelé Muda, est un élargissement de la Yoshinogawa, près de la ville de Yoshino (département de Nara). Tous ces cours d'eau ici nommés se situent dans le bassin de Nara.

195. Il s'agit du Kokin waka rokujō (Poèmes anciens et modernes en six livres).

196. Tachibana no Sai (??-737), dit prince Sai.

197. Il s'agit du poème $\mathrm{n}^{\circ} 1004 \mathrm{du}$ fascicule 6 , cité plus haut.

198. Sunadojō, espèce marine de la famille des loches (Misgurnus anguillicaudatus). Pour ces dernières, voir HIYAMA et YASUDA, Nihon-san gyorui daizukan, op. cit., 1971, p. 74, nº 110.

199. Le « chabot » (voir supra).

200. Il s'agit probablement $\mathrm{du}$ petit poisson d'eau douce communément appelée donko (Odontobutis, Eleotris, ou Mogurnda obscura, selon SIEBOLD, TEMMINCK and SCHLEGEL, Fauna Japonica, 1845), un genre de gobie endémique du Japon (plus particulièrement des rivières du Kansai), de la Corée et de la Chine (voir HIYAMA \& YASUDA, Nihon-san gyorui daizukan, op. cit., p. 205, n²78). Sa chair blanche et délicate est appréciée frite ou grillée. Le Rijksmuseum voor Volkenkunde de 
Leiden en possède une représentation $(34,450 \mathrm{~cm})$ de la main du peintre Kawahara Keiga (1786-1860), destinée à figurer dans la Fauna Japonica (1838) de Siebold, voir NAGASAKI SHIRITSU HAKUBUTSUKAN, Hizō kapitan no Edo korekushon: Oranda jin no Nihon shumi (Collection secrète des directeurs des comptoirs de la VOC: le goût hollandais pour les choses japonaises), Nichiran kōryū 400 shūnen kinen, Nagasaki, Nagasaki shiritsu hakubutsukan, 2000, p. 139. Voir aussi ici.

201. Un des très nombreux noms locaux du donko (note précédente).

202. Probablement une erreur pour « 2 pouces ", les espèces de gobie ne dépassant en général guère les $15-16 \mathrm{~cm}$.

203. Par miko, on entend soit les chamanes ou autres diseuses de bonne aventure, itinérantes le plus souvent, soit les jeunes filles exécutant les danses rituelles dans les sanctuaires shintō ; vu que celles-ci sont vêtues pour l'occasion de longues jupes rouges, c'est à elles que le nom du poisson semble faire référence.

204. Saga désigne une région, aujourd'hui incluse dans la ville de Kyōto, mais anciennement située à la périphérie, au nord-ouest de la capitale.

205. Kōsōgyo : 黄頚魚; quant à ōshigyo 鰪絲魚, cette graphie est introuvable.

206. Kakubutsu est la lecture proposée aujourd'hui pour le poisson du nom de 杜父魚.

207. On note que l'arare-uo est aujourd'hui identifié au tofugyo, aussi appelé kakubutsu (Cottus pollux). Ce qui est certain c'est qu'il appartient à la famille des gobies.

208. Okoze: Inimicus japonicus, genre de poisson de la même famille que le poisson-pierre (Synanceiidae) ; apparenté à la rascasse, équipé d'aiguillons venimeux.

209. Le terme kochi désigne aujourd'hui sans distinction les poissons à la forme aplatie, dotés de grandes nageoires, et vivant dans les fonds marins. En supposant que ce kochi ait un minimum de ressemblance ou de points communs avec les autres poissons évoqués dans cette rubrique, l'identification la plus probable semble être avec Platicephalus spinosus (SIEBOLD, TEMMINCK and SCHLEGEL, Fauna Japonica), la plus petite (une petite dizaine de $\mathrm{cm}$ ) des nombreuses espèces de la famille des platicéphalidés ou «poissons-crocodiles». Selon la nomenclature actuelle, il correspondrait à l'oni-gochi (Onigocia spinosa). 


\section{Fascicule 5}

\section{NOTE DE L'ÉDITEUR}

Ce sommaire, placé en début de fascicule par l'auteur du Meisan zue, fait partie du texte d'origine. Ce sommaire n'a pas vocation à être utilisé pour circuler à l'intérieur du Meisan zue. Pour ce faire, le lecteur pourra utiliser toutes les fonctionnalités propres à l'édition en ligne de la plateforme OpenEdition Books, notamment sa table des matières générale.

\section{Sommaire du fascicule 5}

- Méduse de Bizen

- Chaux d'ōmi

- Céramique d'Imari

- Toile d'Echigo

- Otarie de Matsumae

- Laminaire, la flûte de Kosa

- Entrée dans le port des bateaux chinois

- Débarquement de la divinité Bosa

- Bateaux hollandais

\section{[Produits de la pêche : suite]}

\section{Méduse ${ }^{1}$}

Autres noms : le « seigneur aux yeux d'emprunt "; langue de mer

On en récolte dans de nombreuses provinces mais Bizen ${ }^{2}$ en a fait sa spécialité. Celles que l'on appelle «méduse de Chine » ou «méduse de Corée » sont produites à Hizen On leur donnait ce nom à l'origine car elles étaient importées à Nagasaki. Aujourd'hui on sait les produire dans notre pays mais on continue à les appeler «méduses de Chine ». Voici comment on procède : on les fait macérer et blanchir dans de la chaux et 
de l'alun pour éliminer le sang. Leur couleur devient alors d'une blancheur immaculée. À Bizen, on passe rapidement au feu des feuilles de chêne qu'on pile ensuite dans un mortier, et auxquelles on ajoute de l'eau salée. On y met alors à tremper les méduses. Il existe plusieurs autres espèces de méduse. Parmi ces dernières, les «méduses d'eau " mizukurage, ou celles qui sont de couleur noire ou rouge, contiennent du poison et les pêcheurs ne les prennent pas.

Pour ce qui est de la forme, elles ont l'air d'être enveloppées d'une feuille de lotus bordée de quelque chose qui ressemble à des «pattes». Elles ont une teinte rouge violette, elles n'ont ni yeux ni bouche. Elles ont sous le ventre des sortes de filaments ou d'ouate en forme de traîne. Les crevettes s'y agglutinent immanquablement. On raconte chez nous que les méduses se servent de leurs yeux pour nager. C'est pourquoi on les appelle "seigneurs aux yeux d'emprunt ». Les plus grosses ont la taille d'un baquet, les plus petites celle d'un plateau. Leur goût est fade et on les consomme en les assaisonnant de gingembre ou encore de vinaigre. Vivant généralement dans les eaux boueuses, elles sont nombreuses dans les provinces de Chikuzen ${ }^{4}$ et de Bizen et sont rares à Edo. On les pêche depuis les bateaux aux neuvième et dixième mois à l'aide d'épuisettes lorsqu'elles flottent à la surface de l'eau. Lorsque la mer est agitée, elles sont parfois rejetées sur les plages [voir Pl. 57].

\section{PI. 57 : Méduse de Bizen}

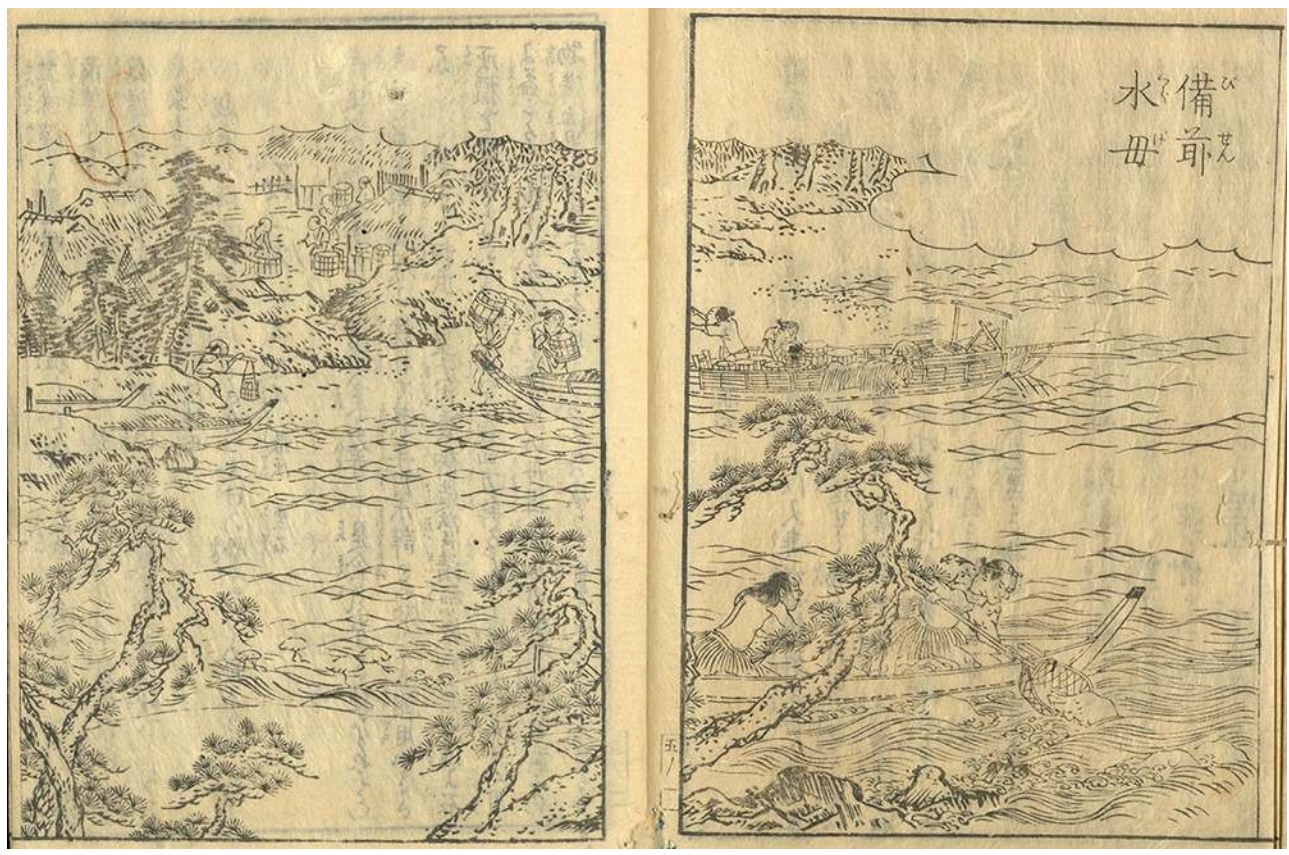

National Institute of Japanese Literature. Lien vers l'illustration

Poème de Minamoto no Nakamasa ${ }^{5}$ dans le Fuboku wakashō (Sélection de poésie japonaise) ${ }^{6}$

Je me consume d'amour passant le temps à attendre

la lune de mer

Dans l'espoir de voir une nuit

Un os de méduse ${ }^{7}$ 


\section{Chaux de pierre ${ }^{8}$}

Autres noms : cendre pour teindre; poudre de cendre ; aseki

4 De nos jours, celle d'Ōmi ${ }^{9}$ est considérée comme de qualité supérieure. Celle de Mino ${ }^{10}$ est d'une qualité équivalente. C'est parce que la terre n'y contient pas de fer. À l'origine, c'est à Yoshino ${ }^{11}$ et à Takahara ${ }^{12}$ dans la province de Yamato ${ }^{13}$ qu'on a commencé à la calciner. On ne peut en dater exactement le début mais son usage est très ancien dans notre empire. Lorsqu'il fonda le palais impérial [de Heiankyō], l'empereur Kanmu ${ }^{14}$ avait fait enduire de chaux une estrade située à proximité du siège impérial dans le pavillon de Pureté et de Fraîcheur ${ }^{15}$ et en avait fait le siège en terre pour accomplir le rite de la salutation des quatre directions ${ }^{16}$. En dehors de cela, elle procure d'infinis services à l'homme. On en recouvre des dizaines de milliers de bateaux et de rames, des quantités de clôtures ; c'est indispensable pour toutes choses contenant de l'eau, des canaux aux ustensiles. C'est un véritable don du ciel. Comme dit le dicton: "à cent lieues ${ }^{17}$ d'une capitale, on trouvera toujours un endroit où l'on extrait la pierre à chaux $»^{18}$.

5 Ce que l'on brûle dans la province d'Ōmi, vers le mont Ibuki ${ }^{19}$, ou encore à Ishibe ${ }^{20}$, c'est de la pierre verte aoishi. Dans la province de Yamashiro ${ }^{21}$, à Kurama ${ }^{22}$, on brûle la pierre de couleur nuit, de qualité inférieure. La pierre de couleur bleue laiteuse se classe derrière celles-ci. On n'extrait que les pierres qui se trouvent à 2 à 3 pieds $^{23} \mathrm{de}$ profondeur. On n'utilise pas celles qui prennent un aspect voilé fümu lorsqu'elles sont extraites. Sarachiyama ${ }^{24}$, au pied du mont Ibuki, est complètement couvert de pierre verte. La pierre marbrée shimasuji est considérée comme de qualité inférieure.

6 Pour l'extraire, on brise la terre avec un coin et on provoque l'effondrement de la montagne sur une hauteur d'environ deux cents travées ${ }^{25}$ à l'aide de leviers et de bouts de bois. En général, la pierre atterrit en morceaux. Ce qui n'est pas brisé n'est pas utilisé. On le charge sur des bateaux pour traverser la rivière Yabu ${ }^{26}$ [voir Pl. 58]. 


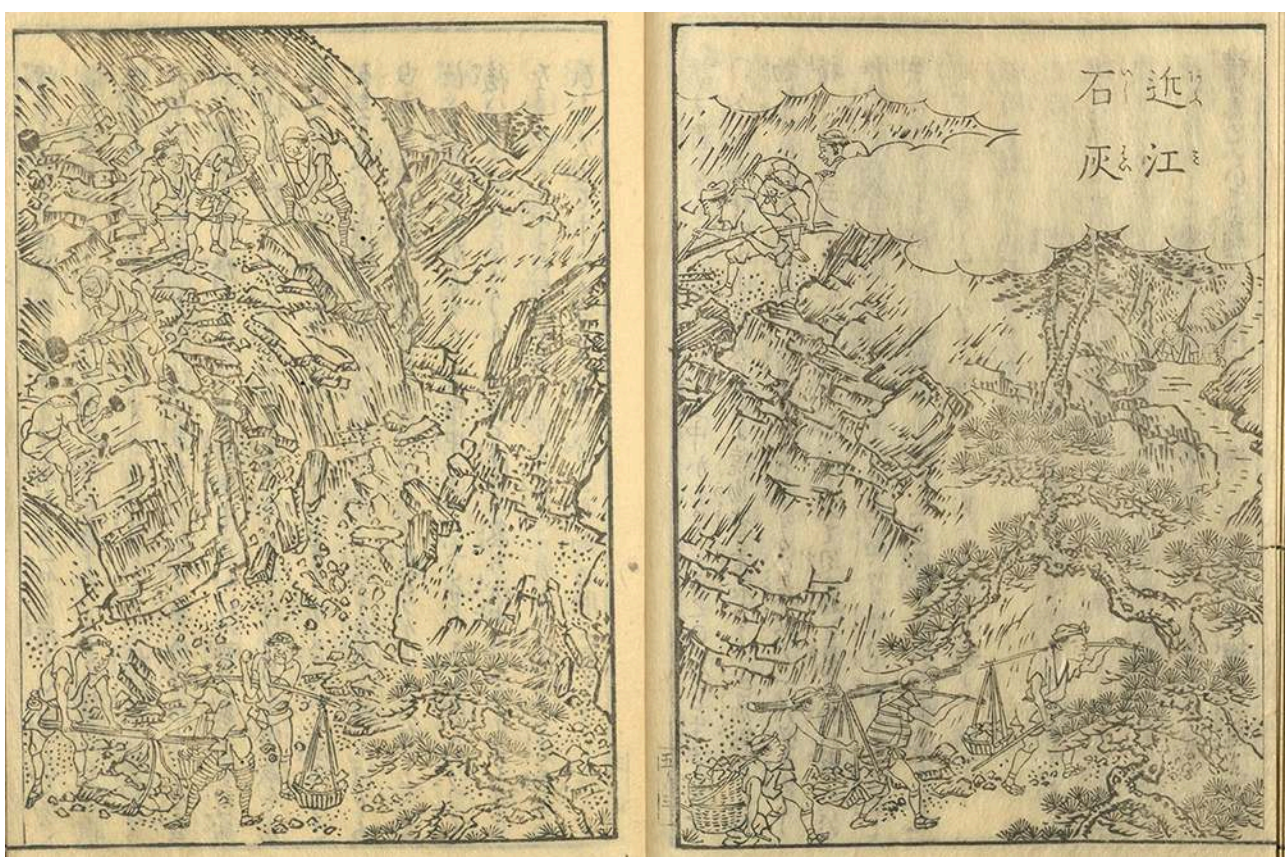

National Institute of Japanese Literature. Lien vers l'illustration

7 La chaux obtenue en brûlant les coquilles d'huîtres est de moindre qualité.

8 Pour la calcination, on utilisera un four d'une hauteur de 3 pieds, d'une circonférence d'environ quatre travées. Il est fait en terre de rizière. Un orifice est prévu au pied du four pour laisser entrer l'air. Tout d'abord, on casse derechef la pierre et on remplit le four entièrement. Au-dessus, on étale du charbon, puis on introduit le feu. On s'assure que la chaleur a gagné le fond du four, puis on éteint le feu. On retire les cendres et on répète plusieurs fois l'opération.

Les fours [à chaux] de Mino sont différents. On l'appelle four en forme de tour (yagura gama). Il est haut d'une toise ${ }^{27}$ avec un diamètre/circonférence de 3 pieds. Il se rétrécit vers le bas. C'est pour que la cendre qui a été placée en entonnoir tombe automatiquement, une fois consumée, au fond de la cavité. On dispose en alternance une couche de pierre et une couche de charbon, on introduit le feu par le bas et on fait monter la chaleur. On ramasse au fur et à mesure par un orifice latéral ce qui est calciné et tombé au fond. De cette façon, on ajoute des nouvelles couches de pierre et de charbon par le haut et la calcination se poursuit sans interruption durant une centaine de jours. C'est la même méthode que celle utilisée en Chine [voir Pl. 59]. Cependant, on n'allume pas le four en été et en hiver. Si après calcination, on laisse [le produit] à l'air libre durant vingt jours, il baigne dans sa chaleur, et se transforme spontanément en poudre sous l'effet du vent ${ }^{28}$. Quand on veut s'en servir tout de suite, on jette de l'eau et cette matière se désagrège instantanément. Cependant, si on opte pour la chaux aérienne, on remplira des ballots de paille avec cette matière, on les exposera dans un endroit aéré et si on les garde assez longtemps, alors, progressivement, la matière verra son poids augmenter et son volume doubler, si bien que les ballots qui étaient lâches au début se tendront à craquer. On dit que la chaux s'humidifie $^{29}$. Ainsi, on laisse s'écouler un an avant de livrer chaque production au marché. Une fois réduite à cet état, la chaux craint beaucoup l'eau. Si on verse de l'eau, elle chauffe aussitôt et on en perd le contrôle. C'est pourquoi on la surveille de près 
dans les bateaux et si on la transporte à dos de bœuf, les bergers gardent toujours une faux sur eux, pour pouvoir rompre aisément les cordes nouées et éviter ainsi qu'une pluie inattendue ne provoque le feu et ne blesse les bêtes.

\section{PI. 59 : Fours à chaux « en forme de tour » de Mino - Four à chaux d'Ōmi}

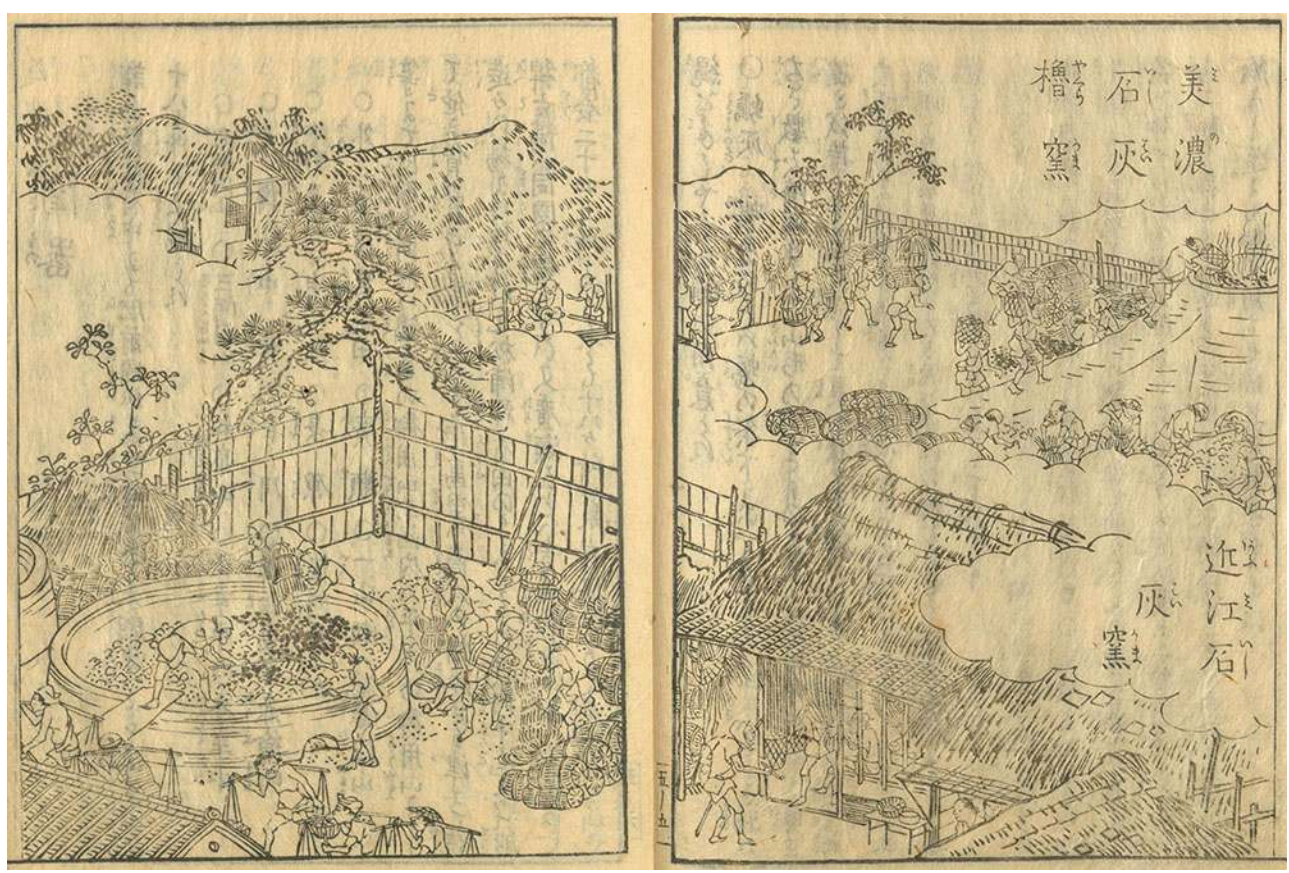

National Institute of Japanese Literature. Lien vers l'illustration

Chaux d'huître : Pour les «chambres d'huîtres » reibō, comme on l'a déjà dit dans le fascicule des huitres ${ }^{30}$, les plus anciennes s'étendent sur plusieurs toises et certaines prennent la forme de collines bosselées. Les habitants des bords de mer ramassent les [coquilles d'huîtres] dans l'eau, munis d'un ciseau et d'un maillet et les font brûler (les huîtres vendues en pharmacie comme médicament sont des coquilles pulvérisées). La chaux utilisée à Ōsaka est en général de ce type, on n'y emploie que rarement la chaux de pierre. C'est pourquoi lorsqu'un marchand de chaux indique sur son enseigne "véritable chaux de pierre", il s'agit de produits de la province d'Ōmi. Le mode de calcination n'est pas différent. Cependant, calciner des palourdes et des conques produit une chaux de qualité très inférieure.

11 Usages de la chaux: Pour renforcer les jointures des bateaux, on imbibe d'huile de paulownia et d'huile de poisson de la soie épaisse et un tissu en soie fine ${ }^{31}$, et on frappe un millier de fois ${ }^{32}$. Pour construire des clôtures et des murs en pierre, on passe d'abord la soie au tamis pour éliminer les grumeaux, on l'imbibe d'eau, on la pétrit et on y ajoute de l'huile. Pour en enduire les murailles, on rajoute des fibres de papier et de paille kamisusa. Pour les réservoirs d'eau, on prend une portion de chaux pour deux portions de sable de rivière passé au tamis pour éliminer les boules de terre, on le délaie avec de l'eau et on le pétrit avant de les fabriquer. Ils sont alors solides, résistants, et ne se corrodent pas. Par ailleurs, on ajoute également de la chaux pour fabriquer des rideaux de séparation ${ }^{33}$ ou des cloisons en papier. Il est impossible d'énumérer tous les usages possibles. 


\section{Céramique $^{34}$}

12 Parmi les nombreuses sortes de céramiques que l'on produit dans les provinces, la céramique d'Imari de la province de Hizen est réputée pour être la meilleure de notre pays. Les quelques dix-huit ateliers que compte ce site de production ont une réputation d'excellence ${ }^{35}$ : la colline d'ōkawachi ${ }^{36}$, celle de Mikawachi ${ }^{37}$, Izumi ${ }^{38}$, Kankōhira, Honkōhira, Ōtaru, Nakataru, Shirakawa, Hiekoba, Akaemachi ${ }^{39}$, Nakanohara, Iwaya, Nagahara, Minamikawara (four du haut et four du bas), Hokao, Kuromuta, Hirose, Ichinose, la colline d'ōhō, etc.

13 Parmi ces derniers, Ōkawachi est l'atelier attitré des Nabeshima ${ }^{40}$; de même, Mikawachi est l'officine du fief de Hirado ${ }^{41}$. Il est défendu de vendre la production de ces ateliers ailleurs. Imari ${ }^{42}$ est un port où convergent les marchands. Ce n'est pas un site de fabrication. La plupart des ateliers sont situés à Arita $^{43}$, dans le district de Matsura. Parmi ces derniers, Nakao, Mitsunomata, Hiekoba sont situés dans la même province de Hizen mais sont rattachés à d'autres domaines. À Hirose, on trouve beaucoup de grès à couverte verte ${ }^{44}$ mais pas de produits de qualité supérieure. Cela fait en tout vingtquatre à vingt-cinq ateliers, dont dix-huit qui jouxtent le mont Izumi, et c'est de cette colline qu'on extrait l'argile.

\section{Terre blanche (shiratsuchi) ${ }^{45}$}

On en extrait à Izumi et elle est renommée dans tout le pays. Elle n'a pas sa pareille dans l'empire. En Chine, on en extrait à cinq ou six endroits différents dans tout le pays $^{46}$. C'est de la terre sans être tout à fait de la terre. C'est de la pierre sans en être tout à fait. La terre blanche a cette particularité d'être extrêmement dure. On la concasse avec un ciseau puis on la pile dans un mortier chinois hydraulique équipé de pilons métalliques (le pilon ${ }^{47}$ a 1 pied environ de largeur, 1 pied et $5-6$ pouces ${ }^{48}$ d'épaisseur, la longueur est d'une travée ${ }^{49}$ et demi environ). Diriger une grande quantité d'eau permet d'activer un plus grand nombre de mortiers. Une fois réduite en poudre, on incorpore deux trois autres sortes d'argile tendre, puis le mélange sera mis à tremper dans un bassin dans l'enceinte de l'habitation. On mélange plusieurs fois et lorsqu'il est homogène, on le passe au tamis ; puis on le transvase dans un autre bassin et on le laisse bien décanter. Ce qui flotte fournit la matière la plus fine. Ce qui est au milieu est employé pour des produits d'usage courant de qualité supérieure. Ce qui se dépose au fond est retiré et n'est pas utilisé. On plaque cette matière obtenue par lévigation (suihi) ${ }^{50}$ contre les parois extérieures du four à biscuit et on se sert de la chaleur du four pour la faire sécher. Au moment propice, on la racle ; à nouveau, on l'imbibe d'une eau pure, puis on la pétrit en forme de pains. On les donne aux potiers. Jusqu'ici, c'était le travail des femmes de la maison [voir Pl. 60]. 


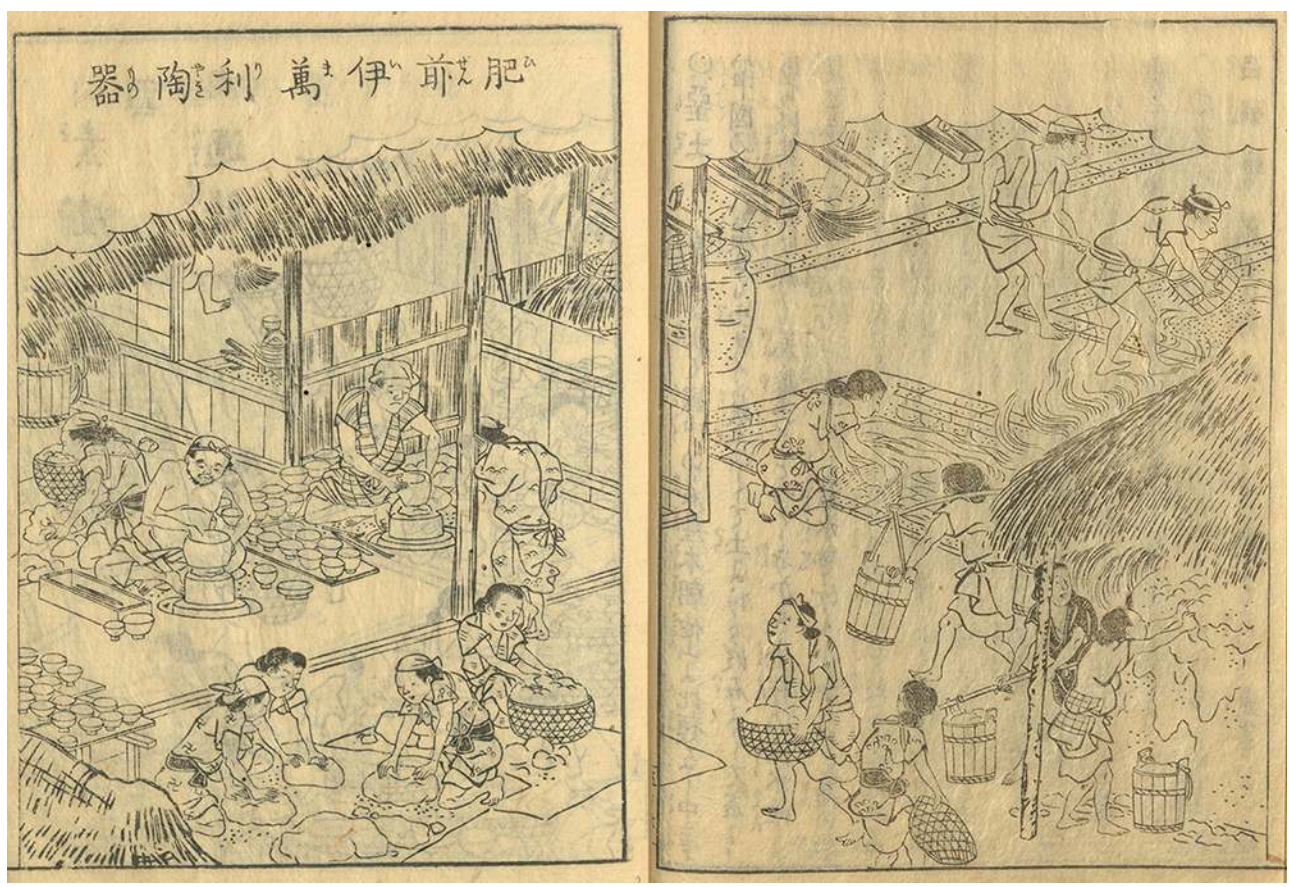

National Institute of Japanese Literature. Lien vers l'illustration

\section{Fabrication des récipients}

D’une manière générale, on distingue deux façons de fabriquer des récipients.

Il y a d'une part les récipients obtenus en " pressant des moules » kataoshi. Entrent dans cette catégorie de nombreux récipients ronds ou carrés, comme par exemple les vases, jarres, brûle-parfum, mais aussi paravents et bougeoirs ${ }^{51}$. Pour ces derniers, on les modèle grossièrement, on les divise ou on les coupe en deux, on pétrit à nouveau cette argile blanche qu'on presse dans le moule. On peut aussi presser le moule contre l'argile. Par ailleurs, la même argile mélangée à de l'« eau de rouille » est utilisée pour enduire les deux moitiés et les coller.

Il y a d'autre part les "récipients ronds ", par quoi on entend les millions de coupes et d'assiettes, petites ou grandes, utilisés au quotidien, qui constituent les neuf dixièmes de la production. Pour façonner ces récipients ronds, il faut d'abord fabriquer un tour (de potier). Il comporte deux disques, placés l'un au-dessus de l'autre. Celui du bas est un peu plus grand. Comme axe du tour, un pieu en bois ${ }^{52}$ est fiché dans le sol à 3 pieds de profondeur et de manière à ce qu'il dépasse de 2 pieds. On façonne [les récipients] en posant l'argile au centre de la roue supérieure. L'artisan actionne avec son pied la roue inférieure qui continue à tourner toute seule pendant un certain temps. Par la pression de ses deux mains, il fait monter l'argile posée sur le disque supérieur. Sans effort, ses doigts pénètrent à l'intérieur du récipient et, tandis que la roue tourne, à l'aide de son pouce placé au fond du récipient, le potier sculpte, à son gré, des modèles variés. Grâce à sa dextérité prodigieuse, il produit en un clin d'œil des quantités de pièces. Des dizaines de milliers de spécimens sont ainsi fabriqués sans la moindre erreur quant à leurs dimensions comme si elles sortaient du même moule. Pour fixer la base des bols et des jattes, il faut les laisser un peu sécher avant de les remettre sur le tour, et façonner 
avec une spatule l'extérieur et l'intérieur de la base. C'est à ce moment qu'on corrigera les fêlures. Pour ce qui est des anses et des becs, ils sont confectionnés séparément et on les fixe avec de l'argile. Enfin, on les met à sécher à l'ombre ${ }^{53}$ jusqu'à ce qu'ils deviennent complètement blancs, puis on les introduit dans le four à biscuit.

Le four à biscuit ressemble, comme le montre l'image, à une chambre de fermentation. On empile les pièces de poterie à l'intérieur. L'ouverture pour le combustible se trouve sur un côté, on utilise des bûches. Quand la bonne chaleur est atteinte, on arrête le feu et on laisse bien refroidir.

On trace les cercles et on peint les motifs puis on enfourne les pièces à nouveau.

Quand les biscuits sont suffisamment refroidis, on les retire du four. On les rince une fois à l'eau, on les essuie et on les frotte avec un chiffon en coton. Pour tracer les cercles, en haut, en bas, à l'intérieur comme à l'extérieur, sur les bols et les jattes, on les pose à nouveau sur le tour, on place le pinceau à l'endroit souhaité puis on fait tourner. On applique les inscriptions et les dessins avant de les recouvrir de deux couches de couverte puis on les met à sécher. On les place dans le four principal pour la cuisson, et à la sortie du feu, les motifs apparaissent. On les sort du four et on les rince une nouvelle fois à l'eau, ce qui parachève le processus. La moindre petite assiette doit, entre le moment où on extrait la terre et celui où elle est achevée, passer par soixantedouze manipulations. Les infimes détails et les étapes sont tellement nombreux qu'on ne peut les énumérer tous ici [voir Pl. 61].

\section{PI. 61 : Céramique d'Imari : four à biscuit ; application de la couverte ; traçage des cercles}

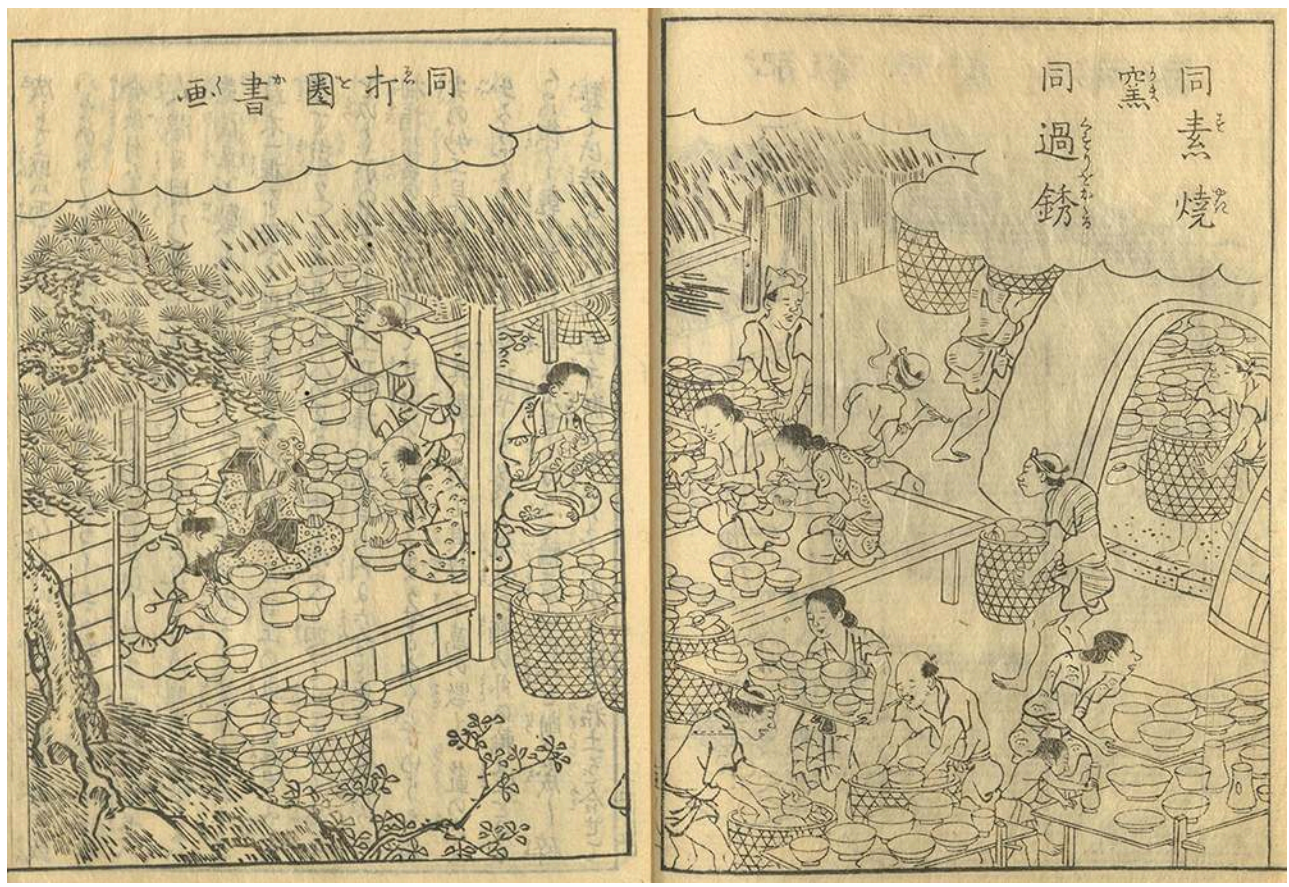

National Institute of Japanese Literature. Lien vers l'illustration

Le four à biscuit se trouve dans l'enceinte de l'habitation. Le four principal est construit à flanc de colline et on n'en trouve jamais en terrain plat. Les chambres du four sont disposées en enfilade, avec une chambre par niveau. L'intérieur est large d'environ 30 tsubo ${ }^{54}$. On en juxtapose jusqu'à six, et on prévoit à chaque jonction une fenêtre pour laisser passer la chaleur. Le feu est cependant alimenté au niveau de chaque four. À 
l'intérieur, il y a des étagères pour poser les récipients. Ces étagères sont en terre. Les récipients sont posés un à un, sans laisser le moindre espace entre eux. On laisse libre une bande étroite sur le côté, pour y placer les bûches. Les bouches de four font 8 pouces de côté sur une hauteur de 2 pieds. Le feu est entretenu durant trois à quatre jours, sans interruption, et on consomme jusqu'à vingt mille bûches par four. Il y a cependant un art d'alimenter le feu et les tarifs de l'ouvrier varient selon ses capacités (il faut veiller à ce que les bûches enfournées ne s'entassent pas). Par ailleurs, il y a, à côté de l'entrée, une trappe de la taille d'une balle à jouer. On en ôte le bouchon de temps en temps pour évaluer la chaleur. Lorsque la cuisson est achevée, on éteint le feu, et on laisse bien refroidir, puis on sort les récipients du four. La production d'une seule fournée peut atteindre cent ballots ${ }^{55}$ [voir Pl. 62].

\section{PI. 62 : Céramique d'Imari : four principal}

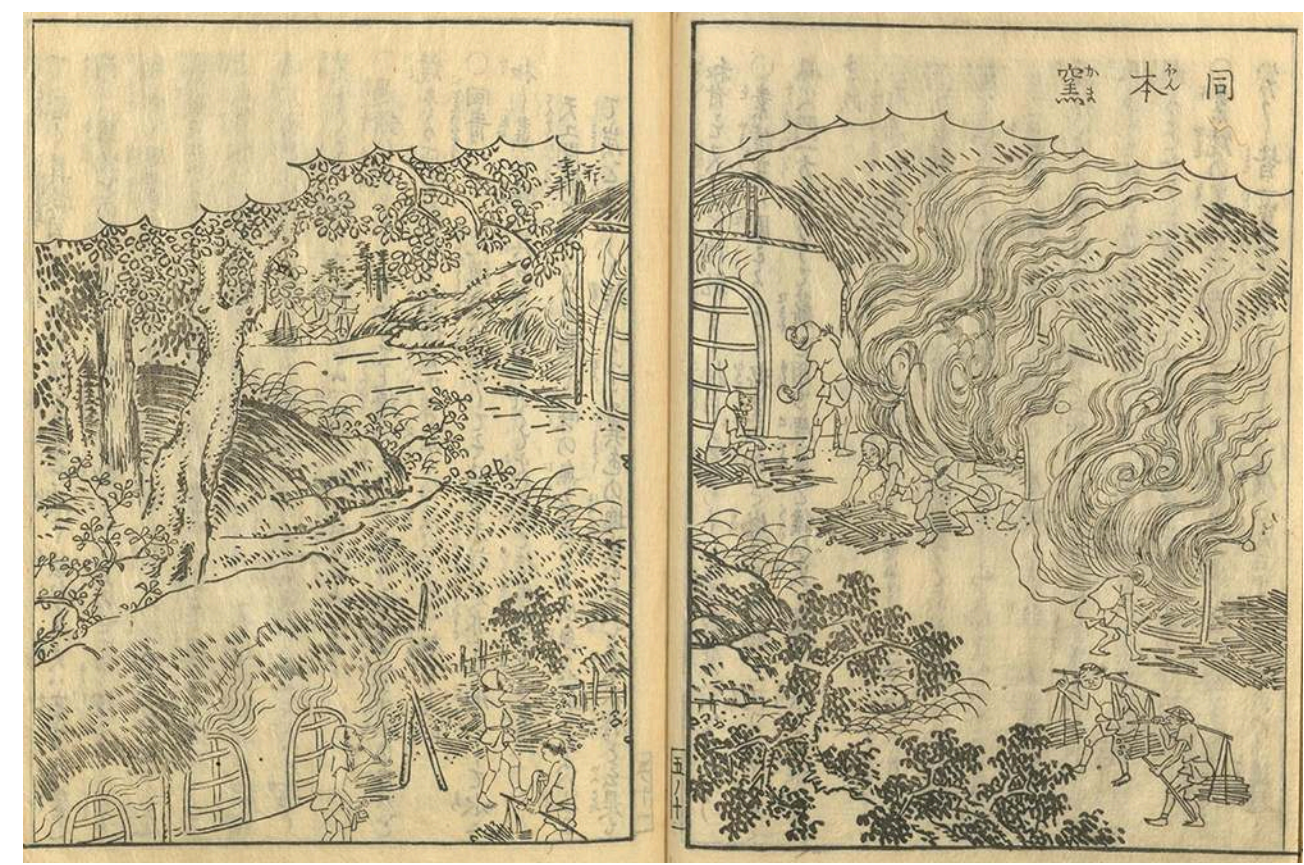

National Institute of Japanese Literature. Lien vers l'illustration

Pour préparer la couverte, on prend la même argile, prélevée à la surface de l'eau, là où elle est la plus claire et de qualité supérieure. On y incorpore de la cendre obtenue en brûlant de l'écorce de l'arbre yushinoki $i^{56}$. Chaque maison a cependant son propre dosage et sa propre composition et il n'y a pas de recette unique.

À l'origine, l'émail bleu était préparé à partir d'un produit importé de Chine de nature inconnue. Il faut le réduire en fine poudre et le délayer dans l'eau. L'émail est tout noir au moment où on l'applique mais après cuisson il vire au bleu vert.

Le Tiangong kaiwu (Exploitation des œuvres de la nature) ${ }^{57}$ indique qu'il s'agit dans tous les cas de produits de la catégorie des «merveilles sans nom » mumyōi $i^{58}$. [D'après ce que nous savons], on trouve dans les montagnes, aux endroits où l'on a fait longtemps brûler du charbon, des concrétions à l'aspect étrange. On les connaît sous le nom de " résine d'arbre médicinal ». C'est un autre nom pour la «merveille sans nom ». On trouve un produit du même nom dans les mines d'argent de la province d'Iwami ${ }^{59}$, mais il ne s'agit pas de la même chose. On l'obtient en lévigeant une poudre violette que l'on 
trouve dans la terre. Elle n'est utilisée que pour arrêter les saignements. Il y a beaucoup de contrefaçons. Pour la «merveille sans nom » dont il est ici question, [le Tiangong kaiwu nous dit qu'] elle se forme à la surface du sol et non en profondeur. Il ne faut pas la chercher à plus de 3 pieds sous terre. Les experts distinguent entre les trois qualités supérieure, moyenne et inférieure. Le produit de qualité supérieure donne à la sortie du four un bleu vif comme le plumage du martin-pêcheur. Celui de qualité moyenne donne une couleur bleu pâle ${ }^{60}$. À l'origine, les produits importés étaient considérés comme de qualité supérieure. Les plus grosses pépites pèsent à peine $1 b u^{61}$. Les plus petites sont fines comme du sable. En outre, il existe une grande diversité de produits de qualité supérieure ou de qualité inférieure.

On désigne par style brocart ${ }^{62}$ les pièces aux «dessins rouges ». L'art d'appliquer les émaux aux cinq couleurs, l'or et l'argent, est un secret d'atelier qu'il est interdit de divulguer. C'est pourquoi on ne le mentionne pas ici. On raconte qu'on les produit à partir de la fameuse « couverte vitreuse ${ }^{63}$.

Les poteries anciennes de Nankin ${ }^{64}$ datent toutes de l'époque où l'on ne connaissait pas la terre blanche. L'argile utilisée était proche de celle des terres cuites ${ }^{65}$ et très tendre. Comme on ajoutait de la poudre de verre à la couverte, des défauts surgissaient naturellement. Ces pièces sont aujourd'hui recherchées sous le nom de «style piqué des vers $»^{66}$ mais pour leur qualité d'usage elles ne valent pas la céramique d'aujourd'hui. Tout l'art de la céramique de Nankin réside dans cette impression que l'émail bleu ressort sur la couverte, mais c'est un effet produit par le verre. Si l'émail bleu apparaît au Japon comme noyé sous la couverte, c'est parce qu'on n'utilise pas de verre. Mais cette céramique s'avère supérieure à l'usage.

À propos de la céramique [ancienne], le Kujiki ${ }^{67}$ fait référence au Ōsue sumi ${ }^{68}$ de l'ancien district de $\mathrm{Chinu}^{69}$. Chinu se trouve dans la province d'Izumi ${ }^{70}$. On y trouve encore aujourd'hui des villages de potiers. Autrefois, on utilisait les plats en terre cuite ou des feuilles pour servir les mets. Aujourd'hui, au palais, on n'utilise que des plats en terre cuite, et ils sont tous façonnés à la main tsukune. On y voit la volonté de maintenir la tradition des objets simples et peu décorés de l'Antiquité. Dans le chapitre «Âge des dieux » du Nihongi (Chronique du Japon), les itsue, itsue no okimono, ou inbe $e^{71}$ sont tous des noms de récipients en terre utilisés pour les offrandes aux dieux. Dans le Wamyōshō (Répertoire ordonné des noms du Yamato), on donne le nom de hiraka à la jarre hotogi et on dit qu'elle sert à recueillir un to ${ }^{72}$ (le to ancien est le masu $^{73}$ d'aujourd'hui) de saké. Le plateau bon ou la jarre hotogi du Engishiki (Règlements de l'ère Engi) ${ }^{74}$ renvoient l'un comme l'autre à des récipients de style archaïque. Au cours des périodes postérieures, on a appelé itsue no okimono les pièces disposées lors du départ des troupes. Enfin, aujourd'hui encore, on utilise le mot ancien inbe pour désigner ces ustensiles anciens. Inbe est aussi le nom du clan qui était préposé à cette céramique. On ne peut dater le début de la poterie d'Imari ${ }^{75}$.

\section{Tissage des toiles ${ }^{76}$}

Le tissage est pratiqué massivement à Nara dans le Yamato $^{77}$, dans les provinces d'Echigo ${ }^{78}$ et d'Ōmi. En particulier, la production d'Echigo est réputée et connue sous le nom de crêpe d'Echigo. La qualité de la fibre végétale ${ }^{79} \mathrm{y}$ est excellente et le filage d'un haut degré de perfection. On ignore quand le tissage a commencé à Echigo mais il est plus ancien qu'à Nara et à Ōmi. En effet, les provinces qui s'égrènent à partir d'Echigo, 
telles que Shinano ${ }^{80}$, Musashi ${ }^{81}$, Shimōsa ${ }^{82}$, Shimotsuke ${ }^{83}$, Hitachi $^{84}$, sont toutes des terroirs où ces plantes à fibre textile poussaient en abondance au point que les noms des provinces en portent la trace. C'est le cas de Shimōsa, Kazusa et Shinano. Kazusa, Shimōsa, prononcées à l'origine kami-fusa et shimo-fusa, étaient donc des provinces de fusa. Or fusa est dérivé d'asa (chanvre). Par ailleurs, hier comme aujourd'hui, asa 麻 (chanvre) est dit shina (fibre du tilleul du Japon) dans le dialecte des provinces de l'est. Quand on raconte que les gens d'Ezo ${ }^{85}$ fabriquent leur ceinture avec l'écorce d'un arbre ayant pour nom shina, il s'agit de la même chose. Shinano vient de shina-nuno ou " toile de shina ", sans doute parce que c'est le nom de la région où elle était principalement tissée. Dans le Wamyōshō, on trouve fréquemment le mot shina ${ }^{86}$ dans les noms des districts de la province de Shinano : Sarashina ${ }^{87}$ (lieu où on expose cette fibre au soleil), Hoshina (lieu où on la met à sécher) ${ }^{88}$, Kurashina ${ }^{89}$ (grenier pour stocker la fibre), Nishina ${ }^{90}$ (lieu où on la mettait à rouir avant de la carder). Par ailleurs, on trouve les noms d'omi ${ }^{91}$ dans les districts d'Ina ou de Sarashina. C'est là qu'on filait la fibre végétale.

Par ailleurs, dans les chants de kagura ${ }^{92}$, on trouve le poème : « Dans la lande de Shinano (shinahara) où l'on fabrique la bourre de tilleul / toi qui viens en visite / toi aussi tu es un dieu ! / Divertissons-nous! $»^{93}$. Par ailleurs, dans les règlements relatifs à l'office des magasins de la cour du Engishiki, les graines de chanvre (aliment consommé à l'époque ancienne ${ }^{94}$ ) de Hitachi, Musashi et Shimōsa sont mentionnées comme denrées proposées en échange par la province de Nagato $0^{95}$. Par ailleurs, les règlements relatifs au département du trésor mentionnent la "toile de Shinano» comme gratification saisonnière de printemps et d'automne accordée au [poste de] bureau du service intérieur. Ce sont là autant de preuves [de la culture de la fibre à Shinano]. La province d'Echigo étant contigüe à celle-ci, l'activité y a tout naturellement été introduite par la suite. À Hitachi, on tisse une étoffe à motifs appelée shizuri ${ }^{96}$.

Dans la province d'Echigo, les maisons sont enfouies sous la neige entre la dixième et la troisième lune [de l'année suivante] si bien que les routes de circulation sont plus élevées que les arêtes des toits. C'est pourquoi, les avant-toits des maisons descendent très bas pour permettre le passage. Pour atteindre les maisons en vis-à-vis, on s'aide souvent de marches en bois ${ }^{97}$ fixées dans la neige pour monter et descendre. Ainsi, partout, dans les montagnes comme dans les vallées, lorsque la neige recouvre toute la végétation, et que l'on est privé des moyens de cultiver, hommes et femmes, jeunes et vieux, se consacrent au tissage, véritable don du ciel pour toute la province. Kashiwazaki ${ }^{98}$ est aujourd'hui une ville située au bord de la mer où affluent les marchands de toile. Un peu plus loin, on trouve également des marchands à Ojiya ${ }^{99}$. C'est tout près de Shinano. Les plantes à fibre textile sont semées en grand nombre autour de Shimotani ${ }^{100}$. Senju ${ }^{101}$ est un haut lieu pour le tissage de motifs de rayures à l'ikat. La ville de Shiozawa ${ }^{102}$ est spécialisée dans l'ikat bleu marine, celle de Tōkamachi ${ }^{103}$ dans les rayures irrégulières ou fantaisie ${ }^{104}$, et les alentours de Horino'uchi ${ }^{105}$ dans le crêpe blanc. Chaque village ne tisse qu'une sorte de rayures et s'y tient. Les grossistes se chargent de réunir ces marchandises pour les vendre aux provinces. 


\section{Semer les graines de chanvre et de ramie, les exposer au soleil, les teindre et les tisser}

Il n'y a pas de terre où la ramie ne pousse pas. Il y a deux manières de la faire pousser : le semis à la volée ou le semis en poquet. On trouve également deux variétés : une verte, une jaune. La plante est moissonnée deux fois l'an. Cependant, selon la terre, une même espèce peut être d'une résistance variable. Celle qui pousse à Ōmi sera plus souple et lisse. Celle qui pousse sur les terres froides des provinces orientales est particulièrement résistante. En ce qui concerne Echigo, il n'y a pas que la résistance du chanvre [qui contribue à la qualité] : sur cette terre éloignée de la capitale, il y a aussi que les gens sont simples et que les ouvriers sont consciencieux.

Le chanvre diffère beaucoup de la ramie : ses feuilles ressemblent à celles de l'érable alors que la ramie est proche du paulownia. La ramie est teillée crue, alors que le chanvre est bouilli avant d'être teillé. C'est ce que l'on appelle le "teillage bouilli» nigoki. Le chanvre-mâle donne des fleurs. C'est le chanvre cerisier sakura asa. La femelle ne donne pas de fleurs mais des graines. Quand on sème, les plantes mâles et femelles se mêlent tout naturellement et poussent les unes à côté des autres. Il s'agit bien du mâle et de la femelle. La ramie est également appelée karamushi. Les plantes sont récoltées le huitième jour de la cinquième lune, quand elles sont hautes d'environ 5 pieds. On considère qu'en brûlant le bas des tiges, la plante n'en sera que plus vigoureuse l'année suivante. On l'appelle aussi Naraso, chanvre de Nara, et il est tissé dans la capitale du sud (Nara). À Echigo, c'est surtout la ramie qu'on cultive. Il y en a une grande variété dans les montagnes. Comme en cas de pluie la ramie teillée pourrit, on s'assurera d'abord que le temps est dégagé avant de procéder. Mais, lors du teillage, on fait macérer la plante dans l'eau, en veillant toutefois à ne pas dépasser vingt heures $k o k u^{106}$. Ces [tiges] de couleur jaune clair sont exposées à l'air, et pour obtenir une couleur blanche, les ouvriers chargés du blanchiment prennent de la cendre de paille [de riz] et de la chaux, versent de l'eau, puis font bouillir [le tout]. Après quoi, ils les exposent au soleil, à nouveau sur des plans inclinés ${ }^{107}$.

Pour filer, l'ouvrière expérimentée se sert d'un rouet actionné par une pédale ashikuruma, ce qui équivaut au travail de trois fileuses. On trie le fil de bonne qualité, qu'on carde finement avant de tisser. Les fils grossiers sont tressés ensemble pour en faire des cordes ou du fil à coudre. Tout cela est principalement le fait d'une maind'œuvre féminine [voir Pl. 63]. Quelques hommes se joignent à elles. C'est pourquoi on se réjouit habituellement dans ces pays de la naissance d'une fille. Lorsqu'une fille a deux ou trois ans, on inspecte le bout de ses doigts pour voir s'ils sont fins ou épais, et s'ils sont fins, toute la province se la disputera. 


\section{PI. 63 : Tissage de toiles à Echigo}

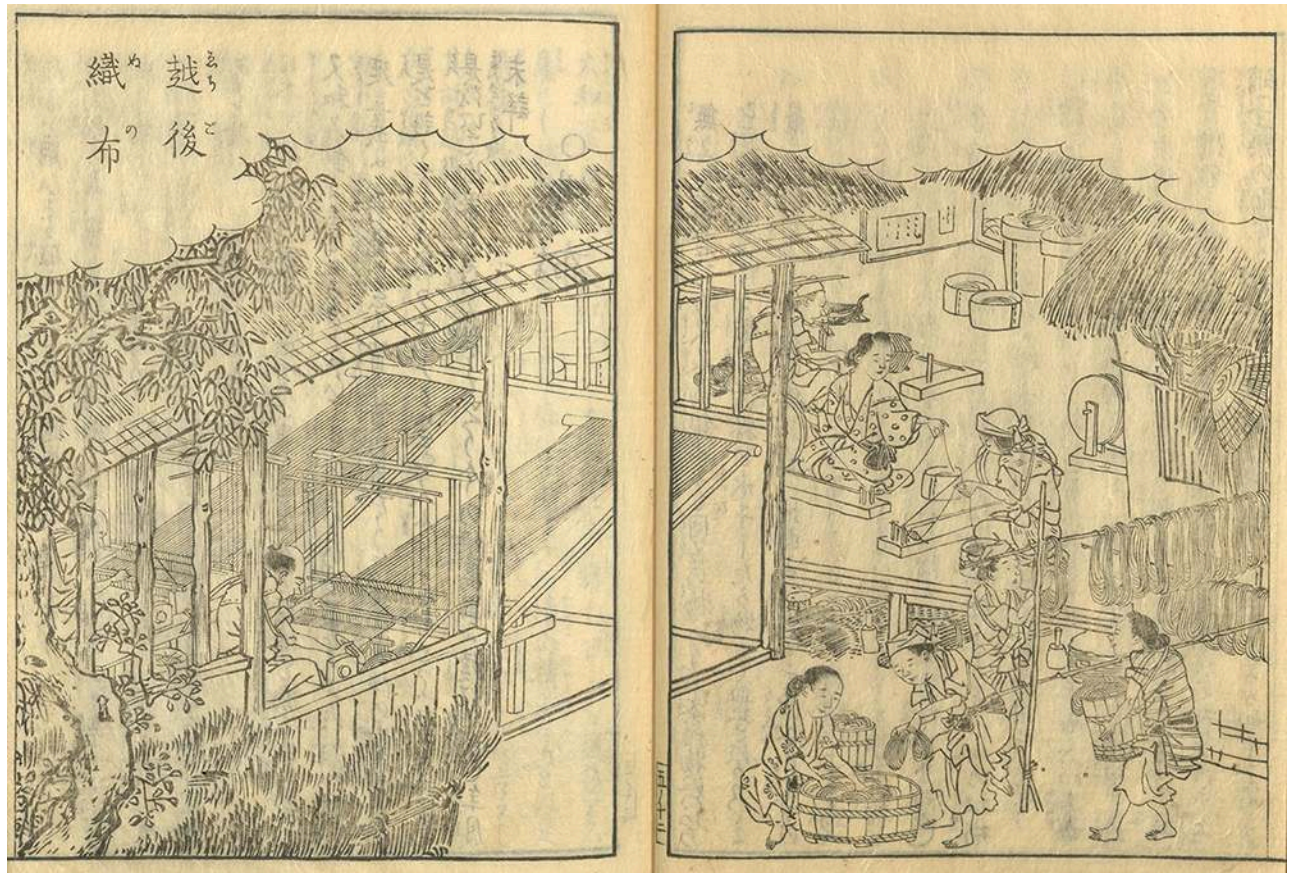

National Institute of Japanese Literature. Lien vers l'illustration

Pour la teinture du fil, le procédé ne diffère pas de celui de Kyōto. Pour les motifs à rayures, le tissu est pressé et lavé dans une eau conservée depuis la veille, puis mise à sécher à l'ombre. Pour fabriquer une étoffe blanche, on la blanchit après le tissage. Pour la blanchir, on la brasse trois à cinq fois dans le mélange de cendre et de chaux ${ }^{108}$ mentionné plus haut. Puis, on l'étale sur la neige, après quoi, on laisse la neige la recouvrir. On remet de la toile sur cette neige, et on procède ainsi par couches successives comme si on construisait une digue. Sous l'effet du soleil, la neige se mettra à fondre, laissant un tissu d'une blancheur parfaite. On le brassera bien une dernière fois dans l'eau [voir Pl. 64]. 
PI. 64 : À Echigo, la toile est exposée à la neige

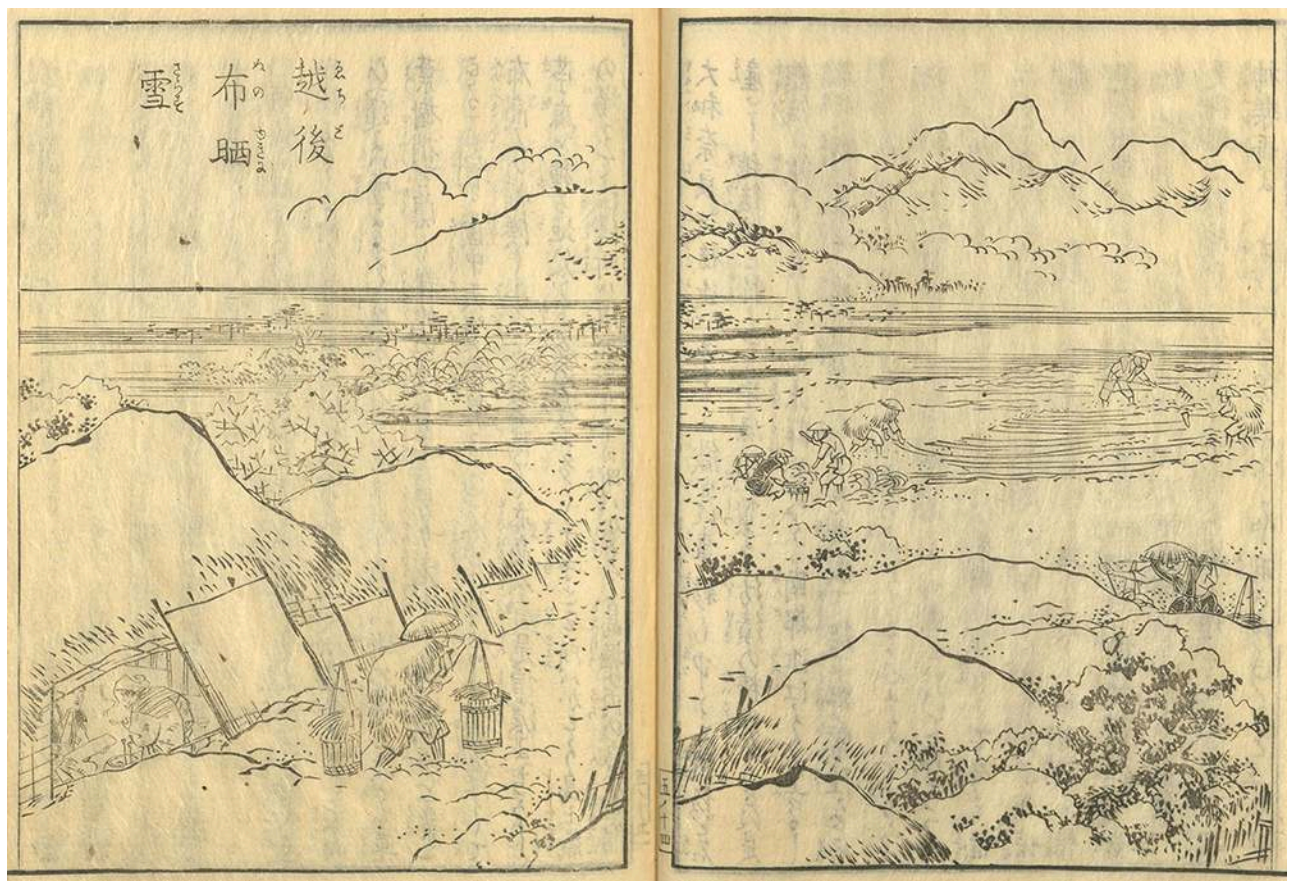

National Institute of Japanese Literature. Lien vers l'illustration

Il y a encore l'explication suivante. Selon les dires des gens de campagne sur les habitudes des marchands d'étoffe, ces derniers distingueraient la finesse et la qualité des étoffes en qualifiant de « $1 g \overline{0}^{109}$ » l'étoffe la plus fine, et de « $2 g \bar{o} »$, « $3 g \bar{o} »$, les qualités suivantes. Cela concernerait les étoffes fabriquées dans les régions montagneuses. « $1 g \bar{o} »$ correspond au sommet de la montagne, les gens y sont d'une extrême simplicité. C'est pourquoi, les dépenses nécessaires pour pourvoir femmes et enfants en nourriture, vêtements et logement durant un an s'élèvent à peine à 50 à 60 monme. C'est le coût du filage pour une pièce d'étoffe fine. On constate ainsi que ce sont les différences dans la vie matérielle qui sont à l'origine de ce que le tissu le plus fin se trouve au sommet (un gō) de la montagne, et qu'il devient plus grossier avec le 2 gō et le $3 g \bar{o}$. De cet exemple, on déduit que les objets et les arts de nos jours sont inférieurs en tout point, et notamment en qualité, à ce qu'ils pouvaient être autrefois, ne valant guère que 2 ou 3 gō selon cette échelle.

\section{Otaries (ottotsujūi) ${ }^{110}$}

Bien que considérée comme une production de Matsumae ${ }^{111}$, on la capture au lieu dit Oshamanbe ${ }^{112}$ dans le territoire d'Ezo. La chasse se déroule en période froide, de la fin de l'année à la deuxième lune. Cependant la prise du printemps ${ }^{113}$ est écartée des tributs car moins adaptée pour la salaison. Il y a à Ezo des postes de traite situés à 70-80 lieues au nord-est de Matsumae. On dit que les routes maritimes peuvent atteindre jusqu'à 700 ou 800 lieues $^{114}$ loin des côtes. Ces postes de traite abritent des comptoirs de marchands de Matsumae, de Mutsu ${ }^{115}$ ou d'Ōmi. Les fonctionnaires de Matsumae s'y rendent les premiers pour superviser les échanges [voir Pl. 66]. 


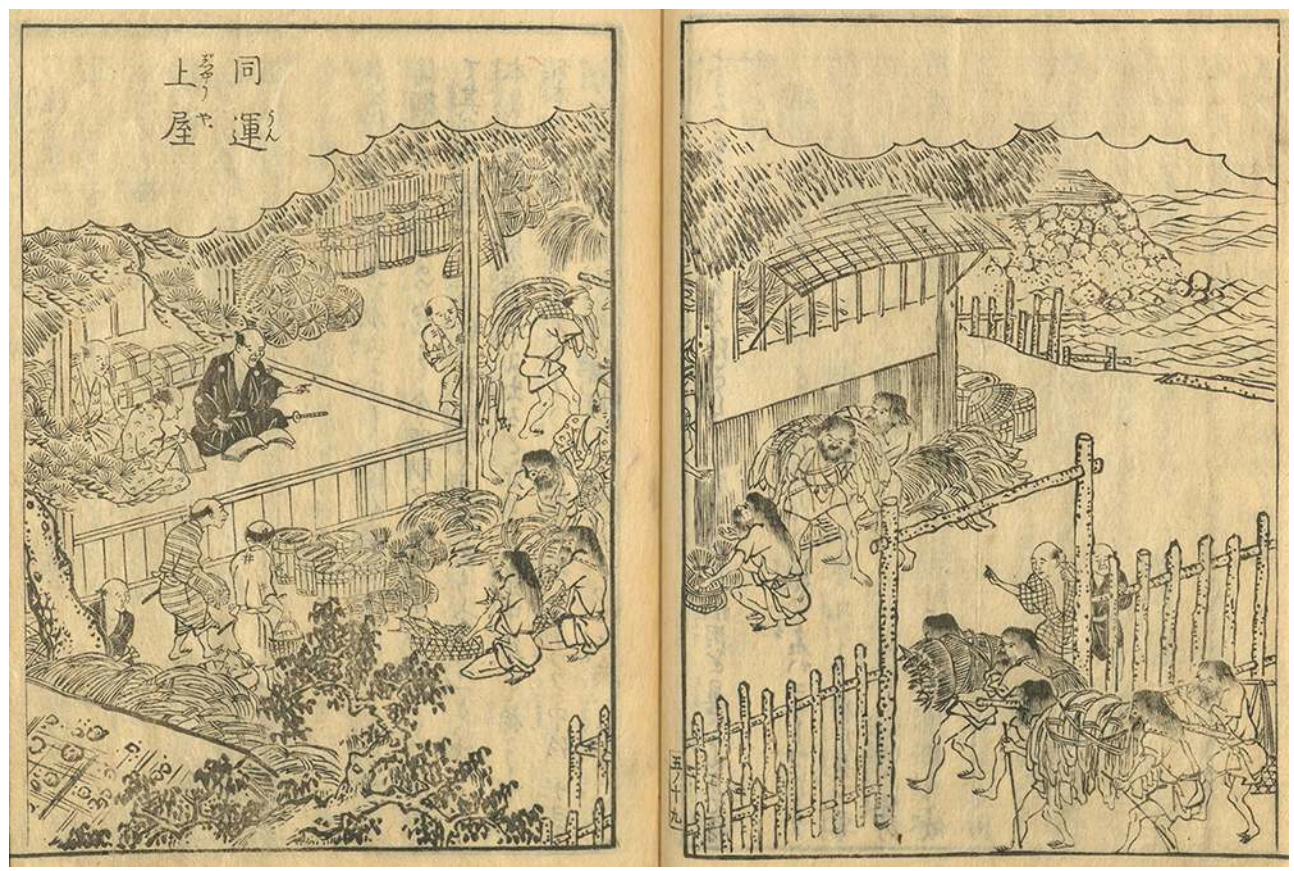

National Institute of Japanese Literature. Lien vers l'illustration

Les marchandises acheminées du Japon sont : le riz, le sel, le kōji, les vêtements usés, le tabac, la vaisselle, etc. Mais il n'y a pas de lames ${ }^{116}$. Par ailleurs, les produits d'Ezo sont la loutre de mer kaiku (littér. « chien de mer»), l'otarie ottotsu, l'ours, la bile d'ours, la peau de daim, la morue, le saumon, la laminaire, l'ormeau, la truite, le hareng ou encore les œufs de hareng. Parmi ces derniers, le «brocart d'Ezo " ezonishiki est un produit de Mandchourie (il s'agit de la Tartarie, proche d'Ezo), acheminé en un lieu appelé Sōya ${ }^{117}$ à Ezo.

Quant aux ours, on pose d'abord un piège au petit, puis, le lendemain, on capture la mère. Le petit est nourri au sein des femmes, et quand il a ses dents, on lui donne à manger. Quand il atteint l'âge adulte, on l'étrangle entre deux rondins; le corps est placé sur une natte, du saké et du poisson sont présentés en offrande, après quoi on récolte la bile et on consomme la chair.

Utiliser le nom d'ottosei ${ }^{118}$ pour l'otarie est une erreur. Le nom du quadrupède est ottotsu. Le ottotsusei, transcrit dans un ouvrage avec les caractères 膃肭臍, désigne les « reins extérieurs » gaijin, autrement dit les testicules de l'otarie. On fait grand cas de ses vertus médicinales mais on évoque rarement sa chair. Ainsi, les produits commercialisés comme "pénis d'otarie " takeri sont en réalité des testicules. On en produit aussi dans les régions de Tsugaru ${ }^{119}$ et $\mathrm{Nanbu}^{120}$, et reconnaître les vrais des faux n'est pas facile. C'est parce qu'il en existe différents spécimens. Bien que «loutre de mer" kaidatsu ou "chien de mer" kaiku passe pour une autre appellation [de l'otarie], ce sont en fait des noms génériques.

On trouve encore le «lion de mer» kaihyō. En japonais, on l'appelle azarashi. Il a une peau tachetée de noir et ressemble à l'otarie. Il doit s'agir de la même espèce que l'ajika ou « daim de mer ». Le kaidatsu ${ }^{121}$ est une loutre kawauso qui vit en mer. Sa morphologie est en tout point similaire à celle de l'otarie. Une manière de les distinguer est d'observer les rangées d'incisives. Celles qui en ont deux sont d'authentiques otaries. 
On dit aussi que les deux rangées d'incisives seraient uniquement des dents supérieures. Il a par ailleurs un orifice sur la tête par lequel il rejette l'eau de mer. Il est difficile à apercevoir car caché par le pelage. On sait qu'on a de vraies otaries, si, en jetant dans l'eau glacée leur chair ou leurs boyaux, ils réchauffent l'eau et l'empêchent de geler.

41 Parmi les produits vendus comme "pénis d'otarie", on trouve des faux fabriqués à l'aide de boyaux. C'est pourquoi ils n'ont pas de poils. On les appelle «takeri de boyau » hyakuhiro takeri. Les authentiques takeri ont environ 3 pouces de long; ils sont rougeâtres et ont des poils à la racine.

Le corps est entièrement gris cendré, identique à celui de la loutre d'eau douce, mais légèrement plus long. Sa tête est petite, semblable à celle d'un chat. Ses moustaches sont très longues. Ses pattes [avant] sont situées de part et d'autre [à gauche et à droite], dans le prolongement de la mâchoire inférieure et ont l'air de grandes nageoires. Ses pattes arrière se placent avant la queue, elles sont longues d'environ 1 pied. Il y a cinq ongles à l'extrémité de chaque patte. La queue est fine. Ces bêtes vivent dans les eaux les plus profondes. On les trouve parfois en train de ronfler sur les rochers au bord de la mer. Elles se laissent aussi dériver au gré du courant, endormies, par troupeau entier. L'une d'entre elles se tient éveillée pour faire le guet, et dès qu'un bateau vient à passer, elle pousse un cri pour avertir le troupeau qui plonge alors dans l'eau. Lorsqu'elles se déplacent, leur corps reste à moitié hors de l'eau. Ce sont d'excellentes nageuses qui fendent énergiquement les vagues.

Ce qui a déjà été dit plus haut s'applique aussi aux loutres de mer. Derrière les produits qui nous arrivent sous le nom d'otaries, beaucoup sont des loutres de mer, et il est difficile de départager le vrai. C'est comme le remède appelé "le grain d'élixir d'or " ichiryū kintan ${ }^{122}$ de la région de Nanbu dont on dit que le plus difficile est de le reconnaître. Comme le Bencao gangmu (Classement raisonné de la materia medica) ${ }^{123}$ indique dans sa rubrique "explications réunies »124 qu'on les trouve dans les mers de l'Est, cela signifie que le produit est rare en Chine et que c'est le Japon qui en est le fournisseur.

À Ezo, celles de grande taille sont appelées neffu, celles de taille moyenne choki, et les petites uneu ${ }^{125}$. Il s'agit là des vraies otaries. Les nageoires sont appelées tetsuhi. On les assimile à des volatiles quand on les compte ${ }^{126}$. À Tsugaru, on récolte les nageoires et on les prépare pour en faire des mets d'accompagnement du saké. Les plus grandes sont appelés to. $\mathrm{Si}$, de nos jours, toto désigne le poisson dans la langue des femmes et des enfants, cela pourrait bien provenir de là. Ce mot existe aussi en Chine.

On trouve dans le Fubokushū, dans le fascicule 18, sous le sujet "rêve", le poème suivant du Tiers ministre Kinugasa (Fujiwara no Ienaga ${ }^{127}$ ), extrait du Concours de poèmes en cent manches de l'an 8 de l'ère Kenchō (1257) :

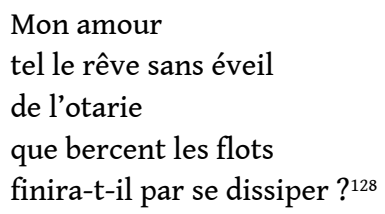

Il s'agit dans le poème de la variété du "cheval de mer »"129 kaiba et ce n'est donc pas la même chose. Par ailleurs, les caractères « âne de mer » kairo employés [dans le poème pour todo] sont lus michi no kawa (fourrure de michi ${ }^{130}$ ) dans le chapitre «Palais du roi dragon » du fascicule consacré à l'Âge des dieux dans le Nihongi (Chronique du Japon). 


\section{La chasse}

Pour les capturer, les gens d'Ezo se déplacent sur des bateaux aux parois cousues de cordes $^{131}$ et lorsqu'ils repèrent un troupeau endormi qui se laisse dériver, ils agitent la queue d'un renard en direction du guetteur. Ils attendent que celui-ci, étranglé par la peur, prenne la fuite pour capturer les bêtes endormies avec des flèches ou des harpons. Leur dextérité est sans égal. Les bateaux sont maniés uniquement en pagayant, sans utiliser de perche [voir Pl. 65].

\section{PI. 65 : Les gens d'Ezo chassent l'otarie}

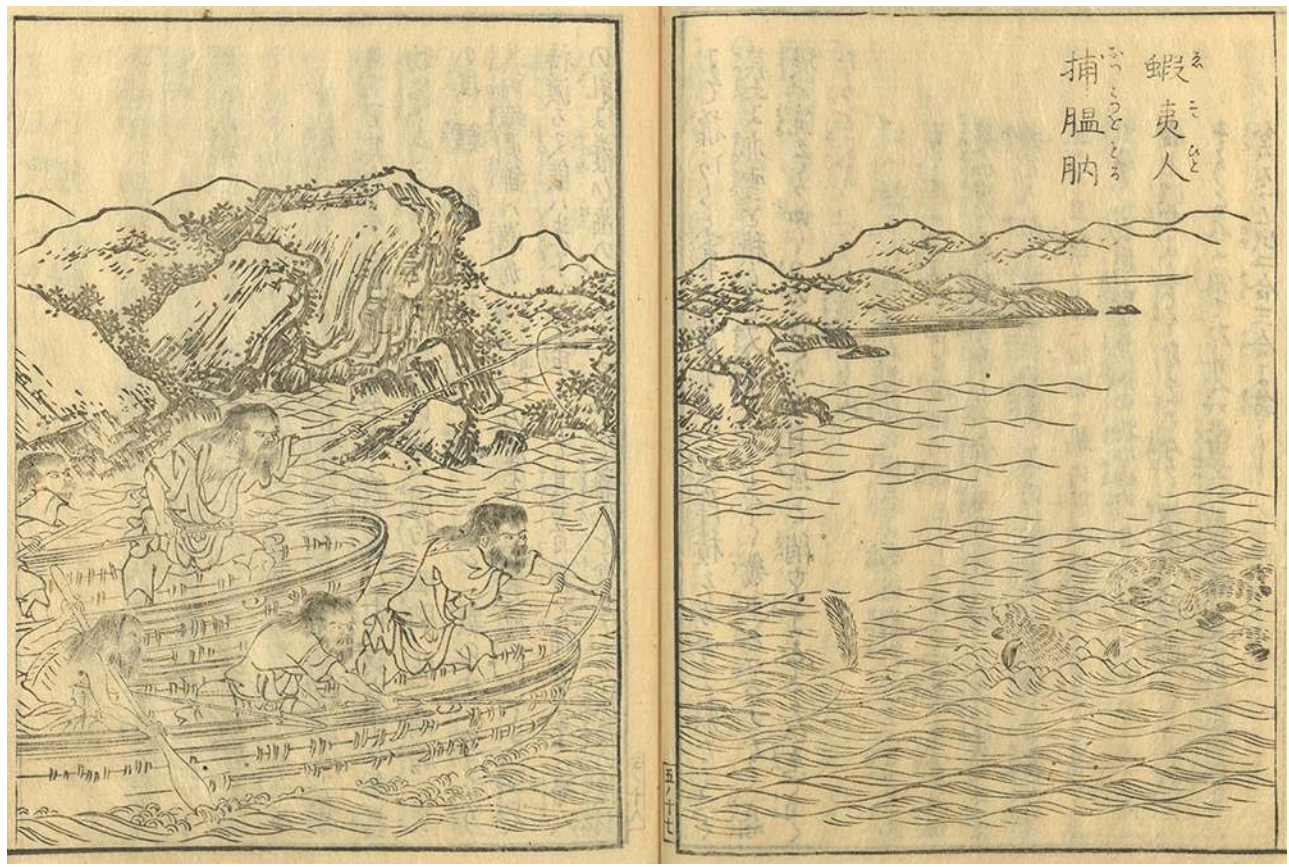

National Institute of Japanese Literature. Lien vers l'illustration

On dit aussi que ottosei désigne la verge hozo ${ }^{132}$. Dès lors, comment se fait-il qu'on l'identifie aux testicules gaijin? Dans un ouvrage, on raconte qu'on s'est adressé aux gens de Matsumae et de Nanbu pour obtenir la fameuse verge hozo mais qu'il n'y avait pas moyen d'en obtenir. À entendre les indigènes de nos jours, hozo et takeri sont très proches l'un de l'autre. C'est pourquoi lorsqu'on prélève le second, on détruit inévitablement le premier et il est perdu. Certains s'interrogent : cela vaut pour le mâle mais la femelle a-t-elle nécessairement un hozo?

\section{Laminaire (konbu)}

le nom japonais est hirome, ou encore kaifu, toile de mer

La récolte a lieu à l'époque du doyō, à la sixième lune ${ }^{133}$, et non toute l'année. Par ailleurs, on en récolte à Matsumae, Esashi ${ }^{134}$, et Hakotachi ${ }^{135}$ à Ezo. Les hommes montent sur des barques et plongent dans l'eau, avec une faucille. Après un certain temps, ils se laissent remonter à la surface, en tenant les laminaires. Les laminaires poussent sur les rochers au fond de la mer et leur longueur varie de 3-4 pieds à dix 
travées. Il arrive qu'on les remonte avec les rochers mais au bout de dix jours elles se détachent toutes seules. On coupe les plus longues à une longueur convenable et on les met à sécher pour une journée sur le sable des plages de Matsumae, sur les toits des maisons et jusque sur les routes, au point qu'il ne reste aucun espace disponible. À la tombée de la nuit, on les rentre et on les entasse dans des huttes. On les recouvre de nattes de paille et, après une nuit, on les retrouve couvertes d'une pellicule de sel : c'est la laminaire brute (on entend dire que les maisons d'Ezo ont des toits de laminaires, cela vient sans doute de ce qu'on les y a vu sécher. Toutes les maisons d'Ezo ont leur toit et leurs murs en planches). Les laminaires rouges sont considérées comme de qualité supérieure. On établit ainsi une légère distinction entre elles.

Par ailleurs, les laminaires que les vagues rejettent sur les rivages vers la huitième ou la neuvième lune sont appelées "laminaires rejetées" yosekonbu. Autrefois, on les transportait à Tsuruga ${ }^{136}$, dans la province d'Echizen ${ }^{137}$, puis de là elles partaient pour la province de Wakasa ${ }^{138}$. Les marchands d'Obama ${ }^{139}$ les transformaient et le résultat était appelé « laminaires de Wakasa ». De Wakasa, elles sont transportées à Kyōto où elles sont à nouveau transformées et prennent le nom de laminaires de Kyō. Ce sont celles qui ont le meilleur goût.

Ces propos ont leur source dans les récits de poètes de haikai itinérants qui se sont rendus à Matsumae. Il ne s'agit pas de choses que j'ai vues de mes propres yeux. À propos des vêtements des gens d'Ezo, j'ai entendu dire qu'ils appréciaient tout particulièrement les vieux kimonos du Japon et que, lors des banquets donnés par les riches chefs de villages, ils posaient en guise d'ornement plusieurs épaisseurs de kimono sur des tonneaux de saké empilés. Par ailleurs, dans ces régions, on tisse avec de l'écorce d'orme ${ }^{140}$. La couleur en est jaune et marbrée. Dans le dialecte local, elle est appelée atsushi, et elle sent particulièrement mauvais. Selon la coutume, le col est croisé gauche sur droite et la ceinture est faite d'écorce de tilleul ${ }^{141}$. Les hommes et les femmes ne se baignent jamais. Leurs sourcils forment un seul trait au-dessus de leurs yeux, et comme ils ne se coupent ni les cheveux, ni même la barbe, ils sont particulièrement longs. Quand ils mangent, ils tiennent les baguettes avec la main gauche et absorbent leur repas en soulevant leur moustache. Ils conservent le saké dans des coffrets hokai ${ }^{142}$, et se servent de bols de riz pour le boire. Ces bols portent tous le motif du tomoe ${ }^{143}$. Je n'en connais pas la raison. Les femmes ont toutes leurs lèvres tatouées et les hommes comme les femmes ont leurs larmes qui coulent du nez. Quand ils s'aventurent hors du village, ils vont pieds nus même par temps de neige. Ils portent un arc rudimentaire. Ils emploient des arcs et des flèches en bois. On dit, par ailleurs, que le poison appelé busu, dont ils enduisent les flèches avec lesquelles ils chassent l'ours ou le cerf, est fabriqué à partir de la racine de gentiane ${ }^{144}$ et de guêpes tuées. Mais on n'emploie pas ce poison pour les otaries.

\section{Un poème de Tameie}

Quand le kosa souffle ${ }^{145}$,

des nuages se forment,

à Ezo,

la lune d'automne ne se montre pas

Par ailleurs le vers de haikai de Jōha :

Soir de printemps à Ezo,

le kosa voile la lune dans le ciel 
Il n'y a personne à ce jour qui sache parfaitement ce qu'est le kosa. Je dessine ici toutefois ce qui m'a été transmis par une personne avec sa légende.

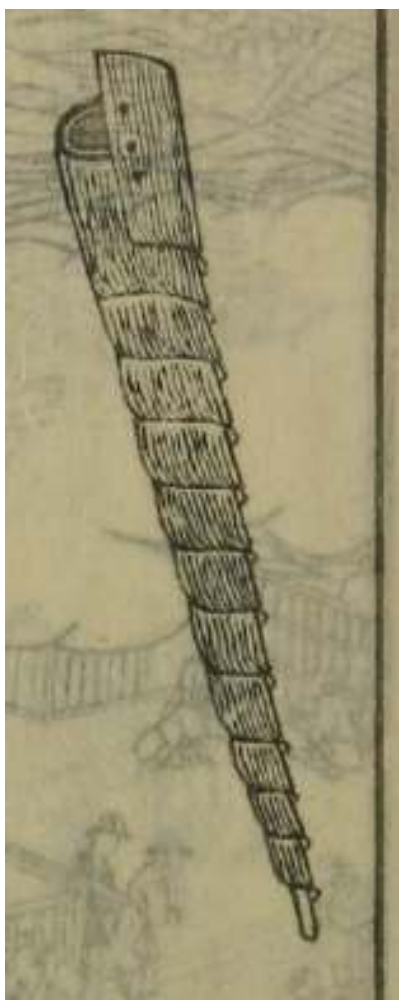

55 «Douze rouleaux» (fabriqué avec de l'écorce d'arbre enroulée. C'est une écorce blanche, semblable à une liane, avec des tâches de couleur bambou. Sa longueur totale est de 1 pied 2 pouces)

À ce qu'il me semble, cela n'est sans doute pas un kosa. C'est une flûte de cette région. Est-ce qu'ils pratiquent la pêche en remplissant cela d'eau de mer et en soufflant vers le ciel, produisant ainsi une brume autour de la lune? On raconte aussi que les gens qui allaient se promener dans les montagnes ou au bord de la mer ramassaient une feuille morte qu'ils enroulaient et dans laquelle ils soufflaient, produisant le son d'une flûte au son mélancolique. On l'appelle aussi kosa.

Bien que le voyage à Ezo de Yoshitsune ${ }^{146}$ transmis par des légendes n'ait pu être prouvé, c'est une thèse légitime. Il existe des caps portant le nom de Benkei ${ }^{147}$ sur les côtes d'Ezo. On dit aussi que l'empereur des Qing est un descendant de Sewa (Genji) ${ }^{148}$, ce qui voudrait dire que Yoshitsune aurait franchi [les terres] en passant par Ezo. D'après ce que l'on raconte, nombreuses en seraient les preuves. De la Tartarie à Ezo, il n'y a pas loin.

\section{Produits étrangers}

Alors qu'à l'époque du Taikō Hideyoshi ${ }^{149}$, les bateaux étrangers accostaient dans le havre de Sakai dans la province d'Izumi, le port d'accostage devint par la suite Hirado dans la province de Hizen. À partir des années Genki (1570-73), on le remplaça par Nagasaki et depuis lors le commerce n'y a jamais cessé. Cette terre était à l'origine couverte de forêts, mais après que l'on a défriché les régions de Tamanoura et Fukae ${ }^{150}$, c'est devenu un port populeux et florissant. Les bateaux chinois proviennent de 
Nankin, Pékin, Hokujū, Chakuchū ${ }^{151}$ et d'autres endroits. Au total, on laisse venir en tout treize bateaux par an. Ils transportent des denrées médicinales, des soieries, du sucre, du papier, des ustensiles, etc. On ne peut en établir la liste complète. On a placé des longues-vues sur les promontoires de $\mathrm{Nomo}^{152}$, Fukahori ${ }^{153}$, et Nishito ${ }^{154}$, à partir desquels on peut surveiller la mer à une distance de 40 lieues. Dès qu'on aperçoit l'ombre d'un navire, des fanions sont hissés l'un après l'autre pour alerter les services du préfet. À l'approche du bateau, l'interprète-aîné, l'interprète-cadet ainsi que les officiels dépêchent une chaloupe pour l'accueillir. Ils montent à bord de la jonque chinoise et, une fois les contrôles de la licence ${ }^{155}$ effectués, on procède à l'accostage et au déchargement de la jonque. Des barques sont mobilisées pour transporter les marchandises jusqu'aux entrepôts situés dans les quartiers nouveaux [voir Pl. 67].

\section{PI. 67 : Arrivée d'une jonque chinoise}

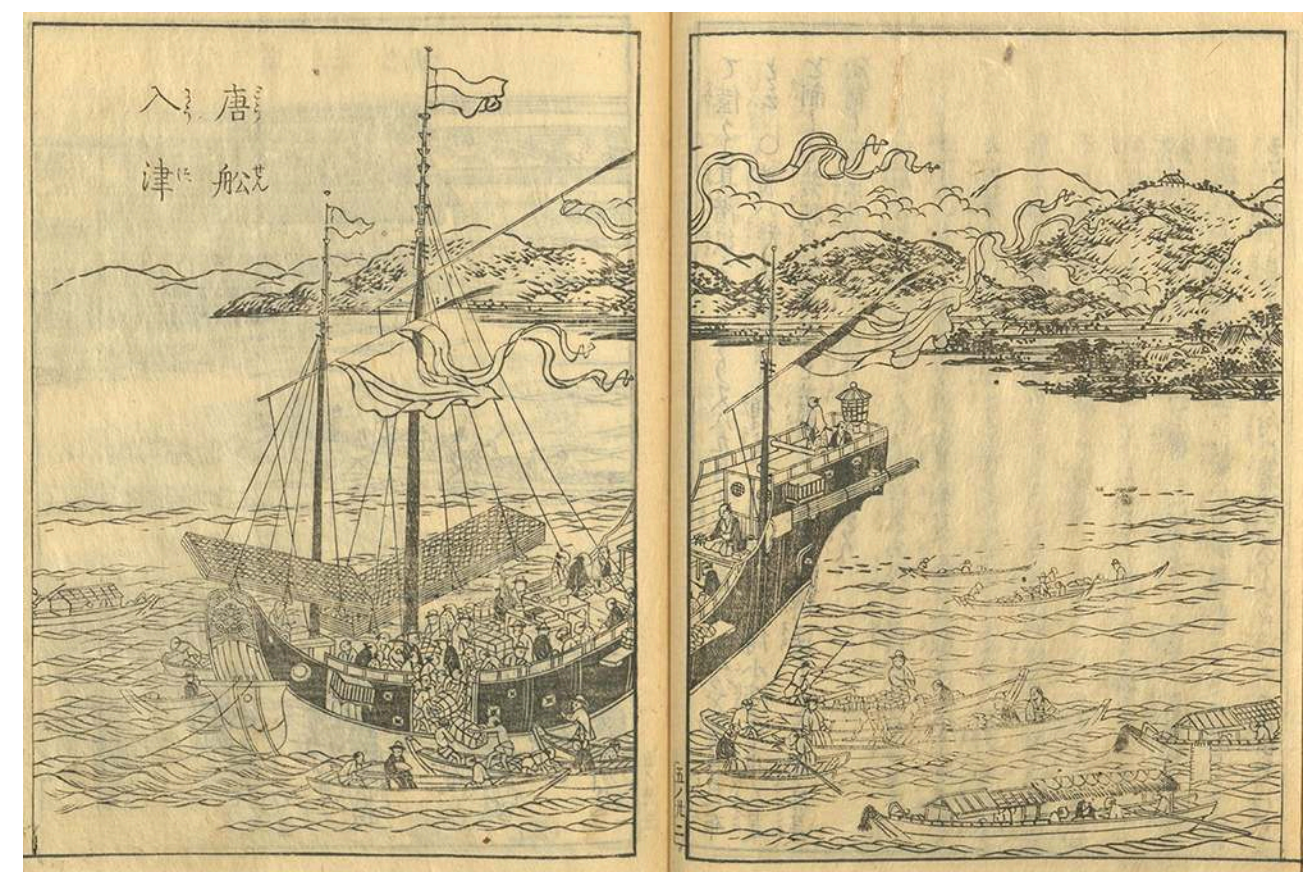

National Institute of Japanese Literature. Lien vers l'illustration

Une fois le déchargement achevé, se déroule ce qu'on appelle le « débarquement de la divinité Mazu $»^{156}$. Dans toutes les jonques chinoises, on vénère une effigie en costume de personnage officiel, appelée Bosa, en tout point semblable à l'« esprit du bateau » funadama $a^{157}$ de notre pays. Cette statue est confiée à un temple de Nagasaki. Cela donne lieu à une procession haute en couleurs, précédée de lanternes allumées en plein jour, qui s'arrête à chaque croisement pour frapper le gong, agiter des bâtons et danser (c'est une erreur de considérer que la statuette représente Guanyu (j. Kan- $\mathrm{u}^{158}$ ) [voir Pl. 68]. Le bateau est amarré en un endroit appelé Umegashima, les gens s'installent dans la Résidence des Chinois ${ }^{159}$ et un banquet est organisé pour fêter leur arrivée à bon port. À cette occasion, les courtisanes des quartiers de Maruyama et de Yoriai se rendent en grand nombre sur les lieux, trouvent leurs clients et les divertissent. Plus tard, au moment du départ, les marchandises exportées, fixées par la loi, sont: holothuries séchées, laminaires, ormeaux séchés, papier, parapluies, laques, ailerons de requin, pachymes ${ }^{160}$, auxquels s'ajoutent des accessoires divers, ou d'autres objets en fonction de la mode du moment. Par ailleurs, la mise en vente des objets chinois est du ressort 
des services officiels, ils sont cédés aux marchands accrédités les plus offrants [voir Pl. 69].

\section{PI. 68 : Débarquement de l'effigie Bosa}

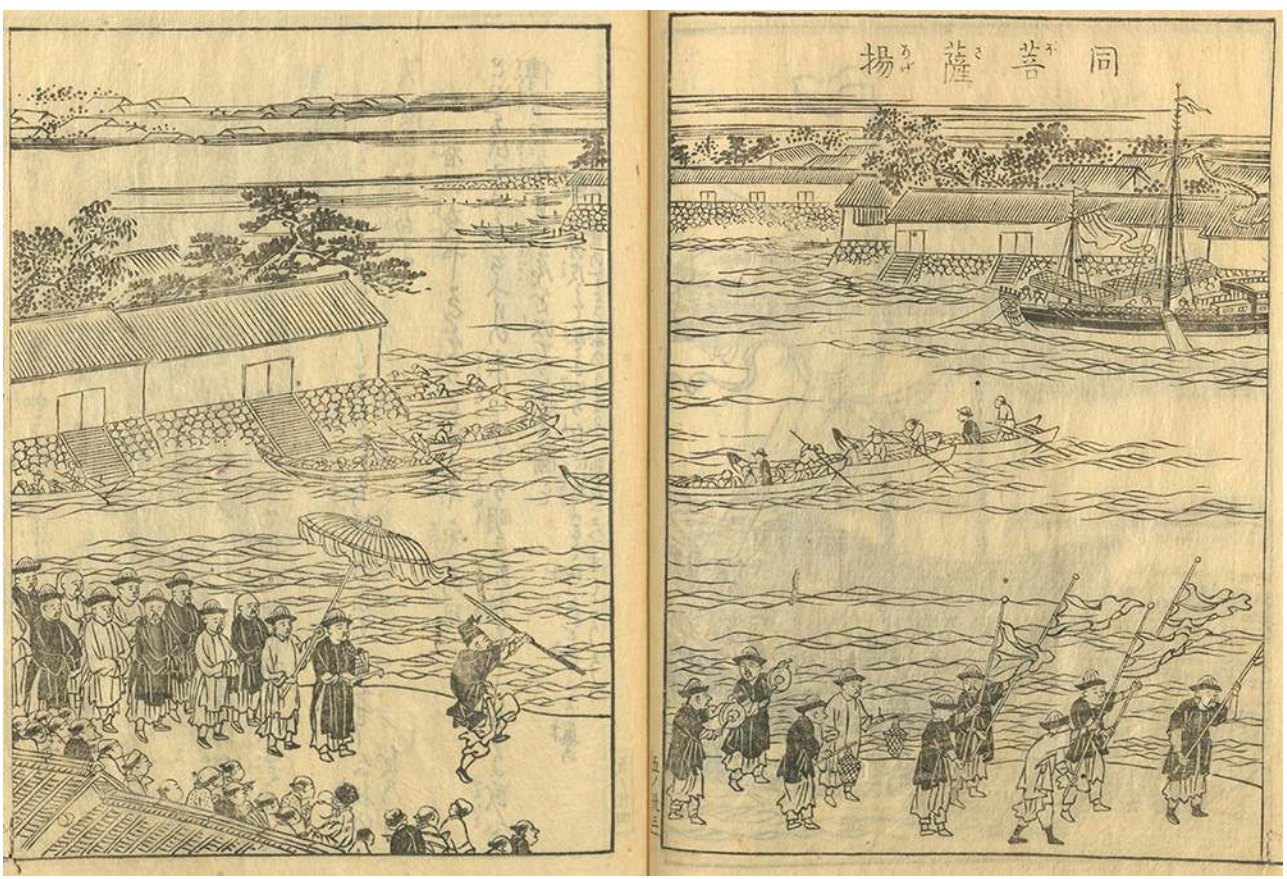

National Institute of Japanese Literature. Lien vers l'illustration

\section{PI. 69 : Résidence des Chinois et entrepôts des quartiers nouveaux à Nagasaki}
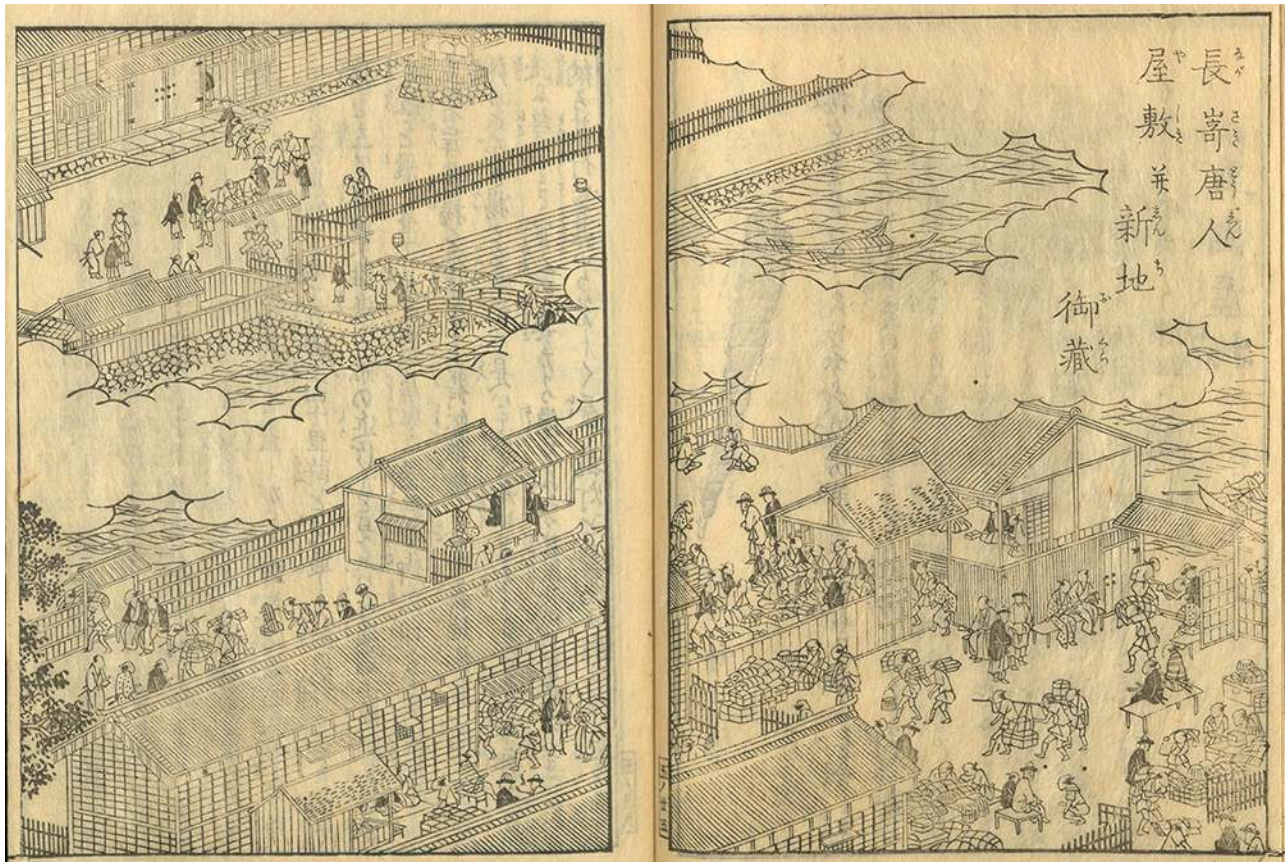

National Institute of Japanese Literature. Lien vers l'illustration 


\section{Bateaux hollandais ${ }^{161}$}

Ils accostent chaque année vers la septième lune. De la même manière, lorsque l'alerte est donnée au moyen de longues-vues, le capitaine hollandais arrivé l'année précédente, accompagné de l'interprète aîné ${ }^{162}$, de l'interprète cadet, et d'un officiel, s'embarquent dans deux chaloupes légères au drapeau hissé, et rament en direction du navire. Ils montent à bord, procèdent aux vérifications de la licence, etc. puis regagnent la terre. Après quoi, le navire tire neuf coups de canon [voir Pl. 70]. Accompagnant le bateau dans sa progression, un grand nombre de chaloupes le guident lentement dans la rade. Arrivé au niveau des postes de surveillance de Nishito et de Marutomachi, il tire sept coups de canon, puis à nouveau, lorsqu'il atteint l'embarcadère de Dejima, il fait résonner neuf coups de canon. À cet instant, le pavillon est hissé sur le navire, et on fait de même à la résidence de Dejima. On appelle cela la «reconnaissance des drapeaux ». Là-dessus, la musique s'élève : le son est extraordinaire. L'ancre est jetée, puis à nouveau dix-huit coups de canon sont tirés. Une fumée noire se dégage, et le bateau disparaît derrière la fumée. Pendant ce temps, on hisse complètement les quarante-huit voiles et on orne le navire de drapeaux placés à dix emplacements différents, si bien qu'au moment où la fumée se dissipe, le navire se dévoile au regard comme s'il avait été reconstruit. Il est d'une splendeur à couper le souffle, c'est véritablement grandiose [voir Pl. 71].

PI. 70 : Résidence hollandaise de Deshima à Nagasaki - Arrivée du bateau hollandais

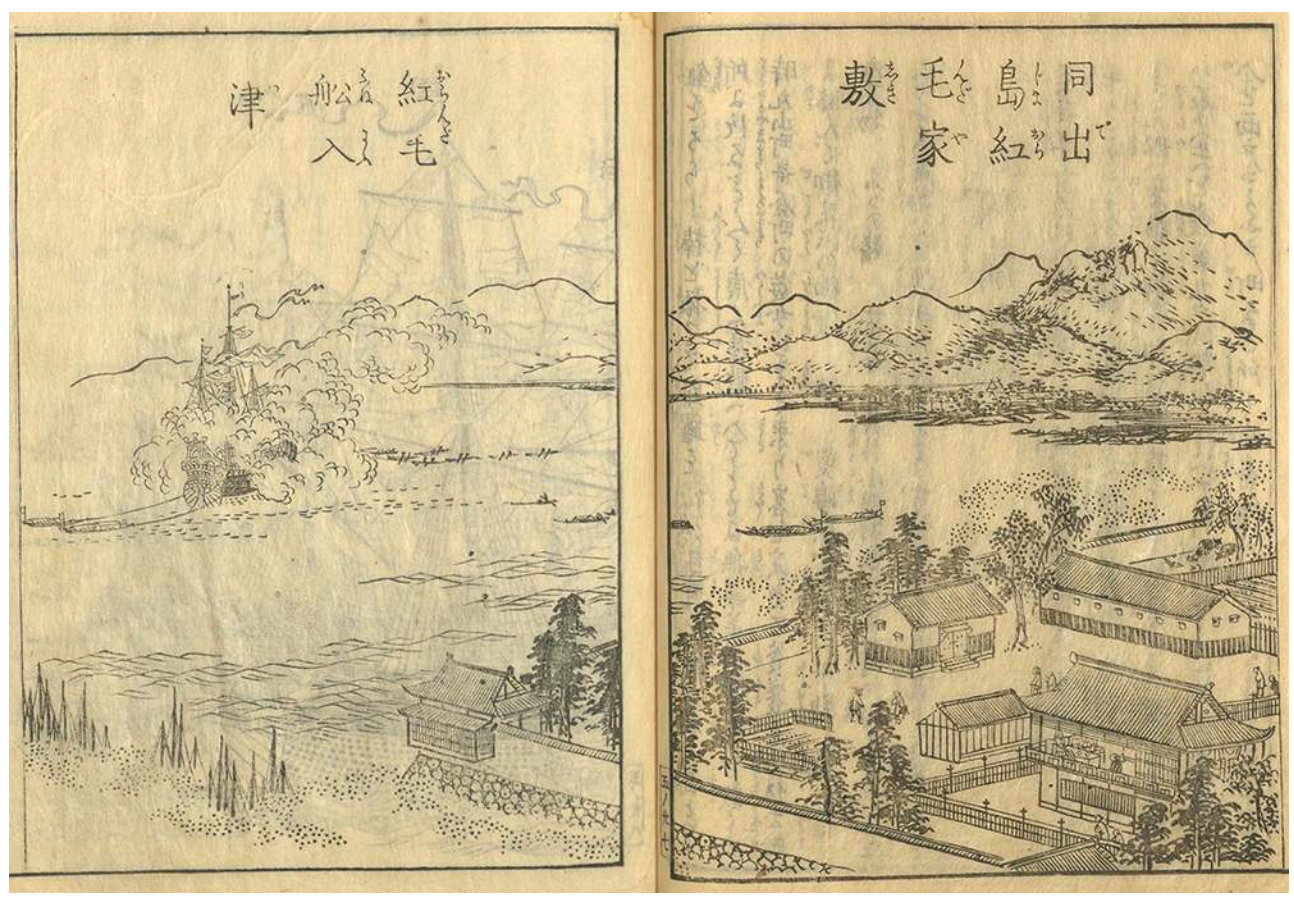

National Institute of Japanese Literature. Lien vers l'illustration 


\section{PI. 71 : Bateau hollandais}

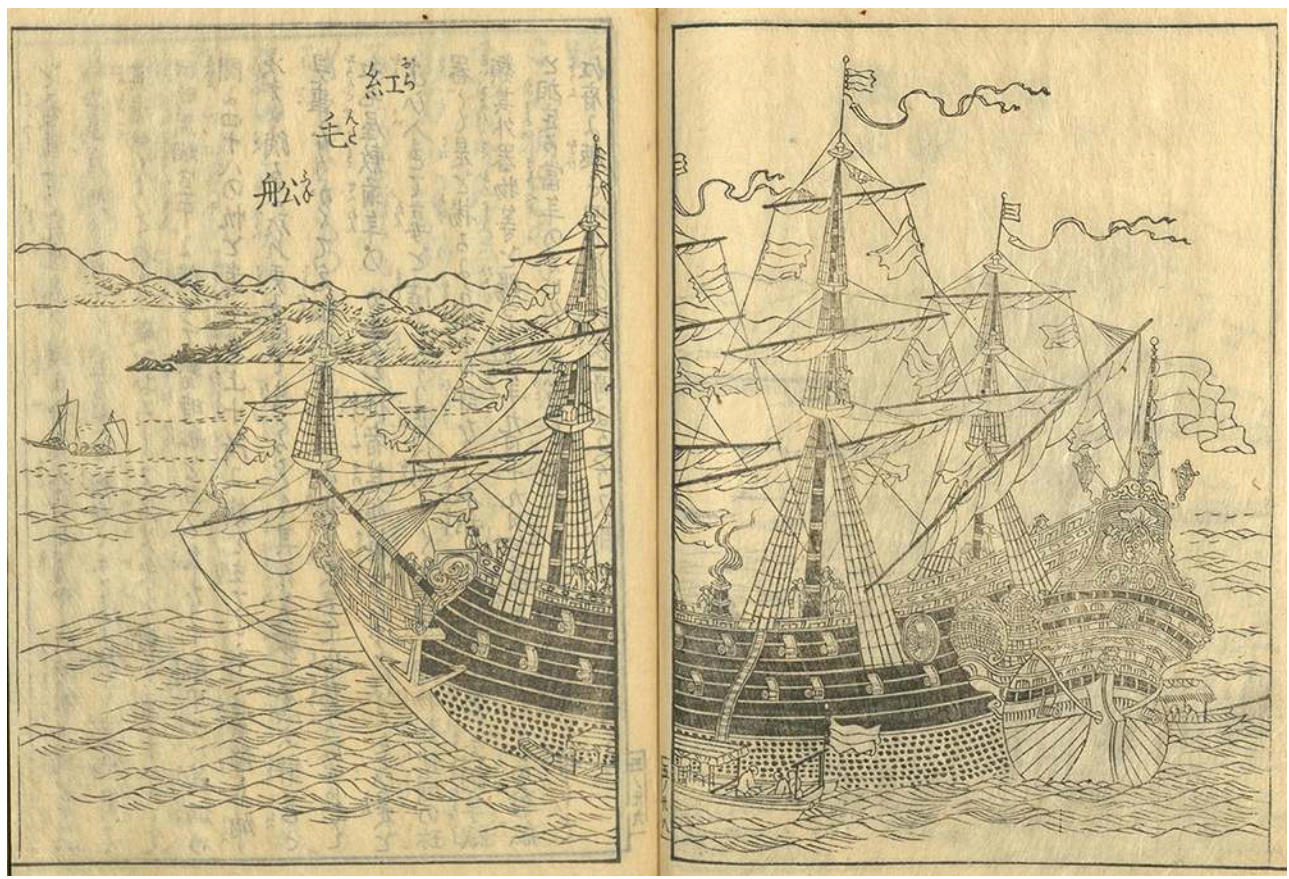

National Institute of Japanese Literature. Lien vers l'illustration

61 C'est ainsi que le capitaine du navire s'embarque dans une chaloupe pour gagner Dejima où il est accueilli par le capitaine de l'année précédente, ses seconds, ainsi que des courtisanes; puis il est guidé à l'intérieur de la résidence où est organisé un banquet. Le chargement est pareillement composé de denrées médicinales, d'accessoires divers, et de curiosités venant de l'étranger. Il lui faut environ quarante jours pour décharger sa cargaison. Pour ce qui est des marchandises exportées, on trouve du cuivre, du papier, des poteries. La date du dix-neuf du neuvième mois est fixée, chaque année, comme date de départ du capitaine précédent. Le capitaine en poste, part le quinzième du premier mois à Edo, chargé de présents pour le shogun. Il revient à Nagasaki au quatrième ou au cinquième mois, puis attend la venue de la relève.

\section{NOTES}

1. La section traite ici d'une espèce particulière de méduse, le Rhopilema esculentum, largement consommée en Asie. Au Japon, on la trouve dans la mer intérieure de Seto et autour de l'île du Kyūshū. La région d'okayama (anciennement province de Bizen) en a fait sa spécialité, d'où le nom de bizen kurage, qu'elle porte aujourd'hui. L'auteur explique ici le mode de préparation de ce mets, macéré, puis séché, et consommé assaisonné. La spécialité est déjà mentionnée par Kaibara Ekken dans son Yamato Honzō (Materia medica du Japon), daté de 1709.

2. Bizen, ancienne province située dans l'actuel département d'okayama. 
3. Hizen, ancienne province, à cheval sur l'actuel département de Nagasaki et le département de Saga.

4. Chikuzen, ancienne province située dans l'actuel département de Fukuoka.

5. Minamoto no Nakamasa (?-?), poète de statut guerrier de la fin de l'époque de Heian.

6. Fuboku wakashō, également désigné dans le livre par Fubokushū. Recueil de plus de 17350 poèmes japonais (waka) compilé en 1310 par Fujiwara no Nagakiyo.

7. Fascicule 27, Poème $n^{\circ} 13093$ «Waga koi wa/uminotsuki o zo/machiwataru/kurage no hone ni/ auyo ariyato » Fuboku wakashō, op. cit., vol. 4, 1987, p. 248.

8. Toute la section consacrée à la chaux s'inspire de la section du même nom du Tiangong kaiwu de Song Yingxing (publié en 1637, voir notre introduction), bien qu'il ne soit pas cité. De nombreux passages reproduisent, à quelques détails près, ceux du livre chinois. C'est le cas pour la description des différents types de pierre que l'on trouve dans la nature, le mode de cuisson à l'aide du four, les différents usages de la chaux (bateaux et murs), ainsi que les parties dédiées à la chaux d'huîtres et les « chambres d'huîtres ». Voir Tiangong kaiwu, op. cit., fascicule 11, vol. 6, ff. 53-57; Tenkō kaibutsu, yABUUCHI (trad. et éd.), op.cit., p. 217-219 et T'ien-kung k'ai-wu, Chinese technology in the seventeenth century, sun E-Tu Zen and suN Shiou-chuan (transl.), University Park, PA, Penn State University Press, p. 201-203.

9. Ōmi, ancienne province située dans l'actuel département de Shiga.

10. Mino, ancienne province située dans l'actuel département de Gifu.

11. Yoshino, nom de district dans la province de Yamato.

12. Les lieux mentionnés peuvent être localisés dans l'actuel département de Nara, dans l'actuel district de Yoshino. Région montagneuse connue depuis les temps anciens pour la fabrication de la chaux. (Voir CHIBA, Nippon sankai meisan meibutsu zue, op.cit., note 2, p. 158).

13. Ancienne province, située dans l'actuel département de Nara.

14. Empereur Kanmu (737-806), l'empereur qui réalisa le transfert de la capitale de Nara à Heian kyō.

15. Seiryōden joue le rôle d'appartements privés de l'empereur à partir du milieu de l'époque Heian. S'y déroulent également un certain nombre de rites dont la salutation des quatre directions.

16. Il s'agit d'un rite de la cour en vigueur aux $\mathrm{x}^{\mathrm{e}}$-XII ${ }^{\mathrm{e}}$ siècles au cours duquel l'empereur effectuait des salutations face aux quatre points cardinaux, en se plaçant dans le jardin situé devant le pavillon Seiryōden. Des nattes y étaient étalées et le lieu était entouré de paravents. À partir d'un certain moment, peut-être l'époque de l'empereur Kammu (r. 781-806), on y a installé une estrade enduite de chaux. Francine Hérail, communication personnelle.

17. La « lieue » est une traduction de l'unité de distance ri. Elle vaut environ $3,9 \mathrm{~km}$.

18. La même phrase est citée par Song Yingxing dans le Tiangong kaiwu. Voir T'ien-kung k'ai-wu, op. cit., p. 201.

19. Le mont Ibuki est situé à la frontière de l'actuel département de Shiga et de celui de Gifu. On en extrait de la pierre de chaux.

20. Ishibe, région montagneuse située dans le district de Kōga dans le département de Shiga.

21. Yamashiro, ancienne province située dans l'actuelle préfecture de Kyōto.

22. Kurama, nom de montagne située dans l'actuelle préfecture de Kyōto.

23. Le pied traduit ici l'unité de longueur shaku, qui vaut environ $30 \mathrm{~cm}$.

24. Nom de lieu non répertorié dans le Nihon rekishi chimei taikei.

25. La travée est une traduction de l'unité de longueur ken, qui vaut environ 1, $818 \mathrm{~m}$. La hauteur évoquée est donc de plus de $360 \mathrm{~m}$.

26. L'éditeur moderne du Nippon sankai meisan zue, Chiba Tokuji, s'interroge sur la raison de cette référence compte tenu de la distance qui sépare cette rivière Yabu de la province de Gifu avec le mont Ibuki.

27. La toise est la traduction de jō. Elle vaut 10 pieds, soit environ $3 \mathrm{~m}$. 
28. La transformation désignée par le terme suika, suggère parallèlement, par le biais de sa graphie et de sa prononciation, que la matière absorbe de l'eau et devient volatile.

29. Le terme fukeru employé pour exprimer cette idée est noté phonétiquement, ce qui donne à penser que l'auteur tient l'information des artisans japonais. Le terme fukeru renvoie à la transformation qui s'opère dans la consistance de la matière par absorption d'humidité, celle-ci devenant plus molle.

30. Voir le fascicule 3, section : «Les huîtres » du présent ouvrage.

31. Le terme utilisé 羅, auquel la lecture usumono (soie fine) est accolée, désigne à l'origine un type de tissage particulier originaire de Chine et en usage au Japon à l'époque ancienne. Il n'est pour ainsi dire plus employé à l'époque d'Edo.

32. Bien que cela ne soit pas mentionné, ce passage est extrait du Tiangong kaiwu, fascicule 11 ( Calcination des pierres»). L'auteur cite le passage en omettant de préciser que la chaux est préalablement mélangée à l'huile, ce qui rend le passage peu compréhensible.

33. Le terme employé, kaishiro, désigne un rideau utilisé en guise de cloison dans la maison. L'idée est de renforcer avec de la chaux la résistance des tissus ou des papiers destinés à des usages répétés.

34. Toute la section s'inspire de la section «Porcelaine blanche» du chapitre «Fabrication de la porcelaine » du Tiangong kaiwu. Assez rarement l'auteur reproduit à l'identique des passages du livre chinois. Il s'agit le plus souvent d'adapter le texte chinois en y introduisant des éléments de contexte japonais. La liste des sites de production et leurs caractéristiques par laquelle s'ouvre la section est directement inspirée de la liste fournie par Song Yingxing pour la Chine. Il existe une traduction en français de cette partie, intitulée "Mémoire sur la porcelaine du Japon » par Johann Hoffmann. Voir Histoire et fabrication de la porcelaine chinoise, Julien Stanislas (trad.), notes par Salvétat Louis Alphonse, augmenté d'un Mémoire sur la porcelaine du Japon traduit du japonais par Hoffman Johann Joseph, Paris, Mallet-Bachelier, 1856.

35. Le fief de Saga a procédé à l'époque d'Edo à un regroupement de villages sous le nom d'Arita gō pour en faciliter l'administration. Le nombre de villages ainsi regroupés est de l'ordre de vingt-deux à vingt-trois. L'auteur relève ici les noms des villages les plus fameux. Le site couvre une surface assez étendue, englobant l'essentiel de la ville d'Arita machi, Nishiarita machi, et la partie sud de la ville d'Imari. Kadokawa chimei jiten, notice « Arita gō ».

36. Ōkawachi est le nom d'un village de l'époque d'Edo situé dans le district de Matsura. Il donne son nom aujourd'hui à un quartier de la ville d'Imari et l'ancien site du four des Nabeshima est classé monument historique.

37. Mikawachi est le nom d'un village situé dans le district de Fujitsu à l'époque d'Edo. Aujourd'hui, après plusieurs refontes administratives, il peut être localisé dans la ville de Kashima, située au sud du département de Saga.

38. Izumi est un village, également appelé Izumi yama, situé dans le district de Matsura, au pied de la montagne Izumi dont est extraite la pierre blanche. Regroupe les ateliers de fabrication de la porcelaine.

39. Akaemachi est une ville située dans le district de Matsura, lieu où se trouvaient réunis les artisans spécialisés dans la fabrication de la porcelaine polychrome appelé dans la région akae (dessins rouges), voir plus loin.

40. Les Nabeshima sont les seigneurs du fief de Saga. La production de céramique d'ōkawachi était depuis le milieu du XVII ${ }^{\mathrm{e}}$ siècle le bien exclusif de la famille Nabeshima qui en redistribuait les pièces à titre de cadeaux. Saga ken no rekishi, p. 97-98.

41. Le fief de Hirado avait sous son administration le district de Matsura dans la province de Hizen et la province d'Iki, tous deux situés dans l'actuel département de Nagasaki. Le fief de Hirado était gouverné par la famille Matsura.

42. Imari est le nom d'une ville, à l'embouchure du fleuve de même nom, dans le district de Matsura, dans la province de Hizen. Imari est aujourd'hui une ville portuaire de plus de 
cinquante mille habitants dont le développement remonte à l'époque d'Edo. La porcelaine fabriquée dans la région était chargée sur des bateaux à Imari et livrée dans les différentes parties du Japon.

43. Arita est une ville située dans le fief de Saga. Elle est encore aujourd'hui une ville réputée pour ses ateliers de céramique, située dans le district de Nishi Matsura dans le département de Saga.

44. En japonais, seijimono. Le terme très répandu en Occident de céladon tend actuellement à être remplacé par l'appellation "grès à couverte verte », plus proche de l'usage anglo-saxon greenware et plus fidèle au chinois qingciqi.

45. Le terme utilisé peut également se lire ado. C'est le terme que l'on trouve dans le Tiangong kaiwu. Il s'agit de la terre blanche, extraite du mont Izumi, qui sert à la fabrication locale de la porcelaine. Il s'agit d'une terre dure, qui est mêlée à une terre plus tendre au cours du processus de fabrication. D'après la note (2), p. 285 d'Histoire et fabrication de la porcelaine chinoise, JULIEN (trad.), op. cit., la pierre dure serait du feldspath.

46. Le Tiangong kaiwu indique et apporte des précisions sur les cinq à six sites de production de la porcelaine en Chine. Il est remarquable que le nom des ateliers du site de Jingdezhen ne soit pas mentionné par l'auteur du Nippon sankai meisan zue. Voir Tenkō kaibutsu, op.cit., p. 140 et T'ien-kung k'ai-wu..., op. cit., p. 146.

47. Les dimensions semblent concerner la poutre plutôt que le pilon.

48. Le pouce est une traduction de l'unité sun. 10 pouces valent 1 pied.

49. Une travée (ken) est égale à 6 pieds.

50. Le terme exact en français n'est pas connu. Suihi désigne le processus consistant à laisser tremper dans l'eau pour récupérer la matière la plus fine en suspension dans le liquide.

51. Le passage décrivant les deux modes de production des récipients est très proche du Taingong kaiwu. Cependant, le livre chinois est beaucoup plus précis dans les étapes de la fabrication. Le caractère lacunaire du texte japonais peut être complété par le livre chinois.

52. Le texte indique la lecture shinki pour cette combinaison de caractères, qui peut également être prononcée maki. Le terme maki renvoie à plusieurs sortes de bois, mais principalement au koyamaki, pin parasol (Sciadopitys verticillata). Le koyamaki est un bois présentant de nombreuses qualités, réputé pour sa résistance, utilisé depuis l'Antiquité, mais dont l'usage s'est raréfié au cours du temps. L'axe du tour de potier faisait donc appel à un matériau précieux et rare. On remercie Mechtild Mertz pour ces informations.

53. Le terme employé, kageboshi, renvoie à un type de séchage où l'objet est placé à l'ombre et exposé à l'air.

54. Le tsubo est une unité de surface correspondant au carré d'une travée (ken). Il vaut environ $3,3 \mathrm{~m}^{2}$.

55. Les marchandises sont emballées dans des sacs de paille appelés hyō. Nous traduisons ce terme par ballot.

56. Il s'agit d'un arbre, plus connu sous le nom d'isunoki (Distylium racemosum). Il pousse dans les régions chaudes, dans la partie occidentale de l'île du Honshū. Le bois est connu pour sa dureté. On en fait des bûches et la cendre obtenue, appelée isubai, est utilisée précisément dans la fabrication de céramique. Les feuilles portent des petites noix de galle qui contiennent du tanin utilisé en teinture. Dans la traduction de Hoffmann, on trouve des notes très étoffées sur la question. Histoire et fabrication de la porcelaine chinoise, JULIEN (trad.), op. cit., p. 290-291.

57. Le Tiangong kaiwu est un ouvrage chinois de l'époque des Ming (préface datée de 1637) attribué à Song Yingxing (1587-vers 1666). Il présente les productions artisanales et les transformations des produits naturels des différentes régions de Chine.

58. Terme générique pour désigner les matières ferreuses ou contenant du manganèse, de couleur noire ou brun foncé. Pyrolusite $\left[\mathrm{MnO}_{2}\right]$. 
59. Mines d'argent situées dans le département de Shimane, dans le district de Nima, ville d'Ōmori.

60. Le passage correspondant dans le Tiangong kaiwu indique qu'avec la qualité inférieure on obtient une couleur brune de terre. Bizarrement, ce complément attendu n'est pas mentionné dans le texte japonais.

61. 1 bu est le dixième de monme, une unité de poids de la monnaie d'argent qui était toujours pesée pour être échangée.

62. La terme japonais nishikide se décompose en : nishiki (brocart) et de (manière). Le brocart renvoie à la polychromie, comme c'est le cas pour le nishikie, les impressions polychromes.

63. Le terme bidoro gusuri employé ici ne se trouve pas dans le Tiangong kaiwu. Bīdoro est un terme d'origine portugaise qui signifie le verre. L'auteur semble se référer à un type de vitrification de la porcelaine d'origine occidentale.

64. Nom par lequel on désignait au Japon la céramique fabriquée dans les fours du Jingdezhen en Chine sous les Ming et les Qing et importée via Nankin.

65. C'est-à-dire sans couverte.

66. Le terme mushikuide indique l'apparition sur la surface de la poterie de zones où la couverte est manquante et où l'on peut voir le matériau brut. Ces irrégularités qui n'étaient au départ que des défauts de fabrication constatés sur la porcelaine de Jingdezhen en sont venues à être recherchées par les maîtres de thé au Japon. Voir Kadokawa Nihon tōji daijiten, p. 1337.

67. Il s'agit du titre abrégé du Kuji hongi, annale historique d'auteur inconnu, dont la date d'écriture se situerait entre 806 et 936. Il retrace les faits se rapportant aux premiers empereurs.

68. Le deuxième caractère entrant dans ce nom est tōo qui signifie la céramique. Il est difficile de conclure s'il s'agit d'un toponyme, mais c'est ce que le texte laisse entendre.

69. Ancien nom de district, situé en bordure de mer, dans la partie orientale de la baie d'Osaka. Il s'étirait de Sakai à Sennan, en passant par Kishiwada.

70. Il convient de ne pas confondre la province d'Izumi mentionnée ici, qui est située dans la partie sud de l'actuelle préfecture d'Osaka, avec le mont Izumi mentionné au début du chapitre qui se situe dans l'île du Kyūshū.

71. Les termes mentionnés ici sont tous des combinaisons de caractères signifiant pot (kame) et purifier (imu).

72. Une unité de volume de liquide correspondant à l'époque d'Edo à 18 litres environ, soit dix fois l'unité appelée shō. Mais il est ici fait référence au to ancien.

73. Le masu est un récipient étalon en bois ou en métal mesurant la quantité d'un produit liquide ou se présentant sous la forme de poudre. Le masu de Kyōto est adopté en 1669 comme valeur de référence pour le pays. Il a 2,7 pouces de profondeur, et le côté de sa section carrée est de 4,9 pouces.

74. Le Engishiki (927), édité par plusieurs auteurs dont Fujiwara no Tokihira (871-909), est un traité précisant les contours de la bureaucratie et le rituel de cour qui complète le code administratif ritsuryō publié en 702.

75. On note que le rôle des artisans coréens invités par les daimyō du Kyūshū n'est à aucun moment mentionné alors que l'on sait qu'il a été essentiel dans le développement de la porcelaine dans la région d'Arita au tournant du XVII ${ }^{\mathrm{e}}$ siècle. Voir NAGAHARA Keiji (éd.), Kōza: Nihon gijutsu no shakaishi (Leçons sur l'Histoire sociale des techniques du Japon), vol. 4, Nihon hyōronsha, 1984, p. 180-183.

76. Dans l'édition moderne du livre, Chiba s'interroge sur les raisons qui ont poussé l'auteur à ignorer le coton produit un peu partout au Japon et notamment dans la région d'Osaka. Plusieurs provinces du centre sont indiquées comme lieux de production de coton dans le Nōgyō zensho (Traité complet d'agriculture), publié en 1696, et l'on peut considérer qu'il constitue une production courante de l'époque. L'intérêt de l'auteur se porte ici sur le crêpe d'Echigo, plus connu aujourd'hui sous le nom de crêpe d'Ojiya et classé au patrimoine culturel immatériel de 
l'UNESCO. La région était déjà connue dès l'antiquité pour sa production d'étoffes de ramie de qualité supérieure. La production du crêpe, qui s'en distingue par son aspect plissé, serait plus tardive et daterait du milieu du XVII ${ }^{\mathrm{e}}$ siècle. Elle aurait connu une croissance remarquable à partir de la fin $\mathrm{du} \mathrm{XVII}^{\mathrm{e}}$ siècle en important la ramie de provinces voisines. À l'époque de notre auteur, elle est à son sommet, alors que Nara, autrefois connu pour ses étoffes, connaît le déclin.

77. Yamato, ancienne province située dans l'actuel département de Nara.

78. Echigo, ancienne province, correspondant grosso modo à l'actuel département de Niigata.

79. Le terme employé mao, se décompose en : ma, également lu karamushi, signifiant « ramie » et o, signifiant «chanvre». Dans ce passage, le terme peut être considéré comme désignant l'ensemble des fibres textiles. Cela comprend aussi le tilleul du Japon appelé shina.

80. Shinano, ancienne province correspondant à l'actuel département de Nagano.

81. Musashi, ancienne province à cheval sur l'actuelle ville de Tokyo, le département de Saitama et le département de Kanagawa.

82. Shimōsa, ancienne province à cheval sur l'actuel département de Chiba et celui d'Ibaragi.

83. Shimotsuke, ancienne province correspondant à l'actuel département de Tochigi.

84. Hitachi, ancienne province correspondant grosso modo à l'actuel département d'Ibaragi.

85. Ezo, région aux frontières indéterminées, située aux portes nord du territoire japonais, et englobant l'actuelle île de Hokkaidō et les îles limitrophes. Les gens d'Ezo sont les Ainu. L'auteur pourrait sous-entendre ici que l'origine du tissage pratiqué à Echigo se trouverait à Ezo.

86. Toile ou fibre du tilleul du Japon.

87. Sarashina est en effet à l'époque d'Edo le nom d'un district situé dans la province de Shinano. Il est aujourd'hui intégré dans la ville de Chikuma, dans le département de Nagano.

88. Hoshina n'est pas un nom de district. Il est à l'époque d'Edo le nom d'un village situé dans le district de Takai, dans la province de Shinano. Le village est désormais un secteur de la ville de Nagano.

89. Kurashina, non plus, n'est pas un nom de district mais un nom de village, situé à l'époque dans le district de Hanishina. Il est localisé aujourd'hui dans la ville de Chikuma dans le département de Nagano.

90. Il n'existe ni district ni village portant ce nom mais le secteur entourant le sanctuaire Nishina shinmeigū et la ville d'ōmachi, dans le département de Nagano, est désigné sous ce nom. Nishina est le nom d'un clan implanté dans la région.

91. Les deux caractères qui composent le terme omi 麻績 signifient respectivement «fibre végétale » et « filer ».

92. Les chants de kagura sont à l'origine des chants et danses exécutés devant les divinités. Ils se fixent comme rites de cour à l'époque Heian. À l'époque d'Edo, les chants de kagura renvoient à

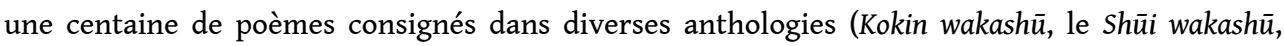
etc.), remontant au $\mathrm{x}^{\mathrm{e}}$ et $\mathrm{xI}^{\mathrm{e}}$ siècle. Voir Dictionnaire historique du Japon, Paris, Maisonneuve \& Larose, 2002, notice kagura.

93. Yū tsukuru/Shinano haraniya/asatatsune/asatatsune. Voir usUDA Jingorō (éd.), Shinpen nihon koten bungaku zenshū 42 Kagurauta, Saibara, Ryōjinhishō, Kanginshū, Tokyo, Shōgakukan, 2000, p. 81. Le poème est ici traduit par Daniel Struve.

94. Le commentateur de l'édition moderne du Meisan zue pense qu'il y a probablement eu confusion et que les graines mentionnées devaient avoir un autre usage. Voir CHIBA T., Nippon sankai meisan zue, p. 172.

95. Nagato, nom d'une ancienne province située dans l'actuel département de Yamaguchi.

96. Le shidori ou shizuri, écrit avec les caractères signifiant "japonais " et "motif » est le nom d'un type de tissage autochotone, largement répandu au Japon, avant l'introduction du brocart chinois. Le terme est mentionné dans les anciens documents du Japon. On suppose qu'il présentait des rayures ou des motifs. 
97. L'auteur semble faire une confusion en employant ici le terme gangi qui désigne habituellement une structure ajoutée aux auvents, alors qu'il a sans doute à l'esprit des raquettes chaussées par les gens de la région pour se déplacer dans la neige.

98. Kashiwazaki est encore de nos jours une ville d'importance située en bordure de mer, dans le département de Niigata.

99. Ojiya est une autre ville du département de Niigata, de moindre envergure que Kashiwazaki, située à $25 \mathrm{~km}$ dans les terres. Le nom d'Ojiya est aujourd'hui étroitement associé à la fabrication du crêpe.

100. Aucun lieu du nom de Shimotani n'a pu être localisé dans cette région.

101. Senju est le nom d'un village qui se trouve aujourd'hui fondu dans la commune de Kawanishimachi, dans le district de Nakauonuma, dans le département de Niigata.

102. Shiozawa correspond à la commune du même nom, situé dans le district de Minamiuonuma.

103. Tōkamachi correspond à la ville du même nom, située dans le département de Niigata. Située dans une cuvette, elle est traversée par le fleuve Shinano.

104. Ce nom est donné à un motif en rayures obtenu par variation d'un motif standard ou encore à un type de rayures qui ne ressemble à rien de connu.

105. Horinouchi est le nom d'un village en bordure de mer, situé aujourd'hui dans la ville de Murakami, à l'extrême nord du département de Niigata.

106. Cette indication est probablement reprise du Tiangong kaiwu qui indique le même chiffre. Yabuuchi l'évalue à cinq heures environ, en se référant probablement à un découpage de la journée en 100 koku à la manière des spécialistes de calendrier. Voir Tenkō kaibutsu, YABUUCHI (trad.), op. cit., p. 6.

107. On note que l'auteur reprend ici mot pour mot des passages du paragraphe consacré aux vêtements d'été dans le Tiangong kaiwu (Exploitation des œuvres de la nature). Les étoffes tissées en fibres de ramie sont en effet considérées comme adaptées pour les vêtements d'été. On retrouve notamment la mention qu'il existe deux types de ramie, que celle-ci pousse partout, qu'on produit trois fois plus avec un rouet à pédale, et la description de la procédure de fabrication. La différence réside dans l'exposition à la neige et au soleil, une spécificité des terres enneigées d'Echigo. Voir Tenkō kaibutsu, op. cit., p. 59.

108. Aku : eau prélevée dans la partie supérieure du mélange obtenu de la cendre et de l'eau.

109. Le gō est employé encore de nos jours comme borne de hauteur sur un chemin de montagne, reliant le pied au sommet. Le chemin est divisé en dix segments selon des critères assez flous, liés à la difficulté de l'ascension, et les gō ou gōme se comptent généralement à partir du point de départ. Cela contredit l'exposé de l'auteur mais il est possible que les pratiques aient évolué. L'emploi est attesté à l'origine pour le mont Fuji.

110. Le terme provient de la langue ainu onnep, transcrit avec les caractères 膃㑂 dans les ouvrages chinois et repris ici. On peut supposer que cette espèce animale était très peu connue par les Japonais des régions centrales et qu'elle pouvait susciter la curiosité des lecteurs. Il s'agit selon toute probabilité du lion de mer de Steller ou de l'otarie de Steller, que l'on trouve précisément près des côtes du Pacifique nord. Les mâles sont plus gros que les femelles et peuvent atteindre 1 tonne pour une longueur de $3 \mathrm{~m}$.

111. Matsumae est le nom de la ville castrale du fief du même nom, située à la pointe sud-ouest de la presqu'île d'Oshima dans l'île de Hokkaidō. Voir l'introduction pour plus de précisions.

112. Le nom est donné phonétiquement. Oshamanbe a été relié à Matsumae par une route en 1799. La commune du même nom est située aujourd'hui à une distance d'environ $100 \mathrm{~km}$ de Matsumae.

113. La deuxième lune est celle de l'équinoxe de printemps.

114. L'estimation donnée ici d'une distance de $2700 \mathrm{~km}$ paraît excessive.

115. Nom d'une ancienne province qui englobe les départements actuels d'Iwate, Miyagi, Aomori, Miyagi et une partie du département d'Akita. 
116. La liste des produits proposés aux populations Ainu montre que les Japonais évitaient de procurer à ces derniers des objets pouvant être utilisés comme armes.

117. Sōya est le nom de la pointe située à l'extrême-nord de l'île de Hokkaidō.

118. Ottosei est le nom du médicament produit à partir de la verge d'otarie, mentionné plus loin dans le texte.

119. Désigne la façade occidentale de l'extrême nord du Honshū.

120. Désigne la façade orientale de l'extrême nord du Honshū.

121. Enhydra lutris, loutre de mer, que l'on ne trouve que dans l'océan pacifique, au nord du Japon.

122. Il s'agit d'un médicament produit dans la région de Nanbu, mais on ne sait pas grand-chose de plus. Chiba indique que selon le Complément du Bencao gangmu (Honzō kōmuku shūi), il s'agirait d'une plante qui serait utilisée dans les pommades à appliquer sur les plaies.

123. Le titre mentionné est Honzō shikkai que nous assimilons au Bencao gangmu.

124. La rubrique jijie ou "Explications réunies" dans le livre de Li Shizhen regroupe des commentaires d'auteurs anciens, cités dans un ordre chronologique. On trouve en effet dans une citation tirée du 臨海志 que l'otarie a pour origine les mers de l'Est.

125. Cette terminologie donnée uniquement phonétiquement donne à penser qu'elle est d'origine ainu.

126. Renvoie au spécificatif numérique wa (ailes) utilisé pour les volatiles.

127. Connu sous ce nom car ayant fait construire une résidence secondaire au pied du mont Kinugasa.

128. Fascicule 36, Poème $n^{\circ} 16978$. «Waga koiwa / todo no nenagare /sameyaranu / yumenarinagara / taeya hatenan », traduit par Daniel Struve. Fuboku wakashō, op. cit., vol. 5, 1988, p. 321.

129. Kaiba est un autre nom pour désigner les éléphants de mer, voire les morses.

130. Michi est l'ancien nom pour l'otarie (ashika).

131. On peut supposer ici qu'il s'agit des nawatojifune, ces petites embarcations dont le socle était fait de rondelles de bois et les rebords d'écorce de bois fixée avec des cordes, d'où le nom de nawatoji fune (bateau relié avec des cordes).

132. Le dernier caractère de ottosei est ici lu hozo, qui signifie pénis. Deux termes sont employés pour pénis, takeri et hozo. La différence de signification, si tant est qu'elle existe, est difficile à saisir. Dans le dernier paragraphe, il est dit que l'un est proche de l'autre, ce qui laisserait entendre que les termes renverraient à deux parties proches mais distinctes de l'animal.

133. C'est-à-dire pendant les dix-huit jours qui précèdent le début de l'automne (risshū), c'est-àdire vers la fin du mois de juillet et au début du mois d'août.

134. Ville castrale située au sud de l'actuel département d'Iwate.

135. Port situé au sud-ouest de la péninsule d'Ojima, dans le Hokkaidō. Se développe à partir du moment où il est intégré dans le domaine du bakufu.

136. Nom d'un port donnant sur la mer du Japon, situé dans l'actuel département de Fukui, qui s'est développé comme lieu de débarquement des marchandises acheminées depuis le nord du Honshū et Ezo vers Kyōto via le lac Biwa.

137. Echizen, ancienne province située au nord de l'actuel département de Fukui.

138. Wakasa, ancienne province située au sud de l'actuel département de Fukui.

139. Obama est une ville castrale du fief du même nom, située dans la province de Wakasa.

140. Oihyō : orme de Mandchourie, de la famille des ulmacées, poussant dans le Hokkaidō. On utilise l'écorce pour fabriquer des cordes, des étoffes et du papier. Nom savant : Ulmus laciniata.

141. Shina. Le nom japonais plus courant est shinanoki. Il s'agit du tilleul japonais (tilia japonica) que l'on trouve dans les régions montagneuses du sud au nord de l'archipel. 
142. Récipient en bois laqué, de forme cylindrique, à trois pieds, utilisé pour transporter des mets, lors de déplacements. La précision fait allusion sans doute à l'usage détourné du récipient qui est fait par les Ainu.

143. Le tomoe est un motif en forme de « virgule".

144. L'ikema est une plante de la famille des gentianacées (Cynanchum caudatum).

145. Il faut comprendre ici, « quand les Ainu respirent ». D'après le Nihon kokugo daijiten, le terme donné ici phonétiquement aurait pour origine le mot ainu husa. Cela renverrait au souffle des gens d'Ezo et à l'épaisse brume qui en résulterait. Il existe de nombreuses interprétations sur le lien entre cette flûte et le terme kosa, mais aucune ne s'impose. Selon l'éditeur moderne du texte, cela résulterait d'un rapprochement entre des noms proches associés à un monde lointain que les Japonais ne connaissaient que par ouï-dire.

146. Minamoto no Yoshitsune (1159-1189) est un guerrier connu comme fin stratège ayant remporté de nombreuses victoires contre les Taira au profit du clan des Minamoto. Son destin tragique, isolé et traqué par son frère Yoritomo, a contribué à la naissance d'une légende autour de sa personne. D'après la légende, il aurait survécu à ses ennemis et se serait échappé à Ezo, voire même en Tartarie.

147. Benkei est un fidèle serviteur de Yoshitsune, auteur de nombreux exploits, qui aurait combattu aux côtés de son maître, jusqu'à son dernier souffle.

148. Les Sewa, famille des Genji dont Yoshitsune est un représentant.

149. C'est une désignation courante de Toyotomi Hideyoshi (1537-1598), l'un des guerriers unificateurs du Japon de la fin du Xvi ${ }^{\mathrm{e}}$ siècle.

150. Tamanoura est le nom d'un centre administratif du territoire placé sous la gouvernance shogunale situé dans l'île de Fukue, elle-même comprise dans l'archipel Gotō. Fukae est le nom d'un village administré par le fief de Shimabara, aujourd'hui intégré dans la ville de Shimabara.

151. Deux noms de lieux chinois donnés ici, non à l'aide de caractères mais phonétiquement. Il est pour cette raison difficile de les identifier. Pour le premier, il pourrait s'agir de Fuzhou (prononcé Fukushū en japonais).

152. Nom d'un village de pêcheurs situé sur la pointe du même nom, à l'extrême sud de la péninsule de Nagasaki. Le village est demeuré sous administration shogunale pendant la période d'Edo.

153. Également lu Fukabori. Nom d'un regroupement de villages placés sous l'administration de Fukahori, vassal des Nabeshima du fief de Saga. Il correspond à l'actuel quartier Fukabori chō de la ville de Nagasaki.

154. Non localisé.

155. Seuls les bateaux munis de licences, appelées goshuin, portant le cachet de l'administration shogunale étaient autorisés à accoster et à commercer à Nagasaki. Ce contrôle très strict sur le commerce extérieur était le moyen trouvé par le shogunat pour réguler le volume de son commerce extérieur.

156. Il s'agit de Mazu (j. Maso), divinité protectrice des marins, dont le culte a débuté dans le Fujian en Chine et s'est étendu dans toute l'Asie du sud-est.

157. Nom d'une divinité protectrice des marins, vénérée au Japon depuis le Moyen Âge. Des amulettes la représentant étaient déposées dans les bateaux.

158. Nom d'un guerrier chinois héroïque de l'époque des trois dynasties, largement vénéré en Chine en tant que Dieu de la guerre.

159. Tōjin yashiki, la résidence réservée aux Chinois, a été construite en 1688. Moins surveillée que Dejima (voir plus bas), la résidence pouvait héberger environ deux mille personnes.

160. Champignon de la catégorie des Polyporaceae utilisé comme remède en médecine chinoise.

161. Les marchands de la Compagnie hollandaise des Indes orientales (VOC) étaient les seuls Européens dont la présence au Japon était autorisée par le bakufu. Ils résidaient dans la presqu'île artificielle de Dejima, rattachée à la ville de Nagasaki par une porte surveillée nuit et 
jour. La relève du personnel se faisait lors de l'arrivée chaque année des bateaux de la Compagnie envoyés de Batavia. Le nombre de bateaux a été réduit à un à la fin du XVIII siècle. Voir Bridging the Divide: 400 years The Netherlands-Japan, Leonard BLussé, Willem REMMELINK, Ivo SMITS (ed.), Teleacnot, Hotei Publishing, 2000, p. 33-54.

162. Les interprètes chargés d'assurer la communication entre l'administration shogunale et les marchands de la Compagnie des Indes orientales étaient classés selon une stricte hiérarchie. Les postes d'« interprète-aîné » ōtsūji et d'« interprète-cadet » kotsūji étaient les plus élevés dans la hiérarchie. 


\section{Postface}

Traduction : Daniel Struve et Annick Horiuchi

1 Ce projet qui, n'était que gouttelettes au bout des branches d'un arbre du vivant de Hose $^{1}$, a pris de l'ampleur au point de se changer en flots abondants de la rivière Minano, grâce au talent de Kangetsu ${ }^{2}$ qui a laissé courir son pinceau.

On chercha à nouer des liens dans les lieux situés dans les provinces éloignées, et, au fil des ans, tout naturellement, les contacts augmentèrent, et les informations qu'on avait d'abord recueillies en faible quantité, finirent par parvenir sans qu'on les sollicite.

3 C'est ainsi que, Rankō ${ }^{3}$, disciple de Kangetsu, par fidélité au souvenir de son maître défunt (Kangetsu), compléta le travail là où il n'était qu'esquissé et je contribuai pour ma part à la correction d'un certain nombre de détails. Finalement, une occasion favorable se présenta, ce qui donna ce magnifique ouvrage en cinq fascicules.

4 Pourquoi le royaume de Silla était-il connu autrefois comme pays aux trésors ? J'ignore les raisons des Anciens, mais, comme le millet dans une rizière qui, tant qu'il est en herbe, ne peut être distingué du riz et se remarque une fois qu'il a poussé, ainsi les richesses s'étant répandues en surabondance dans nos provinces, quelles que soient les occupations auxquelles ils s'adonnent, les gens du peuple, dans le désir de l'emporter sur les autres, ne relâchent pas leurs efforts, explorent toutes les possibilités, et il n'y a pas la moindre occupation qu'ils ne fassent prospérer.

5 Pour reprendre l'image du champ de millet, dans quelle catégorie doit-on ranger tous ces produits des provinces, dans celle du millet ou celle du riz ?

6 Ainsi donc, s'agissant de pays de trésors de l'empire, quels lieux de production laisser de côté ? Lesquels retenir?

7 À force de chercher, nous avons fini par découvrir le rameau de joyaux du mont Penglai ou le coquillage qui facilite l'enfantement (koyasugai) que l'on trouve dans un nid d'hirondelles ${ }^{4}$; trésors lointains que nous avons placés à la fin de l'ouvrage, de manière à ce qu'il soit complet.

8 Achevé à Ōsaka, en l'an dix de l'ère Kansei, année du cheval. 


\section{NOTES}

1. C'est le nom de plume de Hirase Tessai, auteur du Nippon sankai meibutsu zue, dont on a vu qu'il constituait un diptyque avec le Meisan zue (voir notre introduction). Cette postface, qui ne porte aucune signature, confirme le lien très étroit qui lie les deux ouvrages et la possibilité qu'ils aient fait partie d'un même projet qui aurait vu le jour dans les années 1750, puisque la publication du Meibutsu zue date de 1754.

2. Il s'agit donc de Shitomi Kangetsu, auteur des illustrations du Meisan zue, dont on apprend ici qu'il a joué un rôle important dans la conception du Meisan zue.

3. Il s'agit de Nakai Rankō, disciple de Shitomi Kangetsu qui a réalisé les illustrations pour le Meisan zue. Il s'agit d'un milieu de peintres originaires d'Ōsaka, gravitant notamment autour de l'école confucéenne de Kaitokudō, bien connus de Kimura Kenkadō.

4. Ces objets font partie de la liste des trésors réclamés par l'héroïne à ses cinq prétendants, dans le roman Princesse Kaguya, également connu sous le nom de Taketori monogatari (Le conte du coupeur de bambou). Ces trésors sont réputés rares et difficiles d'accès. 


\section{Partie III - Annexes}




\section{Liste des anciennes provinces}

\begin{tabular}{|c|c|c|}
\hline Noms des anciennes provinces & & Noms des départements actuels \\
\hline Aki 安芸 & 18 & Hiroshima 広島 Ouest \\
\hline Awa 安房 & 59 & Chiba千葉 Sud \\
\hline Awa 阿波 & 14 & Tokushima 徳島 \\
\hline Awaji 淡路 & 26 & Hyōgo 兵庫 \\
\hline Bingo 備後 & 20 & Hiroshima 広島 Est \\
\hline Bitchū 備中 & 22 & Okayama 岡山 Est \\
\hline Bizen 備前 & 23 & Okayama 岡山 Sud-Ouest \\
\hline Bungo 豊後 & 5 & Ōita 大分 \\
\hline Buzen 豊前 & 4 & Fukuoka 福岡 Est \\
\hline Chikugo 筑後 & 7 & Fukuoka 福岡 Sud \\
\hline Chikuzen 筑前 & 6 & Fukuoka 福岡 Nord \\
\hline \multirow{2}{*}{ Dewa 出羽 } & 67 & Akita 秋田 \\
\hline & 67 & Yamagata 山形 \\
\hline Echigo 越後 & 65 & Niigata 新潟 \\
\hline Echizen 越前 & 46 & Fukui 福井Est \\
\hline Etchū 越中 & 49 & Toyama 富山 \\
\hline Ezo 蝦夷 & & Hokkaidō北海道 \\
\hline
\end{tabular}




\begin{tabular}{|c|c|c|}
\hline Harima 播磨 & 27 & Hyōgo 兵庫 Sud \\
\hline Hida 飛騨 & 50 & Gifu 岐阜 Est \\
\hline Higo 肥後 & 9 & Kumamoto 熊本 \\
\hline Hitachi 常陸 & 62 & Ibaragi 茨城 Nord \\
\hline Hizen 肥前 & 8 & Saga 佐賀 \\
\hline Hōki 伯耆 & 25 & Tottori 鳥取 Ouest \\
\hline Hyūga 日向 & 3 & Miyazaki 宮崎 \\
\hline Iga 伊賀 & 41 & Mie 三重 Nord \\
\hline Iki 志岐 & 10 & Nagasaki 長崎 \\
\hline Inaba 因幡 & 29 & Tottori 鳥取 Est \\
\hline Ise 伊勢 & 42 & Mie 三重 Sud \\
\hline Iwami 石見 & 19 & Shimane 島根 Ouest \\
\hline Iyo 伊予 & 12 & Ehime 愛媛 \\
\hline Izu 伊豆 & 54 & Shizuoka 静岡 \\
\hline Izumi 和泉 & 34 & Ōsaka 大阪 \\
\hline Izumo 出雲 & 21 & Shimane 島根 Est \\
\hline Ka'i 甲斐 & 56 & Yamashina 山梨 \\
\hline Kaga 加賀 & 47 & Ishikawa 石川 \\
\hline Kawachi 河内 & 35 & Ōsaka 大阪 \\
\hline Kazusa 上総 & 60 & Chiba 千葉 \\
\hline Kii 紀伊 & 36 & Wakayama 和歌山 \\
\hline Kōzuke 上野 & 64 & Gunma 群馬 \\
\hline Mikawa 三河 & 51 & Aichi 愛知 Est \\
\hline Mimasaka 美作 & 24 & Okayama 岡山 Centre \\
\hline Mino 美濃 & 45 & Gifu 岐阜 Ouest \\
\hline \multirow{2}{*}{ Musashi 武蔵 } & 58 & Saitama 埼玉 \\
\hline & 58 & Tōkyō 東京 \\
\hline
\end{tabular}




\begin{tabular}{|c|c|c|}
\hline \multirow{4}{*}{ Mutsu 陸奥 } & 68 & Aomori 青森 \\
\hline & 68 & Iwate 岩手 \\
\hline & 68 & Miyagi 宮城 \\
\hline & 68 & Fukushima 福島 \\
\hline Nagato 長門 & 17 & Yamaguchi 山口Est \\
\hline Noto 能登 & 48 & Ishikawa 石川 \\
\hline Oki 隠岐 & 30 & Shimane 島根 \\
\hline Ōmi 近江 & 40 & Shiga 滋賀 \\
\hline Ōsumi 大隅 & 1 & Kagoshima 鹿児島 Est \\
\hline Owari 尾張 & 44 & Aichi 愛知 Ouest \\
\hline Ryūkyū 琉球 & & Okinawa 沖縄 \\
\hline Sado 佐渡 & 66 & Niigata 新潟 \\
\hline Sagami 相模 & 55 & Kanagawa 神奈川 \\
\hline Sanuki 讃岐 & 15 & Kagawa 香川 \\
\hline Satsuma 薩摩 & 2 & Kagoshima 鹿児島 Ouest \\
\hline \multirow{2}{*}{ Settsu 掑津 } & 33 & Hyōgo 兵庫 Sud-Est \\
\hline & 33 & Ōsaka 大阪 \\
\hline Shima 志摩 & 43 & Mie 三重 \\
\hline \multirow{2}{*}{ Shimōsa 下総 } & 61 & Chiba 千葉 Nord \\
\hline & 61 & Ibaragi 茨城 Sud \\
\hline Shimotsuke 下野 & 63 & Tochigi 杤木 \\
\hline Shinano 信濃 & 57 & Nagano 長野 \\
\hline Suō 周防 & 16 & Yamaguchi 山口Ouest \\
\hline Suruga 駿河 & 53 & Shizuoka 静岡 Est \\
\hline Tajima 但馬 & 28 & Hyōgo 兵庫 Nord Ouest \\
\hline Tanba 丹波 & 32 & Hyōgo 兵庫Nord Est \\
\hline Tango 丹後 & 31 & Kyōto 京都 \\
\hline
\end{tabular}




\begin{tabular}{|l|l|l|}
\hline Tosa 土佐 & 13 & Kōchi 高知 \\
\hline Tōtoumi 遠江 & 52 & Shizuoka 静岡 Ouest \\
\hline Tsushima 対馬 & 11 & Nagasaki 長崎 \\
\hline Wakasa 若狭 & 39 & Fukui 福井 Ouest \\
\hline Yamashiro 山城 & 38 & Kyōto 京都 \\
\hline Yamato 大和 & 37 & Nara 奈良 \\
\hline
\end{tabular}

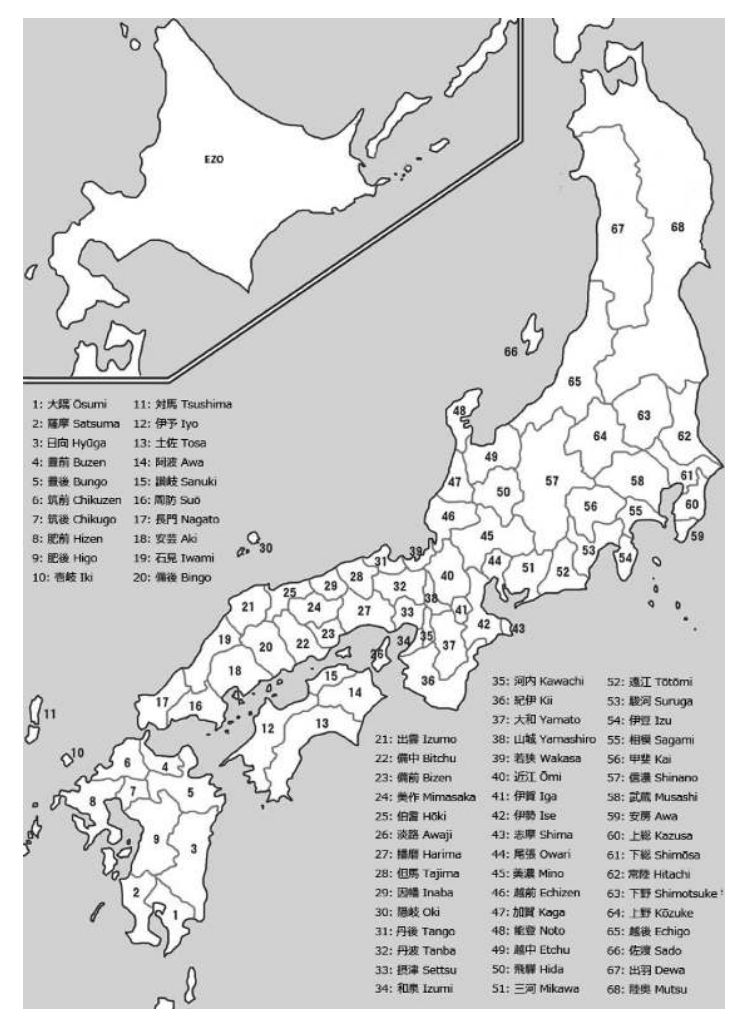

Carte des anciennes provinces du Japon 


\section{Tableau des unités de mesure}

1 Les correspondances données ici n'ont qu'une valeur indicative. Les unités de mesure utilisées à l'époque du Meisan zue présentent une variabilité régionale. Des mesures ont été prises par les dirigeants de l'époque pour unifier certaines unités. Le tableau n'indique que les unités apparaissant dans le texte.

2 Sources : Yoshida Mitsuyoshi, Jinkōki, annoté par Ōya Shin'ichi, Iwanami shoten, 1977 ; Iwanami Nihonshi jiten, 1999, p. 1342.

\begin{tabular}{|c|c|c|c|c|c|}
\hline Mesure & Unité & Conversion & kanji & traduction & Valeur approximative \\
\hline \multirow[t]{6}{*}{ Volume } & koku & $=10$ to & 石、斛 & & 1801 \\
\hline & to & $=10 \mathrm{sho}$ & 斗 & & 181 \\
\hline & shō & $=10 g \bar{o}$ & 升 & & 1,81 \\
\hline & $g \bar{o}$ & & 合 & & $0,18 \mathrm{l} / 180 \mathrm{ml}$ \\
\hline & masu & & 升、妌 & mesure & \\
\hline & hyō (tawara) & & 俵 & sac ou ballot & \\
\hline \multirow[t]{7}{*}{ Longueur, distance } & shaku & & 尺 & pied & $30 \mathrm{~cm}$ \\
\hline & sun & $=0.1$ shaku & 寸 & pouce & $3,03 \mathrm{~cm}$ \\
\hline & $b u$ & $=0.1 \mathrm{sun}$ & 分 & ligne $^{1}$ & $3 \mathrm{~mm}$ \\
\hline & hiro ${ }^{2}$ & $=5$ ou 6 shaku & 広、尋 & brasse & $1,5 \mathrm{~m}$ \\
\hline & jō & $=10$ shaku & 大 & toise & $3 \mathrm{~m}$ \\
\hline & ken & $=6$ shaku & 間 & travée & $1,8 \mathrm{~m}^{3}$ \\
\hline & chō & $=60 \mathrm{ken}$ & 町 & & $109 \mathrm{~m}$ \\
\hline
\end{tabular}




\begin{tabular}{|l|l|l|l|l|l|}
\hline & ri & $=36$ cho & 里 & lieue & $3900 \mathrm{~m}$ \\
\hline surface & tsubo & $=(6 \text { shaku })^{2}$ & 坪 & parcelle & $3306 \mathrm{~m}^{2}$ \\
\hline poids & kin & $=160$ monme & 斤 & livre & $600 \mathrm{~g}$ \\
\hline & monme & $=0.0001$ kanme & 外 & & $3,75 \mathrm{~g}$ \\
\hline & kanme & & 貫目 & & $3,75 \mathrm{~kg}$ \\
\hline
\end{tabular}

\section{NOTES}

1. ligne : en France, ancienne mesure de longueur $=1 / 12$ du pouce

2. Unité utilisée pour mesurer la profondeur de l'eau.

3. Par définition, la travée correspond à la distance entre deux piliers. Elle est de 6 shaku 5 sun au début de l'époque d'Edo, et par la suite, fixée à 6 shaku. 


\section{Bibliographies}

\section{Sources premières}

Bencao gangmu 本草綱目 (Classement raisonné de la materia medica), LI Shizhen 李時珍, copie conservée à la Bibliothèque de la Diète, imprimé en 1590. Accessible en ligne.

Dai Nippon bussan zue 大日本物産図会 (Album illustré des produits du Grand Japon), UTAGAWA Hiroshige (dit Hiroshige III), Tokyo, Ōkura Magobee, 1877.

Engishiki 延喜式 (Règlements de l'ère Engi), Tokyo, Yoshikawa kōbunkan, coll. "Shintei zōho kokushi taikei ", 3 vols., réédition (fukyūban), 1972. vol. 1 : Kōtaishiki, Kōjinshiki, Engishiki zenpen 交 替式·弘仁式·延喜式前篇; vol. 2 : Engishiki chūhen 延喜式中篇; vol. 3 Engishiki kōhen 延喜式後篇.

Fuboku wakashō 夫木和歌抄 (Sélection de poésie japonaise), FUJIWARA Nagakiyo 藤原長清, Kunaichō shoryōbu (éd.), 5 vols., Tokyo, Meiji shoin, coll. « Zushoryō sōkan », 1984-1988.

Gōke shidai 江家次第 ou Gōshidai 江次第 (Déroulement des procédures et des célébrations selon la maison Ōe), vol. 2, Tokyo, Meiji tosho shuppan, coll. « Shintei zōhō kojitsu sōsho », 1951.

Heiji monogatari 平治物語 (Le Dit de Heiji), vol. 31, Tokyo, Iwanami shoten, coll. « Nihon koten bungaku taikei », 1961.

Histoire et fabrication de la porcelaine chinoise, JULIEN Stanislas (trad.), notes par SALVÉTAT Louis Alphonse, augmenté d'un Mémoire sur la porcelaine du Japon traduit du japonais par HOFFMAN Johann Joseph, Paris, Mallet-Bachelier, 1856.

Honchō shokkan 本朝食鑑 (Miroir des aliments de notre empire), HITOMI Hitsudai 人見必大, SHIMADA Isao 島田勇雄 (trad. et éd.), 5 vols., Tokyo, Heibonsha, coll. « Tōyō bunko », 1980.

Ikkaku sankō 一角纂考 (Somme de recherches sur le monocéros; 1795), in Edo kagaku koten sōsho 32 Rikubutsu shinshi kō, Ikkaku sankō kō 江戸科学古典㙚書32 六物新志・稿. 一角纂考・稿, Tokyo, Kōwa shuppan, 1980.

Jinrin kinmōzui 人倫訓蒙図彙 (Dictionnaire illustré de l'humanité), ASAKURA Haruhiko 朝倉治彦 (éd.), Tokyo, Heibonsha, coll. « Tōyō bunko » 519, 1990.

Koji ruien 古事類苑 (Encyclopédie des choses anciennes), Koji ruien kankōkai (éd.), 60 vols., Tokyo, Koji ruien kankōkai, 1931-1936.

Kojiki 古事記 (Chronique des faits anciens), Tokyo, Iwanami shoten, coll. « Nihon shisō taikei » 1, 1958. 
Man.yôshû, SIEFFERT René (trad), Paris, Publications Orientalistes de France/UNESCO, Livres I-III 1997 ; Livres IV-VI 1998 ; Livres VII-IX 2001 ; Livres X-XII 2003.

Man'yōshū 万葉集 (Recueil des dix mille feuilles), SATAKE Akihiro 佐竹昭広, YAMADA Hideo 山田英 雄, KUDō Rikio 工藤力男, ōTANI Masao 大谷雅夫, YAMAZAKI Yoshiyuki 山崎福之 (éd.), vol. 1-4, Tokyo, Iwanami shoten, coll. « Shin Nihon Koten bungaku taikei », 1999-2003.

Nihon shoki, 日本書紀 (Chronique du Japon), Tokyo, Iwanami shoten, coll. « Nihon koten bungaku taikei » 67 et 68, 1993.

Nippon sankai meibutsu zue 日本山海名物図会 (Guide illustré des spécialités des monts et mers du Japon), Tokyo, Meicho kankōkai, 1969 (reproduction de l'édition datée de 1754, introduction de HIGUCHI Hideo 樋口秀雄).

Nippon sankai meisan zue 日本山海名産図会 (Guide illustré des produits renommés des monts et mers du Japon), Tokyo, Meicho kankōkai, 1969 (reproduction de l'édition datée de 1799, introduction de HIGUCHI Hideo 樋口秀雄).

Nippon sankai meisan meibutsu zue 日本山海名産名物図会 (Guides illustrés des produits renommés et des spécialités des monts et mers du Japon), cHIBA Tokuji 千葉徳爾 (éd.), Tokyo, Shakai shisōsha, 1970.

Nihon suido kō, Suido kaiben, zōho kai tsūshōkō 日本水土考・水土解弁・増補華夷通商考 (Réflexions sur le milieu naturel du Japon ; Discours sur l'interprétation au moyen du milieu naturel ; version augmentée des Considérations sur les échanges commerciaux avec la Chine et les barbares), NISHIKAWA Joken 西川如見, IIJIMA Tadao 飯島忠夫 et al. (éd.), Tokyo, Iwanami shoten, coll. « Iwanami bunko », 1944.

Sangi ittō̄ 三義一統 (Trois principes, une autorité), Zoku gunsho ruijū vol. 24a, chap. 681, Gunsho ruijū kanseikai, 1924.

Saigyō Sankashū zenchūkai 西行山家集全注解 (Le Sankashū de Saigyō intégralement annoté), WATABE Tamotsu 渡部保 (éd.), Tokyo, Kazama shobō, 1979.

SIEBOLd Philipp Franz von, TEMMINCK Coenraad Jacob et SCHLEGEL Hermann, Fauna Japonica 2. Pisces : sive description animalium, quae in itinere per Japoniam, jussu et auspiciis superiorum, qui summum in India Batavia imperium tenent, suscepto, annis 1823-1830 collegit, notis observationibus et adumbrationibus illustravit Pisces, Dusselorfiae, Apud Arnz et Socios, 1844. Accessible en ligne.

SIEBOLD Philipp Franz von. Nippon - Archiv zur Beschreibung Japans, Leyden: bei dem Verfasser, 1832-1852, Tokyo, Yūshōdō, 1979.

USUDA Jingorō (éd.), Shinpen nihon koten bungaku zenshū 42, Kagurauta, Saibara, Ryōjinhishō, Kanginshū，新編日本古典文学全集 42, 神楽歌, 催馬楽，梁鹿秘 抄, 䦥吟集, Tokyo, Shōgakukan, 2000.

Shinsarugakuki (Notes sur de nouveaux divertissements comiques), FUJIWARA no Akihira, HÉRAIL Francine (traduit et annoté par), Paris, Les Belles Lettres, 2014.

Shoku Kokin wakash $\bar{u}$ 続古今和歌集 (Suite au Recueil de waka anciens et modernes), in Shinpen Kokka taikan 新編国歌大観・第 1 巻·歌集, vol. 1, Tokyo, Kadokawa shoten, 1983, p. 357-388.

Tenkō kaibutsu 天工開物 (Exploitation des œuvres de la nature), soNG Yingxing 宋應星, YABUUCHI Kiyoshi 藪内清 (trad. et éd.), Tokyo, Heibonsha, coll. « Tōyō bunko » 130, 1988 (première édition 1969).

T'ien-kung k'ai-wu, Chinese technology in the seventeenth century, sUNG Ying-Hsing, sun E-Tu Zen and suN Shiou-chuan (transl.), University Park, PA, Penn State University Press, 1966.

Tiangong kaiwu 天工開物, soNG Yingxing 宋應星, 18 fascicules, (imprimé) 1771, 7 vols., Osaka. Copie conservée à la Bibliothèque de la Diète, Accessible en ligne. 
Tōga 東雅 (Er'ya de l'Est), ARAI Hakuseki 新井白石, ōTSUKI Nyoden 大槻如電 (éd.), vol. 1-20, Tokyo, Yoshikawa Hanshichi, 1903.

Tsurezuregusa 徒然草 (Les heures oisives), in Hōjōki, Tsurezuregusa 方丈記、徒然草, SATAKE Akihiro 佐竹昭広 et кUвотA Jun 洼田淳 (éd.), Tokyo, Iwanami shoten, coll. «Shin Nihon koten bungaku taikei » 39, 1989.

Les heures oisives (Tsurezure-gusa), URABE Kenkō, GROSBOIS Charles et YOSHIDA Tomiko (Traduction et commentaires de), suivi de Notes de ma cabane de moine (Hōjō-ki), KAMO no Chōmei, R. P. Sauveur Candau (trad.), Paris, Gallimard, 1980.

Wakan sansai zue 和漢三才図会 (Encyclopédie sino-japonaise des trois règnes), TERAJIMA Ryōan 寺 島良安, SHIMADA Isao 島田勇雄, TAKESHIMA Atsuo 竹島淳夫, HIGUCHI Motomi 樋口元巳 (trad. et éd.), 18 vols., Tokyo, Heibonsha, coll. « Tōyō bunko », 1985-1991.

Wakan sansai zue 和漢三才四会 (Encyclopédie sino-japonaise des trois règnes), TERAJIMA Ryōan 寺 島良安, 2 vols. (jō et ge), Tokyo, Tōkyō bijutsu, 1970.

Wakoku hyakujo 和国百女 (Cent femmes du Japon), Hishikawa Moronobu 菱川師宣 (illustré par), imprimé en 1695, in Nihon füzoku zue 日本風俗図絵, KUROKAWA Mamichi 黒川真道 (éd.), vol. 1, Nihon fūzoku zue kankōkai, 1915. Copie conservée à la Bibliothèque de la Diète. Accessible en ligne.

Wakoku shoshoku etsukushi 和國諸職繪つくし (Catalogue illustré des métiers du Japon), HISHIKAWA Moronobu 菱川師宣 (illustré par), imprimé en 1685, in Fūzoku zue 日本風俗図絵, KUROKAWA Mamichi 黒川真道 (éd.), vol. 2, Nihon fūzoku zue kankōkai, 1915. Copie conservée à la Bibliothèque de la Diète. Accessible en ligne.

Wamyōshō 和名抄 ou encore Wamyō ruijyushō 倭名類聚鈔 (Répertoire ordonné de noms du Yamato) de Minamoto no Shitagō 源順. Ouvrage datant de 934, réédité à l'époque d'Edo à plusieurs reprises. Édition de Naha Dōen 那波道圓 (Kassho 活所) (1595-1648), 20 fascicules, Osaka, préface datée de 1617. Copie conservée à la Bibliothèque de la Diète. Accessible en ligne.

Yamato honzō 大和本草 (La materia medica du Japon), in Ekiken zenshū 益軒全集 (๕uvres complètes d'Ekiken), KAIBARA Ekiken 貝原益軒, vol. 6, Tokyo, Kokusho kankōkai, 1973, (première édition 1911). Édition en 20 fascicules datée de 1709 conservée à la Bibliothèque de la Diète. Accessible en ligne.

\section{Études}

ABÉ Yoshio, «La fabrication du saké : processus biochimique et procédé technique », in Des mérites comparés du saké et du riz, LEGGERI-BAUER Estelle, BÉRANGER Véronique, BRISSET Claire-Akiko (éd.), Paris, Diane de Selliers, 2015, p. 233-237.

ABÉ Yoshio, "Anthropologie comparée d'une céréale majeure asiatique: le riz », Séminaire à l'EHESS, Centre d'études sur la Chine moderne et contemporaine, 2008-2009, 2009-2010, inédit.

ABE Tokiharu 阿部宗明 (éd.), Genshoku gyorui daizukan 原色魚類大困鑑 (Grand atlas des poissons en couleurs), Tokyo, Hokuryūkan, 1987.

AKABA Masaharu 赤羽正春, Sake Masu 鮭·鱒 (Saumon et Truite), 2 vols., Tokyo, Hōsei daigaku shuppankyoku, coll. « Mono to ningen no bunkashi » 133-1 et 133-2, 2006.

AKIYAMA Takashi 秋山高志 et al. (éd.), Zuroku. Sangyoson seikatsu jiten 図録・山魚村生活事典 (Encyclopédie de la vie dans les villages de montagne et de pêcheurs. Atlas), Tokyo, Kashiwa shobō, 1991.

ANTONI Klaus, Miwa - der heilige Trank, Wiesbaden, Franz Steiner Verlag, 1988. 
ARISAKA Michiko 有坂道子, “Toshi bunjin 都市文人 (Le lettré citadin)” in Chishiki to gakumon o ninau hitobito 知識と学問をになう人々 (Les hommes en charge de la connaissance et des savoirs), чокотА Fuyuhiko (éd.), Tokyo, Yoshikawa kōbunkan, coll. « Mibunteki shūen to Kinsei shakai » 5, 2007, p. 143-174.

ASTON William George (trad), Nihongi Chronicles of Japan, Tokyo, Charles Tuttle Comp., 1972.

BAUMERT Nicolas, Le saké, une exception japonaise, Rennes, Presses Universitaires de Rennes, coll. «Tables des hommes », 2011.

BLUSSÉ Leonard, REMMELINK Willem, SMITS Ivo (ed.), Bridging the Divide: 400 years The NetherlandsJapan, Leiden, Hotei Publishing, 2000.

BriLlat-SAVARIN Jean Anthelme, Physiologie du goût, Paris, A. Sautelet, 1825.

CHIBA Tokuji 千葉徳爾 (éd.), Nippon sankai meisan meibutsu zue 日本山海名産名物図会, Tokyo, Shakai shisōsha, 1970.

Dictionnaire historique du Japon, 2 vols, Paris, Maisonneuve \& Larose, 2002. Accessible en ligne.

ELMAN Benjamin A., From Philosophy to Philology: Intellectual and Social Aspects of Change in Late Imperial China, Cambridge Mass. and London, Harvard University Press, 1984.

GAY Suzanne, The Moneylenders of Late Medieval Kyoto, Honolulu, University of Hawai'i Press, 2001.

HAYAMA Teisaku 葉山禎作 (éd.), Seisan no gijutsu 生産の技術 (Techniques de production), Tokyo, Chūō kōronsha, coll. « Nihon no kinsei » 4, 1992.

HAYEK Matthias, HORIUCHI Annick (ed.), Listen, Copy, Read: Popular Learning in Early Modern Japan, Leiden, Brill, Japanese Studies Library, 2014.

HIYAMA Yoshio 檜山義夫 et YASUDA Fujirō 安田富士郎, Nihon-san gyorui daizukan 日本産魚類大困 鑑 (Grand atlas des poissons produits au Japon), Tokyo, Kōdansha, 1971.

HSU Elisabeth (ed.), Innovation in Chinese medicine, Cambridge, Cambridge University Press, 2001.

HUETZ DE LEMPS Alain, «L'expansion de la pêche japonaise », Cahiers d'outre-mer, vol. 13, $\mathrm{n}^{\circ} 49$, janvier-mars 1960.

INOUE Mitsusada 井上光貞 et al. (éd.)., Ritsuryō 律令, Tokyo, Iwanami Shoten, coll. « Nihon shisō taikei » 3, 1997.

INOUE Tadashi 井上忠, Kaibara Ekiken 貝原益軒, Tokyo, Yoshikawa kōbunkan, 1963, p. 186.

INOUE Tomokatsu 井上智勝, “Kenkadō no zōsho ni tsuite 萧臀堂の蔵書について (À propos de la collection de livres de Kenkadō)", in Kimura Kenkadō : naniwa chi no kyojin. Tokubetsuten botsugo 200 nen kinen 木村萧臀堂: なにわ知の巨人, ŌSAKA REKISHI HAKUBUTSUKAN (éd.), Kyōto, Shibunkaku shuppan, 2003, p. 151-167.

JōJIMA Masayoshi 城島正祥 et SUGITANI Akira 杉谷昭, Saga-ken no rekishi 佐賀県の歴史 (Histoire de la préfecture de Saga), Tokyo, Yamagawa shuppan, coll. « Kenshi shirīzu » 41, 1980.

JORDAN David Starr, TANAKA Shigeo, SNYDER John Otterbein, “A Catalogue of the Fishes of Japan”, Journal of the College of Science, Imperial University of Tokyo, Tokyo, publ. by the University, 1913, $497 \mathrm{p}$.

Kadokawa Nihon tōji daijiten fukyū-ban 角川日本陶磁大辞典・普及版 (Grand dictionnaire Kadokawa des céramiques et porcelaines du Japon, édition grande diffusion), YABE Yoshiaki 矢部良明 et al. (éd.), Tokyo, Kadokawa gakugei shuppan, 2011.

KALLAND Arne, Fishing Villages in Tokugawa Japan, Richmond, Surrey, Curzon Press, 1995.

KAMO No CHŌMEI, Kamo no Chômei Notes sans titre: Mumyôshô : Propos sur les poètes et la poésie. Traduit du japonais et annoté par le Groupe Koten, Claire-Akiko BRISSET, Jacqueline PIGEOT, Daniel STRUVE, Sumie TERADA et Michel VIEILLARD-BARON, Paris, Le Bruit du temps, 2010. 
KAWASHIMA Shūichi 川島秀一, Katsuoryō カツ才漁 (La pêche à la bonite), Tokyo, Hōsei daigaku shuppankyoku, coll. « Mono to ningen no bunkashi » 127, 2005.

KISHINOUYE Kamakichi, "Contributions to the Comparative Study of the So-called Scombroid Fishes", Journal of the College of Agriculture, vol. VIII, n 3, 1923, p. 293-475, 21 pl.

LACHAUD François, "The Scholar and the Unicorn. Antiquarians, Connoisseurs, and Eccentrics in Eighteenth Century Japan", in World Antiquarianism. A Global Perspective, Los Angeles, Getty Publications, 2014, p. 343-371.

MANE Perrine, "Images médiévales de la pêche en eau douce », Journal des savants, juilletdécembre, 1991.

MARCON Federico, The Knowledge of Nature and the Nature of Knowledge in Early Modern Japan, Chicago/London, The University of Chicago Press, 2015.

MERTZ Mechtild, Wood and Traditional Woodworking in Japan, Otsu, Kaiseisha, 2011.

MÉTAILIÉ Georges, “The Bencao ganmu of Li Shizhen: an innovation in natural history?" in Innovation in Chinese Medicine, HSU Elisabeth (ed.), Cambridge, Cambridge University Press, coll. « Needham Research Institute Studies » 3, 2001, p. 221-261.

міно Tadao 三保忠夫, “Riyō-sen Kohon Yōkotsuhō, Rien-sen Shinzō Yōkotsuhō ni tsuite- Kunaichō shoryōbu shozō takasho takakotoba no kenkyū 李瑢撰『古本鷹鶻方』・李爛撰『新増鷹鶻方』 について-宮内庁書陵部所蔵鷹書·鷹詞の研究 (À propos de l'ancien manuscrit Yōkotsuhō de Riyō et de la nouvelle édition augmentée du même ouvrage par Rien : recherches sur les ouvrages de fauconnerie conservés au département des écrits et sépultures de l'Agence impériale et la terminologie de la fauconnerie)", Shinjodai kokubun 神女大国文, nº 25, 2014, p. 23-40.

MIYASHITA Akira 宮下章, Katsuo bushi 鰹節 (La bonite séchée), Tokyo, Hōsei daigaku shuppankyoku, coll. « Mono to ningen no bunkashi » 97, 2000.

MIZUTA Norihisa 水田紀久 (éd.), Mizu no chūō ni ari : Kimura Kenkadō kenkyū 水の中央に在り: 木村 萧葭堂研究 (Une existence au cœur de l'eau : recherches sur Kimura Kenkadō), Tokyo, Iwanami shoten, 2002.

MUUS B. J., DAHLSTRÖM Preben, Guide des poissons de mer et pêche, Paris, Delachaux \& Niestlé, 1981.

NAGAHARA Keiji 永原慶二 (éd.), Kōza: Nihon gijutsu no shakaishi 講座・日本技術の社会史 (Leçons sur l'Histoire sociale des techniques du Japon), vol. 4, Tokyo, Nihon hyōronsha, 1984.

NAGASAKI SHIRITSU HAKUBUTSUKAN 長崎市立博物館 (éd.), Hizō kapitan no Edo korekushon: Oranda jin no Nihon shumi 秘蔵カピタンの江戸コレクション:オランダ人の日本趣味 (Collection secrète des directeurs des comptoirs de la VOC: le goût hollandais pour les choses japonaises), Nichiran kōryū 400 shūnen kinen, Nagasaki, Nagasaki shiritsu hakubutsukan, 2000.

NAKAMURA Shin'ichirō 中村真一郎 (éd.), Kimura Kenkadō no saron 木村萧臀堂のサロン (Le salon de Kimura Kenkadō), Tokyo, Shinchōsha, 2000.

NAKAMURA Takashi 中村喬 (éd.), Chūgoku no shusho 中国の酒書 (Les livres sur l'alcool de riz chinois), Tokyo, Heibonsha, coll. « Tōyō bunko », 1991.

NEEDHAM Joseph, MÉTAILIÉ Georges, Science and Civilisation in China: Biology and Biological Technology, LLOYD Janet (trad.), Cambridge, Cambridge University Press, 2015.

NESAKI Mitsuo 根崎光男, Inu to taka no Edojidai: “Inu kubō” Tsunayoshi to “Taka shōgun" Yoshimune 犬と鷹の江戸時代・〈犬公方〉 綱吉と〈鷹将軍〉吉宗 (L'époque Edo des chiens et des faucons : le Shogun des chiens Tsunayoshi et le Shogun des faucons Yoshimune), Tokyo, Yoshikawa kōbunkan, 2016.

NIHON GYORUI GAKKAI 日本魚類学会 (Société ichthyologique du Japon) (éd.), Nihonsan gyomei daijiten 日本産魚名大辞典 (Grand dictionnaire des noms de poissons japonais), Tokyo, Sanseidō, 1981. 
NIHON GAKUSHIIN NIHON KAGAKUSHI KANKŌKAI 日本学士院・日本科学史刊行会 (éd.), Meiji zen Nihon gyogyō gijutsushi 明治前日本漁業技術史 (Histoire des techniques de pêche du Japon d'avant Meiji), Tokyo, Nihon gakujutsu shinkōkai, 1982 (première édition 1959).

Nihon kokugo daijiten 日本国語大辞典 (Grand dictionnaire de la langue japonaise), deuxième édition, 13 vols. + 1 vol. Supplément, Shōgakukan, 2000-2002.

OKUMURA Shōji 奥村正二, Hiraga Gennai o aruku: Edo no kagaku o tazunete 平賀源内を步く 江戸の 科学を訪礼て (Marcher sur les traces de Hiraga Gennai: à la recherche de la science de l'époque d'Edo), Tokyo, Iwanami shoten, 2003.

ONO Masatoshi 小野正敏, GOMI Fumihiko 五味文产, HAGIHARA Mitsuo 萩原三雄 (éd.), Utage no chūsei : ba, kawarake, kenryoku 宴の中世 : 場•かわらけ・権力 (Le Moyen Âge des banquets : lieux, vaisselle, pouvoir), Tokyo, Kōshi Shoin, 2008.

ŌSAKA REKISHI HAKUBUTSUKAN 大阪歴史博物館 (éd.), Kimura Kenkadō: naniwa chi no kyojin. Tokubetsuten botsugo 200 nen kinen 木村莱荍堂•なにわ知の巨人 特別展 没後二百年記念 (Kimura Kenkadō, le géant du savoir de Naniwa. Exposition à l'occasion du deux centième anniversaire de sa mort), Kyōto, Shibunkaku shuppan, 2003.

PÉRONNY Claude, Les animaux du Man.yō-shū, Paris, Collège de France, Institut des Hautes Études Japonaises, 2007.

SAEGUSA Hiroto 三枝博音, “Tenkō kaibutsu no kenkyū 天工開物の研究 (Recherches sur le Tiangong kaiwu)", in Saegusa Hiroto chosakushū 三枝博音著作集 (Collection d'œuvres de Saegusa Hiroto), vol. 11, Tokyo, Chūō kōronsha, 1973, p. 120-180.

SCHÄFER Dagmar, The Crafting of the 10,000 things: Knowledge and Technology in Seventeenth-Century China, Chicago/London, The University of Chicago Press, 2011.

SHIRAHATA Yōzaburō, "The Printing of Illustrated Travelogues in $18^{\text {th }}$ Century Japan" in Written Texts - Visual Texts, Woodblock Printed Media in Early Modern Japan, FORMANEK Susanne and LINHART Sepp (ed.), Amsterdam, Hotei Publishing, 2005, p. 199-214.

SHIRAI Shōhei 白井祥平, Kai 貝 (Coquillages), 3 vols., Tokyo, Hōsei daigaku shuppankyoku, coll. «Mono to ningen no bunkashi » 83, 1997.

SHŌGAKUKAN SHOKUZAI ZUTEN HENSHŪBU 小学館食材図典編集部 (éd.), Shokuzai zuten 食材図典 (Catalogue illustré des matières culinaires), Tokyo, Shōgakukan, 1995.

SMITH Thomas C., Native Sources of Japanese Industrialization 1750-1920, Berkeley/Los Angeles/ London, University of California Press, 1988.

TAJIMA Yoshiya 田島佳也, “Kinsei kishū no ryōhō no tenkai 近世紀州の漁法の展開 (Développement des techniques de pêche dans la province de Kii à l'époque prémoderne)" in Seisan no gijutsu 生産 の技術 (Techniques de production), HAYAMA Teisaku 葉山禎作 (éd.), Tokyo, Chūō kōronsha, coll. « Nihon no kinsei » 4, 1992, p. 211-278.

TANABE Satoru 田辺悟, Ami 網 (Les filets de pêche), Tokyo, Hōsei daigaku shuppankyoku, coll. «Mono to ningen no bunkashi » 97, 2002.

TANAKA Shigeho 田中茂穂, (Zôho kaitei) Zōho kaitei Nihon-san gyorui zusetsu 増補改訂 日本産魚類 圖説 - Figures and Descriptions of the Fishes of Japan - including Riukiu Islands, Bonin Islands, Formosa, Kurile Islands, Korea, and Southern Sakhalin, Tokyo, Kazama shobō, vol. I-XXX, 1951, vol. XXXI-XLVIII, 1952.

TомiYama Tetsuo (éd.), Fisheries in Japan - Skipjack and Mackerel, Tokyo, Japan Marine Products Photo Materials Association, 1976.

TOMIYAMA Tetsuo (éd.), Fisheries in Japan - Salmonidae, Tokyo, Japan Marine Products Photo Materials Association, 1972. 
TONE Yūtarō 刀禰勇太郎, Tako 蛸(タコ) (La pieuvre), Tokyo, Hōsei daigaku shuppankyoku, coll. «Mono to ningen no bunkashi » 74, 1994.

TSUDA Takeyoshi 津田武美, Genshoku. Nihonkai gyorui zukan 原色日本海魚類困鑑 (Encyclopédie illustrée des poissons de mer), Toyama, Katsura shobō, 1990.

TSUKADA Takashi 塚田孝, “Kimura Kenkadō to Kita Horie go chōme - Kinsei Ōsaka no toshi shakai kōzō to no kanren de 木村萧臀堂と北堀江五丁目一近世大阪の都市社会構造との関連で (Kimura Kenkadō et le quartier de Kita Horie 5 : étude en lien avec celle de la structure sociale de la ville d'Osaka à l'époque prémoderne)", in Ōsaka ni okeru toshi no hatten to kōzō 大阪における都 市の発展と構造, TSUKADA Takashi (éd.), Tokyo, Yamagawa shuppan, 2004, p. 113-137.

TSUKUBA Hisaharu 筑波常治, Nihon no nōsho, nōgyō wa naze kinsei ni hatten shitaka 日本の農書. 農 業はなぜ近世に発展したか (Les traités agricoles du Japon. Pourquoi l'agriculture s'est-elle développée à l'époque prémoderne ?), Tokyo, Chūō kōron, coll. « Chūkō shinsho », 1987.

unschuld Paul Ulrich, Medicine in China. A History of Pharmaceutics. Berkeley/Los Angeles/London, University of California Press, 1986.

WATANABE Masao, Fauna Japonica - Cottidae (Pisces), Tokyo, Biogeographical Society of Japan (publ. Tokyo News Service), 1960.

WINKEL Margarita, Discovering different dimensions: explorations of culture and history in early modern Japan, unpublished PhD Dissertation, Leiden University, 2004.

YABE Ichirō 矢部一郎, Edo no honzō: yakubutsugaku to hakubutsugaku 江戸の本草 : 薬物学と博物 学 (Le honzō d'Edo: pharmacopée et histoire naturelle), Tokyo, Saiensusha, coll. «Raiburari kagakushi » 6, 1984.

YABUUCHI Kiyoshi 薮内清 (éd.), Tenkō kaibutsu no kenkyū. Kyōto daigaku jinbun kagaku kenkyūjo kenkyū hōkoku 天工開物の研究 (Recherches sur le Tiangong kaiwu), Kyōto daigaku jinbun kagaku kenkyūjo kenkyū hōkoku 京都大学人文科学研究所研究報告, Tokyo, Kōseisha kōseikaku, 1953.

YANo Ken'ichi 矢野憲一, Awabi 鮑 (Ormeaux), Tokyo, Hōsei daigaku shuppankyoku, coll. « Mono to ningen no bunkashi » $62,1989$.

YoSHIDA Hajime 吉田元, Sake 酒 (Saké), Tokyo, Hōsei daigaku shuppankyoku, coll. «Mono to ningen no bunkashi »172, 2015.

YoSHIDA Nobuyuki 吉田伸之, “Dentō toshi no shūen 伝統都市の周縁” (À la périphérie de la ville traditionnelle) in Kinsei no kaitai 近世の解体 (Déconstruction du prémoderne), Nihonshi kōza, vol. 7, Tōkyō daigaku shuppankai, 2005.

YUNOKI Manabu 柚木學, Sakezukuri no rekishi 酒造りの歴史 (Histoire de la fabrication du saké), Tokyo, Yūzankaku Shuppan, 1987. 


\section{Liste des œuvres citées dans le Nippon sankai meisan zue}

1 Azuma kagami 東鑑 ou 吾妻鏡 (Miroir de l'Est), chronique du bakufu de Kamakura, en 52 fascicules (1 fascicule manquant), d'auteur inconnu.

2 Bencao gangmu 本草綱目 (Classement raisonné de la materia medica), traité de bencao (j. honzō) composé par LI Shizhen 李時珍 (1518-1593) vers les années 1590-1596, et considéré comme le sommet jamais atteint par les spécialistes du domaine au cours des XVII-XIX ${ }^{e}$ siècles. Il connaît un grand succès au Japon où il sera réédité à plusieurs reprises (j. Honzō kōmoku) et abondamment étudié.

Daizuibian 代醉編 (Substitut à l'ivresse), abréviation de Langye daizuibian 珢㴬代醉編, un ouvrage du lettré Ming, Zhang Dingsi 張鼎思 (1543-1603), en 40 fascicules.

4 Engishiki 延喜式 (Règlements de l'ère Engi), (901-930), 927, édité par Fujiwara no Tokihira 藤原時平 (871-909) et al., en 50 fascicules, expose le détail du fonctionnement de l'administration centrale. Il procure entre autres des informations sur la vie quotidienne de la cour, les rites, les produits agricoles et artisanaux des provinces du Japon.

5 Fubokushū ou Fuboku wakashō 夫木集 ou 夫木和歌抄 (Sélection de poésie japonaise), complétée en 1310 par Fujiwara no Nagakiyo 藤原長清 (dates inconnues), en 36 fascicules, regroupant des poèmes composés sur 596 thèmes poétiques.

Gōke shidai 江家次第 ou Gōshidai 江次第 (Protocole des célébrations selon la maison Ōe), manuel des cérémonies de la cour, de date inconnue, compilé par ōe no Masafusa 大江 匡房 (1041-1111).

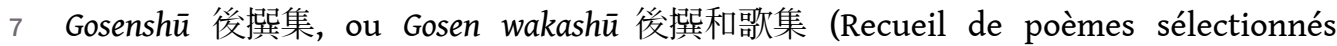
postérieurement), anthologie de poésie japonaise, compilée sur ordre impérial entre 951 et 958, en 20 fascicules, par Ōnakatomi no Yoshinobu 大中臣能宣, Kiyohara no Motosuke 清原元輔, Minamoto no Shitagō 源順, Ki no Tokibumi 紀時文, et Sakanoue no Mochiki 坂上望城.

Heiji monogatari 平治物語 (Récit épique de Heiji), anonyme, date inconnue, élaboration progressive à partir du XIII siècle, récit épique sur les troubles de l'ère Heiji (1159). 
Honchō shokkan 本朝食鑑 (Miroir des aliments du Japon), ouvrage de Hitomi Hitsudai 人 見必大 (vers 1642-1701), en 12 fascicules, achevé en 1692 et imprimé en 1697. Inventorie 442 sortes d'aliments produits au Japon. A comme caractéristique de faire une large place aux produits d'origine animale.

10 Jiupu 酒譜 (Traité du vin), composé vers 1084, ouvrage du fonctionnaire lettré Dou Ping 赎茾 des Song, réunissant diverses légendes et faits historiques sur cette boisson et les pratiques qui l'entourent.

$11 K a^{\prime} i$ tsūshōkō 華夷通商考 (Considérations sur les échanges commerciaux avec la Chine et les barbares), ouvrage de Nishikawa Joken 西川如見 (1648-1724), imprimé en 1708, donnant une description des partenaires commerciaux du Japon et des produits échangés.

12 Kanjikō 冠辞考 (Réflexions sur les mots initiateurs), ouvrage de poétique de Kamo no Mabuchi 賀茂真淵 (1697-1769) en 10 fascicules, imprimé en 1757, commentant 326 mots initiateurs tirés de la Chronique des choses anciennes, de la Chronique du Japon et du Man'yōshū.

13 Kinmō zui 訓蒙圖彙 (Encyclopédie illustrée), dictionnaire illustré de Nakamura Tekisai 中村惕斎 (1629-1702) en 20 fascicules, imprimé en 1666, selon le modèle Ming du Sancai tuhui 三才図絵 (1607). Il procure pour un large spectre d'objets, animaux ou phénomènes, les noms japonais et chinois, complétés d'une illustration et d'une courte description.

14 Kojiki 古事記 (Chroniques des choses anciennes), compilé en 712 par ō no Yasumaro 太 安麻呂 (?-723). C'est le plus ancien ouvrage japonais qui relate les origines divines de l'État du Yamato et les règnes des premiers empereurs.

15 Kokinshū uchigiki 古今集打聴 (Transcription du commentaire $\mathrm{du}$ Kokinsh $\bar{u}$ ), commentaire de l'anthologie impériale Kokin wakashū en 20 fascicules, attribué à Kamo no Mabuchi 賀茂真淵 (1697-1769), dont le texte prononcé oralement aurait été recueilli par son disciple Nomura Tomohiko 野村ともひ子, puis révisé par Ueda Akinari 上田秋 成 (1734-1809) et imprimé en 1789.

16 Kokin waka rokujō 古今和歌六帖 (Poèmes anciens et modernes en six livre). Anthologie de l'époque Heian, en six fascicules, d'éditeur inconnu, ayant vu le jour dans les années 976-987 et réunissant environ 4500 poèmes anciens classés par thème.

17 Kokin wakashū 古今和歌集 (Recueil de poèmes anciens et modernes), compilé vers 913-914 (ou vers 905) par Ki no Tsurayuki 紀貫之 (?-?) et al. Première anthologie impériale du Japon, il compte, dans sa version la plus répandue, 1111 poèmes, répartis en 20 livres et classés selon diverses rubriques.

18 Kujiki ou Kuji hongi 旧事本紀 ou Sendai kujihongi 先代旧事本紀 (Choses anciennes et chroniques essentielles). Annales historiques d'auteur inconnu, datant du milieu du $\mathrm{IX}^{\mathrm{e}}$ siècle, retraçant les événements allant de l'avènement $\mathrm{du}$ monde au règne de l'empereur Suiko. Est également considéré comme un Classique du shintô.

19 Liji 礼記 (Mémoire sur les Rites), l'un des cinq classiques confucéens, compilé par Dai Sheng 戴聖 (dynastie des Han de l'Ouest), rendant compte du rituel des époques des Printemps et Automnes et des Royaumes Combattants. Il contient les règles sur les " procédures au fil du calendrier » yueling 月令, correspondant à un calendrier rituel et agricole. 

auteur), commentaire du Man'yōshū composé par Keichū 契沖 (1640-1701). Il en existe deux versions. La seconde, datée de 1690, est fondée sur une recherche plus rigoureuse sur le plan philologique.

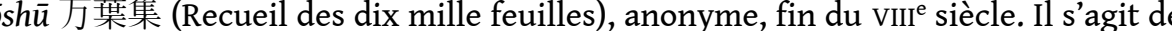
la plus ancienne anthologie de poésie conservée au Japon. La compilation des 4500 poèmes en vingt fascicules connue de nos jours est attribuée à Ōtomo no Yakamochi 大 伴家持 (717 ?-785).

(Livre de Min), traité géographique chinois en 150 fascicules portant sur la province du Fujian, compilé par He Qiaoyuan 何喬遼, achevé en 1520 et imprimé en 1631. 王圻 (1529-1612) et Wang Siyi 王思義. Elle a été complétée en 1607 et publiée en 1609 sous les Ming. Les trois règnes sont le Ciel, la Terre et les Hommes, trois catégories de connaissances développées dans le Classique des Mutations.

Sangi ittō 三義一統 (Trois principes - une autorité), manuel d'étiquette qu'Ogasawara Nagahide 小笠原長秀 (?-1424) aurait écrit à l'intention de la classe guerrière de l'époque Muromachi. Il comprendrait notamment les règles relatives à l'équitation et le tir à l'arc.

Sankashū 山家集 (Recueil de l'ermitage de montagne), vers 1167 à 1185, le plus célèbre des recueils de poésie de Saigyō 西行 (1118-1190), comprenant plus de 1500 poèmes en langue japonaise wabun.

Senchū wamyō ruijushō 䈉注倭名類聚抄 (Répertoire ordonné des noms du Yamato annoté), en 10 fascicules, de Kariya Ekisai 狩谷棭斎 (1775-1835), achevée en 1827, résultat d'une recherche philologique sur l'œuvre de l'époque Heian, Wamyō ruijushō. Shijing 詩経 (Livre des odes ou Classique de la poésie), Shikyō en japonais, recueil de 305 pièces de vers, comprenant des chants religieux, rituels ou populaires, composés entre la dynastie des Zhou de l'Ouest et l'époque des Printemps et Automnes. 
Shiming 釋名 (Explication des noms), dictionnaire chinois en 8 livres, datant de l'époque des Han postérieurs (25-220 ap. J-C). Il existe une incertitude autour de son auteur, Liu Xi 劉熙 étant le plus souvent cité. Source essentielle pour connaître la prononciation du chinois parlé de l'époque.

Shinsarugakuki 新猿楽記 (Notes sur de nouveaux divertissements comiques), œuvre de fiction d'un fascicule, composée par Fujiwara no Akihira 藤原明衡 (989-1066) et comportant des descriptions d'arts de divertissement et de différents métiers avec des listes d'objets et de termes techniques qui leur sont associés.

Shinsen shōjiroku 新撰姓氏録 (Nouveau registre des titres héréditaires et des noms de familles), compilé en 814 puis complété en 815. Il consigne les généalogies des familles, classées selon leurs origines.

Shiwu bencao 食物本草 (Pharmacopée des aliments). Il existe plusieurs ouvrages de ce nom, mais il s'agit sans doute ici de l'ouvrage en 22 fascicules, du médecin Li Gao 李杲 (1180-1251) des Yuan, dans la mesure où une édition corrigée par Li Shizhen a été imprimée en 1638.

Shoku kokinsh $\bar{u}$, abréviation du Shoku kokinwakash $\bar{u}$ 続古今和歌集 (Suite au Recueil des poèmes anciens et modernes), anthologie en 20 fascicules compilée sur ordre impérial par le poète Fujiwara no Teika 藤原定家 en 1265 .

Songshi 年史 (Annales dynastiques des Song), en 496 fascicules, achevée en 1344 sous la direction de Tuo Tuo 脫脫 ou Togto (1313-1355) de l'empereur Shun Di (1320-1370) de la dynastie Yuan.

Songshu 朱書 (Livre des Song), texte historique en 100 fascicules sur la dynastie des Song, l'une des dynasties du sud, composée par Shen Yue 沈約 (441-513) des Liang.

Tiangong kaiwu 天工開物 (Exploitation des œuvres de la nature), ouvrage encyclopédique illustré daté de 1637, présentant les différentes techniques de production de l'époque des Ming, composé par Song Yingxing 柂應星 (vers 1590-vers 1650).

3 Tōga 東雅 (Er'ya de l'Est), dictionnaire en 20 fascicules d'Arai Hakuseki 新井白石 (1657-1725), composé en 1717 et donnant l'étymologie des noms japonais.

Tongŭi pogam 東醫寶鑑 (Le Miroir de la médecine orientale), encyclopédie de médecine coréenne, réalisée sous la direction de Heo Jun 許浚 au XvI ${ }^{\mathrm{e}}$ siècle. L'ouvrage composé de 25 fascicules fut abondamment consulté en Chine et au Japon à l'époque d'Edo.

Tongya 通雅, ouvrage de lexicologie de Fang Yizhi 方以智 (1579-1671) de la dynastie Ming en 52 fascicules, réunissant sa réflexion sur un large spectre de termes, classés selon 25 domaines.

46 Tsurezuregusa 徒然草, essai 《au fil du pinceau » en 2 fascicules du XIV siècle, composé par Yoshida Kenkō 吉田兼好 (dates inconnues). La date de composition n'est pas connue avec certitude, mais des spécialistes la situent autour de 1331.

Unggobang (j. Yōkotsuhō) 鷹鶻方, traité coréen sur l'élevage et le dressage des autours et des faucons, composé par Yi Yong 李瑢, dont l'édition la plus ancienne remonterait à 1444.

Unkonshi 雲根志 (Traité sur les roches), ouvrage en 16 fascicules imprimé en trois séries, entre 1773 et 1801, par le naturaliste japonais Kino'uchi Sekitei 木内石亭 
(1724-1808), répertoriant environ 2000 espèces de roches, de minéraux, de fossiles et objets en pierre.

Wahō ichimanpō 和方一万方 (Dix milles remèdes du Japon), ouvrage de médecine en 41 fascicules de Murai Kinzan 村井琴山 (1733-1815), avec une préface datée de 1781. Il s'agit d'un recueil de remèdes traditionnels du Japon, dans la lignée de l'école de Yoshimasu Tōdō de Kyoto.

Wakan sansai zue 和漢三才圖繪 (Encyclopédie sino-japonaise des trois règnes), encyclopédie illustrée de Terajima Ryōan 寺島良安, imprimée en 1712, en 105 fascicules, sur le modèle de l'encyclopédie chinoise Sancai tuhui.

51 Wamyōshō 和名抄, également Wamyō ruijushō 倭名類聚鈔 (Répertoire ordonné des noms du Yamato), est un dictionnaire de l'époque de Heian de Minamoto no Shitagō 源 順 (911-983), achevé autour de 934, répertoriant environ 2600 termes chinois classés selon différents domaines, allant des phénomènes célestes aux plantes et animaux. Deux versions existent, comptant respectivement 10 et 20 fascicules.

52 Wuli xiaozhi 物理小識 (Aperçu sur le principe des choses), ouvrage encyclopédique en 20 fascicules de Fang Yizhi 方以智（1611 -1671）, reposant sur une classification en quinze domaines (Ciel, Histoire, Divination, Corps humain, Minéraux, Outils, Vêtements, Aliments, Animaux, Végétaux, etc). Il est imprimé à titre posthume en 1664. Wuli lun 物理論 (Discours sur la raison des choses) est un essai métaphysique chinois de Yang Quan 楊泉 (dates inconnues), de l'époque des trois dynasties. Composé originellement de 16 fascicules, seuls des fragments sont conservés.

54 Wuzazu (j. Gozasso) 五雑珇, plus exactement 五雑組 (Les cinq miscellanées), ouvrage de Xie Zhaozhe 謝肇淛 (1567-1624) des Ming, en 16 fascicules, imprimé en 1619, abordant des sujets relevant de la politique, de l'économie, des savoirs, et des arts, classés selon les cinq catégories : Ciel, Terre, Homme, Faits, et Choses.

Xunpu 菌譜 (Traité des champignons), traité composé en 1245, selon la préface, par Chen Renyu 陳仁玉 des Song du sud, décrivant 11 espèces de champignons poussant dans la région du Zhejiang (au sud de Shanghai).

56 Yamato honzō 大和本草 (Materia medica du Japon), traité de honzō de Kaibara Ekiken 貝 原益軒 (1630-1714), imprimé en 1709, réalisé sous l'influence du Bencao gangmu mais dont le propos est recentré sur les espèces naturelles observées au Japon. Composé de 16 fascicules, 2 fascicules annexes, et 2 fascicules d'illustrations. 


\section{Tableau des noms propres}

\begin{tabular}{|c|c|c|}
\hline Ahiko & 阿珥子 & Fascicule 2 \\
\hline Amaterasu (déesse) & あまてらす & Présentation du fascicule 1 \\
\hline Ashikaga Yoshimitsu & 足利義満 & Fascicule 3 \\
\hline Azuma kagami & 東鑑 & Fascicule 2 \\
\hline Bencao gangmu & 本草綱目 & Fascicules 2, 3, 4, 5 \\
\hline Boyi & 伯夷 & Fascicule 2 \\
\hline Buretsu tennō & 武烈天皇 & Fascicule 3 \\
\hline Chen Renyu & 陳仁玉 & Fascicule 2 \\
\hline Chōrakuji & 長楽寺 & Présentation du fascicule 1 \\
\hline Chronique de l'empereur ōjin ōjinki & 応神記 & Fascicule 2 \\
\hline Daijōe & 大嘗会 & Fascicule 1 \\
\hline Daishōki voir Man'yō daishōki & 代匠記 & Fascicule 4 \\
\hline Daizuibian voir Langye daizuibian & 代醉編 & Fascicule 2 \\
\hline Dou Ping & 䆬苹 & Fascicule 1 \\
\hline Du Kang & 杜康 & Fascicule 1 \\
\hline Engishiki & 延喜式 & Fascicules 2, 3, 4 \\
\hline Fubokushū / Fuboku wakashō & 夫木集／夫木和歌抄 & Fascicules 4, 5 \\
\hline Fujiwara no Mototoshi & 藤原基俊 & Fascicule 2 \\
\hline
\end{tabular}




\begin{tabular}{|c|c|c|}
\hline Fujiwara no Nagakiyo & 藤原長清 & Fascicule 5 \\
\hline Gōke shidai & 江家次第 & Fascicule 3 \\
\hline Gosen wakash $\bar{u}$ & 後撰和歌集 & Fascicule 4 \\
\hline Gosensh $\bar{u}$ voir Gosen wakashū & 後撰集 & Fascicule 4 \\
\hline Gōshidai voir Gōke shidai & 江次第 & Fascicule 3 \\
\hline Guanyu (j. Kan’u) & 関羽 & Fascicule 5 \\
\hline Hachiman & 八幡 & Fascicule 3 \\
\hline Han Baosheng & 韓保昇 & Fascicule 3 \\
\hline Heiji monogatari & 平治物語 & Fascicule 3 \\
\hline Hirase Tessai, voir Hose & 平瀬徹斎 & Introduction \\
\hline Hitomi Hitsudai & 人見必大 & Fascicule 4 \\
\hline Hŏ Chun (j. Kyo Shun) & 許浚 & Fascicule 4 \\
\hline Hononinigi (dieu) & ほのににぎ & Présentation du fascicule 1 \\
\hline Honchō shokkan & 本朝食鑑 & Fascicule 4 \\
\hline Honzō kōmoku voir aussi Bencao gangmu & 本草綱目 & Fascicule 4 \\
\hline Honzō shikkai & 本草集解 & Fascicule 5 \\
\hline Hose voir Hirase Tessai & 補世 & Introduction \\
\hline Ikeda Manganjiya & 池田満原頞寺屋 & Fascicule 1 \\
\hline Imagawa Ujiyori & 今川氏頼 & Fascicule 3 \\
\hline Inateraya Masuya & 猪名寺屋升屋 & Fascicule 1 \\
\hline Inō Jakusui & 稲生若水 & Préface, Fascicule 4 \\
\hline Ji Xin & 紀信 & Fascicule 4 \\
\hline Jingū kōgō & 神功皇后 & Fascicule 1 \\
\hline Jinmu (empereur) & 神武 & Présentation du fascicule 1 \\
\hline Jiupu & 酒譜 & Fascicule 1 \\
\hline Jomei tennō & 舒明天皇 & Fascicule 4 \\
\hline Junpu & 菌譜 & Fascicule 2 \\
\hline
\end{tabular}




\begin{tabular}{|c|c|c|}
\hline Ingyō tennō & 允恭天皇 & Fascicule 3 \\
\hline Ise Mitsutada & 伊勢満忠 & Fascicule 3 \\
\hline Kaibara Ekiken & 貝原益軒 & Fascicule 4 \\
\hline Ka'itsūshōkō & 華夷通商考 & Fascicule 2 \\
\hline Kamo no Chōmei & 鴨長明 & Fascicules 2, 4 \\
\hline Kamo no Mabuchi & 賀茂真淵 & Fascicules 1,4 \\
\hline Kanjikō & 冠辞考 & Fascicule 1 \\
\hline Kanmu tennō & 桓武天皇 & Fascicule 5 \\
\hline Kashiragaki zōho kinmō zui & 頭書増補訓蒙圖彙 & Fascicule 4 \\
\hline Keikō tennō & 景行天皇 & Fascicule 4 \\
\hline Kenreimon'in & 建礼門院 & Fascicule 4 \\
\hline Kimura Kōkyō [Kenkadō] & 木村孔恭 [莱葭堂] & Préface \\
\hline Kinmō Zue ou Kinmō zui & 訓蒙圖会／訓蒙困菓 & Fascicule 4 \\
\hline Kō Kansui & 向観水 & Préface \\
\hline Kōbō daishi & 弘法大師 & Fascicule 2 \\
\hline Kojiki & 古事記 & Fascicule 1 \\
\hline Kōfukuji & 興福寺 & Présentation du fascicule 1 \\
\hline Kokin uchigiki ou Kokinshū uchigiki & 古今打聴／古今集打恥 & Fascicule 4 \\
\hline Kokinshū ou Kokin wakashū & 古今集／古今和歌集 & Fascicules 2, 4 \\
\hline Kokin Waka rokujō & 古今和歌六帖 & Fascicules 3, 4 \\
\hline Kujiki & 舊事記 & Fascicule 5 \\
\hline Kuratsukuri No suguri Masuhito & 按作村主益人 & Fascicule 4 \\
\hline Langye daizuibian & 琅躯代醉編 & Fascicule 2 \\
\hline Li Shizhen & 李時珍 & Fascicule 4 \\
\hline Liji & 礼記 & Fascicule 3 \\
\hline Lu Huo & 盧和 & Fascicule 4 \\
\hline Man'yō daishōki & 万葉代匠記 & Fascicule 4 \\
\hline
\end{tabular}




\begin{tabular}{|c|c|c|}
\hline Man'yōshū & 万葉集 & Fascicules 2, 3, 4 \\
\hline Matsunoo no kami & 松の尾之神 & Fascicule 1 \\
\hline Matsuoka Jo'an & 松岡恕庵 & Préface, Fascicule 4 \\
\hline Mazu (j. Maso) & 媽祖 & Fascicule 5 \\
\hline Meibutsu dokudan & 名物独断 & Préface \\
\hline Minamoto no Nakamasa & 源仲正 & Fascicule 5 \\
\hline Minamoto no Yoritomo & 源頼朝 & Fascicule 3 \\
\hline Minshu (j. Binsho) & 閩書 & Fascicule 3 \\
\hline Mukai Genshō & 向井元升 & Préface \\
\hline Mumyōshō & 無名抄 & Fascicules 2, 4 \\
\hline Murakami Yoshiteru & 村上義光 & Fascicule 4 \\
\hline Nakai Rankō & 中井藍江 & Introduction \\
\hline Nakamura Tekisai & 中村惕齊 & Fascicule 4 \\
\hline Nabeshima & 鍋島 & Présentation du fascicule 5 \\
\hline Nihon shoki/Nihongi & 日本書紀／日本紀 & Fascicules $1,2,3,4$ \\
\hline Nintoku tennō & 仁徳天皇 & Fascicules 1,2 \\
\hline Nishiyama Toyokazu & 西山豊一 & Fascicule 1 \\
\hline Ogasawara Nagahide & 小笠原長秀 & Fascicule 3 \\
\hline Ōjin tennō & 応神天皇 & Fascicule 1 \\
\hline Okamoto tennō & 岡本天皇 & Fascicule 4 \\
\hline ōkunitama & 大国魂 & Fascicule 1 \\
\hline Ōmiwa & 大 & Fascicule 1 \\
\hline Ōmononushi & 大物主 & Fascicule 1 \\
\hline Onko jitsuroku ou Onko nichiroku & 温故日録 & Fascicule 4 \\
\hline Ōsazaki (empereur) & 大焦鳥鴺 & Fascicule 1 \\
\hline Ōtomo no Yakamochi & 大伴家持 & Fascicule 3 \\
\hline Qishenlu & 稽神録 & Fascicule 2 \\
\hline
\end{tabular}




\begin{tabular}{|c|c|c|}
\hline Rankō, voir Nakai Rankō & 藍江 & Introduction \\
\hline Rokujō voir Kokin waka rokujō & 六帖 & Fascicule 4 \\
\hline Ruimingli (j. Zuimeirei) & 瑞命禮 & Fascicule 2 \\
\hline Saigyō & 西行 & Fascicules 3,4 \\
\hline Saiyaku dokudan & 採薬独断 & Préface \\
\hline Sancai tuhui (j. Sansai zue) & 三才圖會 & Fascicule 4 \\
\hline Sangi ittōo & 三義一統 & Fascicule 3 \\
\hline Sankai meisan zue & 山海名産困会 & Préface \\
\hline Sankashū & 山家集 & Fascicule 3 \\
\hline Sa'iō ou Tachibana no Sa'i & 佐為王 ; 樀佐為 & Fascicule 4 \\
\hline Seiryōden & 清凉殿 & Fascicule 5 \\
\hline She Du Kang & 葉杜康 & Fascicule 1 \\
\hline Shiaku & 塩飽 & Fascicule 2 \\
\hline Shijing & 詩経 & Fascicule 2 \\
\hline Shimazu & 島津 & Fascicule 4 \\
\hline Shiming & 釈名 & Fascicule 3 \\
\hline Shimokōbe Shūsui & 下河辺拾水 & Fascicule 4 \\
\hline Shinsarugakuki & 新猿楽記 & Fascicule 3 \\
\hline Shitomi Kangetsu & 部関月 & Préface \\
\hline Shō Igan & 松怡顔 & Préface \\
\hline Shoku kokinshū & 続古今集 & Fascicule 2 \\
\hline Shokumotsu honzō & 食物本草 & Fascicule 4 \\
\hline Shōmu tennō & 聖武天皇 & Fascicule 4 \\
\hline Shukun & 酒君 & Fascicule 2 \\
\hline Shuqi & 叔齊 & Fascicule 2 \\
\hline Song Yingxing & 株応星 & Fascicule 5 \\
\hline Songshu & 朱書 & Fascicule 2 \\
\hline
\end{tabular}




\begin{tabular}{|c|c|c|}
\hline Sujin tennō & 崇神天皇 & Fascicule 1 \\
\hline Takeuchi no sukune & 武内宿禰 & Fascicule 1 \\
\hline Tenji tennō & 天智天皇 & Fascicule 3 \\
\hline Tenjin & 天神 & Fascicule 1 \\
\hline Tiangong kaiwu & 天工開物 & Fascicule 5 \\
\hline Tō Jakusui (= Inō Jakusui) & 稲若水 & Préface, Fascicule 4 \\
\hline Tō Hōsui & 島彭水 & Préface \\
\hline Tōga & 東雅 & Fascicules 3,4 \\
\hline Tōi hōkan (cor. Tongŭi pogam) & 東醫珼鑑 & Fascicule 4 \\
\hline Toneri shinnō & 舎人親王 & Fascicule 3 \\
\hline Tongya & 通雅 & Fascicule 2 \\
\hline Toyotomi Hideyoshi & 豊臣秀吉 & Fascicule 5 \\
\hline Tsurezuregusa & 徒然草 & Fascicule 4 \\
\hline Tsushima Tsunenoshin & 津島恒之進 & Préface \\
\hline Ueshima Onitsura & 上島鬼貫 & Fascicule 1 \\
\hline Unkonshi & 雲根志 & Fascicule 2 \\
\hline Wahōsho & 和方書 & Fascicule 2 \\
\hline Wakan sansei zue & 和漢三才圖繪 & Fascicule 4 \\
\hline Wamyōshō ou Wamyō ruijyushō & 倭名抄／倭名類聚抄 & Fascicules 2,4 et 5 \\
\hline Wuli xiaozhi & 物理小識 & Fascicule 2 \\
\hline Wulilun & 物理論 & Fascicule 2 \\
\hline Wuzazu (j. Gozasso) & 五雑珇／五雑組 & Fascicule 4 \\
\hline Xie Zhaozhe & 謝肇淛 & Fascicule 4 \\
\hline Yamato honzō & 大和本草 & Fascicules 3, 4 \\
\hline Yōkotsuhō (cor. Ǔnggolbang) & 鷹鶻方 & Fascicule 2 \\
\hline Yoshida Kenkō & 吉田兼好 & Fascicule 4 \\
\hline Yoshitsune & 義経 & Fascicule 5 \\
\hline
\end{tabular}




\begin{tabular}{|l|l|l|}
\hline Yūryaku tennō & 雄略天皇 & Fascicule 2 \\
\hline Zen & 禅 & Présentation du fascicule 1 \\
\hline Zhu Shunshui (j. Shu Shunsui) & 朱舜水 & Fascicule 4 \\
\hline
\end{tabular}




\section{Glossaire des termes techniques}

1 On trouvera ci-dessous un glossaire contenant les principaux «termes techniques » rencontrés dans le Meisan zue, en englobant sous cette dénomination les noms d'outils, d'ustensiles, de matériaux, d'espèces animales, minérales ou végétales, de métiers, ou encore de procédures ou gestes techniques particuliers. La colonne 4 indique le ou les fascicules de leur occurrence.

2 Le glossaire liste ces termes par ordre alphabétique :

3 - ils figurent en français dans la colonne 1, lorsqu'ils ont été traduits en français. Le lecteur trouvera le terme original transcrit en caractères latins dans la colonne 2, sa graphie originale dans la colonne 3.

4 - ils figurent en japonais lorsqu'ils n'ont pas été traduits ou que seule leur signification littérale a été donnée. On les trouvera alors dans la colonne 2, en caractères latins, et dans la colonne 3, dans leur graphie originale.

5 Pour les noms d'objets très spécifiques, on indique entre parenthèses la famille d'objets à laquelle il appartient ou se rapporte.

\begin{tabular}{|c|c|c|c|}
\hline abeille & hachi & 蜂 & \multirow{8}{*}{ Fascicule 2} \\
\hline - ordinaires & gun'hō & 群蜂 & \\
\hline - ouvrière & saikunin & 細工人 & \\
\hline - de terre & tsuchihachi & 土蜂 & \\
\hline — « eunuque du miel » & mikkan & 蜜宦 & \\
\hline — « marieuse des fleurs » & kabai & 花媒 & \\
\hline — «porteur royal» & $\overline{o y o ̄ d o}$ & 王腰奴 & \\
\hline - «pirate des fleurs » & kazoku & 花賊 & \\
\hline « accroche-pierre » & ishibushi & イシブシ,石伏 & Fascicule 4 \\
\hline
\end{tabular}




\begin{tabular}{|c|c|c|c|}
\hline aérien [chaux] & $f u ̄ k a$ & 風化 & Fascicule 5 \\
\hline affinage final & awasedo & 磨石 & Fascicule 2 \\
\hline \multirow[t]{4}{*}{ aigle } & washi & 熟 & Fascicule 2 \\
\hline & aji & アジ & Fascicule 2 \\
\hline & ajika « daim de mer» & 葦鹿 & Fascicule 5 \\
\hline & ajikamo & 冠鳥 & Fascicule 2 \\
\hline \multirow[t]{6}{*}{ ajouts } & soe & 酘 & Fascicule 1 \\
\hline & akagashira (canard) & 赤頭 & Fascicule 2 \\
\hline & akage (autour) & 赤毛 & Fascicule 2 \\
\hline & akake (autour) & 網掛 & Fascicule 2 \\
\hline & akasashiba (buse) & 赤さしば & Fascicule 2 \\
\hline & akatake (champignon) & 赤菌 & Fascicule 2 \\
\hline \multirow[t]{4}{*}{ akébie } & akebi & 通草 & Fascicule 2 \\
\hline & akoyagai ; & アコヤ貝 & \multirow{2}{*}{ Fascicule 3} \\
\hline & (autre nom) akuya & 阿久夜 & \\
\hline & $a k u$ & 灰汁 & Fascicule 5 \\
\hline alevin & ko & 苗 & \multirow{3}{*}{ Fascicule 4} \\
\hline - de la truite & ayu no ko & 鮎の苗 & \\
\hline - de sardine & isaza & 鶖毛挺 & \\
\hline \multirow[t]{2}{*}{ alun } & myōban & 明礬 & Fascicule 5 \\
\hline & amasagi & アマサギ & Fascicule 4 \\
\hline âne de mer & todo & 海驢 & Fascicule 5 \\
\hline anémone de mer & shasan & 沙蒜 & Fascicule 4 \\
\hline \multirow[t]{4}{*}{ « anguille de mer» } & umi-unagi & 海鰻 & Fascicule 4 \\
\hline & aosashiba (buse) & 青さしば & Fascicule 2 \\
\hline & aotaka (autour) & 蒼鷹 & Fascicule 2 \\
\hline & aoto (pierre) & 青砥 & Fascicule 2 \\
\hline
\end{tabular}




\begin{tabular}{|c|c|c|c|}
\hline & arare-uo « poisson-grêle » & 霰魚 & \multirow{2}{*}{ Fascicule 4} \\
\hline & (autre nom) kakubutsu & カクブツ & \\
\hline & aratamataka (autour) & 新玉鷹 & Fascicule 2 \\
\hline \multirow[t]{3}{*}{ ardoise (voir aoto) } & & & Fascicule 2 \\
\hline & aseki & 亜石 & Fascicule 5 \\
\hline & ashikuruma & 脚車 & Fascicule 5 \\
\hline arsenic & banseki & 䙪石 & Fascicule 2 \\
\hline \multirow[t]{2}{*}{ arsénopyrite } & yoseki & 譽石 & Fascicule 2 \\
\hline & asagi (pierre) & 浅黄 & Fascicule 2 \\
\hline autour & taka & 鷹 & \multirow{5}{*}{ Fascicule 2} \\
\hline - aîné & $s h \bar{o}$ & 兄鷹 & \\
\hline - cadet & tai & 弟鷹 & \\
\hline - grand & ōdaka & 大鷹 & \\
\hline \multirow[t]{4}{*}{- petit } & kotaka & 小鷹 & \\
\hline & awasu, awaseto (pierre) & 磨石 & Fascicule 2 \\
\hline & azarashi & アザラシ & Fascicule 5 \\
\hline & $a z u k i$ & 小豆 & Fascicule 2 \\
\hline bâche de paille & komo & 䳸 & Fascicule 1 \\
\hline $\begin{array}{l}\text { balancier } \quad \text { "pilier } \\
\text { porteur } ~\end{array}$ & otoko-bashira & 男柱 & Fascicule 1 \\
\hline balle à jouer & temari & 手鞠 & Fascicule 5 \\
\hline baquets en bois & oke & 桶 & Fascicule 1 \\
\hline bascule sans pareille & musō gaeshi & 無双返 & Fascicule 2 \\
\hline $\begin{array}{l}\text { base [des récipients en } \\
\text { porcelaine] }\end{array}$ & itozoko & 輪台 & Fascicule 5 \\
\hline bassin & tameike & 溜池 & Fascicule 5 \\
\hline bassins & mizubachi & 水鉢 & Fascicule 2 \\
\hline bateau de halage & amifune & 網船 & Fascicule 3 \\
\hline
\end{tabular}




\begin{tabular}{|c|c|c|c|}
\hline bateau stabilisateur & hikaebune & ひかえ船 & Fascicule 3 \\
\hline & benikin (champignon) & 紅菌 & Fascicule 2 \\
\hline & bidoro & 硝子 & Fascicule 5 \\
\hline & bidoro kusuri, voir couverte vitreuse & & Fascicule 5 \\
\hline bile, vésicule biliaire & kimo & 膽 & \multirow{8}{*}{ Fascicule 2} \\
\hline - d'ours & kumano'i & 熊膽 & \\
\hline variété noire de - & kurote & 黒様 & \\
\hline variété jaune de - & mamenokote & 豆粉様 & \\
\hline variété ambre - & kohakute & 琥珀様 & \\
\hline - d'été & natsuno'i & 夏膽 & \\
\hline - d'hiver & fuyuno’i & 冬膽 & \\
\hline - liquide & $\operatorname{tanju} \bar{u}$ & 膽汁 & \\
\hline blanchir & sarasu & 晒す & Fascicule 5 \\
\hline bleu pâle & bisei & 微青 & Fascicule 5 \\
\hline bleu vert & seiheki & 青碧 & Fascicule 5 \\
\hline \multirow[t]{3}{*}{ bobine } & waku & 轤 & Fascicule 2 \\
\hline & bōchōgyo & 望潮魚 & Fascicule 4 \\
\hline & bon & 盆 & Fascicule 5 \\
\hline bonite & katsuo & 堅魚, 鰹 & \multirow{2}{*}{$\begin{array}{l}\text { Fascicules 3, } \\
4\end{array}$} \\
\hline - séchée & katsuo; katsuobushi & 乾魚 ; 鰹節 & \\
\hline - séchée d'automne & aki-bushi & 秋節 & \multirow[t]{6}{*}{ Fascicule 4} \\
\hline - séchée du printemps & haru-bushi & 春節 & \\
\hline $\begin{array}{l}\text { morceau du ventre de - } \\
\text { séchée }\end{array}$ & hara-bushi & 腹節 & \\
\hline $\begin{array}{l}\text { morceau du dos de la - } \\
\text { séchée }\end{array}$ & sebushi & 替節 & \\
\hline première - & hatsu-gatsuo & 初䚛 & \\
\hline extrait de - & ikari & 煎汁 & \\
\hline
\end{tabular}




\begin{tabular}{|c|c|c|c|}
\hline & (autre nom) nitori & 者取 & \\
\hline (nom ancien) & kogyo " poisson dur» & 固魚 & \\
\hline $\begin{array}{l}\text { (autre nom) «poisson de } \\
\text { pin» }\end{array}$ & shōgyo & 松魚 & \\
\hline - gâteau de riz & mochi gatsuo & 餅鰹 & \\
\hline - à ventre rayé & suji-gatsuo & 縷鰹 & \\
\hline - à cercles & uzuwa-gatsuo & 宇津和鰹 & \\
\hline - à rayures transversales & yokowa-gatsuo & 横輪鰹 & \\
\hline - plate & hira-gatsuo & ヒラ鰹 & \\
\hline tataki de - & tataki & タタキ & \\
\hline & bora & 鰡 & Fascicule 3 \\
\hline & botai & ボタイ & Fascicule 1 \\
\hline boule de terre & dokai & 土塊 & Fascicule 5 \\
\hline bourre de mûrier & $y \bar{u}$ & 木綿 & Fascicule 5 \\
\hline boyaux & hyakuhiro & 百ヒロ & Fascicule 5 \\
\hline brasser & momi'arau & 揉みあらう & Fascicule 5 \\
\hline brocart d'Ezo & Ezo nishiki & 蝦夷錦 & Fascicule 5 \\
\hline brûler [la chaux] & yaku & 燔く; 焼く & Fascicule 5 \\
\hline \multirow[t]{3}{*}{ brume (nom de filet) } & kasumi & 霞 & Fascicule 2 \\
\hline & $b u$ & 分 & Fascicule 5 \\
\hline & bukuryō & 茯苓 & Fascicule 2 \\
\hline \multirow[t]{2}{*}{$\begin{array}{l}\text { bureau du service } \\
\text { intérieur }\end{array}$} & naishi no tsukasa & 内侍司 & Fascicule 5 \\
\hline & buri & 鯔 & Fascicule 3 \\
\hline câble & tenawa & 手縄 & Fascicule 3 \\
\hline calcite & hōkaiseki & 方解石 & Fascicule 2 \\
\hline canard & kamo & 鳥,身 & \multirow[t]{2}{*}{ Fascicule 2} \\
\hline - d'eau & mizugamo & 水鴨 & \\
\hline
\end{tabular}




\begin{tabular}{|c|c|c|c|}
\hline - domestique & ahiru; aisa & 鴨 ; 秋紗 & \\
\hline - à tête verte & aokubi & 青頭 & \\
\hline carder & saku & 破く & Fascicule 5 \\
\hline carex (fil de) & sugaito & すが糸 & Fascicule 2 \\
\hline carpe noire & kokurigyo & 黒鯉魚 & Fascicule 4 \\
\hline carrelet & ami & 網 & Fascicule 4 \\
\hline ceinture & obi & 带 & Fascicule 5 \\
\hline $\begin{array}{l}\text { cendres pour clarifier le } \\
\text { saké }\end{array}$ & sumashi hai & すまし灰 & Fascicule 1 \\
\hline cendre de paille & ine no hai & 稲灰 & Fascicule 5 \\
\hline cendre pour teindre & senkai & 染灰 & Fascicule 5 \\
\hline céramique & yakimono & 陶器 & Fascicule 5 \\
\hline cercle & $w a$ & 圏輪, 圏 & Fascicule 5 \\
\hline chabot & kajika & カジカ 䱃 & \multirow{6}{*}{ Fascicule 4} \\
\hline (nom chinois du -) & tofugyo & 杜夫魚, 杜父魚 & \\
\hline (autre nom) & ishimochi & イシモチ & \\
\hline (autre nom) & kuchinawadonko & クチナワドンコ & \\
\hline (autre nom) & maru & マル & \\
\hline (autre nom) & muko & ムコ & \\
\hline chaleur & $k a k i$ & 火気 & Fascicule 5 \\
\hline chambre d'huîtres & reibō & 蠣房 & Fascicule 5 \\
\hline chambre de fermentation & muro; kōjimuro & 室; 麦米室 & $\begin{array}{l}\text { Fascicules } 1, \\
5\end{array}$ \\
\hline champignon des arbres & mokukin & 木菌 & \multirow{5}{*}{ Fascicule 2} \\
\hline - de la fauvette & uguisu take & 䉆蔦菌 & \\
\hline - « œuf de moineau » & suzume no tamago & 竹莀 & \\
\hline - de roche & sekikin & 石菌 & \\
\hline - de terre & dokin & 土菌 & \\
\hline
\end{tabular}




\begin{tabular}{|c|c|c|c|}
\hline - du tonnerre & raikin & 雷菌 & \\
\hline chanvre & asa & 大麻 & \multirow{2}{*}{ Fascicule 5} \\
\hline - cerisier & sakura asa & サクラアサ & \\
\hline charme & shidenoki & 扶移の樹 & Fascicule 2 \\
\hline charpentier & daikō & 大工 & Fascicule 2 \\
\hline chaux de pierre & ishibai & 石灰 & Fascicule 5 \\
\hline $\begin{array}{l}\text { "Chevesne } \\
\text { ornithorynque » }\end{array}$ & oikawa & オイカワ & Fascicule 4 \\
\hline \multirow[t]{2}{*}{ chèvrefeuille japonais } & nindō & 忍冬 & Fascicule 2 \\
\hline & chichikaburi & 䰹 & Fascicule 4 \\
\hline chien de mer & kaiku & 海狗 & Fascicule 5 \\
\hline \multirow[t]{6}{*}{ chinchard } & aji & 鮯 & Fascicule 3 \\
\hline & chirimen-koayu & チリメン小アユ & Fascicule 4 \\
\hline & chirimen-zako & チリメンザコ & Fascicule 4 \\
\hline & chishin (champignon) & 地岑 & Fascicule 2 \\
\hline & chixi $(\mathrm{j}$. chigeki) & 絺綌 & Fascicule 2 \\
\hline & chō (variété d'aigle) & 鵀 & Fascicule 2 \\
\hline cire d'abeilles & mitsurō & 蜜㩊 & Fascicule 2 \\
\hline cire d'Aizu & Aizurō & 会津蛹 & Fascicule 2 \\
\hline cire blanche d'insecte & chūhakurō & 蟲白蝛 & Fascicule 2 \\
\hline cire jaune & ōrō & 黄蠟 & Fascicule 2 \\
\hline ciseau & nomi ou gennō & 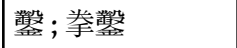 & Fascicule 5 \\
\hline claie & kōshi & 格子 & Fascicule 2 \\
\hline cobalt & seiheki & 青碧 & Fascicule 5 \\
\hline « coffres en vannerie » & fujikori & 葛簏 & Fascicule 2 \\
\hline $\begin{array}{l}\text { concombre de mer, voir } \\
\text { aussi holothurie }\end{array}$ & namako & 生海鼠 & Fascicule 3 \\
\hline conduit en pierre & mizo'ishi & 溝石 & Fascicule 2 \\
\hline
\end{tabular}




\begin{tabular}{|c|c|c|c|}
\hline conque de turbot cornu & sazae & 榮螺 & Fascicule 2 \\
\hline contrefaçon & gibutsu, nisemono & 偽物 & Fascicule 2 \\
\hline \multirow[t]{2}{*}{ convulsion } & kan & 疳 & Fascicule 2 \\
\hline & namima kashiwa (coquillage) & 波間かしわ & Fascicule 3 \\
\hline coquillage-casque & kabutogai & 少貝 & Fascicule 4 \\
\hline corbicula & shijimi & 蜆 & Fascicule 3 \\
\hline corde & nawa & 縄 & Fascicule 5 \\
\hline courtisane-miko & miko-jorō & みこ女郎 & Fascicule 4 \\
\hline couteau à papier & kamitachi & 紙裁 & Fascicule 2 \\
\hline couverte & kusuri; uwagusuri & 銹獎; 上薬 & \multirow{2}{*}{ Fascicule 5} \\
\hline - vitreuse & bìdoro kusuri & 硝子銹 & \\
\hline crabe à mitaines & zugani & ズ蟹 & Fascicule 4 \\
\hline crabe de rivière & zugani & ズガニ & Fascicule 2 \\
\hline crapaud & kawazu & 蛙 & \multirow{2}{*}{ Fascicule 4} \\
\hline voir aussi « grenouille» & gama & 蝦墓 & \\
\hline crêpe & chijimi & 縮 & Fascicule 5 \\
\hline crevette & $e b i$ & 海老;エビ & \multirow{4}{*}{ Fascicule 3} \\
\hline «-dragon » & ryūka & 龍鰕 & \\
\hline " tête de -" & kakai & 蝦鬼 & \\
\hline «- rouge » & $k o ̄ k a$ & 紅鰕 & \\
\hline croissant de lune & tsuki no wa & 月の輪 & Fascicule 2 \\
\hline cuir chamoisé & momigawa & 揉皮 & Fascicule 2 \\
\hline culée de pont & hashidai & 橋台 & Fascicule 2 \\
\hline cuve & oke & 桶 & \multirow{3}{*}{ Fascicule 1} \\
\hline - en bois & toko & 槽 & \\
\hline - de clarification & sumashi no oke & 澄しの桶 & \\
\hline cuveau & hangiri & 半切 & \multirow[b]{2}{*}{ Fascicule 1} \\
\hline - averse & bonburi & ぼんぶり & \\
\hline
\end{tabular}




\begin{tabular}{|c|c|c|c|}
\hline - verseur & ageoke & あげ桶 & \\
\hline cycle d'opérations & shimai & 仕迴 & Fascicule 1 \\
\hline daim & shika & 鹿 & Fascicule 2 \\
\hline dalle (de jardin) & tobiishi & 飛石 & Fascicule 2 \\
\hline dalle & shikiishi & 敷石 & Fascicule 2 \\
\hline décanter & sumasu & 澄す & Fascicule 5 \\
\hline déchirer & saku & 裂く & Fascicule 2 \\
\hline découpe & wari & 割 & Fascicule 2 \\
\hline dégrossir & aratogi & 荒磨 & Fascicule 2 \\
\hline Département du trésor & Ōkurashō & 大蔵省 & Fascicule 5 \\
\hline deutzia & utsugi & 楊櫨 & Fascicule 2 \\
\hline disque & enban & 円盤 & Fascicule 5 \\
\hline district (ancien) & agata & 県 & Fascicule 5 \\
\hline & donguro-haze & どんぐろはぜ & Fascicule 4 \\
\hline dorade & tai & 鯛 & \multirow{3}{*}{ Fascicule 3} \\
\hline - séchée & hidai & 干鯛 & \\
\hline petite - & kodai & 小鯛 & \\
\hline & dorome & 泥目 & Fascicule 4 \\
\hline & doromesako & 湎條魚 & Fascicule 4 \\
\hline $\begin{array}{l}\text { eau conservée depuis la } \\
\text { veille }\end{array}$ & nemizu & 宿水 & Fascicule 5 \\
\hline \multirow[t]{2}{*}{ eau de rouille } & kusurimizu & 銹水 & Fascicule 5 \\
\hline & $e b i$ & 海鰕, 海老 & Fascicule 3 \\
\hline émail bleu & aoe no kusuri & 包青 & Fascicule 5 \\
\hline empreinte de moule & kataoshi & 印器 & Fascicule 5 \\
\hline enceinte de jardin & teireki & 庭彻 & Fascicule 2 \\
\hline encoche & hazu & 筈 & Fascicule 2 \\
\hline
\end{tabular}




\begin{tabular}{|c|c|c|c|}
\hline & engyo & 淵魚 & Fascicule 4 \\
\hline $\begin{array}{l}\text { entrailles, abats, } \\
\text { macéré(e)s dans le sel }\end{array}$ & wata ou hara-wata ; hishio & 腸; 酷 & $\begin{array}{l}\text { Fascicules 3, } \\
4\end{array}$ \\
\hline $\begin{array}{l}\text { entrailles [d'holothurie] } \\
\text { marinées }\end{array}$ & konoko & 海鼠子 & Fascicule 4 \\
\hline épervier & karaami & 唐網 & Fascicule 2 \\
\hline épuisette & tamo (ami) & 夕モ（網） & Fascicule 4 \\
\hline épuisette & tamaami & 網 & Fascicule 5 \\
\hline escargot de mer Noire & akanishi & 赤螺 & Fascicule 4 \\
\hline \multirow[t]{2}{*}{ esprit de cent fleurs } & hyakkasei (miel) & 百花精 & Fascicule 2 \\
\hline & essa’i & 䟺さい & Fascicule 2 \\
\hline \multirow[t]{2}{*}{ essaim } & gunhō & 群蜂 & Fascicule 2 \\
\hline & Esshi (pierre) & 越䃨 & Fascicule 2 \\
\hline étamines de cent fleurs & hyakkazui & 百花芯 & Fascicule 2 \\
\hline exposer au soleil/blanchir & sarasu & 晒す & Fascicule 5 \\
\hline fauchard & naginata & 薙刀 & Fascicule 2 \\
\hline faucher & oru & 折る & Fascicule 5 \\
\hline faucon & hayabusa & 鶻 & Fascicule 2 \\
\hline fer & kaneke ou kinki & 金気 & Fascicule 5 \\
\hline feuilles de chêne & kunugi & 櫪 & Fascicule 5 \\
\hline fibre de papier et de paille & kamisusa & 紙荔 & Fascicule 5 \\
\hline fil à coudre & nui ito & 縫線 & Fascicule 5 \\
\hline filage & bōseki & 約績 & Fascicule 5 \\
\hline filer & yoru & 紡る & Fascicule 5 \\
\hline filer & umu & 紡む & Fascicule 2 \\
\hline filet & $a m i$ & 網 & $\begin{array}{l}\text { Fascicules 2, } \\
3 \text { et } 4\end{array}$ \\
\hline - à canard & kamoami & 鴨羅 & Fascicule 2 \\
\hline
\end{tabular}




\begin{tabular}{|c|c|c|c|}
\hline - basculant & kaeshiami & かえし網 & \\
\hline - brume & kasumi ami & 霞羅 & \\
\hline - dit « tendu» & harikiri ami & はり切編羅 & \\
\hline - en forme de lanterne & chōchin-ami & 提灯羅 & \\
\hline - recouvrant & òten & おうてん & \\
\hline - de pêche & ami & 魚網 & \multirow{7}{*}{ Fascicule 3} \\
\hline - de chalut & teguri ami & 手操網 & \\
\hline - droit & oiami & 追網 & \\
\hline - flottant & nagashi ami & 流し網 & \\
\hline - posé & shiki ami & 敷網 & \\
\hline - traîné & hiki ami & 史網 & \\
\hline - vertical & tateami & 竪網 & \\
\hline $\begin{array}{l}\text { - de rivière manœuvré } \\
\text { par bateau }\end{array}$ & nori-kawaami & 乗川網 & Fascicule 4 \\
\hline fléau & tenbin & 天秤 & Fascicule 4 \\
\hline flotteur & $a b a$ & アバ & $\begin{array}{l}\text { Fascicules 3, } \\
4\end{array}$ \\
\hline flotteur & uke, uki & 泛子 & Fascicule 3 \\
\hline fondation en pierre & hashiraishi & 柱礎 & Fascicule 2 \\
\hline forgeron de sabre & katanakaji & 刀剣鍛冶 & Fascicule 2 \\
\hline fortification & do'i & 土居 & Fascicule 2 \\
\hline four à biscuit & suyakikama & 素暁窯 & Fascicule 5 \\
\hline four en forme de tour & yagura gama & 櫓窯 & Fascicule 5 \\
\hline four principal & honkama & 本窯 & Fascicule 5 \\
\hline \multirow[t]{4}{*}{ fourmi charpentière } & yamaari & 山蟻 & Fascicule 2 \\
\hline & fuji & 葛 & Fascicule 2 \\
\hline & fujigoromo & 葛衣 & Fascicule 2 \\
\hline & fukeru, voir s'humidifier & & Fascicule 5 \\
\hline
\end{tabular}




\begin{tabular}{|c|c|c|c|}
\hline & fukusō & 福草 & Fascicule 2 \\
\hline & fushi/bushi & 節 & Fascicule 3 \\
\hline & fushisō & 不死草 & Fascicule 2 \\
\hline \multirow[t]{3}{*}{ fût } & taru & 樽 & Fascicule 1 \\
\hline & futa'ishi & 蓋石 & Fascicule 2 \\
\hline & futtai & フッタイ & Fascicule 4 \\
\hline \multirow[t]{3}{*}{ gaffe à anguille } & unagikaki & うなぎ择 & Fascicule 4 \\
\hline & gama & 蝦墓 & Fascicule 4 \\
\hline & gangi & 雁木 & Fascicule 5 \\
\hline \multirow[t]{2}{*}{ gangue (de la pierre) } & kawa & 皮 & Fascicule 2 \\
\hline & gigi & ギギ,軌々 & Fascicule 4 \\
\hline gingembre & shōga & 生姜 & Fascicule 4 \\
\hline ginseng & ninjin & 人参 & Fascicule 4 \\
\hline « ginseng marin » & kaijin & 海参 & Fascicule 4 \\
\hline glandes extérieures & gaijin & 外腎 & Fascicule 5 \\
\hline glu & mochi & 榽 & Fascicule 2 \\
\hline glu flottante & nagashimochi & 流䅗 & Fascicule 2 \\
\hline \multirow[t]{2}{*}{ glycine de mer } & kaitōka & 海藤花 & Fascicule 4 \\
\hline & gō, gōme & 合, 合目 & Fascicule 5 \\
\hline gobie & gori & 鮴, 䱃 & \multirow{6}{*}{ Fascicule } \\
\hline (autre nom) & kaisangyo & 鱠残魚 & \\
\hline (autre nom) & shiro'uo & 白魚 & \\
\hline - creuseur de sable & sunabori-haze & 砂堀魦 & \\
\hline (autre nom) & ishibushi & 鰻 & \\
\hline pousser le - & gori-oshi & 鮴押 & \\
\hline \multirow[t]{2}{*}{ gord } & yana & ヤナ & Fascicule 4 \\
\hline & gori-ishibushi & ゴリ石伏 & Fascicule 4 \\
\hline
\end{tabular}




\begin{tabular}{|c|c|c|c|}
\hline & goshikidake (champignon) & 五色芝 & Fascicule 2 \\
\hline grain (de la pierre) & ishime & 石理、文理 & Fascicule 2 \\
\hline graine de chanvre & onomi & 麻の子 & Fascicule 5 \\
\hline Grandes prémices & Daijōe & 大嘗会 & Fascicule 1 \\
\hline grenouille & $\begin{array}{l}\text { kajika } \\
\text { kawazu } \\
\text { tanikugu, ou taniguku }\end{array}$ & $\begin{array}{l}\text { 河鹿 } \\
\text { 蛙 } \\
\text { 谷潜 }\end{array}$ & Fascicule 4 \\
\hline grenouille rouge & akagaeru & 山蛤 & Fascicule 2 \\
\hline $\begin{array}{l}\text { grès à couverte verte ou } \\
\text { céladon }\end{array}$ & seijimono & 青磁物 & Fascicule 5 \\
\hline grumeau & sekkai & 石塊 & Fascicule 5 \\
\hline \multirow[t]{9}{*}{ hache } & ono & 斧 & Fascicule 2 \\
\hline & hadara & はだら & Fascicule 3 \\
\hline & haga-ijime & 羽がいじめ & Fascicule 2 \\
\hline & hago & ハゴ & Fascicule 2 \\
\hline & haitaka (autour) & 鷂 & Fascicule 2 \\
\hline & hamaguri, voir palourde & & Fascicule 4 \\
\hline & haraakago " œufs de ventre rouge " & 腹赤子 & Fascicule 4 \\
\hline & haraka & 鰚魚 & Fascicule 4 \\
\hline & hararago & ハララゴ & Fascicule 4 \\
\hline hareng & nishin & ニシン & $\begin{array}{l}\text { Fascicules 3, } \\
5\end{array}$ \\
\hline \multirow[t]{7}{*}{ haricot des daims } & shikamame & 鹿豆 & Fascicule 2 \\
\hline & haritake (champignon) & 針罩 & Fascicule 2 \\
\hline & hashihiro (canard) & ハシヒロ & Fascicule 2 \\
\hline & hashitae (autour) & 波廝妙 & Fascicule 2 \\
\hline & hatsukusa (autour) & 初種 & Fascicule 2 \\
\hline & hatsutake (champignon) & 初罩 & Fascicule 2 \\
\hline & hayabusa & 隼鶻 & Fascicule 2 \\
\hline
\end{tabular}




\begin{tabular}{|c|c|c|c|}
\hline & heriko & へり子 & Fascicule 4 \\
\hline & hiratake (champignon) & 天花蔁 & Fascicule 2 \\
\hline & hirenaga & ひれなが & Fascicule 3 \\
\hline & hishio & 䤈 & Fascicule 4 \\
\hline & hitofu, futafu, mifu & $\begin{array}{l}\text { 一生 ; 二生 ; 三 } \\
\text { 生 }\end{array}$ & Fascicule 2 \\
\hline & hitori (canard) & ヒトリ & Fascicule 2 \\
\hline & hitotsu ishi « pierre unique » & 一ツ石 & Fascicule 2 \\
\hline & hoka'i & 行器 & Fascicule 5 \\
\hline holothurie & namako & 生海鼠 & \multirow{15}{*}{ Fascicule 4} \\
\hline (autre nom) & shasun & 沙噀 & \\
\hline (autre nom) & shasan & 沙蒜 & \\
\hline (autre nom) & tojun & 塗荀 & \\
\hline (autre nom) & tawarago & 俵子 & \\
\hline (autre nom) & kaijin « ginseng marin » & 海参 & \\
\hline - d'or & kinko & 金海鼠 & \\
\hline entrailles d'-marinées & konowata & 海鼠腹 & \\
\hline - rôtie et séchée & iriko & 謷海鼠 & \\
\hline (autre nom) & kaidanshi & 海男子 & \\
\hline (autre nom) & kaiso & 海蛆 & \\
\hline œuf d'- & konoko & 海鼠子 & \\
\hline en brochette & kushiko & 串海鼠 & \\
\hline - tigrée & torako & 虎海鼠 & \\
\hline \multirow[t]{3}{*}{ - tigrée séchée } & tawarago & 俵子 & \\
\hline & hoya & 老海鼠 & Fascicule 4 \\
\hline & hozo, voir pénis d'otarie & & Fascicule 5 \\
\hline huître & kaki & 牡蠣 & $\begin{array}{l}\text { Fascicules 3, } \\
5\end{array}$ \\
\hline
\end{tabular}




\begin{tabular}{|c|c|c|c|}
\hline (s')humidifier & fukeru & フケル & Fascicule 5 \\
\hline & ibokurai (insecte) & イボクライ & Fascicule 2 \\
\hline & ibota (arbre) & 水蛹樹 & Fascicule 2 \\
\hline & ichigō, nigō, sangō & $\begin{array}{l}\text { 一合, 二合, 三 } \\
\text { 合 }\end{array}$ & Fascicule 5 \\
\hline & ikari (voir bonite) & 煎汁 & Fascicule 4 \\
\hline ikat bleu marine & konkasuri & 紺かすり & Fascicule 5 \\
\hline imbiber & chōka & 調和 & Fascicule 5 \\
\hline \multirow[t]{2}{*}{ impératas } & chigusa & 茅草 & Fascicule 2 \\
\hline & inbe & 忌瓮 & Fascicule 5 \\
\hline \multirow[t]{2}{*}{ incorporer } & ka-suru, chōka & 加スル , 調和 & Fascicule 5 \\
\hline & inokorotake (champignon) & 蜀挌 & Fascicule 2 \\
\hline insecte de la Vitis ficifolia & ebitsuru no mushi & 䫤耎蟲 & Fascicule 2 \\
\hline \multirow[t]{11}{*}{ insecte du saule } & yanagi no mushi & 柳の虫 & Fascicule 2 \\
\hline & iriko, voir holothurie & 㥿海鼠 & Fascicule 4 \\
\hline & Ise'ebi & 伊勢海鰕 & Fascicule 3 \\
\hline & ishibushi & $\begin{array}{l}\text { イシブシ ou 石 } \\
\text { 伏 }\end{array}$ & Fascicule 4 \\
\hline & ishifushi & 䱌 & Fascicule 4 \\
\hline & $\begin{array}{l}\text { ishigurai « mord-pierre »; } \\
\text { (autre nom) donguro }\end{array}$ & $\begin{array}{l}\text { 石ぐらい;ドン } \\
\text { グロ }\end{array}$ & Fascicule 4 \\
\hline & ishimochi « agrippe-pierre » & イシモチ & Fascicule 4 \\
\hline & itaoshiki & 板おしき & Fascicule 4 \\
\hline & itsue & 厳䆙 & Fascicule 5 \\
\hline & itsue no okimono & 厳瓮之置 & Fascicule 5 \\
\hline & iwatake & 石耳, 石芝 & Fascicule 2 \\
\hline jarre & hotogi & 缶 & Fascicule 5 \\
\hline jet & yama ashio & 山足緒 & \\
\hline
\end{tabular}




\begin{tabular}{|c|c|c|c|}
\hline & $\bar{o} o$ & 大緒 & \\
\hline & jijie (j. shūkai) & 集解 & Fascicule 5 \\
\hline & jin (champignon) & 罩 & Fascicule 2 \\
\hline & jitsuya & 地艶 & Fascicule 2 \\
\hline \multirow[t]{16}{*}{ jus de racines de narcisse } & suisenka no ne & 水仙花の根 & Fascicule 2 \\
\hline & $k a$, voir incorporer & & Fascicule 5 \\
\hline & $k a b a i$ « marieuse des fleurs» & 花媒 & Fascicule 2 \\
\hline & kaeri (autour) & 鶭 & Fascicule 2 \\
\hline & kai'oshō « bonze de mer» & 海和尚 & Fascicule 4 \\
\hline & kaiba « cheval de mer» & 海馬 & Fascicule 5 \\
\hline & kaiku; kaidatsu (« loutre de mer ») & 海狗 ; 海獺 & Fascicule 5 \\
\hline & kaifu, voir laminaire & & Fascicule 5 \\
\hline & kaihyō « lion de mer » & 海豹 & Fascicule 5 \\
\hline & karei & 鰈 & Fascicule 3 \\
\hline & kaishiro & 坦代 & Fascicule 5 \\
\hline & kaitōka & 海藤花 & Fascicule 4 \\
\hline & kaitsuburi & 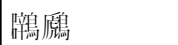 & Fascicule 2 \\
\hline & kajika & 河鹿 & Fascicule 4 \\
\hline & kajin (« champignon domestique ») & 家草 & Fascicule 2 \\
\hline & kaki & 牡蠣 & Fascicule 3 \\
\hline \multirow[t]{7}{*}{ kaki amer } & konerigaki & こねり柿 & Fascicule 2 \\
\hline & kakkoku (puéraire) & 葛穀 & Fascicule 2 \\
\hline & kakubutsu, voir aussi arare no & カクブツ & Fascicule 4 \\
\hline & kameisō & 科名草 & Fascicule 2 \\
\hline & kamosu & 醸す & Fascicule 2 \\
\hline & kamisusa, voir fibre & & Fascicule 5 \\
\hline & kanakine, voir pilon métallique & & Fascicule 5 \\
\hline
\end{tabular}




\begin{tabular}{|c|c|c|c|}
\hline & karamushi & カラムシ & Fascicule 5 \\
\hline & karausu, voir mortier & & Fascicule 5 \\
\hline & kasurijima & かすり島 & Fascicule 5 \\
\hline & kata, voir moule & & Fascicule 5 \\
\hline & katakaeri (autour) & 片かえり & Fascicule 2 \\
\hline & kataoshi, voir empreinte de moule & & Fascicule 5 \\
\hline & kawarijima & かわり島 & Fascicule 5 \\
\hline & kawatake (« champignon cuir ») & 皮曹 & Fascicule 2 \\
\hline & kawazu & 蛙 & Fascicule 4 \\
\hline & kayoitsumi (épervier du Japon) & 通つみ & Fascicule 2 \\
\hline & kazoku « pirates des fleurs » (abeille) & 花䁍 & Fascicule 2 \\
\hline & keōren (racine) & 毛苖蓮 & Fascicule 2 \\
\hline & kiboshi (séchage sur pied) & 生乾し & Fascicule 2 \\
\hline & kihitsu « pinceau de démon » & 鬼筆 & Fascicule 2 \\
\hline & kikai (champignon) & 鬼蓋 & Fascicule 2 \\
\hline & kikurage (champignon) & 木耳 & Fascicule 2 \\
\hline & kin (champignon) & 菌 & Fascicule 2 \\
\hline & kintake (champignon d'or) & 金菌 & Fascicule 2 \\
\hline & kisare (autour) & 木曝 & Fascicule 2 \\
\hline & $\begin{array}{l}\text { kisuko ou kisugo («pêche-madame } \\
\text { argenté ») }\end{array}$ & $\begin{array}{l}\text { キスコ, キスゴ, } \\
\text { 白魚 }\end{array}$ & Fascicule 4 \\
\hline & kitaka (autour) & 黄鷹 & Fascicule 2 \\
\hline & Kitayama-tsumi (épervier du Japon) & 北山つみ & Fascicule 2 \\
\hline & $\begin{array}{l}\text { kitsune no karakasa «ombrelle de } \\
\text { renard» (champignon) }\end{array}$ & $\begin{array}{l}\text { キツネノカラカ } \\
\text { サ }\end{array}$ & Fascicule 2 \\
\hline & kōgyo; (nom japonais) karakako & 䱩魚 ; カラカコ & Fascicule 4 \\
\hline kōji & $k \overline{o j} i$ & 麦匊 & \\
\hline plateaux à - & kōji-buta & 麦米盆 & Fascicule 1 \\
\hline
\end{tabular}




\begin{tabular}{|c|c|c|c|}
\hline \multirow[t]{2}{*}{ chambre de fermentation } & kōjimuro & 麦米室 & \\
\hline & kōkin (champignon) & 香菌 & Fascicule 2 \\
\hline & komadori & こま鳥 & Fascicule 4 \\
\hline & komotake (champignon) & 土菌 & Fascicule 2 \\
\hline & konkasuri & 紺かすり & Fascicule 5 \\
\hline & konohatsumi (épervier du Japon) & 木葉つみ & Fascicule 2 \\
\hline & konoko (« œufs d'holothurie») & 海鼠子 & Fascicule 4 \\
\hline & konori (autour) & 兒鷹 & Fascicule 2 \\
\hline & konowata & 海鼠腹 & Fascicule 4 \\
\hline & koshi marubashi & 腰丸觜 & Fascicule 2 \\
\hline & kōshi (champignon) & 香菰 & Fascicule 2 \\
\hline & kōsōgyo " poisson au front jaune » & 黄頚魚 & Fascicule 4 \\
\hline & kotsugyo & 䰴魚 & Fascicule 4 \\
\hline & koyamagaeri (autour) & 小山かえり & Fascicule 2 \\
\hline & kuchi (oiseau) & 俱知 & Fascicule 2 \\
\hline & kumataka (aigle) & 鵰 & Fascicule 2 \\
\hline & kumatsumi (épervier du Japon) & 熊つみ & Fascicule 2 \\
\hline & kuriishi & 栗石 & Fascicule 2 \\
\hline & kurokamo (canard) & 黒鴨 & Fascicule 2 \\
\hline & $\begin{array}{l}\text { kurokawatake «champignon cuir } \\
\text { noir» }\end{array}$ & 黒皮たけ & Fascicule 2 \\
\hline & kurotsumi (oiseau) & 黒つみ & Fascicule 2 \\
\hline & kusagi no mushi & 常山のむし & Fascicule 2 \\
\hline & kusotsukami (autour) & くそつかみ & Fascicule 2 \\
\hline & kuwatake (champignon) & 荣蕫 & Fascicule 2 \\
\hline & kuzutake " champignon du puéraire" & 葛草 & Fascicule 2 \\
\hline & kyūseigyo " poisson à neuf étoiles » & 九星魚 & Fascicule 4 \\
\hline
\end{tabular}




\begin{tabular}{|c|c|c|c|}
\hline laminaire & hirome ; konbu ; kaifu & $\begin{array}{l}\text { ヒロメ; 昆布; } \\
\text { 海布 }\end{array}$ & \multirow{2}{*}{ Fascicule 5} \\
\hline laminaire rejeté & yosekonbu & 寄せ昆布 & \\
\hline lamproie & yatsume-unagi & 八目鰻 & Fascicule 4 \\
\hline lance & yari & 槍 & Fascicule 2 \\
\hline langouste & $e b i$ & 海鰕 & Fascicule 3 \\
\hline langouste d'Ise & Ise'ebi & 伊勢海鰕 & Fascicule 3 \\
\hline langue de mer & kaizetsu & 海舌 & Fascicule 5 \\
\hline lest & $i w a$ & イワ & Fascicule 4 \\
\hline levain & moto & 院 & Fascicule 1 \\
\hline lévigation & suihi & 水飛 & Fascicule 2 \\
\hline lévigation & suihi & 水干 & Fascicule 5 \\
\hline levure & $k \overline{o j i}$ & 麦米 & Fascicule 5 \\
\hline liane de glycine & fujikazura & 藤かずら & Fascicule 2 \\
\hline liane & tsurukusa & 蔓草 & Fascicule 2 \\
\hline ligne de traîne & haenawa & 延縄 & Fascicule 3 \\
\hline limande & karei & 鰈 & Fascicule 3 \\
\hline $\operatorname{lin}$ & asa & 麻 & Fascicule 2 \\
\hline \multirow[t]{2}{*}{ liquide épais fermenté } & moromi & もろみ; 諸味 & Fascicule 1 \\
\hline & lizangtu & 李蔵㻌 & $\begin{array}{l}\text { Fascicules 3, } \\
4\end{array}$ \\
\hline loche des sables & sunadojō & 砂鯂 & Fascicule 4 \\
\hline macérer & hitasu & 浸す & Fascicule 5 \\
\hline maille & me & 目 & \multirow{2}{*}{ Fascicule 3} \\
\hline maille serrée & hosomono & 細物 & \\
\hline \multirow[t]{3}{*}{ maillet } & tsuchi & 槌 & Fascicule 5 \\
\hline & magamo (canard) & 真鳥 & Fascicule 2 \\
\hline & maitake (champignon) & 舞蕈 & Fascicule 2 \\
\hline
\end{tabular}




\begin{tabular}{|c|c|c|c|}
\hline & maki, voir pieu en bois & & Fascicule 5 \\
\hline & makusotsukami (autour) & まくそつかみ & Fascicule 2 \\
\hline & mannen take (champignon) & 万年たけ & Fascicule 2 \\
\hline & mao, voir ramie & & Fascicule 5 \\
\hline maquereau & $s a b a$ & 鯖 & \multirow{2}{*}{ Fascicule 3} \\
\hline - tacheté du Japon & sawara & 鰆 & \\
\hline marbre blanc & kansuiseki & 寒水石 & Fascicule 2 \\
\hline marbrée & shimasuji & 島筋 & Fascicule 5 \\
\hline & maruhachi & マルハチ & Fascicule 2 \\
\hline & masu & 升 & Fascicule 5 \\
\hline & matsu non koke & 松のこけ & Fascicule 2 \\
\hline & matsutake (champignon) & 松罩 & Fascicule 2 \\
\hline méduse & kurage & 水母 & \multirow{4}{*}{ Fascicule 5} \\
\hline - d'eau & mizukurage & 水水母 & \\
\hline - de Chine & tōkurage & 唐水母 & \\
\hline - de Corée & chōsen kurage & 朝鮮水母 & \\
\hline menuisiers & shōkō & 小工 & Fascicule 2 \\
\hline merveille sans nom & mumyōi & 無名異 & Fascicule 5 \\
\hline méthode dite « des chocs» & uchitsukewari & 打附割 & Fascicule 2 \\
\hline méthode par « levée » & sukuiwari & すくい割 & Fascicule 2 \\
\hline miel & hachimitsu; mitsu & 蜂蜜; 蜜 & \multirow[t]{7}{*}{ Fascicule 2} \\
\hline - de montagne & yamamitsu & 山蜜 & \\
\hline - d'arbre & kimitsu & 木蜜 & \\
\hline - de roche & sekimitsu & 石蜜 & \\
\hline - domestique & kamitsu & 家蜜 & \\
\hline - d'écoulage & taremitsu & たれ蜜 & \\
\hline$-\mathrm{cru}$ & shōmitsu & 生蜜 & \\
\hline
\end{tabular}




\begin{tabular}{|c|c|c|c|}
\hline - de pressage & shibori & 絞り & \\
\hline - cuit & jukumitsu & 熟蜜 & \\
\hline \multirow[t]{3}{*}{ - de pression } & jukumitsu & 熟蜜 & \\
\hline & mikkan, voir abeille & & Fascicule 2 \\
\hline & mikoai & ミコアイ & Fascicule 2 \\
\hline \multirow[t]{4}{*}{ miso } & miso & 味噌 & Fascicule 4 \\
\hline & mizutabi & 水足袋 & Fascicule 2 \\
\hline & mizutake (champignon) & 水たけ & Fascicule 2 \\
\hline & mochi (boulettes) & 餅 & Fascicule 2 \\
\hline modeler & tsukuru & 塑 & Fascicule 5 \\
\hline moisissure de riz & moyashi & 蓝 & Fascicule 1 \\
\hline \multirow[t]{3}{*}{ montagne réservée } & otomeyama & 御留山 & Fascicule 2 \\
\hline & morohaku & 諸白 & Fascicule 1 \\
\hline & morokaeri (autour) & 諸かえり & Fascicule 2 \\
\hline mortier & karausu & 碓 & \multirow{2}{*}{ Fascicule 5} \\
\hline - chinois hydraulique & so'uzukarausu & 添水碓 & \\
\hline morue & tara & 鱈 & Fascicule 5 \\
\hline motifs à rayures à l'ikat & kasurijima & かすり島 & Fascicule 5 \\
\hline moule & igai & 貽貝 & Fascicule 3 \\
\hline moule & kata & 範; 印 & Fascicule 5 \\
\hline moucheture & madara & 斑 & Fascicule 2 \\
\hline \multirow[t]{4}{*}{$\operatorname{moxa}$} & mogusa & 艾綿 & Fascicule 2 \\
\hline & mozu & 鵙 & Fascicule 4 \\
\hline & muchi & 鞭 & Fascicule 2 \\
\hline & muko « poisson-gendre » & ムコ & Fascicule 4 \\
\hline mulet & bora & 鰡 & Fascicule 4 \\
\hline mur d'enceinte & tsuigaki & 築垣 & Fascicule 2 \\
\hline
\end{tabular}




\begin{tabular}{|c|c|c|c|}
\hline mur de jardin & teireki & 庭彻 & Fascicule 2 \\
\hline \multirow[t]{2}{*}{ mur de résidence } & shikii no do'i & 敷居の土居 & Fascicule 2 \\
\hline & murage (autour) & 村毛 & Fascicule 2 \\
\hline \multirow[t]{12}{*}{ murène (japonaise) } & rei, aussi yatsume-unagi & 鱧, aussi八目鰻 & Fascicule 4 \\
\hline & mushikuide & 虫喰出 & Fascicule 5 \\
\hline & mushikuito (pierre) & 虫喰砥 & Fascicule 2 \\
\hline & musō gaeshi & 無双がえし & Fascicule 2 \\
\hline & $n a b a$ & なば & Fascicule 2 \\
\hline & nagatana (à un tranchant) & 菜刀 & Fascicule 2 \\
\hline & nakato (pierre) & 中砥 & Fascicule 2 \\
\hline & namafu & 生麥䢊 & Fascicule 2 \\
\hline & namako & 生海鼠 & Fascicule 4 \\
\hline & nametake (champignon) & 滑草 & Fascicule 2 \\
\hline & namima kashiwa (coquillage) & 波間かしわ & Fascicule 3 \\
\hline & nanba & なんば & Fascicule 2 \\
\hline nasses & eri & エリ & Fascicule 3 \\
\hline \multirow[t]{2}{*}{ nattes de paille } & mushiro & 筵 & Fascicule 3 \\
\hline & nawatojifune & 縄綴船 & Fascicule 5 \\
\hline nid (d'abeille) & su & 脾 & \multirow{3}{*}{ Fascicule 2} \\
\hline séparation des - & suwakare & 領脾 & \\
\hline \multirow[t]{7}{*}{ découpage de - } & su o kiru & 割脾 & \\
\hline & nishikide, voir style & & Fascicule 5 \\
\hline & nitori & 煮取 & Fascicule 4 \\
\hline & nobe & 延條 & Fascicule 2 \\
\hline & nomi, voir ciseau & & Fascicule 5 \\
\hline & noshi & 長鮑 & Fascicule 3 \\
\hline & norige (autour) & のり毛 & Fascicule 2 \\
\hline
\end{tabular}




\begin{tabular}{|c|c|c|c|}
\hline & nori-kawaami & 乗川網 & Fascicule 4 \\
\hline & nosaretaka (autour) & 野嚗鷹 & Fascicule 2 \\
\hline & nukuchi & 暖血 & Fascicule 2 \\
\hline & nunobikidake (champignon) & 布引罩 & Fascicule 2 \\
\hline & ōbaku & 黄柏 & Fascicule 2 \\
\hline œufs de hareng & kazunoko & 数の子 & Fascicule 5 \\
\hline \multirow[t]{3}{*}{$\begin{array}{l}\text { office des magasins de la } \\
\text { cour }\end{array}$} & kuranoryō & 内蔵寮 & Fascicule 5 \\
\hline & ogamo & 小ガモ & Fascicule 2 \\
\hline & ogitake (champignon) & 蒦菌 & Fascicule 2 \\
\hline \multirow[t]{5}{*}{ oie sauvage } & gan & 鴈 & Fascicule 2 \\
\hline & okitsu ikuri & 奥津いくり & Fascicule 2 \\
\hline & (kawa-)okoze « okoze de rivière » & 川オコゼ & Fascicule 4 \\
\hline & omi & 麻績 & Fascicule 5 \\
\hline & onaga (canard) & 尾長 & Fascicule 2 \\
\hline oreille de Judas & kikurage & 木耳 & Fascicule 4 \\
\hline orme & nire & 榆 & Fascicule 2 \\
\hline ormeau & awabi, tokobushi & 鰒,鰒 & \multirow{7}{*}{ Fascicule 3} \\
\hline - battu & uchi awabi & 打あはび & \\
\hline ruban d'- & noshi & 長鮑 & \\
\hline - séché & hoshi awabi & 干鮑 & \\
\hline - émincé & usu awabi & 薄鮑 & \\
\hline \multirow[t]{4}{*}{ perle d'- } & awabi-tama & 鰒玉 & \\
\hline & akoyatama & あこや玉 & \\
\hline & osaki & 尾尖 & Fascicule 2 \\
\hline & ōtaka (autour) & 角鷹 & Fascicule 2 \\
\hline otarie & ottotsu; ottotsujū & 膃肭; 膃肭獣 & Fascicule 5 \\
\hline
\end{tabular}




\begin{tabular}{|c|c|c|c|}
\hline & otometaka (autour) & 乙女鷹 & Fascicule 2 \\
\hline ours & kuma & 熊 & $\begin{array}{l}\text { Fascicules 2, } \\
5\end{array}$ \\
\hline oursin & $\begin{array}{l}\text { uni «bile de mer»; (autre nom) } \\
\text { reiruishi }\end{array}$ & $\begin{array}{l}\text { 海膽； } \\
\text { 靈贏子 }\end{array}$ & Fascicule 4 \\
\hline $\begin{array}{l}\text { ouvrier chargé du } \\
\text { blanchiment }\end{array}$ & sarashiya & 漂工屋 & Fascicule 5 \\
\hline & ōyōdo (abeille) & 王腰奴 & Fascicule 2 \\
\hline palissade & ajiroki & 網代木 & Fascicule 4 \\
\hline palourde & hamaguri & 蛤 & $\begin{array}{l}\text { Fascicules } 3, \\
4,5\end{array}$ \\
\hline - grillée & yaki-hamaguri & 暁蛤 & \multirow{2}{*}{ Fascicule 4} \\
\hline — « averse d'automne» & shigure-hamaguri & 時雨蛤 & \\
\hline papier de Mino & Minogami & 美濃紙 & Fascicule 2 \\
\hline $\begin{array}{l}\text { parfum de neige } \\
\text { (champignon) }\end{array}$ & yukiko & 雪香 & Fascicule 2 \\
\hline $\begin{array}{l}\text { parfum du printemps } \\
\text { (champignon) }\end{array}$ & haruko & 春香 & Fascicule 2 \\
\hline " passage des dieux» & kami-no-miwatari & 神の御渡 & Fascicule 4 \\
\hline passer au tamis & kosu & 漉す & Fascicule 5 \\
\hline passereau & hiyodori & 鷶 & Fascicule 2 \\
\hline paulownia & kiri & 桐 & Fascicule 5 \\
\hline $\begin{array}{l}\text { Pavillon de la Pureté et de } \\
\text { la Fraîcheur }\end{array}$ & Seiryōden & 清凉殿 & Fascicule 5 \\
\hline pêche à la ligne & tsuri & 釣り & Fascicule 3 \\
\hline pelle à mélanger & $k a i$ & 械 & Fascicule 1 \\
\hline pénis d'otarie & takeri; hozo & 陰茎; 臍 & Fascicule 5 \\
\hline penne & oha & 尾羽 & Fascicule 2 \\
\hline perle & shinju; tama & 真珠,玉 & \multirow{2}{*}{ Fascicule 3} \\
\hline - d'ormeau & awabi-tama, akoyatama & 鰒玉 ; あこや玉 & \\
\hline
\end{tabular}




\begin{tabular}{|c|c|c|c|}
\hline perche amie & tomosao & 友竿 & Fascicule 3 \\
\hline perchoir & hoko & 架 & Fascicule 2 \\
\hline pétrir & koneru & 粉る; 埏る & Fascicule 5 \\
\hline pie-grièche (bucéphale) & mozu & 鵙 & Fascicule 4 \\
\hline pièce [pour une étoffe] & $\tan$ & 端 & Fascicule 5 \\
\hline pied & shaku & 尺 & Fascicule 5 \\
\hline piège & otoshi/oshi & 落し/機 & \multirow{3}{*}{ Fascicule 2} \\
\hline - écrasant & oshi/oso & オソ/オシ & \\
\hline - -plafond & tenjōtsuri & 天井釣 & \\
\hline pierre & ishi & 石 & \multirow{10}{*}{ Fascicule 2} \\
\hline - à affûter & to'ishi & 砥嘱 & \\
\hline — d'affûtage concassée & uwahiki & 上引 & \\
\hline - d'aimant ; & jishaku & 慈石 & \\
\hline - dressée yang & yōkiseki & 陽起石 & \\
\hline - en feuille d'arbre & konoha-ishi & 木の葉石 & \\
\hline - ponce & karu-ishi & 浮石 & \\
\hline- rouge & $a k a$ & 赤 & \\
\hline - décoratives & sute-ishi & 捨石 & \\
\hline - unique & hitotsu ishi & 一ツ石 & \\
\hline - verte & aoishi & 青石 & \multirow{2}{*}{ Fascicule 5} \\
\hline - à chaux & ishibai & 石灰 & \\
\hline pieu en bois & maki, shinki & 真木 & Fascicule 5 \\
\hline pieuvre & tako & 章魚 ; 鮹 & \multirow[t]{5}{*}{ Fascicule 4} \\
\hline (autre nom) & kai'oshō « bonze de la mer » & 海和尚 & \\
\hline œufs de - & kaitōka « Glycines de mer» & 海藤花 & \\
\hline œufs de - & heriko & ヘり子 & \\
\hline (autre nom) & rakutei « pieds qui se tortillent» & 絡蹄 & \\
\hline
\end{tabular}




\begin{tabular}{|c|c|c|c|}
\hline (autre nom) & jūya-dako « pieds qui se tortillent » & 十夜蛸 & \\
\hline (- de grande taille) & sekidako & セキ蛸 & \\
\hline (autre nom) & shōhasshōgyo & 小八梢魚 & \\
\hline (autre nom) & hasshōgyo « poisson à huit rameaux » & 八梢魚 & \\
\hline (pot à -) & takotsubo & 蛸劽 & \\
\hline pilon métallique & kanakine & 金杵 & Fascicule 5 \\
\hline plan incliné & nagare & 流れ & Fascicule 5 \\
\hline planchette & buri & フリ & Fascicule 3 \\
\hline plomb (de filet) & iwa & 重石 & Fascicule 3 \\
\hline pluvier & chidori & 千鳥 & Fascicule 4 \\
\hline poche & fukuro & 袋 & Fascicule 3 \\
\hline poinçon de teinturier & katahori & 形周, & Fascicule 2 \\
\hline poisson annuel & nengyo & 年魚 & Fascicule 4 \\
\hline poisson chat & namazu & 鯄 & Fascicule 2 \\
\hline poisson de glace & hio ou hi'uo & 水魚 & Fascicule 4 \\
\hline poisson de roche tacheté & sekihangyo & 石班魚 & \multirow{3}{*}{ Fascicule 4} \\
\hline (autre nom) poisson rouge & aka'uo & 赤魚 & \\
\hline $\begin{array}{l}\text { (autre nom) ventres } \\
\text { rouges }\end{array}$ & akahara & 赤腹 & \\
\hline poisson-hôte & maru & マル & Fascicule 4 \\
\hline poisson poivré & sanshō-uo & 山椒魚 & Fascicule 2 \\
\hline poisson-crocodile & kochiuo & 牛尾魚 & Fascicule 2 \\
\hline poisson miko & miko'uo & 峄こ魚 & \multirow{2}{*}{ Fascicule 4} \\
\hline (nom chinois de -) & ōshigyo & 鮚綵魚 & \\
\hline poivre (du Japon) & sanshō & 山椒 & $\begin{array}{l}\text { Fascicules 2, } \\
4\end{array}$ \\
\hline polissage fin & voir awasu & & Fascicule 2 \\
\hline ponts en pierre & ishibashi & 石橋 & Fascicule 2 \\
\hline
\end{tabular}




\begin{tabular}{|c|c|c|c|}
\hline pot à pieuvre & takotsubo & 蛸壼 & Fascicule 4 \\
\hline pouce & sun & 寸 & Fascicule 5 \\
\hline poudre de cendre & sankai & 散灰 & Fascicule 5 \\
\hline poudre lavée & mizukona & 水粉 & Fascicule 2 \\
\hline poule des bois & maitake (champignon) & マイタケ & Fascicule 2 \\
\hline poulpe ocellé & iidako & 飯蛸 & \multirow{2}{*}{ Fascicule 4} \\
\hline (nom chinois) & bōchōgyo & 望潮魚 & \\
\hline pousse de bambou & takenoko & 笋 & Fascicule 2 \\
\hline poutargue & karasumi & カラスミ & Fascicule 3 \\
\hline praire & asari & あさり & Fascicule 3 \\
\hline presser & momu & 揉む & Fascicule 5 \\
\hline pressoir (à saké) & fune & 醣 & Fascicule 1 \\
\hline puéraire & kuzu & 葛 & \multirow{3}{*}{ Fascicule 2} \\
\hline racine de - & kuzune & 葛根 & \\
\hline poudre de - & kuzuko & 葛粉 & \\
\hline pulvériser & kudakeru & 砕ける & Fascicule 5 \\
\hline ramie & $o ; m a o$ & 苧; 苧麻 & $\begin{array}{l}\text { Fascicules 2, } \\
5\end{array}$ \\
\hline $\begin{array}{l}\text { rayures irrégulières ou } \\
\text { fantaisie }\end{array}$ & kawarijima & かわり島 & Fascicule 5 \\
\hline $\begin{array}{l}\text { récipients obtenus en } \\
\text { pressant un moule }\end{array}$ & kataoshi & 印器 & Fascicule 5 \\
\hline \multirow[t]{3}{*}{ récipients ronds } & enki & 円器 & Fascicule 5 \\
\hline & reiruishi & 靈贏子 & Fascicule 4 \\
\hline & reishi (champignon) & 霊芝 & Fascicule 2 \\
\hline repaire (de l'ours) & suana & 单穴 & Fascicule 2 \\
\hline résine d'arbre médicinal & yakubokukō & 薬木膠 & Fascicule 5 \\
\hline rideau & kaishiro & 坦代 & Fascicule 5 \\
\hline
\end{tabular}




\begin{tabular}{|c|c|c|c|}
\hline rigole & mizo & 溝渠 & Fascicule 2 \\
\hline $\begin{array}{l}\text { rite de la salutation des } \\
\text { quatre directions }\end{array}$ & shihōhai & 四方拝 & Fascicule 5 \\
\hline riz ancien & komai & 古米 & \multirow{3}{*}{ Fascicule 1} \\
\hline - blanchi & hakumai & 白米 & \\
\hline - poli & seimai & 精米 & \\
\hline \multirow[t]{3}{*}{ rocher décoratif } & sute'ishi & 捨石 & Fascicule 2 \\
\hline & rokunin & 六人 & Fascicule 2 \\
\hline & Rokurō dayū (puéraire) & 六郎太夫 & Fascicule 2 \\
\hline $\begin{array}{l}\text { rouet actionné par une } \\
\text { pédale }\end{array}$ & ashikuruma & & Fascicule 5 \\
\hline rouge-gorge & komadori & こま鳥 & Fascicule 4 \\
\hline \multirow[t]{5}{*}{ sac tressé } & kamasu & 蒲簀 & Fascicule 3 \\
\hline & saiso & 菜蔬 & Fascicule 2 \\
\hline & saiwatake (champignon) & 芝菌 & Fascicule 2 \\
\hline & $\begin{array}{l}\text { saiwatake à ramure de daim } \\
\text { rokkakushi }\end{array}$ & 鹿角芝 & \\
\hline & sakaami (filet) & 坂網 & Fascicule 2 \\
\hline saké blanc & shiro-ki & 白酒 & \multirow{10}{*}{ Fascicule 1} \\
\hline - clair & seishu & 清酒 & \\
\hline - d'avant les froids & kanmae-zake & 寒前酒 & \\
\hline - des froids & kanshu & 寒酒 & \\
\hline- fait & seijuku & 成熟した酒 & \\
\hline - d'entre-deux & aishu & 間酒 & \\
\hline - nouveau & shinshu & 新酒 & \\
\hline - noir & kuro-ki & 黒酒 & \\
\hline - supérieur & jōshu & 上酒 & \\
\hline - parfaitement clair & morohaku & 諸白 & \\
\hline
\end{tabular}




\begin{tabular}{|c|c|c|c|}
\hline - trouble & dakushu & 濁酒 & \\
\hline & sakutake (champignon) & さくたけ & Fascicule 2 \\
\hline salamandre & sanshō'io/uo & 䲅；山椒魚 & $\begin{array}{l}\text { Fascicules 2, } \\
4\end{array}$ \\
\hline \multirow[t]{4}{*}{ salpêtre } & shôseki & 硝石 & Fascicule 2 \\
\hline & sanobori & サノボリ & Fascicule 4 \\
\hline & sanshishi & 山桅子 & Fascicule 2 \\
\hline & saohime kaeri (autour) & 佐保姫 & Fascicule 2 \\
\hline sardine & iwashi & 鮙 & \multirow{3}{*}{$\begin{array}{l}\text { Fascicules 3, } \\
4\end{array}$} \\
\hline alevin de - & iwashi no ko & 鮙の苗 & \\
\hline (autre nom de l'alevin) & isaza & 鶖毛挺 & \\
\hline \multirow[t]{4}{*}{ sarrasin } & soba & 䓂麦 & $\begin{array}{l}\text { Fascicules 2, } \\
4\end{array}$ \\
\hline & saru no koshikake (champignon) & 胡孫眼 & Fascicule 2 \\
\hline & sashiba & 䲴鳩 & Fascicule 2 \\
\hline & satoochitaka (autour) & 里落鷹 & Fascicule 2 \\
\hline sauce de soja & shōyu & 㽜油 & Fascicule 4 \\
\hline saule & yanagi & 柳 & Fascicule 2 \\
\hline saumon & sake & 鮭 & \multirow{2}{*}{ Fascicule 4} \\
\hline \multirow[t]{3}{*}{ œufs de - } & hararago & ハララゴ & \\
\hline & sawara & 鰆 & Fascicule 3 \\
\hline & sazae & 栄螺 & Fascicule 3 \\
\hline sécher à l'ombre & kageboshi & 陰乾 & Fascicule 5 \\
\hline seiche & $i k a$ & 烏賊 & $\begin{array}{l}\text { Fascicules 3, } \\
4\end{array}$ \\
\hline - en rubans & ika noshi & 烏賊のし & Fascicule 3 \\
\hline \multirow[t]{2}{*}{$\begin{array}{l}\text { «seigneur aux yeux } \\
\text { d'emprunt" }\end{array}$} & shakugankō & 借眼公 & Fascicule 5 \\
\hline & seihekishoku & 青碧色 & Fascicule 5 \\
\hline
\end{tabular}




\begin{tabular}{|c|c|c|c|}
\hline & seijimono, voir grès à couverte verte & & Fascicule 5 \\
\hline & sekidako & セキ蛸 & Fascicule 4 \\
\hline & sekishi (champignon) & 石芝 & Fascicule 2 \\
\hline & sekka & 石瘕 & Fascicule 2 \\
\hline semis à la volée & mine & 撒子 & Fascicule 5 \\
\hline semis en poquet & wake'ue & 分頭 & Fascicule 5 \\
\hline & sen (champignon) & & Fascicule 2 \\
\hline senne & fukuro-ami & 袋網 & Fascicule 4 \\
\hline sériole & buri & 鯔 & Fascicule 3 \\
\hline \multirow[t]{6}{*}{ serpent (grand) } & uwabami & 蟒 & Fascicule 4 \\
\hline & shasan & 沙蒜 & Fascicule 4 \\
\hline & shibaoshi & シバオシ & Fascicule 2 \\
\hline & shichiseigyo « Poisson à sept étoiles » & 七星魚 & Fascicule 4 \\
\hline & shichōtake (champignon) & 仕丁たけ & Fascicule 2 \\
\hline & shii (arbre) & 椎 & Fascicule 2 \\
\hline \multirow[t]{10}{*}{ shiitake } & shiitake (champignon) & 香罩 & Fascicule 2 \\
\hline & shikiden (nom d'un filet) & シキデン & Fascicule 2 \\
\hline & shimafuku (canard) & 島フク & Fascicule 2 \\
\hline & shimeji (champignon) & 玉菒 & Fascicule 2 \\
\hline & shina & シナ & Fascicule 5 \\
\hline & shinano-nuno & 信濃布 & Fascicule 5 \\
\hline & shiokara & 塩辛 & Fascicule 4 \\
\hline & shirataka (autour) & 白鷹 & Fascicule 2 \\
\hline & shiratsuchi, voir terre blanche & & Fascicule 5 \\
\hline & shiro (nom pour l'ours) & 子路 & Fascicule 2 \\
\hline
\end{tabular}




\begin{tabular}{|c|c|c|c|}
\hline & shiroiyo (pierre) & 白伊予 & Fascicule 2 \\
\hline & shiromizu & 米泔 & Fascicule 2 \\
\hline & shiro'uo & 䞣面佟魚 & Fascicule 4 \\
\hline & shizuri & 倭文 & Fascicule 5 \\
\hline & shitasashiba (buse) & 下さしば & Fascicule 2 \\
\hline & shōgyo « poisson de pin» & 松魚 & Fascicule 4 \\
\hline & shojin (champignon) & 處䓥 & Fascicule 2 \\
\hline & shōhasshōgyo & 小八梢魚 & Fascicule 4 \\
\hline & shutō & 酒盗 & Fascicule 4 \\
\hline siège en terre & doseki & 土席 & Fascicule 5 \\
\hline silice voir bidoro & & & Fascicule 5 \\
\hline sirène & ningyo & 人魚 & Fascicule 2 \\
\hline site de production & jōba & 上場 & Fascicule 5 \\
\hline soie & kinu & 絹 & \multirow{2}{*}{ Fascicule 5} \\
\hline- fine & usumono & 羅 & \\
\hline \multirow[t]{2}{*}{ soja (fèves de) } & daizu & 大豆 & Fascicule 4 \\
\hline & sokosashiba (buse) & 底さしば & Fascicule 2 \\
\hline sole & hirame & ヒラメ & Fascicule 3 \\
\hline \multirow[t]{2}{*}{ somites } & fukurin & 幅輪 & Fascicule 3 \\
\hline & sōmakuri & 䊍まくり & Fascicule 2 \\
\hline sophoras du Japon & enji & 槐 & Fascicule 2 \\
\hline stalactites & shōnyūseki & 鐘乳石 & Fascicule 2 \\
\hline stéatite & katsuseki & 滑石 & Fascicule 2 \\
\hline \multirow[t]{4}{*}{ style brocart } & nishikide & 錦様 & Fascicule 5 \\
\hline & sudaka & 巣鷹 & Fascicule 2 \\
\hline & sugitake (champignon) & 杉菌 & Fascicule 2 \\
\hline & suichō & スイチョウ & Fascicule 4 \\
\hline
\end{tabular}




\begin{tabular}{|c|c|c|c|}
\hline & suihi, voir lévigation & & Fascicule 5 \\
\hline & suika, voir transformation & & Fascicule 5 \\
\hline & suiton (boulettes en potage) & 水湎 & Fascicule 2 \\
\hline & sukuri-bune & スクリ舩 & Fascicule 4 \\
\hline & sumawari (autour) & 巣廻 & Fascicule 2 \\
\hline & sutaka (autour) & 巣鷹 & Fascicule 2 \\
\hline & tai & 鯛 & Fascicule 3 \\
\hline \multirow[t]{5}{*}{ tailleur } & sekku & 石工 & Fascicule 2 \\
\hline & taka-kai & 養鷹 & Fascicule 2 \\
\hline & takae & タカヘ & Fascicule 2 \\
\hline & takanawa & 高縄 & Fascicule 2 \\
\hline & takeri, voir pénis d'otarie & & Fascicule 5 \\
\hline \multirow[t]{2}{*}{ takeri de boyau } & hyakuhiro takeri & 百ヒロタケリ & Fascicule 5 \\
\hline & tako & 章魚 & Fascicule 4 \\
\hline tamis & furui ou ikaki & 篩; 飯籮 & Fascicule 5 \\
\hline \multirow[t]{3}{*}{ tanière } & hora & 洞 & Fascicule 2 \\
\hline & tanikugu, ou taniguku & 谷潜 & Fascicule 4 \\
\hline & tara & 鱈 & Fascicule 5 \\
\hline teillage & hagu & 剥ぐ ; 破折 & Fascicule 5 \\
\hline teillage bouilli & nigoki & 煮ゴキ & Fascicule 5 \\
\hline terre blanche & shiratsuchi & 亜土 ; 白亜 & Fascicule 5 \\
\hline terre cuite & kawarake & 土器 & Fascicule 5 \\
\hline thon & shibi; maguro & 鮁;まぐろ & $\begin{array}{l}\text { Fascicules 3, } \\
4\end{array}$ \\
\hline- roi & $\bar{o}^{\prime} i$ & 王鮁 & \multirow{3}{*}{ Fascicule 3} \\
\hline - cadet & shuku'i & 叔鮁 & \\
\hline - enfant & mejika & 鮥子 & \\
\hline
\end{tabular}




\begin{tabular}{|c|c|c|c|}
\hline- noir & kuroshibi & 黒鮁 & \\
\hline \multirow[t]{10}{*}{ tisser } & oru & 織る & Fascicule 2 \\
\hline & tiyu (jap. teigyo) & 䱱魚 & Fascicule 2 \\
\hline & to & 斗 & Fascicule 5 \\
\hline & tobinagashi & 飛流 & Fascicule 2 \\
\hline & todo & 海驢 & Fascicule 5 \\
\hline & tofugyo & 杜夫魚; 杜父魚 & \multirow{5}{*}{ Fascicule 4} \\
\hline & (autre nom) ishimochi & イシモチ & \\
\hline & (autre nom) kuchinawadonko & クチナワドンコ & \\
\hline & (autre nom) maru & マル & \\
\hline & (autre nom) muko & ムコ & \\
\hline \multirow[t]{2}{*}{ toise } & $j \bar{o}$ & 丈 & Fascicule 5 \\
\hline & tōnaga (canard) & トウ長 & Fascicule 2 \\
\hline $\begin{array}{l}\text { transformation sous l'effet } \\
\text { de l'air }\end{array}$ & suika & 吹化 & Fascicule 5 \\
\hline travail à la main & temoto & 手元 & Fascicule 1 \\
\hline travée & ken & 間 & Fascicule 5 \\
\hline treuil & rokuro & 轆轤 & Fascicule 3 \\
\hline triton & imori & 水蜊蜴 & Fascicule 2 \\
\hline trône & utena & 台 & Fascicule 2 \\
\hline trubles & tamaami & たま網 & Fascicule 3 \\
\hline truite & masu & 鱒 & $\begin{array}{l}\text { Fascicules 4, } \\
5\end{array}$ \\
\hline ancien nom de - & haraaka « ventre rouge» & 腹赤 & \\
\hline (autre nom) & oikawa & オイカワ & \\
\hline truite ayu & ayu & 鮎 & \\
\hline alevin de - & ayu no ko & 鮎の苗 & Fascicule 4 \\
\hline (autre nom) & nengyo & 年魚 & \\
\hline
\end{tabular}




\begin{tabular}{|c|c|c|c|}
\hline & tsubanage (autour) & つばな毛 & Fascicule 2 \\
\hline & tsubo & 坪 & Fascicule 5 \\
\hline & tsukune & 塑 & Fascicule 5 \\
\hline & tsumi & 躝鷂 ou 鵰 & Fascicule 2 \\
\hline & tsuzura & 葛籠 & Fascicule 2 \\
\hline tue-verrues & ibo-otoshi & イボおとし & Fascicule 2 \\
\hline \multirow[t]{6}{*}{ turbo cornu } & sazae & 栄螺 & Fascicule 3 \\
\hline & uchi'oroshi (autour) & 打おろし & Fascicule 2 \\
\hline & uchigumori (pierre) & 内量 & Fascicule 2 \\
\hline & umu & 績む & Fascicule 5 \\
\hline & ushinokawatake (champignon) & 地耳 & Fascicule 2 \\
\hline & usumono, voir soie & & Fascicule 5 \\
\hline \multirow[t]{3}{*}{ ustensiles } & kibutsu & 器物 & Fascicule 2 \\
\hline & usuba (à deux tranchants) & 薄刃 & Fascicule 2 \\
\hline & uwabiki, uwahiki & 上わ引 ;上引 & Fascicule 2 \\
\hline vandoise (« japonaise ») & haraaka, haraka & 腹赤 & Fascicule 4 \\
\hline vasque & hachi & 鉢 & \multirow{2}{*}{ Fascicule 2} \\
\hline - à ablution & chōzubachi & 手水鉢 & \\
\hline ver de terre & mimizu & 虾蚓 & Fascicule 4 \\
\hline verre & bidoro & 硝子 & Fascicule 5 \\
\hline vigne sauvage & nobudō & 野葡萄 & Fascicule 2 \\
\hline $\begin{array}{l}\text { vin exquis parfumé au } \\
\text { curcuma }\end{array}$ & ukkinkō & 㯊金香 & Fascicule 2 \\
\hline vinaigre de riz & $s u$ & 酢 & Fascicule 1 \\
\hline voilé [aspect] & fūmu & 風霧 & Fascicule 5 \\
\hline & wakataka (autour) & 黄鷹 & Fascicule 2 \\
\hline & warabi & 薕根 & Fascicule 2 \\
\hline
\end{tabular}




\begin{tabular}{|c|c|c|c|}
\hline & washi & 熟 & Fascicule 2 \\
\hline & watage (autour) & 綿毛 & Fascicule 2 \\
\hline & $\begin{array}{l}\text { yakubokukō, voir résine d'arbre } \\
\text { médicinal }\end{array}$ & & Fascicule 5 \\
\hline & yamadonko & 山どんこ & Fascicule 4 \\
\hline & yamasare (autour) & 山曝 & Fascicule 2 \\
\hline & $\begin{array}{l}\text { yatsume-unagi «anguille à huit } \\
\text { yeux» }\end{array}$ & 八目鰻 & Fascicule 4 \\
\hline & yoshifuku (canard) & ヨシフク & Fascicule 2 \\
\hline & yotaka (autour) & 惰鳥 & Fascicule 2 \\
\hline & yushinoki & 蚊子木 & Fascicule 5 \\
\hline & yuzu & 柚 & Fascicule 2 \\
\hline zizanie & makomo & 真菰 & Fascicule 2 \\
\hline & zunashi & 頭無 & Fascicule 2 \\
\hline
\end{tabular}




\section{Tableau des toponymes}

\begin{tabular}{|c|c|c|}
\hline Aburata & 油田 & Fascicule 2 \\
\hline Aizu & 会津 & Fascicule 2 \\
\hline Akaemachi & 赤絵町 & Fascicule 5 \\
\hline Akamagaseki & 赤間関 & Fascicule 4 \\
\hline Akashi & 明石 & Fascicule 4 \\
\hline Akashi & 赤石 & Fascicule 3 \\
\hline Akasuga & 赤すが & Fascicule 4 \\
\hline Aki & 安芸 & Fascicules 2, 3 \\
\hline Akoya & あこや & Fascicule 3 \\
\hline Amakusa (pierre) & 天草 & Fascicule 2 \\
\hline Amano-hashidate & 天の橋立 & Fascicule 3 \\
\hline Aokaya (pierre) & 青茅 & Fascicule 2 \\
\hline Aomiko (pierre) & 青神子 & Fascicule 2 \\
\hline Arima & 有馬 & Fascicules 2, 4 \\
\hline Asanogawa & 浅野川 & Fascicule 4 \\
\hline Ashinoyama (pierre) & 芦野山 & Fascicule 2 \\
\hline Atago & 愛宕 & Fascicule 1 \\
\hline Awa & 阿波 & Fascicules 2, 3, 4 \\
\hline
\end{tabular}




\begin{tabular}{|c|c|c|}
\hline Awa & 安房 & Fascicule 4 \\
\hline Awaji & 淡路 & Fascicules 1,3 \\
\hline Bingo & 備後 & Fascicule 2 \\
\hline Biwa-ko (lac) & 琵琶湖 & Fascicule 4 \\
\hline Bungo & 豊後 & Fascicules $1,3,4$ \\
\hline Bizen & 備前 & Fascicule 5 \\
\hline Buzen & 豊前 & Fascicule 4 \\
\hline Chamiko (pierre) & 茶神子 & Fascicule 2 \\
\hline Chikuma & 千曲 & Fascicule 5 \\
\hline Chikuzen & 筑前 & Fascicules 2, 3, 4 \\
\hline Chinu & 茅涥 & Fascicule 5 \\
\hline Chita & 知多 & Fascicule 3 \\
\hline Chōshū & 長州 & Fascicule 4 \\
\hline Chūgoku & 中国 & Fascicule 2 \\
\hline Corée Chōsen & 朝鮮 & Fascicule 2 \\
\hline Daikuchi (pierre) & 台口 & Fascicule 2 \\
\hline Dejima & 出島 & Fascicule 5 \\
\hline Dewa & 出羽 & Fascicule 2 \\
\hline Ebishi & エヒシ & Fascicule 3 \\
\hline Echigo & 越後 & Fascicules 2, 4, 5 \\
\hline Echizen (pierre) & 越前 & Fascicules $1,2,3,4,5$ \\
\hline Edo & 江戸 & Fascicules $1,3,5$ \\
\hline Emata & 夏股 & Fascicule 3 \\
\hline Esashi & 江刺 & Fascicule 5 \\
\hline Etchū & 越中 & Fascicules 2, 4 \\
\hline Europe (l'ouest de) & & Fascicule 2 \\
\hline Ezo & 蝦夷 & Fascicules 2, 5 \\
\hline
\end{tabular}




\begin{tabular}{|c|c|c|}
\hline Fuchū & 府中 & Fascicule 2 \\
\hline Fukae & 深江 & Fascicule 5 \\
\hline Fukahori ou Fukabori & 深堀 & Fascicule 5 \\
\hline Fukusaki & 福崇 & Fascicule 4 \\
\hline Fukushima & 福島 & Fascicule 4 \\
\hline Funai & 船井 & Fascicule 1 \\
\hline Fushimi & 伏見 & Fascicule 4 \\
\hline Futami & 二見 & Fascicules 3, 4 \\
\hline Fuyu (mont) & 夫餘山 & Fascicule 2 \\
\hline Gotō & 五島 & Fascicules 3,4 \\
\hline Hakone & 箱根 & Fascicule 2 \\
\hline Hakotachi & 箱館 & Fascicule 5 \\
\hline Hakozaki & 箱崎 & Fascicule 3 \\
\hline Hamajizō & 浜地蔵 & Fascicule 4 \\
\hline Hanishina & 埴科 & Fascicule 5 \\
\hline Harayama & 原山 & Fascicule 2 \\
\hline Harima & 播磨 & Fascicules 1, 2, 3, 4 \\
\hline Hida & 飛騨 & Fascicule 4 \\
\hline Hiekoba & 稗古場 & Fascicule 5 \\
\hline Higo & 肥後 & Fascicule 2 \\
\hline Himeji & 姫路 & Fascicule 1 \\
\hline Hirado (fief) & 平戸 & Fascicules $3,4,5$ \\
\hline Hirae & 平江 & Fascicule 4 \\
\hline Hirao (pierre) & 平尾 & Fascicule 2 \\
\hline Hirose & 広瀬 & Fascicule 5 \\
\hline Hiroshima & 広島 & Fascicule 3 \\
\hline Hitachi & 常陸 & Fascicules 4,5 \\
\hline
\end{tabular}




\begin{tabular}{|c|c|c|}
\hline Hizen (province) & 肥前 & Fascicules 2, 3, 5 \\
\hline Hokao & 外尾 & Fascicule 5 \\
\hline Honkōhira & 本幸平 & Fascicule 5 \\
\hline Hori no uchi & 堀の内 & Fascicule 5 \\
\hline Hoshina & 穂科 & Fascicule 5 \\
\hline Hyōgo & 兵庫 & Fascicules 1,3 \\
\hline Hyūga & 日向 & Fascicules 2, 4 \\
\hline Ibuki & 伊吹 & Fascicule 5 \\
\hline Ichinose & 一の瀬 & Fascicule 5 \\
\hline Ide & 井堤 & Fascicule 4 \\
\hline Idenokuro (pierre) & 井手黒 & Fascicule 2 \\
\hline Ieno'ura & 家の浦 & Fascicule 2 \\
\hline Ikeda & 池田 & Fascicule 1 \\
\hline Iki (province) & 壱岐 & Fascicule 5 \\
\hline Imari (port) & 伊万里 & Fascicule 5 \\
\hline Imazu & 今津 & Fascicule 1 \\
\hline Ina & 伊奈 & Fascicule 5 \\
\hline Inaba & 因幡 & Fascicule 2 \\
\hline Inagawa & 猪名川 & Fascicule 1 \\
\hline Ine & イネ & Fascicule 3 \\
\hline Inokura (pierre) & 猪倉 & Fascicule 2 \\
\hline Isayagawa & いさや川 & Fascicule 4 \\
\hline Ise & 伊勢 & Fascicules 2, 3 \\
\hline Ishibe & 石部 & Fascicule 5 \\
\hline Ishiyama & 石山 & Fascicule 2 \\
\hline Italie & & Fascicule 2 \\
\hline Itami & 伊丹 & Fascicule 1 \\
\hline
\end{tabular}




\begin{tabular}{|c|c|c|}
\hline Itsushiki (Isshiki) & 一色 & Fascicule 4 \\
\hline Iwami & 石見 & Fascicules 2, 5 \\
\hline Iwatani (pierre) & 岩谷 & Fascicule 2 \\
\hline Iwaya & 岩屋 & Fascicule 5 \\
\hline Iyatani & 弥谷 & Fascicules 2,4 \\
\hline Iyo & 伊予 & Fascicule 2 \\
\hline Izu & 伊豆 & Fascicule 4 \\
\hline Izumi (province) & 和泉 & Fascicules 2, 3, 4, 5 \\
\hline Izumi (mont) & 和泉山 & Fascicule 5 \\
\hline Izumo & 出雲 & Fascicule 2 \\
\hline Jinzugawa & 神通川 & Fascicule 4 \\
\hline Jōkeiji (pierre) & 常慶寺 & Fascicule 2 \\
\hline Ka'i & 甲斐 & Fascicule 2 \\
\hline Kaga & 加賀 & Fascicules 1, 2, 4 \\
\hline Kamakura & 鎌倉 & Fascicules 3,4 \\
\hline Kametsu & 亀津 & Fascicule 4 \\
\hline Kami-Kurokawa & 上黒川 & Fascicule 2 \\
\hline Kaminami ou Kannamigawa & 神奈備川 & Fascicule 4 \\
\hline Kamogawa & 加茂川 & Fascicules 2,4 \\
\hline Kanazawa & 金沢 & Fascicule 4 \\
\hline Kankōhira & 上幸平 & Fascicule 5 \\
\hline Kanzaki & 神崇 & Fascicule 2 \\
\hline Karatsu (pierre) & 唐津 & Fascicule 2 \\
\hline Karuizawa & 軽井沢 & Fascicule 2 \\
\hline Karutomura & かると村 & Fascicule 2 \\
\hline Kashima & 鹿島 & Fascicule 5 \\
\hline Kashiwazaki & 柏崎 & Fascicule 5 \\
\hline
\end{tabular}




\begin{tabular}{|c|c|c|}
\hline Kasuga (mont) & 春日山 & Fascicule 2 \\
\hline Kawachi & 河内 & Fascicule 2 \\
\hline Kawara & 川原 & Fascicule 2 \\
\hline Kayaganaka (pierre) & 茅が中 & Fascicule 2 \\
\hline Kazusa & 上総 & Fascicule 4 \\
\hline Keishi & 京師 & Fascicule 5 \\
\hline Kii & 紀伊 & Fascicules 2, 3, 4 \\
\hline Kinai & 紋内 & Fascicules 2, 3 \\
\hline Kinkasan ou Kinkazan & 金花山 & Fascicule 4 \\
\hline Kiseto & 吉瀬戸 & Fascicule 3 \\
\hline Kisogawa & 木曾川 & Fascicule 4 \\
\hline Kitakaze & 北風 & Fascicule 1 \\
\hline Kitanakajima & 北中島 & Fascicule 2 \\
\hline Kiyokigawara & 清河原 & Fascicule 4 \\
\hline Kogawa & 粉川 & Fascicule 1 \\
\hline Komogai & 熊川 & Fascicule 2 \\
\hline Kōmura & こう村 & Fascicule 2 \\
\hline Kōnoike & 鴻池 & Fascicule 1 \\
\hline Kotō & 江東 & Fascicules 4,5 \\
\hline Kōya & 高野 & Fascicule 2 \\
\hline Kōzuke & 上野 & Fascicule 2 \\
\hline Kumano & 熊野 & Fascicules 2, 3, 4 \\
\hline Kunimura & 国先 & Fascicule 3 \\
\hline Kunisaki & 国村 & Fascicule 3 \\
\hline Kurama & 鞍馬 & Fascicules 2, 5 \\
\hline Kurashina & 倉科 & Fascicule 5 \\
\hline Kurokawa & 黒川 & Fascicule 2 \\
\hline
\end{tabular}




\begin{tabular}{|c|c|c|}
\hline Kuromuta & 黒牟田 & Fascicule 5 \\
\hline Kusatsu & 草津 & Fascicule 3 \\
\hline Kuwana & 桑名 & Fascicule 4 \\
\hline Kyōto & 京都 & Fascicules 1,3 \\
\hline Kyūshū & 九州 & Fascicule 2 \\
\hline Marutomachi & まる戸町 & Fascicule 5 \\
\hline Maruyama & 丸山 & Fascicule 5 \\
\hline Matsumae (ville) & 松前 & Fascicules 2, 5 \\
\hline Matsura (district) & 松浦 & Fascicule 5 \\
\hline Mikage & 御影 & Fascicule 2 \\
\hline Mikawa & 三河 & Fascicules 2, 3, 4 \\
\hline Mikawachi yama & 三河内山 & Fascicule 5 \\
\hline Mikogahama (pierre) & 神子が浜 & Fascicule 2 \\
\hline Mikogahama (pierre) & 神子浜 & Fascicule 2 \\
\hline Mimasaka & 美作 & Fascicule 2 \\
\hline Min & 閩 & Fascicule 2 \\
\hline Minamikawara & 南河原 & Fascicule 5 \\
\hline Minamura (pierre) & 南村 & Fascicule 2 \\
\hline Minano gawa & みなの川 & Postface \\
\hline Mino (province) & 美濃 & Fascicules 2, 5 \\
\hline Miura & 三浦 & Fascicule 4 \\
\hline Miwa & 三輪山 & Fascicule 1 \\
\hline Miyakawa (pierre) & 宮川 & Fascicule 2 \\
\hline Miyazu & 宮津 & Fascicule 3 \\
\hline Mizuno'e & 水の江 & Fascicule 4 \\
\hline Monkuchi (pierre) & 紋口 & Fascicule 2 \\
\hline Mont Ise & 伊勢山 & Fascicule 2 \\
\hline
\end{tabular}




\begin{tabular}{|c|c|c|}
\hline Monzen (pierre) & 門前 & Fascicule 2 \\
\hline Mozuno & 百舌野 & Fascicule 2 \\
\hline Muko & 武庫 & Fascicule 2 \\
\hline Munakata & 宗像 & Fascicule 3 \\
\hline Musashi (province) & 武蔵 & Fascicules $3,4,5$ \\
\hline Mutsu & 陸奥 & Fascicules 2, 3, 4, 5 \\
\hline Mutsuta ou Muda & 六田 & Fascicule 4 \\
\hline Nada & 灘 & Fascicule 1 \\
\hline Nagahama & 長浜 & Fascicule 4 \\
\hline Nagahara & 長原 & Fascicule 5 \\
\hline Nagasaki & 長崎 & Fascicule 5 \\
\hline Nagasu & 長渚 & Fascicule 4 \\
\hline Nagatani (pierre) & 長谷 & Fascicule 2 \\
\hline Nagato & 長門 & Fascicule 5 \\
\hline Nakamura (pierre) & 中村 & Fascicule 2 \\
\hline Nakanohara & 中野原 & Fascicule 5 \\
\hline Nakataru & 中樽 & Fascicule 5 \\
\hline Nakura (pierre) & 名倉 & Fascicule 2 \\
\hline Namase & 生瀬 & Fascicule 2 \\
\hline Namerikawa & 滑川 & Fascicule 4 \\
\hline Nanbu & 南部 & Fascicules 4,5 \\
\hline Naniwa & 浪花 & Fascicule 4 \\
\hline Nankin & なんき & Fascicule 5 \\
\hline Nara & 奈良 & Fascicules $1,3,5$ \\
\hline Nara, Nanto & 南都 & Fascicule 2 \\
\hline Narutaki (pierre) & 鳴滝 & Fascicule 2 \\
\hline Nebukawa & 袮ぶ川 & Fascicule 2 \\
\hline
\end{tabular}




\begin{tabular}{|c|c|c|}
\hline Nishina & 仁科 & Fascicule 5 \\
\hline Nishinomiya & 西宮 & Fascicules 1,4 \\
\hline Nishito & 西戸 & Fascicule 5 \\
\hline Nomo & 野茂（野母） & Fascicule 5 \\
\hline Noto & 能登 & Fascicules 3,4 \\
\hline Obama & 小浜 & Fascicule 5 \\
\hline Ōbuchi (pierre) & 大淵 & Fascicule 2 \\
\hline Ochiai & 落合 & Fascicule 4 \\
\hline Odawara & 小田原 & Fascicule 4 \\
\hline Ogawa & 小川 & Fascicule 1 \\
\hline Ōgitani (pierre) & 扇谷 & Fascicule 2 \\
\hline Ōhara & 大原 & Fascicule 4 \\
\hline Ōhōyama & 應法山 & Fascicule 5 \\
\hline Ojiya & 小千谷 & Fascicule 5 \\
\hline Okamoto & 岡本 & Fascicule 4 \\
\hline ōkawachi yama & 大河内山 & Fascicule 5 \\
\hline Omashi & 御座 & Fascicule 3 \\
\hline Ōmi & 近江 & Fascicules 2, 3, 4, 5 \\
\hline Ōmura & 大村 & Fascicules 3,4 \\
\hline Ōno & 大野 & Fascicule 3 \\
\hline Ōsaka & 大阪 & Fascicules 1, 2, 3, 5 \\
\hline Ōsawa & 大沢 & Fascicule 2 \\
\hline Oshamanbe & オシャマンベ & Fascicule 5 \\
\hline Ōsuesumi & 大陶祗 & Fascicule 5 \\
\hline Ōtaru & 大樽 & Fascicule 5 \\
\hline Ōtsume & 大爪 & Fascicule 2 \\
\hline Owari & 尾張 & Fascicules 1, 2, 3, 4 \\
\hline
\end{tabular}




\begin{tabular}{|c|c|c|}
\hline Oyamada & 小山田 & Fascicule 2 \\
\hline Ōzu & 大洲 & Fascicule 4 \\
\hline Paekche & 百済 & Fascicule 2 \\
\hline Pékin & 北京 & Fascicule 5 \\
\hline Penglai & 蓬躚 & Postface \\
\hline Saga & 嵯峨 & Fascicule 2 \\
\hline Saga (fief) & 佐賀 & Fascicule 5 \\
\hline Sagami & 相模 & Fascicules 1, 2, 4 \\
\hline Sahogawa & 佐保川 & Fascicule 4 \\
\hline Saiki (pierre) & 佐伯 & Fascicule 2 \\
\hline Sakai & 堺 & Fascicule 5 \\
\hline Saku, (île de) & 柵の島, 佐久 & Fascicule 4 \\
\hline San'indō & 山陰道 & Fascicule 2 \\
\hline Sanctuaire de pierre Ishi no hōden & 石の宝殿 & Fascicule 2 \\
\hline Sanjūsangendō & 三十三間堂 & Fascicule 4 \\
\hline Sanuki & 讃岐 & Fascicules 2, 3, 4 \\
\hline Sarachiyama & 更地山 & Fascicule 5 \\
\hline Sarashina & 更科 & Fascicule 5 \\
\hline Satsuma & 薩摩 & Fascicules 2,4 \\
\hline Satsuma-Shimazu & 薩摩島津 & Fascicule 4 \\
\hline Sayo (montagne) & 小夜 & Fascicule 2 \\
\hline Seikanji & 清閑寺 & Fascicule 2 \\
\hline Senju & 千手 & Fascicule 5 \\
\hline Settsu & 摂津 & Fascicules 2, 4 \\
\hline Shang (les quatre vieillards chenus du mont) & 商山 & Fascicule 2 \\
\hline Shikazaki & 鹿埼 & Fascicule 2 \\
\hline Shikoku & 四国 & Fascicules 2, 4 \\
\hline
\end{tabular}




\begin{tabular}{|c|c|c|}
\hline Shima & 志摩 & Fascicule 3,4 \\
\hline Shimashimo (district) & 島下 & Fascicule 2 \\
\hline Shimazu & 島津 & Fascicule 4 \\
\hline Shimizu & 清水 & Fascicule 4 \\
\hline Shimōsa & 下総 & Fascicule 5 \\
\hline Shimotani & 下谷 & Fascicule 5 \\
\hline Shimotsuke & 下野 & Fascicules 2, 5 \\
\hline Shinano & 信濃 & Fascicules 2, 5 \\
\hline Shinobu & しのぶ & Fascicule 2 \\
\hline Shinshū (Shinano) & 信州 (信濃) & Fascicule 4 \\
\hline Shintōgawa/Jinzugawa & 神道川 & Fascicule 4 \\
\hline Shioichi & 汐市 & Fascicule 2 \\
\hline Shiozawamachi & 塩沢町 & Fascicule 5 \\
\hline Shirakawa & 白川 & Fascicules 2, 5 \\
\hline Shire (pierre) & 白谷 & Fascicule 2 \\
\hline Shiro'uma (pierre) & 白馬 & Fascicule 2 \\
\hline Shōdo, (île de) & 小豆島 & Fascicules 2, 4 \\
\hline Shouyang (Mont) & 首陽 & Fascicule 2 \\
\hline Somata (pierre) & 杣田 & Fascicule 2 \\
\hline Sōya & 宗谷 & Fascicule 5 \\
\hline Sumiyoshi & 住吉 & Fascicules 2,4 \\
\hline Suruga & 駿河 & Fascicules $1,2,4$ \\
\hline Suwa-no-umi & 諏訪の海 & \\
\hline Kami-Suwa & 上諏訪 & Fascicule 4 \\
\hline Shimo-Suwa & 下諏訪 & \\
\hline Tajima & 但馬 & Fascicules 2, 3, 4 \\
\hline Takaa'i & 鷹合 & Fascicule 2 \\
\hline Taka'i (village) Taka'i no mura & 鷹甘邑 & Fascicule 2 \\
\hline
\end{tabular}




\begin{tabular}{|c|c|c|}
\hline Takagamine & 鷹が峯 & Fascicule 2 \\
\hline Takahara & 高原 & Fascicule 5 \\
\hline Takai & 高井 & Fascicule 5 \\
\hline Takao & 高尾 & Fascicule 2 \\
\hline Takasago & 高砂 & Fascicule 4 \\
\hline Takatsuki & 高槻 & Fascicule 1 \\
\hline Tamakina & 玉杵 & Fascicule 4 \\
\hline Tamanoura & 玉之浦 & Fascicule 5 \\
\hline Tanagura & 棚倉 & Fascicule 4 \\
\hline Tanakami & 田上 & Fascicule 4 \\
\hline Tanba & 丹波 & Fascicules $1,2,3$ \\
\hline Tango & 丹後 & Fascicules 1, 2, 3 \\
\hline Tatsugahashi & 龍ヶ端 & Fascicule 2 \\
\hline Tatsuyama & 立山 & Fascicule 2 \\
\hline Tatsuyama & 龍山 & Fascicule 2 \\
\hline Tengu (Pic du) Tengumine & 天狗峰 & Fascicule 2 \\
\hline Tennōji & 天王寺 & Fascicule 2 \\
\hline Teshima & 豊島 & Fascicule 2 \\
\hline Tōbu & 東武 & Fascicule 4 \\
\hline Tōjinyashiki & 唐人屋敷 & Fascicule 5 \\
\hline Tōkamachi & 十日町 & Fascicule 5 \\
\hline Tokkai & 年遣 & Fascicule 2 \\
\hline Tokonoyama & 床山 & Fascicule 4 \\
\hline Tomisawa & 富沢 & Fascicule 2 \\
\hline Tomita & 富田 & Fascicule 4 \\
\hline Torigai & 鳥飼 & Fascicule 2 \\
\hline Tosa & 土佐 & Fascicules 2, 4 \\
\hline
\end{tabular}




\begin{tabular}{|c|c|c|}
\hline Tosawa (pierre) & 戸沢 & Fascicule 2 \\
\hline Tōse & 塔世 & Fascicule 3 \\
\hline Tōshi (île) & 答志 & Fascicule 3 \\
\hline Tōtōmi & 遠江 & Fascicule 4 \\
\hline Toyama & 富山 & Fascicule 4 \\
\hline Toyota & 豊田 & Fascicule 4 \\
\hline Tsugaru & 津軽 & Fascicules 2, 5 \\
\hline Tsuruga & 敦賀 & Fascicules $1,3,5$ \\
\hline Tsukushi & 筑紫 & Fascicule 1 \\
\hline Tsushima & 対馬 & Fascicule 2 \\
\hline Tsuzumigataki & 鼓ヶ滝 & Fascicule 4 \\
\hline Ubara (Mubara) & 菟原 & Fascicule 2 \\
\hline Ujigawa & 宇治川 & Fascicule 4 \\
\hline Umegashima & 梅が島 & Fascicule 5 \\
\hline Uranoshima & 浦島 & Fascicule 4 \\
\hline Utsunomiya & 宇都宮 & Fascicule 2 \\
\hline Wada & 和田 & Fascicules 2, 4 \\
\hline Waka & 和歌 & Fascicule 3 \\
\hline Wakasa & 若狭 & Fascicules $3,4,5$ \\
\hline Wagu & 和具 & Fascicule 3 \\
\hline Yamakata & 山方 & Fascicule 1 \\
\hline Yamashiro & 山城 & Fascicules $2,4,5$ \\
\hline Yamata & 矢俣 & Fascicule 2 \\
\hline Yamato & 大和 & Fascicules $1,2,5$ \\
\hline Yangquan & 陽泉 & Fascicule 2 \\
\hline Yase & 八瀬 & Fascicule 4 \\
\hline Yodo & 淀 & Fascicule 1 \\
\hline
\end{tabular}




\begin{tabular}{|l|l|l|}
\hline Yokomakura & 横枕 & Fascicule 4 \\
\hline Yoriai & 寄合 & Fascicule 5 \\
\hline Yosa & 与謝 & Fascicule 3 \\
\hline Yosami (domaine royal de) & 依羅 & Fascicule 2 \\
\hline Yoshino & 吉野 & Fascicule 2 \\
\hline Yoshino & 芳野 & Fascicule 5 \\
\hline Yoshinogawa & 吉野川 & Fascicule 4 \\
\hline Yufune (pierre) & 湯船 & Fascicule 2 \\
\hline Zentsūji & 善通寺 & Fascicule 2 \\
\hline
\end{tabular}

